\title{
Hanford Site Ground-Water Monitoring for April Through June 1987
}

\author{
J. C. Evans \\ P. J. Mitchell \\ D. I. Dennison
}

January 1988

Prepared for the U.S. Department of Energy under Contract DE-AC06-76RLO 1830

Pacific Northwest Laboratory

Operated for the U.S. Department of Energy by Battelle Memorial Institute 


\title{
DISCLAIMER
}

This report was prepared as an account of work sponsored by an agency of the United States Government. Neither the United States Government nor any agency thereof, nor Battelle Memorial Institute, nor any of their employees, makes any warranty, expressed or implied, or assumes any legal liability or responsibility for the accuracy, completeness, or usefulness of any information, apparatus, product, or process disclosed, or represents that its use would not infringe privately owned rights. Reference herein to any specific commercial product, process, or service by trade name, trademark, manufacturer, or otherwise, does not necessarily constitute or imply its endorsement, recommendation, or favoring by the United States Government of any agency thereof, or Battelle Memorial Institute. The views and opinions of authors expressed herein do not necessarly state or reflect those of the United States Government or any agency thereof, or Battelle Memorial Institute.

\author{
PACIFIC NORTHWEST LABORATORY \\ operated by \\ BATTELLE MEMORIAL INSTITUTE \\ for the \\ UNITED STATES DEPARTMENT OF ENERGY \\ under Contract DE-AC06-76RLO 1830
}

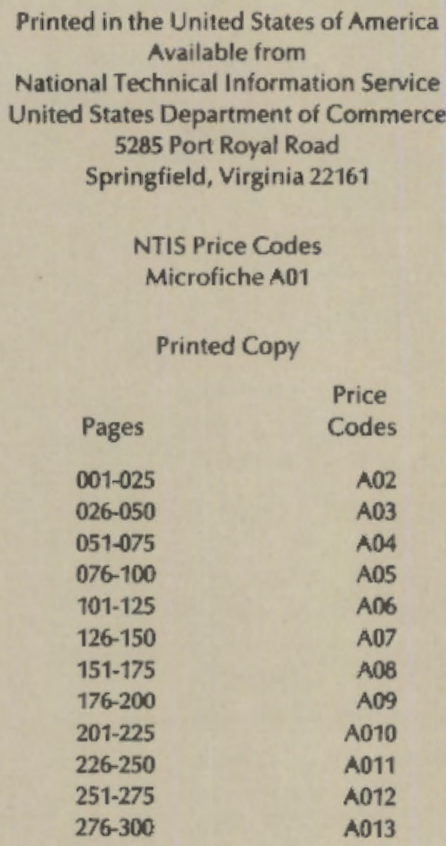



J. C. Evans
P. J. Mitchell
D. I. Dennison

January 1988

Prepared for

the U.S. Department of Energy

under Contract DE-AC06-76RLO 1830

Pacific Northwest Laboratory

Richland, Washington 99352 


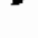




\section{SUMA-ARY}

In a continuing effort for the U.S. Department of Energy (DOE), Pacific Northwest Laboratory (PNL) is conducting ground-water monitoring at the Hanford Site. Results for monitoring by PNL and Westinghouse Hanford Company (WHC) during April-June 1987 show that certain regulated hazardous materials and radionuclides exist in Hanford Site ground waters. The presence of regulated constituents in the ground water derives both from site operations and from natural sources (for some constituents). The major contamination problems defined by recent monitoring activities are 1) carbon tetrachloride in the 200 West Area; 2) cyanide in and north of the 200 East Area; 3) hexavalent chromium contamination in the 100B, $100 \mathrm{D}, 100 \mathrm{~K}$, and $100 \mathrm{H}$ Areas;

4) chlorinated hydrocarbons in the vicinity of the Central Landfi11;

5) uranium at the 216-U-1 and 216-U-2 cribs in the 200 West Area; 6) tritium across the site; and 7) nitrate across the site. The distribution of hazardous materials related to site operations is more limited than the distribution of tritium and nitrate. 



\section{ACKNOWLEDGMENTS}

The authors wish to thank D. R. Sherwood and A. G. Law (WHC) for carefully reviewing this report, D. J. Bates for preparation of tables and report review, and S. B. Moore for map preparation. 
1 


\section{CONTENTS}

SUMMARY ............................... i

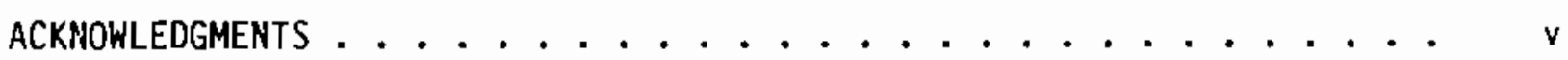

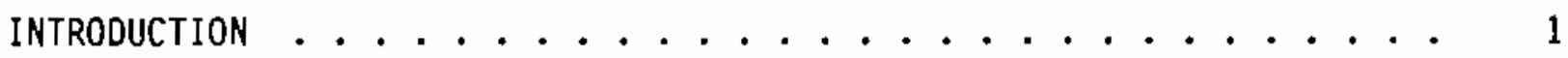

SAMPLING AND ANALYSIS .................... 5

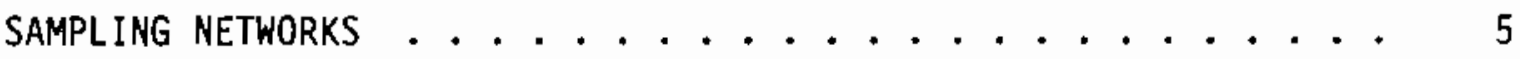

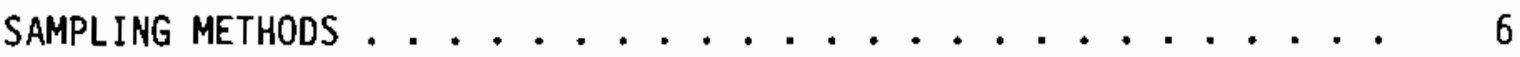

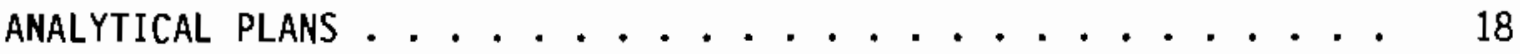

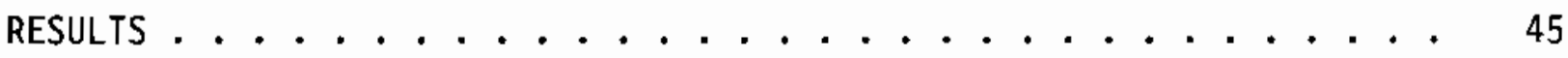

CARBON TETRACHLORIDE IN THE 200 WEST AREA ......... 45

CYANIDE IN AND NORTH OF THE 200 EAST AREA ......... 47

HEXAVALENT CHROMIUM IN THE 100 AREAS . . . . . . . . . 49

CHLORINATED HYDROCARBONS NEAR THE CENTRAL LANDFILL . . . . . 50

URANIUM AT THE 216-U-1 AND 216-U-2 CRIBS IN THE 200 WEST AREA . . 51

TRITIUM ACROSS THE SITE .................... 51

Tritium Monitoring Results for $1986 \ldots . . . . . .51$

Tritium Monitoring Results During April through June 1987 . . 52

NITRATE ACROSS THE SITE .................... 55

Nitrate Monitoring Results for $1986 \ldots . . . . . . .56$

Nitrate Monitoring Results During April through June 1987 . . 56

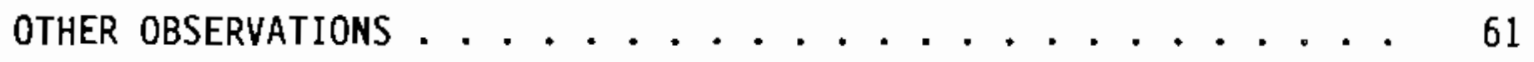

Acetone ........................ 61

Amnonia ......................... 61

Hexavalent Chromium in the 200 west and 600 Areas ..... 61

Fluoride ..................... 62

Chlorinated Hydrocarbons near the 300 Area ...... 62 
Methyl Ethyl Ketone ............... 62

Methylene Chloride ............... 62

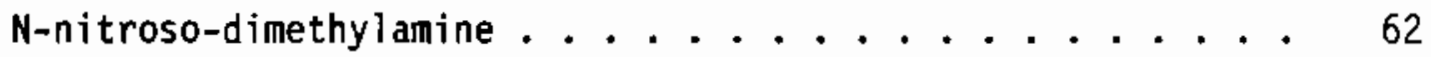

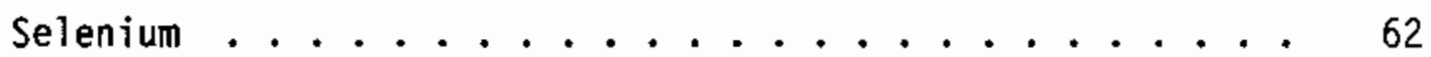

Total Organic Halogens .............. 63

Cyanide in the 200 West Area ........... 63

Gross Alpha Activity, Uranium, and Plutonium ...... 63

Gross Beta Activity. . . . . . . . . . . 66

Strontium-90 ....................... 68

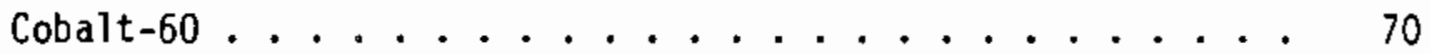

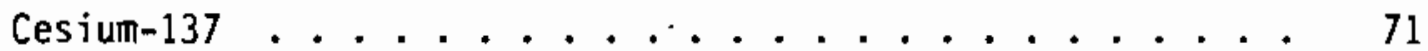

Ruthenium-106 . . . . . . . . . . . . 72

Antimony-125 ...................... 72

Technetium-99 . . . . . . . . . . . . . 72

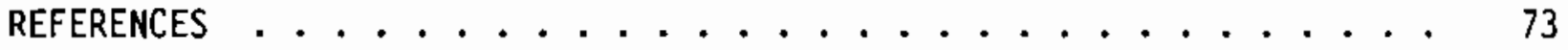

APPENDIX A - DATA LISTINGS . . . . . . . . . . . A.I

APPENDIX B - DRINKING WATER STANDARDS AND DERIVED
CONCENTRATION GUIDES ......... B.1 


\section{FIGURES}

1 Location of the Hanford Site .............

2 Location of Site-Wide Ground-Water Monitoring Wells

Sampled During April-June 1987 ........... 7

3 Location of Ground-Water Monitoring Wells in the $100 \mathrm{H}$

Area Sampled During April-June 1987 ........... 9

4 Location of Ground-Water Monitoring Wells in the $100 \mathrm{~N}$ Area

Sampled During April-June 1987 ........... 10

5 Location of Ground-Water Monitoring Wells in the 200 East

Area Sampled During April-June 1987 ............ 11

6 Location of Ground-Water Monitoring Wells in the 200 West

Area Sampled During April-June 1987 . . . . . . . . . . 13

7 Location of Ground-Water Monitoring Wells in the 300 Area

Sampled During April-June 1987 . . . . . . . . 15

8 Water-Table Elevations for the Unconfined Aquifer

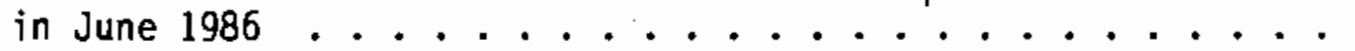

9 Tritium Concentrations in Wells $1-\mathrm{N}-2,1-\mathrm{N}-3,1-\mathrm{N}-4$,

$1-\mathrm{N}-5$, and $1-\mathrm{N}-14$, in 1986 and the First Half of $1987 \ldots \ldots$

10 Tritium Concentrations in wells 6-26-15A, 6-27-8,

6-31-31, and 6-32-22, in 1986 and the First Half of $1987 \ldots 54$

11 Nitrate Concentrations in Wells 1-H4-4, 1-H4-9,

1-H4-12A, and 1-H4-12B, in 1986 and the First Half of $1987 \ldots$

12 Nitrate Concentrations in Well 1-H3-1, in 1986 and the First Half of $1987 \ldots \ldots$. . . . . . . . . 58

13 Nitrate Concentrations in Wells $1-\mathrm{N}-14,1-\mathrm{N}-23,1-\mathrm{N}-36$, 1-N-37, and 1-N-45, August 1986 Through June $1987 \ldots \ldots$

14 Nitrate Concentrations in Wells 6-32-43, 6-33-42, and 6-37-43, July 1986 Through June 1987 .......... 60

15 Uranium Concentrations in Wells 2-W19-3, 2-W19-9, 2-W19-11, 2-W19-15, and 2-W19-16 for the First Haif of $1987 \ldots \ldots$. . .

16 Alpha Concentrations in Wells 2-W19-3, 2-W19-9, 2-W19-11, 2-W19-15, and 2-W19-16 for the First Half of 1987 . . . . . .

17 Uranium Concentrations in Wells 2-W19-17, 2-W19-18, and 2-W19-19

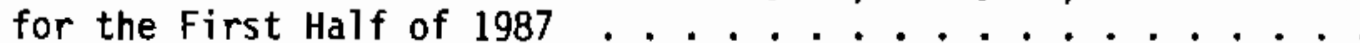


18 Alpha Concentrations in Wells 2-w19-17, 2-w19-18, and 2-W19-19

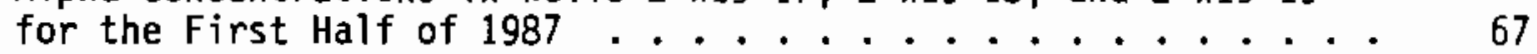

19 Uranium Concentrations in Well 1-F8-1 for the First Half of $1987 \quad 68$

20 Strontium-90 Concentrations in wells 6-53-48B, 6-54-48, and 6-54-49 for the First Half of $1987 \ldots . . . . . . .696$

21 Cobalt-60 Concentrations in Wells 1-N-4, 1-N-31, 1-N-32, and $1-\mathrm{N}-33$ for 1986 and the First Half of $1987 \ldots 70$

22 Cobalt-60 Concentrations in Wells $1-\mathrm{N}-27,1-\mathrm{N}-30,1-\mathrm{N}-37$, and $1-\mathrm{N}-39$ for 1986 and the First Half of $1987 \ldots . . . . . .271$ 


\section{TABLES}

1 Key to Constituent and Constituent Group Names Used in

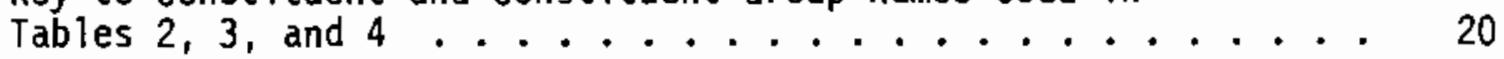

2 Analytical Plan for Site-Wide Chemical Monitoring Wells . . . . 30

3 Analytical Plan for Compliance Monitoring Wells . . . . . . . . 34

4 Analytical Plan for Site-Wide Radiological (plus Nitrate)

Monitoring Wells Not in the Site-Wide Chemical or Compliance

Monitoring Networks ................ . . 37

5 Estimated Background Levels for Selected Constituents

in Hanford Ground Water . . . . . . . . . . . . . . . . 46 



\section{INTRODUCTION}

The ground water on the Hanford Site in southeastern Washington State (Figure 1) is sampled to monitor the distribution of radionuclides and other hazardous materials and to evaluate the impact of past and present site operations on the environment. The Pacific Northwest Laboratory (PNL) program monitors contaminants in the ground water and their migration to other pathways (e.g., the Columbia River). The monitoring well network is designed to meet the intent of U.S. Department of Energy (DOE) Orders 5480.1 and 5484.1, which are applicable to environmental monitoring. DOE Order 5480.1, Chapter XI, lists the permissible concentrations of radionuclides applicable to the discharge of liquid effluent in controlled and uncontrolled areas. This order also requires that the environment be monitored sufficiently to ensure that the radiation doses the public is exposed to are as low as reasonably achievable (ALARA). OOE Order 5480.1, Chapter XII, requires that DOE cooperate with the U.S. Environmental Protection Agency (EPA) and state, interstate, and local agencies in the prevention, control, and abatement of environmental pollution. Annual reports, e.g., Environmental Monitoring at Hanford for 1986 (PNL 1987), are published by PNL to document all surface and subsurface monitoring activities at the site, as well as measured and calculated doses to the public.

Results from 1986 monitoring by PNL indicated that tritium, nitrate, and certain mobile radionuclides released to the ground-water system continued to migrate slowly, i.e., over tens of years, toward the Columbia River (PNL. 1987). Plumes of some of these constituents emanate from operating areas within the Hanford Site and enter the river through springs and the river bed at downgradient locations.

The objectives of site-wide chemical and radiological monitoring are to 1) determine the distribution of radionuclides and nitrate ion to define the extent of impacted ground water; 2) relate the distribution of these constituents to site operations; 3 ) establish background concentrations for naturally occurring regulated hazardous materials; and 4) identify hazardous chemicals in the ground water that resulted from site operations. 


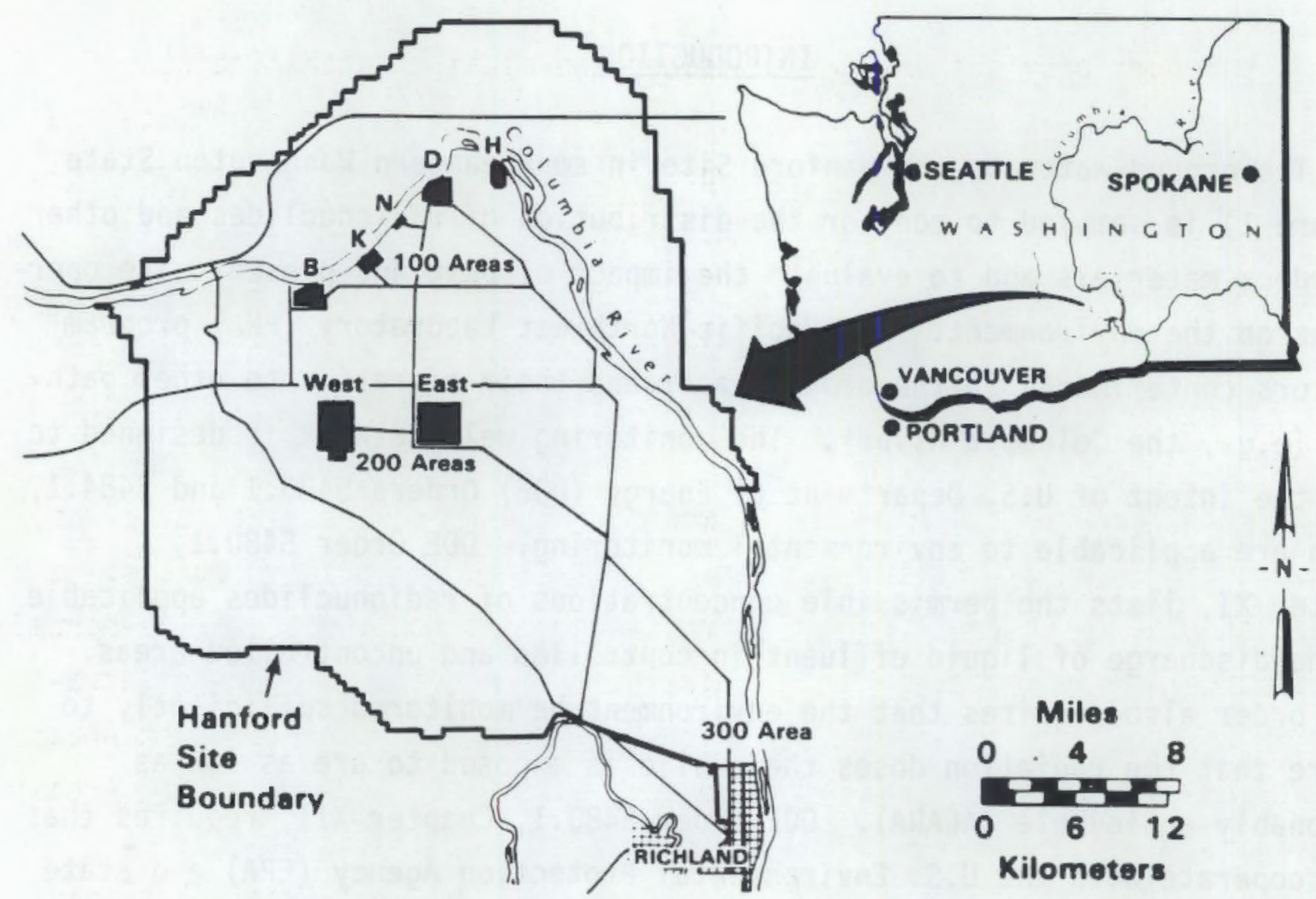

FIGURE 1. Location of the Hanford Site

Other ground-water monitoring activities at the Hanford Site are also being conducted by PNL or Westinghouse Hanford Company (WHC) for compliance with the Resource Conservation and Recovery Act (RCRA) and/or Washington Administrative Code (WAC) 173-303. These activities include sampling programs at the 183-H Solar Evaporation Basins in the 100H Area, Transportable Grout Facility, 300-Area Process Trenches, Solid Waste Landfill (SWL), and Non-Radioactive Dangerous Waste Landfill (NRDW). (The SWL and the NRDW together will be referred to as the Central Landfill.) The results of these studies are discussed briefly in this report and will be reported later in more detail elsewhere. These compliance monitoring results (primarily for chemicals) are valuable in determining the total impact of Hanford Site operations on ground water. Most of the radiological results included in Appendix A were provided by PNL's site-wide or WHC's operational (in and around the 200 Areas) monitoring programs. 
This report provides brief discussions and detailed data listings of results for ground-water monitoring at the Hanford Site during April through June of 1987. More detailed discussions of the hydrology and geology of the site, operational activities, sampling, analysis, and distributions of average constituent concentrations during 1986 are included in the most recent annual report by PNL (1987). Also, Law, Serkowski, and Schatz (1987) have reported 1986 radiological monitoring results for the 200 Areas and some of the surrounding 600 Area.

The following text describes the monitoring networks, analytical plans, and monitoring results for all Hanford ground-water monitoring programs. Because of their high concentrations, regulatory implications, or wide-spread distribution, seven contaminants are discussed in detail: 1) carbon tetrachloride in the 200 West Area; 2) cyanide in and north of the 200 East Area; 3) hexavalent chromium contamination in the $100 \mathrm{~B}, 100 \mathrm{D}, 100 \mathrm{~K}$, and $100 \mathrm{H}$ Areas; 4) chlorinated hydrocarbons in the vicinity of the Central Landfill; 5) uranium at the $216-\mathrm{U}-1$ and $216-\mathrm{U}-2$ cribs in the 200 West Area; 6) tritium across the site; and 7) nitrate across the site. The potential implications of several other observations are also discussed briefly. Appendix A includes all results reported above detection levels for chemicals, and those radiochemical results for which the result is larger than the counting error. The three large tables in Appendix A contain all available chemical and radiological results for 1 ) site-wide chemical monitoring; 2) site-specific monitoring for compliance with RCRA and/or WAC; and 3) site-wide radiological and nitrate monitoring for wells not included in the first two tables. Both PNL's and WHC's results are included in all tables. 


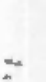

$=$ 


\section{SAMPLING AND ANALYSIS}

\section{SAMPLING NETWORKS}

The ground-water monitoring networks at the Hanford Site include both site-wide and site-specific networks. The current PNL site-wide radiological sampling network consists of 391 wells, 316 of which were sampled this quarter. In the 200 Areas and surrounding 600 Area, PNL sampled 153 wells so that the Operating Contractor (WHC) could monitor site-specific waste disposal. Although an overlap in wells exists between the two networks, each program analyzed the well samples for different constituents. In general, PNL complemented WHC's site-specific radiological monitoring near active and inactive waste-disposal sites by including those constituents that could possibly migrate from other inactive waste facilities.

A subset of both the PNL and WHC radiological networks is used for sitewide chemical sampling by PNL. Over 100 chemical sampling wells were selected primarily for their proximity to known active and inactive chemical disposal areas in the 100,200, and 600 Areas and on the basis of the waste inventories compiled in the report titled "Draft Phase I Installation Assessment of Inactive Waste Disposal at Hanford."(a) Areas covered by RCRA compliance monitoring, such as the 300 Area and the $100 \mathrm{H}$ Area, were not included in the site-wide chemical monitoring network, although their monitoring results are included in the data listings in Appendix A. Only wells containing submersible pumps were chosen to allow sufficient purging of wells prior to sampling. (It is not physically feasible to equip all sampling wells in the 200 Areas with pumps.) Nineteen more wells were sampled in conjunction with a DOE Headquarters Environmental Audit Team (DOE/HQ-EAT) during June 1987.

The site-wide monitoring network is shown in Figure 2. Detailed maps of site-specific monitoring well networks for the $100 \mathrm{H}, 100 \mathrm{~N}, 200$ East, 200 West and 300 Areas are included in Figures 3, 4, 5, 6, and 7, respectively. Those wells used for site-wide chemical or compliance sampling are noted in each figure. Most wells sample the unconfined or shallow aquifer; wells that
(a)
U.S. Department of Energy, 1986, Richland, Washington. 
are constructed to sample from confined aquifers are noted in all figures. Water table elevations for the unconfined aquifer in June 1986 are shown in Figure 8 . The general directions of ground-water flow can be determined from Figure 8 by drawing flow lines perpendicular to the equipotential contours and moving from high to low contours.

\section{SAMPLING METHODS}

Samples from the unconfined aquifer were collected in wells that are typically screened between 3 and $6 \mathrm{~m}$ (10 and $20 \mathrm{ft}$ ) below the water table. This sampling approach has been justified because maximum concentrations for some radionuclides were measured near the top of the aquifer at a few Hanford Site locations in a study by Eddy, Myers, and Raymond (1978).

Wells fitted with submersible pumps $(0.63 \mathrm{~L} / \mathrm{s}$ or $10 \mathrm{gal} / \mathrm{min})$ were sampled after pumping for a long enough time (at least $20 \mathrm{~min}$ ) to allow temperature, $\mathrm{pH}$, and specific conductivity to equilibrate. This purging process ensures that any standing water in the well has been removed, allowing collection of a sample that is representative of the ground water near the well. A stainless steel sampling tee was then connected to the pump discharge line. One side of the tee consisted of a $0.476-\mathrm{cm}(3 / 16-\mathrm{in}$. critical orifice discharging to a $0.635-\mathrm{cm}(1 / 4-i n$.$) Teflon sampling line.$ Excess water was discharged through a ball valve on the other branch of the tee. This arrangement allowed the pump discharge to be throttled back sufficiently to provide a continuous water column, while providing some pressure relief to avoid damage to the header pipe. Samples for volatile organic analyses were taken with zero head space and sealed immediately with a septum-sealed cap. For filtered trace metals, a disposable, 0.45-micron pore-sized filter pack was connected to the Teflon sampling line. The filter was prepurged with $500 \mathrm{ml}(0.13 \mathrm{gal})$ of well water and then a sample was collected in a plastic bottle. Trace metal samples and some radiochemical samples were preserved by acidification at the time of collection. All samples were placed in numbered ice chests immediately after sampling and

- Teflon is the registered tradename for a fluorocarbon resin product of E. I. DuPont de Nemours and company, Wilmington, Delaware. 


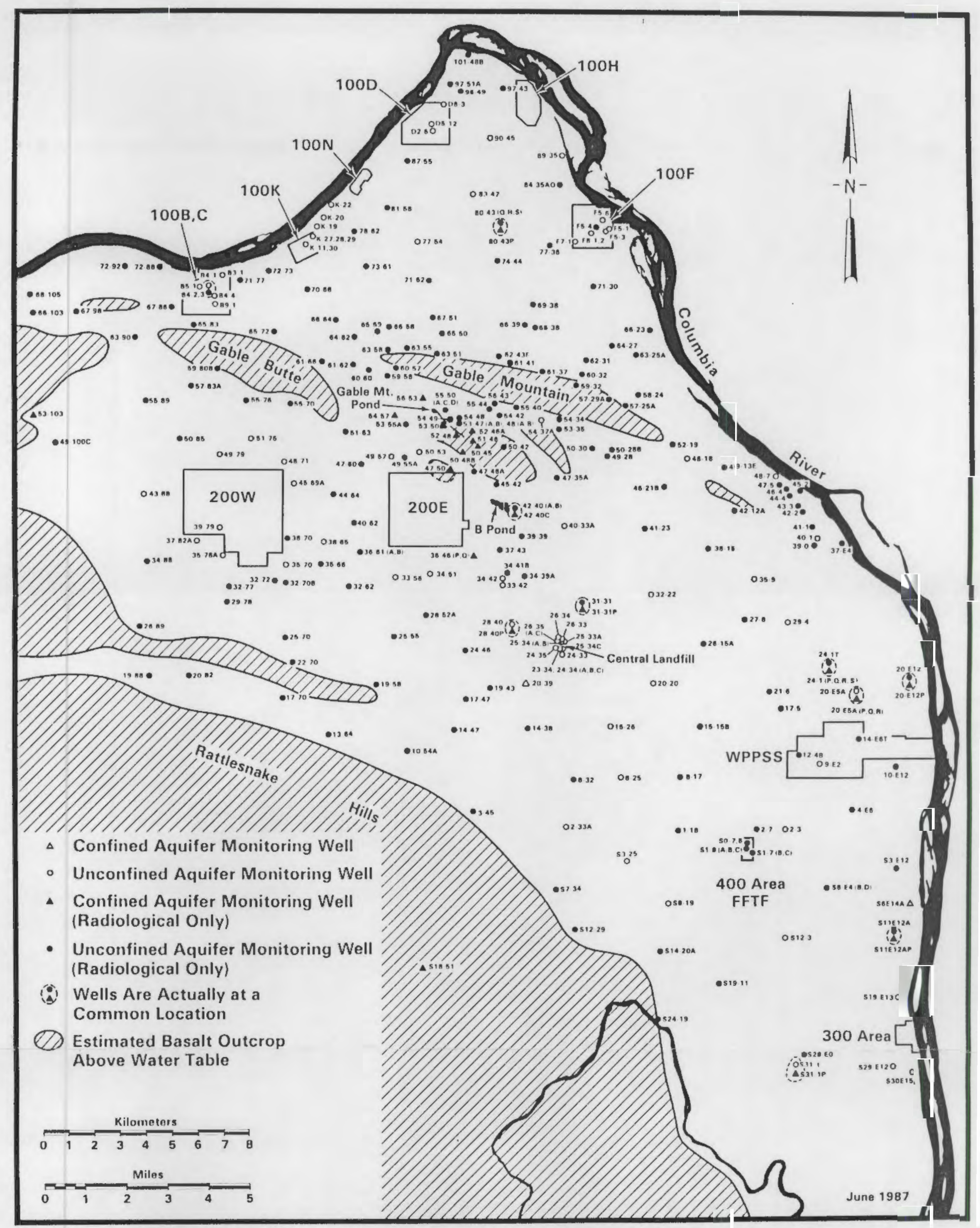

FIGURE 2. Location of Site-Wide Ground-Water Monitoring Wells Sampled During April-June 1987 (see Figures 2-6 for wells in the 100H, 100N, 200 East, 200 West and 300 Areas) 


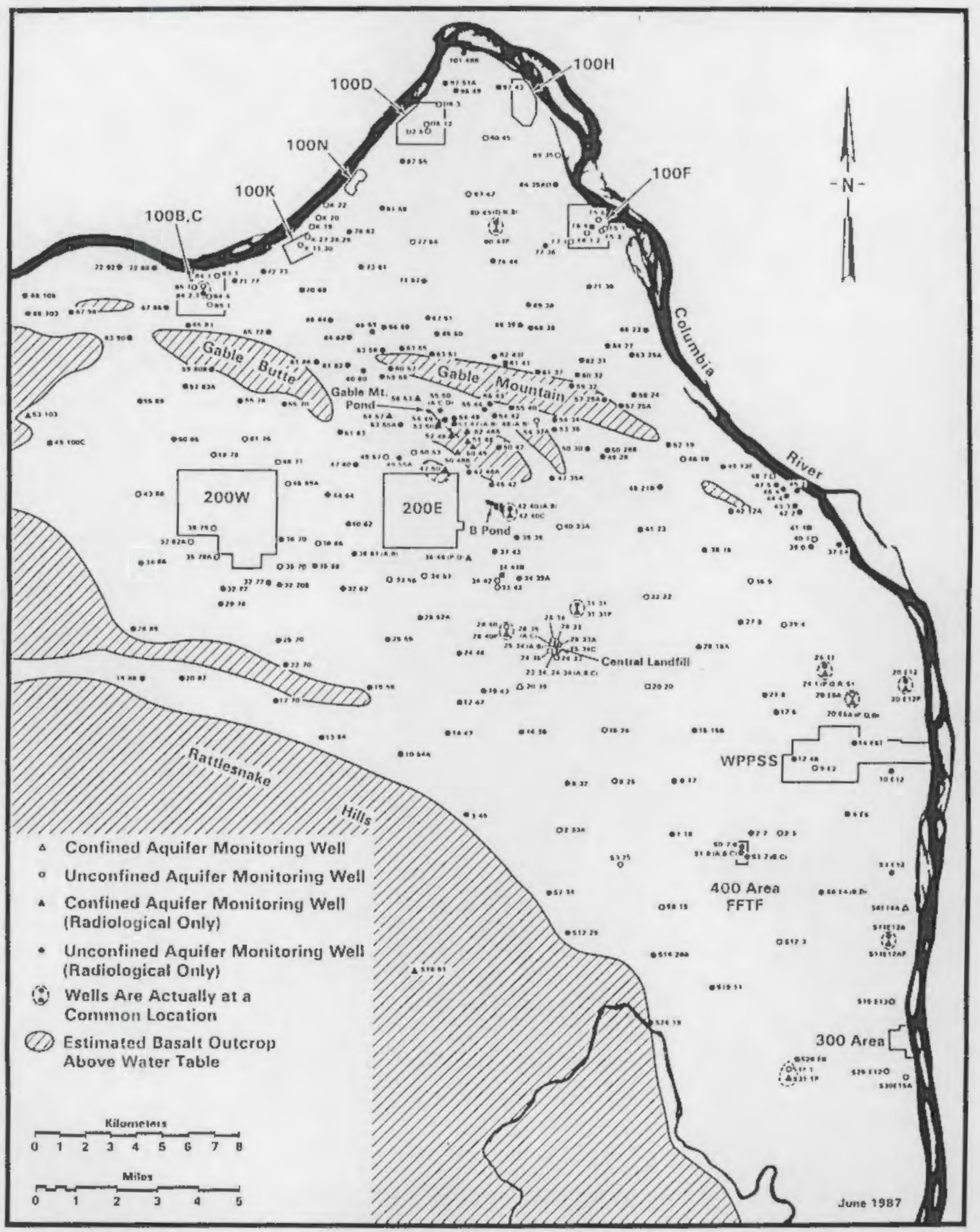

FIGURE 2. Location of Site-Wide Ground-Water Monitoring Wells Sampled During Apri)-June 1987 (see Figures 2-6 for wells in the 100H, 1004, 200 East, 200 hest and 300 Areas) 


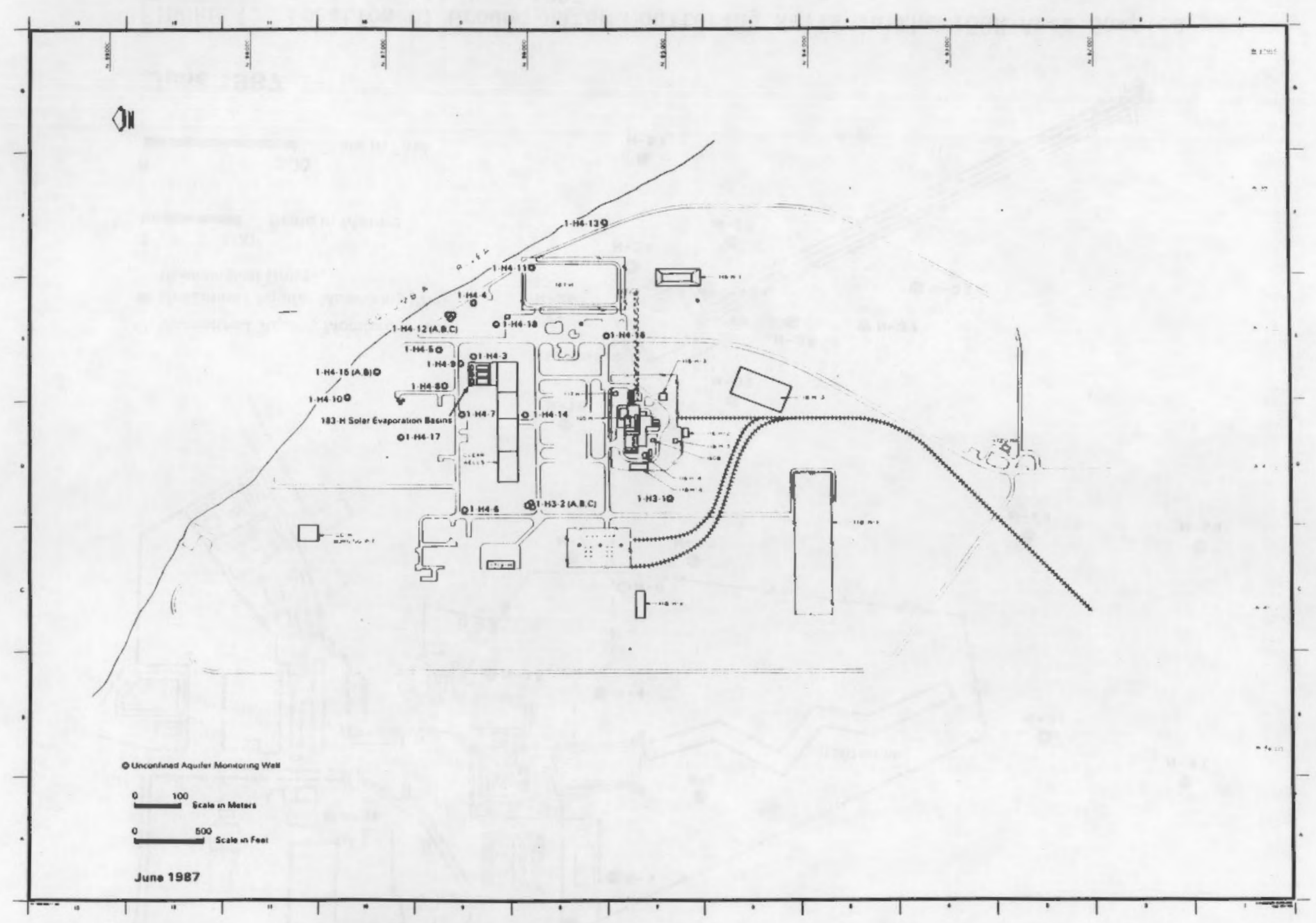

FIGURE 3. Location of Ground-Water Monitoring Wells in the $100 \mathrm{H}$ Area Sampled During April-June 1987 


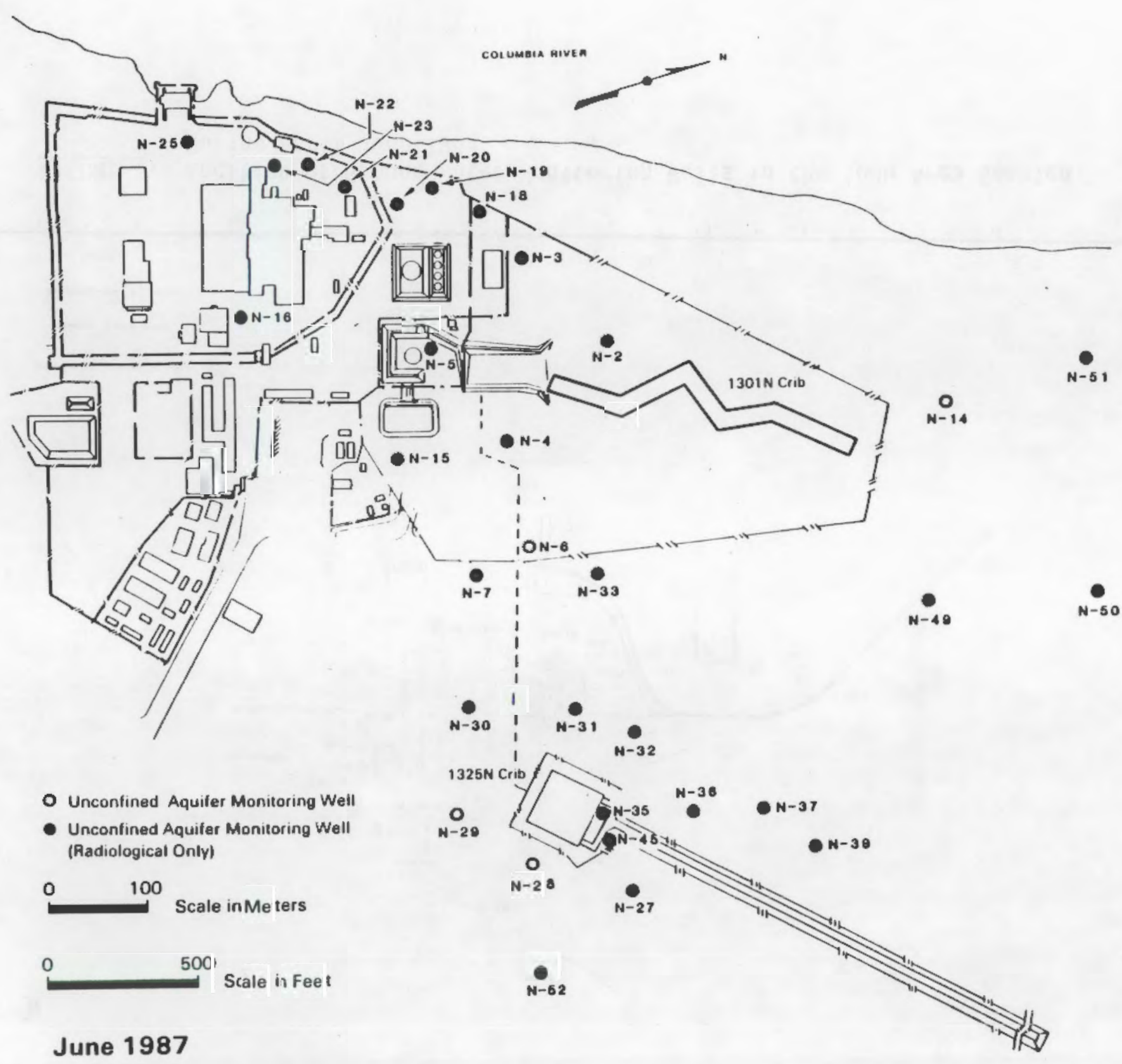

FIGURE 4. Location of Ground-Water Monitoring Wells in the 100N Area Sampled During April-June 1987 


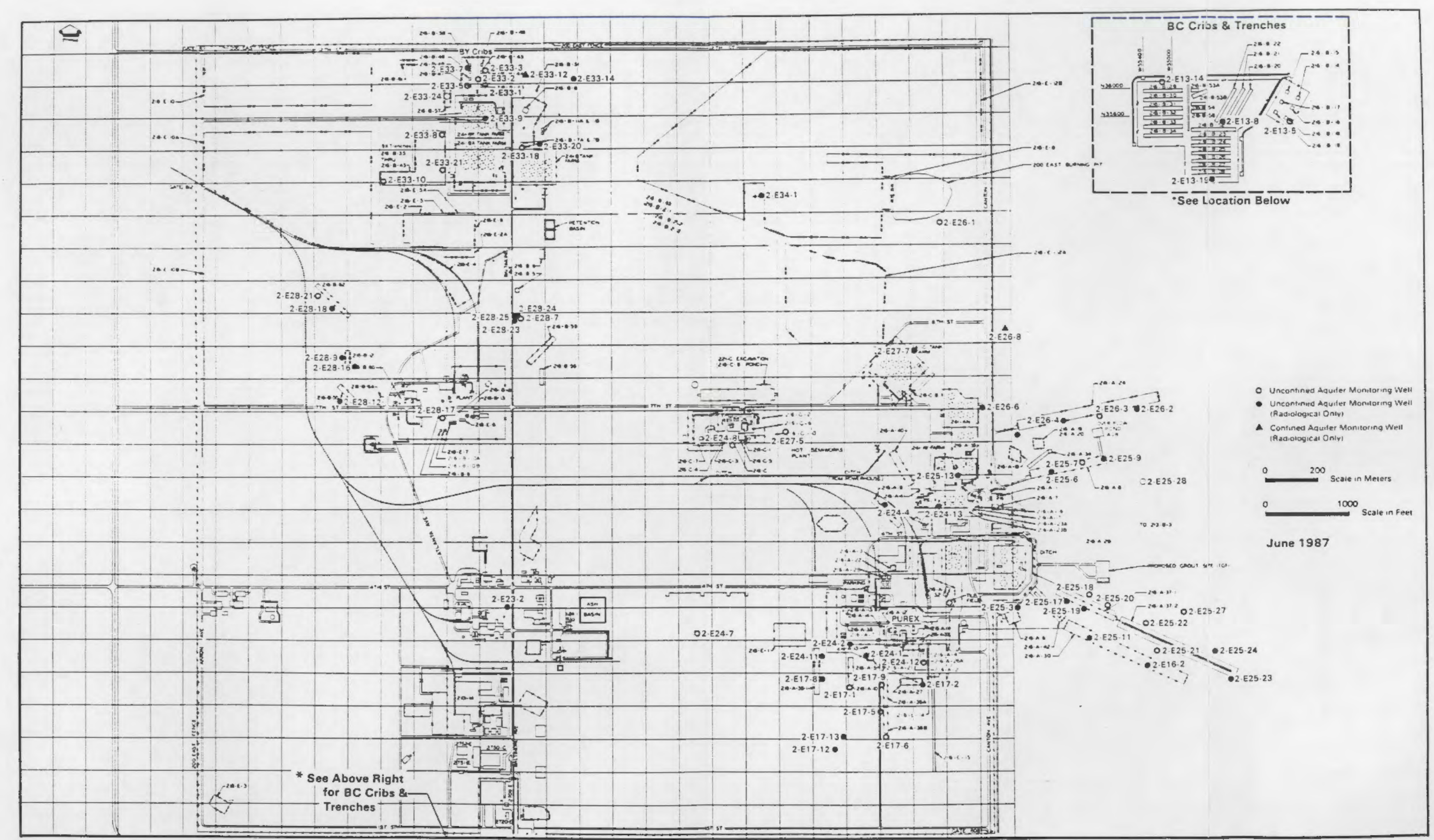

FIGURE 5. Location of Ground-Water Monitoring Wells in the 200 East Area Sampled During April-June 1987 



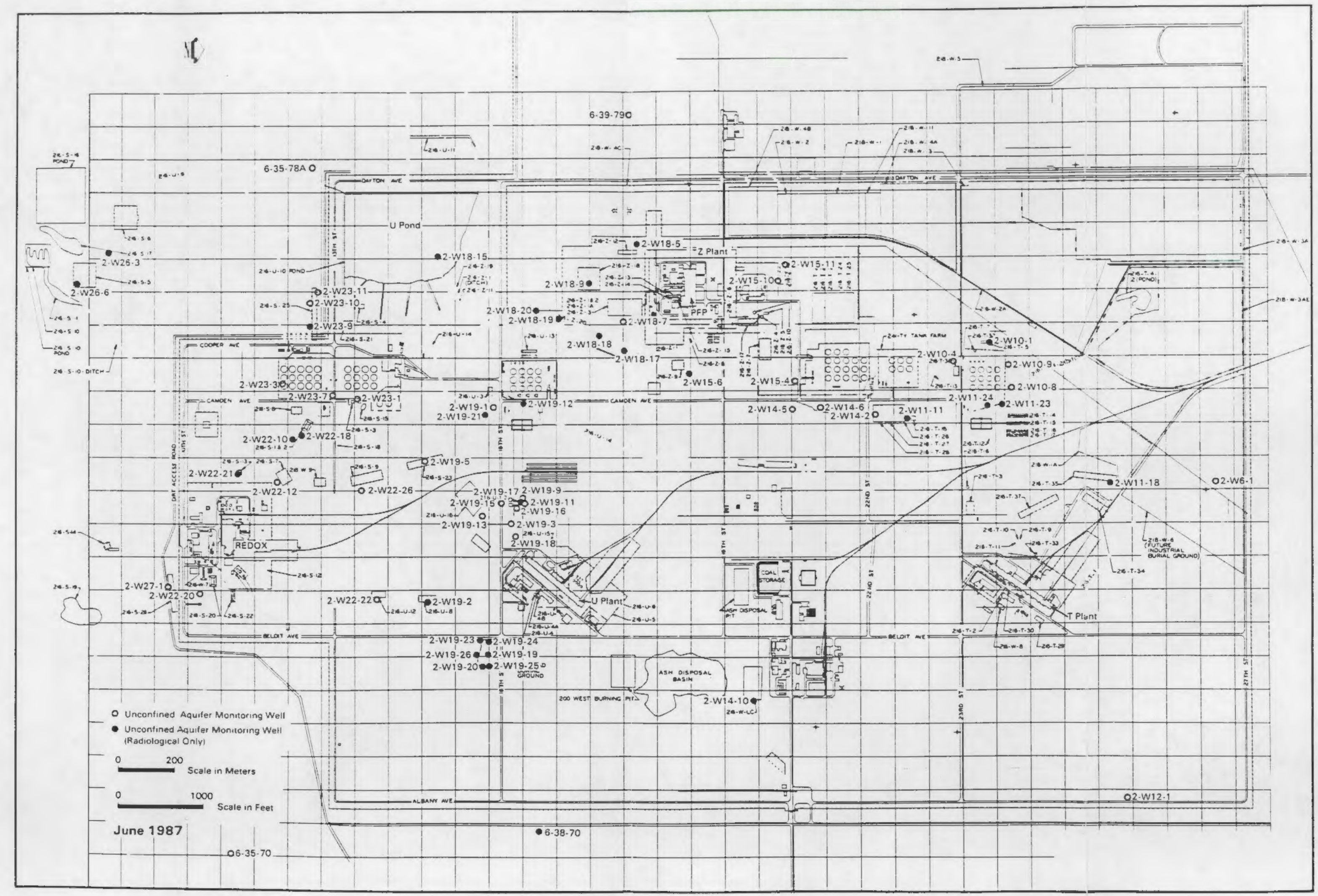

FIGURE 6. Location of Ground-Water Monitoring Wells in 200 West Area Sampled During April-June 1987 



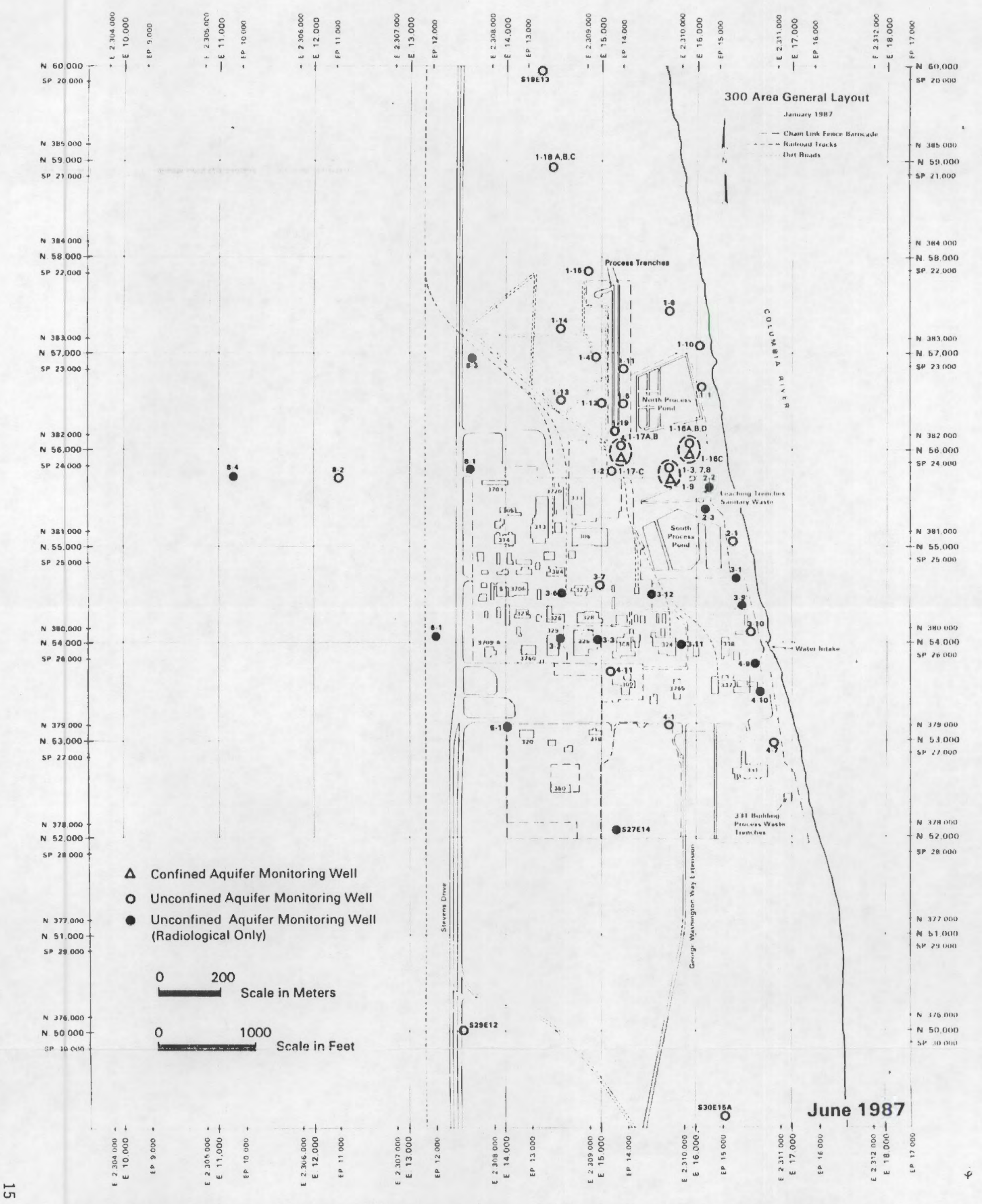

FIGURE 7. Location of Ground-Water Monitoring Wells in the 300 Area Sampled During April-June 1987 



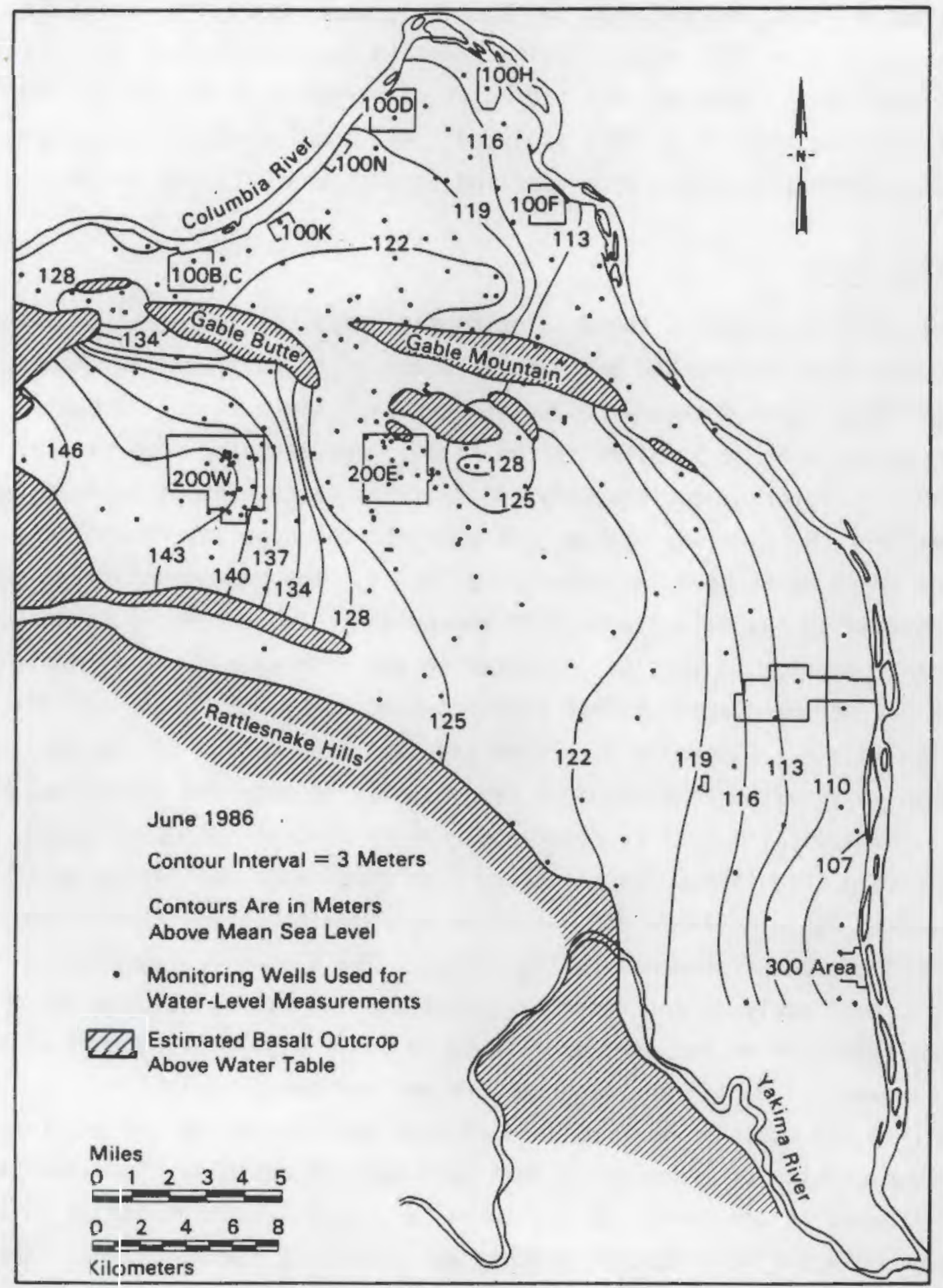

FIGURE 8. Water-Table Elevations for the Unconfined Aquifer in June 1986 (Schatz and Jensen 1986) 
were transferred the same day or early the next morning to the analytical subcontractor, U.S. Testing (UST) of Richland, Washington, for immediate analysis of species with short holding times (e.g., for nitrate and volatile organic analyses). Samples were stored at no warmer than $4^{\circ} \mathrm{C}\left(39^{\circ} \mathrm{F}\right)$ from the time of sampling until they were analyzed. All samples were tracked by chain-of-custody procedures from sampling through analysis and disposal.

\section{ANALYTICAL PLANS}

This section contains tables listing the constituents for which samples taken during the quarter had been analyzed and reported by UST at the time of writing. Some planned sample collection and analyses were not conducted because of unavoidable problems in the field. For example, some samples could not be filtered; consequently, no filtered trace element analyses were performed for those wells. Table 1 is a key to the constituent and constituent group names used in Tables 2, 3, and 4 . The same constituent names are also used in the data listings in Appendix A. Table 2 shows the constituents analyzed for in samples collected in the site-wide chemical monitoring network during the quarter (those wells co-sampled by DOE/HQ-EAT are footnoted in Table A.1, Appendix A). When the data from DOE/HQ-EAT become available, a detailed comparison of results will be made and documented as part of the project's quality assurance/quality control (QA/QC) program. The analyses conducted in samples collected from compliance monitoring wells are indicated in Table 3 (Table A.2, Appendix A, shows which wells were sampled at each of the compliance monitoring sites). The site-wide radiological (plus nitrate) analyses for those wells outside the site-wide chemical or compliance monitoring networks are listed in Table 4 . The frequency of sampling or specific monitoring programs are not indicated in Tables 2, 3, and 4 to simplify the tables. Most wells were sampled once during the quarter, with the exception of a few wells that were sampled monthly. Some analyses in 200-Area wells are conducted semiannually. Compliance monitoring in the $100 \mathrm{H}$ and 300 Areas is conducted monthly and bimonthly, respectively. Sample collection dates are included in the data tables in Appendix A. 
Analyses were performed by UST following EPA-approved procedures (USEPA 1982) or other standard methods. In a few cases for which standard methods were not available, methods were developed and documented by PNL. Specific conductance, $\mathrm{pH}$, and temperature were measured in the field at the time of collection, in accordance with documented procedures. The analytical procedures have been described elsewhere (PNL 1987, Appendix D). 
IABLE 1. Key to Constituent and Constituent Group Names Used in Tables 2, 3, and 4

Individual Constituents

\begin{tabular}{|c|c|c|}
\hline Name in Tables & Units & Full Name \\
\hline ALKALIN & $\mathrm{ppb}$ & Total alkalinity in $\mathrm{ppb} \mathrm{CaCO}_{3}$ \\
\hline$A M-241$ & $\mathrm{pCi} / \mathrm{L}$ & Americium-241 \\
\hline AMMONIU & $\mathrm{ppb}$ & Anmonium ion \\
\hline ARSENIC & $\mathrm{ppb}$ & Arsenic \\
\hline BETA & $\mathrm{pCj} / \mathrm{L}$ & Gross beta \\
\hline BROMIDE & $\mathrm{ppb}$ & Bromide \\
\hline CITRUSR & $\mathrm{ppb}$ & Citrus red \\
\hline COLIFRM & $\operatorname{mpn}(a)$ & Coliform bacteria \\
\hline CONDFLD & $\boldsymbol{\mu} \operatorname{mh} \mathrm{h}$ & Specific conductance \\
\hline CYANIDE & $\mathrm{ppb}$ & Cyanide \\
\hline DIOXIN & $\mathrm{ppb}$ & Dioxin \\
\hline ETHYGLY & $\mathrm{ppb}$ & Ethylene glycol \\
\hline FARSENI & $\mathrm{ppb}$ & Arsenic, filtered \\
\hline FLEAD & $\mathrm{ppb}$ & Lead, filtered \\
\hline FMERCUR & $\mathrm{ppb}$ & Mercury, filtered \\
\hline FSELENI & $\mathrm{ppb}$ & Selenium, filtered \\
\hline FTHALLI & $\mathrm{ppb}$ & Thallium, filtered \\
\hline HNI TRAT & $\mathrm{ppb}$ & Nitrate, high detection limit \\
\hline LEADGF & $\mathrm{ppb}$ & Lead (graphite furnace) \\
\hline LHYORAZ & $\mathrm{ppb}$ & Hydrazine, low detection limit \\
\hline LOALPHA & $\mathrm{pCi} / \mathrm{L}$ & Gross alpha \\
\hline LPHENOL & $\mathrm{ppb}$ & Phenol, low detection limit \\
\hline MERCURY & $\mathrm{ppb}$ & Mercury \\
\hline NITRI TE & $\mathrm{ppb}$ & Nitrate \\
\hline PERCHLO & $\mathrm{ppb}$ & Perchlorate \\
\hline PHFIELD & & $\mathrm{pH}$ (measured in field) \\
\hline RADIUM & $\mathrm{pCi} / \mathrm{L}$ & Radium-226 \\
\hline SELENUM & $\mathrm{ppb}$ & Selenium \\
\hline SR 90 & $\mathrm{PCi} / \mathrm{L}$ & Strontium-90 \\
\hline SULFIDE & $\mathrm{ppb}$ & Sulfide \\
\hline TC & $\mathrm{ppb}$ & Total carbon \\
\hline TC-99 & $\mathrm{pCi} / \mathrm{L}$ & Technetium-99 \\
\hline TDS & $\mathrm{ppm}$ & Total dissolved solids \\
\hline THALIUM & $\mathrm{ppb}$ & Thallium \\
\hline TOC & $\mathrm{ppb}$ & Total organic carbon \\
\hline TOX & $\mathrm{ppb}$ & Total organic halogen \\
\hline TOXLDL & $\mathrm{ppb}$ & Total organic halogen, low detection limi \\
\hline TRITIUM & $\mathrm{pCi} / \mathrm{L}$ & Tritium \\
\hline U & $\mathrm{pCi} / \mathrm{L}$ & Total uranium \\
\hline & $\mu g / L(b)$ & Total uranium \\
\hline
\end{tabular}

(a) $m p n=$ most probable number.

(b) $\mu \mathrm{g} / \mathrm{L}=\mathrm{ppb}$. 
TABLE 1. (contd)

Group: DIRAQIN $=$ Direct Aqueous Injection

Name in Tables Units Full Name

$1,1-D I M$

1,2-DIM

ACETILE

ACRYIDE

ALLYLAL

CHLACET

CHLORAL

CHLPROP

CYANBRO

CYANCHL

CYANOGN

DICPROP

ETHCARB

ETHCYAN

ETHOXID

FLUOROA

GLYCIDY

HYDRAZI

ISOBUTY

METZINE

PARALDE

PROPYL

PROPYNO

\begin{tabular}{l} 
Units \\
\hline$p p b$ \\
$p p b$ \\
$p p b$ \\
$p p b$ \\
$p p b$ \\
$p p b$ \\
$p p b$ \\
$p p b$ \\
$p p b$ \\
$p p b$ \\
$p p b$ \\
$p p b$ \\
$p p b$ \\
$p p b$ \\
$p p b$ \\
$p p b$ \\
$p p b$ \\
$p p b$ \\
$p p b$ \\
$p p b$ \\
$p p b$ \\
$p p b$ \\
$p p b$
\end{tabular}

1,1-dimethy Thydraz ine

1,2-dimethyl hydraz ine

Acetonitrile

Acrylamide

Allyl alcohol

Chloroacetaldehyde

Chloral

3-chloropropionitrile

Cyanogen bromide

Cyanogen chloride

Cyanogen

Dichloropropanol

Ethyl carbamate

Ethyl cyanide

Ethylene oxide

Fluoroacetic acid

Glycidylaldehyde

Hydrazine

Isobutyl alcohol

Methyl hydrazine

Paraldehyde

$\mathrm{N}$-propylamine

2-propyn-1-ol

Group: GAMMA = Gamna Scan

Name in Tables Units

C0-60

CS- 137

$\mathrm{RU}-103$

$\mathrm{RU}-106$

SB-125

$\mathrm{pCi} / \mathrm{L}$

$\mathrm{PCi} / \mathrm{L}$

$\mathrm{pCi} / \mathrm{L}$

$\mathrm{pCi} / \mathrm{L}$

$\mathrm{pCi} / \mathrm{L}$

Full Name

Cobalt-60

Cesium-137

Rutheni um-103

Ruthen i um-106

Antimony-125

Group: HERB $=$ Herbicides

Name in Tables Units

Full Name

$\begin{array}{lll}2,4,5 T P & \mathrm{ppb} & 2,4,5-\mathrm{TP} \text { silvex } \\ 2,4-D & \mathrm{ppb} & 2,4-\mathrm{D}\end{array}$

Group: HERBE $=$ Herbicides, enhanced (HERB plus the following)

Name in Tables Units

Full Name

2,4,5-T $\quad$ ppb $2,4,5-\mathrm{T}$


TABLE 1. (contd)

Group: $I$ ICPMT $=$ ICP Metals

Name in Tables Units

Ful1 Name

$\begin{array}{lll}\text { ALUMNUM } & \mathrm{ppb} & \text { Aluminum, unfiltered } \\ \text { BARIUM } & \mathrm{ppb} & \text { Barium, unfiltered } \\ \text { CADMIUM } & \mathrm{ppb} & \text { Cadmium, unfiltered } \\ \text { CALCIUM } & \mathrm{ppb} & \text { Calcium, unfiltered } \\ \text { CHROMUM } & \mathrm{ppb} & \text { Chromium, unfiltered } \\ \text { COPPER } & \mathrm{ppb} & \text { Copper, unfiltered } \\ \text { IRON } & \mathrm{ppb} & \text { Iron, unfiltered } \\ \text { MAGNES } & \mathrm{ppb} & \text { Magnesium, unfiltered } \\ \text { MANGESE } & \mathrm{ppb} & \text { Manganese, unfiltered } \\ \text { NICKEL } & \mathrm{ppb} & \text { Nickel, unfiltered } \\ \text { POTASUM } & \mathrm{ppb} & \text { Potassium, unfiltered } \\ \text { SILVER } & \mathrm{ppb} & \text { Silver, unfiltered } \\ \text { SODIUM } & \mathrm{ppb} & \text { Sodium, unfiltered } \\ \text { VANADUM } & \mathrm{ppb} & \text { Vanadium, unfiltered } \\ \text { ZINC } & \mathrm{ppb} & \text { Zinc, unfiltered }\end{array}$

Group: ICPMTE $=$ ICP Metals, enhanced (ICPMT plus the following)

Names in Tables Units

Full Name

$\begin{array}{lll}\text { ANTIONY } & \text { ppb } & \text { Antimony, unfiltered } \\ \text { BERYLAM } & \text { ppb } & \text { Berylliam, unfiltered } \\ \text { OSMIUM } & \text { ppb } & \text { Osmium, unfiltered } \\ \text { STRONUM } & \text { ppb } & \text { Strontium, unfiltered }\end{array}$

Group: ICPMTF $=$ ICP Metals, filtered

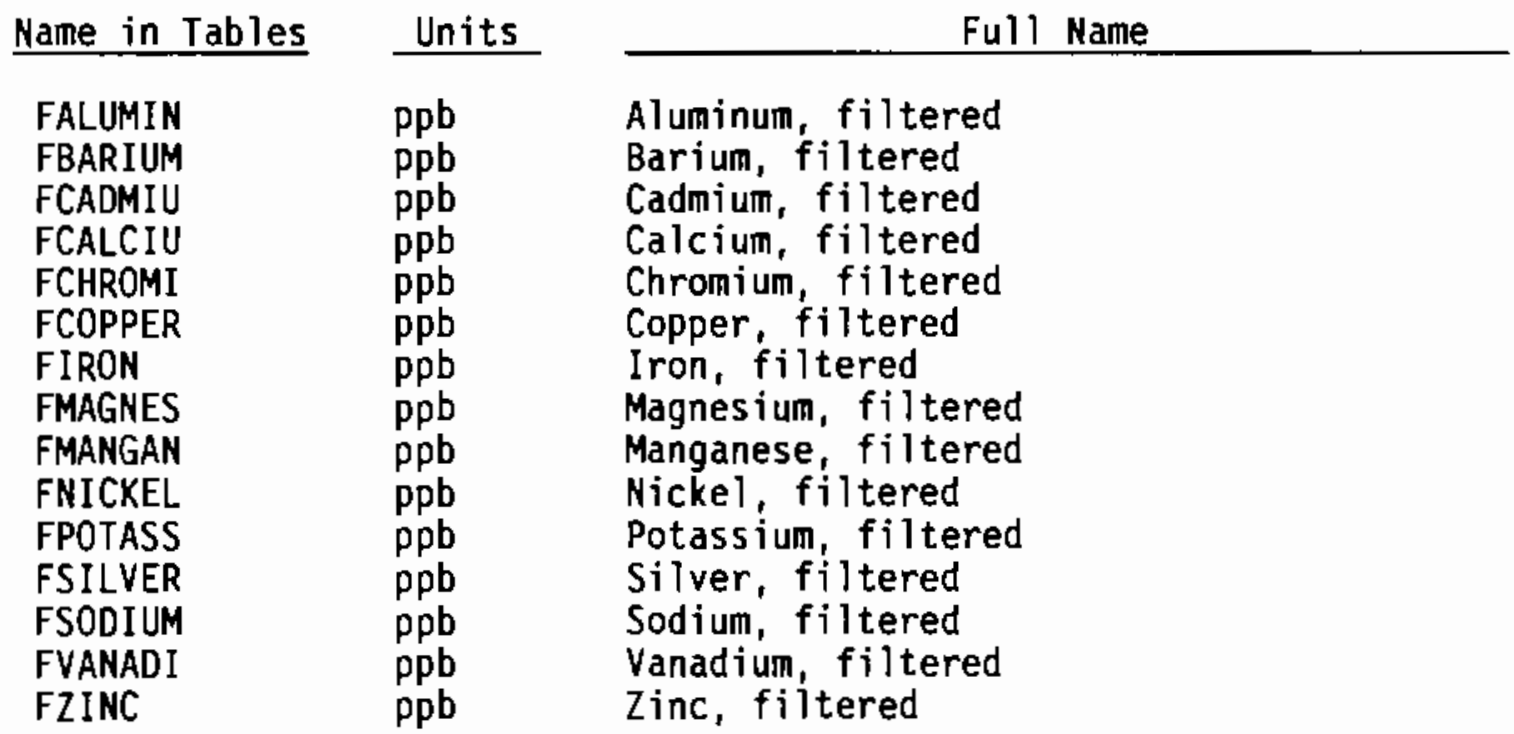


TABLE 1. (contd)

Group: ICPMTFE $=$ ICP Metals, filtered, enhanced (ICPMTF plus the following)

Name in Tables Units Full Name

$\begin{array}{lll}\text { FANTIMO } & \mathrm{ppb} & \text { Antimony, filtered } \\ \text { FBERYLL } & \mathrm{ppb} & \text { Berylitium, filtered } \\ \text { FOSMIUM } & \mathrm{ppb} & \text { 0smium, filtered } \\ \text { FSTRONT } & \mathrm{ppb} & \text { Strontium, filtered }\end{array}$

Group: IONS $=$ Ions

Name in Tables Units

Full Name

$\begin{array}{lll}\text { CHLORID } & \mathrm{ppb} & \text { Chloride } \\ \text { FLUORID } & \mathrm{ppb} & \text { Fluoride } \\ \text { NITRATE } & \mathrm{ppb} & \text { Nitrate } \\ \text { PHOSPHA } & \mathrm{ppb} & \text { Phosphate } \\ \text { SULFATE } & \mathrm{ppb} & \text { Sulfate }\end{array}$

Group: $P C B S=$ Polychlorinated Biphenyls

Name in Tables Units Full Name

$\begin{array}{lll}\text { AR1016 } & \mathrm{ppb} & \text { Arochlor } 1016 \\ \text { AR1221 } & \mathrm{ppb} & \text { Arochlor } 1221 \\ \text { AR1232 } & \mathrm{ppb} & \text { Arochlor } 1232 \\ \text { AR1242 } & \mathrm{ppb} & \text { Arochlor } 1242 \\ \text { AR1248 } & \mathrm{ppb} & \text { Arochlor } 1248 \\ \text { AR1254 } & \mathrm{ppb} & \text { Arochlor } 1254 \\ \text { AR1260 } & \mathrm{ppb} & \text { Arochlor } 1260\end{array}$

Group: $\quad$ PEST $=$ Pesticides

Name in Tables Units

Full Name

$\begin{array}{lll}\text { ENDRIN } & \mathrm{ppb} & \text { Endrin } \\ \text { METHLOR } & \mathrm{ppb} & \text { Methoxychlor } \\ \text { TOXAENE } & \mathrm{ppb} & \text { Toxaphene } \\ \mathrm{a}-\mathrm{BHC} & \mathrm{ppb} & \text { Alpha-BHC } \\ \mathrm{b}-\mathrm{BHC} & \mathrm{ppb} & \text { Beta-BHC } \\ \mathrm{d}-\mathrm{BHC} & \mathrm{ppb} & \text { Delta-BHC } \\ \mathrm{g}-\mathrm{BHC} & \mathrm{ppb} & \text { Ganma-BHC }\end{array}$

Group: PESTE $=$ Pesticides, enhanced (PEST plus the following)

\begin{tabular}{|c|c|c|}
\hline Name in Tables & Units & Full Name \\
\hline $\begin{array}{l}\text { ALDRIN } \\
\text { CHLLATE } \\
\text { CHLOANE }\end{array}$ & $\begin{array}{l}\mathrm{ppb} \\
\mathrm{ppb} \\
\mathrm{ppb}\end{array}$ & $\begin{array}{l}\text { Aldrin } \\
\text { Chlorobenzilate } \\
\text { Chlordane }\end{array}$ \\
\hline
\end{tabular}


TABLE 1. (contd)

\begin{tabular}{lll} 
DDD & $\mathrm{ppb}$ & DDD \\
DDE & $\mathrm{ppb}$ & DDE \\
DDT & $\mathrm{ppb}$ & DDT \\
DIELRIN & $\mathrm{ppb}$ & Dieldrin \\
ENDO1 & $\mathrm{ppb}$ & Endosulfan I \\
END02 & $\mathrm{ppb}$ & Endosulfan II \\
HEPTIDE & $\mathrm{ppb}$ & Heptchlor epoxide \\
HEPTLOR & $\mathrm{ppb}$ & Heptachlor \\
Group: PHOSPST & $=$ Phosphorus \\
\hline
\end{tabular}

\begin{tabular}{|c|c|c|}
\hline Name in Tables & Units & Full I Name \\
\hline $\begin{array}{l}\text { CARBPHT } \\
\text { DIMETHO } \\
\text { DISULFO } \\
\text { METHPAR } \\
\text { PARATHI } \\
\text { TETEPYR }\end{array}$ & $\begin{array}{l}\mathrm{ppb} \\
\mathrm{ppb} \\
\mathrm{ppb} \\
\mathrm{ppb} \\
\mathrm{ppb} \\
\mathrm{ppb}\end{array}$ & $\begin{array}{l}\text { Carbophenothion } \\
\text { Dimethoate } \\
\text { Disul foton } \\
\text { Methy parathion } \\
\text { Parathion } \\
\text { Tetraethylpyrophosphate }\end{array}$ \\
\hline
\end{tabular}

Group: PU-ISO $=$ Plutonium Isotopes

\begin{tabular}{|c|c|c|}
\hline Name in Tables & Units & Full Name \\
\hline $\begin{array}{l}\text { PU-238 } \\
\text { PU39-40 }\end{array}$ & $\begin{array}{l}\mathrm{pCi} / \mathrm{L} \\
\mathrm{pCi} / \mathrm{L}\end{array}$ & $\begin{array}{l}\text { Plutoni ium-238 } \\
\text { Plutonium-239, Plutonium-240 }\end{array}$ \\
\hline Name in Tables & Units & Full Name \\
\hline $\begin{array}{l}\text { 12-dben } \\
\text { 1234TE } \\
\text { 1235TE } \\
\text { 123TRI } \\
\text { 13-dben } \\
\text { 135TRI } \\
\text { 14-dben } \\
\text { HEXACHL } \\
\text { HEXCBEN } \\
\text { KEROSEN } \\
\text { NAPHTHA } \\
\text { PENTCHB } \\
\text { PHENOL } \\
\text { TETRCHB } \\
\text { TRICHLB }\end{array}$ & $\begin{array}{l}\mathrm{ppb} \\
\mathrm{ppb} \\
\mathrm{ppb} \\
\mathrm{ppb} \\
\mathrm{ppb} \\
\mathrm{ppb} \\
\mathrm{ppb} \\
\mathrm{ppb} \\
\mathrm{ppb} \\
\mathrm{ppb} \\
\mathrm{ppb} \\
\mathrm{ppb} \\
\mathrm{ppb} \\
\mathrm{ppb} \\
\mathrm{ppb}\end{array}$ & $\begin{array}{l}\text { 1,2-dichlorobenzene } \\
\text { 1,2,3,4-tetrachlorobenzene } \\
\text { 1,2,3,5-tetrachlorobenzene } \\
\text { 1,2,3-trichlorobenzene } \\
\text { 1,3-dichlorobenzene } \\
\text { 1,3,5-trichlorobenzene } \\
\text { 1,4-dichlorobenzene } \\
\text { Hexachlorophene } \\
\text { Hexachlorobenzene } \\
\text { Kerosene } \\
\text { Naphthalene } \\
\text { Pentachlorobenzene } \\
\text { Phenol } \\
\text { 1,2,4,5-tetrachlorobenzene } \\
\text { 1,2,4-trichlorobenzene }\end{array}$ \\
\hline
\end{tabular}


IABLE 1. (contd)

Group: SEMVOLE = Semi-volatile Organics, enhanced (SEMVOL plus the following)

Name in Tables Units Full Name

$1-$ napha

2-napha

24-dchp

24-dint

$245-\operatorname{trp}$

$246-\operatorname{trp}$

26-dchp

26-dint

ACEFENE

ACETOPH

AMI I SOX

AMINOYL

AMITROL

ANILINE

ARAMITE

AURAMIN

BENDICM

BENDINE

BENTHOL

BENZAAN

BENZBFL

BENZCAC

BENZCHL

BENZJFL

BENZOPY

BIS2CHE

BIS2CHM

BIS2EPH

BIS2ETH

BROPHEN

BUTBENP

BUTDINP

CHALETH

CHLANIL

CHLCRES

CHLEPOX

CHLNAPH

CHLNAPZ

CHLPHEN

CHRYSEN

CRESOLS

CYCHDIN

DIBAEPY

DIBAHAC

DIBAHAN $\mathrm{ppb}$

$\mathrm{ppb}$

$\mathrm{ppb}$

$\mathrm{ppb}$

$\mathrm{ppb}$

$\mathrm{ppb}$

ppb

$\mathrm{ppb}$

$\mathrm{ppb}$

$\mathrm{ppb}$

ppb

$\mathrm{ppb}$

$\mathrm{ppb}$

$\mathrm{ppb}$

ppb

$\mathrm{ppb}$

$\mathrm{ppb}$

$\mathrm{ppb}$

$\mathrm{ppb}$

$\mathrm{ppb}$

$\mathrm{ppb}$

$\mathrm{ppb}$

$\mathrm{ppb}$

$\mathrm{ppb}$

$\mathrm{ppb}$.

$\mathrm{ppb}$

$\mathrm{ppb}$

$\mathrm{ppb}$

$\mathrm{ppb}$

$\mathrm{ppb}$

$\mathrm{ppb}$

$\mathrm{ppb}$

$\mathrm{ppb}$

$\mathrm{ppb}$

$\mathrm{ppb}$

$\mathrm{ppb}$

$\mathrm{ppb}$

$\mathrm{ppb}$

$\mathrm{ppb}$

$\mathrm{ppb}$

$\mathrm{ppb}$

$\mathrm{ppb}$

$\mathrm{ppb}$

$\mathrm{ppb}$

$\mathrm{ppb}$ 1-naphthylamine

2-naph thyl amine

2,4-dich Torophenol

2,4-dinitrotoluene

2,4,5-trichlorophenol

2,4,6-trichlorophenol

2,6-dichlorophenol

2,6-dinitrotoluene

2-acetyl aminofluorene

Acetophenone

5-(aminomethy 1$)-3-i$ soxazolol

4-aminobypheny

Amitrole

Aniline

Aramite

Auramine

Benzene, dichloromethy?

Benzidine

Benzenethoil

Benz [a] anthracene

Benzo [b] fluoranthene

Benz [c] acridine

Benzyl chloride

Benzo[j]fluoranthene

Benzo[a]pyrene

Bis (2-chloroethyl) ether

Bis(2-chloroethoxy) methane

Bis(2-ethylhexy1) phthalate

Bis(2-chloroi sopropy 1) ether

4-bromopheny 1 phenyl ether

Butyl benzyl phthalate

2-sec-buty 1-4,6-dinitrophenol

Chloroalkyl ethers

P-chloroaniline

P-chloro-m-cresol

1-chloro-2,3-epoxypropane

2-chloronaphthalene

Chlornaphazine

2-chlorophenol

Chrysene

Cresols

2-cyclohexy 1-4,6-din itropheno]

Dibenzo[a,e]pyrene

Dibenz $[a, h]$ acridine

Dibenz $[a, h]$ anthracene 
IABLE 1. (contd)

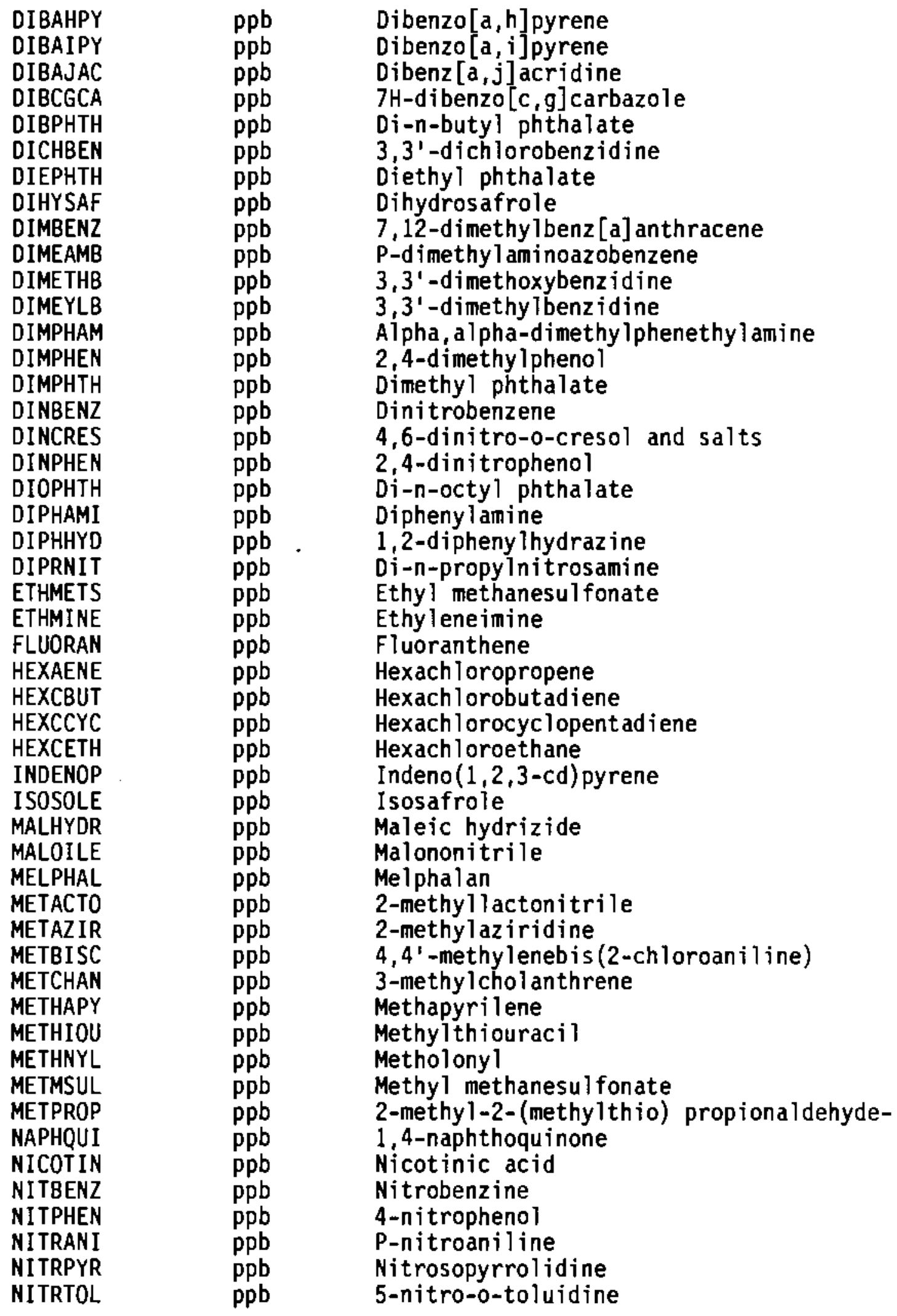


IABLE 1. (contd)

\begin{tabular}{|c|c|c|}
\hline $\begin{array}{l}\text { NNIBUTY } \\
\text { NNIDIEA } \\
\text { NNIDIEY } \\
\text { NNIDIME } \\
\text { NNIMETH } \\
\text { NNIMORP } \\
\text { NNINICO } \\
\text { NNIPIPE } \\
\text { NNIURET } \\
\text { NNIVINY } \\
\text { OTOLHYD } \\
\text { PBENZQU } \\
\text { PENTCHN } \\
\text { PENTCHP } \\
\text { PHENINE } \\
\text { PHENTIN } \\
\text { PHTHEST } \\
\text { PICOLIN } \\
\text { PRONIDE } \\
\text { RESERPI } \\
\text { RESORCI } \\
\text { SAFROL } \\
\text { STRYCHN } \\
\text { SYMTRIN } \\
\text { TETRCHP } \\
\text { THIONOX } \\
\text { THIURAM } \\
\text { TOLUDIA } \\
\text { TRIPHOS } \\
\text { TRISPHO } \\
\text { WARFRIN }\end{array}$ & $\begin{array}{l}\rho p b \\
p p b \\
p p b \\
\rho p b \\
p p b \\
p p b \\
p p b \\
p p b \\
\rho p b \\
\rho p b \\
p p b \\
p p b \\
p p b \\
\rho p b \\
\rho p b \\
\rho p b \\
p p b \\
p p b \\
p p b \\
p p b \\
p p b \\
\rho p b \\
p p b \\
p p b \\
\rho p b \\
p p b \\
p p b \\
p p b \\
p p b \\
p p b \\
\rho p b\end{array}$ & 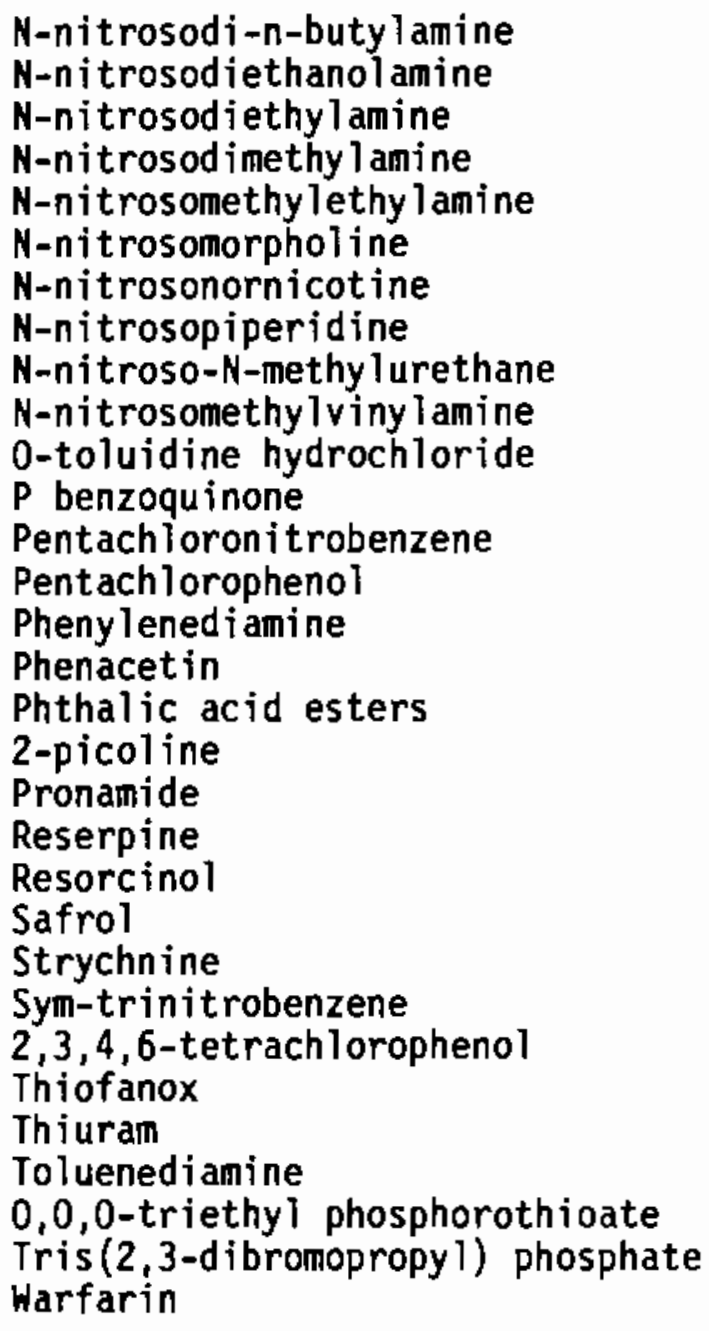 \\
\hline
\end{tabular}

\begin{tabular}{|c|c|c|}
\hline Name in Tables & Units & Full Name \\
\hline $\begin{array}{l}\text { ACETREA } \\
\text { CHLOREA } \\
\text { DIETRO } \\
\text { ETHYREA } \\
\text { NAPHREA } \\
\text { PHENREA } \\
\text { THIOURA }\end{array}$ & $\begin{array}{l}\rho p b \\
p p b \\
p p b \\
p p b \\
p p b \\
p p b \\
p p b\end{array}$ & $\begin{array}{l}\text { 1-acetyl-2-thiourea } \\
1-(0-c h l o r o p h e n y l) \text { thiourea } \\
\text { Diethylstilbesterol } \\
\text { Ethylenethiourea } \\
\text { 1-naphthyl-2-thiourea } \\
\text { N-phenylthiourea } \\
\text { Thiourea }\end{array}$ \\
\hline
\end{tabular}




\section{TABLE 1. (contd)}

Group: U-ISO = Uranium Isotopes

\begin{tabular}{|c|c|c|}
\hline Name in Tables & Units & \\
\hline $\begin{array}{ll}\text { U } & 234 \\
\text { U } & 235 \\
\text { U } & 238\end{array}$ & $\begin{array}{l}\mathrm{pCi} / \mathrm{L} \\
\mathrm{pCi} / \mathrm{L} \\
\mathrm{pCi} / \mathrm{L}\end{array}$ & $\begin{array}{l}\text { Uranium-234 } \\
\text { Uranium-235 } \\
\text { Uranium-238 }\end{array}$ \\
\hline
\end{tabular}

Group: VOLORG $=$ Volatile Organics

Name in Tables Units

$1,1,1-\mathrm{T}$

$1,1,2-T$

CHLFORM

M-XYLE

METHONE

METHYCH

OPXYLE

PERCENE

TETRANE

TRICENE ppb

$\mathrm{ppb}$

$\mathrm{ppb}$

$\mathrm{ppb}$

$\mathrm{ppb}$

$\mathrm{ppb}$

$\mathrm{ppb}$

$\mathrm{ppb}$

$\mathrm{ppb}$

$\mathrm{ppb}$
Ful1 Name

Full Name

1,1,1-trichloroethane

1,1,2-trichloroethane

Chloroform

Xylene-m

Methyl ethyl ketone

Methylene chloride

Xylene-0,p

Perchloroethylene

Tetrachloromethane (carbon tetrachloride)

Trichloroethylene (1,2-trichloroethene)

Group: VOLORGE = Volatile Organics, enhanced (VOLORG plus the following)

Name in Tables Units

$1,1-D I C$

$1,2-D I C$

$1112-\mathrm{tc}$

$1122-$ tc

$123-\operatorname{trp}$

ACROLIN

ACRYILE

BENZENE

BISTHER

BROMONE

BROMORM

CARBIDE

CHLBENZ

CHLTHER

CHMTHER

CROTONA

DIBRCHL

DIBRETH

DIBRMET

DIBUTEN

DICDIFM

DICETHY

DICPANE ppb

$\mathrm{ppb}$

$\mathrm{ppb}$

$\mathrm{ppb}$

$\mathrm{ppb}$

$\mathrm{ppb}$

$\mathrm{ppb}$

$\mathrm{ppb}$

$\mathrm{ppb}$

$\mathrm{ppb}$

$\mathrm{ppb}$

$\mathrm{ppb}$

$\mathrm{ppb}$

$\mathrm{ppb}$

$\mathrm{ppb}$

$\mathrm{ppb}$

$\mathrm{ppb}$

$\mathrm{ppb}$

$\mathrm{ppb}$

$\mathrm{ppb}$

$\mathrm{ppb}$

$\mathrm{ppb}$

$\mathrm{ppb}$
Fult Name

1,1-dichloroethane

1,2-dichloroethane

$1,1,1,2$-tetrachlorethane

$1,1,2,2$-tetrachlorethane

1,2,3-trichloropropane

Acrolein

Acrylonitrile

Benzene

$B$ is (chloromethyl) ether

Bromoacetone

Bromoform

Carbon disulfide

Chlorobenzene

2-chloroethyl vinyl ether

Chloromethyl methyl ether

Crotonaldehyde

1,2-dibromo-3-chloropropane

1,2-dibromoethane

Dibromomethane

1,4-dichloro-2-butene

Dichlorod if luoromethane

1,1-dichloroethylene

1,2-dichloropropane 
TABLE 1. (contd)

$\begin{array}{lll}\text { DICPENE } & \mathrm{ppb} & \text { 1,3-dichloropropene } \\ \text { DIETHY } & \mathrm{ppb} & \text { Diethylarsine } \\ \text { DIOXANE } & \mathrm{ppb} & \text { Dioxane } \\ \text { ETHMETH } & \mathrm{ppb} & \text { Ethyl methacrylate } \\ \text { FORMALN } & \mathrm{ppb} & \text { Formalin } \\ \text { HYDRSUL } & \mathrm{ppb} & \text { Hydrogen sulfide } \\ \text { IODOMET } & \mathrm{ppb} & \text { Iodomethane } \\ \text { METACRY } & \mathrm{ppb} & \text { Methyl methacrylate } \\ \text { METHACR } & \mathrm{ppb} & \text { Methacrylonitrile } \\ \text { METHBRO } & \mathrm{ppb} & \text { Methyl bromide } \\ \text { METHCHL } & \mathrm{ppb} & \text { Methyl chloride } \\ \text { METHTHI } & \mathrm{ppb} & \text { Methanethiol } \\ \text { NNDIEHY } & \mathrm{ppb} & \text { N, N-diethylhydrazine } \\ \text { PENTACH } & \mathrm{ppb} & \text { Pentachioroethane } \\ \text { PYRIDIN } & \mathrm{ppb} & \text { Pyridine } \\ \text { TOLUENE } & \mathrm{ppb} & \text { Toluene } \\ \text { TRANDCE } & \mathrm{ppb} & \text { Trans-1,2-dichloroethene } \\ \text { TRCMEOL } & \mathrm{ppb} & \text { Trichloromethanethiol } \\ \text { TRCMFLM } & \mathrm{ppb} & \text { Trichloromonofluoromethane } \\ \text { TRCPANE } & \mathrm{ppb} & \text { Trichloropropane } \\ \text { VINYIDE } & \mathrm{ppb} & \text { Vinyl chloride }\end{array}$


TABLE 2. Analytical Plan for Site-Wide Chemical Monitoring Wells

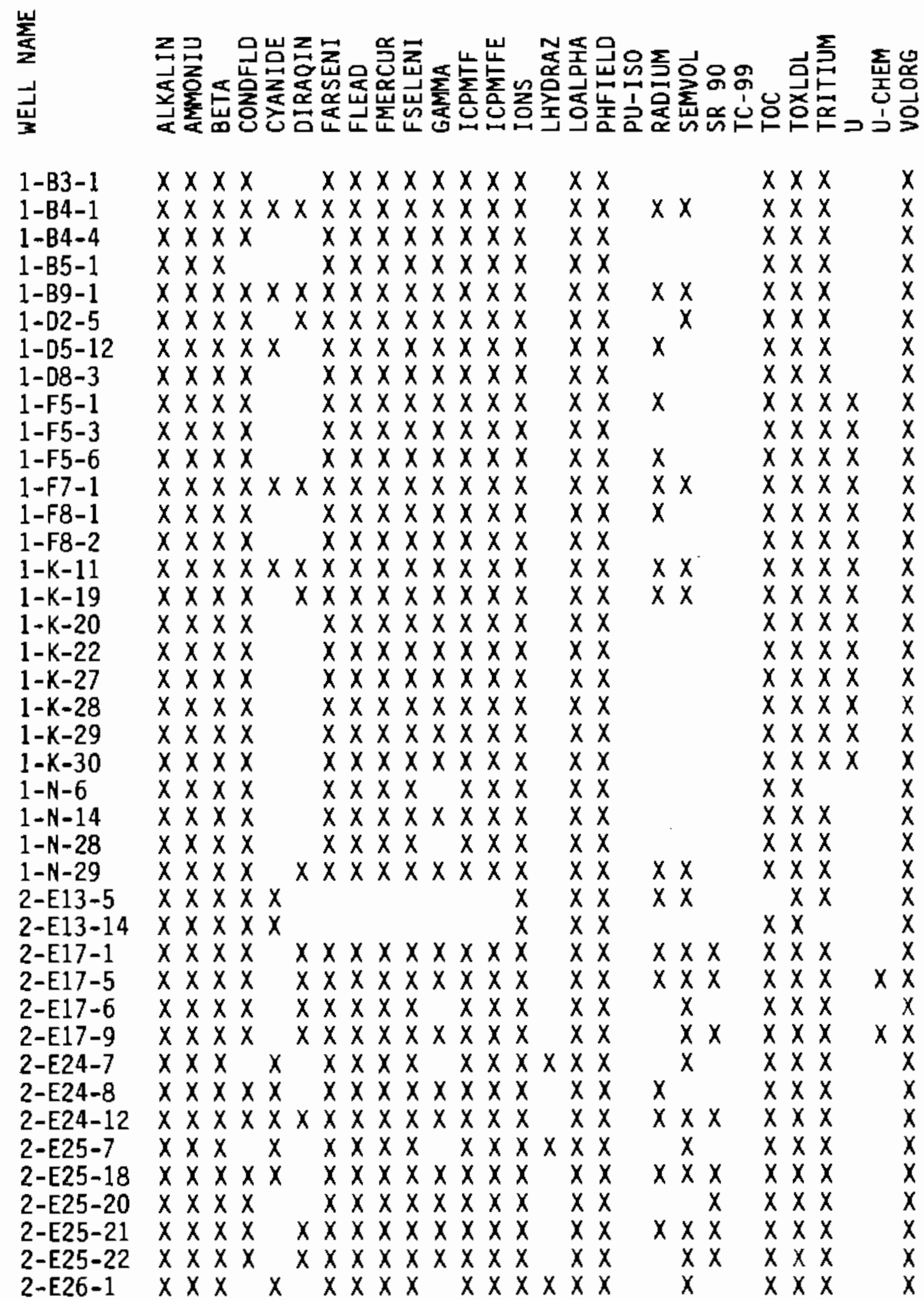


TABLE 2. (contd)

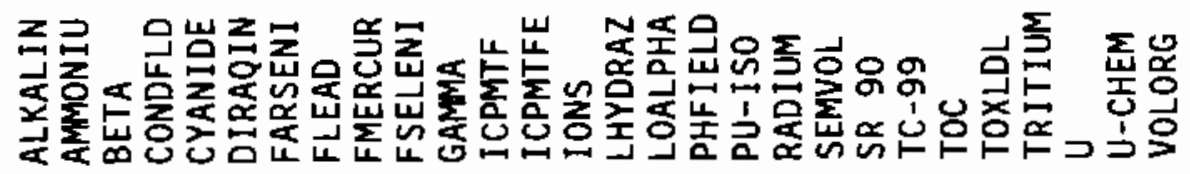

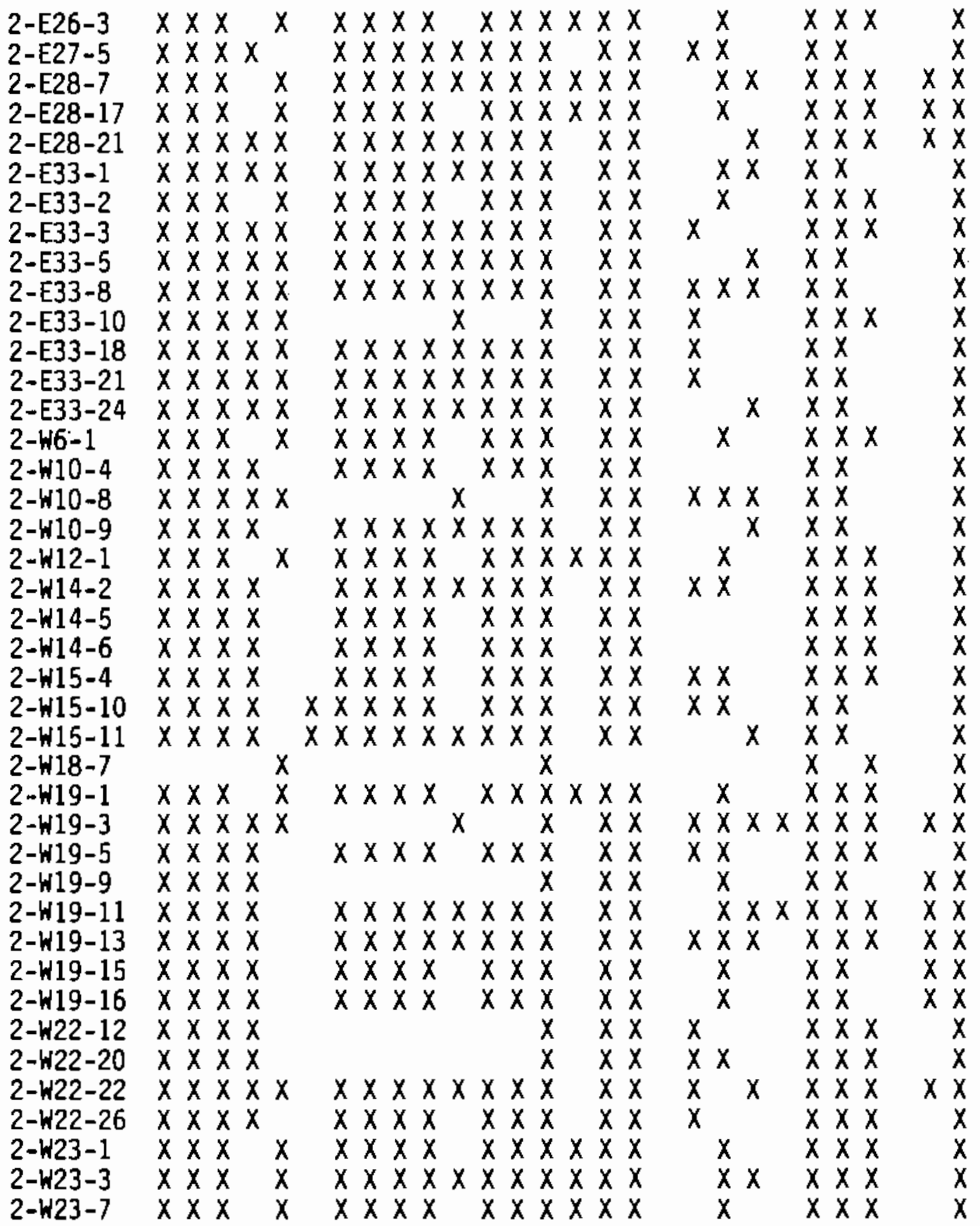


TABLE 2. (contd)

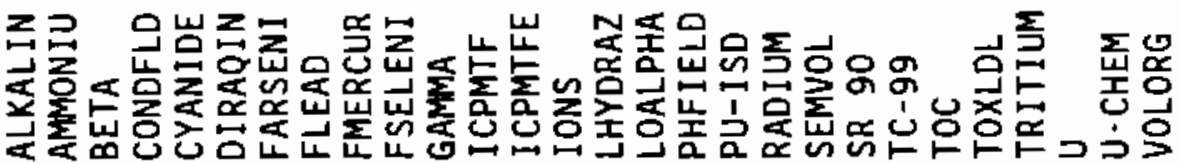

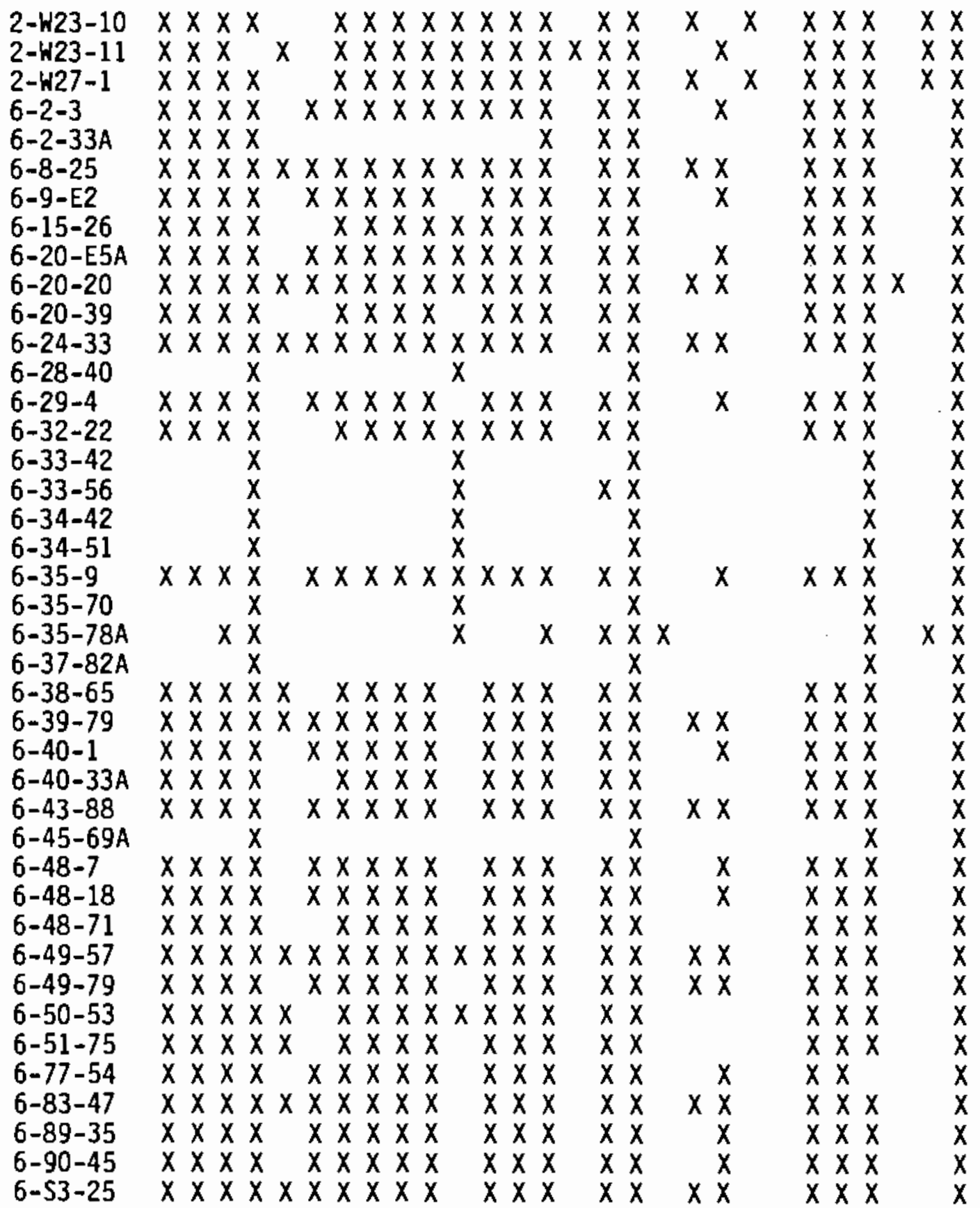


TABLE 2. (contd)

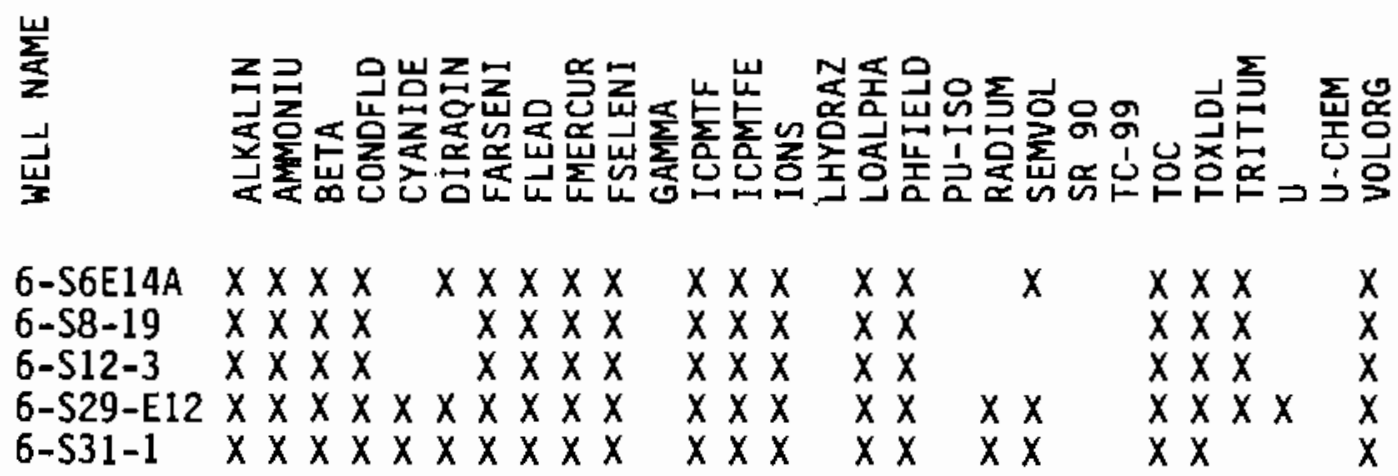


TABLE 3. Analytical Plan for Compliance Monitoring Wells

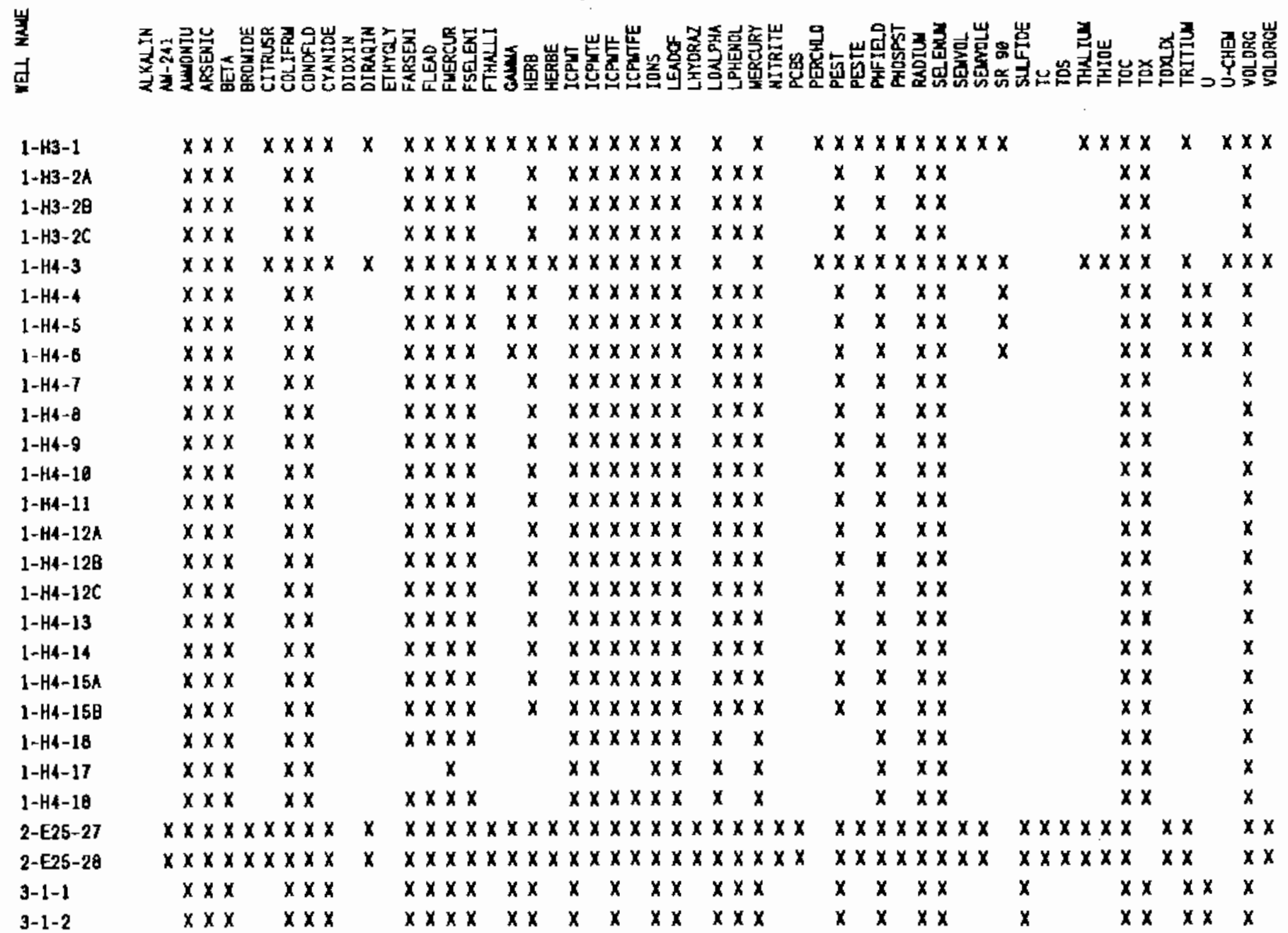


IABLE 3. (contd)

崖

\begin{tabular}{|c|c|c|c|c|c|c|c|c|}
\hline $3-1-3$ & $x \times x$ & $x \times x$ & $x \times x \times$ & $x \times$ & $x$ & $x$ & $x \times$ & $x \times$ \\
\hline $3-1-4$ & $x \times x$ & $x \times x$ & $x \times x \times$ & $x \times$ & $x$ & $x$ & $x \times$ & \\
\hline $3-1-5$ & $x \times x$ & $\times \times \times \times \times \times \times$ & $x \times \times \times)$ & $\times \times \times x$ & $6 \times x$ & $x \times$ & $x \times$ & $x$ \\
\hline $3-1-6$ & $x \times x$ & $x \times x$ & $x \times x \times$ & $x \times$ & $x$ & $x$ & $x x$ & \\
\hline $3-1-7$ & $x \times x$ & $x \times x$ & & $x$ & $x$ & & $x \times$ & \\
\hline $3-1-8$ & $x \times x$ & $x \times x$ & $x \times \times x$ & $x$ & $x$ & $x$ & $x \times$ & $x$ \\
\hline $3-1-9$ & $x \times x$ & $x \times x$ & $x \times x \times$ & $x$ & $x$ & $x$ & $x \times$ & \\
\hline $3-1-10$ & $x \times x$ & $x \times x$ & $x \times x \times$ & $x$ & $x$ & $x$ & $x \times$ & \\
\hline $3-1-11$ & $x \times x$ & $\times \times x$ & $x \times x \times$ & $x$ & $x$ & $x$ & $x \times$ & \\
\hline $3-1-12$ & $x \times x$ & $x \times x$ & $x \times x \times$ & $x$ & $x$ & $x$ & $x \times$ & \\
\hline $3-1-13$ & $x \times x$ & $x \times x$ & $\times \times \times \times$ & $x$ & $x$ & $x$ & $x \times$ & \\
\hline $3-1-14$ & $x \times x$ & $x \times x$ & $x \times \times x$ & $x$ & $x$ & $x$ & $x \times$ & \\
\hline $3-1-15$ & $x \times x$ & $x \times x$ & $x \times \times x$ & $x$ & $x$ & $x$ & $x \times$ & \\
\hline $3-1-18 A$ & $x \times x$ & $x \times x$ & $x \times \times x$ & $x$ & $x$ & $x$ & $x \times$ & \\
\hline $3-1-180$ & $x \times x$ & $x \times x$ & $x \times x \times$ & $x$ & $x$ & $x$ & $x x$ & \\
\hline $3-1-18 C$ & $x \times x$ & $x \times x$ & $x \times x \times$ & $x$ & $x$ & $x$ & $x \times$ & \\
\hline $3-1-160$ & $x \times x$ & $x \times x$ & & $x$ & $x$ & & $x \times$ & \\
\hline $3-1-17 A$ & $x \times x$ & $x \times x$ & $x \times x \times$ & $x$ & $x$ & $x$ & $x \times$ & \\
\hline $3-1-178$ & $x \times x$ & $x \times x$ & $x \times x \times$ & $x$ & $x$ & $x$ & $x \times$ & \\
\hline $3-1-17 c$ & $x \times x$ & $x x x$ & $x \times x \times$ & $x$ & $x$ & $x$ & $x \times$ & \\
\hline $3-1-18 A$ & $x \times x$ & $x \times x$ & $x \times x \times$ & $x$ & $x$ & $x$ & $x x$ & \\
\hline $3-1-188$ & $x \times x$ & $x \times x$ & $x \times x \times$ & $x$ & $x$ & $x$ & $x \times$ & \\
\hline $3-1-18 C$ & $x \times x$ & $x \times x$ & $x \times x \times$ & $x$ & $x$ & $x$ & $x \times$ & $x$ \\
\hline $3-1-19$ & $x \times x$ & $x \times x$ & $x \times x \times$ & $x$ & $x$ & $x$ & $x \times$ & \\
\hline $3-2-1$ & & $x \times x$ & $x \times \times x$ & $x \times$ & $x$ & $x$ & $x \times$ & \\
\hline $3-3-7$ & & $x \times x$ & $x \times \times x$ & $x \times$ & $x$ & $x$ & $x x$ & \\
\hline
\end{tabular}

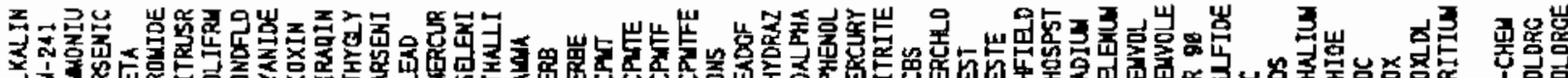

w

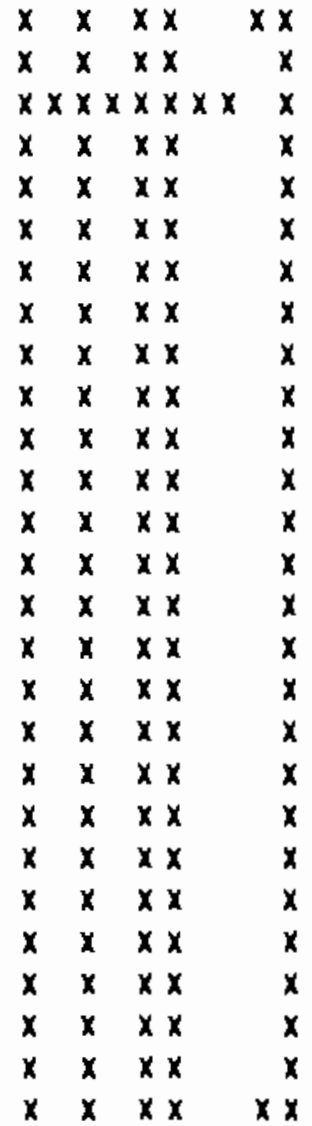

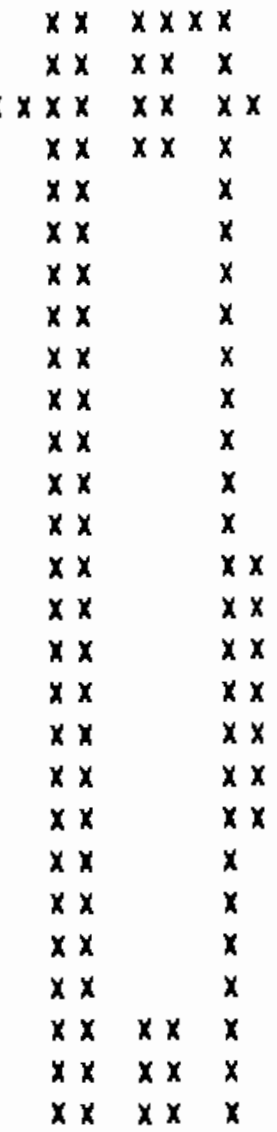


IABLE 3. (contd)

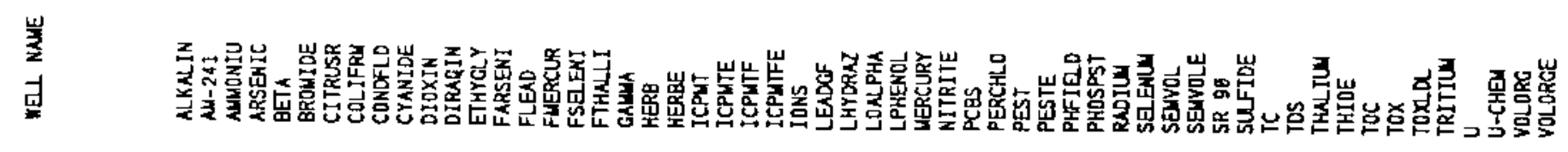

\begin{tabular}{|c|c|c|c|c|c|c|c|c|c|c|c|c|c|c|c|c|c|}
\hline $3-4-1$ & & $x \times x$ & $x \times x$ & $x \times x \times$ & $x \times$ & $x$ & $x$ & $x \times$ & $x \times x$ & & $x$ & $x$ & $x \times$ & $x$ & $x \times$ & $x \times$ & $x$ \\
\hline $3-4-7$ & & $x \times x$ & $x \times x$ & $x \times \times \times$ & $x \times$ & $x$ & $x$ & $x \times$ & $x \times x$ & & $x$ & $x$ & $x \times$ & $x$ & $x \times$ & $x \times$ & $x$ \\
\hline $3-4-11$ & & $x \times x$ & $x \times x$ & $x \times \times x$ & $x$ & $x$ & $x$ & $x \times$ & $x \times x$ & & $x$ & $x$ & $x \times$ & $x$ & $x \times$ & & $x$ \\
\hline $3-8-2$ & & $x \times x$ & $x \times x$ & $x \times \times x$ & $x x$ & $x$ & $x$ & $x \times$ & $x \times x$ & & $x$ & $x$ & $x \times$ & $x$ & $x \times$ & $x \times$ & $x$ \\
\hline $6-S 19-\mathrm{E} 13$ & & $x \times x$ & $\times \times \times \times \times \times x$ & $x \times \times \times x$ & $x \times x$ & $x \times$ & $x \times$ & $x \times$ & $x \quad x$ & & $x \times$ & $x \times$ & $x \times \times \times x$ & $x$ & $x \times x \times$ & $x \times x$ & $x \times$ \\
\hline $6-S 39 E 15 A$ & & $x \times x$ & $x \times x$ & $x \times \times x$ & $x$ & $x$ & $x$ & $x \times$ & $x \times x$ & & $x$ & $x$ & $x \times$ & $x$ & $x \times$ & $x \times$ & $\mathbf{x}$ \\
\hline $6-23-34$ & $x$ & $x \times x$ & $x \times x$ & $x \times x \times$ & $x \times$ & $x \times$ & $x \times$ & $x x$ & $x \quad x$ & $x$ & $x \times$ & $x x$ & $x \times x$ & & $x \quad x$ & & $x \times$ \\
\hline $6-24-34 A$ & $x$ & $x \times x$ & $x \times x$ & $x \times x \times$ & $x \times$ & $x \times$ & $\times x$ & $x \times$ & $x$ & $x$ & $x \times$ & $x \times$ & $x \times x$ & & $x$ & & $x \times$ \\
\hline $8-24-318$ & $x$ & $x \times x$ & $x \times x$ & $x \times x \times$ & $x x$ & $x \times$ & $\times x$ & $\times x$ & $x$ & $x$ & $x \times$ & $x x$ & $x \times x$ & & $x$ & & $x \times$ \\
\hline $8-24-34 C$ & $x$ & $x \times x$ & $x \times x$ & $x \times \times x$ & $x \times$ & $x \times x$ & $x \times$ & $x x$ & $x$ & $x$ & $x x$ & $x \times$ & $x \times x$ & & $x$ & & $x \times$ \\
\hline $6-24-35$ & $x$ & $x \times x$ & $x \times x$ & $x \times x \times$ & $x \times$ & $x \times$ & $x \times$ & $x \times$ & $x$ & $x$ & $x x$ & $x x$ & $x \times x$ & & $x$ & & $x \times$ \\
\hline $6-25-34 C$ & $x$ & $x \times x$ & $x \times x$ & $x \times x \times$ & $x \times$ & $\times x$ & $x \times$ & $x \times$ & $x \dot{x}$ & $x$ & $x \times$ & $x \times$ & $x \times x$ & & $x \quad x$ & & $x \times$ \\
\hline $0-25-33 A$ & & $x x$ & $x \times$ & $x \times x \times$ & $x$ & $x$ & $x$ & $x \times$ & $x \times x$ & & $x$ & $x$ & $x \times x$ & $x$ & $x \times$ & & $x \times$ \\
\hline $8-25-34 A$ & & $x x$ & $x x$ & $x \times x \times$ & $x$ & $x$ & $x$ & $x \times$ & $x \times x$ & & $x$ & $x$ & $x \times x$ & $x$ & $x \times$ & & $x \times$ \\
\hline $6-25-348$ & & $x \times$ & $x \times$ & $x \times x \times$ & $x$ & $x$ & $x$ & $x \times$ & $x \times x$ & & $x$ & $x$ & $x \times x$ & $x$ & $x \times$ & & $x \times$ \\
\hline $8-28-33$ & & $x \times$ & $x \times$ & $x \times x \times$ & $x$ & $x$ & $x$ & $x \times$ & $x \times x$ & & $x$ & $x$ & $x \times x$ & $x$ & $x \times$ & & $x \times$ \\
\hline $8-26-34$ & & $x x$ & $x \times$ & $x \times x \times$ & $x$ & $x$ & $x$ & $x \times$ & $x \times x$ & & $x$ & $x$ & $x \times x$ & $x$ & $x \times$ & & $x x$ \\
\hline $6-28-35 A$ & & $x \times$ & $x \times$ & $x \times x \times$ & $x$ & $x$ & $x$ & $x \times$ & $x \times x$ & & $x$ & $x$ & $x \times x$ & $x$ & $x \times$ & & $x \times$ \\
\hline $8-28-35 C$ & & $x \times$ & $x x$ & $x \times x \times$ & $x$ & $x$ & $x$ & $x x$ & $x \times x$ & & $x$ & $x$ & $x \times x$ & $x$ & $x \times$ & & $x \times$ \\
\hline
\end{tabular}


TABLE 4. Analytical Plan for Site-Wide Radiological (plus Nitrate) Monitoring Wells Not in the Site-Wide Chemical or Compliance Monitoring Networks

Well name BETA GAMMA NITRATE LOALPHA PU-ISO SR 90 TC-99 TRITIUM U U-CHEM U-ISO

\begin{tabular}{|c|c|c|c|c|c|c|c|}
\hline \multicolumn{2}{|l|}{$1-84-2$} & $x$ & \multicolumn{3}{|l|}{$x$} & \multicolumn{2}{|l|}{$x$} \\
\hline \multirow{2}{*}{\multicolumn{2}{|c|}{$\begin{array}{l}1-B 4-3 \\
1-F 5-4\end{array}$}} & $x$ & $x$ & & & $x$ & \\
\hline & & $x$ & $x$ & & & $x$ & $x$ \\
\hline \multicolumn{2}{|l|}{$1-\mathrm{N}-2$} & $x$ & $x$ & & $\mathrm{X}$ & $x$ & \\
\hline \multicolumn{2}{|l|}{$1-\mathrm{N}-3$} & $x$ & & & $x$ & $x$ & \\
\hline \multicolumn{2}{|l|}{$1-N-4$} & $x$ & $x$ & & $x$ & $x$ & \\
\hline \multicolumn{2}{|l|}{$1-N-5$} & $x$ & $\dot{x}$ & & $x$ & $x$ & \\
\hline \multicolumn{2}{|l|}{$1-N-15$} & $x$ & $x$ & & $x$ & $x$ & \\
\hline \multicolumn{2}{|l|}{$1-N-16$} & $x$ & $x$ & & $x$ & $x$ & \\
\hline \multicolumn{2}{|l|}{$1-N-18$} & $x$ & $\ddot{x}$ & & $x$ & $x$ & \\
\hline \multicolumn{2}{|l|}{$1-N-19$} & $x$ & $x$ & & $x$ & $x$ & \\
\hline \multicolumn{2}{|l|}{$1-N-20$} & $x$ & $x$ & & $x$ & $x$ & \\
\hline \multicolumn{2}{|l|}{$1-N-21$} & $x$ & $x$ & & $x$ & $x$ & \\
\hline \multicolumn{2}{|l|}{$1-N-22$} & $x$ & $x$ & & $x$ & $\mathrm{x}$ & \\
\hline \multicolumn{2}{|l|}{$1-N-23$} & $x$ & $x$ & & $x$ & $\mathrm{x}$ & \\
\hline \multicolumn{2}{|l|}{$1-N-25$} & $x$ & $x$ & & $x$ & $x$ & \\
\hline $1-N-27$ & & $x$ & $x$ & & $x$ & $x$ & \\
\hline $1-N-30$ & & $x$ & $x$ & & $x$ & $x$ & \\
\hline $1-N-31$ & & $x$ & $\ddot{x}$ & & $x$ & $x$ & \\
\hline $1-N-32$ & & $\hat{x}$ & $\ddot{x}$ & & $\ddot{x}$ & $x$ & \\
\hline $1-N-33$ & & $x$ & $x$ & & $x$ & $x$ & \\
\hline $1-N-36$ & & $x$ & $x$ & & $x$ & $x$ & \\
\hline $1-N-37$ & & $x$ & $x$ & & $x$ & $x$ & \\
\hline $1-N-39$ & & $x$ & $x$ & & & $x$ & \\
\hline $1-N-45$ & & $x$ & $x$ & . & $x$ & $x$ & \\
\hline $1-N-49$ & & & $x$ & & $x$ & $x$ & \\
\hline $1-N-50$ & & & $\hat{x}$ & & $\hat{x}$ & $\hat{x}$ & \\
\hline $1-N-51$ & & & $\hat{x}$ & & $\hat{x}$ & $\ddot{x}$ & \\
\hline $1-N-52$ & & & $\hat{x}$ & & $\ddot{x}$ & $\ddot{x}$ & \\
\hline $2-E 13-8$ & $x$ & $x$ & & & & & \\
\hline $2-E 13-19$ & $x$ & & & & & & \\
\hline $2-E 16-2$ & $x$ & $x$ & $x$ & $x$ & $x$ & $x$ & \\
\hline $2-E 17-2$ & $x$ & & $x$ & $x$ & & $X$ & \\
\hline $2-E 17-8$ & $\hat{x}$ & $x$ & $\hat{x}$ & & $X$ & $\hat{x}$ & \\
\hline $2-E 17-12$ & $\hat{x}$ & $\hat{x}$ & $\hat{x}$ & $x$ & $\ddot{x}$ & $\ddot{x}$ & $\mathrm{x}$ \\
\hline $2-E 17-13$ & $x$ & $x$ & $x$ & $\ddot{x}$ & $x$ & $x$ & $x$ \\
\hline $2-E 23-2$ & $x$ & & $x$ & & & $x$ & \\
\hline $2-E 24-1$ & $x$ & $x$ & $x$ & & $x$ & $x$ & \\
\hline $2-E 24-2$ & $x$ & $\ddot{x}$ & $x$ & $x$ & $x$ & $x$ & \\
\hline $2-E 24-4$ & $x$ & $x$ & $x$ & & $x$ & $x$ & \\
\hline 2-E24-11 & $\hat{x}$ & $\hat{x}$ & $\hat{x}$ & & $\hat{x}$ & $\hat{x}$ & \\
\hline $2-E 24-13$ & $x$ & $x$ & $x$ & & $x$ & $x$ & \\
\hline $2-E 25-3$ & $\hat{x}$ & & & & & & \\
\hline $2-E 25-6$ & $\hat{x}$ & $x$ & $x$ & $x$ & $x$ & $x$ & \\
\hline $2-E 25-9$ & $\hat{x}$ & $x$ & $\dot{x}$ & $x$ & $\hat{x}$ & $x$ & \\
\hline $2-E 25-11$ & $\dot{x}$ & $x$ & $\ddot{x}$ & $\ddot{x}$ & $x$ & $\ddot{x}$ & \\
\hline
\end{tabular}


TABLE 4. (contd)

Well name BETA GAMMA NITRATE LOALPHA PU-ISO SR 90 TC-99 TRITIUM U U-CHEM U-ISO

\begin{tabular}{|c|c|c|c|c|c|c|c|c|c|c|c|}
\hline 2-E25-13 & $x$ & & $x$ & & & & & & & & \\
\hline $2-E 25-17$ & $x$ & $x$ & $x$ & $x$ & & $x$ & & $x$ & & & \\
\hline 2-E25-19 & $x$ & $x$ & $x$ & $x$ & & $x$ & & $x$ & & & \\
\hline $2-E 25-23$ & $x$ & $x$ & $x$ & $x$ & & & & $x$ & & & \\
\hline $2-E 25-24$ & $\dot{x}$ & $x$ & $x$ & $x$ & & & & $x$ & & & \\
\hline $2-E 26-2$ & $x$ & & $x$ & & & & & $x$ & & & \\
\hline $2-E 26-4$ & $x$ & & $x$ & & & & & $x$ & & & \\
\hline 2-E26-6 & $\hat{x}$ & $x$ & $\ddot{x}$ & $x$ & & $x$ & & $x$ & & & \\
\hline 2-E26-8 & & & $x$ & & & & & $x$ & & & \\
\hline $2-E 27-7$ & $x$ & & $x$ & $x$ & & & & & & & \\
\hline 2-E28-9 & $x$ & & & $x$ & & & & & & $x$ & \\
\hline $2-E 28-12$ & $x$ & $x$ & & & & & & $x$ & & & \\
\hline $2-E 28-16$ & $x$ & & & $x$ & & & & & & $x$ & \\
\hline 2-E28-18 & $x$ & $x$ & $x$ & $x$ & & & & $x$ & & $x$ & \\
\hline $2-E 28-23$ & $x$ & $x$ & $x$ & $x$ & & $x$ & & & & $\hat{x}$ & \\
\hline $2-E 28-24$ & $x$ & $x$ & & $x$ & & $x$ & & & & $x$ & \\
\hline 2-E28-25 & $\hat{x}$ & $\hat{x}$ & & $x$ & & $\hat{x}$ & & & & $\hat{x}$ & \\
\hline $2-E 33-7$ & $\hat{x}$ & $\hat{x}$ & & & & $\hat{x}$ & & & & & \\
\hline $2-E 33-9$ & $\ddot{x}$ & $\ddot{x}$ & $x$ & & & $x$ & & $x$ & & & \\
\hline $2-E 33-12$ & & $x$ & $x$ & & $x$ & $x$ & & $x$ & $x$ & & \\
\hline $2-E 33-14$ & & $x$ & $x$ & & $x$ & $x$ & & $x$ & $x$ & & \\
\hline $2-E 33-20$ & $x$ & $x$ & $\dot{x}$ & & $x$ & $x$ & & $x$ & $x$ & & \\
\hline $2-E 34-1$ & $x$ & $x$ & & $x$ & & & & $x$ & & & \\
\hline $2-W 10-1$ & $x$ & $x$ & & & & $x$ & & & & & \\
\hline $2-w 11-11$ & $\ddot{x}$ & $x$ & & $x$ & & $x$ & & & & & \\
\hline $2-W 11-18$ & $x$ & $x$ & & & & & & & & & \\
\hline $2-W 11-23$ & $\hat{x}$ & $\hat{x}$ & $x$ & $x$ & & $x$ & & & & & \\
\hline $2-W 11-24$ & $x$ & $\mathrm{x}$ & $x$ & $\hat{x}$ & & $x$ & & & & & \\
\hline $2-W 14-10$ & $x$ & $x$ & $\hat{x}$ & $\ddot{x}$ & & $\ddot{x}$ & & $x$ & & & \\
\hline $2-W 15-6$ & $\ddot{x}$ & & $\ddot{x}$ & $\ddot{x}$ & & & & & & & \\
\hline $2-W 18-5$ & $x$ & & & $x$ & & & & & & & \\
\hline $2-W 18-9$ & $x$ & & $x$ & $x$ & & & & & & & \\
\hline $2-W 18-15$ & $x$ & $x$ & $x$ & $x$ & & & & $x$ & & $x$ & \\
\hline $2-W 18-17$ & $x$ & $x$ & & $x$ & & & & $\mathrm{x}$ & & & \\
\hline 2-W18-18 & $\hat{x}$ & $\hat{x}$ & $x$ & $\hat{x}$ & & & & $\hat{x}$ & & & \\
\hline $2-W 18-20$ & $x$ & $x$ & $\hat{x}$ & $x$ & & & & $x$ & & & \\
\hline $2-W 19-2$ & $x$ & $x$ & $x$ & $x$ & & $x$ & $x$ & $x$ & & $x$ & \\
\hline $2-W 19-12$ & $x$ & $x$ & $x$ & $x$ & & $x$ & & $x$ & & $x$ & \\
\hline $2-W 19-17$ & $x$ & & $x$ & $x$ & & & & & & $x$ & \\
\hline $2-W 19-18$ & $x$ & & $x$ & $x$ & & & & & & $x$ & \\
\hline 2-W19-19 & $x$ & $x$ & $x$ & $x$ & & $x$ & & $x$ & & $x$ & \\
\hline $2-W 19-20$ & $x$ & $x$ & $\dot{x}$ & $x$ & & $x$ & & $x$ & & $x$ & \\
\hline $2-W 19-21$ & $x$ & $x$ & $x$ & $x$ & $x$ & $x$ & & $x$ & & $x$ & $\mathrm{x}$ \\
\hline $2-W 19-23$ & $\hat{x}$ & $\hat{x}$ & $\hat{x}$ & $\hat{x}$ & $\hat{x}$ & $\hat{x}$ & & $\ddot{x}$ & & $x$ & $\mathrm{x}$ \\
\hline $2-W 19-24$ & $\hat{x}$ & $\hat{x}$ & $\hat{x}$ & $\hat{x}$ & $\hat{x}$ & $x$ & $x$ & $x$ & & $\hat{x}$ & $\ddot{x}$ \\
\hline $2-W 19-25$ & $\hat{x}$ & $x$ & $x$ & $x$ & $x$ & $x$ & $x$ & $x$ & & $\dot{x}$ & $x$ \\
\hline $2-W 19-26$ & $x$ & $x$ & $x$ & $x$ & $x$ & $x$ & & $x$ & & $x$ & $x$ \\
\hline
\end{tabular}


TABLE 4. (contd)

Well name BETA GAMMA NITRATE LOALPHA PU-ISO SR 90 TC-99 TRITIUM U U-CHEM U-ISO

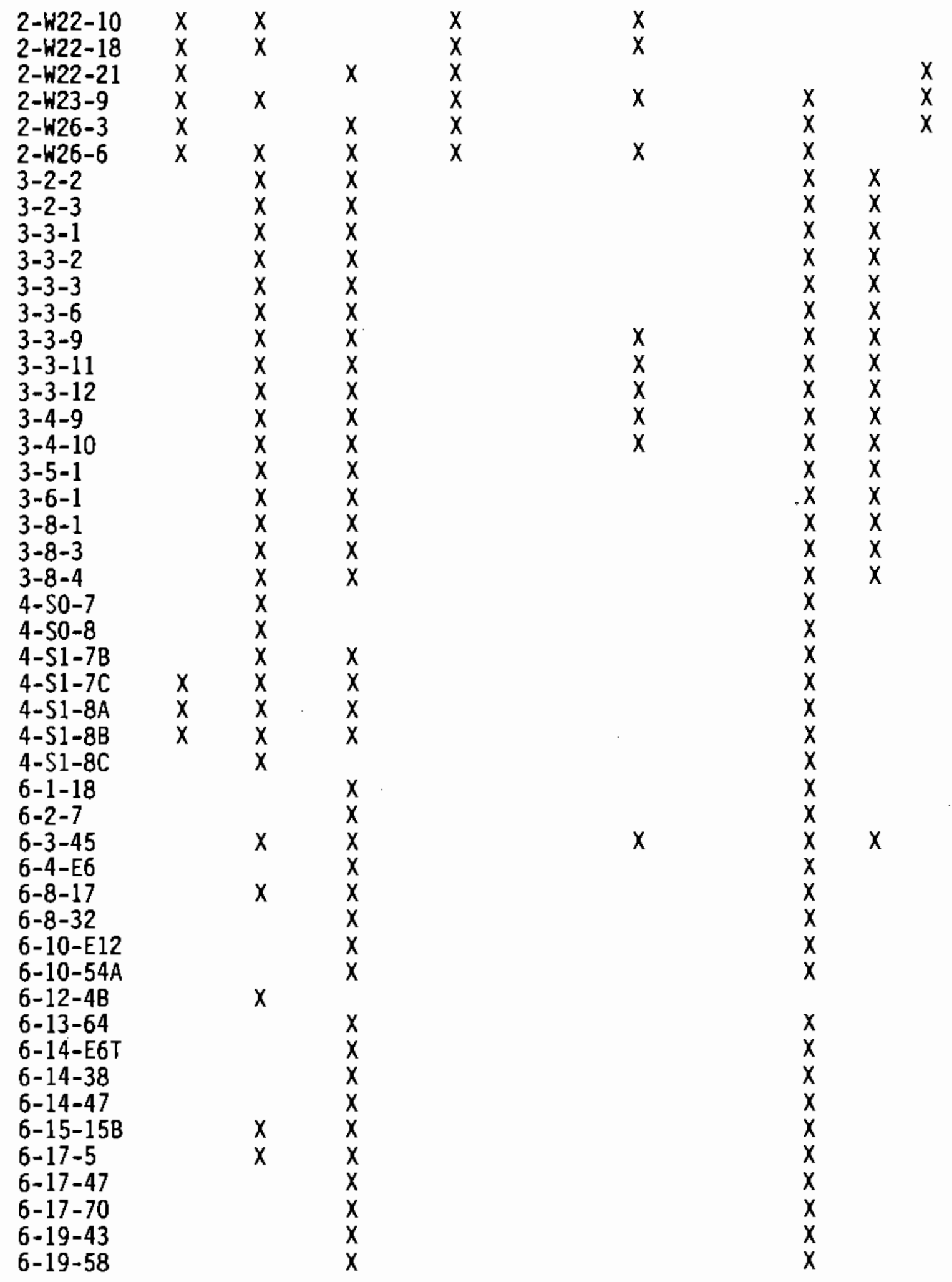


IABLE 4. (contd)

Well name BETA GAMMA NITRATE LOALPHA PU-ISO SR 90 TC-99 TRITIUM U U-CHEM U-ISO

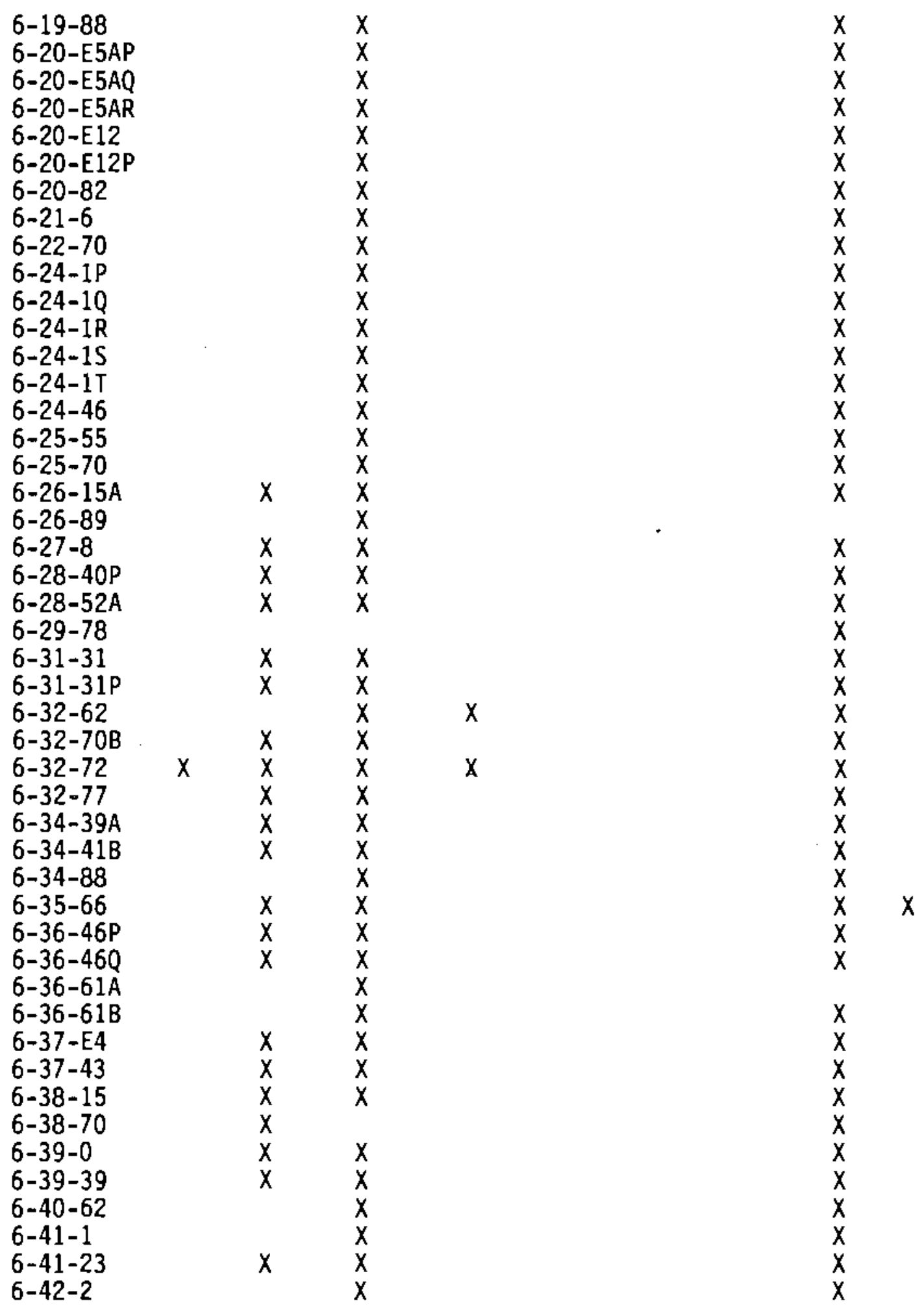


TABLE 4. (contd) Well name BETA GAMMA NITRATE LOALPHA PU-ISO SR 90 TC-99 TRITIUM U U-CHEM U-ISO
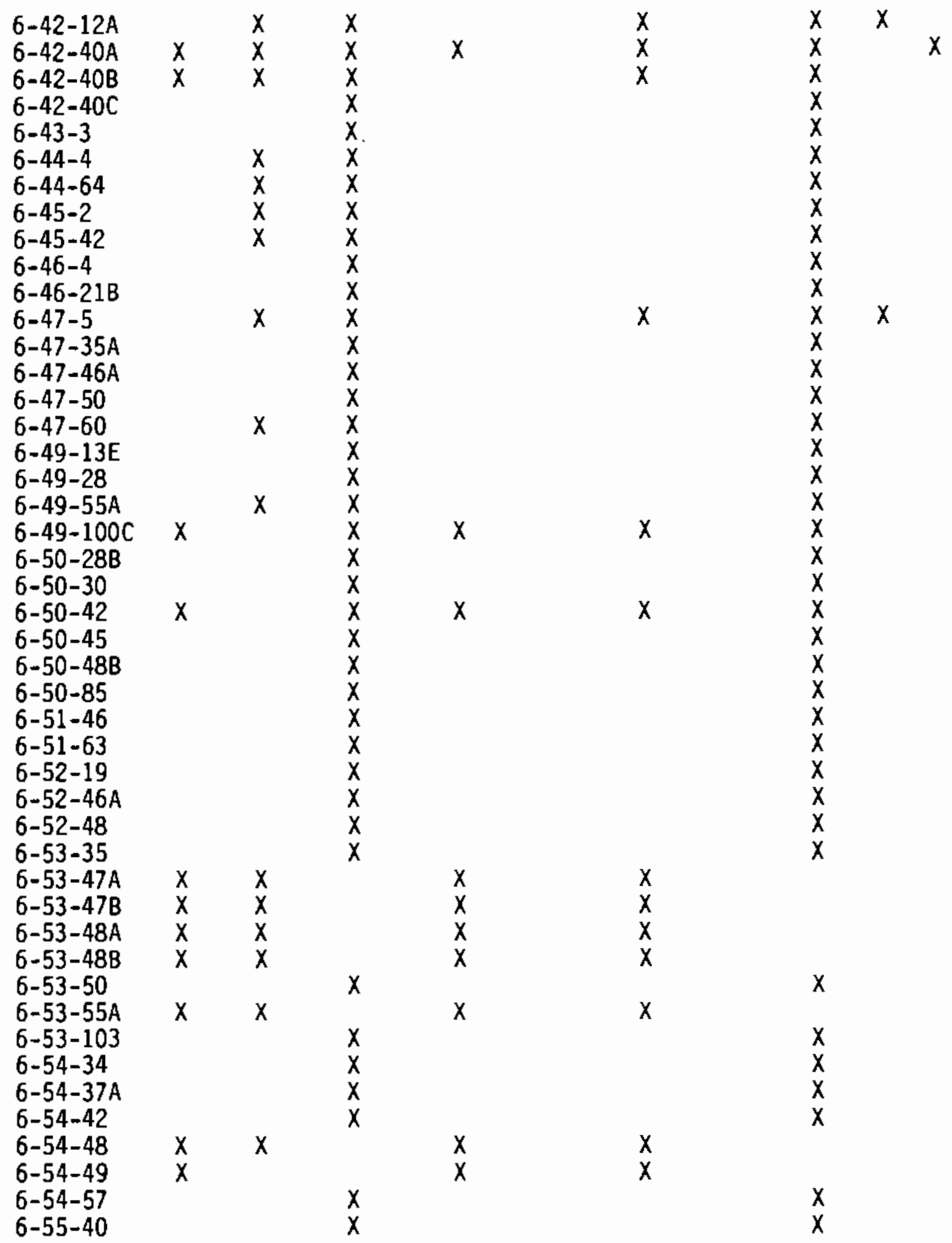
TABLE 4. (contd)

Well name BETA GAMMA NITRATE LOALPHA PU-ISO SR 90 TC-99 TRITIUM U U-CHEM U-ISO

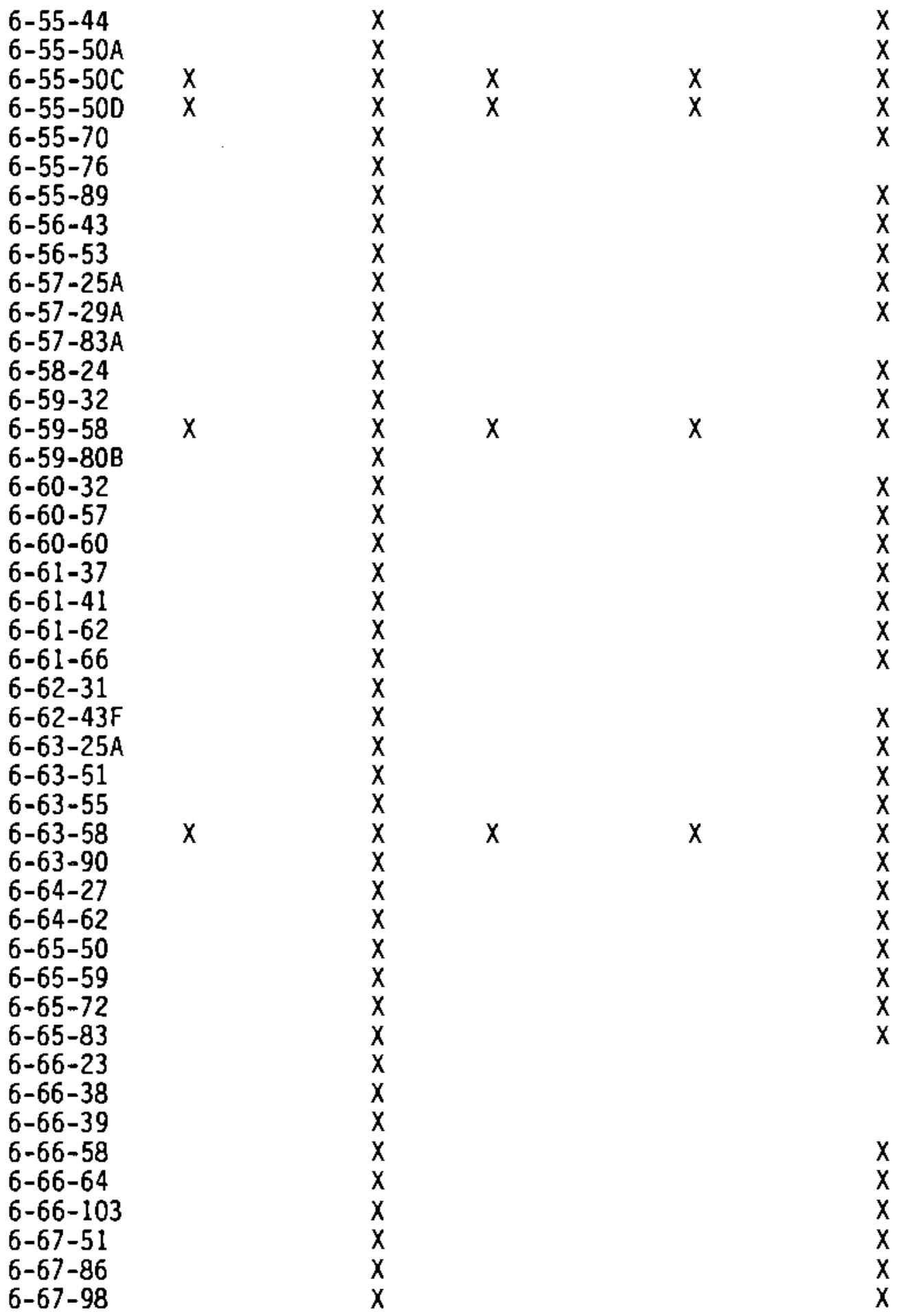


TABLE 4. (contd)

Well name BETA GAMMA NITRATE LOALPHA PU-ISO SR-90 TC-99 TRITIUM U U-CHEM U-ISO

$6-68-105$
$6-69-38$
$6-70-68$
$6-71-30$
$6-71-52$
$6-71-77$
$6-72-73$
$6-72-88$
$6-72-92$
$6-73-61$
$6-74-44$
$6-77-36$
$6-78-62$
$6-80-43 P$
$6-80-430$
$6-80-43 R$
$6-80-43 S$
$6-81-58$
$6-84-35 A 0$
$6-87-55$
$6-96-49$
$6-97-43$
$6-97-51 A$
$6-101-48 B$
$6-S 3-E 12$
$6-S 6-E 4 B$
$6-S 6-E 4 D$
$6-S 7-34$
$6-S 11 E 12 A$
$6-S 11 E 12 A P$
$6-S 12-29$
$6-S 14-20 A$
$6-S 18-51$
$6-S 19-11$
$6-S 24-19$
$6-S 27-E 14$
$6-\$ 28-E 0$
$6-531-1 P$
6
6

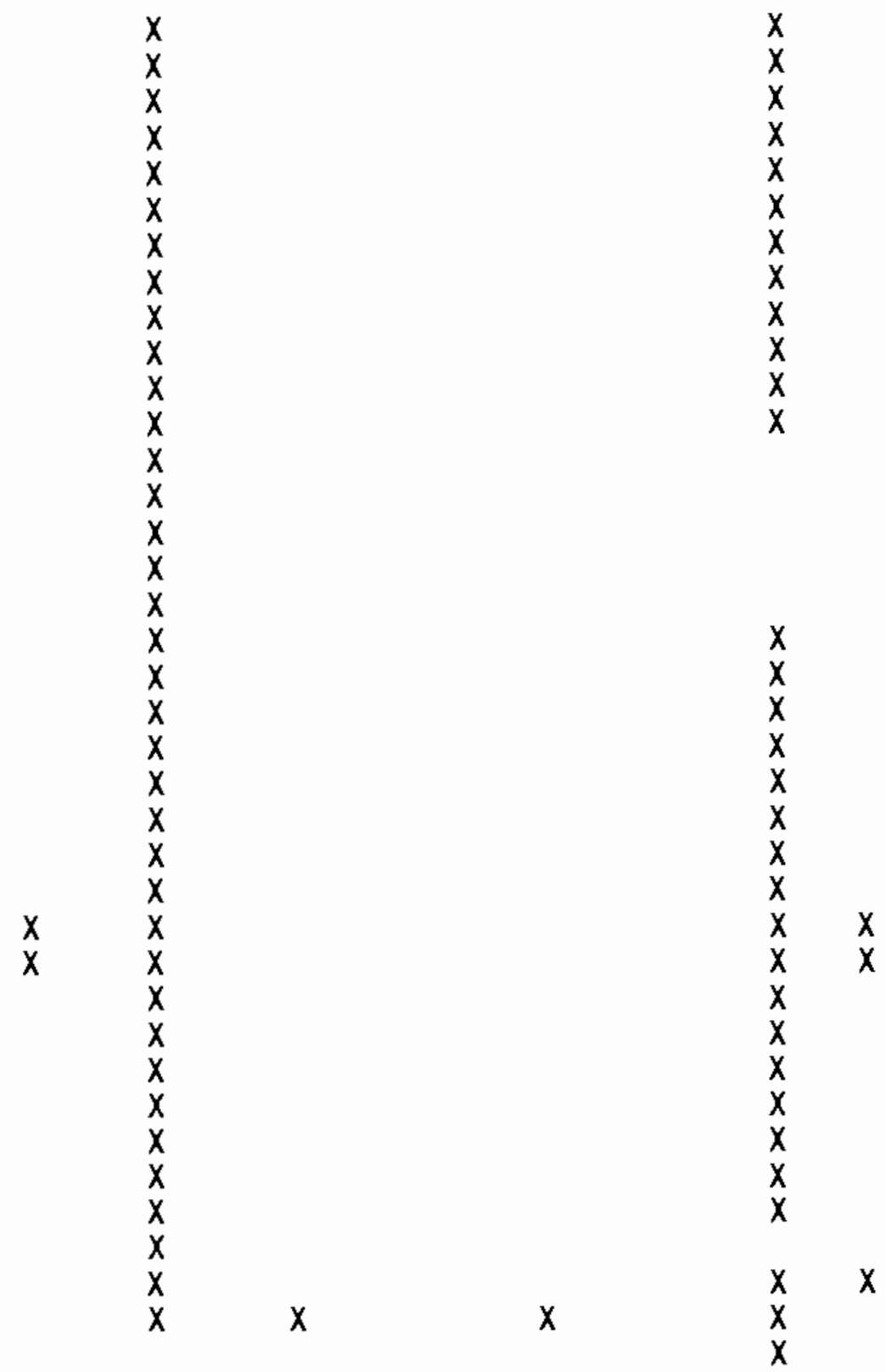




\section{RESULTS}

Resuits of both PNL and WHC ground-water monitoring programs are discussed in this section. A historical perspective for all contaminants can be found in past environmental monitoring reports by PNL and the operating contractor (previously Rockwell Hanford Operations). The most recent reports are by PNL (1987) and Law, Serkowski, and Schatz (1987).

Because of their high concentrations, regulatory implications, or widespread distribution, the following contaminants in Hanford ground water have been identified for discussion here: 1) carbon tetrachloride in the 200 West Area; 2) cyanide in and north of the 200 East Area; 3) hexavalent chromium in the $100 \mathrm{~B}, 100 \mathrm{D}, 100 \mathrm{~K}$, and $100 \mathrm{H}$ Areas; 4) chlorinated hydrocarbons near the Central Landfi11; 5) uranium at the $216-\mathrm{U}-1$ and $216-\mathrm{U}-2$ cribs in the 200 west Area; 6) tritium across the site; and 7) nitrate across the site. The potential implications of several other observations are also discussed briefly. Although ground water is not used as a public drinking water supply, all results are discussed relative to the EPA drinking water standard (DWS) and to the DOE derived concentration guide (DCG), where appropriate (see Appendix B). Radionuclide concentrations in Hanford ground water are under administrative control by DOE Order 5480.1 which contains the DCGs. Table 5 lists estimated background levels for selected constituents in areas unaffected by Hanford Site operations.

\section{CARBON TETRACHLORIDE IN THE 200 WEST AREA}

Several wells have shown measurable concentrations of carbon tetrachloride $\left(\mathrm{CCl}_{4}\right)$ in the environs of the 200 West Area. The contamination has been detected beneath most of the 200 west Area, except for the southeast corner near the Reduction Oxidation Plant (REDOX) (Figure 6). The plume extends a short distance into the 600 Area on the east and west sides of the 200 West Area. The extent of spread to the north is not wide; however, a concentration of $220 \mathrm{ppb}$ was found near the north fence line (in well 2-w6-1), so some spread beyond the fence is likely. For $\mathrm{CCl}_{4}$, the maximum concentration limit (MCL), or target concentration, for remediation under the Comprehensive Environmental Response, Compensation and Liability 
TABLE 5. Estimated Background Levels for Selected Constituents in Hanford Ground Water

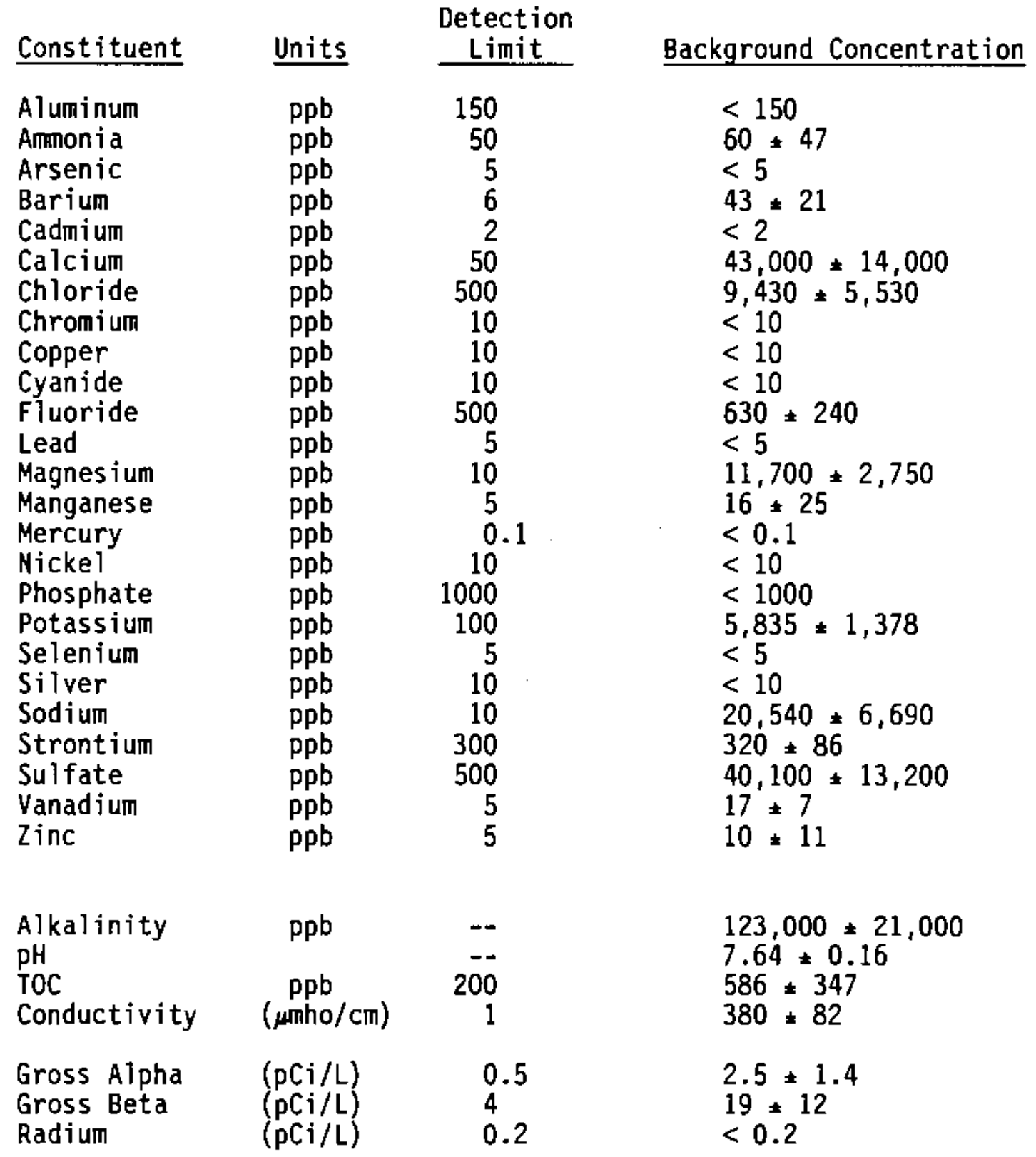

Act (CERCLA) and the Superfund Amendments and Reauthorization Act (SARA) of 1986 is $5 \mathrm{ppb}$. A maximum concentration of 3,210 ppb (well 2-W15-11) was found near the Plutonium Finishing Plant (PFP) (Figure 6). Also, trichloroethylene was found in seven wells in the 200 west Area at concentrations ranging from 10 to $38 \mathrm{ppb}$. This species appears to be associated with the carbon tetrachloride plume. 
The source of the $\mathrm{CCl}_{4}$ is suspected to be past disposal of waste to the soil at the PFP. During operations that began in 1955 and extended through 1973, degraded solvents from various solvent extraction processes were disposed to the soil column. The primary carrier used in those solventextraction processes was $\mathrm{CCl}_{4}$. The practice of disposing of solvent to the soil was discontinued in 1973. Current plant operation employs a method of treating and recycling in which degraded solvent is packaged in sorbent material and sent to a retrievable storage facility in the 200 West Area soljd-waste burial ground.

Many of the soil column facilities (cribs) used by the PFP through 1973 received solvent material. Most of these facilities are near the inactive $U$ Pond location. The flow of ground water in this area is radially outward from the ground-water mound that still exists beneath the $U$ Pond location. This flow pattern may account for the distribution of contamination found beneath the 200 West Area.

No obvious, immediate public health implications are associated with the $\mathrm{CCl}_{4}$ plume. The ground water in question is not currently a source of public drinking water and is not likely to become one in the foreseeable future. The edge of the plume is several kilometers from the Columbia River. Dispersion and dilution are likely to reduce the concentrations of $\mathrm{CCl}_{4}$ significantly by the time they reach the river. Therefore, the long-term public health issue is associated primarily with eventual site abandonment, operational concerns, and associated regulatory considerations under CERCLA/SARA or the RCRA $3004(u)$ statute on continuing releases to the environment.

\section{CYANIDE IN AND NORTH OF THE 200 EAST AREA}

Sampling for chemicals in March 1987 included several 600-Area wells directly north of the 200 East Area. Cyanide was detected in two wells, 6-49-57 and 6-50-53. More extensive sampling during this quarter (April to June) confirmed those preliminary results. A third well, 2-E33-5, inside the 200 East Area was also found to be contaminated with cyanide. In addition, a field test taken during sampling by the DOE/HQ-EAT has indicated the presence of free cyanide in well 6-50-53, just north of the 200 East Area (Figure 2). The presence of cyanide was accompanied by corresponding increases in 
cobalt-60 $\left({ }^{60} \mathrm{Co}\right)$ and gross beta activity in the ground water over a one-year period. Although ${ }^{80} \mathrm{Co}$ is normally relatively immobile in the Hanford subsurface environment, it appears to be chemically complexed and mobilized by cyanide. The maximum concentration of cyanide detected was $460 \mathrm{ppb}$. The extent of the contamination is currently limited to about $2.5 \mathrm{~km}^{2}\left(1 \mathrm{mi}^{2}\right)$; however, the exact extent of the plume is difficult to determine because there are few wells in the area. No fixed MCL or DWS for cyanide currently exists.

The cyanide distribution indicates a plume with its probable source at the BY Cribs (Figure 6). Cyanide is a waste product of the cesium precipitation processes that took place during uranium reclamation operations in the mid-1950s. The reagent used, nickel ferrocyanide, was added in excess of the stoichiometric ratio. The solids and liquids of the waste from this process were separated by cascading the supernate through a series of storage tanks. The supernatant liquor from the final tank was sent to a group of cribs, including the BY Cribs, BX Trenches, BC Cribs, and BC Trenches (Figure 6).

Unambiguous identification of free cyanide has not yet been made by either UST or PNL. The chemical analysis method employed by UST and DOE/HQ-EAT has been reported to be unresponsive to iron complexes of cyanide (USEPA 1982); however, a series of ferrocyanide and ferricyanide standards recently analyzed by UST showed quantitative recovery of the added complexed cyanide. The field test method was found to respond to ferricyanide, but not to ferrocyanide.

Although this was the first analysis of cyanide performed in the 600 Area, historical results for other contaminants indicate that a plume has moved into the area of well 6-50-53 within the last 18 months. Recent changes in waste-management practices, primarily increased liquid disposal to $B$ Pond because of the restart of the Plutonium Uranium Extraction Plant (PUREX) operation (Figure 5), and decreased disposal to Gable Mountain Pond (Law, Serkowski, and Schatz 1987) (Figure 2), has caused some of the flow paths from beneath the 200 East Area to shift to the north. 
No obvious, immediate public health hazards are associated with the cyanide plume. The ground water in the area is not currently a source of drinking water and is not likely to become a source in the foreseeable future. The edge of the plume is several kilometers from the Columbia River. Migration rates to the river are likely to be similar to those of other anionic species or tritium, and depend heavily on waste-management practices in the future. Dispersion and dilution should significantly reduce the cyanide concentration by the time the plume reaches the river. In addition, cyanide may be naturally oxidized to cyanate by bacterial or chemical oxidation. Therefore, long-term public health issues are associated primarily with eventual site abandonment and associated regulatory considerations under CERCLA/SARA or RCRA 3004(u).

HEXAVALENT CHROMIUM IN THE 100 AREAS

During operation of the production reactors in the 100 Areas, sodium dichromate was used to control oxidation of aluminum parts. Chromic acid was also used to decontaminate dumny fuel-elements. 0isposal of these materials to a number of cribs and other liquid-disposal facilities has resilted in ground-water contamination throughout the 100 Areas. All wells in the $100 \mathrm{H}$ Area showed measurable concentrations of hexavalent chromium. In most cases, measurable concentrations were above the DWS of $50 \mathrm{ppb}$. The highest level of chromium contamination $(1,690 \mathrm{ppb})$ was found in well 1-D5-12 in the 1000 Area. Some chromium contamination was also observed in the $100 \mathrm{~K}$ and $100 \mathrm{~B}$ Areas. Although no chromium contamination was observed in the $100 \mathrm{~F}$ Area, only a few wells have been sampled there. Chromium usage and waste disposal at the $100 \mathrm{~F}$ Area were similar to those in the other 100 Areas. No chromium contamination has been observed in the $100 \mathrm{~N}$ Area because the use of sodium dichromate was discontinued in the mid-1970s at the $N$ Reactor.

The ground water affected by the chromium contanination is not currently used as a drinking-water supply. Chromium entering the Columbia River is rapidly diluted to insignificant levels. The primary public health issues for the ground water are eventual site abandonment and associated regulatory considerations under CERCLA/SARA or RCRA $3004(u)$. 
CHLORINATED HYDROCARBONS NEAR THE CENTRAL LANDFILL

Ground-water monitoring conducted by PNL in March 1987 demonstrated the presence of measurable amounts (21 ppb) of 1,1,1-trichloroethane in well 6-24-33 located approximately $300 \mathrm{~m}$ (1000 ft) downgradient from the Hanford Site Central Landfill (Figure 2). As a result of that finding, volatile organic analyses were performed on samples from the recently completed compliance monitoring wells at the SWL and NRDW, which together comprise the Central Landfill. Several chlorinated hydrocarbons, of which 1,1,1-trichloroethane was the most abundant, were found in all six SWL wells; the highest concentration was $56 \mathrm{ppb}$. A trace of 1,1,1-trichloroethane was also found in the NRDW well closest to the SWL. Results were below detection limits in the other six NROW wells. The areal distribution of the contaminants suggest that the SWL was the source. The SWL wells also contained lesser amounts of three other constituents: 1,1,2-trichloroethylene, 1,1-dichloroethane, and perchloroethylene. The relative proportions of the four components were approximately the same for those SWL wells with the highest concentrations and for well 6-24-33. A second set of samples taken in April 1987 confirmed these findings. The specific source of the contamination at the SWL is unknown; several possible sources are under investigation.

The ground water affected by the chlorinated hydrocarbon contamination is not currently used as a drinking-water supply. The levels found are relatively low and the extent of contamination limited. Public health concerns are minor because by the time the ground water reaches the Columbia River or other areas of water use, dispersion and dilution will have significantly reduced the concentrations. However, this contamination may impact the issuance of an operating permit for the SWL under solid-waste disposal regulations. The closed section of the SWL was operated before the current standards and applicable federal and state regulations were developed. Landfill operation as of May 1987 complied with all current federal and local requirements. The regulatory ramifications are unknown at this time. 
URANIUM AT THE 216-U-1 AND 216-U-2 CRIBS IN THE 200 WEST AREA

Ground-water monitoring near the 216-U-1 and 216-U-2 cribs in February 1985 detected increases in uranium concentrations from 200 to $72,000 \mathrm{pCi} / \mathrm{L}$ and in nitrate concentrations from 6 to $1,500 \mathrm{ppm}$ in the previous 2 months (Law, Serkowski, and Schatz 1987). The 216-U-1 and 216-U-2 cribs received an estimated $4,000 \mathrm{~kg}$ of uranium and 1.2 million $\mathrm{kg}$ of nitrate between 1952 and 1967. Perched water resulting from 1iquid discharges to the nearby 216-U-16 crib, which began receiving wastes in July 1984, provided the liquid that transported the uranium from the unsaturated zone beneath the 216-U-1 and 216-U-2 cribs to the water table (see Figure 6 for crib locations). Unsealed well casings and discontinuities in a confining layer are thought to have created a pathway for the uranium-contaminated liquid to reach the ground water. To prevent further contamination, three existing wells were grouted, and the disposal of waste water to the $216-U-16$ crib was discontinued. From June to November 1985, ground water was pumped from an existing well and uranium was removed. The treatment reduced the uranium concentration in the pumping well from 72,000 to $17,000 \mathrm{pCi} / \mathrm{L}$. Nitrates were not removed from the pumped water. Since November 1985, the uranium and nitrate concentrations have remained constant, although contamination continues to spread with general ground-water movement.

\section{TRITIUM ACROSS THE SITE}

Large amounts of tritium are released to the ground annually. For example, $7,040 \mathrm{Ci}$ of tritium were released to the ground from 200 Area facilities during 1986 (Law, Serkowski, and Schatz 1987). Approximately 25 billion liters of liquid effluent were released to the ground in the same area during 1986 (PNL 1987).

Tritium Monitoring Results for 1986

Past monitoring results have shown that tritium is present in ground water, and it appears to be the most mobile radionuclide at the Hanford Site. As a result, tritium reflects the extent of contamination in the ground water from fuel production and reprocessing operations. A contour map of the 1986 
distribution of average tritium concentrations in the unconfined aquifer, resulting from 40 years of Site operations, has been provided elsewhere (PNL 1987, Fig. 3.12).

Tritium Monitoring Results During April through June 1987

Tritium concentrations greater than the DWS of $20,000 \mathrm{pCi} / \mathrm{L}$ were detected in portions of the 100B, 100F, 100K, 100N, 200 East, 200 West, and 600 Areas.

As in the past, the highest tritium concentration within the 100 Areas was observed in well $1-K-30(634,000 \mathrm{pCi} / \mathrm{L})$. The concentrations in that well during the first half of 1987 were similar to those detected in 1986. Tritium concentrations in the $100 \mathrm{~F}$ Area appear to be stabilizing or declining; concentrations in wells 1-F5-4 and 1-F8-1, which had risen above the DWS in 1986, decreased, to concentrations below the DWS during this quarter. Only the concentration in well 1-B4-1 in the $100 \mathrm{~B}$ Area $(20,500 \mathrm{pCi} / \mathrm{L})$ exceeded the DWS during the quarter.

Changes in the tritium distribution beneath the 100N Area (Figure 4) were evident, presumably a result of the shutdown of reactor operations in January. Tritium concentrations in all wells near the $1325 \mathrm{~N}$ Liquid Waste Disposal Facility (LWDF) (Figure 4) and in most other 100N-Area wells decreased during the first half of 1987. The only wells that experienced significant increases in tritium concentrations were wells $1-\mathrm{N}-2,1-\mathrm{N}-3,1-$ $\mathrm{N}-4,1-\mathrm{N}-5$, and $1-\mathrm{N}-14$ (Figure 9). In addition, samples collected in May from wells $1-\mathrm{N}-49,1-\mathrm{N}-50,1-\mathrm{N}-51$, and $1-\mathrm{N}-52$ contained relatively high tritium concentrations, between 50,000 and $200,000 \mathrm{pCi} / \mathrm{L}$; no conclusions concerning changes in concentrations at these wells can be made, because they were sampled only once.

Continued movement of tritium plumes emanating from the 200 Areas may be inferred from variations in concentrations of tritium in the wells within those plumes. The widespread plume that extends from the southeast portion of the 200 East Area to the Columbia River continues to move slowly, i.e., over tens of years, toward the river. Tritium concentrations in wells near the center of the plume (e.g., wells 6-26-15A, 6-27-8, 6-31-31, and 6-32-22) continue to decrease (Figure 10), while concentrations in wells nearer the 


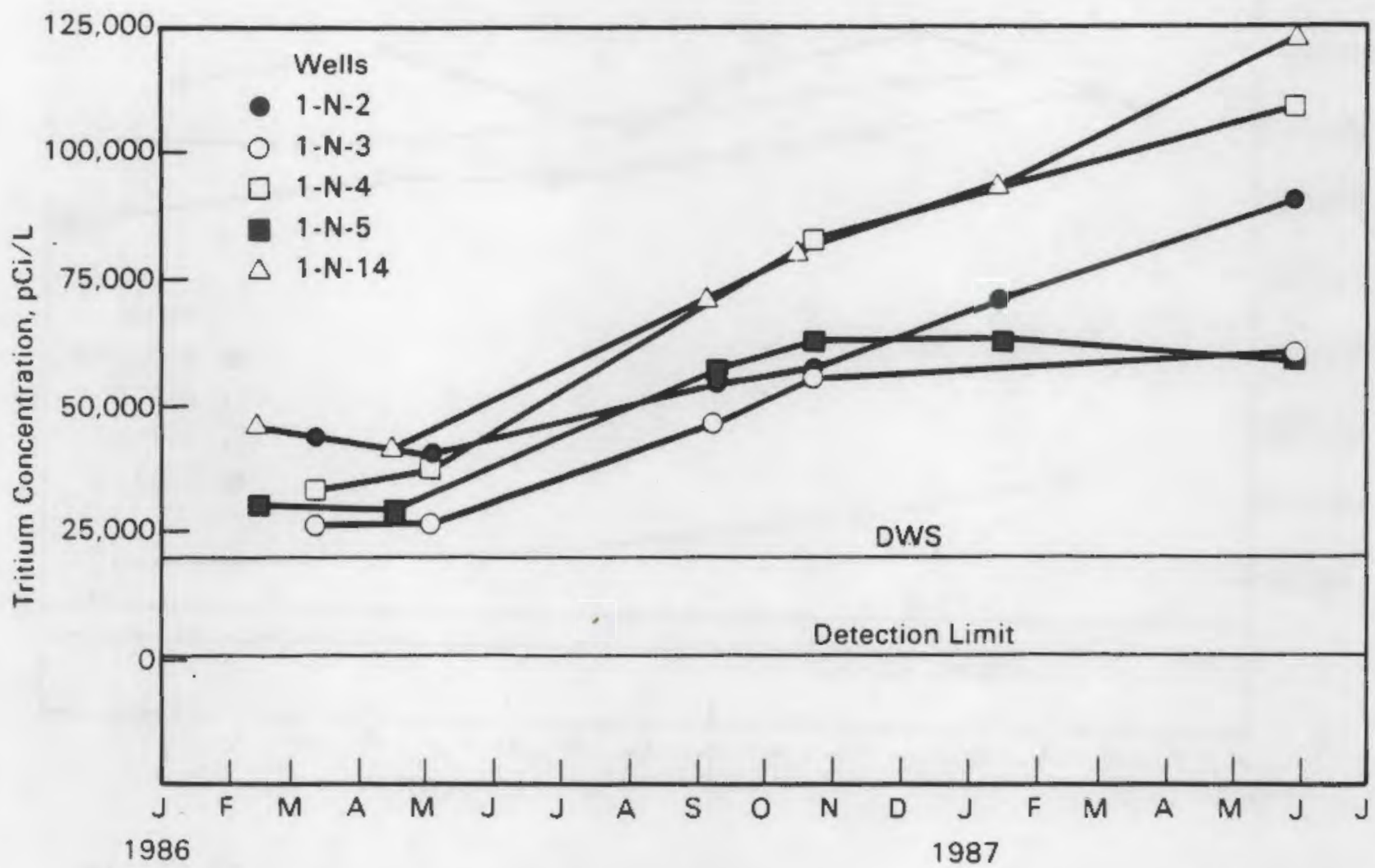

FIGURE 9. Tritium Concentrations in Wells $1-N-2,1-N-3,1-N-4,1-N-5$, and $1-\mathrm{N}-14$, in 1986 and the First Half of 1987

river (e.g., 6-29-4, 6-35-9, 6-39-0, and 6-40-1) remain at relatively high levels or continue to increase, ranging from about 140,000 to $240,000 \mathrm{pCi} / \mathrm{L}$. In addition, this plume appears to be extending farther to the south. Tritium concentrations in wel1s 6-S3-E12, 6-S11-E12A, and 6-S19-E13 increased to concentrations of 5,$140 ; 3,190$; and $5,540 \mathrm{pCi} / \mathrm{L}$ from average concentrations of 4,$400 ; 1,900$; and $4,700 \mathrm{pCi} / \mathrm{L}$, respectively in 1986 .

Tritium concentrations in wells near several facilities receiving waste from PUREX and in some 600-Area wells downgradient from the plant suggest that a plume of increased concentration is emanating from the 200 East Area. Tritium concentrations greater than the $\mathrm{OCG}(2,000,000 \mathrm{pCi} / \mathrm{L})$ are present in wells near the 216-A-10 and 216-A-37-1 cribs, which receive process condensate effluents from PUREX and the 242-A Evaporator, respectively. These wells include 2-E17-1, 2-E17-5, 2-E17-8, 2-E17-9, 2-E17-12, 2-E17-13, 2-E24-1 and 2-E24-2 near the 216-A-10 crib; and well 2-E25-19 near the 216-A-37-1 


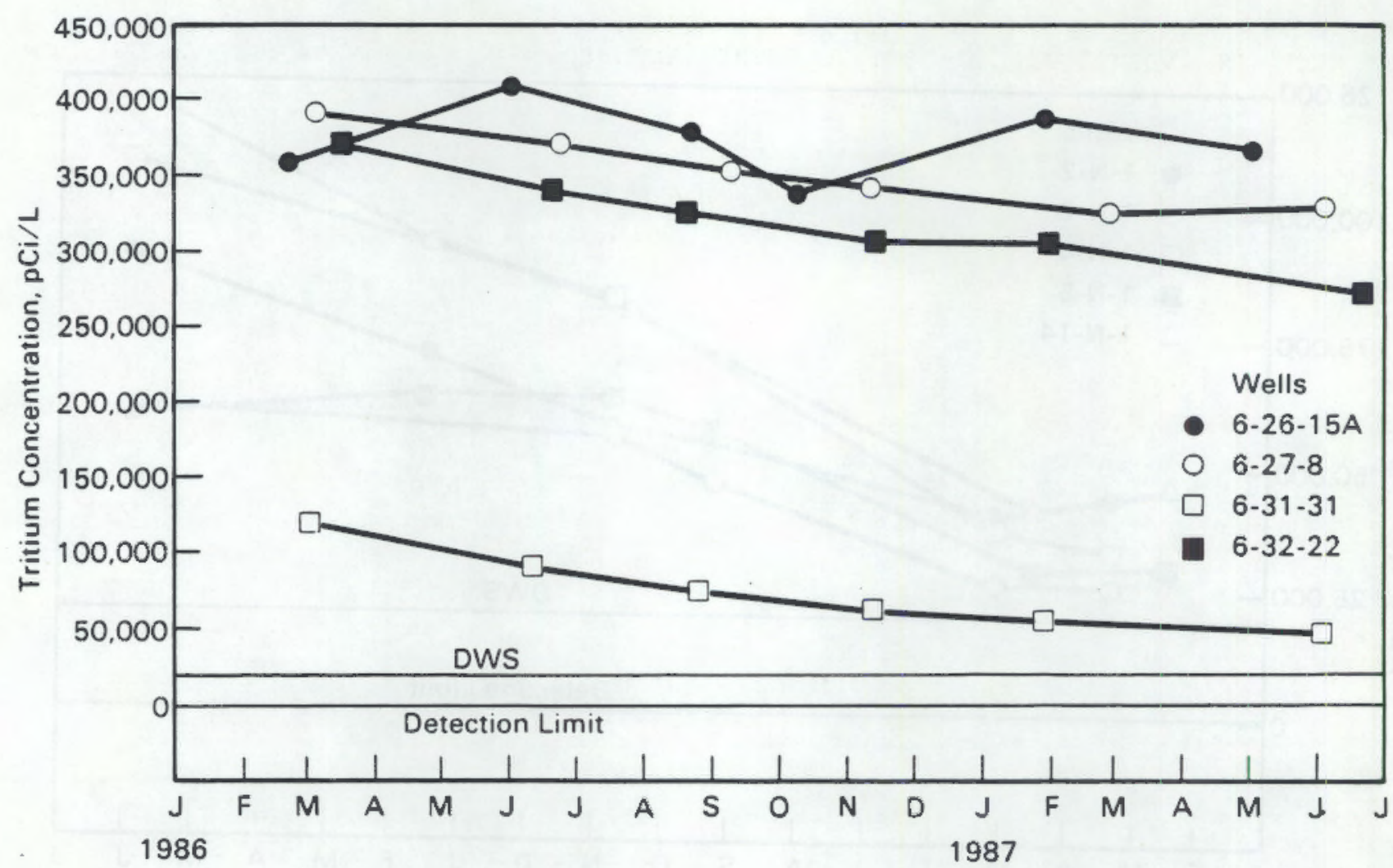

FIGURE 10. Tritium Concentrations in Wells 6-26-15A, 6-27-8, 6-31-31, and 6-32-22, in 1986 and the First Half of 1987

crib (Figure 5). Concentrations in wells near the 216-A-10 crib ranged from less than the $D C G$ to $11,300,000 \mathrm{pCi} / \mathrm{L}$ (at well 2-E24-1) during the quarter. Well 2-E25-19 near the 216-A-37-1 crib had a maximum concentration of $6,560,000 \mathrm{pCi} / \mathrm{L}$. These increases in tritium were expected (U.S. DOE 1983).

Tritium concentrations in downgradient wells 6-32-43 (sampled last quarter) and 6-33-42 have been increasing since mid-1986, up to 259,000 and $280,000 \mathrm{pCi} / \mathrm{L}$, respectively, this quarter. Tritium concentrations in nearby wells 6-34-42 and 6-34-41B have recently increased several thousand $\mathrm{pCi} / \mathrm{L}$ to 88,500 and $56,100 \mathrm{pCi} / \mathrm{L}$, respectively. Because only two wells (6-36-46P and 6-36-46Q, which are completed in a deeper aquifer) exist in the unconfined aquifer between these four wells and the 200 East Area, migration of this plume towards the southeast may be undetected.

The tritium plume that emanated from REDOX in the southeast corner of the 200 West Area (Figure 6) continues to spread slowly northward. This 
movement is demonstrated by the apparent increase in tritium concentrations in well 6-40-62, from $62,800 \mathrm{pCi} / \mathrm{L}$ (last quarter) to $65,100 \mathrm{pCi} / \mathrm{L}$, and by the continued decrease in concentrations in well 6-35-70, from $1,440,000 \mathrm{pCi} / \mathrm{L}$ (1ast quarter) to $1,330,000 \mathrm{pCi} / \mathrm{L}$. Tritium concentrations in wel1 2-W22-9 appear to be decreasing slowly, indicating that the small area of tritium concentrations greater than the $\operatorname{DCG}(2,000,000 \mathrm{pCi} / \mathrm{L})$ within this plume may be moving away from the 200 West Area. An additional area of relatively high concentrations of tritium is present near the $216-\mathrm{S}-25$ crib in the southwest corner of the 200 West Area, as indicated by the concentrations in wells 2-W23-9 and 2-W23-10, which remain well above the DWS $(20,000 \mathrm{pCi} / \mathrm{L})$.

Northward movement of the tritium plume in the northern portion of the 200 West Area is indicated by the continued increase in concentrations in well 2-W6-1, to over $52,000 \mathrm{pCi} / \mathrm{L}$, and the concurrent decrease in concentrations in wells 2-W10-5, 2-W14-2, 2-W14-5, 2-W14-6, and 2-W15-4.

Tritium concentrations within the plume between Gable Butte and Gable Mountain at wells 6-60-60, 6-61-62, 6-64-62, and 6-66-64 (Figure 2) continue to increase slowly, ranging from 6,000 to $8,000 \mathrm{pCi} / \mathrm{L}$. In addition, wells $6-65-72,6-71-77$, and $6-72-73$, which lie between this plume and the Columbia River to the northwest, are displaying increased tritium concentrations, between 3,000 and $4,000 \mathrm{pCi} / \mathrm{L}$. Whether these increases are related to this tritium plume or possibly to plumes emanating from facilities in other locations is unknown. Tritium may be emanating from retired fuel storage basins north of the 200 Areas or past disposal sites near the 100B Area. Additional monitoring wells may be needed in this area to answer these questions.

\section{NITRATE ACROSS THE SITE}

The EPA does not consider nitrate to be a hazardous substance under CERCLA scoring for Superfund remedial action because so many nonoperational sources of nitrate exist across the country (e.g., agricultural, nonpoint sources). Nitrate contamination is a concern at Hanford, however, because of its source (Hanford operations) and wide distribution.

Nitrate is associated with process condensate and other liquid wastes discharged to the ground (PNL 1987). The extent of nitrate contamination in 
the unconfined aquifer reflects the extensive use of nitric acid in chemical reprocessing and decontamination operations.

Nitrate Monitoring Results for 1986

PNL (1987) has reported that nitrate plumes are shaped differently from the tritium plumes, primarily because nitrate concentrations are not reduced with time by radioactive decay, as are tritium concentrations. A contour map of 1986 average nitrate concentrations in the unconfined aquifer has been presented by PNL (1987). The nitrate plumes are also derived from a variety of sources, both natural and manmade. As a result, nitrate is not as easily traced back to isolated sources.

Nitrate Monitoring Results During April through June 1987

Nitrate is analyzed for most wells in all monitoring networks and was measured at concentrations greater than the DWS [45 ppm(a) as nitrate ion] in wells in all of the 100 Areas, except for the 100B Area, and in the 200 Areas.

Nitrate concentrations in 1000 Area wells were similar to 1986 levels. We1ls 1-02-5 and 1-D5-12, with average concentrations of 88.7 and $73.4 \mathrm{ppm}$, respectively, remained above the DWS.

Three of the wells sampled in the 100F area remained above the DWS. Concentrations in well 1-F8-1 continued to increase, reaching a concentration of $191 \mathrm{ppm}$ in June. Concentrations in wells 1-F7-1 and 1-F8-2 averaged 88 and $99 \mathrm{ppm}$, respectively. Well 1-F5-4 showed a concentration of $52.5 \mathrm{ppm}$.

Wells $1-\mathrm{K}-11,1-\mathrm{K}-19$, and $1-\mathrm{K}-30$ in the $100 \mathrm{~K}$ Area contained nitrate in concentrations above the DWS. Concentrations averaged 50,60, and $55 \mathrm{ppm}$, respectively.

Nitrate concentrations greater than the DWS were measured in six $100 \mathrm{H}$ Area wells: $1-\mathrm{H} 3-1,1-\mathrm{H} 4-3,1-\mathrm{H} 4-4,1-\mathrm{H} 4-9,1-\mathrm{H} 4-12 \mathrm{~A}$, and 1-H4-12B

(Figures 11 and 12). All but well 1-H3-1 are located between the 183-H Solar Evaporation Basins (Figure 3 ) and the Columbia River. Nitrate concentrations in well 1-H4-3 have declined slightly from 1986 levels, while those in

(a) $\mathrm{ppm}=\mathrm{ppb} / 1000$. 


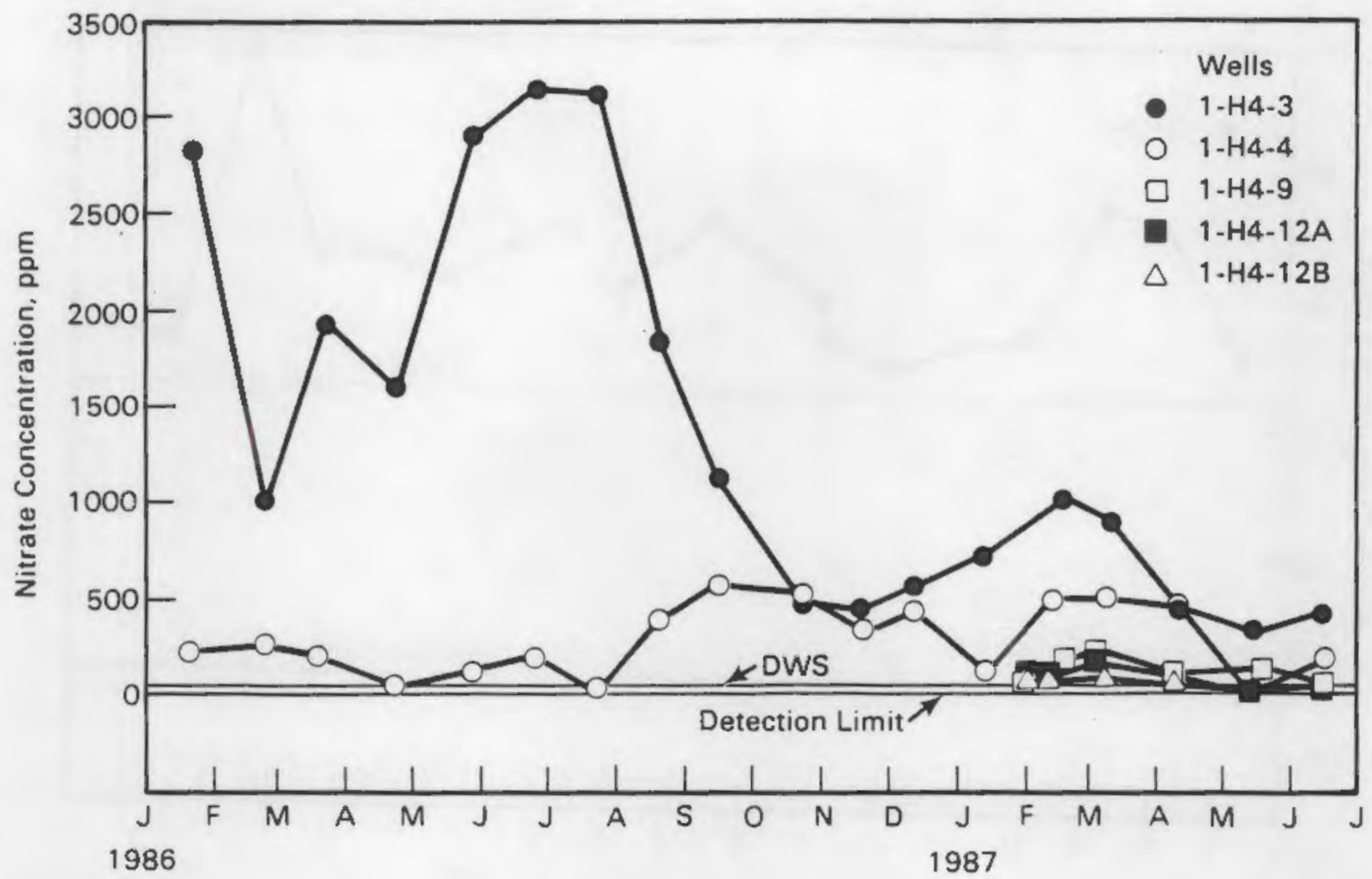

FIGURE 11. Nitrate Concentrations in Wells 1-H4-4, 1-H4-9, 1-H4-12A, and 1-H4-12B, in 1986 and the First Half of 1987

wells 1-H3-1 and 1-H4-4 are relatively unchanged. Concentrations of nitrate in well 1-H4-9 were relatively constant at levels above the DWS. Although nitrate concentrations in wells $1-\mathrm{H} 4-12 \mathrm{~A}$ and $1-\mathrm{H} 4-12 \mathrm{~B}$ were above the DWS in early 1987, the concentrations measured in May and June were slightly below the DWS.

In general, nitrate concentrations in most $100 \mathrm{~N}$-Area wells have declined since January. However, concentrations in wells $1-\mathrm{N}-14,1-\mathrm{N}-23,1-\mathrm{N}-36$, $1-\mathrm{N}-37$, and $1-\mathrm{N}-45$ have increased in 1987, with concentrations greater than the DWS for wells $1-\mathrm{N}-36,1-\mathrm{N}-37$, and $1-\mathrm{N}-45$ (Figure 13). Wells $1-\mathrm{N}-5$, $1-\mathrm{N}-7,1-\mathrm{N}-27,1-\mathrm{N}-30,1-\mathrm{N}-31,1-\mathrm{N}-32$, and $1-\mathrm{N}-39$ had previously contained nitrate in concentrations above the DWS but fell below that level in this quarter.

The highest concentrations of nitrate in the 200 East Area are found in wells in the southeast corner, near PUREX. In general, the wells that 


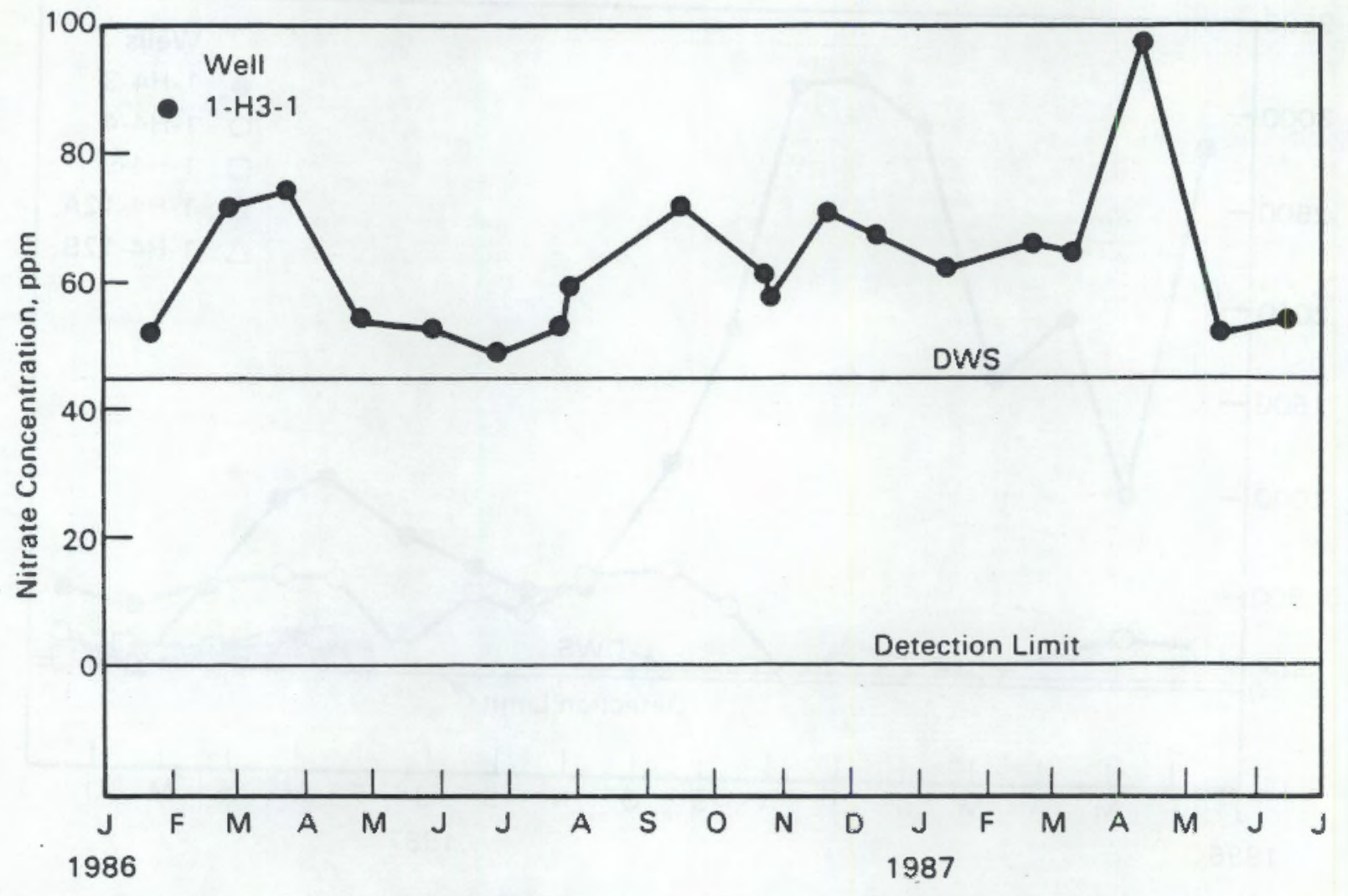

FIGURE 12. Nitrate Concentrations in Well 1-H3-1, in 1986 and the First Half of 1987

contain the highest concentrations of nitrate also contain the highest tritium concentrations (wells 2-E17-1, 2-E17-2, 2-E17-8, 2-E17-9, 2-E24-1, 2-E24-2, 2-E24-11, 2-E25-13, 2-E25-19, and 2-E25-20). These wells are located near cribs $216-A-10$ and $216-A-37-1$ (Figure 5), which receive process condensate effluent from PUREX and the 242-A evaporator, respectively. The maximum nitrate concentration measured in the 200 East Area was in well 2-E24-1 (555 ppm). Concentrations of nitrate in nearby wells 6-32-43, 6-3342, and 6-37-43 are increasing, yet still below the DWS (Figure 14). A similar increase in tritium concentrations in those wells has been noted, and a nitrate plume as well as a tritium plume may be emanating from the southeast portion of the 200 East Area. Well 2-E28-18 in the northwest portion of the 200 East Area continues to contain nitrate in concentrations greater than the DWS, with an average concentration of $59 \mathrm{ppm}$. Concentrations in wells 6-49-55A and 6-50-53, located just north of the 200E Area, have continued to increase to concentrations of 198 and $346 \mathrm{ppm}$, respectively. 


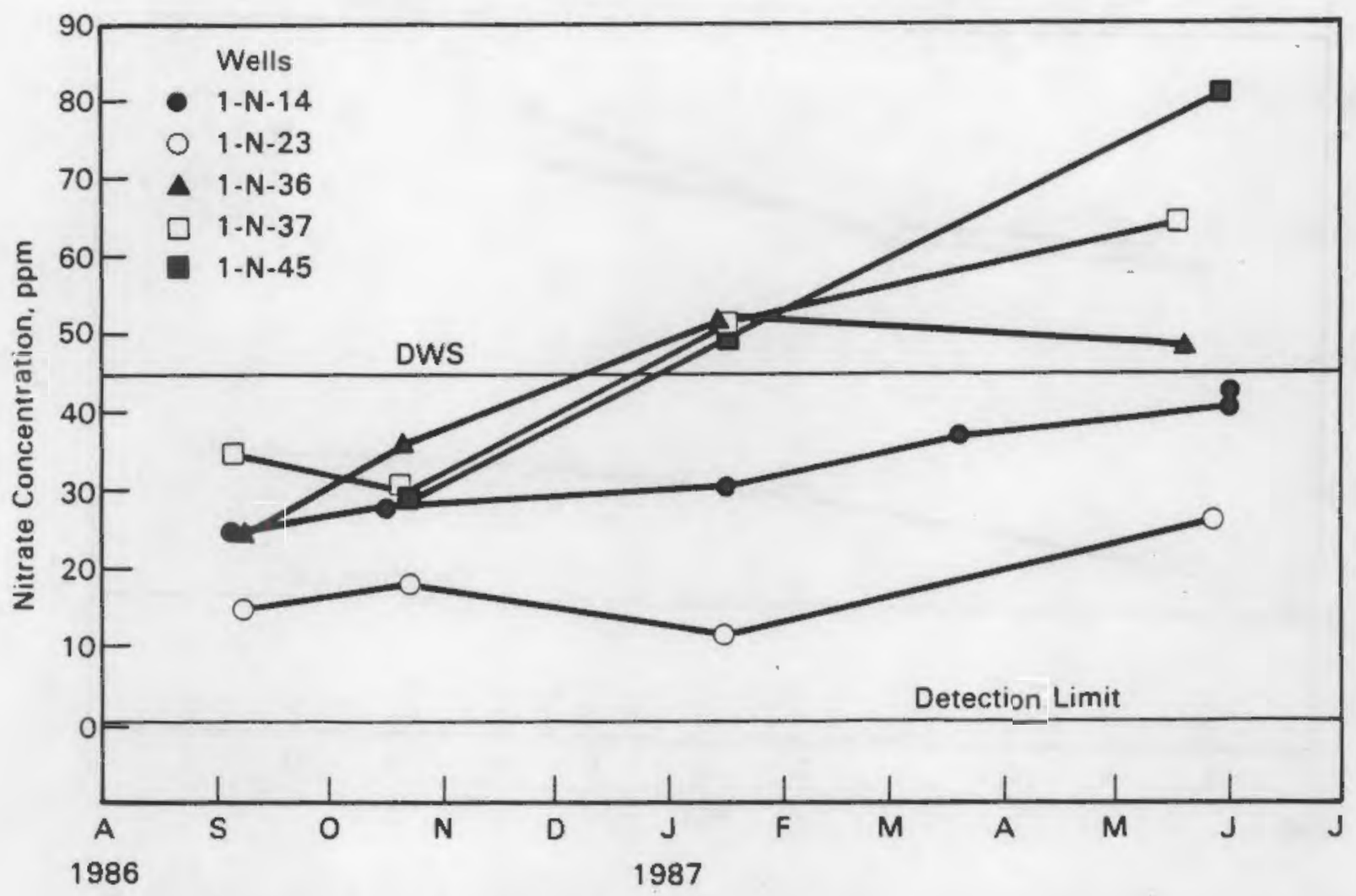

FIGURE 13. Nitrate Concentrations in Wells $1-N-14,1-N-23,1-N-36$, 1-N-37, and 1-N-45, August 1986 Through June 1987

The highest levels of nitrate detected in the 200 West Area are concentrated in two general areas: near the $U$ plant and between the $T$ Plant and the $Z$ Plant. Nitrate concentrations above the DWS are found in wells in the area surrounding the U Plant (i.e., wells with the "2-W19" prefix) (Figure 6). Wells 2-W19-19, 2-W19-20, and 2-W19-24 contained the highest nitrate levels detected on the Hanford Site during the first half of 1987 , with average concentrations of 1,$460 ; 1,060$; and $1,270 \mathrm{ppm}$, respectively. Wells 2-W19-2, 2-W19-23, 2-W19-25, and 2-W19-26 in the same area also contained nitrate concentrations at least 100 times greater than the DWS.

Wells in the northwest portion of the 200 West Area also contained nitrate in concentrations above the DWS. These wells (2-W10-4, 2-W11-23, 2W11-24, 2-W14-5, and 2-W15-4; Figure 6) are located in an area between the $\mathrm{T}$ Plant and the $\mathrm{Z}$ Plant. Concentrations in that area ranged from $83 \mathrm{ppm}$ in well 2-W14-10 to $680 \mathrm{ppm}$ in well 2-W15-4. 


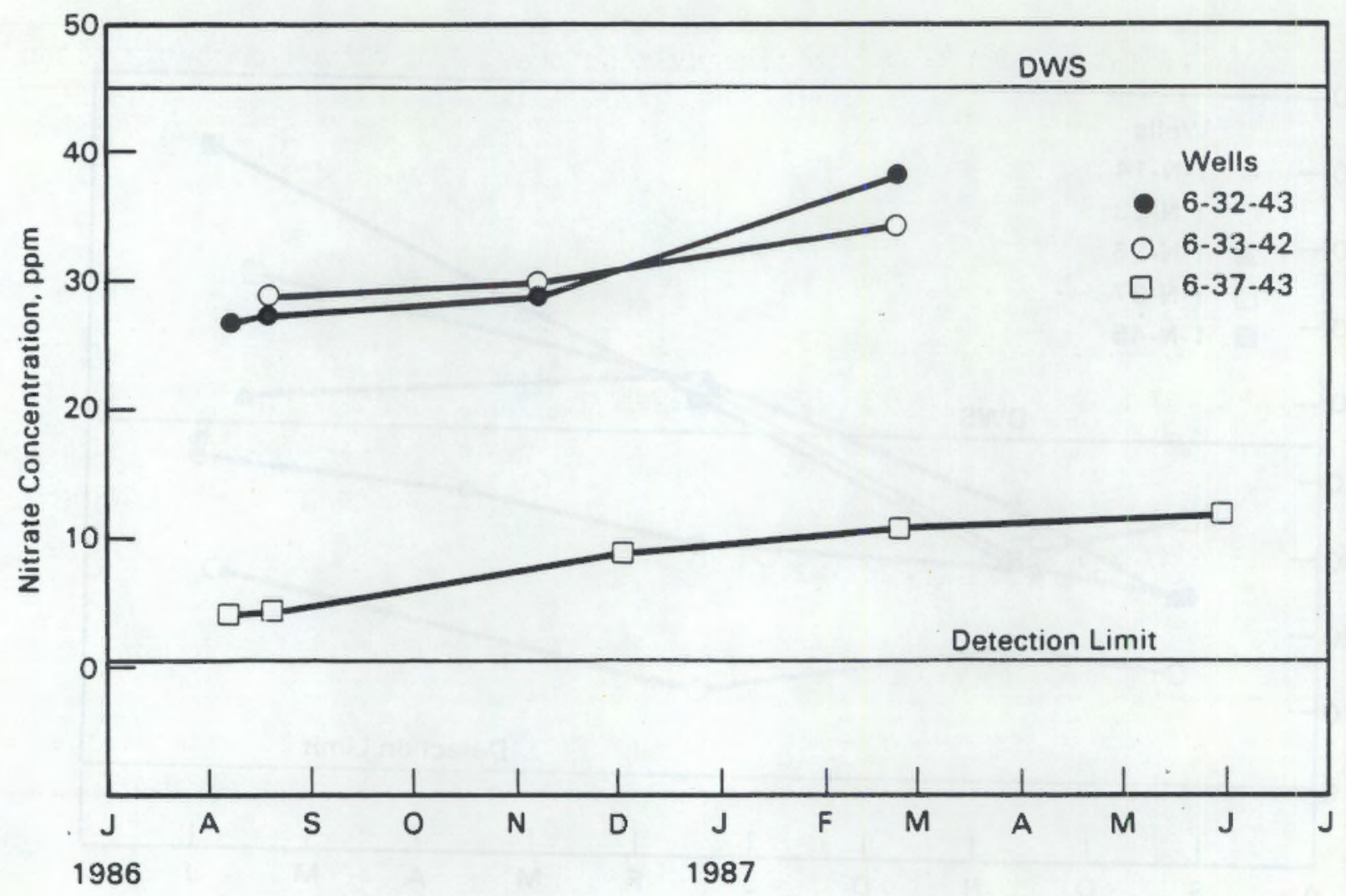

FIGURE 14. Nitrate Concentrations in Wells 6-32-43, 6-33-42, and 6-37-43, July 1986 Through June 1987

0ther 200-West-Area wells (2-W6-1, 2-W10-9, 2-W12-1, 2-W14-2, 2-W15-10, 2-W15-11, 2-W22-20, and 2-W27-1) contained nitrate concentrations greater than the DWS, ranging from 102 to $350 \mathrm{ppm}$.

Nitrate concentrations in 300-Area wells remained at levels equal to or less than those measured in 1986 . The only 300-Area well with nitrate concentrations greater than the DWS was 3-5-1, with a concentration of $45.7 \mathrm{ppm}$, which is relatively unchanged from 1986 concentrations.

Nitrate concentrations in wells in the 400 Area (Figure 2) and most of the 600 Area were similar to those measured in 1986. Nitrate in well 6-17-70, which was above the DWS in 1986, declined to below that level. Nitrate concentrations in the large plume located between the 200 East Area and the Columbia River remained relatively unchanged from those reported for 
1986. Wells in the 600 Area not already mentioned that showed concentrations above the DWS, ranging from 47 to $147 \mathrm{ppm}$, include 6-17-5, 6-38-15, 6-38-65, $6-44-64,6-49-57$, and 6-77-36.

\section{OTHER OBSERVATIONS}

Several other chemical and radiological species detected at levels that may be related to Hanford Site operations are discussed below. The order in which contamination issues are listed is arbitrary. The conclusions are tentative in some cases. However, they are included here because the potential exists for some of these constituents to develop into future issues of concern.

Acetone

Acetone has been detected in seven wells distributed widely across the site. No discernible pattern exists. The acetone may be some type of contamination during sampling or laboratory analysis.

Ammonia

Twenty-nine wells show evidence of ammonia. Anmonia and ammonium salts have been used in a variety of applications on the site. Two monitoring locations are worth noting because both are in operating areas. Wells $1-\mathrm{N}-28$ and $1-\mathrm{N}-29$ in the $100 \mathrm{~N}$ Area have ammonia levels of $1,470 \mathrm{ppb}$ and $3,630 \mathrm{ppb}$, respectively. The same two wells also showed detectable levels of phosphate. The materials detected probably derived from a decontamination solution containing anmonium phosphate. Wells 2-E28-18 and 2-E28-21 in the 200 East Area show maximum anmonia levels of $890 \mathrm{ppb}$ and $234 \mathrm{ppb}$, respectively. Both are near crib 216-B-62, which has received ammoniacal PUREX process condensate from B Plant. Ammonia is also used in the process to remove the zirconium alloy cladding from fuel for the N Reactor.

Hexavalent Chromium in the 200 west and 600 Areas

Some evidence of chromium contamination is evident in three wells near the T Plant and in two wells in the Z Plant Area of the 200 West Area (Figure 6). The source of the contamination is believed to be decontamination waste shipped from the 300 Area. Chromium was also detected in 
well 6-83-47 (45 ppb). That well, which is located near the 100 Areas cannot be tied to any known sources of chromium contamination.

\section{Fluoride}

A number of closely spaced wells in the 200 West Area show elevated levels of fluoride, ranging up to $12,500 \mathrm{ppb}$ in well 2-W15-4. These wells are downgradient of the $216-Z-9$ crib, which has received an estimated 210 metric tons of aluminum fluoride nitrate since operations at the Hanford Site began. (a)

Chlorinated Hydrocarbons near the 300 Area

Several chlorinated hydrocarbons, including 1,2-dichloroethylene and trichloroethylene, have been detected in samples taken from three wells downgradient of the 300 Area process trenches (Figure 7 ).

Methyl Ethyl Ketone

Methyl ethyl ketone was detected in one well (2-E13-5) at a maximum level of $50 \mathrm{ppb}$. The significance of the observation is unknown; however, the species has been detected twice in the same well.

Methylene Chloride

Methylene chloride has been detected occasionally but rarely in the same well twice. Therefore, its appearance is probably associated with laboratory contamination, a well-known problem with that species because of its volatile nature combined with its wide use in laboratory chemical extraction.

$\mathrm{N}$-nitroso-dimethylamine

Well 2-15-4 showed positive indications of $\mathrm{N}$-nitroso-dimethylamine. It is not known whether this species exists in the ground water or is laboratory contamination.

$\underline{\text { Selenium }}$

On two successive measurements, selenium was detected in we11 2-W27-1 at levels five times as great as the DWS (10 ppb). No explanation can

(a) U.S. Department of Energy, 1986, "Oraft Phase I Installation Assessment of Inactive Waste Disposal at Hanford," Richland, Washington. 
be given for the finding because no use of selenium at the Hanford Site has been documented.

\section{Total Organic Halogens}

A number of wells in the 100 Areas showed the presence of total organic halogens not accompanied by corresponding chlorinated hydrocarbons. This anomaly requires further investigation.

Cyanide in the 200 West Area

Cyanide has been detected in two wells in the 200 West Area (2-W12-1 at $34 \mathrm{ppb}$ and 2-W18-7 at $15 \mathrm{ppb})$. These wells are adjacent to several trenches that also received ferrocyanide wastes.

\section{Gross Alpha Activity, Uranium, and Plutonium}

Levels of gross alpha activity were elevated in wells in several areas. Site-wide radiological monitoring results showed levels generally consistent with those reported for 1986 (PNL 1987). Alpha analyses newly added to sitewide chemical and RCRA-compliance monitoring provided information for areas not previously monitored for alpha. The DWS for alpha is $15 \mathrm{pCi} / \mathrm{L}$, not including uranium. Several wells in and around the 200 Areas had gross alpha activity greater than the DWS. Wells in the 200 East Area with average gross alpha concentrations greater than the DWS included 2-E28-18 (64.4 pCi/L), 2-E28-21 (49.2 pCi/L), and 2-E28-23 (48.5 pCi/L). However, these wells contained uranium in concentrations of 80,73 , and $42.1 \mathrm{ppb},(\mathrm{a})$ respectively, which may account for the gross alpha levels measured. The DCG for uranium-234 is $500 \mathrm{pCi} / \mathrm{L}$. In March, plutonium-239 and plutonium-240 $\left({ }^{239}, 248 \mathrm{Pu}\right)$ were also detected in well 2-E28-23 at a concentration of $12.8 \mathrm{pCi} / \mathrm{L}$ and account for part of the gross alpha activity in that well. Smith (1980) has reported that plutonium was injected into the 216-B-5 reverse well during 1945 to 1947 . The $\mathrm{DCG}$ for ${ }^{239,248} \mathrm{Pu}$ is $300 \mathrm{pCi} / \mathrm{L}$.

The highest levels of gross alpha activity were found in wells 2-W19-3, 2-w19-9, 2-w19-11, 2-W19-15, 2-W19-16, 2-W19-17, and 2-W19-18 in the 200 west Area, which are associated with the uranium plume from the $216-\mathrm{U}-1$ and

(a) The conversion factor from $\mathrm{ppb}$ uranium to $\mathrm{pCi} / \mathrm{L}$ uranium is approximately 0.679 (Law, Serkowski, and Schatz 1987). 
216-U-2 cribs, described earlier, and in wells 2-W19-19, 2-W19-23, 2-W19-24, 2-W19-25, and 2-W19-26, which are near an effluent line to the inactive 216$\mathrm{U}-8 \mathrm{crib}$ and the active $216-\mathrm{U}-12 \mathrm{crib}$. All of these wells contained uranium at levels that would account for the gross alpha activity measurements. (Plutonium was not detected in these wells.) Both the gross alpha activity and the uranium concentrations in most of these wells continue to decrease slightly during 1987 (Figures 15 through 18).

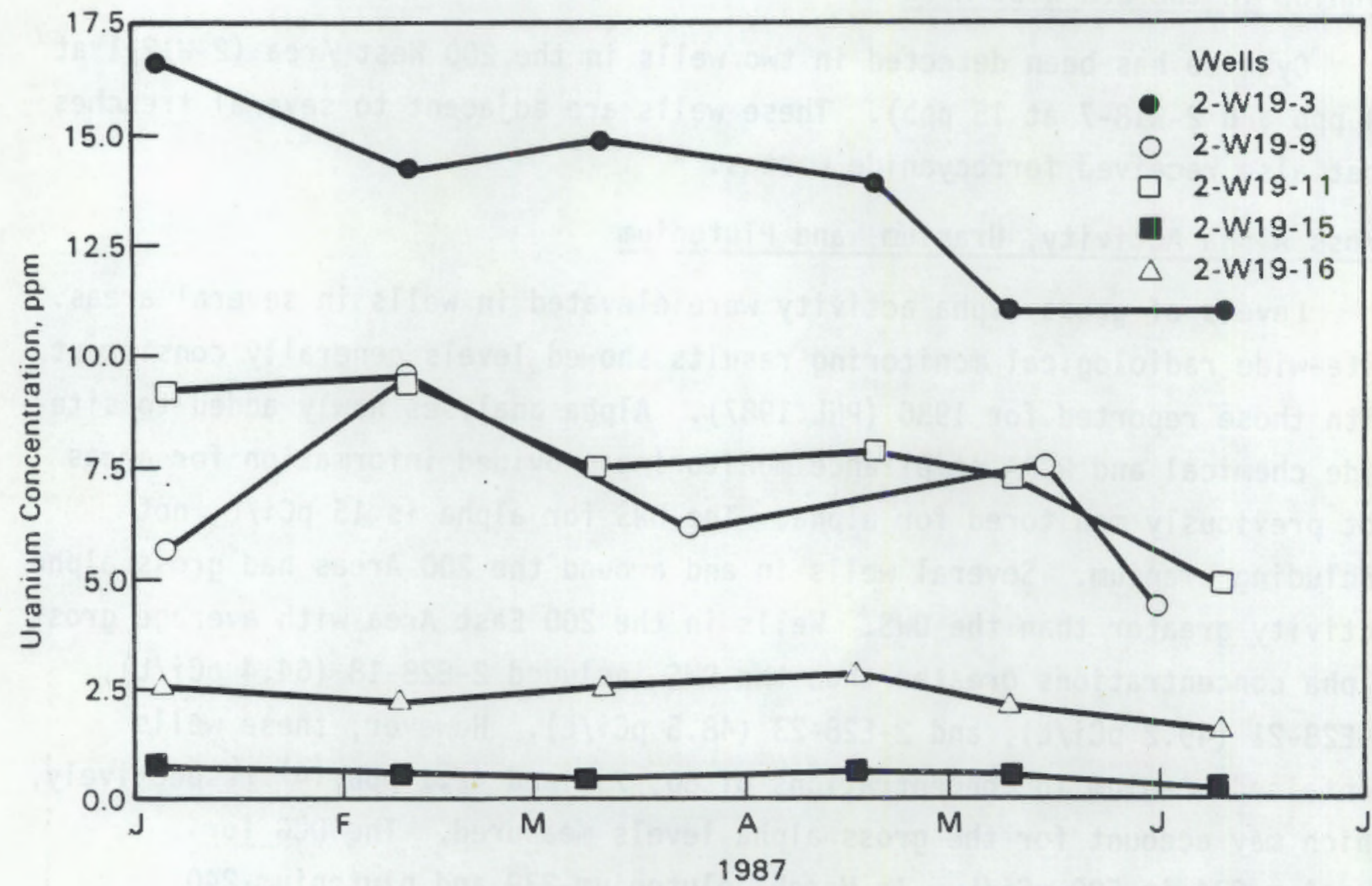

FIGURE 15. Uranium Concentrations in Wells 2-W19-3, 2-W19-9, 2-W19-11, 2-W19-15, and 2-W19-16 for the First Half of 1987 
Uranium concentrations in the 100F, $100 \mathrm{H}, 100 \mathrm{~K}$, and 300 Areas were similar to those measured in 1986. Uranium in well 1-F8-1 in the 100F Area has increased steadily since January (Figure 19). Uranium concentrations in wells 3-1-4 and 3-1-5 also increased in early 1987. In samples taken in April, uranium concentrations remained relatively high in well 3-1-5 $(58.2 \mathrm{pCi} / \mathrm{L})$ but had begun to decline in well $3-1-4$, from $36 \mathrm{pCi} / \mathrm{L}$ in January 1987 to $23.2 \mathrm{pCi} / \mathrm{L}$. Several other isolated elevated gross alpha measurements are noteworthy. These include wells 2-E24-12 (17 pCi/L), 2-E28-18 (34 $\mathrm{pCi} / \mathrm{L}), 2-\mathrm{E} 28-21(54 \mathrm{pCi} / \mathrm{L})$, and $6-512-3(63 \mathrm{pCi} / \mathrm{L})$. Wells 2-E28-18 and 2-E28-21 are near the 218-8-62 crib (Figure 5), and also show associated uranium concentrations. Well $6-\$ 12-3$ is located in an isolated part of the

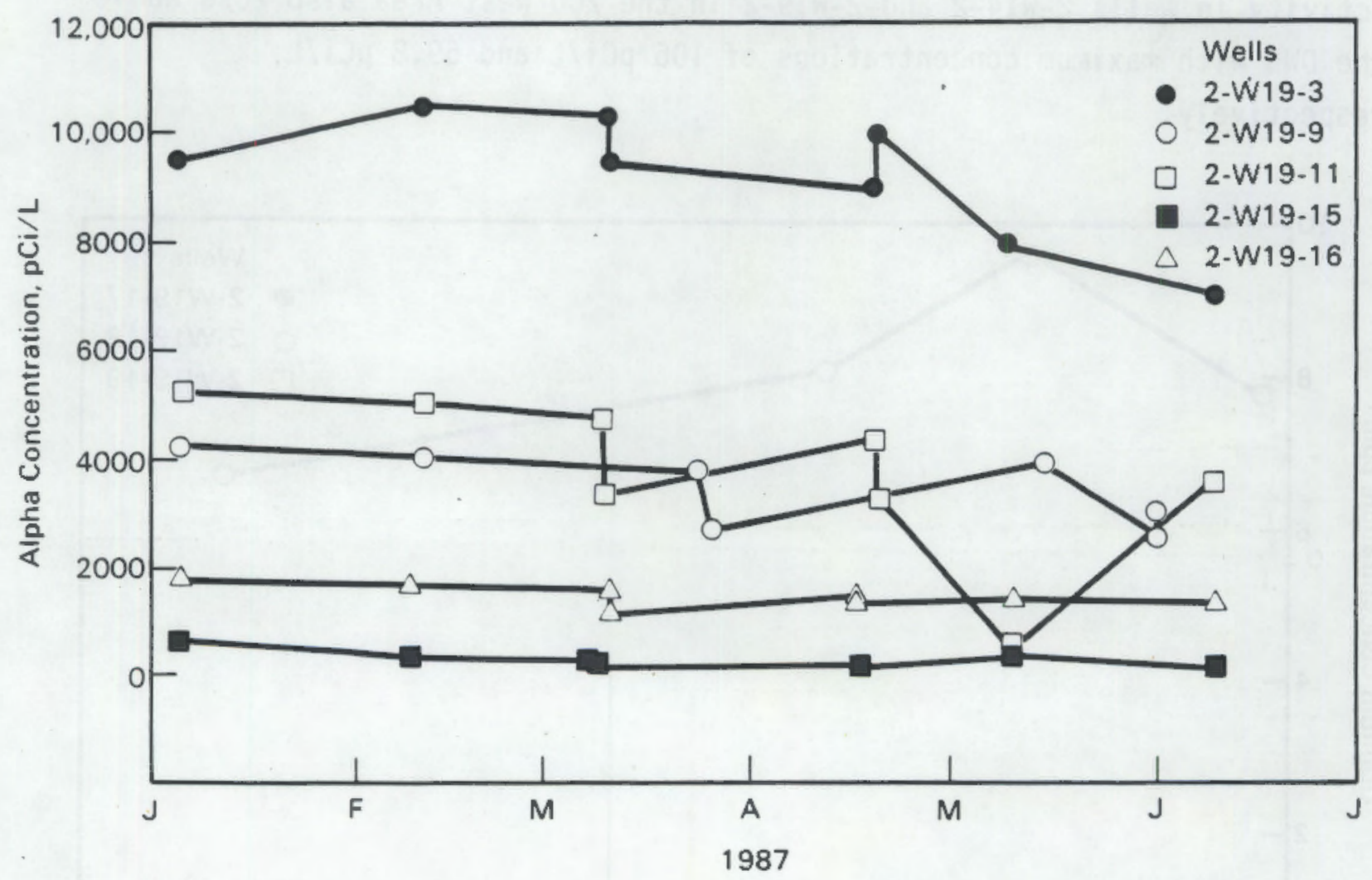

FIGURE 16. Alpha Concentrations in Wells 2-W19-3, 2-W19-9, 2-W19-11, 2-W19-15, and 2-W19-16 for the First Half of 1987 
600 Area approximately halfway between the Fast Flux Test Facility (FFTF) and the 300 Area (Figure 2). The origin of the high gross alpha level in this well is not obvious and is a matter of concern.

\section{Gross Beta Activity}

Gross beta activity in most wells except for the 200 East Area was similar to or less than that measured in 1986. Several wells in the 200 Areas and the surrounding 600 Area continued to have gross beta activity greater than the DWS $(50 \mathrm{pCi} / \mathrm{L})$. Activity in wells 2-E13-19, 2-E17-6, and 2-E24-1 in the 200 East Area had previously been below the DWS and rose above that limit during the first half of 1987. The maximum levels observed in those three wells were $63.1 \mathrm{pCi} / \mathrm{L}, 87.1 \mathrm{pCi} / \mathrm{L}$, and $62.3 \mathrm{pCi} / \mathrm{L}$, respectively. Activity in wells 2-W14-2 and 2-W19-2 in the 200 West Area also rose above the DWS with maximum concentrations of $106 \mathrm{pCi} / \mathrm{L}$ and $69.8 \mathrm{pCi} / \mathrm{L}$, respectively.

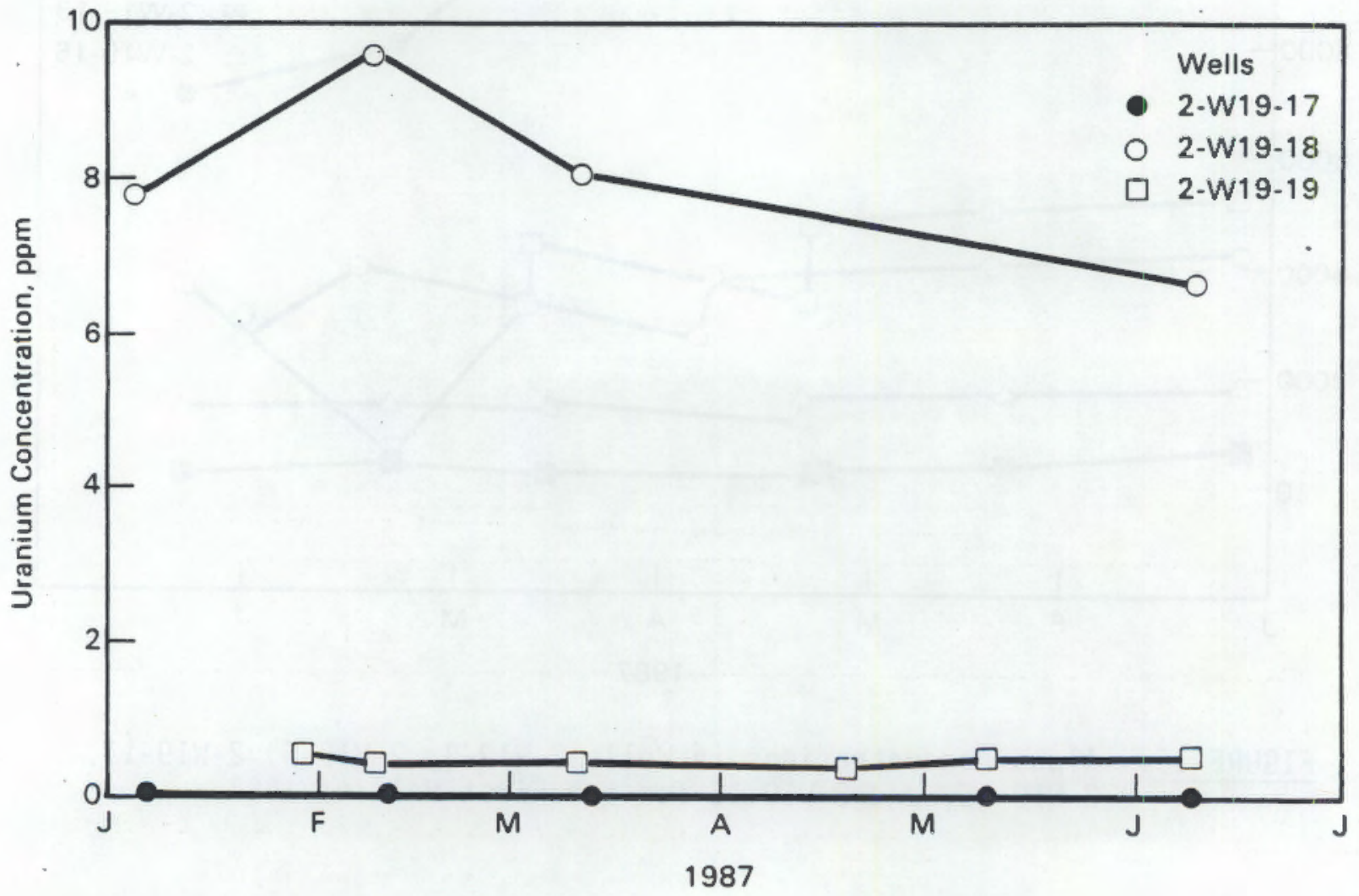

FIGURE 17. Uranium Concentrations in Wells 2-W19-17, 2-W19-18, and 2-W19-19 for the First Half of 1987 
Gross beta activity remained above or had risen above the DWS in several wells near Gable Mountain Pond (wells 6-53-47A, 6-53-47B, 6-53-48B, 6-54-48, and 6-54-49) (Figure 2), ranging from 94 to $531 \mathrm{pCi} / \mathrm{L}$. These wells contain strontium-90 ( $\mathrm{gl} \mathrm{Sr}$ ) in concentrations above the OWS (8 $\mathrm{pCi} / \mathrm{L})$, which would account for most of the gross beta activity measured. Strontium-90 concentrations have increased in three of these wells $(6-53-48 \mathrm{~B}, 6-54-48$, and 6-54-49) in the recent past (Figure 20). The gross beta activity in well 6-40-1 near the Columbia River measured $64.9 \mathrm{pCi} / \mathrm{L}$; this well will be analyzed for ${ }^{9} \mathrm{Sr}$ in the future. Two wells north of the 200 East Area showed gross beta activity levels of $174 \mathrm{pCi} / \mathrm{L}(6-49-57)$ and $1,240 \mathrm{pCi} / \mathrm{L}(6-50-53)$; ${ }^{90} \mathrm{Sr}$ was not measured in these wells. Gamma scans revealed ${ }^{80} \mathrm{Co}$ concentrations of 25.2 and $275 \mathrm{pCi} / \mathrm{L}$, respectively. The $\mathrm{DCG}$ for ${ }^{60} \mathrm{Co}$ is $5,000 \mathrm{pCi} / \mathrm{L}$.

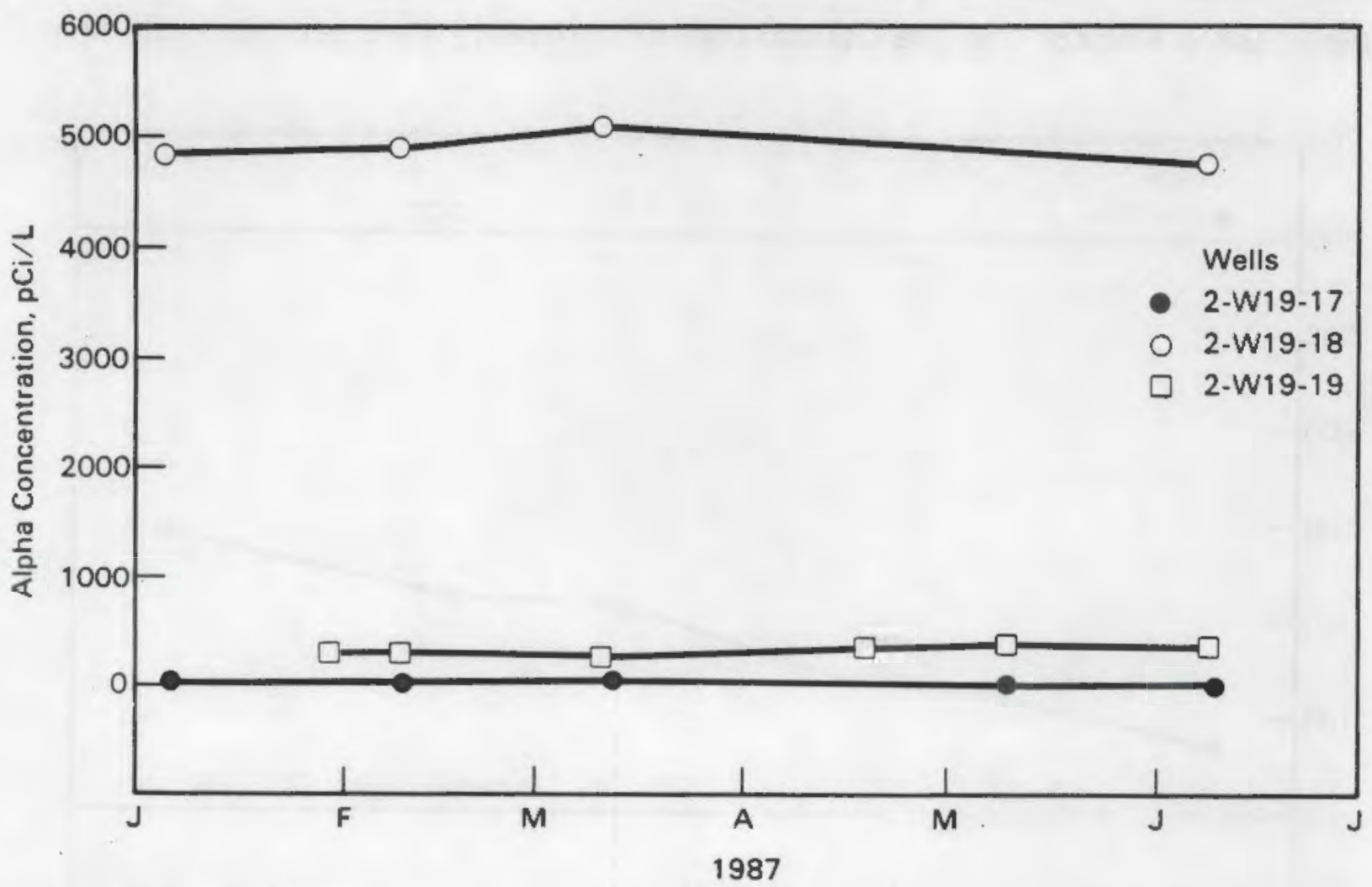

FIGURE 18. Alpha Concentrations in Wells 2-W19-17, 2-W19-18, and 2-W19-19 for the First Half of 1987 


\section{$\underline{\text { Strontium-90 }}$}

Concentrations of ${ }^{96} \mathrm{Sr}$ were also above the DWS in $100 \mathrm{H}-, 100 \mathrm{~N}-$, 200-East-, and 200-West-Area wells. Well 1-H4-4 in the 100 H Area showed a concentration of $8.05 \mathrm{pCi} / \mathrm{L}$, which is slightly above the DWS $(8 \mathrm{pCi} / \mathrm{L})$. The DCG for ${ }^{90} \mathrm{Sr}$ is $1,000 \mathrm{pCi} / \mathrm{L}$. In those $100 \mathrm{~N}$-Area wells that have been sampled since January, ${ }^{90} \mathrm{Sr}$ concentrations have generally declined slightly. Concentrations above the OWS were present in wells $1-\mathrm{N}-2,1-\mathrm{N}-3,1-\mathrm{N}-4,1-\mathrm{N}-5$, $1-N-14,1-N-18,1-N-19,1-N-20,1-N-27,1-N-29,1-N-31,1-N-33,1-N-37$, $1-N-39$, and $1-N-45$. Five of these wells $(1-N-2,1-N-3,1-N-14,1-N-39$, and $1-\mathrm{N}-45)$ showed concentrations near or above the DCG $(1000 \mathrm{pCi} / \mathrm{L})$. The highest concentrations have been reported from wells $1-\mathrm{N}-2$ and $1-\mathrm{N}-3$, downgradient from the 1301N LWDF, and from well 1-N-45 near the 1325N LWDF (Figure 4). In the other $100 \mathrm{~N}$-Area wells that contain ${ }^{91} \mathrm{Sr}$ above the DWS, concentrations are generally well below the DCG. Concentrations in wells between the $\mathrm{N}$ Reactor and the Columbia River generally have been and continue

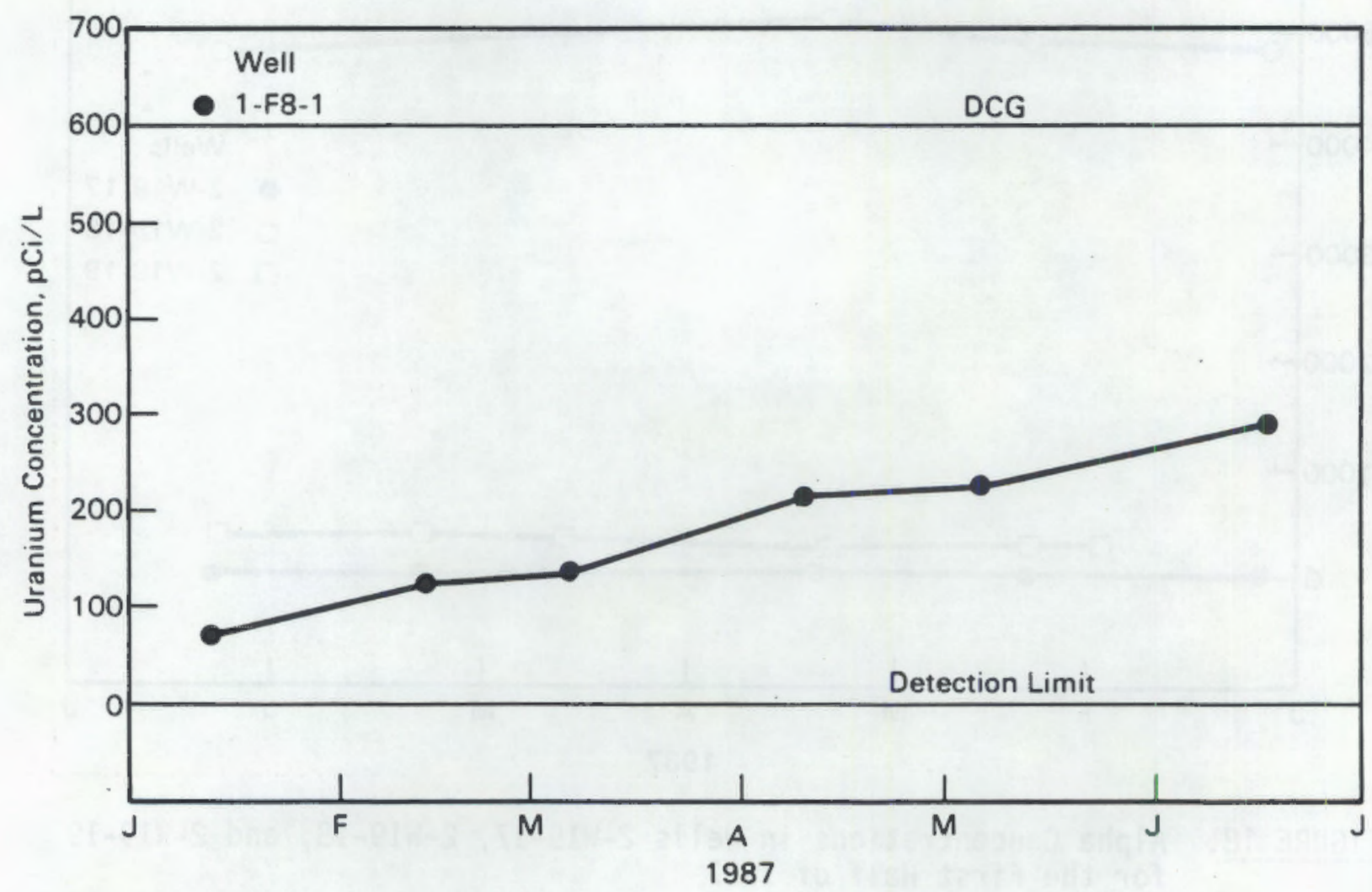

FIGURE 19. Uranium Concentrations in Well 1-F8-1 for the First Half of 1987 
to be below both the DWS and the detection limit. Wells $1-N-21,1-N-22$, $1-\mathrm{N}-23$, and $1-\mathrm{N}-24$ have contained concentrations slightly above the DWS on previous occasions but generally remain below. Concentrations in wells $1-N-2$ and 1-N-3 appear to have peaked in 1986 and are currently declining. The decline is presumably a result of discontinuing disposal of materials containing ${ }^{90} \mathrm{Sr}$ to the $1301 \mathrm{~N}$ LWDF. It is difficult to link trends in ${ }^{90} \mathrm{Sr}$ concentrations in wells near the $1325 \mathrm{~N}$ LWDF with disposal operations at that facility. Strontium-90 concentrations in some of the wells (e.g., 1-N-33 and $1-N-31)$ were higher before use of that facility began in September 1985 , whereas concentrations in other wells have increased since that time (e.g., wells $1-N-27,1-N-29,1-N-36$, and $1-N-45)$.

Several 200-East-Area wells contained ${ }^{90} \mathrm{Sr}$ in concentrations greater than the DWS. The highest concentrations were found northeast of the $B$ Plant in wells 2-E28-7 (85.7 $\mathrm{pCi} / \mathrm{L}), 2-\mathrm{E} 28-23(6,910 \mathrm{pCi} / \mathrm{L}), 2-\mathrm{E} 28-24(186 \mathrm{pCi} / \mathrm{L})$,

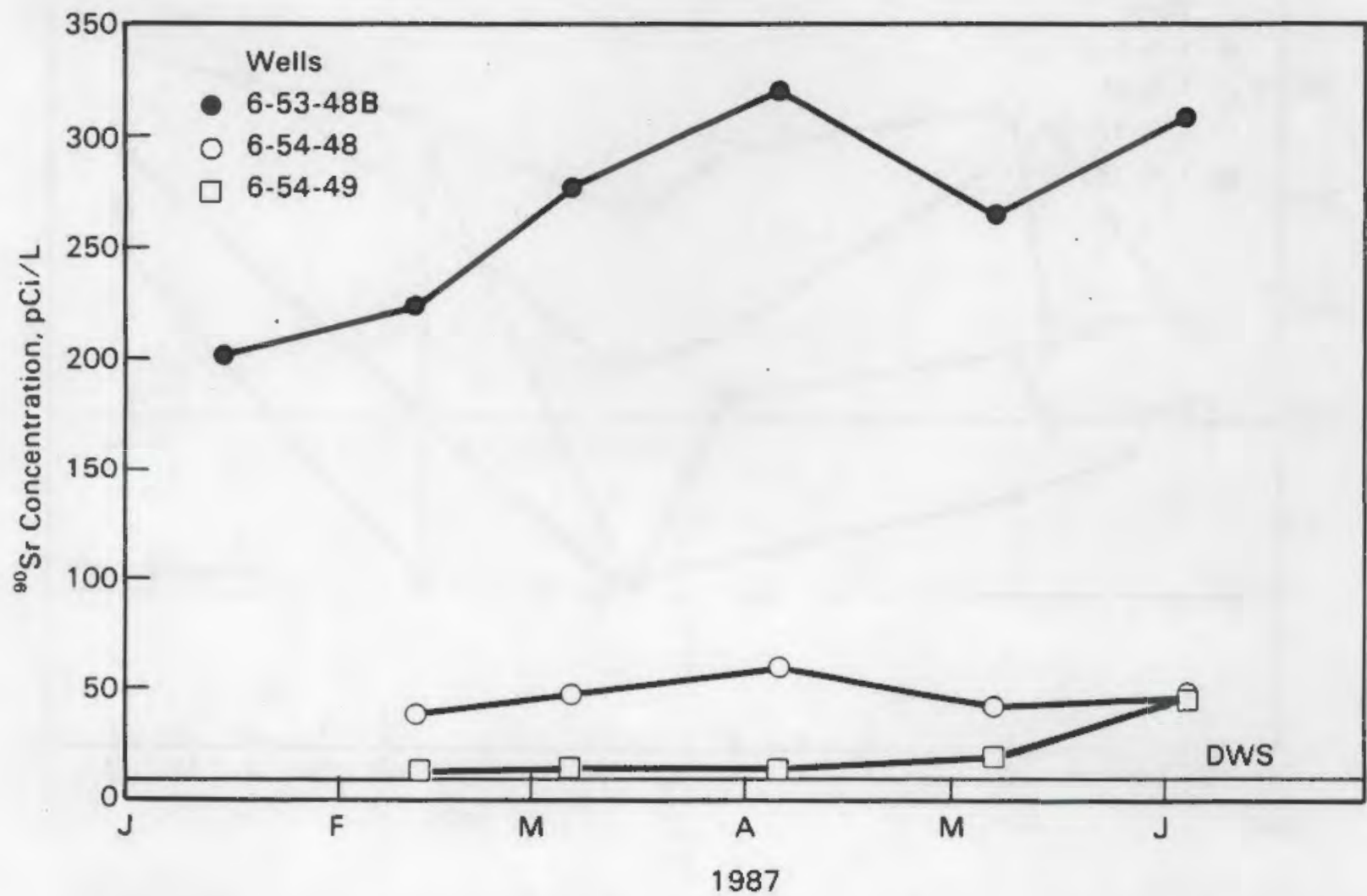

FIGURE 20. Strontium-90 Concentrations in Wells 6-53-488, 6-54-48, and 6-54-49 for the First Half of 1987 
and 2-E28-25 $(3,490 \mathrm{pCi} / \mathrm{L})$. The concentration in these wells can be attributed to wastes discharged to the 216-B-5 reverse well during 1945 to 1947 (Smith 1980). This concentration in well 2-E28-23 was similar to those measured in 1986 (about $7,000 \mathrm{pCi} / \mathrm{L}$ ); the other wells were first sampled in March 1987. The only 200 -West-Area wells that contained ${ }^{98} \mathrm{Sr}$ in concentrations above the DWS were wells 2-W19-2, 2-W22-10, and 2-W22-18 at $15.8 \mathrm{pCi} / \mathrm{L}$, $40.6 \mathrm{pCi} / \mathrm{L}$, and $12 \mathrm{pCi} / \mathrm{L}$, respectively.

\section{Cobalt -60}

A $11{ }^{60} \mathrm{Co}$ results were near or below the detection 1 imit $(20 \mathrm{pCi} / \mathrm{L})$, except in the 100N Area and in an isolated portion of the 200 East Area and the adjacent 600 Area. Concentrations of ${ }^{80} \mathrm{Co}$ in most $100 \mathrm{~N}$-Area wells stabilized or declined slightly from 1986 levels. However, ${ }^{86} \mathrm{Co}$ concentrations in wells $1-\mathrm{N}-4,1-\mathrm{N}-31,1-\mathrm{N}-32$, and $1-\mathrm{N}-33$ increased in samples

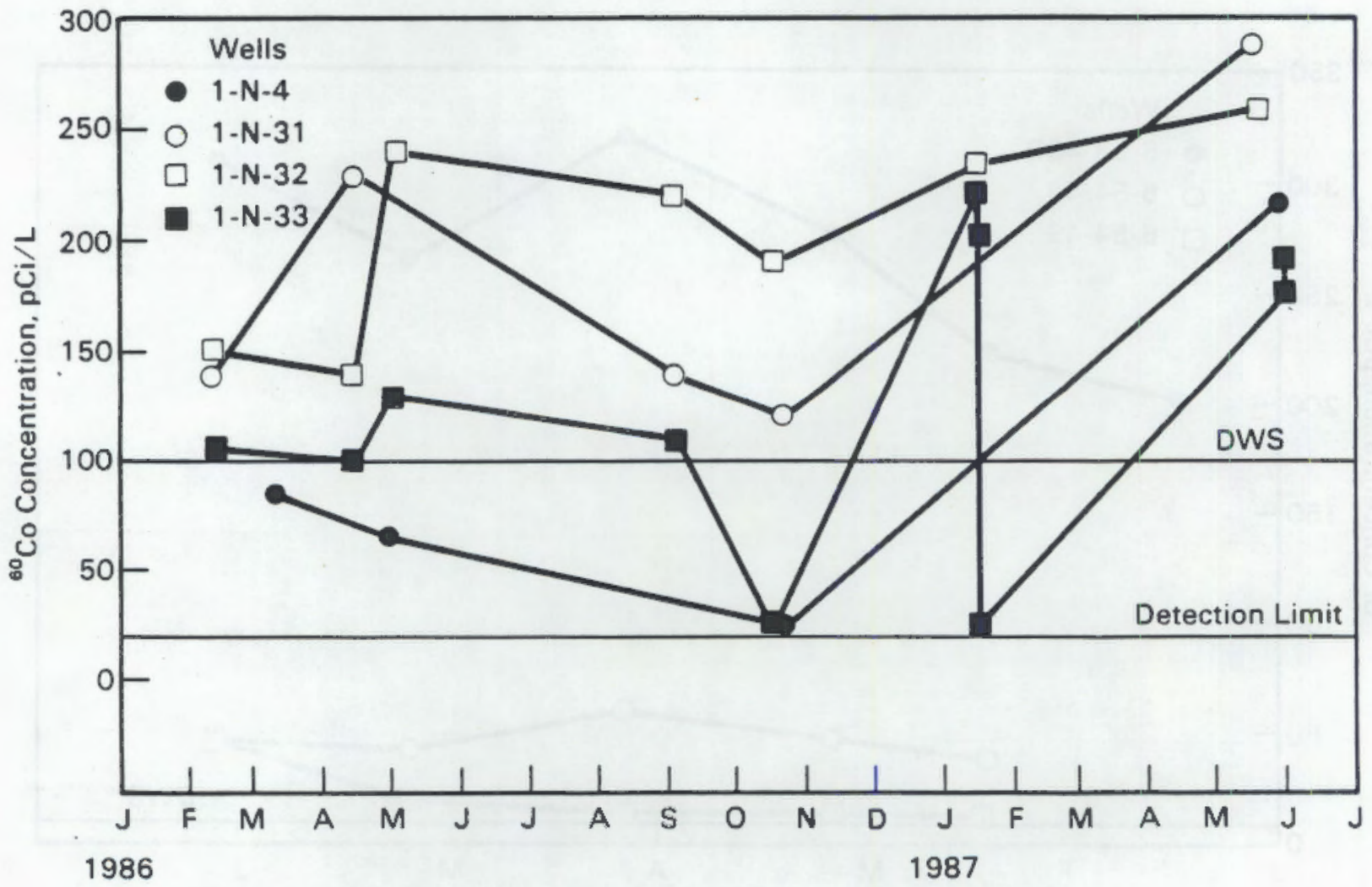

FIGURE 21. Cobalt-60 Concentrations in Wells 1-N-4, 1-N-31, 1-N-32, and 1-N-33 for 1986 and the First Half of 1987 
collected in May (Figure 21). These wells and several other 100N-Area Wells contained in ${ }^{80} \mathrm{Co}$ at levels above the DWS (100 pCi/L) (Figure 22). The DCG for ${ }^{86} \mathrm{Co}$ is $5,000 \mathrm{pCi} / \mathrm{L}$. The concentration of ${ }^{80} \mathrm{Co}$ in well 2-E33-7, located in the northern portion of the 200 East Area, increased to $75 \mathrm{pCi} / \mathrm{L}$ in May. Wells 6-49-55A and 6-50-53, which are immediately north of well 2-E33-7, continue to contain ${ }^{60} \mathrm{Co}$ in concentrations much above the DWS, at 206 and $275 \mathrm{pCi} / \mathrm{L}$, respectively. The transport of ${ }^{80} \mathrm{Co}$ to these wells is probably attributable to complexing by cyanide, which was discussed earlier.

\section{Cesium-137}

Concentrations of cesium-137 $\left({ }^{137} \mathrm{Cs}\right)$ were below the detection limit (22.5 pCi/L) in all wells except 2-E28-23. That well continued to contain ${ }^{137} \mathrm{CS}$ in concentrations averaging $2,490 \mathrm{pCi} / \mathrm{L}$, which is well above the DWS $(200 \mathrm{pCi} / \mathrm{L})$. The $\mathrm{DCG}$ for ${ }^{137} \mathrm{Cs}$ is $3,000 \mathrm{pCi} / \mathrm{L}$.

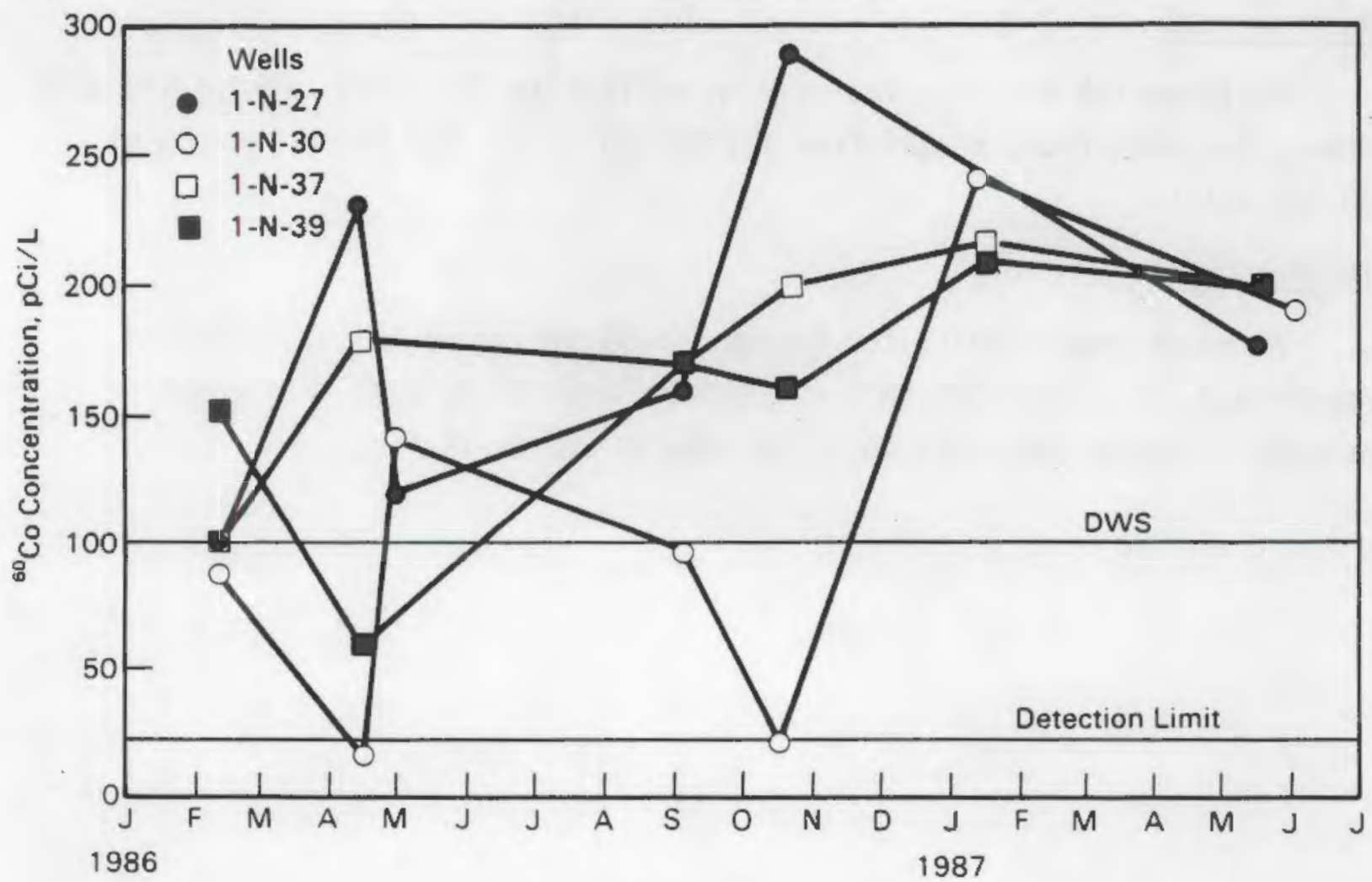

FIGURE 22. Cobalt-60 Concentrations in Wells $1-N-27,1-N-30,1-N-37$, and 1-N-39 for 1986 and the First Half of 1987 
Ruthenium-106

Because of its short half-life (367 days), ruthenium-106 ( $\left.{ }^{168} \mathrm{Ru}\right)$ is principally detected in wells located in areas where reactors are operating and fuels being processed, such as the $100 \mathrm{~N}$ Area and the 200 East Area near PUREX. Concentrations greater than the OWS $(30 \mathrm{pCi} / \mathrm{L})$ but less than the DCG $(6,000 \mathrm{pCi} / \mathrm{L})$ were detected in $100 \mathrm{~N}$ - and 200-East-Area wells. Ruthenium-106 concentrations in most $100 \mathrm{~N}$-Area wells have remained above the DWS; however, they have generally decreased from those measured in 1986 to below the analytical laboratory's detection limit of $173 \mathrm{pCi} / \mathrm{L}$. The $\mathrm{OCG}$ for ${ }^{166} \mathrm{Ru}$ is $6,000 \mathrm{pCi} / \mathrm{L}$. Well 6-38-65, which is located between the 200 west and 200 East Areas (Figure 2), had reached a ${ }^{166} \mathrm{Ru}$ concentration of $560 \mathrm{pCi} / \mathrm{L}$ in 1986. It declined to a concentration slightly above the DWS during this quarter. Ruthenium-106 levels above the DWS were also reported in wells in all operational areas. However, the counting error was at least $75 \%$ of the analytical result, making any interpretation of the results suspect.

Antimony -125

Antimony-125 was detected only in wells near the $1325 \mathrm{~N}$ LWDF in the $100 \mathrm{~N}$ Area. Concentrations ranged from 119 to $295 \mathrm{pCi} / \mathrm{L}$, far below the DCG of $60,000 \mathrm{pCi} / \mathrm{L}$.

Technetium-99

Although some results for technetium-99 are shown in the tables of Appendix $A$, discussion of this constituent will be delayed until next quarter's report when more complete results are available. 


\section{$\underline{\text { REFERENCES }}$}

Eddy, P. A., D. A. Myers, and J. R. Raymond. 1978. Vertical Contamination in the Unconfined Ground Water at the Hanford Site, Washington. PNL-2724, Pacific Northwest Laboratory, Richland, Washington.

Law, A. G., J. A. Serkowski, and A. L. Schatz. 1987. Results of the Separations Area Ground-Water Monitoring Network for 1986. RH0-RE-SR-87-24P. Rockwell Hanford Operations, Richland, Washington.

Pacific Northwest Laboratory (PNL). 1987. Environmental Monitoring at Hanford for 1986. PNL-6120, Pacific Northwest Laboratory, Richland, Washington.

Schatz, A. L., and E. J. Jensen. 1986. Unconfined Water-Table Map. SD-WM-TI-273, Rockwell Hanford Operations, Richland, Washington.

Smith, R. M. 1980. 216-B-5 Reverse Well Characterization Study. RH0-ST-37, Rockwell Hanford Operations, Richland, Washington.

U.S. Department of Energy. 1983. Final Environmental Impact Statement, Operations of PUREX and Uranium Oxide Facilities. DOE/EIS-0089, U.S. Department of Energy, Washington, D.C.

U.S. Environmental Protection Agency (USEPA). 1982. Test Methods for Evaluating Solid Waste. 2nd ed. SW-846. Office of Solid Waste and Emergency Response, Washington, D.C. 
.

; 
APPENDIX A

DATA LISTINGS 
APPENDIX A

\section{DATA LISTINGS}

Each monitoring well sampled on the Hanford Site from April through June 1987 is placed into one of three groups. The first group contains site-wide chemical monitoring wells (Table A.1); those wells also sampled by the DOE/HQ-EAT are footnoted. The second group contains wells in compliance monitoring networks (Table A.2). The third group contains additional sitewide radiological and nitrate monitoring wells that were not in either of the other two networks (Table A.3).

Tables A.4 through A.6 contain computer-generated Tistings of analytical results for these three groups of wells. A key to the constituent names used in Tables A.4 through A.6 is given in Table A.7. Some constituents appear more than once in the list in different forms, e.g., NITRATE and HNITRATE are both nitrate ion, but HNITRATE has a higher detection limit. The constituents $U$ and U-CHEM are both total uranium, but in units of $\mathrm{pCi} / \mathrm{L}$ and $\mathrm{ppb}(\mathrm{a})$, respectively. The constituent names CHROMUM and FCHROMI represent chromium analyses done on unfiltered and filtered samples, respectively.

Tables A.4 through A.6 contain all chemical results (above detection levels) and radiochemical results (for which the result is larger than the counting error). Blank spaces in the tables or constituents missing from the first column indicate that the results did not meet these criteria. The letters "NR" in the tables indicate that the analysis was not requested or the results had not yet been reported by the laboratory at the time of writing. Tables 2 through 4 of the text can be used to confirm which analyses were performed for each of the wells.

(a) The conversion from $\mathrm{ppb}$ uranium to $\mathrm{pC} i / \mathrm{L}$ uranium is approximately 0.679 (Law, Serkowski, and Schatz 1987). 
IABLE A.1. Site-Wide Chemical Monitoring Wells

$\begin{array}{llll}1-B 3-1(a) & 2-E 24-8 & 2-W 18-7(a) & 6-34-42 \\ 1-84-1 & 2-E 24-12 & 2-W 19-1(a) & 6-34-51 \\ 1-B 4-4 & 2-E 25-7(a) & 2-W 19-3 & 6-35-9 \\ 1-B 5-1(a) & 2-E 25-18 & 2-W 19-5 & 6-35-70 \\ 1-B 9-1 & 2-E 25-20 & 2-W 19-9 & 6-35-78 A \\ 1-D 2-5 & 2-E 25-21 & 2-W 19-11 & 6-37-82 A \\ 1-D 5-12 & 2-E 25-22 & 2-W 19-13 & 6-38-65 \\ 1-D 8-3 & 2-E 26-1(a) & 2-W 19-15 & 6-39-79 \\ 1-F 5-1 & 2-E 26-3(a) & 2-W 19-16 & 6-40-1 \\ 1-F 5-3(a) & 2-E 27-5 & 2-W 22-12 & 6-40-33 A \\ 1-F 5-6 & 2-E 28-7(a) & 2-W 22-20 & 6-43-88 \\ 1-F 7-1 & 2-E 28-17(a) & 2-W 22-22 & 6-45-69 A \\ 1-F 8-1 & 2-E 28-21 & 2-W 22-26 & 6-48-7 \\ 1-F 8-2(a) & 2-E 33-1 & 2-W 23-1(a) & 6-48-18 \\ 1-K-11 & 2-E 33-2(a) & 2-W 23-3(a) & 6-48-71 \\ 1-K-19 & 2-E 33-3 & 2-W 23-7(a) & 6-49-57 \\ 1-K-20 & 2-E 33-5 & 2-W 23-10 & 6-49-79 \\ 1-K-22 & 2-E 33-8 & 2-W 23-11(a) & 6-50-53 \\ 1-K-27 & 2-E 33-10 & 2-W 27-1 & 6-51-75 \\ 1-K-28 & 2-E 33-18 & 6-2-3 & 6-77-54 \\ 1-K-29 & 2-E 33-21 & 6-2-33 A & 6-83-47 \\ 1-K-30 & 2-E 33-24 & 6-8-25 & 6-89-35 \\ 1-N-6 & 2-W 6-1(a) & 6-9-E 2 & 6-90-45 \\ 1-N-14 & 2-W 10-4 & 6-15-26 & 6-53-25 \\ 1-N-28 & 2-W 10-8 & 6-20-E 5 A & 6-S 6 E 14 A \\ 1-N-29 & 2-W 10-9 & 6-20-20 & 6-S 8-19 \\ 2-E 13-5 & 2-W 12-1(a) & 6-20-39 & 6-S 12-3 \\ 2-E 13-14 & 2-W 14-2 & 6-24-33 & 6-529-E 12 \\ 2-E 17-1 & 2-W 14-5 & 6-28-40 & 6-531-1 \\ 2-E 17-5 & 2-W 14-6 & 6-29-4 & \\ 2-E 17-6 & 2-W 15-4 & 6-32-22 & \\ 2-E 17-9 & 2-W 15-10 & 6-33-42 & \\ 2-E 24-7(a) & 2-W 15-11 & 6-33-56 & \\ & & & \end{array}$

(a) Well co-sampled by DOE/HQ-EAT. 
TABLE A.2. Compliance Monitoring Wells

\begin{tabular}{|c|c|}
\hline $\begin{array}{l}183-H \text { Solar Evaporation Basins } \\
1-H 3-1 \\
1-H 3-2 A \\
1-H 3-2 B \\
1-H 3-2 C \\
1-H 4-3 \\
1-H 4-4 \\
1-H 4-5 \\
1-H 4-6 \\
1-H 4-7 \\
1-H 4-8 \\
1-H 4-9 \\
1-H 4-10 \\
1-H 4-11 \\
1-H 4-12 A \\
1-H 4-12 B \\
1-H 4-12 C \\
1-H 4-13 \\
1-H 4-14 \\
1-H 4-15 A \\
1-H 4-15 B \\
1-H 4-16 \\
1-H 4-17\end{array}$ & 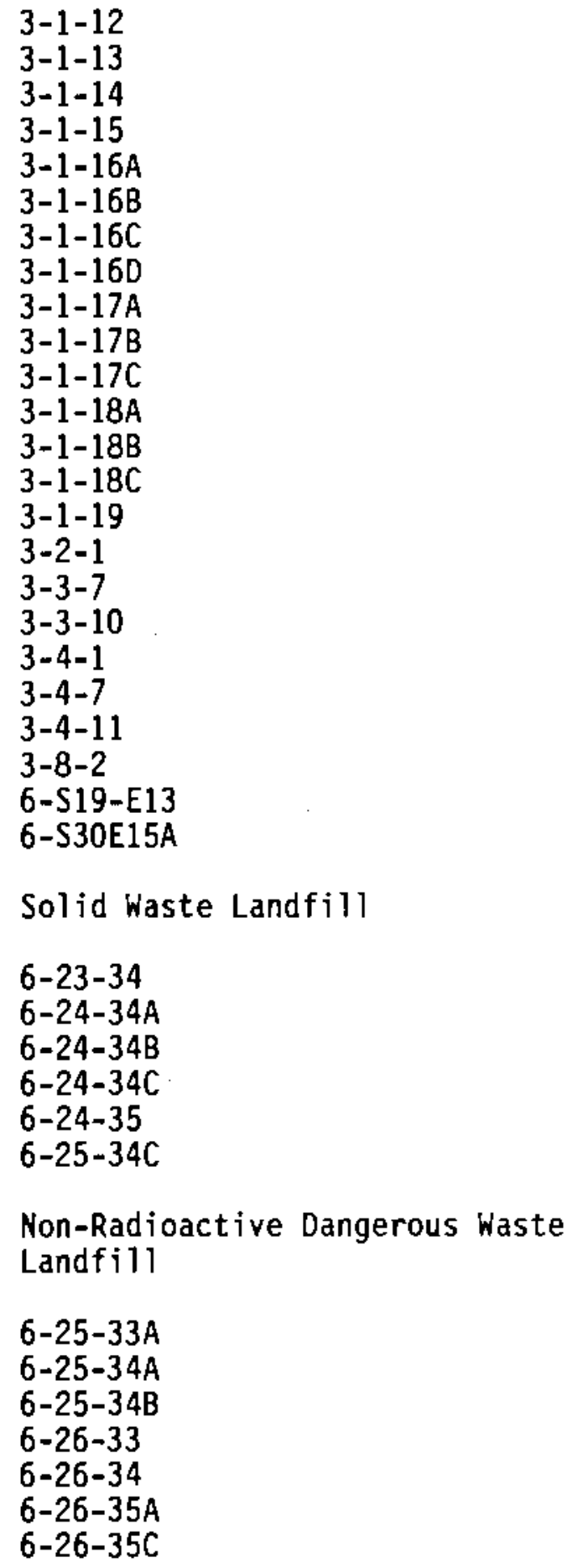 \\
\hline
\end{tabular}


TA8LE A.3. Wells in the Site-wide Radiological (Plus Nitrate) Monitoring Network That are Not Part of the Site-wide Chemical or Compliance Networks (Tables A.1 or A.2)

\begin{tabular}{|c|c|c|c|}
\hline $\begin{array}{l}1-B 4-2 \\
1-B 4-3 \\
1-F 5-4 \\
1-N-2 \\
1-N-3 \\
1-N-4 \\
1-N-5 \\
1-N-15 \\
1-N-16 \\
1-N-18 \\
1-N-19 \\
1-N-20 \\
1-N-21 \\
1-N-22 \\
1-N-23 \\
1-N-25 \\
1-N-27 \\
1-N-30 \\
1-N-31 \\
1-N-32 \\
1-N-33 \\
1-N-36 \\
1-N-37 \\
1-N-39 \\
1-N-45 \\
1-N-49 \\
1-N-50 \\
1-N-51 \\
1-N-52 \\
2-E 13-8 \\
2-E 13-19 \\
2-E 16-2 \\
2-E 17-2 \\
2-E 17-8 \\
2-E 17-12 \\
2-E 17-13 \\
2-E 23-2 \\
2-E 24-1 \\
2-E 24-2 \\
2-E 24-4 \\
2-E 24-11 \\
2-E 24-13 \\
2-E 25-3 \\
2-E 25-6\end{array}$ & $\begin{array}{l}2-E 25-9 \\
2-E 25-11 \\
2-E 25-13 \\
2-E 25-17 \\
2-E 25-19 \\
2-E 25-23 \\
2-E 25-24 \\
2-E 26-2 \\
2-E 26-4 \\
2-E 26-6 \\
2-E 26-8 \\
2-E 27-7 \\
2-E 28-9 \\
2-E 28-12 \\
2-E 28-16 \\
2-E 28-18 \\
2-E 28-23 \\
2-E 28-24 \\
2-E 28-25 \\
2-E 33-7 \\
2-E 33-9 \\
2-E 33-12 \\
2-E 33-14 \\
2-E 33-20 \\
2-E 34-1 \\
2-W 10-1 \\
2-W 11-11 \\
2-W 11-18 \\
2-W 11-23 \\
2-W 11-24 \\
2-W 14-10 \\
2-W 15-6 \\
2-W 18-5 \\
2-W 18-9 \\
2-W 18-15 \\
2-W 18-17 \\
2-W 18-18 \\
2-W 18-20 \\
2-W 19-2 \\
2-W 19-12 \\
2-W 19-17 \\
2-W 19-18 \\
2-W 19-19 \\
2-W 19-20\end{array}$ & $\begin{array}{l}2-W 19-21 \\
2-W 19-23 \\
2-W 19-24 \\
2-W 19-25 \\
2-W 19-26 \\
2-W 22-10 \\
2-W 22-18 \\
2-W 22-21 \\
2-W 23-9 \\
2-W 26-3 \\
2-W 26-6 \\
3-2-2 \\
3-2-3 \\
3-3-1 \\
3-3-2 \\
3-3-3 \\
3-3-6 \\
3-3-9 \\
3-3-11 \\
3-3-12 \\
3-4-9 \\
3-4-10 \\
3-5-1 \\
3-6-1 \\
3-8-1 \\
3-8-3 \\
3-8-4 \\
4-S 0-7 \\
4-S 0-8 \\
4-S 1-7 B \\
4-S 1-7 C \\
4-S 1-8 A \\
4-S 1-8 B \\
4-S 1-8 C \\
6-1-18 \\
6-2-7 \\
6-3-45 \\
6-4-E 6 \\
6-8-17 \\
6-8-32 \\
6-10-E 12 \\
6-10-54 A \\
6-12-4 B \\
6-13-64\end{array}$ & $\begin{array}{l}6-14-E 6 T \\
6-14-38 \\
6-14-47 \\
6-15-15 B \\
6-17-5 \\
6-17-47 \\
6-17-70 \\
6-19-43 \\
6-19-58 \\
6-19-88 \\
6-20-E 5 A P \\
6-20-E 5 A R \\
6-20-E 12 \\
6-20-E 12 P \\
6-20-82 \\
6-21-6 \\
6-22-70 \\
6-24-1 P \\
6-24-1 R \\
6-24-1 S \\
6-24-1 T \\
6-24-46 \\
6-25-55 \\
6-25-70 \\
6-26-15 A \\
6-26-89 \\
6-27-8 \\
6-28-40 P \\
6-28-52 A \\
6-29-78 \\
6-31-31 \\
6-31-31 P \\
6-32-62 \\
6-32-70 B \\
6-32-72 \\
6-32-77 \\
6-34-39 A \\
6-34-41 B \\
6-34-88 \\
6-35-66 \\
6-36-46 P \\
6-36-61 A \\
6-36-61 B \\
6-37-E 4\end{array}$ \\
\hline
\end{tabular}


IABLE A.3. (contd)

$6-37-43$
$6-38-15$
$6-38-70$
$6-39-0$
$6-39-39$
$6-40-62$
$6-41-1$
$6-41-23$
$6-42-2$
$6-42-12 A$
$6-42-40 A$
$6-42-40 B$
$6-42-40 C$
$6-43-3$
$6-44-4$
$6-44-64$
$6-45-2$
$6-45-42$
$6-46-4$
$6-46-21 B$
$6-47-5$
$6-47-35 A$
$6-47-46 A$
$6-47-50$
$6-47-60$
$6-49-13 E$
$6-49-28$
$6-49-55 A$
$6-49-100 C$
$6-50-28 B$
$6-50-30$
$6-50-42$
$6-50-45$
$6-50-48 B$
$6-50-85$

$\begin{array}{lll}6-51-46 & 6-59-58 & 6-71-30 \\ 6-51-63 & 6-59-80 B & 6-71-52 \\ 6-52-19 & 6-60-32 & 6-71-77 \\ 6-52-46 A & 6-60-57 & 6-72-73 \\ 6-52-48 & 6-60-60 & 6-72-88 \\ 6-53-35 & 6-61-37 & 6-72-92 \\ 6-53-47 A & 6-61-41 & 6-73-61 \\ 6-53-47 B & 6-61-62 & 6-74-44 \\ 6-53-48 A & 6-61-66 & 6-77-36 \\ 6-53-48 B & 6-62-31 & 6-78-62 \\ 6-53-50 & 6-62-43 F & 6-80-43 P \\ 6-53-55 A & 6-63-25 A & 6-80-43 R \\ 6-53-103 & 6-63-51 & 6-80-43 S \\ 6-54-34 & 6-63-55 & 6-81-58 \\ 6-54-37 A & 6-63-58 & 6-84-35 A 0 \\ 6-54-42 & 6-63-90 & 6-87-55 \\ 6-54-48 & 6-64-27 & 6-96-49 \\ 6-54-49 & 6-64-62 & 6-97-43 \\ 6-54-57 & 6-65-50 & 6-97-51 A \\ 6-55-40 & 6-65-59 & 6-101-48 B \\ 6-55-44 & 6-65-72 & 6-S 3-E 12 \\ 6-55-50 A & 6-65-83 & 6-S 6-E 4 B \\ 6-55-50 C & 6-66-23 & 6-S 6-E 4 D \\ 6-55-50 D & 6-66-38 & 6-S 7-34 \\ 6-55-70 & 6-66-39 & 6-S 11 E 12 A \\ 6-55-76 & 6-66-58 & 6-S 11 E 12 A P \\ 6-55-89 & 6-66-64 & 6-S 12-29 \\ 6-56-43 & 6-66-103 & 6-S 14-20 A \\ 6-56-53 & 6-67-51 & 6-S 18-51 \\ 6-57-25 A & 6-67-86 & 6-S 19-11 \\ 6-57-29 A & 6-67-98 & 6-S 24-19 \\ 6-57-83 A & 6-68-105 & 6-S 27-E 14 \\ 6-58-24 & 6-69-38 & 6-S 28-E 0 \\ 6-59-32 & 6-70-68 & 6-S 31-1 P \\ & & \end{array}$


TABLE A.4. Results for Site-Wide Chemical Monitoring Wells

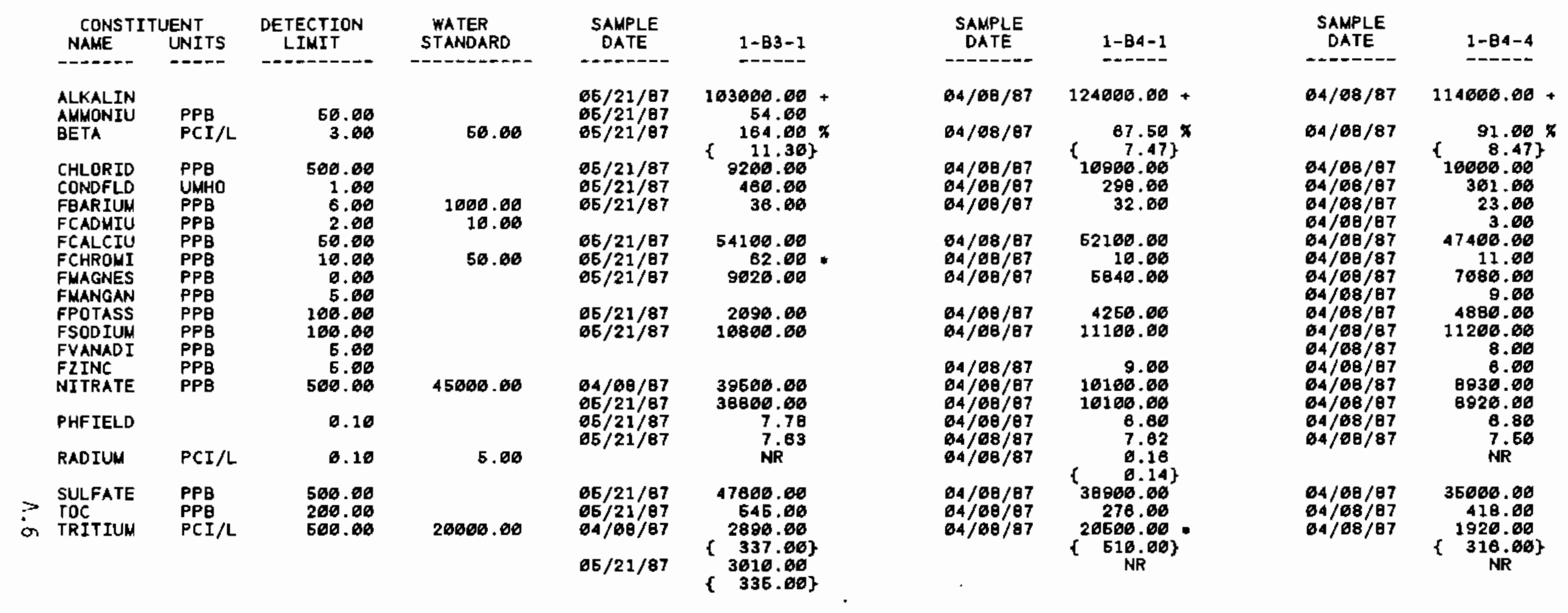

* - VAlUe exceeds primary dRINKINg mater standaro.

- VALUE EXCEEDS PRDPOSED PRIMARY DRINKING WATER STANDARO.

* - VALUE EXCEEDS SCREENING LEVEL FOR FURTHER INVESTIGATION.

* - DETECTION LIMIT WAS NOT AVAILABLE FOR COMPARISON

NR - ANALYSIS NOT REQUESTED OR NOT YET REPORTED

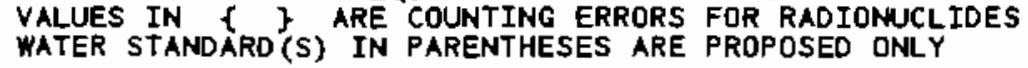


IABLE A.4. (contd)

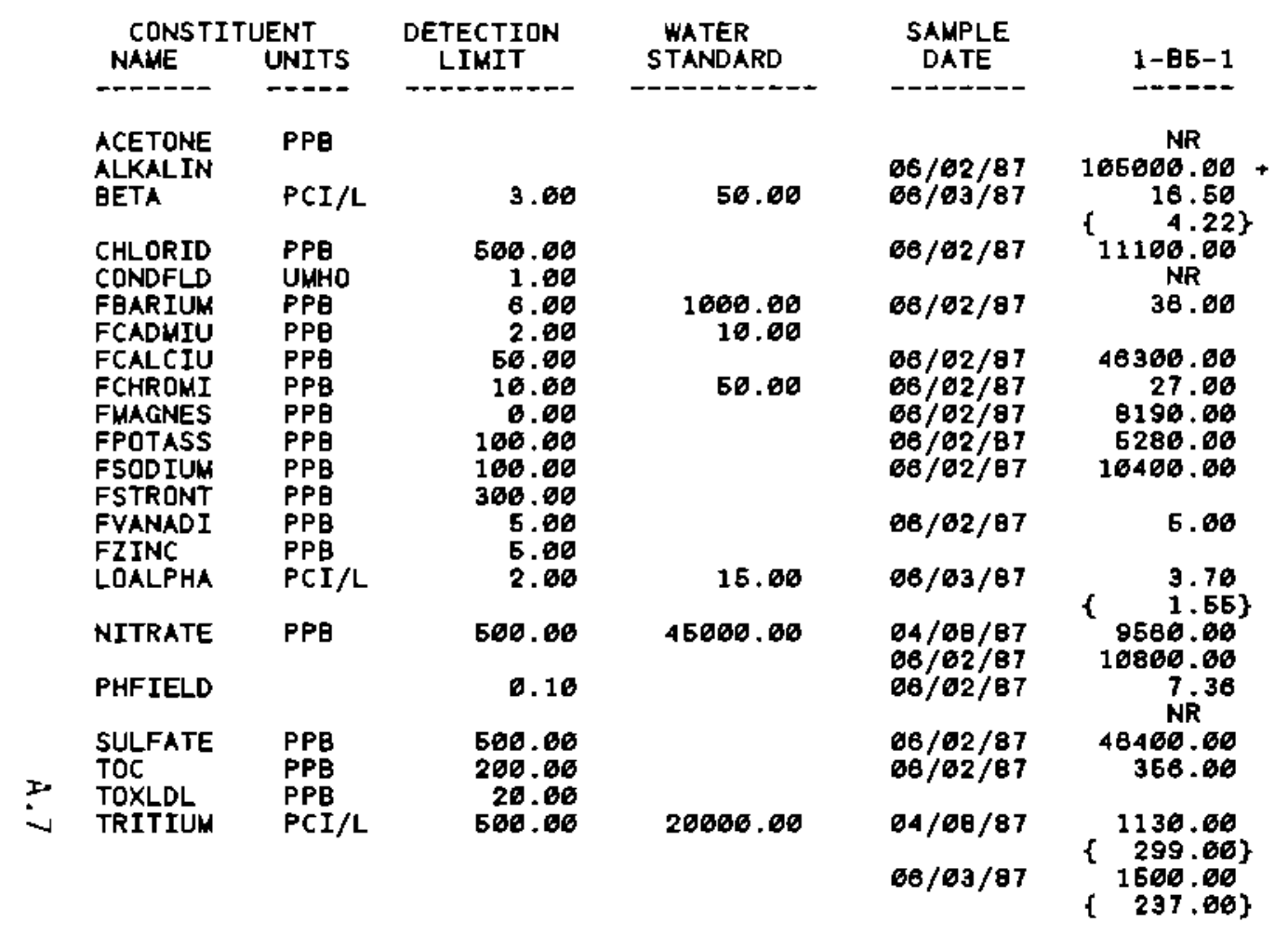

\begin{tabular}{|c|c|}
\hline $\begin{array}{c}\text { SAMPLE } \\
\text { DATE }\end{array}$ & $1-89-1$ \\
\hline 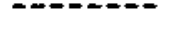 & $\cdots$ \\
\hline $\begin{array}{l}04 / 08 / 87 \\
64 / 68 / 87 \\
04 / 08 / 87\end{array}$ & $\begin{array}{r}85.00 \\
111600.00 \\
24.70\end{array}$ \\
\hline $\begin{array}{l}04 / 08 / 87 \\
04 / 68 / 87 \\
64 / 69 / 87 \\
04 / 08 / 87 \\
64 / 68 / 87 \\
64 / 08 / 87 \\
04 / 08 / 87 \\
64 / 68 / 87 \\
64 / 08 / 87\end{array}$ & $\begin{array}{r}20100.00 \\
430.00 \\
15.00 \\
2.00 \\
62300.00 \\
16.00 \\
12600.00 \\
7990.00 \\
14200.00\end{array}$ \\
\hline $\begin{array}{l}04 / 68 / 87 \\
04 / 08 / 87\end{array}$ & $\begin{array}{l}12.00 \\
13.00\end{array}$ \\
\hline $\begin{array}{l}04 / 08 / 87 \\
04 / 68 / 87 \\
04 / 08 / 87 \\
04 / 68 / 87 \\
04 / 68 / 87 \\
04 / 68 / 87\end{array}$ & $\begin{array}{r}24800.00 \\
26360.00 \\
7.10 \\
7.56 \\
72800.00 \\
769.06\end{array}$ \\
\hline $04 / 08 / 87$ & $\left\{\begin{array}{c}1250.00 \\
366.66 \\
\mathrm{NR}\end{array}\right\}$ \\
\hline
\end{tabular}

SALPLE
DATE
-

$\begin{array}{cc} & N R \\ 64 / 21 / 87 & 108000.00 \\ 64 / 21 / 87 & 0.95\end{array}+$

$64 / 21 / 87$

$64 / 21 / 87$
$04 / 21 / 87$

$04 / 21 / 87$

$04 / 21 / 87$

04/21/87

$04 / 21 / 87$

$64 / 21 / 67$

$04 / 21 / 87$

$04 / 21 / 87$

$64 / 21 / 87$

$64 / 21 / 87$

$04 / 21 / 87$

$64 / 21 / 87$
$64 / 21 / 87$

$64 / 21 / 87$

$64 / 21 / 87$

$64 / 21 / 87$
$04 / 21 / 87$

NR $\left\{\begin{array}{r}3.89\} \\ 23700.09\end{array}\right.$

B85. 00

93.00

95600.00

219.00

27800.06

8030.66

14800.00

823.80

12.06

12.06

77500.000 7.46

201000.06

1230.06

38.56

19760.00

\{591.06\} 99800.66 .

* - VALUE EXCEEDS PRIMARY DRINKING WATER STANDARD.

* - VALUE EXCEEDS SCREENING LEVEL FOR FURTHER INVESTIGATION.

- - DETECTION LINIT WAS NOT AVAILABLE FOR COMPARISON

NR - ANALYSIS NDT REQUESTED OR NOT YET REPORTED

VALUES IN \{ $\}$ ARE COUNTING ERRORS FOR RADTONUCLIDES

WATER STANDARD (S) IN PARENTHESES ARE PROPOSED ONLY 
TABLE A.4. (contd)

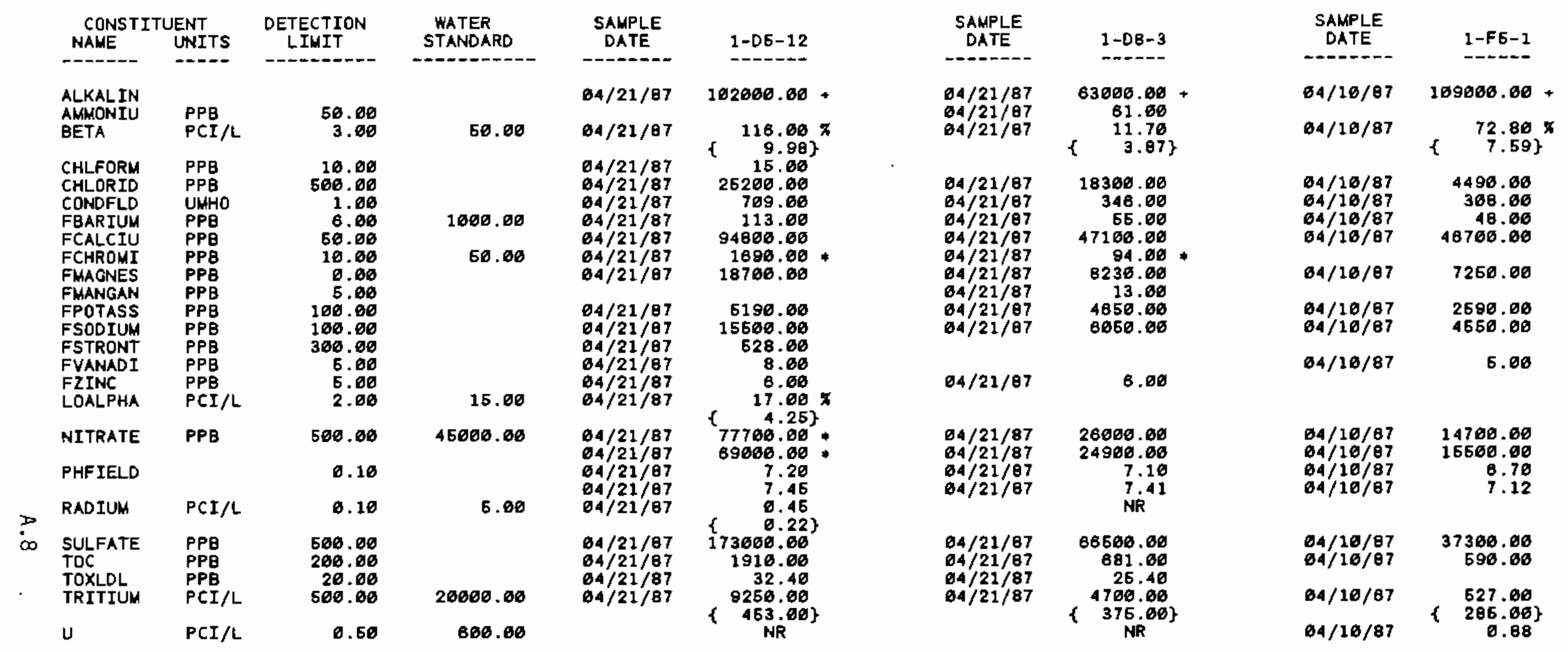

- VALUE eXCEEDS PRIMARY DRINKING waTER STANDARD.

- VALUE EXCEEDS PROPOSED PRIMARY DRINKING WATER STANDARD.

+ - DETECTION LIMIT WAS NOT AVAILABLE FOR COMPARISON

NR - ANALYSIS NOT REQUESTED OR NOT YET REPORTED

VALUES IN \{ \} ARE COUNTING ERRORS FOR RADIONUCLIDES 
TABLE A.4. (contd)

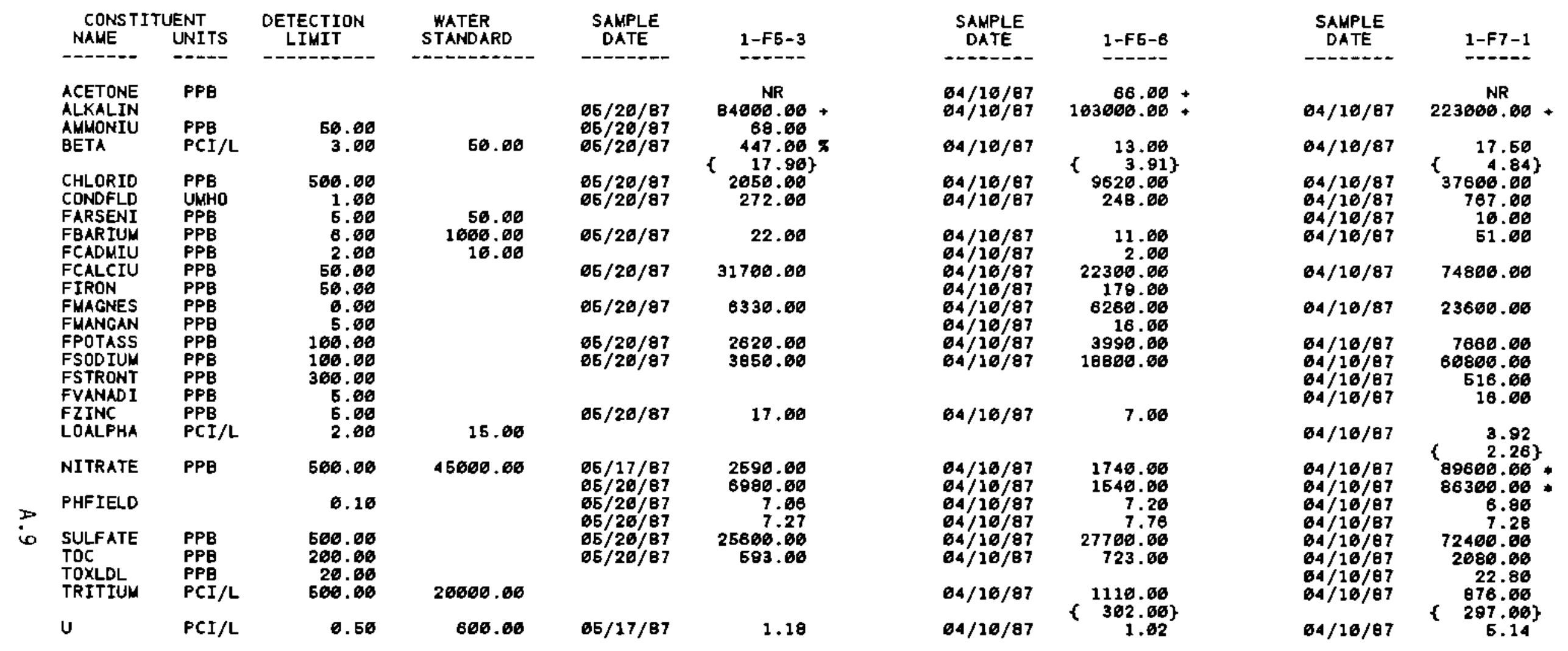

* - VALUE EXCEEDS PRIMARY DRINKING waTER STANDARo.

- VALUE EXCEEDS PROPOSED PRIMARY DRINKING WATER STANDARD.

- VALUE EXCEEDS SCREENING LEVEL FOR FURTHER INVESTIGATION.

+ DETECTION LIMIT WAS NOT AVAILABLE FOR COMPARISON

NR - ANALYSIS NOT REQUESTED OR NOT YET REPORTED

VALUES IN $\{$ ' $\}$ ARE COUNTING ERRORS FOR RADIONUCLIDES 
IABLE A.4. (contd)

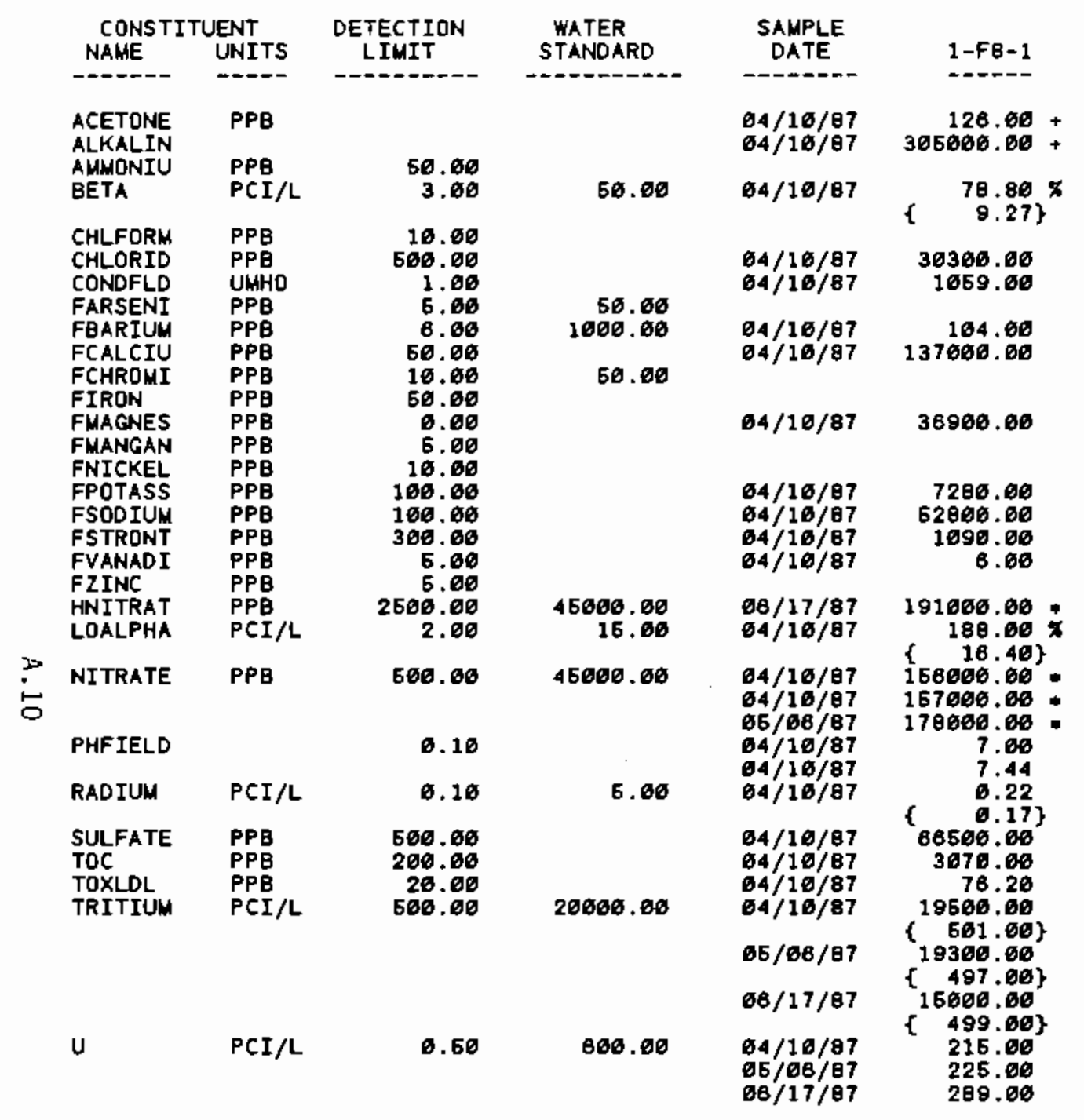

\begin{tabular}{|c|c|}
\hline $\begin{array}{l}\text { SAMPLE } \\
\text { DATE }\end{array}$ & $1-F 8-2$ \\
\hline $\begin{array}{l}06 / 21 / 87 \\
65 / 21 / 87 \\
65 / 21 / 87\end{array}$ & 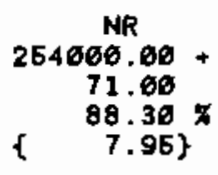 \\
\hline $\begin{array}{l}65 / 21 / 87 \\
05 / 21 / 87\end{array}$ & $\begin{array}{r}11460.00 \\
863.00\end{array}$ \\
\hline $\begin{array}{l}65 / 21 / 87 \\
05 / 21 / 87 \\
05 / 21 / 87 \\
05 / 21 / 87 \\
65 / 21 / 87 \\
05 / 21 / 87 \\
05 / 21 / 87 \\
05 / 21 / 87 \\
65 / 21 / 87 \\
65 / 21 / 87 \\
05 / 21 / 87 \\
65 / 21 / 87\end{array}$ & $\begin{array}{r}48.06 \\
95600.66 \\
21.00 \\
187.06 \\
24700.06 \\
18.00 \\
18.00 \\
7886.60 \\
28306.06 \\
884.00 \\
7.06 \\
13.06\end{array}$ \\
\hline $65 / 21 / 87$ & \\
\hline $\begin{array}{l}65 / 17 / 87 \\
65 / 17 / 87 \\
05 / 21 / 87 \\
65 / 21 / 87 \\
05 / 21 / 87\end{array}$ & $\begin{array}{c}99306.06 \\
89100.06 \\
98606 . .06 \\
7.86 \\
7.71 \\
\mathrm{NR}\end{array}$ \\
\hline $\begin{array}{l}05 / 21 / 87 \\
65 / 21 / 97 \\
65 / 21 / 87 \\
05 / 17 / 87\end{array}$ & $\begin{array}{r}53706.06 \\
2176.06 \\
48.30 \\
2910.06\end{array}$ \\
\hline $65 / 17 / 87$ & $\begin{array}{r}338.66\} \\
3136.06 \\
\{\quad 343.60\}\end{array}$ \\
\hline $65 / 21 / 87$ & $\begin{array}{r}2206.06 \\
+\end{array}$ \\
\hline $\begin{array}{l}05 / 17 / 87 \\
65 / 17 / 87\end{array}$ & $\begin{array}{c}81.50 \\
89.60 \\
\mathrm{NR}\end{array}$ \\
\hline
\end{tabular}

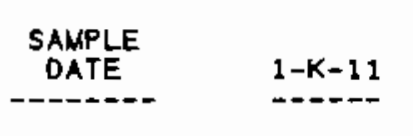

NR

$04 / 23 / 87 \quad 131000.00$.

$64 / 23 / 97$

$04 / 23 / 87$

$64 / 23 / 87$

$04 / 23 / 87$

$04 / 23 / 87$

$64 / 23 / 87$

$04 / 23 / 87$

$04 / 23 / 07$

$04 / 23 / 87$

$04 / 23 / 87$
$04 / 23 / 87$

$04 / 23 / 87$
$04 / 23 / 87$

$04 / 23 / 87$

$64 / 23 / 87$

$64 / 23 / 87$

$04 / 23 / 87$

$64 / 23 / 67$

$04 / 23 / 87$

$64 / 23 / 87$

$04 / 23 / 87$

$04 / 23 / 87$ 16.50
$3.89\}$
16.06

6760.06

447.00

16.00

30.00

41700.00

29.06

11800.00

5916.06

33200.00

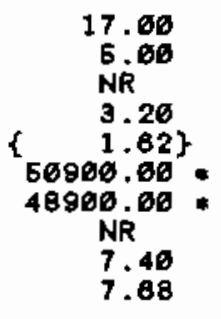

47700.00

1000.00

22.86

1876.00

\{ 315.00$\}$

NR

NR

$64 / 23 / 87$

* - VAlue exceeds PRIMARy dRINKINg water standaro.

* - VALUE EXCEEDS PROPOSED PRIMARY DRINKING WATER STANDARD

* VALUE EXCEEDS SCREENING LEVEL FOR FURTHER INVESTIGATION.

+ - DETECTION LIMIT WAS NOT AVAILABLE FOR COMPARISON

NR - ANALYSIS NOT REQUESTED OR NOT YET REPORTED

VALUES IN \{\} ARE COUNTING ERRORS FOR RADIONUCLIDES

WATER STANDARD (S) IN PARENTHESES ARE PROPOSED ONLY 
TABLE A.4. (contd)

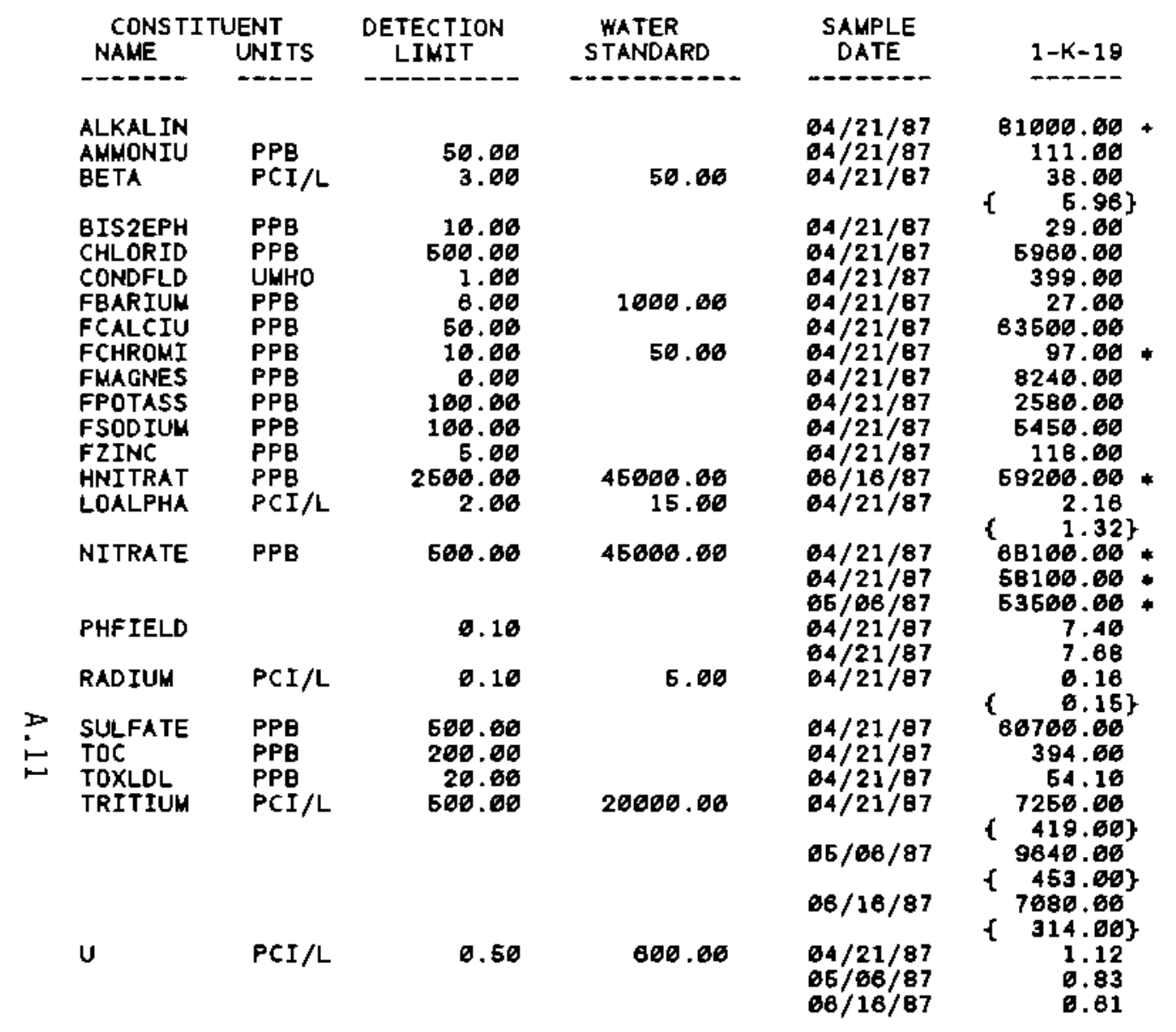

\begin{tabular}{|c|c|}
\hline $\begin{array}{l}\text { SAMPLE } \\
\text { DATE }\end{array}$ & $1-K-2 \theta$ \\
\hline $04 / 23 / 87$ & 85000.00 \\
\hline \multirow[t]{2}{*}{$\begin{array}{l}04 / 23 / 87 \\
04 / 23 / 87 \\
64 / 23 / 87 \\
04 / 23 / 87 \\
04 / 23 / 87 \\
04 / 23 / 87 \\
04 / 23 / 87 \\
04 / 23 / 87\end{array}$} & $\begin{array}{c}37 . B 0 \\
5.75\} \\
N R \\
4548.00 \\
311.00 \\
18.00 \\
47700.00 \\
146.00 \\
7850.00 \\
3180.00 \\
4480.00\end{array}$ \\
\hline & NR \\
\hline $\begin{array}{l}04 / 23 / 87 \\
04 / 23 / 87\end{array}$ & $\begin{array}{l}21800.00 \\
22600.00\end{array}$ \\
\hline $\begin{array}{l}04 / 23 / 87 \\
04 / 23 / 87\end{array}$ & $\begin{array}{l}7.80 \\
7.73 \\
\mathrm{NR}\end{array}$ \\
\hline $\begin{array}{l}04 / 23 / 87 \\
04 / 23 / 87\end{array}$ & $\begin{array}{r}51800.00 \\
327.00\end{array}$ \\
\hline \multirow[t]{2}{*}{$64 / 23 / 87$} & $\begin{array}{c}1210.66 \\
30 \sigma .06\} \\
N R\end{array}$ \\
\hline & NR \\
\hline $04 / 23 / 87$ & $\begin{array}{l}1.29 \\
\text { NR } \\
\text { NR }\end{array}$ \\
\hline
\end{tabular}

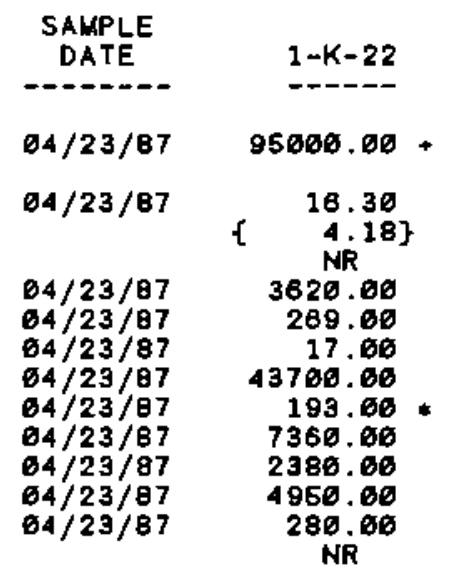

$64 / 23 / 87$

$64 / 23 / 87$

$84 / 23 / 87$

$04 / 23 / 87$
$04 / 23 / 87$

$04 / 23 / 87$

$64 / 23 / 87$

$64 / 23 / 87$

$04 / 23 / 87$

3400.00

3860.60

3560.00

7.56
7.72

NRे

42200.00

564.60

2240.00

( 322.66$\}$

$\left\{\begin{array}{r}299.60\} \\ \text { f }\end{array}\right.$

NR

$64 / 23 / 87$

$04 / 23 / 87$
1.17
1.00

- yalue exceeds primary dRINking water standard

- VALUE EXCEEDS PROPOSED PRIMARY DRINKING HATER STANDARD.

* - VALUE EXCEEDS SCREENING LEVEL FOR FURTHER INVESTIGATION.

+ - DETECTION LIMIT WAS NOT AVAILABLE FOR COMPARISON

NR - ANALYSIS NOT REQUESTED OR NOT YET REPORTED

VALUES IN \& \} ARE COUNTING ERRORS FOR RADIONUCLIDES

WATER STANDARD(S) IN PARENTHESES ARE PROPOSED ONLY 
TABLE A.4. (contd)

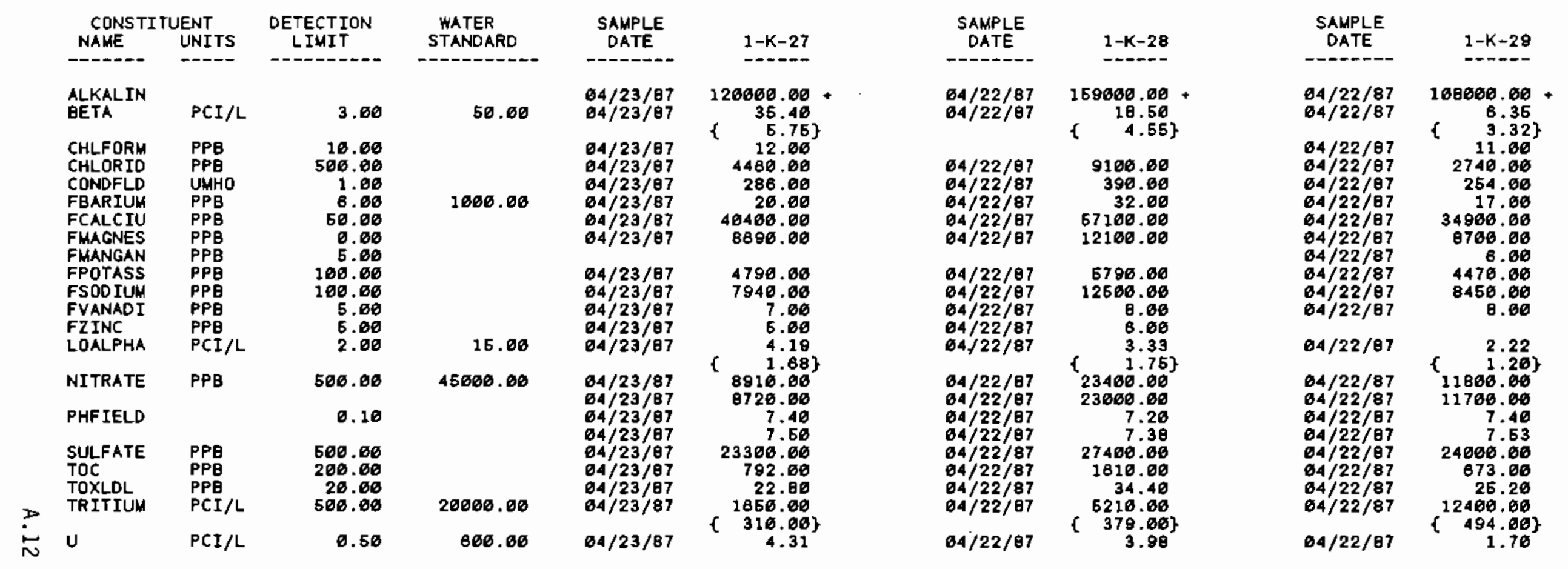

- - value exceeds primary drinking water standard.

- VALUE EXCEEDS PROPOSED PRIMARY DRINKING WATER STANDARD.

* - VALUE EXCEEDS SCREENING LEVEL FOR FURTHER INVESTIGATION.

+ - DETECTION LIMIT WAS NOT AVAILABLE FOR COMPARISON

NR - ANALYSIS NOT REQUESTED OR NOT YET REPORTED

VALUES IN \{ $\}$ ARE COLNTING ERRORS FOR RADIONUCLIDES
WATER STANDARO(S) IN PARENTHESES ARE PROPOSED ONLY 
TABLE A.4. (contd)

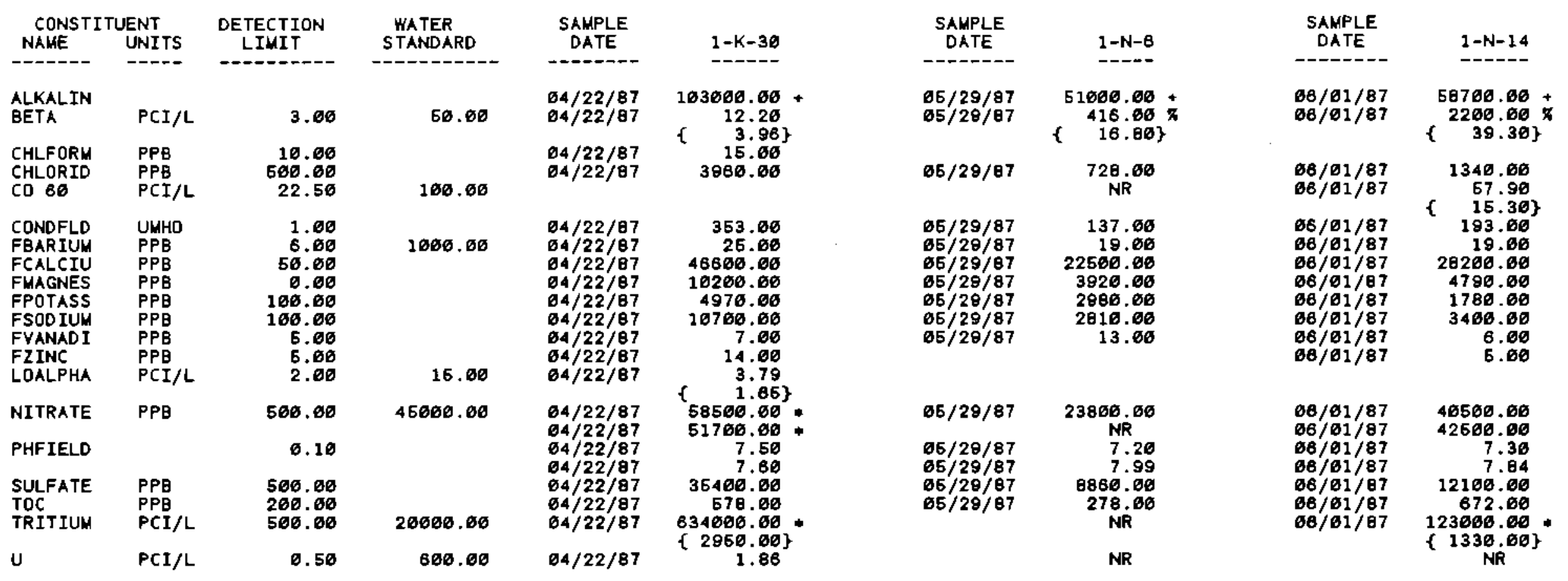

- - VALUe exCEEDS PRIMARY DRINKING water standard.

- VALUE EXCEEDS PROPOSED PRIMARY DRINKING WATER STANDARD

* - VALUE EXCEEDS SCREENING LEVEL FOR FURTHER INVESTIGATION.

+ - DETECTION LIMIT WAS NOT AVAILABLE FOR COMPARISON

NR - ANALYSIS NOT REQUESTED OR NOT YET REPORTED

VALUES IN \{\} ARE COUNTING ERRORS FOR RADIONUCLIDES

WATER STANDARD (S) IN PARENTHESES ARE PROPOSED ONLY 
TABLE A.4. (contd)

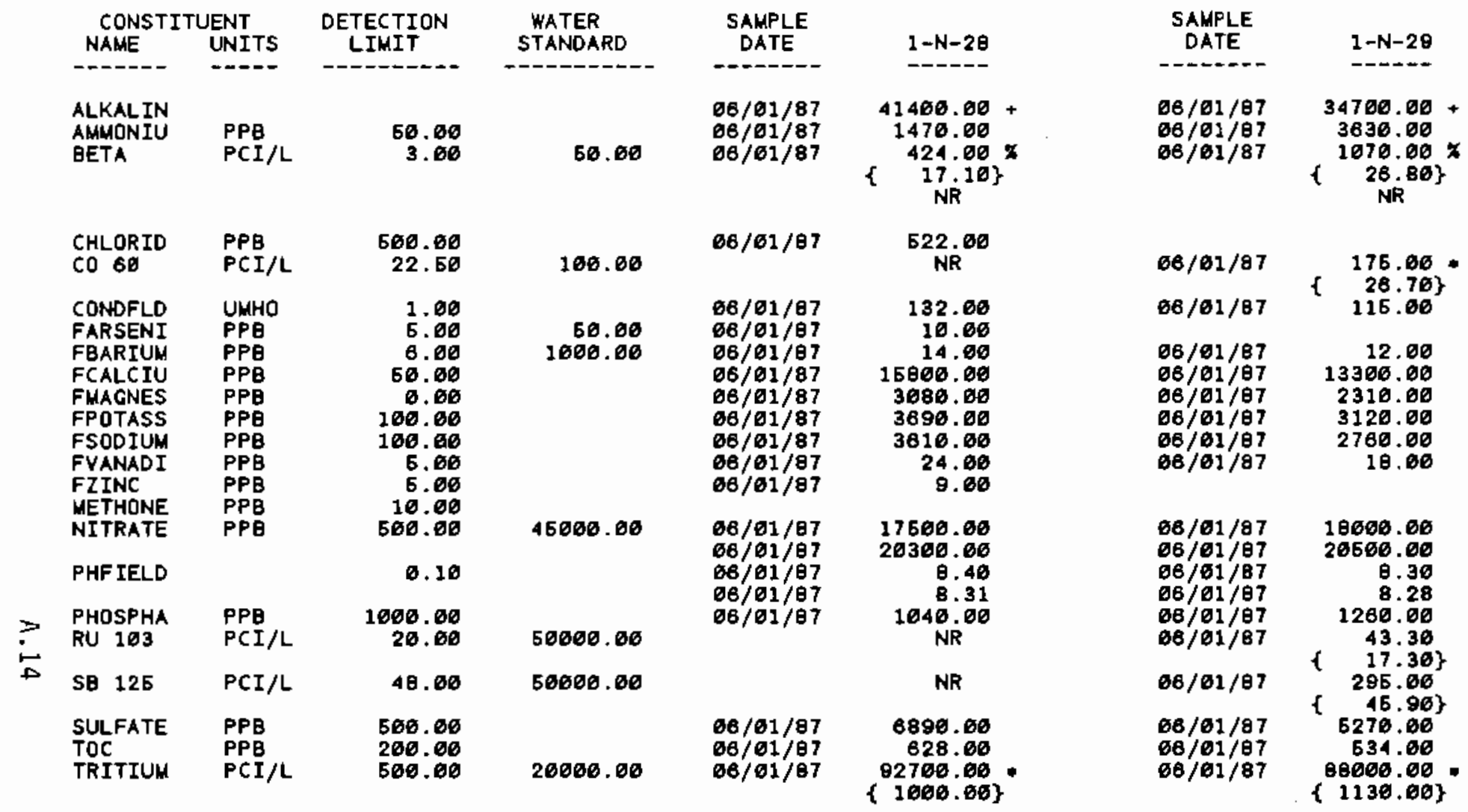

SAMPLE DATE

2-E13-6

$66 / 22 / 97$

$05 / 22 / 87$

$06 / 22 / 87$

$66 / 22 / 87$

$05 / 22 / 97$

58600.00 67.00

$\{\quad 3.59\}$

$\begin{array}{r}8.84 \\ \{\quad 2.34 \\ \hline\end{array}$

7070.00

$65 / 22 / 87$

408.00

NR

NR

NR

NR

NR

NR

NR

$06 / 22 / 87$

$66 / 22 / 87$

60.06

10700.00

NR

$05 / 22 / 97$

6.80
7.62

NR

NR

$06 / 22 / 87 \quad 40000.00$

- - VALUE EXCEEDS PRIMARY DRINKINg waTER STANDARD.

* - VALUE EXCEEDS PROPOSED PRIMARY DRINKING WATER STANDARD.

* - VALUE EXCEEDS SCREENING LEVEL FOR FURTHER INVESTIGATION.

+ - DETECTION LIMIT WAS NOT AVAILABLE FOR COMPARISON

NR - ANALYSIS NOT REQUESTED OR NOT YET REPORTED

VALUES IN \{ \} ARE COUNTING ERRORS FOR RADIONUCLIDES

WATER STANDARD (S) IN PARENTHESES ARE PROPOSED ONLY 
TABLE A.4. (contd)

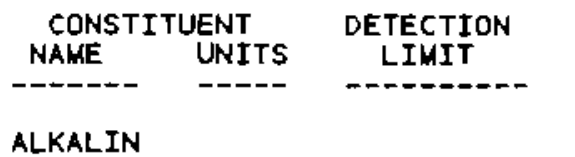

ALKALIN AMMONIU BETA

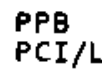

50.00

WATER

STANDARD

SAMPLE

DATE

2-E13-14

65/22/87

$05 / 22 / 87$
$06 / 22 / 87$

$05 / 22 / 87$

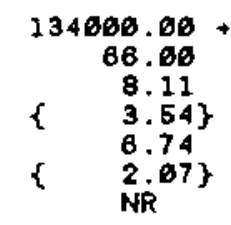

NR

CHLORID

PPB

UNHO
PPB

CONDFL

FARSENI

FBARIUM

FCALCIU

FMAGNES

FPOTASS

FSTRONT

FSTRONT

FVANAD

LOALPHA

PPB

PPB

PPB

PPB

PPB

PPB

PCI/L

500.00

1.00

5.00

6.09

50.00

0.00
100.00
100.00

300.00

300.00
5.00

5.00
2.00

50.00

品

\begin{tabular}{|c|c|c|}
\hline NITRATE & PPB & 600.00 \\
\hline PHF IELD & & 0.10 \\
\hline RADIUM & $\mathrm{PCI} / \mathrm{L}$ & 0.10 \\
\hline SR $9 \emptyset$ & $\mathrm{PCI} / \mathrm{L}$ & 6.00 \\
\hline $\begin{array}{l}\text { SULFATE } \\
\text { TOC } \\
\text { TOXLDL } \\
\text { TRITIUM }\end{array}$ & $\begin{array}{l}\text { PPB } \\
\text { PPB } \\
\text { PPB } \\
\text { PCI/L }\end{array}$ & $\begin{array}{r}500.00 \\
200.00 \\
20.00 \\
500.00\end{array}$ \\
\hline
\end{tabular}

50.00

1000.00

$05 / 22 / 87$

$05 / 22 / 87$

7300.00

350.00

NR

NR

NR

NR

NR

NR

15.00

65/22/87

$\left\{\begin{array}{l}2.61 \\ 1.38\end{array}\right\}$

NR

NR

46000.00

$65 / 22 / 87$

11900.00

NR

NR

6.60

05/22/87

$06 / 22 / 87$

7.65

NR

Q.

$05 / 22 / 87$

38700.00

b5/22/87

27.90

20000.00

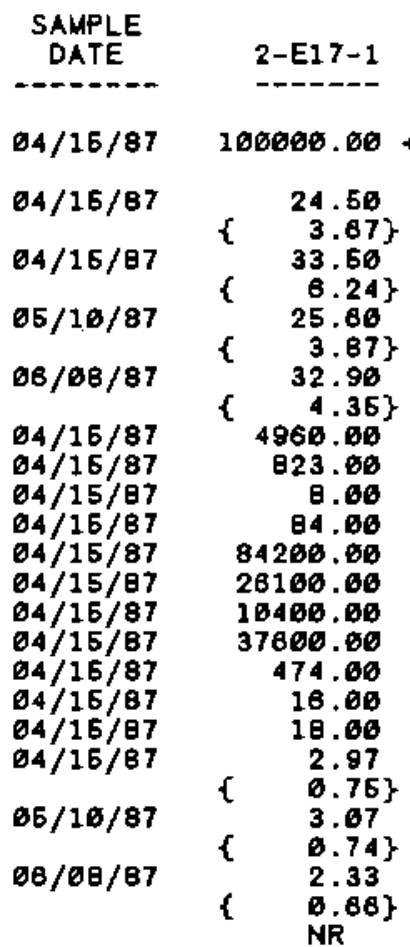

04/16/87 376000.00.

$04 / 16 / 87 \quad 382006.06$.

$06 / 10 / 87 \quad 246000.66$.

$68 / 80 / 87359000.06=$

$04 / 16 / 87$

$04 / 16 / 87$

$84 / 16 / 87$

$04 / 16 / 87$

$04 / 16 / 87$
$04 / 16 / 87$

$04 / 16 / 87$

7.26

7.46

$\{\quad 0.14\}$

6.36
$\quad 1.46\}$

43000.60
450.00

$7690000.60 *$
$\{23606.00\}$

SAMPLE DATE

2-E17-5

$04 / 09 / 8798000.00+$

$04 / 02 / 87 \quad 56.90 \%$

$04 / 09 / 87$ \{ 59.60$\}$

$06 / 10 / 87\left\{\begin{array}{l}7.59\} \\ 60.897 x\end{array}\right.$

6/פB/B7 $\{5.37\}$

$94 / 00 / 87$

$\{\quad 5.17\}$

830.68

$04 / 00 / 87 \quad 39.60$

64/09/87

$04 / 89 / 87$

$04 / 69 / 87$

12960.00

22100.00

21.06

$04 / 02 / 87$

$64 / 09 / 87$

$06 / 10 / 87$

$08 / 08 / 87$

$04 / 02 / 87$

$04 / 09 / 87$

$65 / 10 / 87$

$96 / 08 / 87$

$04 / 09 / 87$

$64 / 09 / 87$

$04 / 09 / 87$

9410987

$64 / 09 / 87$

29800.60
783.00

$04 / 02 / 87 \quad 4210000.00 *$

- - VAlUe EXCEEDS PRIMARY DRINKING water STANDARD.

- VALUE EXCEEDS PROPOSED PRIMARY DRINKING WATER STANDARD.

* - VALUE EXCEEDS SCREENING LEVEL FOR FURTHER INVESTIGATION.

- DETECTION LIMIT WAS NOT AVAILABLE FOR COMPARISON

NR - ANALYSIS NOT REQUESTED OR NOT YET REPORTED

VALUES IN \{\} ARE COUNTING ERRORS FOR RADIONUCLIDES

WATER STANDARD?(S) IN PARENTHESES ARE PROPOSED ONLY 
TABLE A.4. (contd)

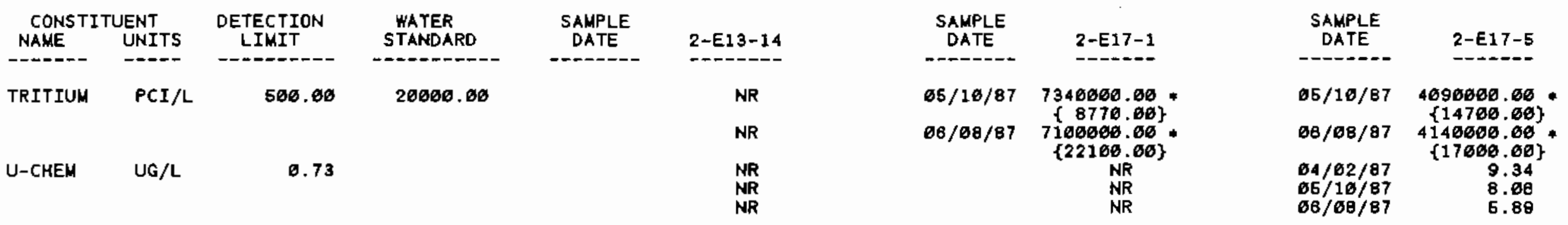

* - VALUE EXCEEDS SCREENING LEVEL FOR FURTHER INVESTIGATION.

+ - DETECTION LIMIT WAS NOT AVAILABLE FOR COMPARISON

NR - ANALYSIS NOT REQUESTED OR NOT YET REPORTED

NALUES IN \{ $\}$ ARE COUNTING ERRORS FOR RADIONUCLIDES 
TABLE A.4. (contd)

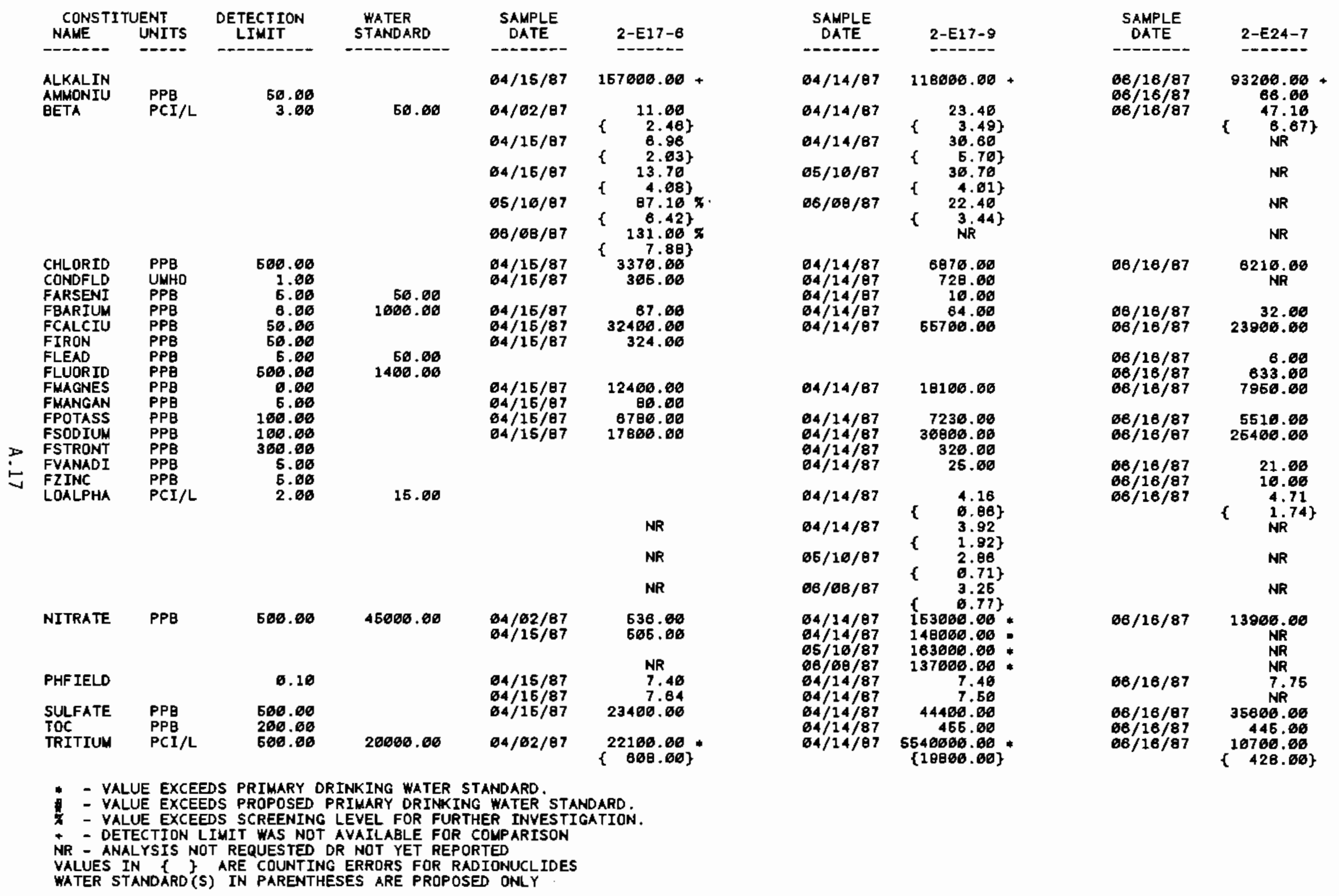


TABLE A.4. (contd)

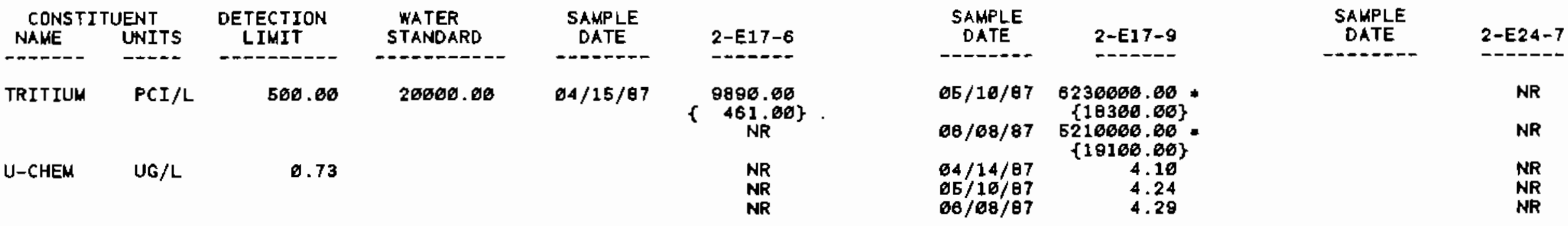

- VALUE EXCEEDS SCREENING LEVEL FOR FURTHER INVESTIGATION.

+ - DETECTION LIMIT WAS NOT AVAILABLE FOR COMPARISON

NR - ANALYSIS NOT REQUESTED OR NOT YET REPORTED

WALUES IN $\{$ f ARE COUNTING ERRORS FOR RADIONUCLIDES 
TABLE A.4. (contd)

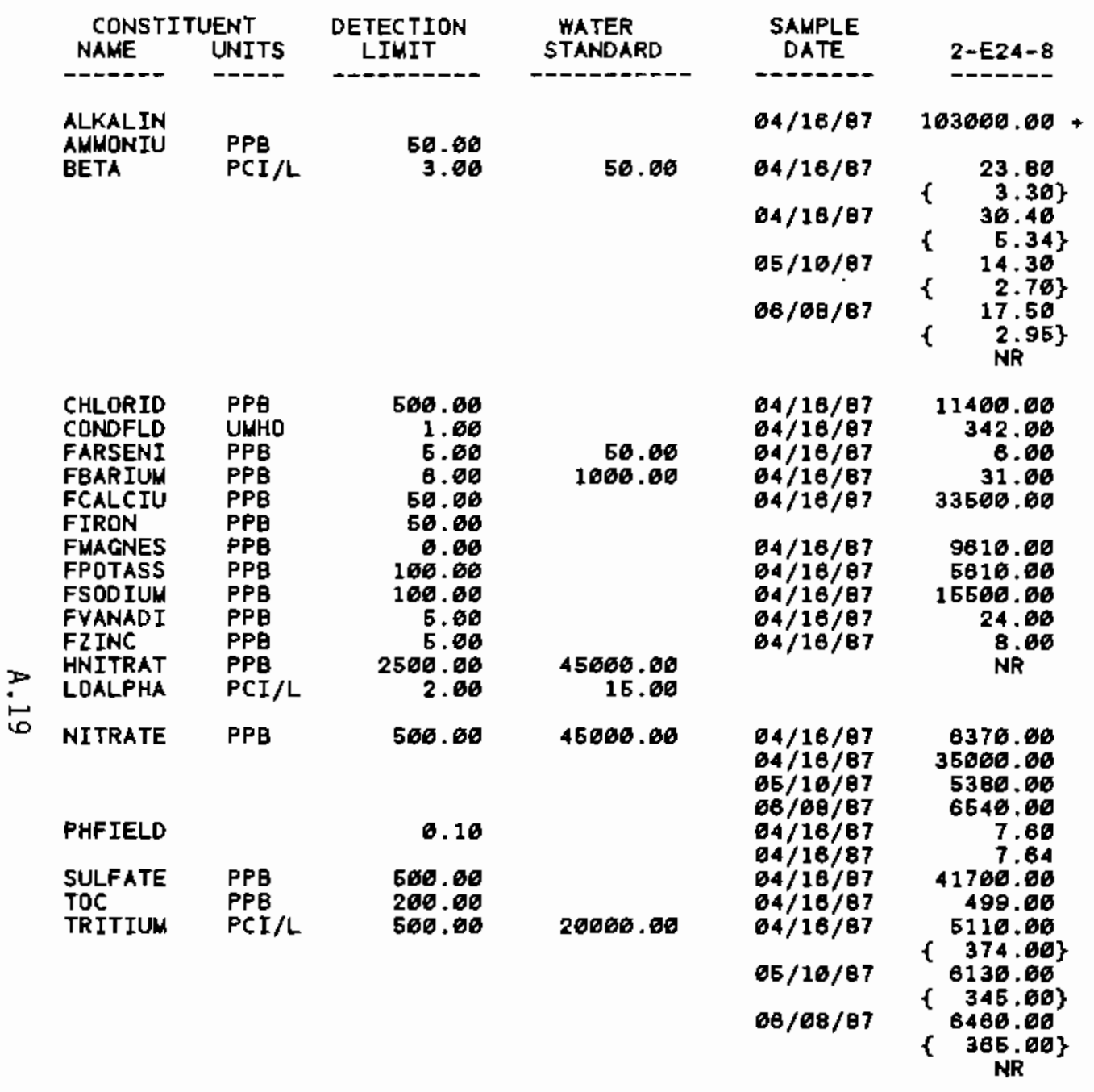

\begin{tabular}{|c|c|}
\hline $\begin{array}{l}\text { SAMPLE } \\
\text { DATE }\end{array}$ & $2-E 24-12$ \\
\hline$-2=0-10$ & \\
\hline $04 / 18 / 87$ & $131060.00+$ \\
\hline $04 / 01 / 87$ & 28.68 \\
\hline $64 / 16 / 87$ & $\begin{array}{r}3.76\} \\
21.76\end{array}$ \\
\hline $04 / 16 / 87$ & $\begin{array}{r}3.23\} \\
32.70\end{array}$ \\
\hline $65 / 27 / 87$ & $\begin{array}{c}5.53\} \\
41.36\end{array}$ \\
\hline $06 / 17 / 87$ & $\begin{array}{c}4.56\} \\
35.20\end{array}$ \\
\hline $\begin{array}{l}64 / 16 / 87 \\
64 / 16 / 87 \\
64 / 16 / 87 \\
64 / 16 / 87 \\
04 / 16 / 87\end{array}$ & $\begin{array}{r}4.26\} \\
4740.06 \\
385.00 \\
7.06 \\
44.00 \\
43300.00\end{array}$ \\
\hline $\begin{array}{l}04 / 16 / 87 \\
04 / 16 / 87 \\
04 / 16 / 87 \\
04 / 16 / 87\end{array}$ & $\begin{array}{r}12206.06 \\
6180.60 \\
21200.06 \\
25.00\end{array}$ \\
\hline $\begin{array}{l}06 / 17 / 87 \\
64 / 16 / 87\end{array}$ & $\begin{array}{r}62760.00 \\
16.50\end{array}$ \\
\hline $\begin{array}{l}04 / 61 / 87 \\
64 / 16 / 87 \\
64 / 16 / 87 \\
65 / 27 / 87 \\
64 / 16 / 87 \\
64 / 16 / 87 \\
84 / 16 / 87 \\
64 / 16 / 87 \\
64 / 61 / 87\end{array}$ & $\begin{array}{r}80006.06 \\
63000.06 \\
46000.06 \\
68200.00 \\
7.10 \\
7.37 \\
32900.06 \\
401.06 \\
1210000.00\end{array}$ \\
\hline $04 / 16 / 87$ & $\begin{array}{l}4130.00\} \\
3600.00 \\
7860.00\}\end{array}$ \\
\hline $05 / 27 / 87$ & $\begin{array}{c}124006 \theta .06 \\
\{0256.06\}\end{array}$ \\
\hline $86 / 17 / 87$ & $\begin{array}{c}1170000.00 \\
\{9080.00\}\end{array}$ \\
\hline
\end{tabular}

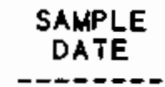

$6 / 17 / 87$

$66 / 17 / 87$
$66 / 17 / 67$ 66/16/日7

$04 / 01 / 87 \quad 28.68 \quad 06 / 16 / 87$

00400.00 69.66

$\begin{array}{r}9.94 \\ \{\quad 3.72\} \\ \hline\end{array}$ NR

NR

NR

NR

$06 / 17 / 87$

$42 \theta 0.00$

NR

$68 / 17 / 87$

$66 / 17 / 87$

$66 / 17 / 87$

66/17/87

$66 / 17 / 87$

B6/17/87

$08 / 17 / 87$

ถ⿻/17/67

11.00

2228.60

58.00

68.00

6420.60

14906.00

32.00

NR

$66 / 17 / 87$

2040.00

NR

NR

$68 / 17 / 87$

$66 / 17 / 87$

$06 / 17 / 87$

$06 / 16 / 97$ 7.04

NR

8100.00
525.00

6810.00

$\{\quad 340.06\}$

NR

NR

NR

* - VAlUe eXCEeds PRIMARY DRINKINg water STANDARD.

* - VALUE EXCEEDS PROPOSED PRIMARY DRINKING WATER STANDARD.

* - VALUE EXCEEDS SCREENING LEVEL FOR FURTHER INVESTIGATION.

+ - DETECTION LIMIT WAS NOT AVAILABLE FOR COMPARISON

NR - ANALYSIS NOT REQUESTED OR NOT YET REPORTED

VALUES IN \{ \} ARE COUNTING ERRORS FOR RADIONUCLIDES

WATER STANDARD'S) IN PARENTHESES ARE PROPOSED ONLY 
TABLE A.4. (contd)

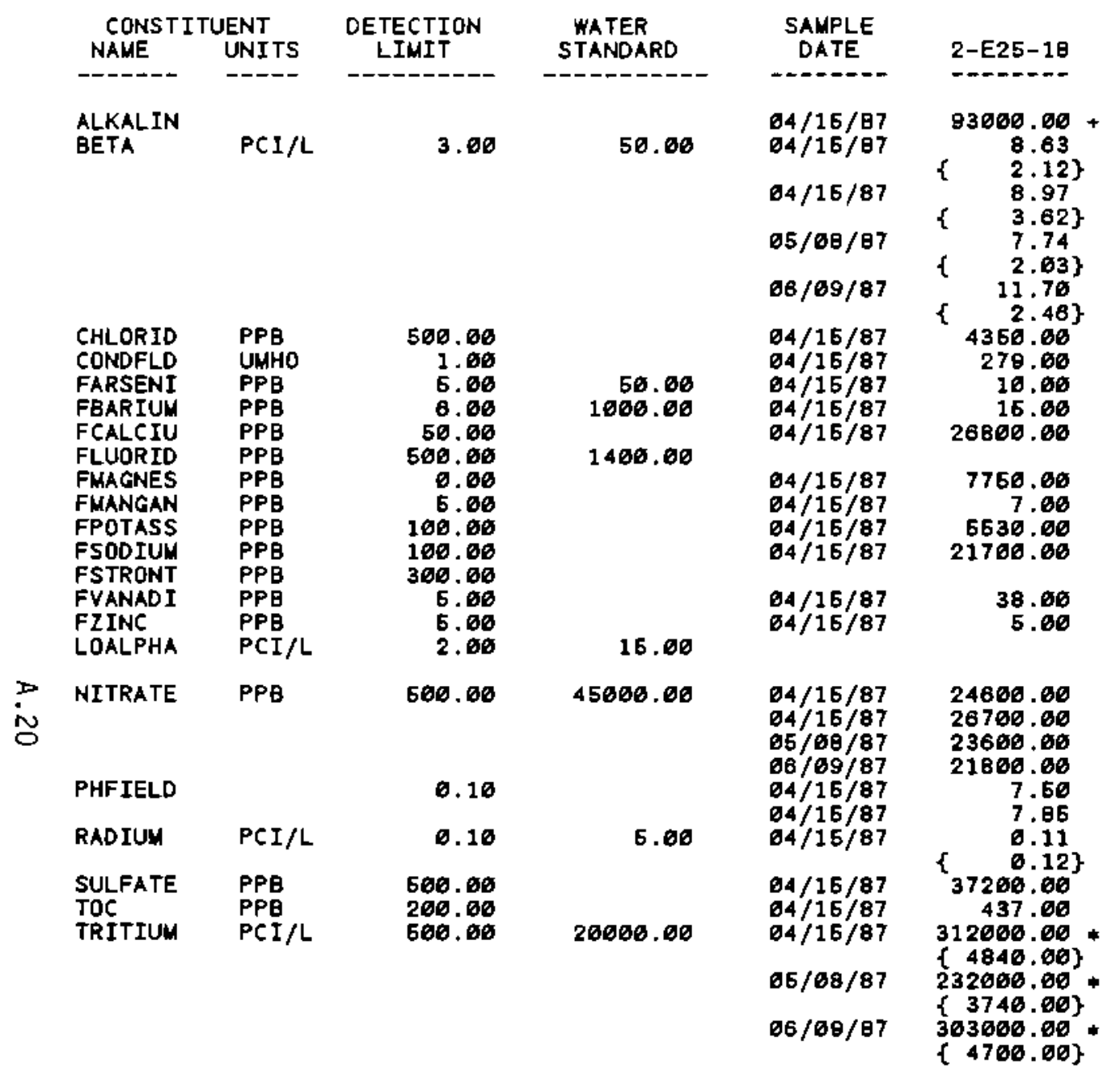

\begin{tabular}{|c|c|}
\hline $\begin{array}{l}\text { SAMPLE } \\
\text { DATE }\end{array}$ & $2-E 25-2 \theta$ \\
\hline $\begin{array}{l}64 / 13 / 87 \\
84 / 13 / 87\end{array}$ & $\begin{array}{c}111000.00 \\
13.30\end{array}+$ \\
\hline $64 / 13 / 87$ & $\begin{array}{r}2.84\} \\
19.70\end{array}$ \\
\hline & $4.84\}$ \\
\hline $05 / 08 / 87$ & 13.40 \\
\hline $66 / 69 / 87$ & $\begin{array}{r}2.86\} \\
17.80\end{array}$ \\
\hline $\begin{array}{l}64 / 13 / 87 \\
64 / 13 / 87 \\
64 / 13 / 87 \\
64 / 13 / 87 \\
64 / 13 / 87 \\
64 / 13 / 87 \\
64 / 13 / 87\end{array}$ & $\begin{array}{r}6700.00 \\
657.00 \\
5.00 \\
58.00 \\
67600.00 \\
613.00 \\
16600.00\end{array}$ \\
\hline $\begin{array}{l}64 / 13 / 87 \\
64 / 13 / 87 \\
64 / 13 / 97 \\
64 / 13 / 87 \\
04 / 13 / 87 \\
66 / 80 / 87\end{array}$ & $\begin{array}{r}12200.00 \\
47800.00 \\
301.00 \\
48.00 \\
8.00 \\
2.11\end{array}$ \\
\hline $\begin{array}{l}64 / 13 / 87 \\
64 / 13 / 87 \\
65 / 68 / 87 \\
66 / 69 / 87 \\
64 / 13 / 97 \\
64 / 13 / 87\end{array}$ & $\begin{array}{c}1680.84\} \\
168006.006 \\
150000.00 \\
196000.00 \\
7.30 \\
7.47 \\
\mathrm{NR}\end{array}$ \\
\hline $\begin{array}{l}64 / 13 / 87 \\
64 / 13 / 87 \\
04 / 13 / 87\end{array}$ & $\begin{array}{r}78000.00 \\
423.00 \\
633000.00\end{array}$ \\
\hline $06 / 08 / 87$ & 767000.00 \\
\hline $06 / 09 / 87$ & $\begin{array}{l}734000.00 \\
\{7330.00\}\end{array}$ \\
\hline
\end{tabular}

$04 / 13 / 87$ $64 / 13 / 87$

$64 / 13 / 87$

$66 / 09 / 87$

$131000.00+$

( 11.00

2.46

$\{\quad 3.92\}$ \{ 2.48$\}$

$04 / 13 / 87$

$04 / 13 / 87$

$64 / 13 / 87$

$64 / 13 / 67$

$04 / 13 / 87$

$64 / 13 / 87$

$64 / 13 / 87$

$04 / 13 / 87$

$64 / 13 / 87$

3930.00

314.00

6.60

24.00

24100.00

698.00

6516.00

8.00
910.00

37806 .

$04 / 13 / 87$

70.00

$94 / 13 / 87$

$04 / 13 / 87$
$04 / 13 / 87$

$04 / 13 / 87$
$06 / 08 / 87$

0730.00

12406.00

10200.00

NR

$64 / 13 / 87$
$64 / 13 / 87$

6.80
7.81

$04 / 13 / 87$

$64 / 13 / 87$

46300.00

414.00

$4 \quad 481.00$

$06 / 09 / 87$

9890.00

$\{408.60\}$

- VALUE eXCEEDS PRIMARY DRINKINg water STANDARD.

* VALUE EXCEEDS PROPOSED PRIMARY DRINKING WATER STANDARD.

* - VALUE EXCEEDS SCREENING LEVEL FOR FURTHER INVESTIGATION.

* - DETECTION LIMIT WAS NOT AVAILABLE FOR COMPARISON

NR - ANALYSIS NOT REQUESTED OR NOT YET REPORTED

VALUES IN \{ $\}$ ARE COUNTING ERRORS FOR RADIONUCLIDES

WATER STANDARD(S) IN PARENTHESES ARE PROPOSED ONLY 
TABLE A.4. (contd)

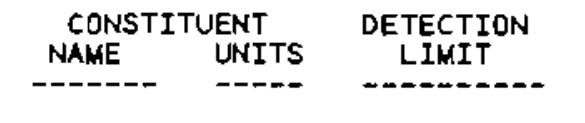

WATER
STANDARD

ALKALIN

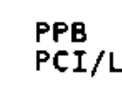

50.00

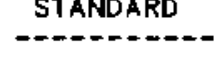

BETA

PPB
PCI/L

3.60

56.60

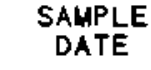

$04 / 15 / 87$

$04 / 16 / 87$

$64 / 16 / 87$

$65 / 17 / 87$

$06 / 09 / 87$

$04 / 16 / 87$

$04 / 16 / 87$

$04 / 16 / 87$

$04 / 16 / 87$

$04 / 16 / 87$

$04 / 16 / 87$

$04 / 16 / 87$

$04 / 16 / 87$

$04 / 16 / 87$

$04 / 16 / 87$

$64 / 15 / 87$
$05 / 17 / 87$

$06 / 17 / 87$

$88 / 69 / 87$
$04 / 16 / 87$

$04 / 16 / 87$

$04 / 15 / 87$

$04 / 15 / 97$
$04 / 15 / 87$

$\begin{array}{ll}\text { TRITIUM } & \text { PCI/L }\end{array}$

500.00

600.06

20000.00

06/17/87

$66 / 09 / 87$

$84 / 16 / 87$

UNKNOWN PPB

0.06
TOC
0500000
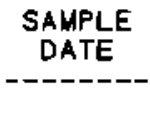

$06 / 16 / 87$
$06 / 16 / 87$

$66 / 16 / 87$

6.12
$\{\quad 1.77\}$

$\begin{array}{r}10.60 \\ \quad 3.65 \\ \hline\end{array}$

3.63
1.53

$\{\quad 1.57\}$

\{ $\begin{array}{r}1.83\} \\ 4036.08\end{array}$

242.00

242.00
11.00

16.00

21500.00

6140.00

5700.00

21860.06

67.00

9.00

4320.06

4320.00

4710.08

260.00

7.48

27600.00

4770.00

$\left\{\begin{array}{l}374.06\} \\ 4750\end{array}\right.$

$\left\{\begin{array}{r}4750.00 \\ 322.00\}\end{array}\right.$

5646.00

$\left\{\begin{array}{r}342.00\} \\ 41.00\end{array}\right.$

66/16/97

$06 / 16 / 97$

96/16/87

08/16/87

$\theta 6 / 16 / \theta 7$

$06 / 18 / 67$

ต6/16/87

ø6/16/97

$06 / 16 / 87$

$66 / 16 / 97$
$66 / 16 / 87$
$06 / 16 / 07 \quad 6900.60$
2-E26-1

$94200.06+$

83. 10

$\left.\begin{array}{l}9.14 \\ 3.96\end{array}\right\}$
$N R$

NR

NR

NR

SAMPLE

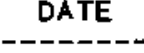

2-E26-3

$66 / 16 / 87$

$68 / 16 / 87$
$68 / 16 / 87$

05700.00

69.00

7.17

NR 88$\}$

NR

NR

$96 / 26 / 97$

4120.00

NR

68/16/87

$8 / 18 / 97$

$86 / 16 / 97$

$06 / 16 / 97$

$06 / 16 / 97$

06/18/97

$06 / 16 / 97$

ब6/16/87

5090.00

NR

NR

${ }_{\mathrm{NR}}^{\mathrm{N}} \mathrm{72}$

NR

302.00

16600.00
$\{\quad 512.00$

NR

NR

Q.00

20100.00

5800.00

0

4380.00

1660.60

38.06

1780.00

NR

NR

$66 / 16 / 87$

7.72

NRे

$66 / 16 / 87$

$66 / 16 / 87$

$06 / 16 / 87$

529.06

3320.00

( 284.06)

NR

NR

NR

- VALUe eXCEEDS PRIMARY DRINKINg water STANDARD.

- VALUE EXCEEDS PROPOSED PRIMARY DRINKING waTER STANDARD

* - VALUE EXCEEDS SCREENING LEVEL FOR FURTHER INVESTIGATION.

+ - OETECTIDN LIMIT WAS NOT AVAILABLE FOR CONPARISON

NR - ANALYSIS NOT REQUESTED OR NOT YET REPDRTED

VALUES IN \{ $\}$ ARE COUNTING ERRORS FOR RADIONUCLIDES

WATER STANDARD (S) IN PARENTHESES ARE PROPOSED ONLY 
TABLE A.4. (contd)

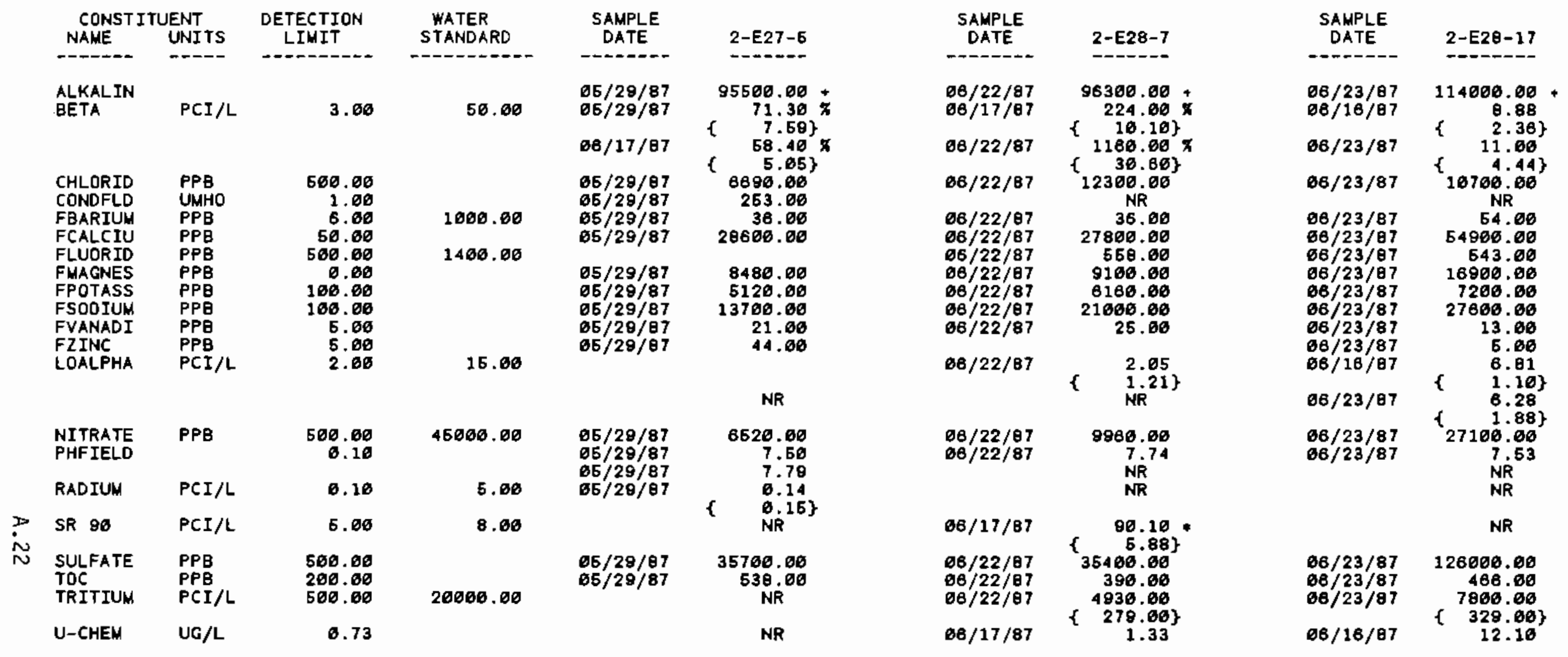

- - VALUE EXCEEDS PRIMARY DRINKINg hater standaRd.

* - VALUE EXCEEDS PROPOSED PRIMARY DRINKING WATER STANDARO.

* - VALUE EXCEEDS SCREENING LEVEL FOR FURTHER INVESTIGATION.

+ - DETECTION LINIT WAS NOT AVAILABLE FOR COMPARISON

NR - ANALYSIS NOT REQUESTED OR NOT YET REPORTED

VALUES IN \{ \} ARE COUNTING ERRORS FOR RADIONUCLIDES

WATER STANDARD (S) IN PARENTHESES ARE PROPOSED ONLY 
TABLE A.4. (contd)

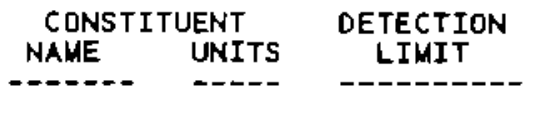

HATER

ALKALIN

ALKALIN

BETA

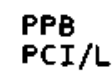

50.00

STANDARD

SAMPLE

-..--

$64 / 14 / 87$

$04 / 14 / 87$

$118060.00+$

127.00

50.00

$04 / 14 / 87$

$66 / 17 / 87$

$66 / 10 / 87$

CHLORID

PPB

FARSENI

FBARIUM

FCALCIU

FLUORID PPB

FMAGNES

FPOTASS

FSILVER

FVANADI

FZINC

iv

w
$64 / 14 / 87$

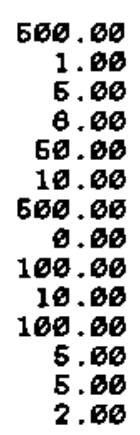

50.00

1000.00

50.00

1400.00

50.00

PPB

PPB

PPB

PCI/L

2.00

15.00

$04 / 14 / 87$

$64 / 14 / 87$

$04 / 14 / 87$

$64 / 14 / 97$

$64 / 14 / 87$

$04 / 14 / 87$

$04 / 14 / 87$

$04 / 14 / 87$
$04 / 24 / 87$

$04 / 14 / 67$

05/17/87

96/16/87

$04 / 14 / 87$

$04 / 14 / 67$

$06 / 17 / 87$

$68 / 10 / 87$

$04 / 14 / 87$

$04 / 14 / 87$

$64 / 14 / 8$

$04 / 14 / B 7$

$64 / 14 / 87$

$05 / 17 / 87$

ø6/10/87

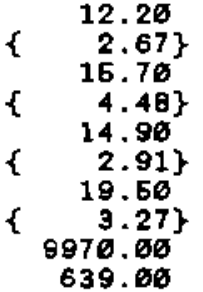

40.00

63800.00

16000.00

4910.06

24700.00

18.00
8.00

$63.96 \%$

( 3.11$\}$

38. $50 \mathrm{x}$

\{ 5.89\}

$60.00 \%$

( 2.98$\}$

\{ 3.01$\}$

44100.00

38400.00

44400.00

7.30

7.38

332.00

( 380.00$\}$

6310.00

$\left\{\begin{array}{r}348.00\} \\ 6740.00\end{array}\right.$

6740.00
$\quad 344.00\}$

\begin{tabular}{|c|c|}
\hline $\begin{array}{l}\text { SAMPLE } \\
\text { DATE }\end{array}$ & $2-E 33-1$ \\
\hline $\begin{array}{l}\sigma 5 / 19 / 87 \\
66 / 19 / 87 \\
65 / 19 / 87\end{array}$ & $\begin{array}{r}98000.00+ \\
64.00 \\
138.00 \times\end{array}$ \\
\hline $66 / 19 / 87$ & 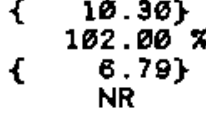 \\
\hline
\end{tabular}

NR

65/19/87

$65 / 19 / 87$

ธ5 $/ 19 / 87$

$65 / 19 / 67$

$65 / 19 / 67$

ब5/19/87

$06 / 19 / 67$

B5/19/87

б5/19/87

ब5/19/87

ธ6/19/87

2260.00

268.00

10.00

21.00

25960.00

11.00

675.00

7510.00

4680.60

10.00

36100.00

32.00
5.00

NR

NR

NR

$06 / 19 / 87$

52360 .

NR

NR

7.60

$65 / 19 / 87$

$06 / 19 / 87$

$5 / 19 / 87$

$65 / 19 / 87$

348.00

NR

NR

NR

SAMPLE

$06 / 23 / 8795600.00$ +

$68 / 23 / 87$

$\left\{\begin{array}{l}81.107 \\ \text { NR }\end{array}\right.$

NR

NR

$68 / 23 / 87$

3260.00

NR

$66 / 23 / 87$

$68 / 23 / 87$

$66 / 23 / 87$

$66 / 23 / 87$

$86 / 23 / 87$

$68 / 23 / 97$

$06 / 23 / 87$

$68 / 23 / 87$

10.00

23900.06

861.66

8070.00

4780.00

31900.00

27.00

NR

NR

NR

$\$ 6 / 23 / 87 \quad 39400.00$

NR

NR

NR
7.61

$68 / 23 / 87$

$06 / 23 / 87$

$80 / 23 / 87$

$$
\text { NR }
$$

30100.00

623.06

808.00
$178.00\}$

NR

NR

* - value exceeds primary drinking hater standard.

- VALUE EXCEEDS PROPOSED PRIMARY DRINKING WATER STANDARD.

* - VALUE EXCEEDS SCREENING LEVEL FOR FURTHER INVESTIGATION.

* - DETECTION LIMIT WAS NOT AVAILABLE FOR COMPARISON

NR - ANALYSIS NOT REQUESTED OR NOT YET REPORTED

VALUES IN \{\} ARE COUNTING ERRORS FOR RADIONUCLIDES

WATER STANDARD(S) IN PARENTHESES ARE PROPOSED ONLY 
TABLE A.4. (contd)

\begin{tabular}{|c|c|c|c|c|c|c|c|c|c|}
\hline $\begin{array}{l}\text { CONSTI } \\
\text { NAME }\end{array}$ & $\begin{array}{l}\text { UENT } \\
\text { UNITS }\end{array}$ & $\begin{array}{l}\text { DETECTION } \\
\text { LIMIT }\end{array}$ & $\begin{array}{l}\text { WATER } \\
\text { STANDARD }\end{array}$ & $\begin{array}{l}\text { SAMPLE } \\
\text { DATE }\end{array}$ & $2-E 28-21$ & $\begin{array}{l}\text { SAMPLE } \\
\text { DATE }\end{array}$ & $2-E 33-1$ & $\begin{array}{l}\text { SAMPLE } \\
\text { DATE }\end{array}$ & 2-E $33-2$ \\
\hline U-CHEM & UG/L & 0.73 & & $\begin{array}{l}04 / 14 / 87 \\
65 / 17 / 87 \\
66 / 16 / 87\end{array}$ & $\begin{array}{l}89.46 \\
79.30 \\
88.00\end{array}$ & & $\begin{array}{l}\text { NR } \\
\text { NR } \\
\text { NR }\end{array}$ & & $\begin{array}{l}\text { NR } \\
\text { NR } \\
\text { NR }\end{array}$ \\
\hline
\end{tabular}

* - VALUE EXCEEDS PRIMARY DRINKING WATER STANDARD.

* - VALUE EXCEEDS PROPOSED PRIMARY DRINKING HATER STANDARD.

* - VALUE EXCEEDS SCREENING LEVEL FOR FURTHER INVESTIGATION.

+ DETECTION LIMIT WAS NOT AVAILABLE FOR COMPARISON

NR - ANALYSIS NOT REQUESTED OR NOT YET REPORTED

VALUES IN $\{$ \& ARE COUNTING ERRORS FOR RADIDNUCLIDES

WATER STANDARD(S) IN PARENTHESES ARE PROPOSED ONLY 
TABLE A.4. (contd)

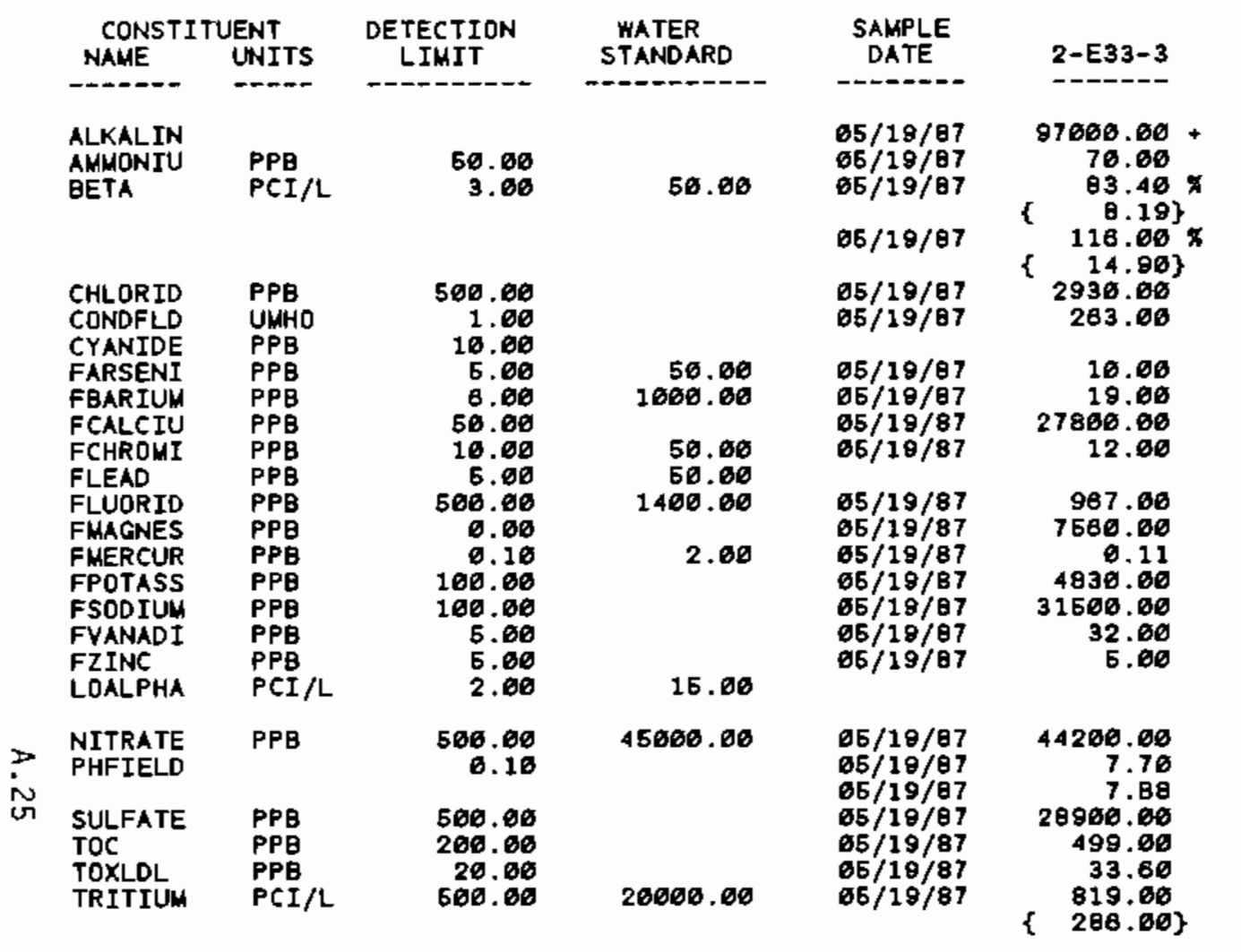

\begin{tabular}{|c|c|}
\hline $\begin{array}{c}\text { SAMPLE } \\
\text { DATE }\end{array}$ & $2-E 33-6$ \\
\hline $\begin{array}{l}\emptyset 5 / 19 / 87 \\
05 / 19 / 87 \\
65 / 19 / 87\end{array}$ & $\begin{array}{r}92000.00 \\
71.00 \\
309.00\end{array}$ \\
\hline $05 / 19 / 87$ & $\begin{array}{l}16.20\} \\
293.00\end{array}$ \\
\hline $\begin{array}{l}06 / 19 / 87 \\
65 / 19 / 87 \\
65 / 19 / 87 \\
05 / 19 / 87 \\
65 / 19 / 87 \\
06 / 19 / 97\end{array}$ & $\begin{array}{r}4480.60 \\
260.00 \\
20.00 \\
0.00 \\
30.00 \\
30000.00\end{array}$ \\
\hline $\begin{array}{l}65 / 19 / 87 \\
05 / 19 / 87 \\
65 / 19 / 97\end{array}$ & $\begin{array}{r}5.00 \\
580.00 \\
0786.00\end{array}$ \\
\hline $\begin{array}{l}\delta 5 / 19 / 87 \\
65 / 19 / 87 \\
65 / 19 / 87 \\
\sigma 6 / 19 / 87\end{array}$ & $\begin{array}{r}5130.00 \\
24900.00 \\
28.00 \\
6.00\end{array}$ \\
\hline \multirow[t]{2}{*}{$\begin{array}{l}65 / 19 / 87 \\
65 / 19 / 87 \\
06 / 19 / 87 \\
05 / 19 / 87 \\
05 / 19 / 87\end{array}$} & $\begin{array}{r}35200.06 \\
7.50 \\
7.70 \\
38600.06 \\
364.06\end{array}$ \\
\hline & NR \\
\hline
\end{tabular}

SAMPLE DATE 2-E33-8

$05 / 22 / 07$ $05 / 22 / 07$ $05 / 22 / 97$ $05 / 22 / 87$
$05 / 22 / 87$ $05 / 22 / 07$ क5/22/87 $05 / 22 / 67$

06/22/07

ต5/22/07 $06 / 22 / 87$ ०5/22/87 ๑5/22/87

D5/22/0 7 $05 / 22 / 97$ $65 / 22 / 87$ $05 / 22 / 87$

NR $101600.00+$

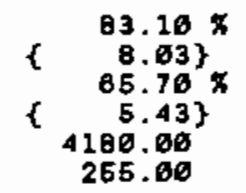
265.60

\subsection{0} 36400.66

9350.00

5000.00 12900.00 19.00 5.00 2.10
$1.15\}$ 8250.00 8. 30 7.71
7908.90 368.00 NR

* - VALUE EXCEEDS PRIMARY DRINKING waTER STANDARD

- VALUE EXCEEDS PROPOSED PRIMARY DRINKING WATER STANDARD.

- VALUE EXCEEDS SCREENING LEVEL FOR FURTHER INVESTIGATION.

+ - DETECTION LIUIT WAS NOT AVAILABLE FOR COMPARISON

NR - ANALYSIS NOT REQUESTED OR NOT YET REPORTED

VALUES IN \{\} ARE COUNTING ERRORS FOR RADIONUCLIDES

WATER STANDARD(S) IN PARENTHESES ARE PROPOSED ONLY 
TABLE A.4. (cont d)

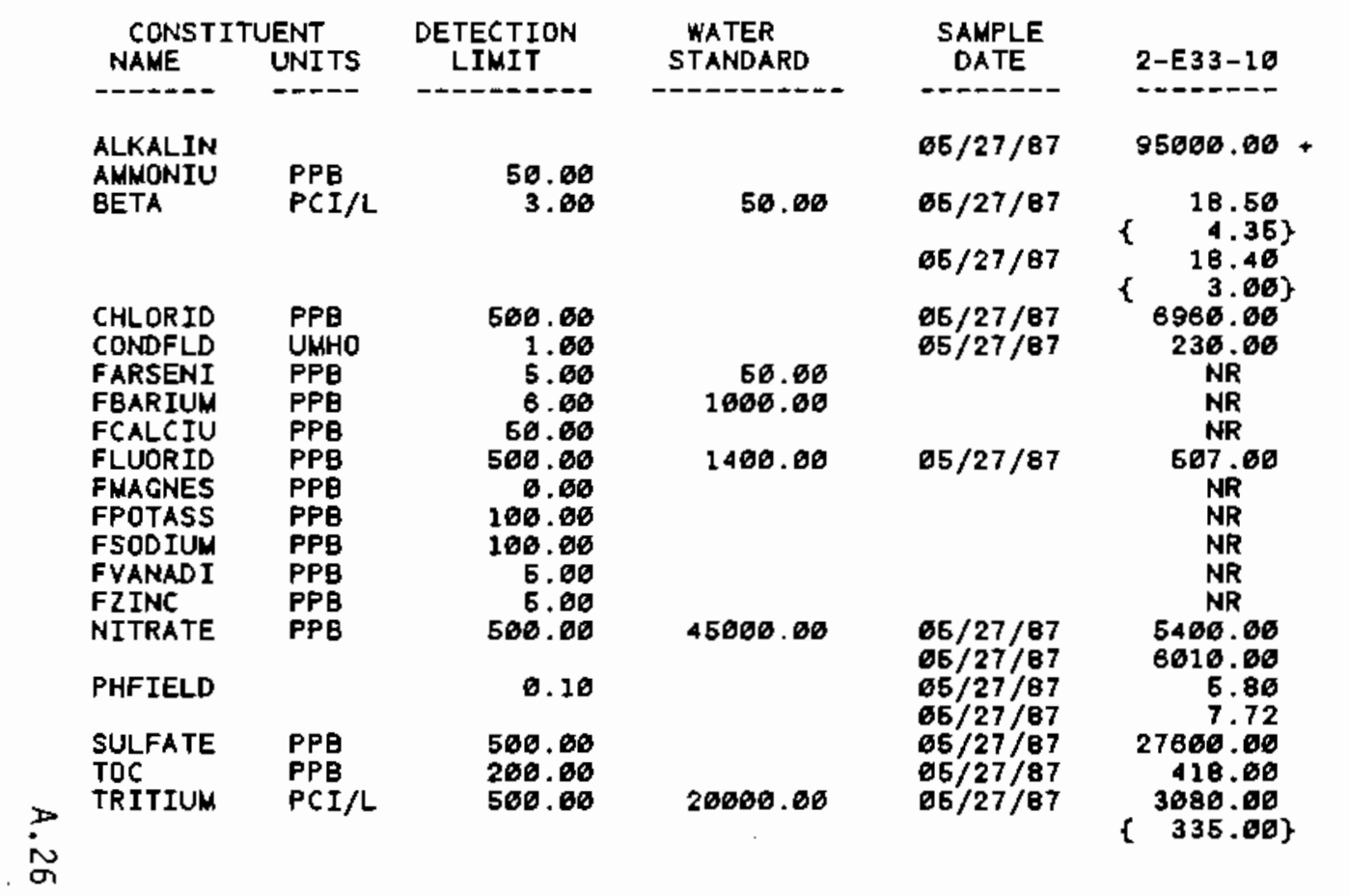

\begin{tabular}{|c|c|}
\hline $\begin{array}{l}\text { SAMPLE } \\
\text { DATE }\end{array}$ & $2-E 33-1 \theta$ \\
\hline $\begin{array}{l}65 / 19 / 87 \\
65 / 19 / 87 \\
65 / 19 / 87\end{array}$ & $\begin{array}{c}94600.00 \\
59.00 \\
11.50\end{array}$ \\
\hline $65 / 13 / 87$ & $\begin{array}{ll}\{\quad 3.83 \\
\{\quad \\
\{.85 \\
\{\end{array}$ \\
\hline $\begin{array}{l}66 / 19 / 87 \\
66 / 19 / 87 \\
65 / 19 / 87 \\
65 / 19 / 87 \\
66 / 19 / 87\end{array}$ & $\begin{array}{r}2230.00 \\
221.00 \\
8.00 \\
24.00 \\
27700.00\end{array}$ \\
\hline $\begin{array}{l}65 / 19 / 87 \\
65 / 19 / 67 \\
65 / 19 / 67 \\
06 / 19 / 87 \\
06 / 19 / 87 \\
65 / 19 / 87\end{array}$ & $\begin{array}{r}9480.00 \\
4860.60 \\
14700.60 \\
28.60 \\
5.60 \\
16700.00\end{array}$ \\
\hline $\begin{array}{l}65 / 19 / 87 \\
65 / 19 / 87 \\
65 / 18 / 87 \\
65 / 19 / 67\end{array}$ & $\begin{array}{r}7.06 \\
7.04 \\
27600.06 \\
412.00 \\
\mathrm{NR}\end{array}$ \\
\hline
\end{tabular}

SAMPLE

$65 / 27 / 87$

06/27/87

$05 / 27 / 87$

$06 / 27 / 87$

$65 / 27 / 87$

$65 / 27 / 87$

$65 / 27 / 67$

ब5/27/87

$05 / 27 / 87$

$05 / 27 / 87$

$05 / 27 / 87$

$06 / 27 / 87$
$65 / 27 / 87$

65/27/87

$65 / 27 / 87$

$65 / 27 / 67$

$65 / 27 / 87$

$05 / 27 / 87$
2-E33-21

$102000.00+$

\begin{tabular}{l}
9.95 \\
\hline
\end{tabular}

$3.49\}$
8.89

\{ $\begin{array}{r}2.24\} \\ 4610.08\end{array}$ 232.00

5.00

29.60

27600.00

8720.00

5230.00

12400.00

20.00

8.00

980.00

NR

6.00

7.69
24700.00

696.00

NR

- - Value exceeds primary drinking water stanoaro.

- VALUE EXCEEDS PROPOSED PRIMARY DRINKING WATER STANDARD.

* - VALUE EXCEEDS SCREENING LEVEL FOR FURTHER INVESTIGATION.

+ - DETECTION LIMIT WAS NOT AVAILABLE FOR COMPARISON

NR - ANALYSIS NOT REQUESTED OR NOT YET REPORTED

VALUES IN \& $)$ ARE COUNTING ERRORS FOR RADIONUCLIDES

WATER STANDARO(5) IN PARENTHESES ARE PROPOSED ONLY. 
TABLE A.4. (cont $d$ )

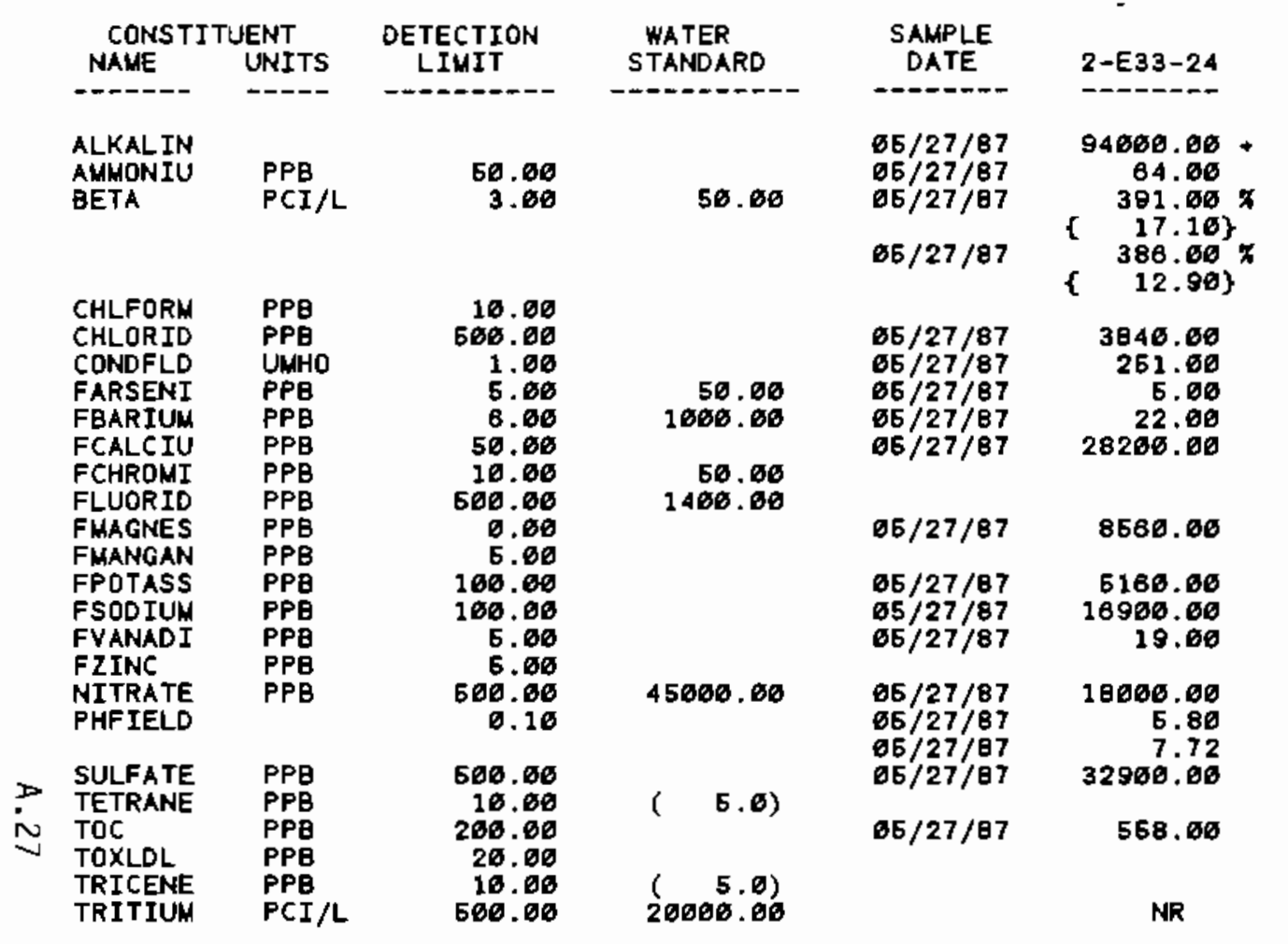

\begin{tabular}{|c|c|}
\hline $\begin{array}{l}\text { SAMPLE } \\
\text { DATE }\end{array}$ & $2-W B-1$ \\
\hline $\begin{array}{l}06 / 10 / 87 \\
06 / 10 / 87 \\
06 / 10 / 87\end{array}$ & $\begin{array}{r}114600.00 \\
165.06 \\
20.16\end{array}$ \\
\hline & $\left\{\quad \begin{array}{l}5.38 \\
N R\end{array}\right.$ \\
\hline $66 / 16 / 87$ & $\underset{N R}{8750.00}$ \\
\hline $\begin{array}{l}\theta 6 / 10 / 87 \\
66 / 10 / 87 \\
6 \theta / 10 / 87\end{array}$ & $\begin{array}{r}46.00 \\
66700.00 \\
46.00\end{array}$ \\
\hline $\begin{array}{l}66 / 10 / 87 \\
66 / 10 / 87 \\
66 / 10 / 87 \\
66 / 16 / 87 \\
66 / 16 / 87 \\
66 / 10 / 87 \\
66 / 16 / 87 \\
66 / 16 / 87\end{array}$ & $\begin{array}{r}24400.00 \\
14.00 \\
4900.00 \\
13200.00 \\
17.00 \\
6.00 \\
205000.00 \\
7.65\end{array}$ \\
\hline $\begin{array}{l}66 / 10 / 87 \\
66 / 10 / 87 \\
66 / 10 / 87 \\
66 / 10 / 87\end{array}$ & $\begin{array}{c}\text { NR } \\
33100.00 \\
220.00 \\
814.00 \\
165.00\end{array}$ \\
\hline $06 / 16 / 87$ & $\begin{array}{r}62300.00 \\
\left\{\begin{array}{r}868.00\}\end{array}\right.\end{array}$ \\
\hline
\end{tabular}

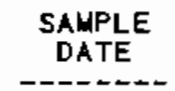

$65 / 18 / 87$

$65 / 18 / 87$ $65 / 18 / 87$

$65 / 18 / 87$

$65 / 18 / 87$ ๑5/18/87 $65 / 18 / 67$ $65 / 18 / 67$ $06 / 18 / 97$ 65/18/87 $06 / 18 / 97$ 65/18/67

$65 / 18 / 97$ $05 / 18 / 87$ $05 / 18 / 87$ $65 / 18 / 87$
$65 / 18 / 87$ 65/18/87 $65 / 18 / 87$
$65 / 18 / 87$ $65 / 18 / 87$
$65 / 18 / 97$ $65 / 18 / 87$
$66 / 18 / 87$ $65 / 18 / 87$ $65 / 18 / 87$ $66 / 19 / 87$
$66 / 18 / 87$
2-พ10-4

$158000.60+$ 57.00

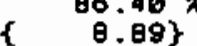
$50.86 \%$ 17.223 13500.08 882.60 31.06 24800.00 3098.00 7750.06

3050.00 152000.00 64.00 229000.00 . 7.90 63000.00

2210.00 425.00 3130.60 32.00

* - Value exceeds primary drinking water standard.

- VALUE EXCEEDS PROPOSED PRIMARY DRINKING WATER STANDARD.

* VALUE EXCEEDS SCREENING LEVEL FOR FURTHER INVESTIGATION.

* - DETECTION LIMIT WAS NOT AVAILABLE FOR COUPARISON

NR - ANALYSIS NOT REQUESTED OR NOT YET REPORTED

VALUES IN \{ \} ARE CDUNTING ERRORS FOR RADIONUCLIDES
WATER STANDARO(S) IN PARENTHESES ARE PROPOSED ONLY 
TABLE A.4. (contd)

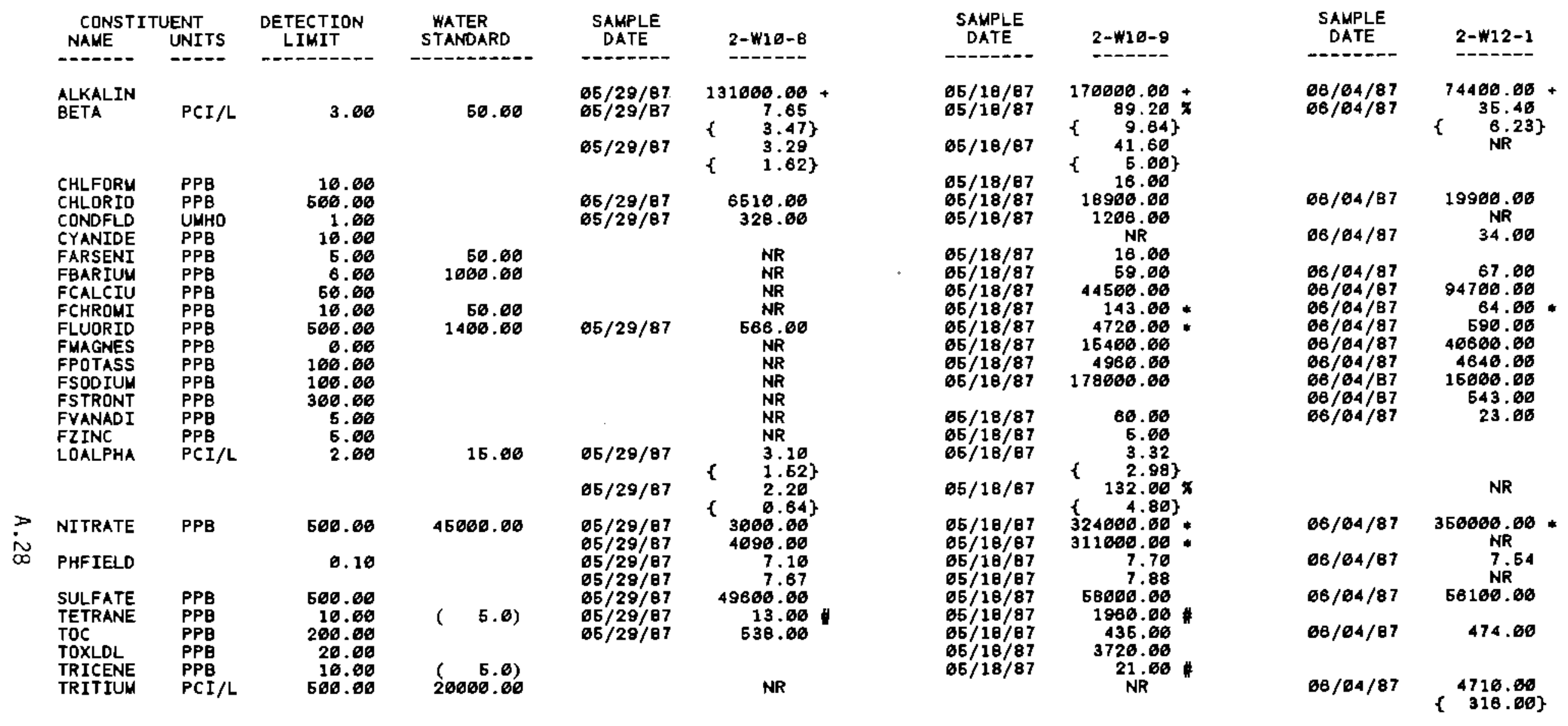

* - VAlue exceeds PRimary orinking water standaro.

- VALUE EXCEEDS PROPOSED PRIMARY DRINKING WATER STANDARD.

* VALUE EXCEEDS SCREENING LEVEL FOR FURTHER INVESTIGATION.

- - DETECTION LIUIT WAS NOT AVAILABLE FOR COMPARISON

NR - ANALYSIS NOT REQUESTED OR NOT YET REPORTED

VALUES IN $\{$ \& ARE COUNTING ERRORS FOR RADIONUCLIDES

WATER STANDARD'S) IN PARENTHESES ARE PROPOSED ONLY 
TABLE A.4. (contd)

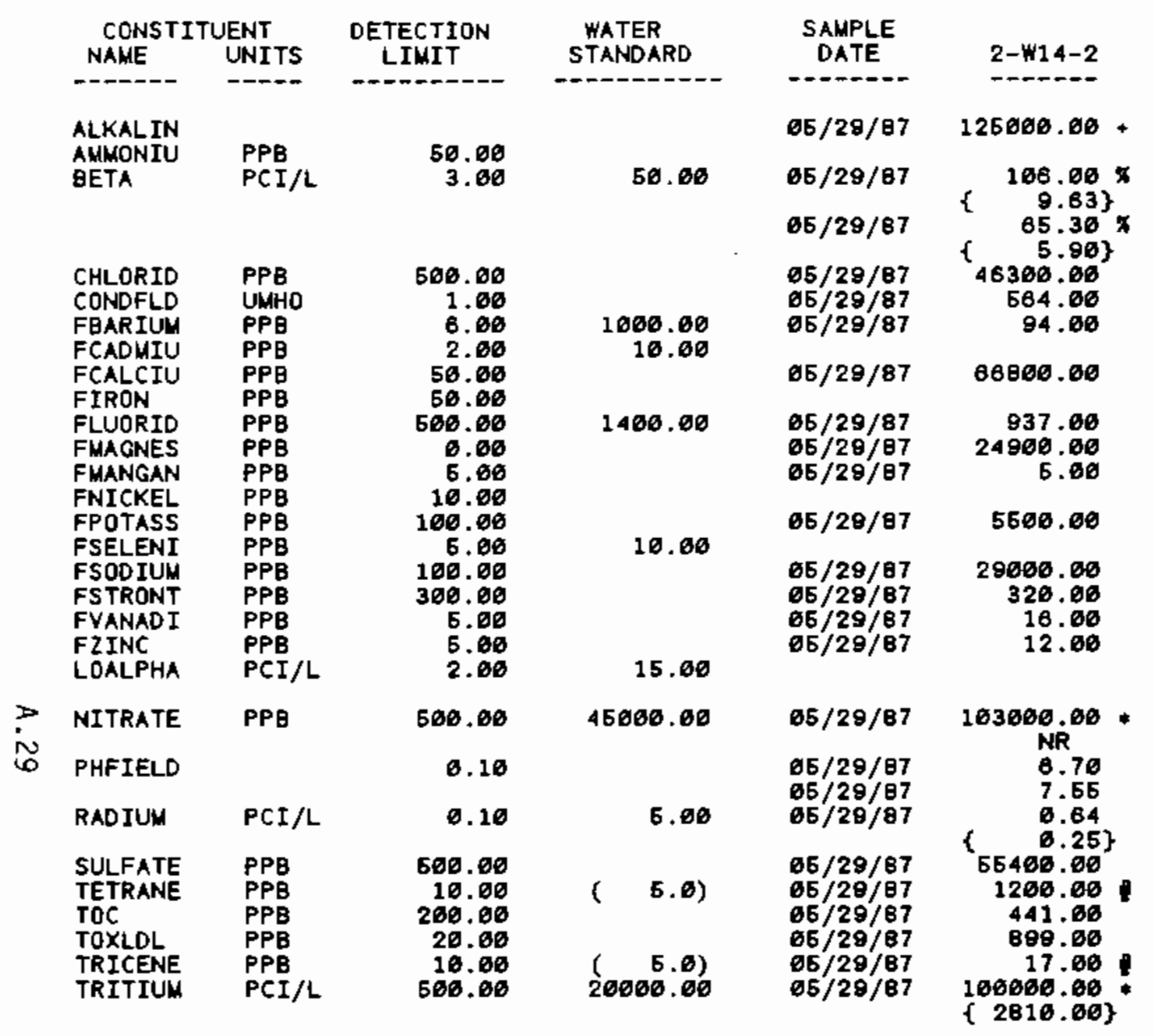

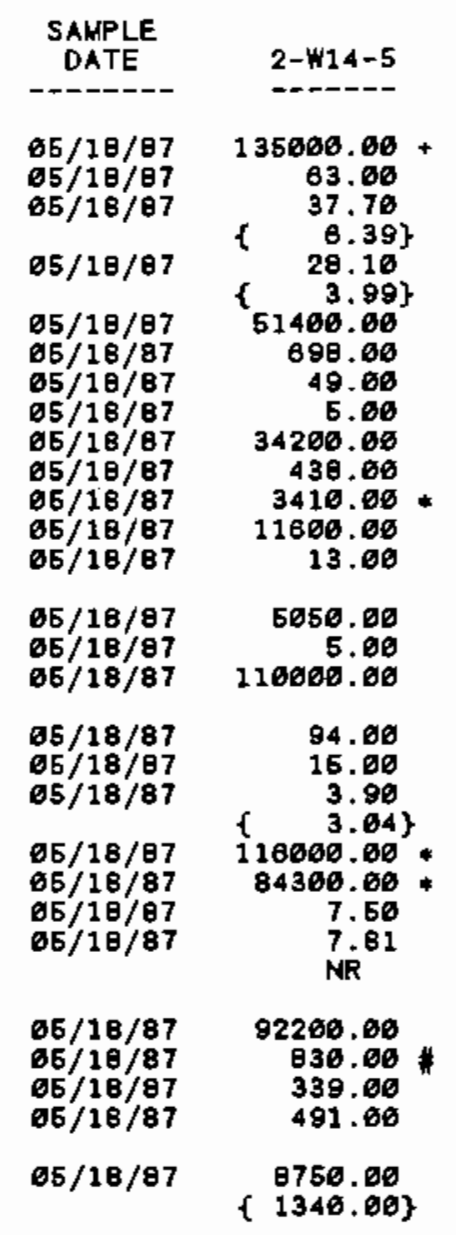

\begin{tabular}{|c|c|}
\hline $\begin{array}{c}\text { SAMPLE } \\
\text { DATE }\end{array}$ & $\begin{array}{l}2-W 14-6 \\
-\end{array}$ \\
\hline $65 / 29 / 87$ & $125000.00+$ \\
\hline $\begin{array}{l}65 / 29 / 87 \\
05 / 29 / 87\end{array}$ & \multirow{2}{*}{$\begin{array}{r}22.60 \\
\{\quad 5.12\} \\
16.30 \\
\{\quad 3.65\} \\
49000.00 \\
657.00 \\
58.00\end{array}$} \\
\hline $\begin{array}{l}05 / 29 / 87 \\
06 / 29 / 87 \\
65 / 29 / 87\end{array}$ & \\
\hline $\begin{array}{l}06 / 29 / 87 \\
65 / 29 / 87 \\
65 / 29 / 87 \\
06 / 29 / 87 \\
65 / 29 / 87 \\
65 / 29 / 87 \\
65 / 29 / 87\end{array}$ & $\begin{array}{r}45200.06 \\
228.00 \\
3800.00 \\
15506.00 \\
13.00 \\
21.00 \\
5460.00\end{array}$ \\
\hline $66 / 29 / 87$ & 66800.00 \\
\hline $\begin{array}{l}65 / 29 / 87 \\
65 / 29 / 87 \\
65 / 29 / 87\end{array}$ & \multirow{2}{*}{$\begin{array}{r}33.06 \\
6.06 \\
3.79 \\
2.67\} \\
\{\quad 4406.00 \\
42600.06 \\
6.70 \\
7.62 \\
\text { NR }\end{array}$} \\
\hline $\begin{array}{l}05 / 29 / 87 \\
05 / 29 / 87 \\
05 / 29 / 87 \\
05 / 29 / \theta 7\end{array}$ & \\
\hline $\begin{array}{l}85 / 29 / 87 \\
65 / 28 / 87 \\
65 / 29 / 87 \\
86 / 29 / 87\end{array}$ & $\begin{array}{r}62700.00 \\
610.00 \\
1200.00 \\
293.00\end{array}$ \\
\hline $05 / 28 / 87$ & $\left\{\begin{array}{c}31100.60 \\
676.60\}\end{array}\right.$ \\
\hline
\end{tabular}

* - Value exceeds primary dRINKINg water standard.

- VALUE EXCEEDS PROPOSED PRIMARY DRINKING WATER STANDARD.

* - VALUE EXCEEDS SCREENING LEVEL FOR FURTHER INVESTIGATION.

+ - DETECTION LIMIT WAS NOT AVAILABLE FOR COMPARISON

NR - ANALYSIS NOT REQUESTED OR NOT YET REPORTED

VALUES IN \{ $\}$ ARE COUNTING ERRORS FOR RADIONUCLIDES

WATER STANDARD (S) IN PARENTHESES ARE PROPOSED ONLY 
TABLE A.4. (contd)

\begin{tabular}{|c|c|c|c|c|c|}
\hline $\begin{array}{l}\text { CONSTI } \\
\text { NAME }\end{array}$ & $\begin{array}{l}\text { JENT } \\
\text { UNI TS }\end{array}$ & $\begin{array}{l}\text { DETECTION } \\
\text { LIMIT }\end{array}$ & $\begin{array}{l}\text { WATER } \\
\text { STANDARD }\end{array}$ & $\begin{array}{l}\text { SAMPLE } \\
\text { DATE }\end{array}$ & $2-W 16-4$ \\
\hline $\begin{array}{l}\text { ALKALIN } \\
\text { ALIMONIU } \\
\text { BETA }\end{array}$ & $\begin{array}{l}\text { PPB } \\
\text { PCI/L }\end{array}$ & $\begin{array}{r}50.00 \\
3.00\end{array}$ & 50.00 & $\begin{array}{l}65 / 2 \theta / \theta 7 \\
65 / 28 / 87 \\
65 / 2 \theta / 87 \\
05 / 2 \theta / 87\end{array}$ & 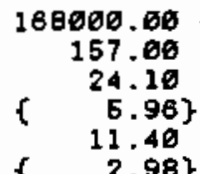 \\
\hline $\begin{array}{l}\text { CHLFORM } \\
\text { CHLORID } \\
\text { CDNDFLD } \\
\text { FARSENI } \\
\text { FBARIUM } \\
\text { FCALCIU } \\
\text { FCHROMI }\end{array}$ & $\begin{array}{l}\text { PPE } \\
\text { PPE } \\
\text { UMHO } \\
\text { PPB } \\
\text { PPB } \\
\text { PPB } \\
\text { PPB }\end{array}$ & $\begin{array}{r}10.00 \\
500.00 \\
1.00 \\
5.00 \\
6.00 \\
50.00 \\
10.00\end{array}$ & $\begin{array}{r}50.00 \\
1000.00 \\
50.00\end{array}$ & $\begin{array}{l}05 / 28 / 87 \\
65 / 28 / 87 \\
65 / 28 / 87 \\
65 / 2 \theta / 87 \\
65 / 2 \theta / 87 \\
65 / 2 \theta / 87\end{array}$ & $\begin{array}{r}17.00 \\
13900.00 \\
1768.00 \\
12.00 \\
60.00 \\
39060.00\end{array}$ \\
\hline $\begin{array}{l}\text { FIRON } \\
\text { FLUORID } \\
\text { FMAGNES } \\
\text { FNANGAN } \\
\text { FPOTASS } \\
\text { FSODIUM } \\
\text { FSTRONT }\end{array}$ & $\begin{array}{l}\text { PPB } \\
\text { PPB } \\
\text { PPB } \\
\text { PPB } \\
\text { PPB } \\
\text { PPB } \\
\text { PPB }\end{array}$ & $\begin{array}{r}50.00 \\
500.00 \\
0.00 \\
5.00 \\
100.00 \\
100.00 \\
300.00\end{array}$ & 1400.00 & $\begin{array}{l}05 / 2 \theta / 87 \\
65 / 28 / 87 \\
65 / 28 / \theta 7 \\
66 / 28 / 87 \\
65 / 2 \theta / 87 \\
66 / 28 / 87\end{array}$ & $\begin{array}{r}58.00 \\
12500.00 \\
11160.00 \\
19.00 \\
7110.00 \\
361000.00\end{array}$ \\
\hline $\begin{array}{l}\text { FVANADI } \\
\text { LOALPHA }\end{array}$ & $\begin{array}{l}\mathrm{PPB} \\
\mathrm{PCI} / \mathrm{L}\end{array}$ & $\begin{array}{l}5.00 \\
2.00\end{array}$ & 16.00 & $\begin{array}{l}05 / 28 / 87 \\
05 / 28 / 87\end{array}$ & $\begin{array}{r}209.00 \\
7.68\end{array}$ \\
\hline NITRATE & PPB & 600.60 & 45000.00 & $\begin{array}{l}\emptyset 5 / 28 / 87 \\
65 / 28 / \theta 7\end{array}$ & $\begin{array}{l}677000.00 \\
680000.00\end{array}$ \\
\hline $\begin{array}{l}\text { NNIDINE } \\
\text { PHF IELD }\end{array}$ & PPE & $\begin{array}{r}10.00 \\
0.10\end{array}$ & & $\begin{array}{l}65 / 28 / 87 \\
65 / 28 / 87 \\
65 / 28 / 87\end{array}$ & $\begin{array}{r}40.00 \\
6.00 \\
7.63\end{array}$ \\
\hline $\begin{array}{l}\text { SULFATE } \\
\text { TETRANE } \\
\text { TOC } \\
\text { TOXLDL } \\
\text { TRICENE } \\
\text { TRITIUM }\end{array}$ & $\begin{array}{l}\text { PPB } \\
\text { PPB } \\
\text { PPB } \\
\text { PPB } \\
\text { PPB } \\
\text { PCI/L }\end{array}$ & $\begin{array}{r}500.00 \\
10.00 \\
200.00 \\
20.00 \\
10.00 \\
600.00\end{array}$ & 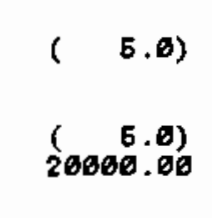 & $\begin{array}{l}65 / 2 \theta / 87 \\
65 / 28 / 87 \\
65 / 28 / 87 \\
65 / 28 / 87 \\
65 / 28 / 87 \\
\nabla 5 / 28 / 87\end{array}$ & $\begin{array}{r}38200.00 \\
1700.00 \\
709.00 \\
1060.00 \\
13.00 \\
261000.00 \\
\text { \{960.00) }\end{array}$ \\
\hline
\end{tabular}

\begin{tabular}{|c|c|}
\hline $\begin{array}{l}\text { SAMPLE } \\
\text { DATE }\end{array}$ & $2-w 15-10$ \\
\hline $06 / 28 / 87$ & $112000.00+$ \\
\hline $85 / 2 \theta / 87$ & 23.10 \\
\hline $06 / 28 / 87$ & $\begin{array}{r}5.84\} \\
21.60\end{array}$ \\
\hline $\begin{array}{l}06 / 28 / 87 \\
05 / 28 / 87 \\
65 / 2 \theta / 87\end{array}$ & $\begin{array}{r}3.43\} \\
24.00 \\
16200.00 \\
511.00\end{array}$ \\
\hline $\begin{array}{l}86 / 28 / 87 \\
85 / 28 / 87 \\
85 / 28 / 87\end{array}$ & $\begin{array}{r}54.06 \\
54300.06 \\
10.00\end{array}$ \\
\hline $05 / 28 / 87$ & 17600.00 \\
\hline $\begin{array}{l}65 / 28 / 87 \\
85 / 29 / 87\end{array}$ & $\begin{array}{r}4860.00 \\
18600.00\end{array}$ \\
\hline$B 5 / 28 / 87$ & 22.00 \\
\hline $\begin{array}{l}65 / 28 / 87 \\
05 / 28 / 87\end{array}$ & $\begin{array}{l}162000.00 \% \\
107000.00\end{array}$ \\
\hline $\begin{array}{l}65 / 2 \theta / 87 \\
65 / 2 \theta / 87 \\
65 / 28 / 87 \\
65 / 28 / 87 \\
65 / 28 / 87 \\
65 / 28 / 87 \\
65 / 28 / 87\end{array}$ & $\begin{array}{c}8.70 \\
7.76 \\
39600.00 \\
2748.00 \\
369.00 \\
2806.06 \\
18.00 \\
\text { NR }\end{array}$ \\
\hline
\end{tabular}

SAMPLE
OATE
-

$65 / 28 / 87$

$65 / 28 / 87$

$05 / 28 / 87$

$65 / 28 / 87$

$65 / 28 / 87$

$65 / 28 / 87$

$05 / 28 / 87$

$65 / 28 / 87$
$65 / 28 / 87$

$05 / 28 / 97$

$\triangle 5 / 28 / 87$

$05 / 28 / 87$

$65 / 28 / 87$

$06 / 28 / 87$

$\theta 5 / 2 \theta / 87$

$05 / 28 / 87$

$06 / 28 / 87$

$65 / 28 / 87$

65/28/87

$65 / 28 / 8$

$66 / 28 / 8$

$05 / 28 / 87$

$65 / 28 / 87$

$65 / 28 / 87$

$05 / 28 / 87$
2-พ15-11

$124000.00+$

21.90

5.13
15.96

[ 3.11$\}$

36.06
15108.06

674.08

69.80

78000.00

0

22206.

5590.00

27400.00

328.00

23.00

2.26
$\{\quad 0.63\}$

141000.06 .

121006.06

NR

9.08
7.65

64800. DE

3210.00 528.06

3300.03

10,00

- - VALUE EXCEEDS PRIMARY DRINKING WATER STANDARD.

- VALUE EXCEEDS PROPOSED PRIMARY DRINKING WATER STANDARD.

* VALUE EXCEEDS SCREENING LEVEL FOR FURTHER INVESTIGATION.

+ - DETECTION LIMIT WAS NOT AVAILABLE FOR COMPARISON

NR - ANALYSIS NOT REQUESTED OR NOT YET REPORTED

VALUES IN $\{$ f ARE COUNTING ERRORS FOR RADIONUCLIDES

WATER STANDARO (S) IN PARENTHESES ARE PROPOSED ONLY 
TABLE A.4. (cont d)

\begin{tabular}{|c|c|c|c|c|c|}
\hline $\begin{array}{l}\text { CONSTI } \\
\text { NALEE }\end{array}$ & $\begin{array}{l}\text { JENT } \\
\text { UNITS }\end{array}$ & $\begin{array}{l}\text { DETECTION } \\
\text { LIMIT }\end{array}$ & $\begin{array}{l}\text { WATER } \\
\text { STANDARD }\end{array}$ & $\begin{array}{l}\text { SAMPLE } \\
\text { DATE }\end{array}$ & $2-W 1 B-7$ \\
\hline & & & & -- & \\
\hline \multirow[t]{4}{*}{$\begin{array}{l}\text { ALKALIN } \\
\text { BETA }\end{array}$} & $\mathrm{PCI} / \mathrm{L}$ & 3.60 & 50.00 & & $\begin{array}{l}\text { NR } \\
\text { NR }\end{array}$ \\
\hline & & & & & NR \\
\hline & & & & & NR \\
\hline & & & & & NR \\
\hline
\end{tabular}

$\begin{array}{ll} & \text { CHLFORM } \\ & \text { CHLORID } \\ & \text { CONDFLD } \\ & \text { CYANIDE } \\ & \text { FARSENI } \\ & \text { FBARIUH } \\ & \text { FCALCIU } \\ & \text { FIRON } \\ & \text { FLUORID } \\ & \text { FMAGNES } \\ & \text { FMANGAN } \\ & \text { FMERCUR } \\ & \text { FPOTASS } \\ & \text { FSODIUM } \\ & \text { FVANADI } \\ & \text { LOALPHA } \\ \omega & \end{array}$

\begin{tabular}{|c|c|}
\hline $\begin{array}{l}\text { PPB } \\
\text { PPB } \\
\text { UMHO } \\
\text { PPB } \\
\text { PPB } \\
\text { PPB } \\
\text { PPB } \\
\text { PPB } \\
\text { PPB } \\
\text { PPB } \\
\text { PPB } \\
\text { PPB } \\
\text { PPB } \\
\text { PPB } \\
\text { PPB } \\
\text { PCI/L }\end{array}$ & $\begin{array}{r}10.00 \\
506.00 \\
1.06 \\
10.00 \\
5.00 \\
6.00 \\
50.00 \\
56.00 \\
500.00 \\
6.00 \\
5.00 \\
0.10 \\
100.00 \\
100.00 \\
5.06 \\
2.00\end{array}$ \\
\hline
\end{tabular}

$\begin{array}{rr} & 66 / 02 / 87 \\ 66 / 62 / 87 \\ 50.66 & 66 / 02 / 87 \\ 1000.66 & \\ 1460.60 & 66 / 62 / 87 \\ 2.00 & \\ 15.00\end{array}$

13.00
2000.00

NR

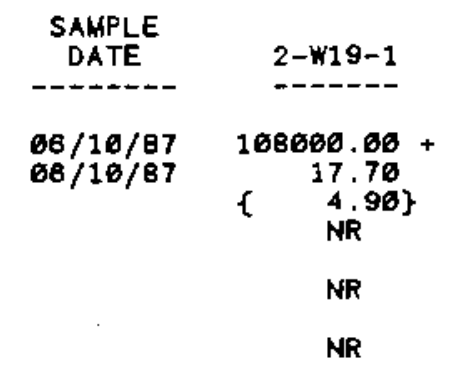

$66 / 10 / 87$

$\begin{array}{llrr}\text { NITRATE } & \text { PPB } & 500.60 & 45000.00 \\ \text { PHFIELD } & & 6.10 & \\ \text { RADIUN } & \text { PCI/L } & 6.10 & 5.60 \\ \text { SR } 96 & \text { PCI/L } & 6.00 & 0.60 \\ \text { SULFATE } & \text { PPB } & 600.60 & \end{array}$

$\begin{array}{ll}\text { SULFATE } & \text { PPB } \\ \text { TC-99 } & \text { PCI/L }\end{array}$

160000.00
15.26

NR

NR

1140.60

NR

NR

NR

NR

NR

NR

NR

NR

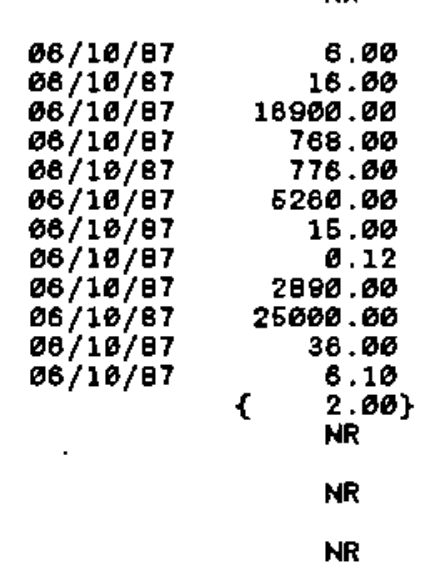

$06 / 10 / 87$

NR
NR
NR
NR
NR
NR
NR

$66 / 62 / 87$

$\begin{array}{ll} & \text { NR } \\ \text { NR } \\ \text { NR } \\ 06 / 10 / 87 & \text { B. } \\ & \text { NR } \\ & \text { NR } \\ & \text { NR }\end{array}$

$06 / 10 / 87$
10600.00

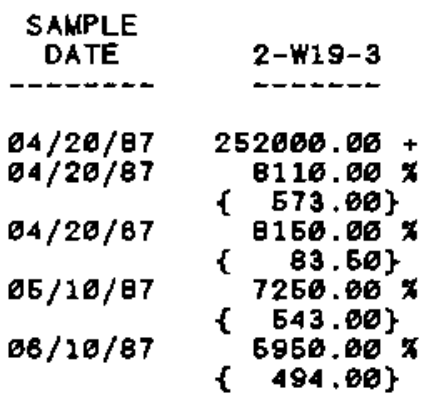

$04 / 26 / 87$ $04 / 20 / 87$

\subsection{0}

704.00

NR

NR

NR

NR

NR

NR

NR

$04 / 26 / 87$

$04 / 20 / 87$

$05 / 10 / 87$

$66 / 10 / 87$

$04 / 20 / 87$

$64 / 20 / 87$

$65 / 16 / 87$

$68 / 10 / 87$

$64 / 20 / 87$

$64 / 26 / 87$

$64 / 26 / 87$

$04 / 26 / 8$

$04 / 26 / 87$
$66 / 10 / 87$

* - VALUE EXCEEDS PRIMARY DRINKING WATER STANDARD.

- VALUE EXCEEDS PROPOSED PRIMARY DRINKING WATER STANDARD.

* - VALUE EXCEEDS SCREENING LEVEL FOR FURTHER INVESTIGATION.

* DETECTION LIMIT WAS NOT AVAILABLE FOR COMPARISON

NR - ANALYSIS NOT REQUESTED OR NOT YET REPORTED

VALUES IN \{ \} ARE COUNTING ERRORS FOR RADIONUCLIDES

WATER STANDARD (5) IN PARENTHESES ARE PROPOSED ONLY 
TABLE A.4. (contd)

\begin{tabular}{|c|c|c|c|c|c|c|c|c|c|}
\hline $\begin{array}{l}\text { CONSTI } \\
\text { NAME }\end{array}$ & $\begin{array}{l}\text { UENT } \\
\text { UNITS }\end{array}$ & $\begin{array}{l}\text { DETECTION } \\
\text { LIMIT }\end{array}$ & $\begin{array}{l}\text { WATER } \\
\text { STANDARD }\end{array}$ & $\begin{array}{l}\text { SAMPLE } \\
\text { DATE }\end{array}$ & $2-W 18-7$ & $\begin{array}{l}\text { SAMPLE } \\
\text { DATE }\end{array}$ & $2-W 19-1$ & $\begin{array}{l}\text { SAMPLE } \\
\text { DATE }\end{array}$ & $2-w 19-3$ \\
\hline 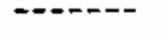 & ---- & $----m--0-$ & $-x+--0-\infty-1$ & $-\infty-----$ & $---\infty-\infty$ & $-+-m-2$ & $--0-0-0$ & $-+\infty-\infty-1$ & ------- \\
\hline $\begin{array}{l}\text { TETRANE } \\
\text { TOC } \\
\text { TOXLDL } \\
\text { TRITIUM }\end{array}$ & $\begin{array}{l}\text { PPB } \\
\text { PPB } \\
\text { PPB } \\
\text { PCI/L }\end{array}$ & $\begin{array}{r}10.00 \\
200.00 \\
20.00 \\
500.00\end{array}$ & $\begin{array}{l}(5 . \sigma) \\
20000.00\end{array}$ & $\begin{array}{l}66 / 02 / 87 \\
06 / 02 / 87\end{array}$ & $\begin{array}{c}18.00 \\
1130.00 \\
\mathrm{NR}\end{array}$ & $06 / 10 / 07$ & 820.00 & $\begin{array}{l}04 / 20 / 87 \\
04 / 20 / 87 \\
04 / 20 / 87 \\
04 / 20 / 87\end{array}$ & $\begin{array}{r}53.00 \\
541.00 \\
45.40 \\
1280.00\end{array}$ \\
\hline & & & & & NR & & NR & $05 / 10 / 87$ & 1300.00 \\
\hline & & & & & NR & & NR & $06 / 16 / 87$ & $\begin{array}{r}1820.00 \\
256.00\}\end{array}$ \\
\hline U-CHEM & UG $/ L$ & 0.73 & & & $\begin{array}{l}\text { NR } \\
\text { NR } \\
\text { NR }\end{array}$ & & $\begin{array}{l}\text { NR } \\
\text { NR } \\
\text { NR }\end{array}$ & $\begin{array}{l}64 / 26 / 87 \\
65 / 16 / 87 \\
68 / 16 / 87\end{array}$ & $\begin{array}{l}13900.00 \\
11000.00 \\
11000.00\end{array}$ \\
\hline
\end{tabular}

* - VAlue exceEds PRIMARY dRINKING hater STANDARD.

* VALUE EXCEEDS PROPOSED PRIMARY DRINKING HaTER STANDARD

* - VALUE EXCEEDS SCREENING LEVEL FOR FURTHER INVESTIGATION.

* - DETECTION LIMIT WAS NOT AVAILABLE FOR COMPARISON

NR - ANALYSIS NOT REQUESTED OR NOT YET REPORTED

VALUES IN \{ $\}$ ARE COUNTING ERRORS FOR RADIONUCLIDES

WATER STANDARD(S) IN PARENTHESES ARE PROPOSED ONLY 
TABLE A.4. (cont $d$ )

\begin{tabular}{|c|c|c|c|c|c|}
\hline $\begin{array}{l}\text { CONSTI } \\
\text { NAME }\end{array}$ & $\begin{array}{l}\text { JENT } \\
\text { UNITS }\end{array}$ & $\begin{array}{l}\text { DETECTION } \\
\text { LIMIT }\end{array}$ & $\begin{array}{l}\text { WATER } \\
\text { STANDARD }\end{array}$ & $\begin{array}{l}\text { SAMPLE } \\
\text { DATE }\end{array}$ & 2-พ19-5 \\
\hline \multirow[t]{3}{*}{$\begin{array}{l}\text { ALKALIN } \\
\text { BETA }\end{array}$} & \multirow[t]{3}{*}{ PCI/L } & \multirow[t]{3}{*}{3.00} & \multirow[t]{3}{*}{50.00} & $\begin{array}{l}06 / 61 / 87 \\
66 / 01 / 87\end{array}$ & $\begin{array}{r}98800.00 \\
21.86\end{array}$ \\
\hline & & & & $86 / 01 / 87$ & $\left\{\begin{array}{c}29.40 \\
N .67\}\end{array}\right.$ \\
\hline & & & & & NR \\
\hline
\end{tabular}

$\begin{array}{ll}\text { CHLORID } & \text { PPB } \\ \text { CONDFLD } & \text { UHHO } \\ \text { FARSENI } & \text { PPB } \\ \text { FEARIUM } & \text { PPB } \\ \text { FCALCIU } & \text { PPB } \\ \text { FCOPPER } & \text { PPB } \\ \text { FIRON } & \text { PPB } \\ \text { FLUORID } & \text { PPB } \\ \text { FMAGNES } & \text { PPB } \\ \text { FMANGAN } & \text { PPE } \\ \text { FPOTASS } & \text { PPB } \\ \text { FSODIUM } & \text { PPB } \\ \text { FVANADI } & \text { PPB } \\ \text { FZINC } & \text { PPB } \\ \text { LOALPHA } & \text { PCI/L } \\ & \end{array}$

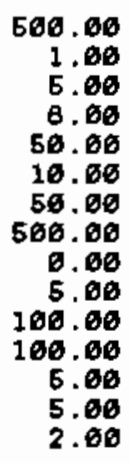

$\omega$

$\left\{\begin{array}{l}6.01 \\ \quad 2.06\end{array}\right\}$

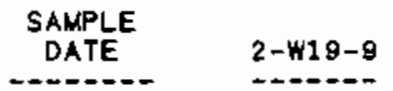

$06 / 01 / 87$

$65 / 15 / 67$

$66 / 61 / 67$

$06 / 01 / 87$

$06 / 01 / 87 \quad 4900.00$

232.60

50.00
1000.00

(1300.6)

1400.00

$06 / 01 / 87$

$08 / 01 / 87$

$08 / 01 / 8$

$68 / 61 / 87$
$06 / 01 / 97$
$06 / 01 / 87$

$06 / 61 / 87$

$06 / 81 / 87$

$68 / 01 / 87$
$66 / 01 / 87$

15.60

19.00

197.08

8440.00

21.06

3240.00

23400.00

16.06
8.06
6.01
2.05

NR

$06 / 01 / 87$

21800.00

525. 00

NR
NR
NR

NR

68/01/87 $\quad$ NR

NR

NR

NR

NR

NR
NR

NR

\begin{tabular}{|c|c|c|c|c|c|}
\hline NITRATE & PPB & 500.00 & 45000.00 & $\begin{array}{l}66 / 61 / 97 \\
66 / 61 / 87\end{array}$ & $\begin{array}{c}4900.00 \\
\text { E520.00 } \\
\text { NR } \\
\text { NR }\end{array}$ \\
\hline PHF IELD & & 6.10 & & $\begin{array}{l}06 / 01 / 87 \\
66 / 01 / 87\end{array}$ & $\begin{array}{l}7.66 \\
7.82\end{array}$ \\
\hline RADIUN & $\mathrm{PCI} / \mathrm{L}$ & 0.10 & 5.00 & $08 / 01 / 87$ & $\begin{array}{r}0.65 \\
5 \quad 0.27\end{array}$ \\
\hline $\begin{array}{l}\text { SULFATE } \\
\text { TC-99 }\end{array}$ & $\begin{array}{l}\mathrm{PPB} \\
\mathrm{PCI} / \mathrm{L}\end{array}$ & $\begin{array}{r}500.00 \\
15.00\end{array}$ & 100000.00 & $06 / 01 / 87$ & 21306.06 \\
\hline $\begin{array}{l}\text { TETRANE } \\
\text { TOC }\end{array}$ & $\begin{array}{l}\text { PPB } \\
\text { PPB }\end{array}$ & $\begin{array}{r}10.00 \\
260.60\end{array}$ & $5 . \boldsymbol{\sigma})$ & $68 / 01 / 07$ & 342.00 \\
\hline
\end{tabular}

- VALUE EXCEEDS PRIMARY DRINKING HATER STANDARD.

\# - VALUE EXCEEDS PROPOSED PRIMARY DRINKING WATER STANDARD

* VALUE EXCEEDS SCREENING LEVEL FOR FURTHER INVESTIGATION.

+ - DETECTION LIMIT WAS NOT AVAILABLE FOR COMPARISON

NR - ANALYSIS NOT REQUESTED OR NOT YET REPORTED

VALUES IN \{ \} ARE COUNTING ERRORS FOR RADIONUCLIDES

WATER STANDARD'(S) IN PARENTHESES ARE PROPOSED ONLY
SAMPLE

DATE

2-W19-11

$161000.00+$

4700.60

(441.06)

$1756.06 \%$

$\left\{\begin{array}{c}37.16\} \\ 3890.00 \times\end{array}\right.$

$463.66\}$

$05 / 15 / 87$

$66 / 01 / 87$

$3910.00 \%$

\{ 258.00$\}$

$\left\{\begin{array}{c}2600.60 \\ 49.60\}\end{array}\right.$

$86 / 61 / 87$

$2920.00 x$

(223.00\}

NR

$06 / 15 / 87 \quad 13980.00$

$68 / 01 / 97 \quad 136000.00$.

$08 / 01 / 87 \quad 126000.00$.

$06 / 01 / 87$

ด8/61/87

26080.00

7.20

7.48

$66 / 01 / 87$

46900.60

NR

$06 / 01 / 87$
$6 B / 61 / 87$

56.00
457.06
$04 / 20 / 87 \quad 211000.00$

$8970.00 \%$

$04 / 20 / 87$ f 400.00

$65 / 16 / 87$

$66 / 10 / 87$

$04 / 20 / 87$

$64 / 20 / 87$

$04 / 20 / 87$

$04 / 20 / 87$

$64 / 26 / 87$

$64 / 26 / 67$

$64 / 26 / 87$

$64 / 20 / 87$

$64 / 26 / 87$

$04 / 20 / 87$

$65 / 16 / 87$

$08 / 16 / 87$

$64 / 26 / 87$

$04 / 26 / 87$

$65 / 16 / 87$

$68 / 10 / 87$

$04 / 20 / 87$

$04 / 20 / 97$

4460.00
$59.30\}$

$59.30\}$
$3270.08 \%$

( 100.00$\}$

$4560.00 \%$

(234.08\})

710.00

6.00

$\begin{array}{r}50.06 \\ \hline\end{array}$

50100.06

11.00

18300.00

5700.06

74900.00

20.00

( $4360.00 \%$

$3280.00 \%$

$\{\quad 59.00\}$

$520.60 x$

$3826.00 \times$

$259.00)$

136000.00 .

118000.00 .

7.76

7 .

$64 / 26 / 87$

$96 / 10 / 87$

$64 / 26 / 87$

$64 / 26 / 87$
63200.00

3200.00

( $\quad 31.80\}$ 512.60
$04 / 20 / 87$ 
TABLE A.4. (contd)

\begin{tabular}{|c|c|c|c|c|c|c|c|c|c|}
\hline $\begin{array}{l}\text { CONSTI } \\
\text { NAME }\end{array}$ & $\begin{array}{l}\text { UENT } \\
\text { UNI TS }\end{array}$ & $\begin{array}{l}\text { OETECTION } \\
\text { LIUIT }\end{array}$ & $\begin{array}{l}\text { WATER } \\
\text { STANDARD }\end{array}$ & $\begin{array}{c}\text { SAMPLE } \\
\text { DATE }\end{array}$ & $2-W 19-6$ & $\begin{array}{l}\text { SAMPLE } \\
\text { DATE }\end{array}$ & $2-w 1 \theta-9$ & $\begin{array}{l}\text { SAMPLE } \\
\text { DATE }\end{array}$ & $2-W 19-11$ \\
\hline$---\infty-\infty$ & ---- & 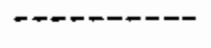 & - - & ------- & $--\infty-\infty$ & $--\infty-\infty$ & $m--\infty$ & ------- & $\ldots . .$. \\
\hline \multirow[t]{3}{*}{$\begin{array}{l}\text { TOXLDL } \\
\text { TRITIUK }\end{array}$} & $\begin{array}{l}\mathrm{PPB} \\
\mathrm{PCI} / \mathrm{L}\end{array}$ & $\begin{array}{r}20.00 \\
500.00\end{array}$ & 20000.00 & & & $06 / 01 / 87$ & ${ }_{\mathrm{NR}}^{48.30}$ & $\begin{array}{l}64 / 20 / 87 \\
04 / 20 / 87\end{array}$ & $\begin{array}{r}68.00 \\
922.00 \\
+\quad 291.00\}\end{array}$ \\
\hline & & & & & NR & & NR & $65 / 10 / 87$ & 1080.00 \\
\hline & & & & & NR & & NR & $66 / 16 / 87$ & $\begin{array}{r}1716.00 \\
\{56.097\end{array}$ \\
\hline U-CHEW & UG/L & 0.73 & & & $\begin{array}{l}\text { NR } \\
\text { NR } \\
\text { NR }\end{array}$ & $\begin{array}{l}65 / 16 / 87 \\
86 / 61 / 87\end{array}$ & $\begin{array}{c}7550.00 \\
4230.00 \\
\text { NR }\end{array}$ & $\begin{array}{l}64 / 20 / 87 \\
06 / 16 / 87 \\
66 / 10 / 87\end{array}$ & $\begin{array}{l}7820.00 \\
7250.00 \\
4780.00\end{array}$ \\
\hline
\end{tabular}

* - VALUE EXCEeds PRIMARY DRINKINg water STANDARD.

* VALUE EXCEEDS PROPOSED PRIMARY DRINKING WATER STANDARD.

* - VALUE EXCEEDS SCREENING LEVEL FOR FURTHER INVESTIGATION.

- DETECTION LIMIT HAS NOT AVAILABLE FOR COMPARISON

NR - ANALYSIS NOT REQUESTED OR NOT YET REPORTED

VALUES IN \{\} ARE COUNTING ERRORS FOR RADIONUCLIDES

WATER STANDARD(S) IN PARENTHESES ARE PROPOSED ONLY 
IABLE A.4. (contd)

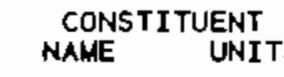

NAME UNITS

ALKALIN

BETA

PCI/L

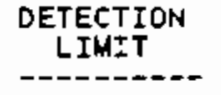

STANOARD

SAMPLE DATE

2-W19-13

$169000.00+$

\begin{tabular}{|c|c|}
\hline $\begin{array}{l}04 / 2 \theta / 87 \\
84 / 2 \theta / 87\end{array}$ & $\begin{array}{r}169060.00 \\
18.10\end{array}$ \\
\hline $04 / 20 / 87$ & $\begin{array}{c}3.12\} \\
22.00\end{array}$ \\
\hline $66 / 10 / 87$ & $\begin{array}{r}4.87\} \\
11.68\end{array}$ \\
\hline $86 / 16 / 87$ & $\begin{array}{r}2.56\} \\
10.00\end{array}$ \\
\hline $\begin{array}{l}04 / 20 / 87 \\
04 / 20 / 87 \\
04 / 26 / 87 \\
04 / 26 / 87\end{array}$ & 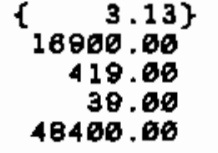 \\
\hline $04 / 20 / 87$ & 14700.00 \\
\hline $04 / 2 \theta / 87$ & 4000.00 \\
\hline $\begin{array}{l}\theta 4 / 2 \theta / 87 \\
\theta 4 / 2 \theta / 87 \\
04 / 2 \theta / 87 \\
84 / 2 \theta / 87\end{array}$ & $\begin{array}{r}27600.00 \\
27.00 \\
10.00 \\
11.00\end{array}$ \\
\hline $04 / 20 / 87$ & $\begin{array}{c}1.41\} \\
20.90\end{array}$ \\
\hline $06 / 10 / 87$ & \\
\hline $06 / 16 / 87$ & \\
\hline $\begin{array}{l}64 / 2 \theta / 87 \\
64 / 20 / 87 \\
66 / 10 / 87 \\
66 / 16 / 87 \\
64 / 20 / 87 \\
64 / 2 \theta / 87 \\
64 / 20 / 87 \\
64 / 20 / 87 \\
64 / 20 / 87 \\
64 / 26 / 87 \\
64 / 20 / 87 \\
65 / 10 / 87 \\
66 / 10 / 87\end{array}$ & $\begin{array}{r}23200.00 \\
22000.00 \\
22400.00 \\
21700.00 \\
7.60 \\
7.00 \\
60000.00 \\
20.00 \\
324.00 \\
31.00 \\
13.90 \\
18.20 \\
13.30\end{array}$ \\
\hline
\end{tabular}

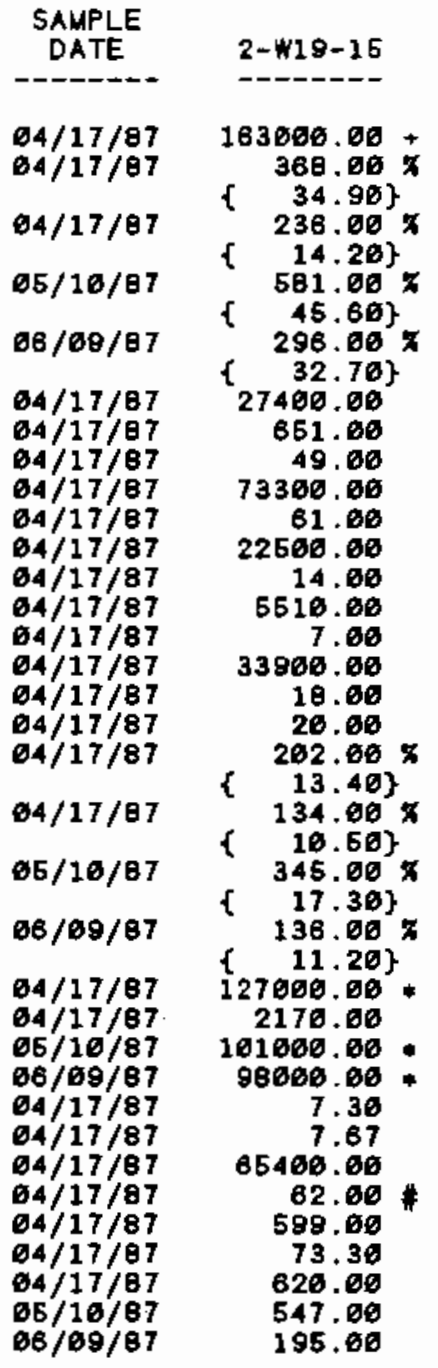

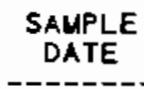

$64 / 17 / 67$

$04 / 17 / 87$

$64 / 17 / 67$

$05 / 16 / 87$

$06 / 10 / 87$

$04 / 17 / 87$

$04 / 17 / 67$

$04 / 17 / 87$

4/17/87

$04 / 17 / 87$

$04 / 17 / 87$

$04 / 17 / 87$

$64 / 17 / 87$

$4 / 17 / 87$

$04 / 17 / 87$

$05 / 10 / 87$

06/16/87

$64 / 17 / 87$

$04 / 17 / 87$

$05 / 10 / 87$

$06 / 10 / 87$

$04 / 17 / 87$

$04 / 17 / 87$

0411787

$04 / 17 / 87$

$04 / 17 / 87$

$04 / 17 / 87$

$4 / 17 / 87$

$04 / 17 / 87$

$66 / 16 / 87$
2-พ19-16

$148000.66+$

$2130.00 \%$

$\left\{\begin{array}{l}306.06\} \\ 644.00 \times\end{array}\right.$

\{ 20.66\}

$1700.00 \times$

$\left\{\begin{array}{l}276.00\} \\ \text {. }\end{array}\right.$

1860.00
$271.06\}$

14806.06

483.00

45.00

42100.06

12600.00

4680.66

47700.60

16.60

12.00

\{ 1490.60 \%

$\left\{\begin{array}{r}169.00\} \\ 1326.00\end{array}\right.$

$\{32.40\}$

$1420.00 x$

( 163.00$\}$

$1310.00 \times$

( 156.00$\}$

4440.00

51806.06 *

48800.06 .

7.38

34690

101.00

425.00

84.20

2840.00

1630.00

- VALUE EXCEEDS PRIMARY DRINKINg water STANDARD.

- VALUE EXCEEDS PROPOSED PRIMARY DRINKING WATER STANDARD

* - VALUE EXCEEDS SCREENING LEVEL FOR FURTHER INVESTIGATION.

+ - DETECTION LIUIT WAS NOT AVAILABLE FOR COMPARISON

NR - ANALYSIS NOT REQUESTED OR NOT YET REPORTED

VALUES IN \{\} ARE COUNTING ERRORS FOR RADIONUCLIDES

WATER STANDARD (S) IN PARENTHESES ARE PROPOSED ONLY 
TABLE A.4. (contd)

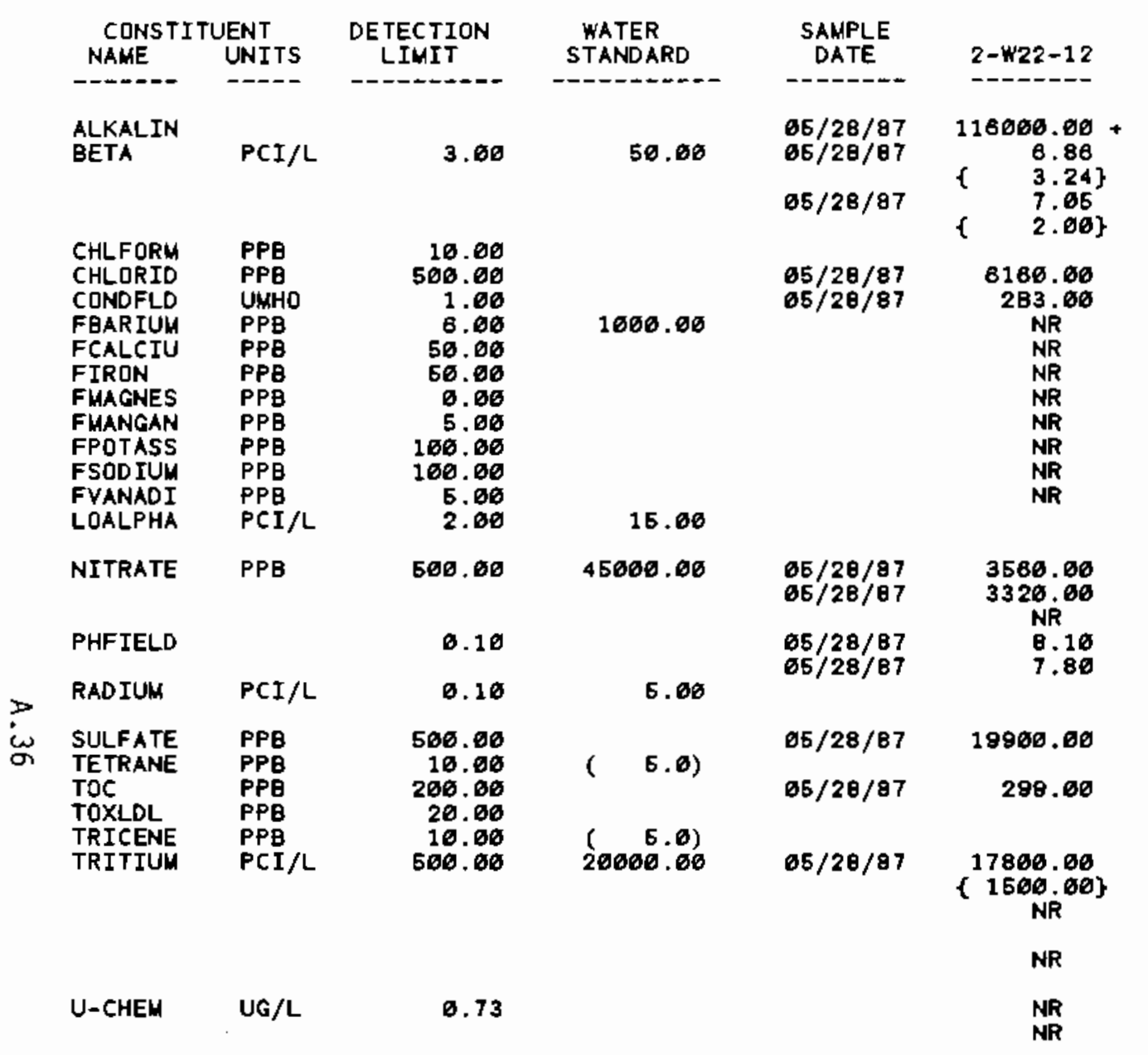

\begin{tabular}{|c|c|}
\hline $\begin{array}{l}\text { SAMPLE } \\
\text { DATE }\end{array}$ & $2-\$ 22-2 \theta$ \\
\hline $\begin{array}{l}85 / 28 / 87 \\
85 / 28 / 87\end{array}$ & $\begin{array}{c}133000.00+ \\
49.80\end{array}$ \\
\hline $05 / 28 / 87$ & $\left\{\begin{array}{r}8.56 \\
42.86\end{array}\right.$ \\
\hline $\begin{array}{l}06 / 28 / 87 \\
05 / 28 / 67\end{array}$ & $\begin{array}{r}4.93\} \\
12.06 \\
29700.08\end{array}$ \\
\hline $65 / 28 / 67$ & $\begin{array}{c}\text { O25. } 0 \varnothing \\
\text { NR } \\
N R \\
N R \\
N R \\
N R \\
N R \\
N R \\
N R \\
N R \\
N R\end{array}$ \\
\hline $05 / 2 \theta / 87$ & $16.36 \%$ \\
\hline $06 / 28 / 87$ & $\begin{array}{c}123600.60 \\
N R\end{array}$ \\
\hline $\begin{array}{l}65 / 28 / 87 \\
05 / 28 / 87 \\
65 / 28 / 87\end{array}$ & $\begin{array}{l}7.66 \\
7.36 \\
1.33\end{array}$ \\
\hline $\begin{array}{l}05 / 2 \theta / 87 \\
05 / 29 / 87 \\
05 / 2 \theta / 87 \\
05 / 2 \theta / 87 \\
05 / 28 / 87 \\
05 / 2 \theta / 87\end{array}$ & 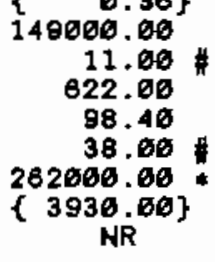 \\
\hline & NR \\
\hline & $\begin{array}{l}\mathrm{NR} \\
\mathrm{NR}\end{array}$ \\
\hline
\end{tabular}

\begin{tabular}{|c|c|}
\hline $\begin{array}{l}\text { SAMPLE } \\
\text { DATE }\end{array}$ & $2-W 22-22$ \\
\hline $\begin{array}{l}04 / 18 / 87 \\
04 / 16 / 07\end{array}$ & $\begin{array}{r}108000.60 \\
5.38 \\
.38\end{array}$ \\
\hline $06 / 10 / 87$ & $\begin{array}{ll}\{ & 3.23\} \\
\{\quad & 4.75 \\
\{1.78\}\end{array}$ \\
\hline $\begin{array}{l}04 / 16 / 87 \\
64 / 16 / 87 \\
64 / 16 / 87 \\
64 / 16 / 87 \\
64 / 16 / 87 \\
64 / 16 / 87 \\
64 / 16 / 87 \\
04 / 18 / 87 \\
64 / 18 / 87 \\
04 / 16 / 87\end{array}$ & $\begin{array}{r}15200.00 \\
305.00 \\
23.00 \\
32006.00 \\
70.00 \\
16300.00 \\
15.00 \\
3730.00 \\
13200.00 \\
22.06\end{array}$ \\
\hline $\begin{array}{l}64 / 16 / 87 \\
64 / 16 / 87 \\
68 / 16 / 87 \\
64 / 16 / 87 \\
04 / 16 / 87\end{array}$ & $\begin{array}{r}1460.00 \\
942.00 \\
2620.00 \\
7.30 \\
7.44\end{array}$ \\
\hline $04 / 18 / 87$ & 19800.00 \\
\hline $04 / 16 / 87$ & 298.00 \\
\hline $04 / 16 / 87$ & 2050.00 \\
\hline $05 / 10 / 87$ & 2050.00 \\
\hline$\theta 8 / 10 / \theta 7$ & $\begin{array}{r}314.06\} \\
1880.06\end{array}$ \\
\hline $\begin{array}{l}64 / 16 / 87 \\
65 / 16 / 87\end{array}$ & $\left\{\begin{array}{c}241.06\} \\
0.76 \\
0.73\end{array}\right.$ \\
\hline
\end{tabular}

* - VALUE EXCEEDS PRIMARY DRINKING water STANDARD.

\# - VALUE EXCEEDS PROPOSED PRIMARY DRINKING HATER STANDARD.

* - VALUE EXCEEDS SCREENING LEVEL FOR FURTHER INVESTIGATION.

* - DETECTION LIMIT WAS NOT AVAILAELE FOR COMPARISON

NR - ANALYSIS NOT REQUESTED OR NOT YET REPORTED

VALUES IN \{ $\}$ ARE COUNTING ERRORS FOR RADIONUCLIDES

WATER STANDARD(S) IN PARENTHESES ARE PROPOSED ONLY 
TABLE A.4. (cont $d)$

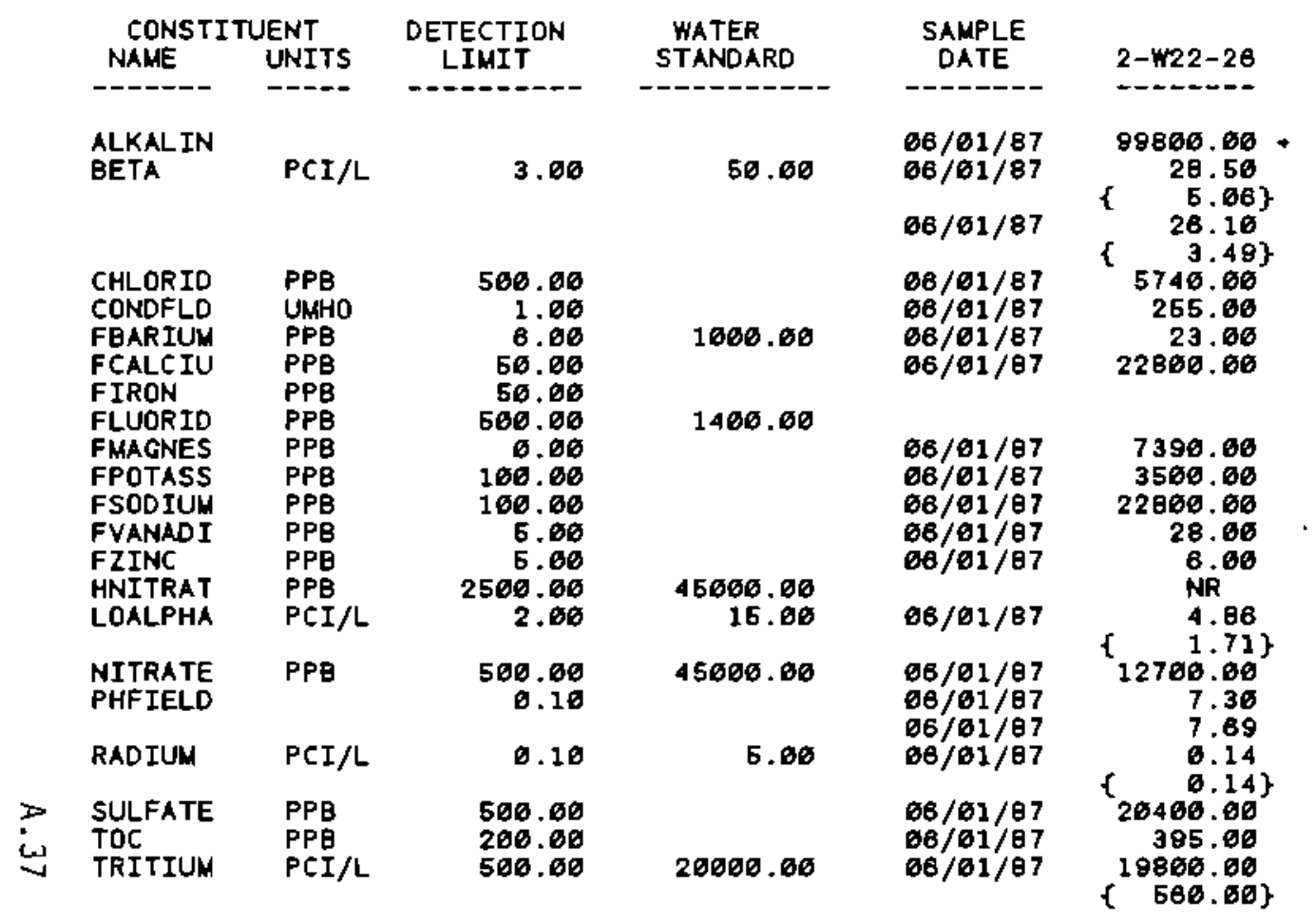

\begin{tabular}{|c|c|}
\hline $\begin{array}{l}\text { SAMPLE } \\
\text { DATE }\end{array}$ & $2-w 23-1$ \\
\hline & \\
\hline $\begin{array}{l}68 / 99 / 87 \\
66 / 09 / 87\end{array}$ & 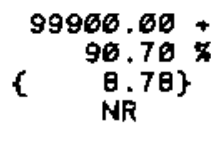 \\
\hline $08 / 98 / 97$ & 4860.60 \\
\hline 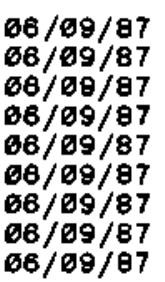 & $\begin{array}{r}29.00 \\
10000.00 \\
74.00 \\
671.00 \\
6300.60 \\
3460.00 \\
23800.06 \\
22.00 \\
135.06\end{array}$ \\
\hline $66 / 08 / 87$ & $\begin{array}{l}\text { NR } \\
\text { B.64 }\end{array}$ \\
\hline $\begin{array}{l}06 / 69 / 87 \\
06 / 09 / 87\end{array}$ & $\begin{array}{c}\text { B630.60 } \\
7.78 \\
\text { NR } \\
\text { NR }\end{array}$ \\
\hline $\begin{array}{l}66 / 89 / 87 \\
08 / 09 / 87\end{array}$ & $\begin{array}{r}17100.00 \\
560.00\end{array}$ \\
\hline
\end{tabular}

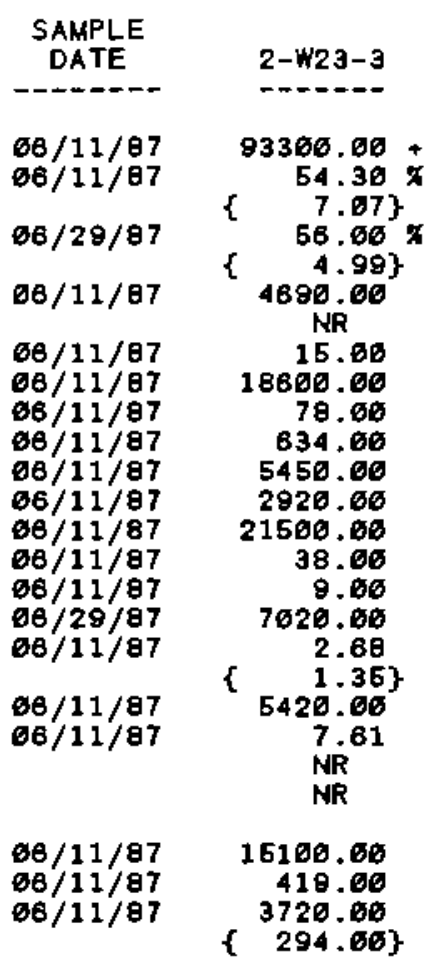

- - Value exceeds primary dRINKINg hater standard.

* - VALUE EXCEEDS PROPOSED PRIMARY DRINKING WATER STANDARD.

* - VALUE EXCEEDS SCREENING LEVEL FOR FURTHER INVESTIGATION.

- DETECTION LIMIT WAS NOT AVAILABLE FOR COMPARISON

NR - ANALYSIS NOT REQUESTED OR NOT YET REPORTED

VALUES IN \{\} ARE COUNTING ERRORS FOR RADIONUCLIDES

WATER STANDARD(S) IN PARENTHESES ARE PROPOSED ONLY 
TABLE A.4. (contd)

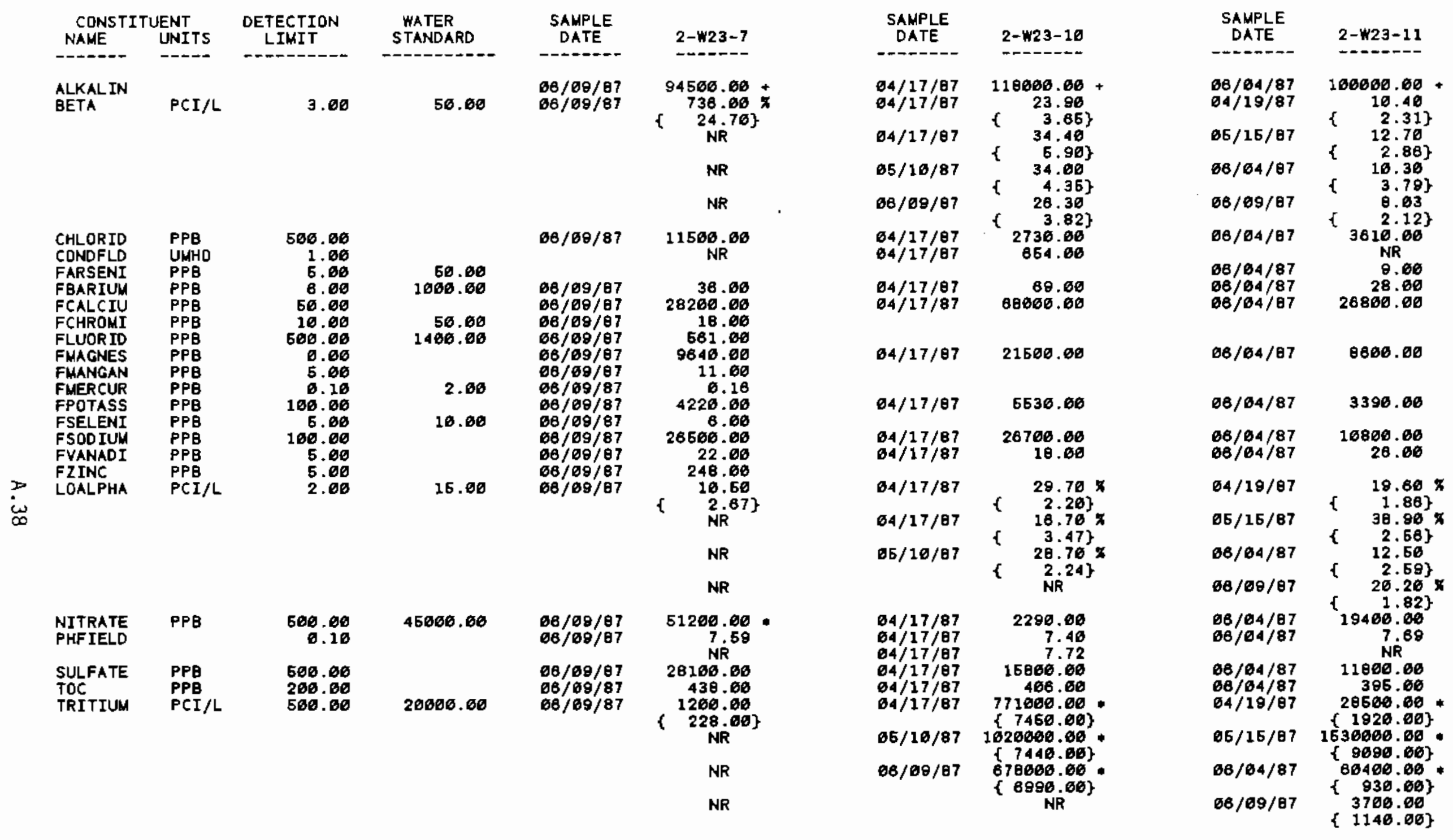

* - VALUE EXCEEDS PRIMARY DRINKING WaTER STANDARD.

* - VALUE EXCEEDS PROPOSED PRIMARY DRINKING WATER STANDARD.

- VALUE EXCEEDS SCREENING LEVEL FOR FURTHER INVESTIGATION.

4 - DETECTION LIMIT WAS NOT AVAILABLE FOR COMPARISON

NR - ANALYSIS NOT REQUESTED OR NOT YET REPORTED

VALUES IN \{ $\}$ ARE COUNTING ERRORS FOR RADIONUCLIDES

WATER STANDARD(S) IN PARENTHESES ARE PROPDSED ONLY 
TABLE A.4. (contd)

\begin{tabular}{|c|c|c|c|c|c|c|c|c|c|}
\hline $\begin{array}{l}\text { CONSTI } \\
\text { NAME }\end{array}$ & $\begin{array}{l}\text { UENT } \\
\text { UNITS }\end{array}$ & $\begin{array}{l}\text { DETECTION } \\
\text { LIMIT }\end{array}$ & $\begin{array}{l}\text { HATER } \\
\text { STANDARD }\end{array}$ & $\begin{array}{l}\text { SAMPLE } \\
\text { DATE }\end{array}$ & $2-H 23-7$ & $\begin{array}{l}\text { SAMPLE } \\
\text { DATE }\end{array}$ & $2-W 23-1 \theta$ & $\begin{array}{l}\text { SAMPLE } \\
\text { DATE }\end{array}$ & $2-123-11$ \\
\hline$--0 .-0$ & $--\infty$ & 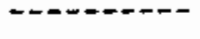 & ---------- & ------- & $-----\infty$ & $-\infty+\cdots$ & $----\infty$ & $\cdots+-. .-1$ & $--0-0-0$ \\
\hline U-CHEM & UG/L & 6.73 & & & $\begin{array}{l}\text { NR } \\
\text { NR } \\
\text { NR }\end{array}$ & $\begin{array}{l}64 / 17 / 07 \\
06 / 16 / 07\end{array}$ & $\begin{array}{l}42.38 \\
61.10 \\
\text { NR }\end{array}$ & $\begin{array}{l}04 / 19 / 07 \\
06 / 15 / 07 \\
66 / 89 / 07\end{array}$ & $\begin{array}{l}28.30 \\
31.80 \\
24.30\end{array}$ \\
\hline
\end{tabular}


TABLE A.4. (contd)

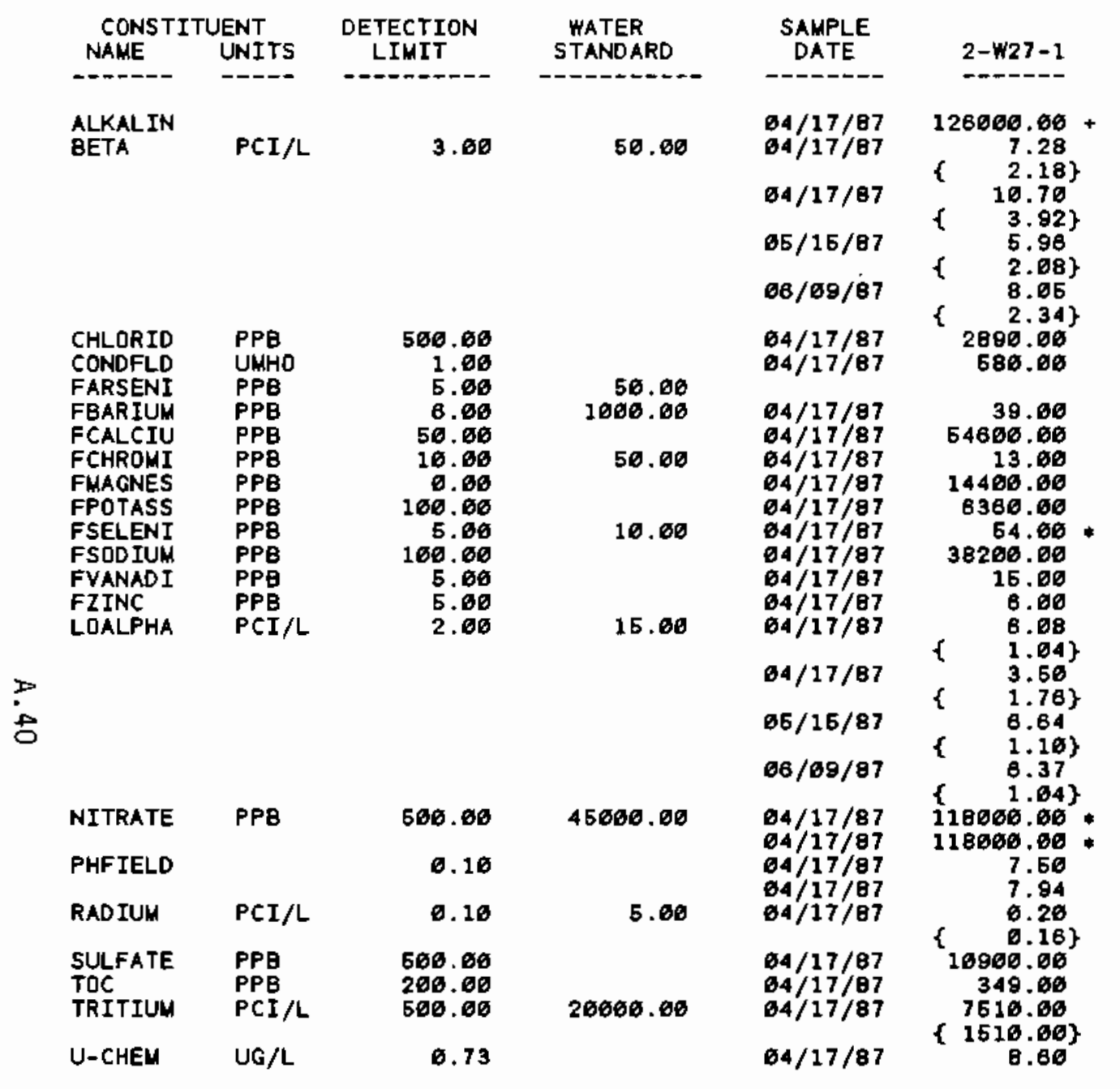

\begin{tabular}{|c|c|}
\hline $\begin{array}{l}\text { SAMPLE } \\
\text { DATE }\end{array}$ & $6-2-3$ \\
\hline \multirow[t]{4}{*}{$\begin{array}{l}66 / 03 / 87 \\
66 / 03 / 87\end{array}$} & $\begin{array}{r}118000.00 \\
38.60\end{array}$ \\
\hline & \\
\hline & NR \\
\hline & NR \\
\hline
\end{tabular}

$06 / 03 / 87$

$08 / 03 / 87$

$68 / 03 / 87$

$66 / 63 / 87$

$06 / 63 / 87$

$68 / 63 / 87$

66/03/87

$06 / 03 / 87$

\subsection{0 \\ 364.00 \\ 5.00}

47468.60

12000.00

6480.06

20200.00

13.66

NR

NR

NR

$66 / 63 / 87$

28800.60

${ }_{7.26}$

$06 / 03 / 87$

7.26
NR

$66 / 03 / 87$

51800.00

$06 / 03 / 87$

163000.00

NR

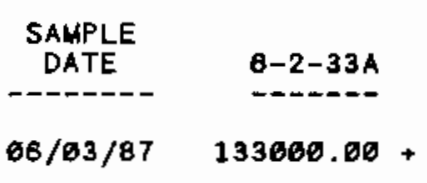

NR

NR

NR

$06 / 03 / 97$

$68 / 03 / 87$

5900.06

311.00

NR

NR

NR

NR

NR

NR

NR

$88 / 63 / 87$

3.69
$1.48\}$

NR

NR

NR

$06 / 63 / 87$

3230.66

NR

$68 / 63 / 87$

8.90

NR

$66 / 63 / 87$

$68 / 03 / 87$

32760.06

638.06

NR

- VAlUe exceeds pRIMARY dRINKINg hater standard

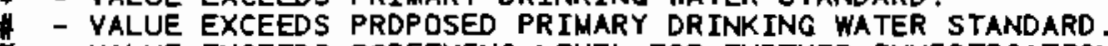

* - VALUE EXCEEDS SCREENING LEVEL FOR FURTHER INVESTIGATION.

+ - DETECTION LIMIT WAS NOT AVAILABLE FOR COMPARISON

NR - ANALYSIS NOT REQUESTED DR NOT YET REPORTED

VALUES IN $\{$ f ARE COUNTING ERRORS FOR RADIONUCLIDES

WATER STANDARD'(S) IN PARENTHESES ARE PROPOSED ONLY 
TABLE A.4. (contd)

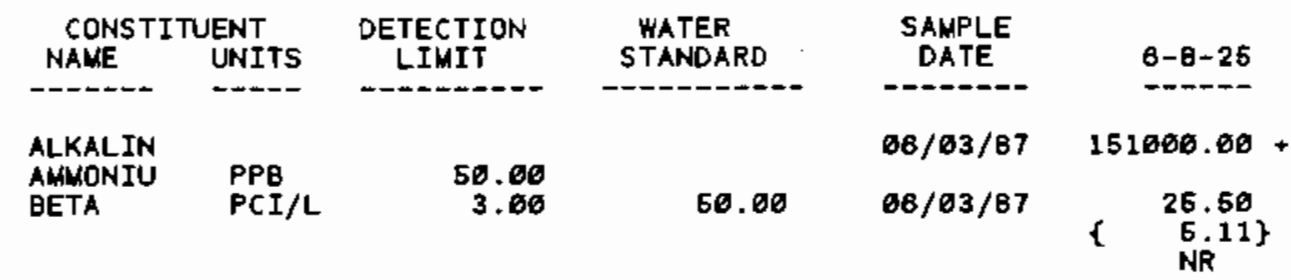

$\begin{array}{ll}\text { CHLORID } & \text { PPB } \\ \text { CONDFLD } & \text { UMHO } \\ \text { FARSENI } & \text { PPB } \\ \text { FBARIUM } & \text { PPB } \\ \text { FCALCIU } & \text { PPB } \\ \text { FLUORID } & \text { PPB } \\ \text { FNAGNES } & \text { PPB } \\ \text { FNANGAN } & \text { PPB } \\ \text { FPOTASS } & \text { PPB } \\ \text { FSODIUN } & \text { PPB } \\ \text { FVANADI } & \text { PPB } \\ \text { FZINC } & \text { PPB } \\ \text { HXDECAC } & \text { PPB } \\ \text { LOALPHA } & \text { PCI/ }\end{array}$

500.00
1.00
5.00
8.00
50.00
500.00
0.00
5.00
100.00
100.00
5.00
5.00
2.00

50.00
1000.00
1400.00
16.00
46000.00

0.10

NITRATE NONACD

$\stackrel{+}{t}$

$\begin{array}{lll}\text { SULFATE } & \text { PPB } & 500.00 \\ \text { TOC } & \text { PPB } & 200.00 \\ \text { TRITIUM } & P C I / L & 500.00\end{array}$

UNKNOWN
PPB
0.00
20000.00

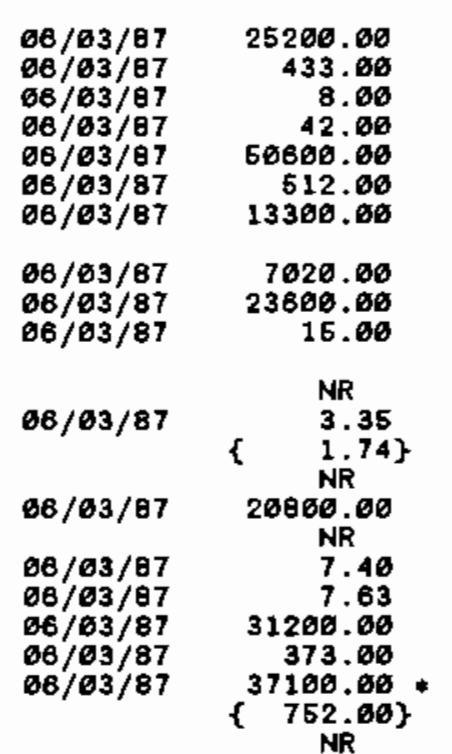

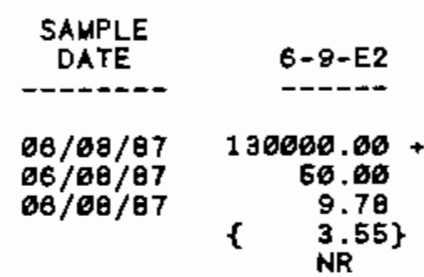

$06 / 06 / 87$

$06 / 08 / 87$

$06 / 08 / 87$

$06 / 08 / 87$

$68 / 08 / 87$

66/08/67

๑6/ø日/87

$66 / 06 / 87$

68/0日/97

B6/60/07

$06 / 00 / \theta 7$

66/06/97

NR
$68 / 88 / 87$

$06 / 80 / 87$

๑6/0日/07

$06 / 06 / 67$

$66 / 60 / 87$

4600.60

305.00

14.60
45.60

24900.00

544.00

10200.00

22.06

8050.00

23900.00

5.00

59.06 +

$23.00+$

1940.00

$36.00+$

7.48
7.67

29200.00

1700.00

68/06/87
NR

72.08

\section{SAMPLE} DATE

$06 / 02 / 87$

$06 / 02 / 87$

øe/02/87

06/02/07 $66 / 02 / 87$

$06 / 02 / 87$

$06 / 02 / 87$
$66 / 02 / \theta 7$

อ6/02/87

๒6/ $12 / 07$

$06 / 02 / 07$

$68 / 02 / 87$

$06 / 02 / 87$

$66 / 02 / 07$

$06 / 02 / 07$

68/02/07

06/02/07

$06 / 02 / 87$

06/02/87

$66 / 02 / 87$

$06 / 82 / 87$
6-15-26

147000.00 +

26.10
$\{\quad 5.20\}$

27.18
$\{\quad 3.76\}$ 9440.80 416.00 B. 00 50.00 49600.00 573.00

12960.00

6000.00 24800.60 17.00 9.60 NR

3.58
$1.75\}$ 23500 . 7.36 7.59

51300.00 386.00 60400.00 $\{933.00\}$ 64800.00. $\{974.80\}$

* - VALUE EXCEEDS PRIMARY DRINKING water STANDARD

- VALUE EXCEEDS PROPOSED PRIMARY DRINKING WATER STANDARD.

- VALUE EXCEEDS SCREENING LEVEL FOR FURTHER INVESTIGATION.

+ - DETECTION LIMIT WAS NOT AVAILABLE FOR COMPARISON

NR - ANALYSIS NOT REQUESTED OR NOT YET REPORTED

VALUES IN $\{$ f ARE COUNTING ERRORS FDR RADIONUCLIOES

WATER STANDARD(S) IN PARENTHESES ARE PROPOSED ONLY 
IABLE A.4. (contd)

\begin{tabular}{|c|c|c|c|c|c|c|c|c|c|}
\hline $\begin{array}{c}\text { CONSTI } \\
\text { NAME }\end{array}$ & $\begin{array}{l}\text { UENT } \\
\text { UNITS }\end{array}$ & $\begin{array}{l}\text { DETECTION } \\
\text { LIMIT }\end{array}$ & $\begin{array}{l}\text { WATER } \\
\text { STANDARD }\end{array}$ & $\begin{array}{c}\text { SAMPLE } \\
\text { DATE }\end{array}$ & $6-26-E 5 A$ & $\begin{array}{c}\text { SAMPLE } \\
\text { DATE }\end{array}$ & $6-2 \theta-2 \theta$ & $\begin{array}{l}\text { SAMPLE } \\
\text { DATE }\end{array}$ & $8-28-39$ \\
\hline $\begin{array}{l}\text { ALKALIN } \\
\text { GETA }\end{array}$ & $\mathrm{PCI} / \mathrm{L}$ & 3.06 & 50.00 & $\begin{array}{l}66 / 09 / 87 \\
66 / 69 / 87\end{array}$ & $\begin{array}{c}114666.606+ \\
26.26 \\
\{\quad 4.59\} \\
\text { NR }\end{array}$ & $\begin{array}{l}66 / 62 / 87 \\
66 / 62 / 87 \\
06 / 02 / 87\end{array}$ & $\begin{array}{c}111000.00 \\
44.00 \\
\{\quad 8.31\} \\
44.36 \\
4 \quad 4.809\end{array}$ & $\begin{array}{l}06 / 11 / 87 \\
06 / 11 / 07\end{array}$ & $\begin{array}{c}125006.66 \\
5.86 \\
\{\quad 3.86\} \\
\quad \mathrm{NR}\end{array}$ \\
\hline $\begin{array}{l}\text { CHLORIO } \\
\text { CONDFLD } \\
\text { FARSENI }\end{array}$ & $\begin{array}{l}\text { PPB } \\
\text { UMHO } \\
\text { PPB }\end{array}$ & $\begin{array}{r}500.00 \\
1.06 \\
5.00\end{array}$ & 50.00 & $\begin{array}{l}66 / 89 / 87 \\
66 / 69 / 87 \\
86 / 68 / 87\end{array}$ & $\begin{array}{r}8750.06 \\
337.00 \\
5.00\end{array}$ & $\begin{array}{l}08 / 02 / \theta 7 \\
\otimes B / \theta 2 / \theta 7 \\
\theta B / \oslash 2 / \theta 7\end{array}$ & $\begin{array}{r}14806.06 \\
386.06 \\
12.00\end{array}$ & $\begin{array}{l}66 / 11 / 87 \\
06 / 11 / 87\end{array}$ & $\begin{array}{r}4748.66 \\
270.06\end{array}$ \\
\hline $\begin{array}{l}\text { FBARIUM } \\
\text { FCALCIU }\end{array}$ & & $\begin{array}{r}8.06 \\
50.06 \\
50.08\end{array}$ & 1000.00 & $\begin{array}{l}06 / 108 / 87 \\
06 / 68 / 87\end{array}$ & $\begin{array}{r}58.00 \\
40900.00\end{array}$ & $\begin{array}{l}08 / 02 / 87 \\
08 / 02 / \theta 7\end{array}$ & $\begin{array}{r}68.00 \\
51300.00\end{array}$ & $\begin{array}{l}66 / 11 / 87 \\
66 / 11 / 87\end{array}$ & $\begin{array}{r}36.00 \\
36106.00 \\
84.90\end{array}$ \\
\hline $\begin{array}{l}\text { FLUDRID } \\
\text { FMAGNES } \\
\text { FMANGAN }\end{array}$ & $\begin{array}{l}\text { PPB } \\
\text { PP } \\
\text { PPE }\end{array}$ & $\begin{array}{r}506.00 \\
6.00 \\
5.00\end{array}$ & 1400.00 & $08 / 09 / 87$ & 18500.00 & $\begin{array}{l}66 / 02 / 87 \\
66 / 02 / 87\end{array}$ & $\begin{array}{r}804.00 \\
11106.00\end{array}$ & $\begin{array}{l}06 / 11 / 87 \\
06 / 11 / 87 \\
06 / 11 / 87\end{array}$ & $\begin{array}{r}585.00 \\
105 \boxminus \emptyset .06 \\
18.00\end{array}$ \\
\hline $\begin{array}{l}\text { FPOTASS } \\
\text { FSODIUU } \\
\text { FVANADI } \\
\text { FZINC }\end{array}$ & $\begin{array}{l}P P E \\
P P B \\
P P B\end{array}$ & $\begin{array}{r}100.00 \\
100.06 \\
5.00 \\
5.00\end{array}$ & & $\begin{array}{l}66 / 69 / 87 \\
68 / 69 / 87 \\
66 / 69 / 87\end{array}$ & $\begin{array}{r}5206.060 \\
12300.00 \\
18.06\end{array}$ & $\begin{array}{l}66 / 02 / 87 \\
66 / 02 / 87 \\
66 / 02 / 87 \\
06 / 02 / 87\end{array}$ & $\begin{array}{r}7520.06 \\
22100.06 \\
17.00 \\
6.80\end{array}$ & $\begin{array}{l}06 / 11 / 87 \\
06 / 11 / 87 \\
06 / 11 / 87\end{array}$ & $\begin{array}{r}48100.00 \\
16406.00 \\
6.00\end{array}$ \\
\hline LOALPHA & $\mathrm{PCI} / \mathrm{L}$ & 2.06 & 15.00 & & & $06 / 02 / 87$ & $\quad 2.59$ & $08 / 11 / 87$ & 2.77 \\
\hline $\begin{array}{l}\text { NITRATE } \\
\text { PHFIELD }\end{array}$ & PPB & $\begin{array}{r}600.00 \\
0.16\end{array}$ & $45 \boxminus \sigma \sigma . \sigma 6$ & $\begin{array}{l}66 / 99 / 87 \\
66 / 69 / 87 \\
66 / 19 / 87\end{array}$ & $\begin{array}{r}24800.00 \\
7.30 \\
7.56\end{array}$ & $\begin{array}{l}06 / 02 / 87 \\
06 / 02 / 87 \\
06 / 02 / 87\end{array}$ & $\begin{array}{r}38606.00 \\
7.60 \\
7.67\end{array}$ & $\begin{array}{l}06 / 11 / \theta 7 \\
08 / 11 / 87 \\
06 / 11 / 87\end{array}$ & $\begin{array}{r}447.66 \\
7.46 \\
7.72\end{array}$ \\
\hline $\begin{array}{l}\text { SULFATE } \\
\text { TOC }\end{array}$ & $\begin{array}{l}\text { PPB } \\
\text { PPB }\end{array}$ & $\begin{array}{l}600.60 \\
200.00\end{array}$ & & $\begin{array}{l}06 / 09 / 87 \\
06 / 68 / 87\end{array}$ & $\begin{array}{r}30500.00 \\
425.06\end{array}$ & $\begin{array}{l}66 / 02 / 87 \\
66 / \oplus 2 / 87\end{array}$ & $\begin{array}{r}57900.00 \\
591.00\end{array}$ & $\begin{array}{l}\theta 6 / 11 / 87 \\
\theta 6 / 11 / 87\end{array}$ & $\begin{array}{r}27200.06 \\
284.00\end{array}$ \\
\hline TRITIUM & $\mathrm{PC} \overline{\mathrm{I}} / \mathrm{L}$ & 500.00 & 20000.60 & $06 / 08 / 07$ & 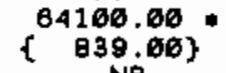 & $66 / 62 / 87$ & $\begin{array}{l}195000.60 \\
\{1460.60\}\end{array}$ & & \\
\hline $\mathrm{u}$ & PCI /L & 0.60 & 600.00 & & & $06 / 02 / 87$ & $3.61^{\circ}$ & & NR \\
\hline
\end{tabular}

* - VALUE EXCEEDS PRIMARY DRINKING WaTER STANDARD.

- VALUE EXCEEDS PROPOSED PRIMARY DRINKING WATER STANDARD.

* - VALUE EXCEEDS SCREENING LEVEl FOR FURTHER INVESTIGATION.

+ - DETECTION LIMIT WAS NOT AVAILABLE FOR COMPARISON

NR - ANALYSIS NOT REQUESTED OR NOT YET REPORTED

VALUES IN \{ \} ARE COUNTING ERRORS FOR RAOIONUCLIDES

WATER STANDARD (S) IN PARENTHESES ARE PROPOSED ONLY 
TABLE A.4. (contd)

\begin{tabular}{|c|c|c|c|c|c|c|c|c|c|}
\hline $\begin{array}{l}\text { CONSTI } \\
\text { NAME }\end{array}$ & $\begin{array}{l}\text { JENT } \\
\text { UNITS }\end{array}$ & $\begin{array}{l}\text { DETECTION } \\
\text { LIMIT }\end{array}$ & $\begin{array}{l}\text { WATER } \\
\text { STANDARD }\end{array}$ & $\begin{array}{l}\text { SAMPLE } \\
\text { DATE }\end{array}$ & $6-24-33$ & $\begin{array}{l}\text { SAMPLE } \\
\text { DATE }\end{array}$ & $6-28-40$ & $\begin{array}{l}\text { SAMPLE } \\
\text { DATE }\end{array}$ & $6-28-4$ \\
\hline $\begin{array}{l}1,1,1-T \\
\text { ALKALIN } \\
\text { BETA }\end{array}$ & $\begin{array}{l}\text { PPB } \\
\mathrm{PCI} / \mathrm{L}\end{array}$ & $\begin{array}{r}10.06 \\
3.06\end{array}$ & $\begin{array}{r}(200.0) \\
50.00\end{array}$ & $\begin{array}{l}\emptyset 5 / 14 / 87 \\
96 / 14 / 87 \\
05 / 14 / 87 \\
06 / 02 / 87\end{array}$ & $\begin{array}{c}17.00 \\
189000.00 \\
23.00 \\
\{\quad 4.08\} \\
\left\{\begin{array}{r}23.20 \\
\{\quad 3.65\}\end{array}+\right.\end{array}$ & & $\begin{array}{l}\text { NR } \\
\text { NR } \\
\text { NR }\end{array}$ & $\begin{array}{l}06 / 09 / 87 \\
06 / 09 / 87\end{array}$ & $\begin{array}{c}120000.00+ \\
37.60 \\
\{\quad 5.61\} \\
\text { NR }\end{array}$ \\
\hline $\begin{array}{l}\text { CHLORID } \\
\text { CONDFLD } \\
\text { FARSENI } \\
\text { FBARIUW } \\
\text { FCALCIU } \\
\text { FWAGNES } \\
\text { FPOTASS } \\
\text { FSODIUM } \\
\text { FVANADI } \\
\text { LOALPHA }\end{array}$ & $\begin{array}{l}\text { PPB } \\
\text { UNHO } \\
\text { PPB } \\
\text { PPB } \\
\text { PPE } \\
\text { PPB } \\
\text { PPE } \\
\text { PPB } \\
\text { PPB } \\
\text { PCI/L }\end{array}$ & $\begin{array}{r}500.00 \\
1.00 \\
5.00 \\
8.00 \\
50.00 \\
0.00 \\
100.00 \\
100.00 \\
5.00 \\
2.00\end{array}$ & 16.00 & $\begin{array}{l}06 / 14 / 87 \\
65 / 14 / 87 \\
\\
65 / 14 / 87 \\
65 / 14 / 87 \\
65 / 14 / 87 \\
65 / 14 / 87 \\
85 / 14 / 87 \\
05 / 14 / 87 \\
05 / 14 / 87\end{array}$ & $\begin{array}{r}7730.00 \\
442.00 \\
66.00 \\
63760.00 \\
15000.00 \\
7330.00 \\
24000.00 \\
16.00 \\
3.60 \\
1.91\}\end{array}$ & $85 / 12 / 87$ & $\begin{array}{c}\text { NR } \\
338.60 \\
\text { NR } \\
\text { NR } \\
\text { NR } \\
\text { NR } \\
\text { NR } \\
\text { NR } \\
\text { NR } \\
\text { NR }\end{array}$ & $\begin{array}{l}66 / 09 / 87 \\
66 / 69 / 87 \\
66 / 09 / 67 \\
66 / 69 / 67 \\
66 / 69 / 87 \\
66 / 69 / 87 \\
66 / 69 / 67 \\
66 / 69 / 87 \\
66 / 69 / 87 \\
06 / 69 / 67\end{array}$ & $\begin{array}{r}6890.00 \\
370.00 \\
6.00 \\
66.00 \\
46900.00 \\
16400.00 \\
5970.00 \\
14400.00 \\
13.00 \\
4.24 \\
1.61\}\end{array}$ \\
\hline $\begin{array}{l}\text { NITRATE } \\
\text { PHFIELD }\end{array}$ & PPB & $\begin{array}{r}560.00 \\
0.10\end{array}$ & 46000.00 & $\begin{array}{l}05 / 14 / 87 \\
65 / 14 / 87 \\
65 / 14 / 87\end{array}$ & $\begin{array}{r}26400.00 \\
6.98 \\
7.26\end{array}$ & $86 / 12 / 67$ & $\begin{array}{l}\text { NR } \\
7.60 \\
\text { NR }\end{array}$ & $\begin{array}{l}06 / 89 / 87 \\
66 / 09 / 87 \\
06 / 09 / 87\end{array}$ & $\begin{array}{r}36360.00 \\
7.30 \\
7.69\end{array}$ \\
\hline $\begin{array}{l}\text { SULFATE } \\
\text { TOC } \\
\text { TOXLDL } \\
\text { TRITIUM }\end{array}$ & $\begin{array}{l}\text { PPB } \\
\text { PPE } \\
\text { PPB } \\
\text { PCI/L }\end{array}$ & $\begin{array}{r}500.00 \\
200.00 \\
20.00 \\
500.00\end{array}$ & 20000.00 & $\begin{array}{l}65 / 14 / 87 \\
66 / 14 / 87 \\
65 / 14 / 87 \\
65 / 14 / 87\end{array}$ & 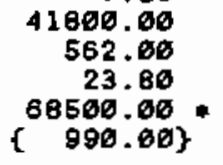 & $65 / 12 / 67$ & $\begin{array}{c}\text { NR } \\
N R \\
N R \\
11200.00 \\
479.00\}\end{array}$ & $\begin{array}{l}66 / 09 / 87 \\
66 / 09 / 87 \\
66 / 09 / 87\end{array}$ & $\begin{array}{c}32000.00 \\
411.00 \\
135000.00 \\
\{1210.00\}\end{array}$ \\
\hline
\end{tabular}

* - VALUE EXCEEDS PRIMARY DRINKING WATER STANDARD.

F - VALUE EXCEEDS PROPOSED PRIMARY DRINKING HATER STANDARD.

* - VALUE EXCEEDS SCREENING LEVEL FOR FURTHER INVESTIGATION.

- - DETECTION LIMIT WAS NOT AVAILABLE FOR COMPARISON

NR - ANALYSIS NOT REQUESTED DR NOT YET REPORTED

VALUES IN \{ $\}$ ARE COUNTING ERRORS FOR RADIONUCLIOES

HATER STANDARD (S) IN PARENTHESES ARE PROPOSED ONLY 
TABLE A.4. (contd)

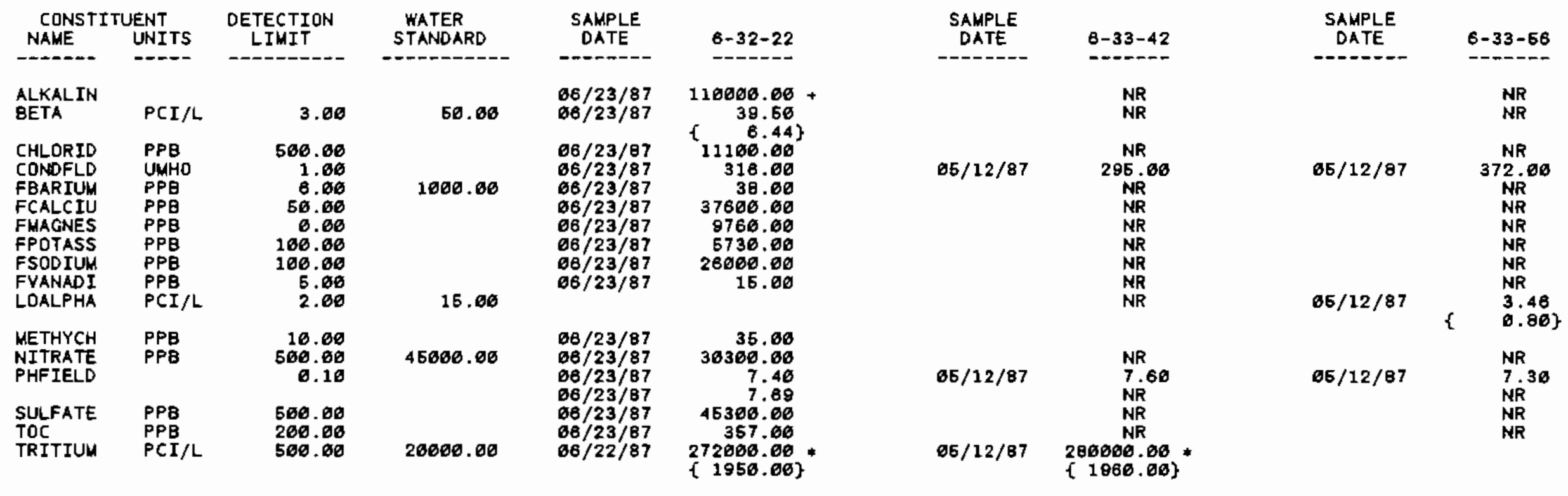

- - VALUE EXCEEDS PRIMARY DRINKING WATER STANDARD.

- VALUE EXCEEDS PROPOSED PRIMARY DRINKING WATER STANDARD.

* VALUE EXCEEDS SCREENING LEVEL FOR FURTHER INYESTIGATION.

+ - DETECTION LIMIT WAS NOT AVAILABLE FOR COMPARISON

NR - ANALYSIS NOT REQUESTED OR NOT YET REPORTED

VALUES IN \& \% ARE COUNT ING ERRORS FOR RADIONUCLIDES

WATER STANDARD(S) IN PARENTHESES ARE PROPOSEO ONLY 
TABLE A.4. (contd)

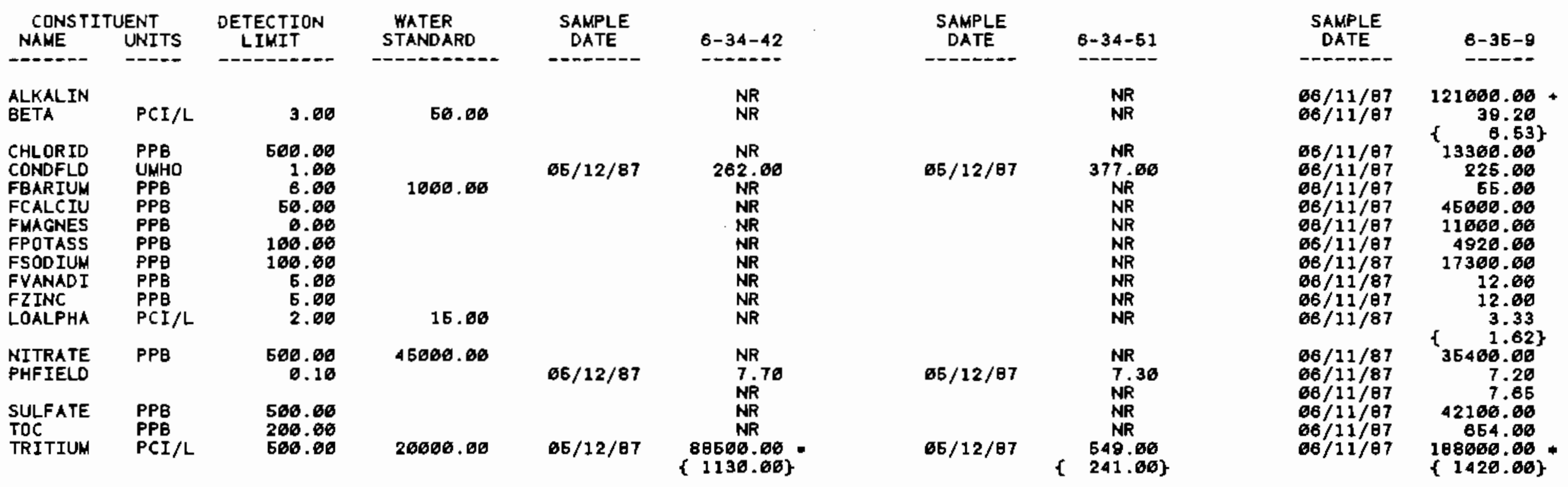


TABLE A.4. (contd)

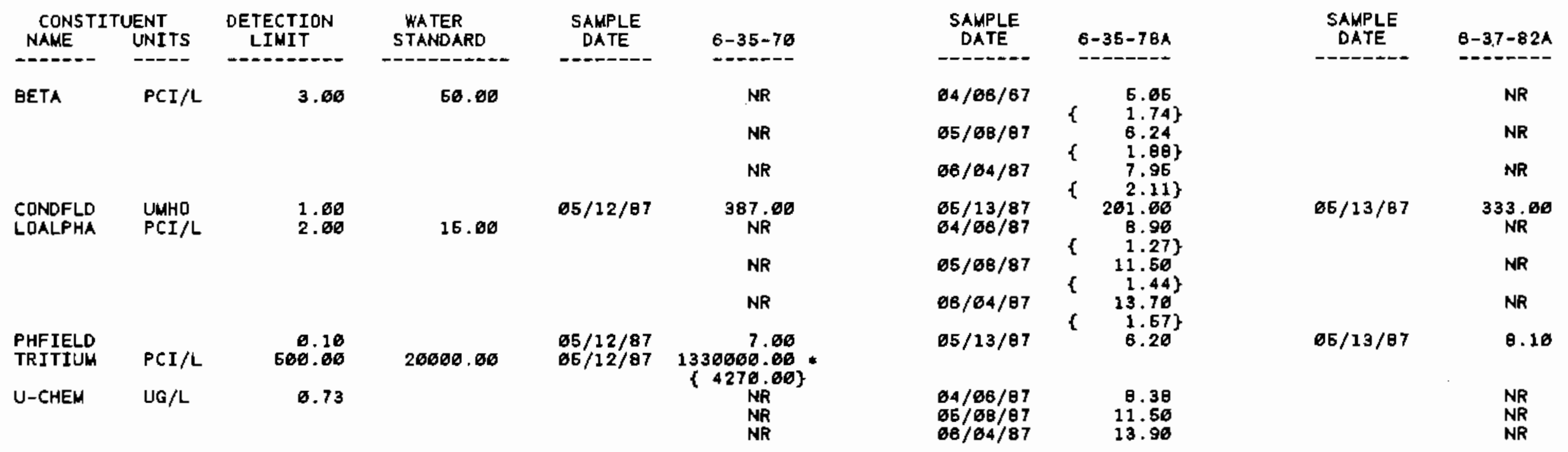

i

* - VALUE EXCEEDS PRIMARY DRINKINg wateR STANDARD.

* - VALUE EXCEEDS PROPOSED PRIMARY DRINKING WATER STANDARD.

* - VALUE EXCEEDS SCREENING LEVEL FOR FURTHER INVESTIIGATION.

NR - DETECTION LIMIT WAS NOT AVAILABLE FOR COMPARISON

NR - ANALYSIS NOT REQUESTED OR NOT YET REPORTED

$\checkmark A L U E S$ IN \{\} ARE COUNTING ERRORS FOR RADIONUCLIDES

WATER STANDARD'S) IN PARENTHESES ARE PROPOSED ONLY 
IABLE A.4. (contd)

\begin{tabular}{|c|c|c|c|c|c|}
\hline \multicolumn{2}{|c|}{$\begin{array}{l}\text { CONSTITUENT } \\
\text { NAME }\end{array}$} & $\begin{array}{c}\text { DETECTION } \\
\text { LIMIT }\end{array}$ & $\begin{array}{l}\text { WATER } \\
\text { STANDARD }\end{array}$ & $\begin{array}{l}\text { SAMPLE } \\
\text { DATE }\end{array}$ & $6-38-85$ \\
\hline$-\cdots$ & --- & - & - - - & $------\infty$ & ------- \\
\hline $\begin{array}{l}\text { ALKALIN } \\
\text { BETA }\end{array}$ & $\mathrm{PCI} / \mathrm{L}$ & 3.00 & 50.00 & $\begin{array}{l}65 / 13 / 87 \\
65 / 13 / 87\end{array}$ & $\begin{array}{c}129000.00 \\
18.90\end{array}$ \\
\hline $\begin{array}{l}\text { CHLORID } \\
\text { CONDFLD } \\
\text { FBARIUM } \\
\text { FCALCIU }\end{array}$ & $\begin{array}{l}\text { PPB } \\
\text { UMHO } \\
\text { PPB } \\
\text { PPB }\end{array}$ & $\begin{array}{r}500.00 \\
1.00 \\
8.00 \\
50.00\end{array}$ & 1000.00 & $\begin{array}{l}05 / 13 / \theta 7 \\
05 / 13 / 87 \\
05 / 13 / 87 \\
65 / 13 / \theta 7\end{array}$ & $\begin{array}{r}8810.00 \\
594.00 \\
82.00 \\
66500.00\end{array}$ \\
\hline $\begin{array}{l}\text { FUAGNES } \\
\text { FPOTASS } \\
\text { FSODIUM } \\
\text { FSTRONT } \\
\text { FYANADI } \\
\text { F ZINC } \\
\text { LOALPHA }\end{array}$ & $\begin{array}{l}\text { PPB } \\
\text { PPB } \\
\text { PPB } \\
\text { PPB } \\
\text { PPB } \\
\text { PPB } \\
\text { PCI/L }\end{array}$ & $\begin{array}{r}0.00 \\
100.00 \\
100.00 \\
300.00 \\
5.00 \\
5.00 \\
2.00\end{array}$ & 15.00 & $\begin{array}{l}05 / 13 / 87 \\
65 / 13 / 87 \\
65 / 13 / 87 \\
05 / 13 / 87 \\
05 / 13 / 87 \\
05 / 13 / 87\end{array}$ & $\begin{array}{r}20600.00 \\
8460.00 \\
25100.00 \\
311.00 \\
29.00 \\
0.00\end{array}$ \\
\hline NITRATE & PPB & 500.00 & 45000.00 & $66 / 13 / 87$ & $147000.00 \%$ \\
\hline PHFIELD & & 0.10 & & $\begin{array}{l}05 / 13 / 87 \\
05 / 13 / 87\end{array}$ & 7.50 \\
\hline SULFATE & $\begin{array}{l}P P B \\
P P B\end{array}$ & 500.00 & $5,8)$ & $05 / 13 / 87$ & 28100.00 \\
\hline $\begin{array}{l}\text { TOC } \\
\text { TOXLDL } \\
\text { TRITIUN }\end{array}$ & $\begin{array}{l}\text { PPB } \\
\text { PPB } \\
\text { PCI/L }\end{array}$ & $\begin{array}{r}200.00 \\
20.00 \\
500.00\end{array}$ & 20000.00 & $\begin{array}{l}65 / 13 / 87 \\
65 / 13 / 97 \\
65 / 13 / 87\end{array}$ & $\begin{array}{r}1320.00 \\
28.20 \\
344800.00 \\
\{2100.00\}\end{array}$ \\
\hline
\end{tabular}

\begin{tabular}{|c|c|}
\hline $\begin{array}{l}\text { SAMPLE } \\
\text { DATE }\end{array}$ & $6-39-79$ \\
\hline & - \\
\hline $\begin{array}{l}05 / 14 / 87 \\
05 / 14 / 87\end{array}$ & $\begin{array}{r}97606.00 \\
11.00\end{array}$ \\
\hline $\begin{array}{l}06 / 14 / 97 \\
06 / 14 / \theta 7 \\
06 / 14 / \theta 7 \\
65 / 14 / \theta 7\end{array}$ & $\begin{array}{r}2490.00 \\
199.00 \\
19.00 \\
19800.00\end{array}$ \\
\hline $\begin{array}{l}05 / 14 / 87 \\
05 / 14 / 87 \\
05 / 14 / 87\end{array}$ & $\begin{array}{r}5340.00 \\
2830.00 \\
21600.06\end{array}$ \\
\hline $06 / 14 / 87$ & 17.06 \\
\hline $66 / 14 / 87$ & 5.95 \\
\hline $05 / 14 / 87$ & 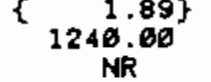 \\
\hline $\begin{array}{l}05 / 14 / 87 \\
65 / 14 / 87 \\
05 / 14 / 97 \\
65 / 14 / 97 \\
66 / 14 / 67 \\
06 / 14 / 67\end{array}$ & $\begin{array}{r}9.20 \\
7.02 \\
14100.00 \\
112.00 \\
457.00 \\
81.40\end{array}$ \\
\hline
\end{tabular}

\begin{tabular}{|c|c|}
\hline $\begin{array}{l}\text { SAMPLE } \\
\text { DATE }\end{array}$ & $6-40-1$ \\
\hline --ー-ー-ー & 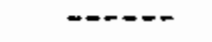 \\
\hline $\begin{array}{l}06 / 09 / 87 \\
06 / 69 / 87\end{array}$ & $\begin{array}{r}122000.000+ \\
84.90 *\end{array}$ \\
\hline $\begin{array}{l}66 / 09 / 87 \\
66 / 08 / 87 \\
66 / 69 / 87 \\
66 / 69 / 87 \\
66 / 69 / 87 \\
66 / 89 / 87 \\
68 / 69 / 87 \\
66 / 69 / 87 \\
66 / 69 / 87 \\
66 / 89 / 87\end{array}$ & $\begin{array}{r}11400.00 \\
379.00 \\
67.00 \\
43000.00 \\
510.00 \\
12900.00 \\
5640.00 \\
19000.00 \\
303.00 \\
14.00\end{array}$ \\
\hline $06 / 08 / 87$ & 4.65 \\
\hline $\begin{array}{l}66 / 68 / 87 \\
66 / 09 / 87 \\
66 / 89 / 87 \\
68 / 69 / 87 \\
66 / 69 / 87\end{array}$ & $\begin{array}{r}1.70\} \\
40100.00 \\
39200.00 \\
6.90 \\
7.72 \\
38200.00\end{array}$ \\
\hline $06 / 0 \theta / \theta 7$ & 774.60 \\
\hline $06 / 09 / 87$ & $\begin{array}{l}238000.00 \\
\{1836.00\}\end{array}$ \\
\hline
\end{tabular}

- - VALUE EXCEEDS PRIMARY DRINKING WATER STANDARD.

- VALUE EXCEEDS PROPOSED PRIMARY DRINKING WATER STANDARD.

- VALUE EXCEEDS SCREENING LEVEL FOR FURTHER INVESTIGATION.

+ - DETECTION LIMIT WAS NOT AVAILABLE FOR COMPARISON

NR - ANALYSIS NOT REQUESTED OR NOT YET REPORTED

VALUES IN $\{$ A ARE COUNTING ERRORS FOR RADIONUCLIDES

WATER STANDARD(S) IN PARENTHESES ARE PROPOSED ONLY 
TABLE A.4. (contd)

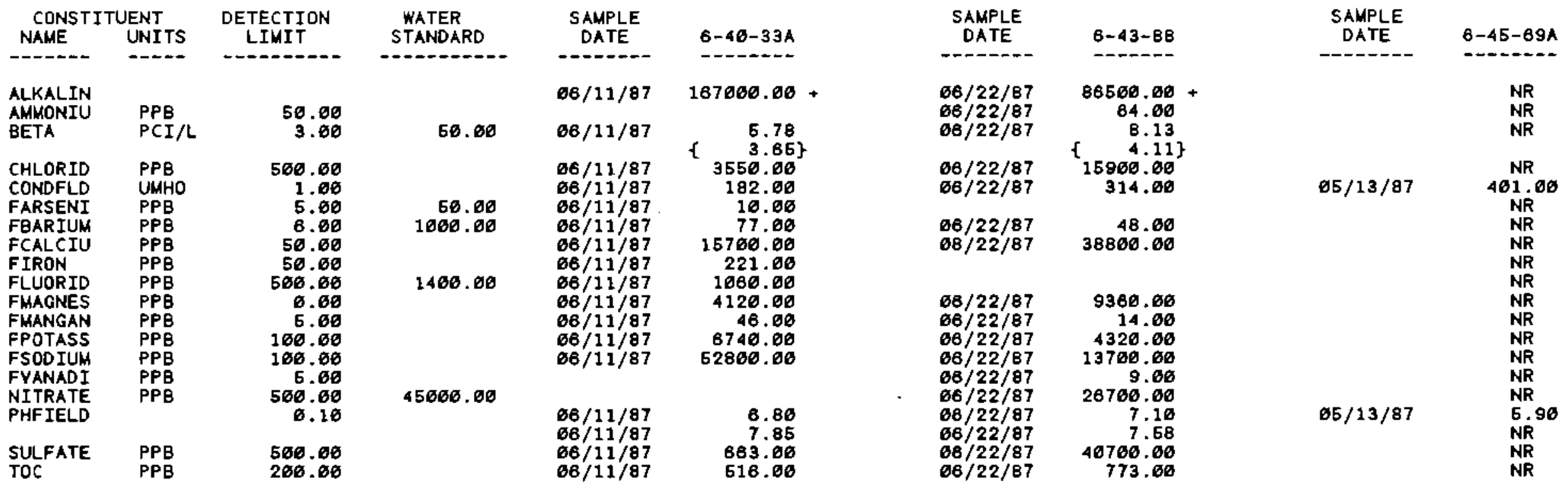

$\stackrel{+}{\infty}$

* - VALUE EXCEEDS PRIMARY DRINKING WATER STANDARD.

* - VALUE EXCEEDS PROPOSED PRIMARY DR INKING WATER STANDARD.

* - VALUE EXCEEDS SCREENING LEVEL FOR FURTHER INVESTIGATION.

+ - DETECTION LIMIT WAS NOT AYAILABLE FOR COMPARISON

NR - ANALYSIS NOT REQUESTED OR NOT YET REPORTED

VALUES IN $f\}$ ARE COUNTING ERRORS FOR RADIONUCLIDES

WATER STANDARD (S) IN PARENTHESES ARE PROPOSED ONLY 
TABLE A.4. (contd)

\begin{tabular}{|c|c|c|c|c|c|}
\hline \multicolumn{2}{|c|}{ CONSTITUENT } & $\begin{array}{l}\text { DETECTION } \\
\text { LINIT }\end{array}$ & $\begin{array}{l}\text { WATER } \\
\text { STANDARD }\end{array}$ & $\begin{array}{l}\text { SANPLE } \\
\text { DATE }\end{array}$ & $6-48-7$ \\
\hline --ー---- & ---- & $--------\infty$ & $\cdots+----\infty$ & $----\infty$ & ----- \\
\hline $\begin{array}{l}\text { ALKALIN } \\
\text { AUMONIU } \\
\text { BETA }\end{array}$ & $\begin{array}{l}\text { PPB } \\
\text { PCI/L }\end{array}$ & $\begin{array}{r}50.00 \\
3.00\end{array}$ & 56.00 & $\begin{array}{l}06 / 23 / 87 \\
06 / 23 / 87\end{array}$ & $\begin{array}{r}87100.00 \\
93.00\end{array}$ \\
\hline $\begin{array}{l}\text { CHLORIO } \\
\text { CONDFLD } \\
\text { FBARIUN }\end{array}$ & $\begin{array}{l}\text { PPE } \\
\text { UNHO } \\
\text { PPB }\end{array}$ & $\begin{array}{r}500.00 \\
1.00 \\
6.00\end{array}$ & 1000.00 & $\begin{array}{l}06 / 23 / 87 \\
66 / 23 / 87 \\
06 / 23 / 87\end{array}$ & $\begin{array}{r}3410.00 \\
181.00 \\
29.00\end{array}$ \\
\hline $\begin{array}{l}\text { FCALCIU } \\
\text { FIRON } \\
\text { FMAGNES } \\
\text { FPOTASS } \\
\text { FSODIUN }\end{array}$ & $\begin{array}{l}\text { PPB } \\
\text { PPE } \\
\text { PPB } \\
\text { PPB } \\
\text { PPB }\end{array}$ & $\begin{array}{r}50.00 \\
50.00 \\
0.00 \\
100.00 \\
100.00\end{array}$ & & $\begin{array}{l}06 / 23 / 87 \\
66 / 23 / 87 \\
68 / 23 / 87 \\
66 / 23 / 87 \\
06 / 23 / 87\end{array}$ & $\begin{array}{r}27200.00 \\
53.00 \\
7180.00 \\
1856.00 \\
8810.00\end{array}$ \\
\hline $\begin{array}{l}\text { FVANADI } \\
\text { FZINC } \\
\text { LOALPHA }\end{array}$ & $\begin{array}{l}\text { PPB } \\
\text { PPB } \\
\text { PCI/L }\end{array}$ & $\begin{array}{r}5.00 \\
5.00 \\
2.00\end{array}$ & 15.00 & $06 / 23 / 87$ & 0.00 \\
\hline $\begin{array}{l}\text { NITRATE } \\
\text { PHFIELD }\end{array}$ & PPB & $\begin{array}{r}506.00 \\
0.10\end{array}$ & 46000.00 & $\begin{array}{l}06 / 23 / 97 \\
06 / 23 / 97\end{array}$ & $\begin{array}{r}4596.00 \\
7.00 \\
7.39\end{array}$ \\
\hline $\begin{array}{l}\text { SULFATE } \\
\text { TOC }\end{array}$ & $\begin{array}{l}\text { PPB } \\
\text { PPB }\end{array}$ & $\begin{array}{l}500.00 \\
260.00\end{array}$ & & $\begin{array}{l}86 / 23 / 87 \\
66 / 23 / 87\end{array}$ & $\begin{array}{r}20000.00 \\
2120.00\end{array}$ \\
\hline
\end{tabular}

\begin{tabular}{|c|c|}
\hline $\begin{array}{l}\text { SAMPLE } \\
\text { DATE }\end{array}$ & $6-48-18$ \\
\hline $66 / 12 / 87$ & 121060.60 \\
\hline $66 / 12 / 87$ & $\begin{array}{r}8.83 \\
\{\quad 3.80\}\end{array}$ \\
\hline $\begin{array}{l}66 / 12 / 97 \\
66 / 12 / 87 \\
66 / 12 / 87 \\
66 / 12 / 87 \\
66 / 12 / 87\end{array}$ & $\begin{array}{r}16200.00 \\
309.00 \\
30.00 \\
2.00 \\
46860.00\end{array}$ \\
\hline $\begin{array}{l}08 / 12 / 87 \\
66 / 12 / 67 \\
66 / 12 / 87 \\
06 / 12 / 87 \\
68 / 12 / 87 \\
66 / 12 / 87 \\
08 / 12 / 87\end{array}$ & $\begin{array}{r}14000.00 \\
8350.00 \\
15000.00 \\
301.00 \\
17.06 \\
19.60 \\
2.25\end{array}$ \\
\hline $\begin{array}{l}66 / 12 / 87 \\
66 / 12 / 87 \\
66 / 12 / 87 \\
06 / 12 / 87\end{array}$ & $\begin{array}{r}6000.00 \\
7.20 \\
7.67 \\
54300.00\end{array}$ \\
\hline
\end{tabular}

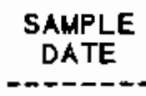

$66 / 23 / 87$

$06 / 23 / 87$

$08 / 23 / 87$
$08 / 23 / 87$

$\$ 6 / 23 / 87$

$66 / 23 / 87$

$06 / 23 / 87$

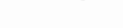

b8/23/87

$68 / 23 / 87$

$86 / 23 / 97$

06723787

$68 / 23 / 87$
6-48-71

104000.00

20.76
$\{\quad 5.16\}$

6930.06

258.60

28.00

$32600.6 B$

12900.00

10900.00

27.66

24100.00

7.30

7.69
28700.60

301.00

is

- - Value exceeds primary dRINKINg hater standaro.

- VALUE EXCEEDS PROPOSED PRIMARY DRINKING WATER STANDARO.

* - VALUE EXCEEDS SCREENING LEVEL FOR FURTHER INVESTIGATION.

- - DETECTION LINIT WAS NOT AVAILABLE FOR COMPARISON

NR - ANALYSIS NOT REQUESTED OR NOT YET REPORTED

VALUES IN \{ $\}$ ARE COUNTING ERRORS FOR RADIONUCLIDES

WATER STANDARO(S) IN PARENTHESES ARE PROPOSED ONLY 
TABLE A.4. (contd)

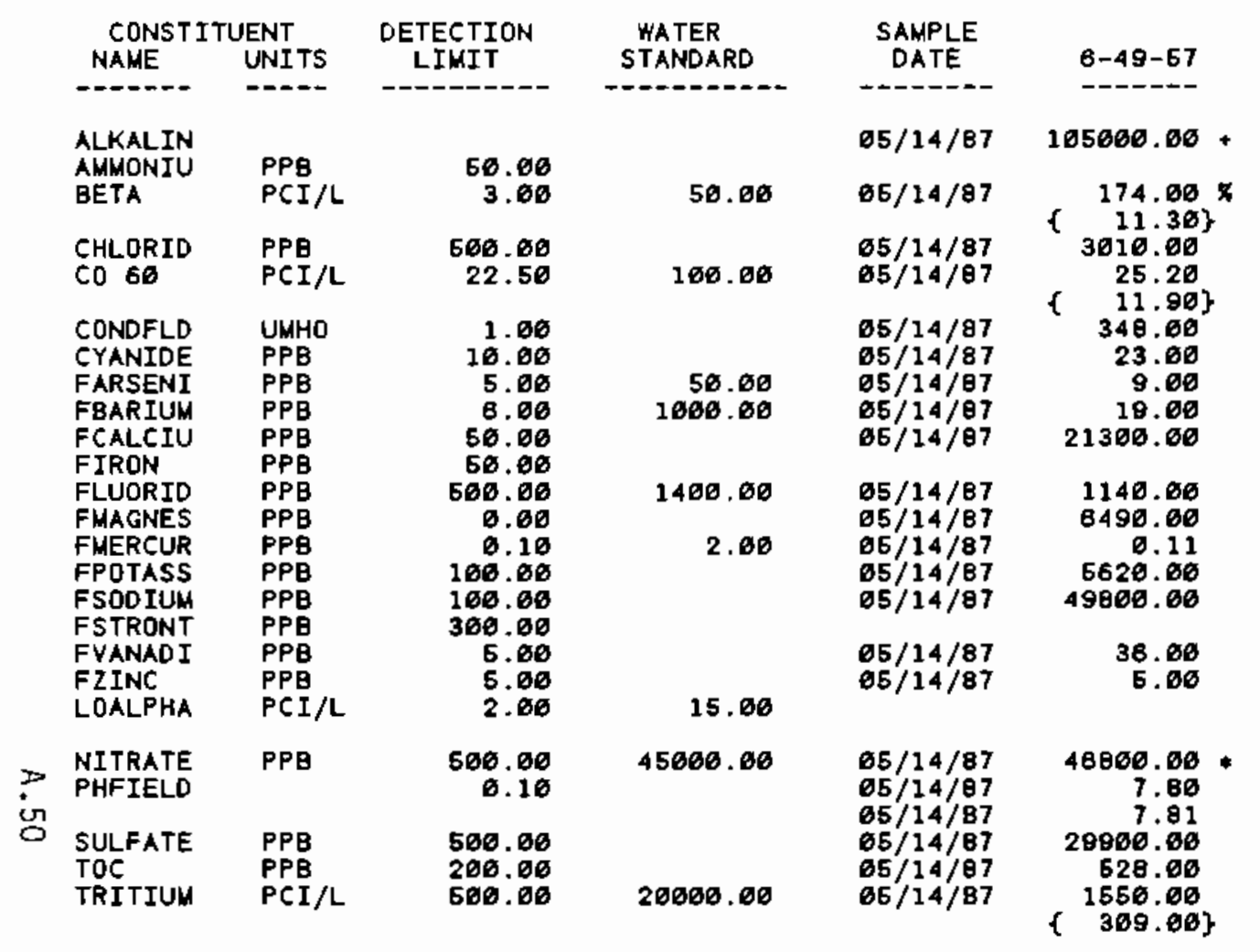

\begin{tabular}{|c|c|}
\hline $\begin{array}{c}\text { SAMPLE } \\
\text { DATE }\end{array}$ & $6-49-79$ \\
\hline $86 / 18 / 87$ & $100000.00+$ \\
\hline $65 / 18 / 87$ & 7.64 \\
\hline $06 / 18 / 87$ & ${ }_{\mathrm{NR}}^{8050}$ \\
\hline $65 / 18 / 87$ & ${ }_{\mathrm{NR}}^{400.00}$ \\
\hline $\begin{array}{l}05 / 18 / 87 \\
05 / 18 / 87\end{array}$ & $\begin{array}{r}2 \theta .00 \\
43600.00\end{array}$ \\
\hline $05 / 18 / 87$ & 14400.00 \\
\hline $\begin{array}{l}05 / 18 / 87 \\
05 / 18 / 87\end{array}$ & $\begin{array}{l}3840.00 \\
8930.00\end{array}$ \\
\hline $\begin{array}{l}85 / 18 / 87 \\
65 / 10 / 87\end{array}$ & $\begin{array}{r}24.00 \\
5.00\end{array}$ \\
\hline $\begin{array}{l}65 / 18 / 87 \\
65 / 18 / 87 \\
65 / 18 / 87 \\
85 / 18 / 87 \\
65 / 18 / 87\end{array}$ & $\begin{array}{r}42700.00 \\
8.00 \\
7.96 \\
45200.00 \\
213.00\end{array}$ \\
\hline
\end{tabular}

SAMPLE DATE $6-68-53$

$65 / 15 / 87$ $65 / 15 / 87$ 85/15/87

$65 / 15 / 87$ $65 / 15 / 87$ $05 / 16 / 87$
$05 / 15 / 87$ อ5/15/87 $05 / 15 / 87$
$05 / 15 / 87$ $05 / 15 / 87$ $05 / 15 / 87$ 05/16/87 D6/15/87 05/16/97 อ6/16/87 66/16/87

65/16/87 $65 / 16 / 87$
$06 / 16 / 87$ $66 / 16 / 87$ $05 / 16 / 87$ $65 / 16 / 87$
$06 / 16 / 87$ 66000.00 + 72.00 $1240.00 \times$ ( 32.30$)$ 40100.00 275.00
$33.46\}$ $\left\{\begin{array}{r}33.46\} \\ 1349.08\end{array}\right.$ 1349.60
460.00 58.60 173000.00 167.00 49400.00 12300.00 51300.00 737.00 14.00 12.00 $\left\{\begin{array}{c}2.18 \\ 1.94\}\end{array}\right.$ 346000.06 7.88 386000.00 727.00 $\left\{\begin{array}{c}1400.00 \\ 306.00\end{array}\right.$

* - VALUE EXCEEDS PRIMARY DRINKING WATER STANDARD.

* VALUE EXCEEDS PROPDSED PRIMARY DRINKING WATER STANDARD.

* - VALUE EXCEEDS SCREENING LEVEL FOR FURTHER INVESTIGATION.

+ - DETECTION LIMIT WAS NDT AVAILABLE FOR COMPARISON

NR - ANALYSIS NOT REQUESTED OR NOT YET REPORTED

VALUES IN \& $\}$ ARE COUNTING ERRORS FOR RADIONUCLIDES

WATER STANDARD(S) IN PARENTHESES ARE PROPOSEO ONLY 
TABLE A.4. (contd)

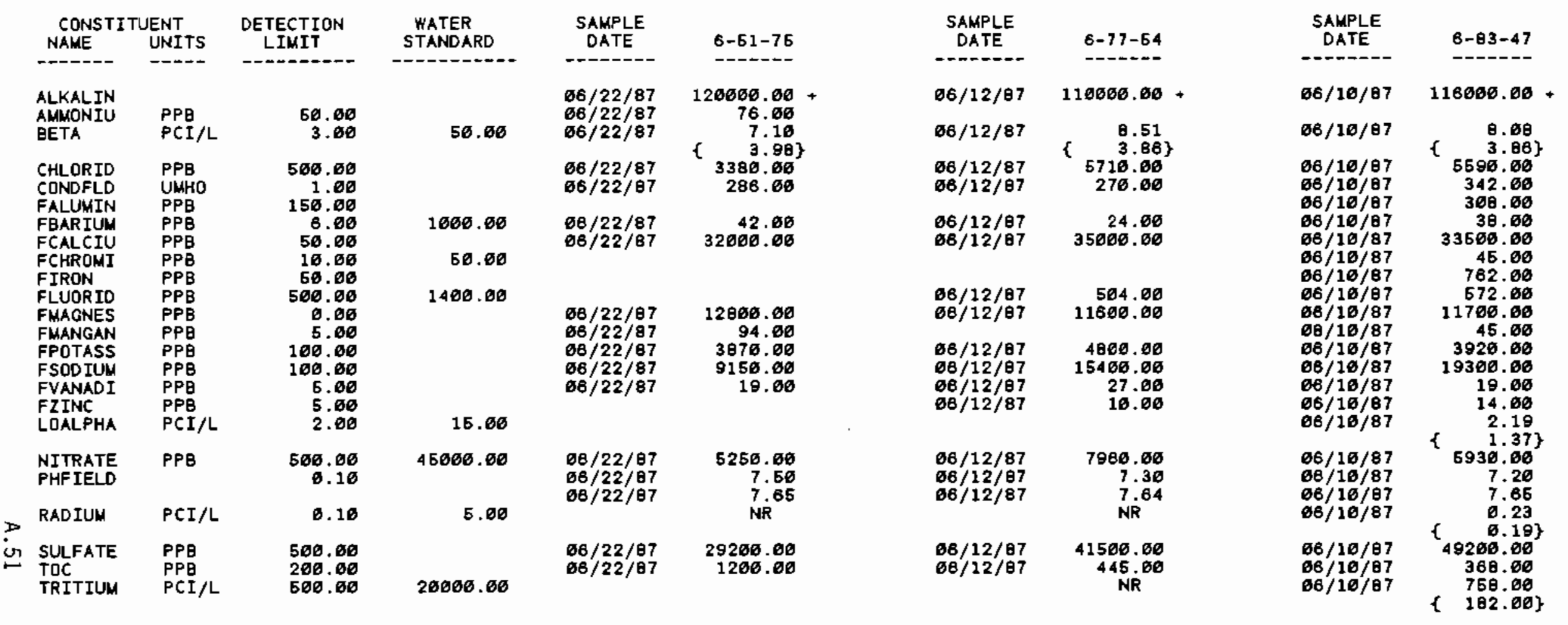

* - VALUE EXCEEDS PRIMARY DRINKING WATER STANDARD.

* - VALUE EXCEEDS PROPOSED PRIMARY DRINKING WATER STANDARD.

* - VALUE EXCEEDS SCREENING LEVEL FOR FURTHER INVESTIGATION.

+ - DETECTION LIMIT WAS NOT AVAILABLE FOR COMPARISON

NR - ANALYSIS NOT REQUESTED OR NOT YET REPORTED

VALUES IN \{ $\}$ ARE COUNTING ERRORS FDR RADIONUCLIDES

WATER STANOARD (5) IN PARENTHESES ARE PROPOSED ONLY 
TABLE A.4. (contd)

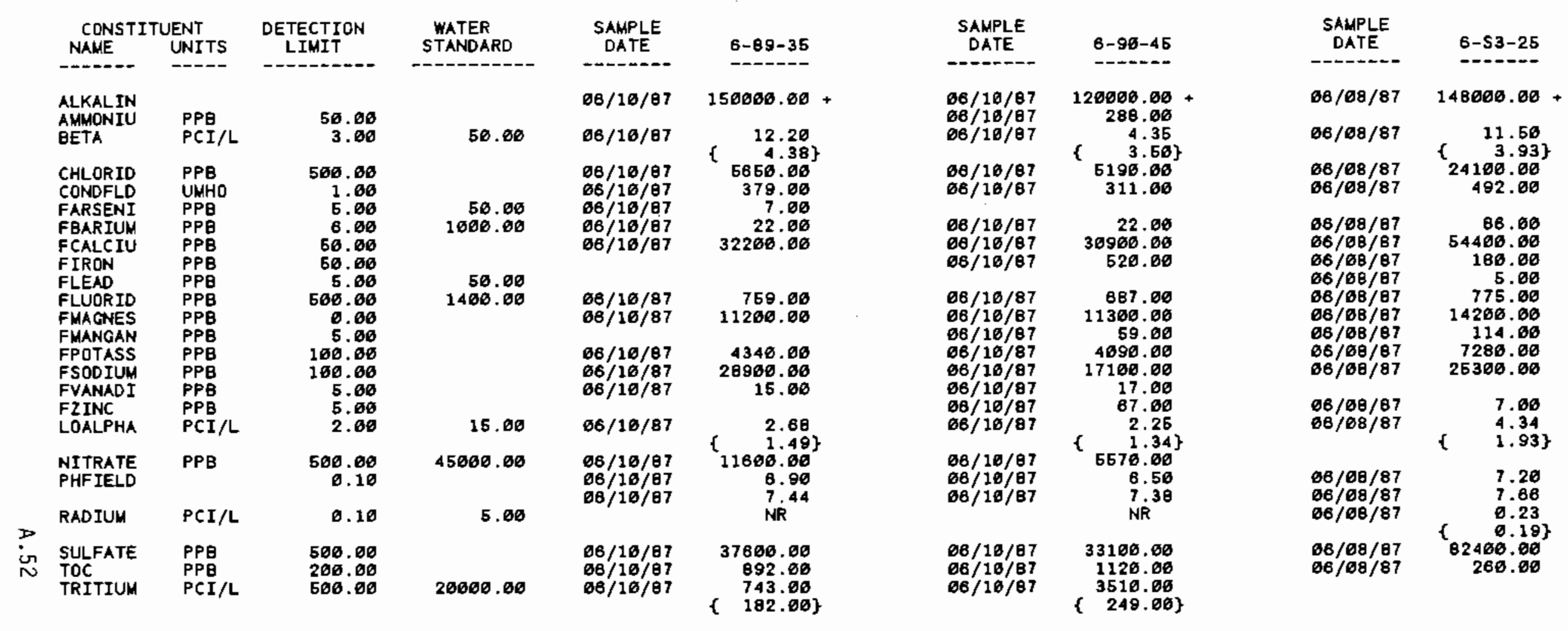

* - value exceeds primary drinking water standard.

* - VALUE EXCEEDS PROPOSEd PRIMARY dRINKING WaTER STANDARD.

* - VALUE EXCEEDS SCREENING LEVEL FOR FURTHER INVESTIGATION.

+ - DETECTION LIMIT WAS NOT AVAILAQLE FOR COMPARISON

NR - ANALYSIS NOT REQUESTED OR NOT YET REPORTED

VALUES IN \{\} ARE COUNTING ERRORS FOR RADIONUCLIDES

HATER STANDARD'(S) IN PARENTHESES ARE PROPOSED ONLY 
TABLE A.4. (cont d)

\begin{tabular}{|c|c|c|c|c|c|c|c|c|c|}
\hline $\begin{array}{l}\text { CONSTI } \\
\text { NAME } \\
-\end{array}$ & $\begin{array}{l}\text { JENT } \\
\text { UNITS }\end{array}$ & $\begin{array}{l}\text { DETECTION } \\
\text { LIMIT }\end{array}$ & $\begin{array}{l}\text { WATER } \\
\text { STANDARD }\end{array}$ & $\begin{array}{l}\text { SAMPLE } \\
\text { DATE }\end{array}$ & 6-S6E14A & $\begin{array}{l}\text { SAMPLE } \\
\text { DATE }\end{array}$ & 6-\$8-19 & $\begin{array}{l}\text { SAMPLE } \\
\text { DATE }\end{array}$ & $\begin{array}{l}6-\$ 12-3 \\
- \\
-\end{array}$ \\
\hline $\begin{array}{l}\text { ALKALIN } \\
\text { BETA } \\
\text { CHLORID } \\
\text { CONDFLD } \\
\text { FARSENI } \\
\text { FBARIUM } \\
\text { FCALCIU } \\
\text { FLUDRID } \\
\text { FMAGNES } \\
\text { FMANGAN } \\
\text { FPOTASS } \\
\text { FSELENI } \\
\text { FSODIUM } \\
\text { FVANADI } \\
\text { FZINC } \\
\text { LOALPHA } \\
\text { NITRATE } \\
\text { PHFIELD } \\
\text { SULFATE } \\
\text { TOC }\end{array}$ & $\begin{array}{l}\text { PCI/L } \\
\text { PPB } \\
\text { UMHO } \\
\text { PPB } \\
\text { PPB } \\
\text { PPB } \\
\text { PPB } \\
\text { PPB } \\
\text { PPB } \\
\text { PPB } \\
\text { PPB } \\
\text { PPB } \\
\text { PPB } \\
\text { PPB } \\
\text { PCI/L } \\
\text { PPB } \\
\text { PPB } \\
\text { PPB }\end{array}$ & $\begin{array}{r}3.00 \\
500.00 \\
1.00 \\
5.00 \\
8.00 \\
50.00 \\
500.00 \\
0.00 \\
5.00 \\
100.00 \\
5.00 \\
100.00 \\
5.00 \\
5.00 \\
2.00 \\
500.00 \\
0.10\end{array}$ & $\begin{array}{r}50.60 \\
1000.00 \\
1400.00 \\
16.00 \\
16.00 \\
46000.00\end{array}$ & 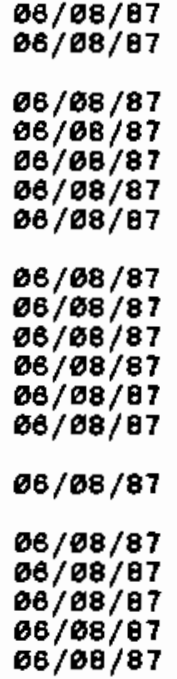 & 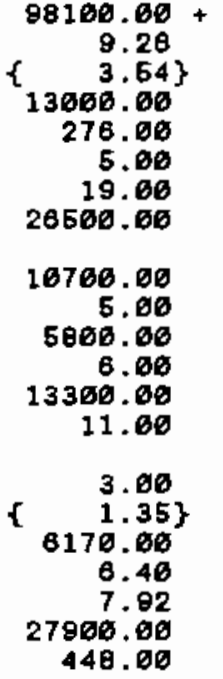 & 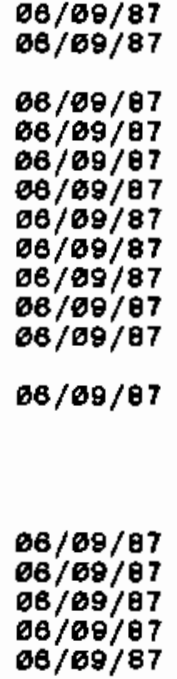 & $\begin{array}{c}163000.00 \\
11.10 \\
3.03\} \\
22000.00 \\
393.00 \\
10.00 \\
62.06 \\
37200.00 \\
1460.06 \\
9580.06 \\
12.00 \\
7730.00 \\
33200.00\end{array}$ & 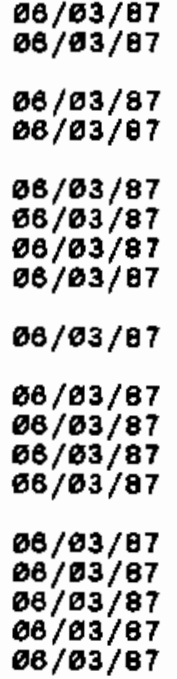 & 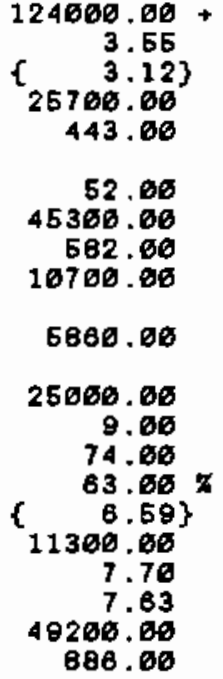 \\
\hline
\end{tabular}

- - VALUe EXCEEDS PRIMARY DRINKING water STANDARD.

* - VALUE EXCEEDS PROPOSED PRIMARY DRINKING WATER STANDARD.

+ - DETECTION LIMIT WAS NOT AVAILABLE FOR COMPARISON

NR - ANALYSIS NOT REQUESTED OR NOT YET REPORTED

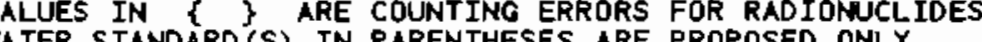


TABLE A.4. (contd)

\begin{tabular}{|c|c|c|c|c|c|c|c|}
\hline $\begin{array}{l}\text { CONSTI } \\
\text { NAME }\end{array}$ & $\begin{array}{l}\text { JENT } \\
\text { UNITS }\end{array}$ & $\begin{array}{l}\text { DETECTION } \\
\text { LIMIT }\end{array}$ & $\begin{array}{l}\text { WATER } \\
\text { STANDARD }\end{array}$ & $\begin{array}{l}\text { SAMPLE } \\
\text { DATE }\end{array}$ & $6-\$ 29-E 12$ & $\begin{array}{l}\text { SAMPLE } \\
\text { DATE }\end{array}$ & $8-531-1$ \\
\hline $\begin{array}{l}\text { ALKALIN } \\
\text { AMMONIU } \\
\text { BETA }\end{array}$ & $\begin{array}{l}\mathrm{PPB} \\
\mathrm{PCI} / \mathrm{L}\end{array}$ & $\begin{array}{r}56.60 \\
3.00\end{array}$ & 60.66 & $68 / 68 / 87$ & $\begin{array}{c}146000.00+ \\
14.90\end{array}$ & $\begin{array}{l}66 / 22 / 87 \\
66 / 22 / 87 \\
66 / 22 / 87\end{array}$ & $\begin{array}{r}89300.00 \\
70.06 \\
5.50\end{array}$ \\
\hline $\begin{array}{l}\text { CHLORID } \\
\text { CONDFLD } \\
\text { FBARIUM } \\
\text { FCALCIU }\end{array}$ & $\begin{array}{l}\text { PPB } \\
\text { UMHO } \\
\text { PPB } \\
\text { PPB }\end{array}$ & $\begin{array}{r}500.06 \\
1.06 \\
6.08 \\
50.08\end{array}$ & 1000.00 & $\begin{array}{l}66 / 68 / 87 \\
66 / 08 / 87 \\
66 / 08 / 87 \\
66 / \oplus 6 / 87\end{array}$ & $\begin{array}{r}13260.00 \\
417.60 \\
47.00 \\
49800.00\end{array}$ & $\begin{array}{l}66 / 22 / 87 \\
66 / 22 / 87 \\
66 / 22 / 87 \\
66 / 22 / 87\end{array}$ & $\begin{array}{r}564 \theta .00 \\
22 \theta .06 \\
19.00 \\
2740 \theta .00\end{array}$ \\
\hline $\begin{array}{l}\text { FLUORID } \\
\text { FNAGNES } \\
\text { FPOTASS } \\
\text { FSDDIUN } \\
\text { FVANADI } \\
\text { FZ }\end{array}$ & $\begin{array}{l}\text { PPB } \\
P P B \\
P P B \\
P P B \\
P P B \\
P P R\end{array}$ & $\begin{array}{r}500.00 \\
0.00 \\
100.00 \\
100.00 \\
5.00 \\
5.00\end{array}$ & 1468.08 & $\begin{array}{l}06 / 06 / 87 \\
66 / 08 / 87 \\
06 / 08 / 87 \\
66 / 06 / 87 \\
66 / 06 / 87 \\
66 / 98 / 87\end{array}$ & $\begin{array}{r}514.00 \\
10860.00 \\
6060.00 \\
22400.00 \\
8.00 \\
15.90\end{array}$ & $\begin{array}{l}06 / 22 / 87 \\
06 / 22 / 87 \\
06 / 22 / 87 \\
06 / 22 / 67\end{array}$ & $\begin{array}{r}6200.00 \\
4280.06 \\
8990.06 \\
15.00\end{array}$ \\
\hline $\begin{array}{l}\text { METHYCH } \\
\text { NITRATE } \\
\text { PHF IELD }\end{array}$ & $\begin{array}{l}\mathrm{PPB} \\
\mathrm{PPE}\end{array}$ & $\begin{array}{r}20.00 \\
500.00 \\
0.10\end{array}$ & $\triangle 5000 . \varnothing \varnothing$ & $\begin{array}{l}66 / 08 / 87 \\
66 / 68 / 87\end{array}$ & $\begin{array}{r}29900.00 \\
7.20\end{array}$ & $\begin{array}{l}06 / 22 / 87 \\
66 / 22 / 87 \\
66 / 22 / 87\end{array}$ & $\begin{array}{r}44.00 \\
3430.06 \\
7.36\end{array}$ \\
\hline RADIUM & PCI/L & 0.10 & 5.00 & $66 / 98 / 87$ & 0.19 & $06 / 22 / 87$ & 0.11 \\
\hline $\begin{array}{l}\text { SULFATE } \\
\text { TOC } \\
\text { TOXLDL } \\
U\end{array}$ & $\begin{array}{l}\text { PPE } \\
\text { PPB } \\
\text { PPB } \\
\text { PCI/L }\end{array}$ & $\begin{array}{r}500.00 \\
200.00 \\
26.00 \\
\emptyset .60\end{array}$ & 660.00 & $\begin{array}{l}06 / 08 / 87 \\
66 / 08 / 87 \\
66 / 08 / 87\end{array}$ & $\begin{array}{r}38360.00 \\
487.00 \\
2.37\end{array}$ & $\begin{array}{l}\theta 6 / 22 / 87 \\
06 / 22 / 87 \\
06 / 22 / 67\end{array}$ & $\begin{array}{c}17700.00 \\
1380.00 \\
60.60 \\
\mathrm{NR}\end{array}$ \\
\hline
\end{tabular}

- - value exceeds primary dRINking hater standard.

- VALUE EXCEEDS PROPOSED PRIMARY DRINKING WATER STANDARD.

- DETECTION LIMIT WAS NOT AVAILABLE FOR COMPARISON

NR - ANALYSIS NOT REQUESTED OR NOT YET REPORTED

VALUES IN $\{$ ? ARE COUNTING ERRORS FOR RADIONUCLIDES
WATER STANDARD'(S) IN PARENTHESES ARE PROPOSED ONLY 
TABLE A.5. Results for Compliance Monitoring Hells

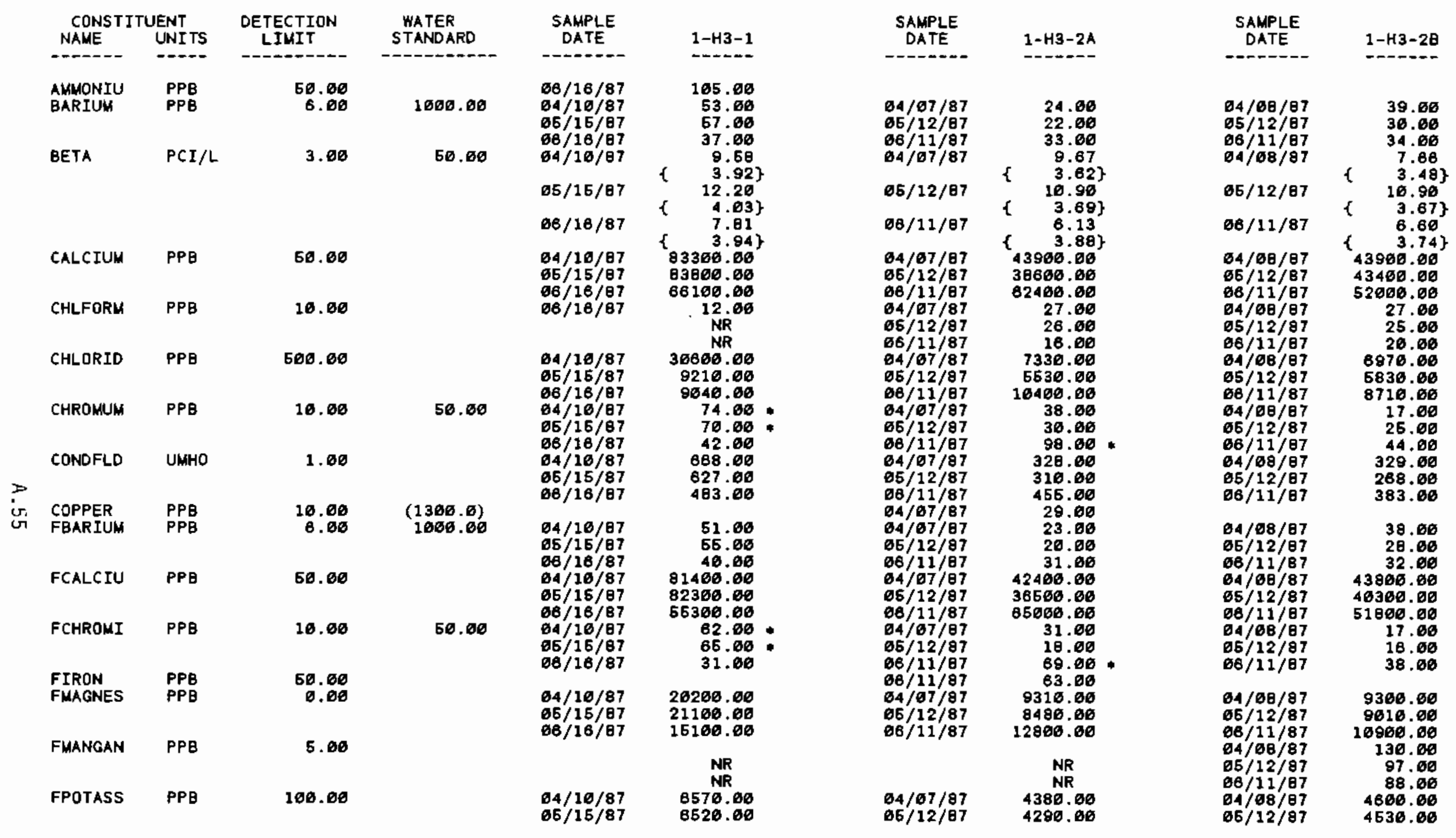

* - VAlUE EXCEEDS PRIMARY DRINKINg waTeR STANDARD.

- VALUE EXCEEDS PROPOSED PRIMARY DRINHING WATER STANDARD.

作

NR - ANALYSIS NOT REQUESTED OR NOT YET REPORTED

VALUES IN \{\} ARE COUNTING ERRORS FOR RADIONUCLIDES

WATER STANDARD'(S) IN PARENTHESES ARE PROPOSED ONLY 
IABLE A.5. (contd)

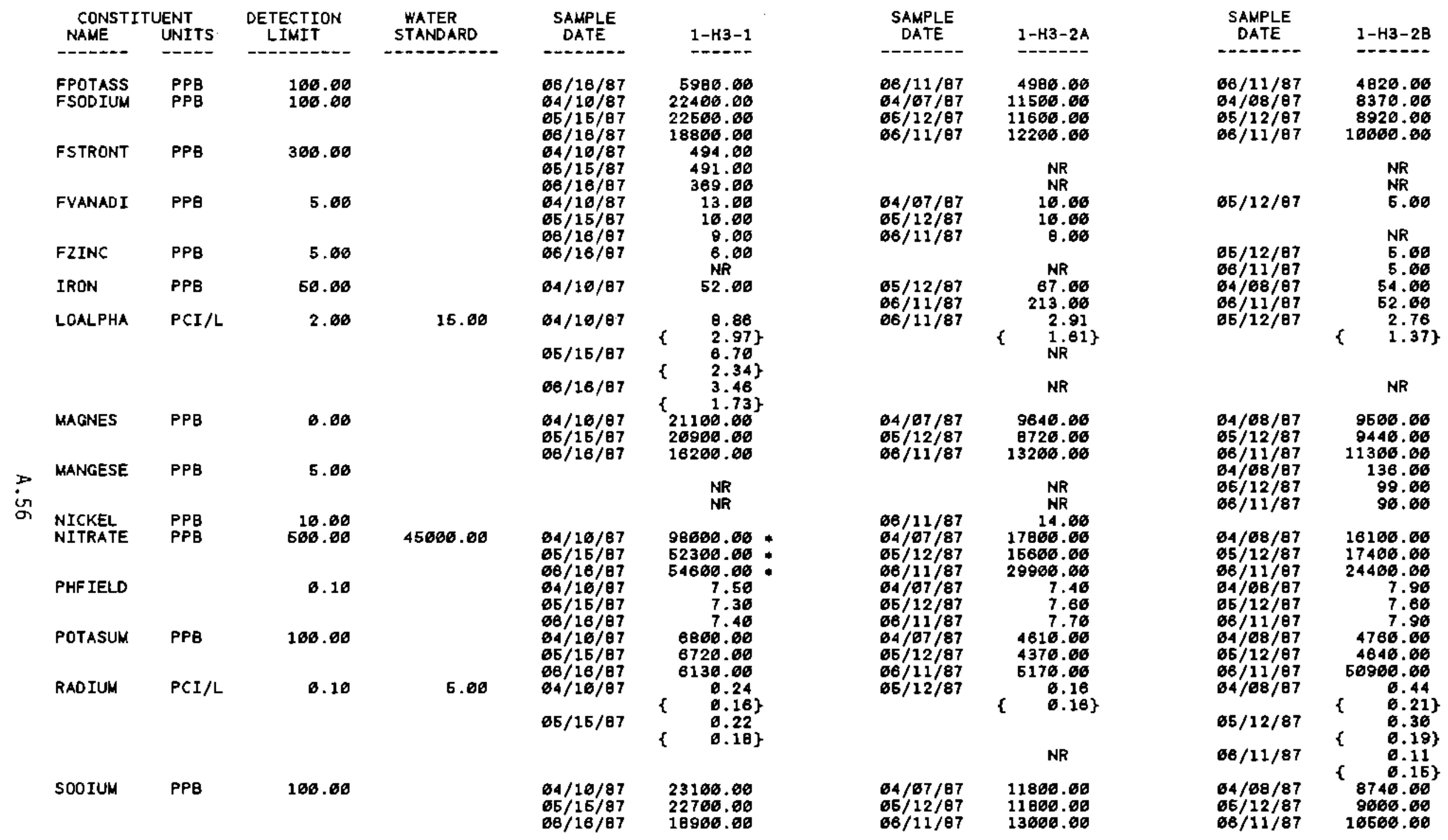

* - Value exceeds primary dRINKING water standard.

- VALUE EXCEEDS PROPOSED PRIMARY DRINKING WATER STANDARD.

- VALUE EXCEEDS SCREENING LEVEL FOR FURTHER INVESTIGATION.

+ - DETECTION LIMIT WAS NOT AVAILABLE FOR COMPARISON

- ANALYSIS NDT REQUESTED OR NOT YET REPORTED

VALUES IN \{ $\}$ ARE COUNTING ERRORS FOR RADIONUCLIDES 
IABLE A.5. (contd)

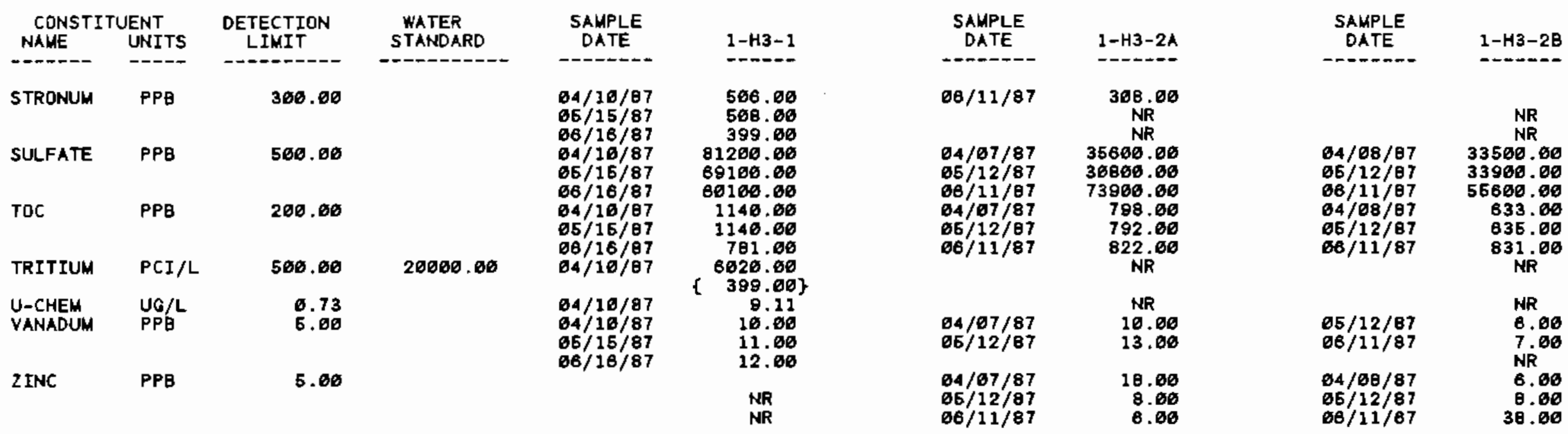

- - VAlUe eXCEEDS PRIMARY DRINKING WATER STANDARD.

* - VALUE EXCEEDS PROPOSED PRIMARY DRINKING WATER STANDARD.

* - VALUE EXCEEDS SCREENING LEVEL FOR FURTHER INVESTIGATION.

+ - DETECTIDN LIMIT WAS NOT AVAILABLE FOR COMPARISON

NR - ANALYSIS NOT REQUESTED OR NOT YET REPORTED

VALUES IN $\{$ t ARE COUNTING ERRORS FOR RADTONUCLIDES

WATER STANDARD(S) IN PARENTHESES ARE PROPOSEO ONLY 
IABLE A.5. (contd)

\begin{tabular}{|c|c|c|c|c|c|c|c|c|c|}
\hline $\begin{array}{l}\text { CONSTI } \\
\text { NAME }\end{array}$ & $\begin{array}{l}\text { UENT } \\
\text { UNITS }\end{array}$ & $\begin{array}{c}\text { OETECTION } \\
\text { LIMIT }\end{array}$ & $\begin{array}{c}\text { WATER } \\
\text { STANDARD }\end{array}$ & $\begin{array}{l}\text { SAMPLE } \\
\text { DATE }\end{array}$ & $1-\mathrm{H}_{3}-2 \mathrm{C}$ & $\begin{array}{c}\text { SAMPLE } \\
\text { DATE }\end{array}$ & $1-\mathrm{H} 4-3$ & $\begin{array}{l}\text { SAMPLE } \\
\text { DATE }\end{array}$ & $1-\mathrm{H}_{4}-4$ \\
\hline $\begin{array}{l}\text { ALUMNUM } \\
\text { AMMONIU }\end{array}$ & $\begin{array}{l}\text { PP日 } \\
\text { PPG }\end{array}$ & $\begin{array}{r}150.06 \\
50.00\end{array}$ & & $\begin{array}{l}64 / 06 / 87 \\
64 / 06 / 87 \\
65 / 11 / 87 \\
66 / 11 / 87\end{array}$ & $\begin{array}{l}607.00 \\
335.00 \\
115.06 \\
110.06\end{array}$ & $04 / 09 / 87$ & $\begin{array}{c}347.00 \\
\text { NR } \\
\text { NR }\end{array}$ & $06 / 16 / 87$ & $\begin{array}{l}64.00 \\
N R \\
N R\end{array}$ \\
\hline ARSENIC & PPB & 5.00 & 50.00 & $60 / 11 / 07$ & ${ }_{\mathrm{NR}}^{5.00}$ & $\begin{array}{l}65 / 15 / 87 \\
06 / 15 / 87\end{array}$ & $\begin{array}{l}8.00 \\
5.00\end{array}$ & $\begin{array}{l}04 / 16 / 87 \\
06 / 16 / 87\end{array}$ & $\begin{array}{l}5.00 \\
5.00\end{array}$ \\
\hline BARIUM & PPB & 6.00 & 1000.00 & $\begin{array}{l}04 / 06 / 87 \\
66 / 11 / 87 \\
06 / 11 / 87 \\
04 / 06 / 87\end{array}$ & $\begin{array}{l}17.00 \\
23.06 \\
23.06 \\
12.76\end{array}$ & $\begin{array}{l}04 / 09 / 87 \\
05 / 15 / 87 \\
06 / 15 / 87 \\
04 / 09 / 87\end{array}$ & $\begin{array}{c}28.00 \\
20.00 \\
39.00 \\
687.60 .7\end{array}$ & $\begin{array}{l}64 / 16 / 87 \\
65 / 14 / 87 \\
66 / 16 / 87 \\
64 / 16 / 87\end{array}$ & $\begin{array}{r}79.06 \\
22.60 \\
54.06 \\
431.00 \%\end{array}$ \\
\hline BETA & $\mathrm{PCI} / \mathrm{L}$ & 3.00 & 50.00 & $\begin{array}{l}64 / 06 / 87 \\
05 / 11 / 87\end{array}$ & $\begin{array}{r}12.78 \\
3.84\} \\
9.22 \\
3 \quad 3.48\}\end{array}$ & $\begin{array}{l}04 / 09 / 87 \\
65 / 15 / 87\end{array}$ & $\begin{array}{c}667.60 \times \\
23.46\} \\
236.60 \times \\
\{\quad 33.90\}\end{array}$ & $05 / 14 / 87$ & $\begin{array}{c}431.00 \% \\
26.006 \\
6.80 \\
3.11\}\end{array}$ \\
\hline & & & & $06 / 11 / 87$ & $\begin{array}{l}4.72 \\
3.44\}\end{array}$ & $66 / 15 / 87$ & 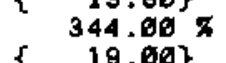 & $06 / 16 / 87$ & $\left\{\begin{array}{l}153.067 \\
\{\quad 11.86\}\end{array}\right.$ \\
\hline CALCIUM & PPB & 50.00 & & $\begin{array}{l}64 / 08 / 87 \\
65 / 11 / 87 \\
06 / 11 / 87\end{array}$ & $\begin{array}{l}25800.00 \\
26200.06 \\
25600.00\end{array}$ & $\begin{array}{l}04 / 09 / 87 \\
65 / 15 / 87 \\
06 / 15 / 87\end{array}$ & $\begin{array}{l}20300.60 \\
11380.00 \\
15860.00\end{array}$ & $\begin{array}{l}04 / 10 / 87 \\
65 / 14 / 87 \\
06 / 16 / 87\end{array}$ & $\begin{array}{l}51800.06 \\
27606.06 \\
37706.06\end{array}$ \\
\hline CHLFORM & PPB & 10.00 & & $04 / 08 / 87$ & 17.60 & $\begin{array}{l}64 / 69 / 87 \\
65 / 15 / 97\end{array}$ & $\begin{array}{l}24.00 \\
22.00\end{array}$ & $04 / 10 / 87$ & 10.00 \\
\hline CHLORID & PPB & 500.00 & & $\begin{array}{l}64 / 06 / 87 \\
65 / 11 / 87 \\
06 / 11 / 87\end{array}$ & $\begin{array}{l}7850.00 \\
2640.00 \\
3240.00\end{array}$ & $\begin{array}{l}08 / 16 / 87 \\
64 / 90 / 87 \\
06 / 15 / 97 \\
06 / 16 / 87\end{array}$ & $\begin{array}{r}13166.06 \\
7256.06 \\
8956.06 \\
312.99\end{array}$ & $\begin{array}{l}64 / 10 / 87 \\
65 / 14 / 87 \\
66 / 16 / 87\end{array}$ & $\begin{array}{l}6656.06 \\
1286.06 \\
4486.06\end{array}$ \\
\hline CHROHUM & PPB & 10.00 & 50.00 & & $\begin{array}{l}\mathrm{NR} \\
\mathrm{NR}\end{array}$ & $\begin{array}{l}64 / 09 / 87 \\
65 / 15 / 97 \\
66 / 15 / 97\end{array}$ & $\begin{array}{l}313.60 \\
298.00\end{array}$ & $\begin{array}{l}04 / 10 / 87 \\
65 / 14 / 87 \\
96 / 16 / 87\end{array}$ & $\begin{array}{l}378.06 \\
28.06\end{array}$ \\
\hline CONDFLD & UMHO & 1.00 & & $\begin{array}{l}64 / 06 / 87 \\
65 / 11 / 87 \\
66 / 11 / 87\end{array}$ & $\begin{array}{l}284.00 \\
187.00 \\
281.00\end{array}$ & $\begin{array}{l}64 / 09 / 87 \\
65 / 15 / 67 \\
68 / 15 / 87\end{array}$ & $\begin{array}{r}1467.06 \\
910.06 \\
1102.06\end{array}$ & $\begin{array}{l}04 / 10 / 87 \\
65 / 14 / 87 \\
06 / 16 / 87\end{array}$ & $\begin{array}{r}1058.00 \\
215.06 \\
573.06\end{array}$ \\
\hline COPPER & PPB & 10.00 & $(1300.0)$ & $\begin{array}{l}04 / 66 / 87 \\
65 / 11 / 87 \\
66 / 11 / 87\end{array}$ & $\begin{array}{l}28.00 \\
21.00 \\
27.00\end{array}$ & $\begin{array}{l}64 / 99 / 87 \\
65 / 15 / 67 \\
68 / 15 / 87\end{array}$ & $\begin{array}{l}18.06 \\
16.00 \\
10.00\end{array}$ & & $\begin{array}{l}\text { NR } \\
\text { NR }\end{array}$ \\
\hline $\begin{array}{l}\text { FALUMIN } \\
\text { FARSENI }\end{array}$ & $\begin{array}{l}\mathrm{PPB} \\
\mathrm{PPB}\end{array}$ & $\begin{array}{r}150.00 \\
5.00\end{array}$ & 50.00 & $04 / 68 / 87$ & 453.00 & $64 / 69 / 07$ & 5.00 & & \\
\hline & & & & & $\begin{array}{l}\text { NR } \\
\text { NR }\end{array}$ & $\begin{array}{l}06 / 15 / 67 \\
98 / 15 / 87\end{array}$ & $\begin{array}{l}9.00 \\
6.00\end{array}$ & & $\begin{array}{l}\text { NR } \\
\text { NR }\end{array}$ \\
\hline FBARIUM & PPB & 6.0 & 1000.00 & $\begin{array}{l}64 / 96 / 87 \\
65 / 11 / 87 \\
68 / 11 / 87\end{array}$ & $\begin{array}{l}17.00 \\
24.00 \\
36.00\end{array}$ & $\begin{array}{l}04 / 89 / 97 \\
05 / 15 / 87 \\
06 / 15 / 87\end{array}$ & $\begin{array}{l}27.00 \\
14.00 \\
24.00\end{array}$ & $\begin{array}{l}64 / 10 / 87 \\
66 / 14 / 97 \\
68 / 16 / 97\end{array}$ & $\begin{array}{l}80.00 \\
27.00 \\
36.00\end{array}$ \\
\hline FCALCIU & PPB & 50.00 & 50.00 & $\begin{array}{l}64 / 06 / 87 \\
65 / 11 / 87 \\
06 / 11 / 87 \\
64 / 06 / 87\end{array}$ & $\begin{array}{r}24200.00 \\
26306.00 \\
20800.00 \\
11.00\end{array}$ & $\begin{array}{l}04 / 69 / 97 \\
95 / 15 / 87 \\
68 / 15 / 87 \\
04 / 69 / 87\end{array}$ & $\begin{array}{r}19800.00 \\
11200.60 \\
15200.06 \\
288.60 .\end{array}$ & $\begin{array}{l}04 / 16 / 87 \\
65 / 14 / 87 \\
06 / 16 / 87 \\
04 / 10 / 87\end{array}$ & $\begin{array}{r}53700.00 \\
25700.00 \\
33100.00 \\
378.00 .\end{array}$ \\
\hline \multicolumn{10}{|c|}{ 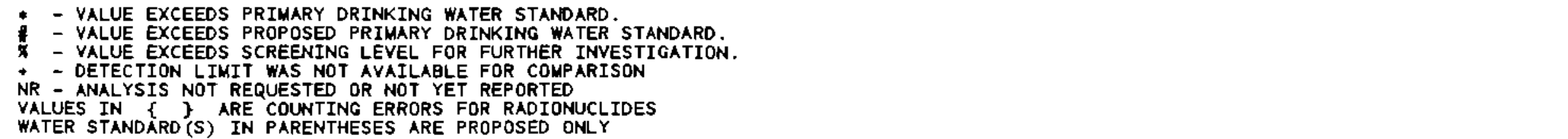 } \\
\hline
\end{tabular}


IABLE A.5. (contd)

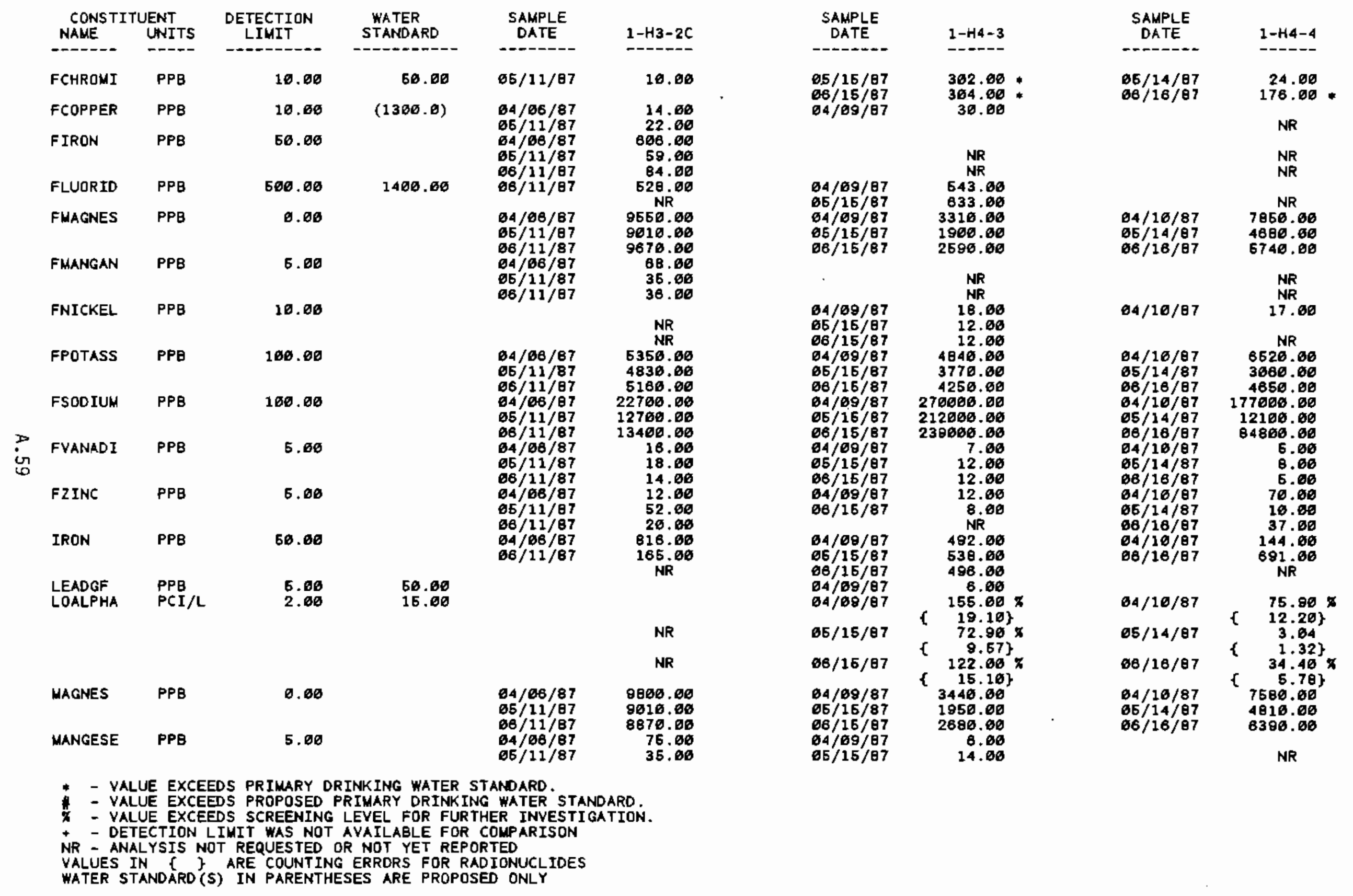


TABLE A.5. (contd)

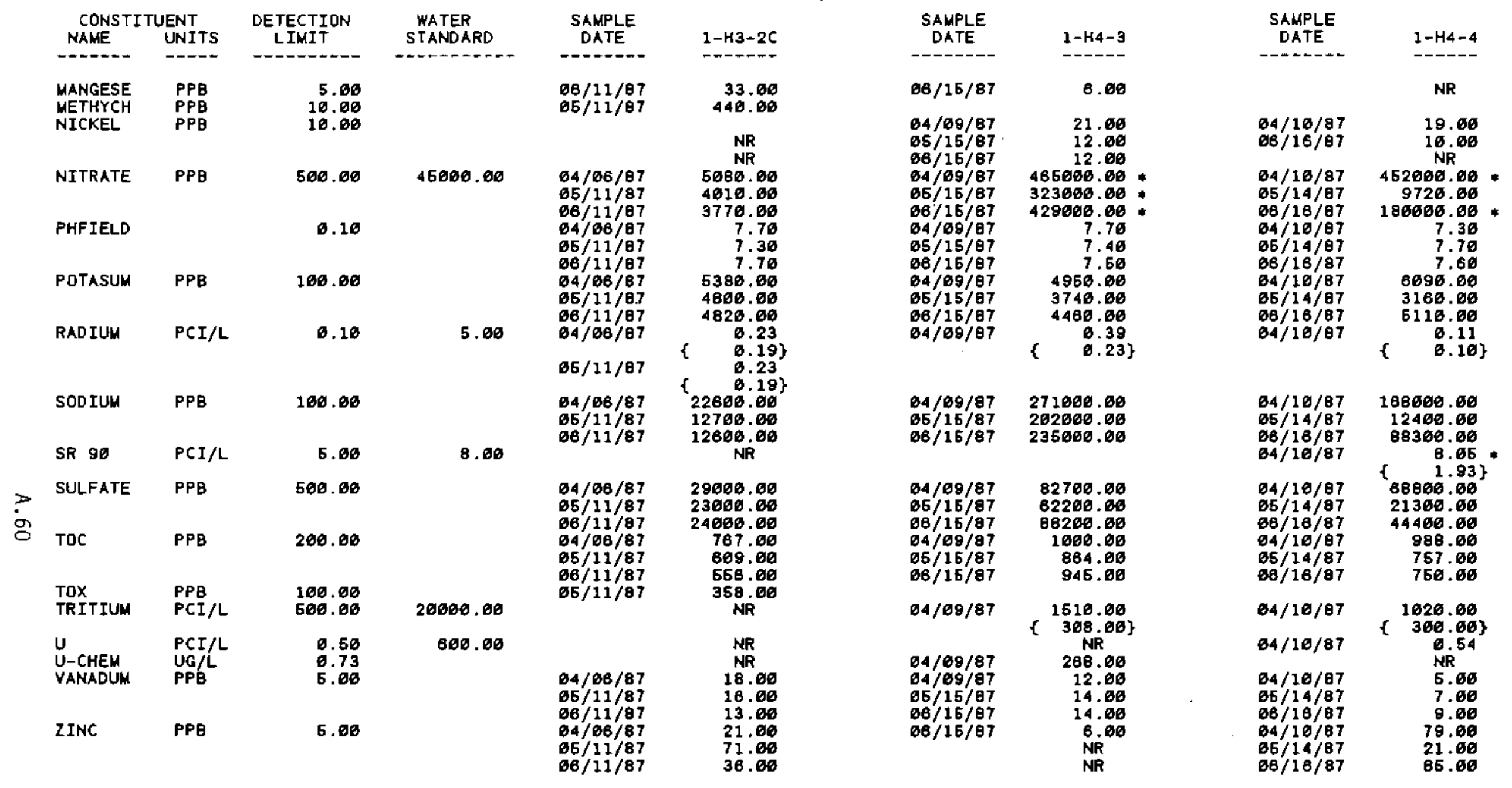

- - Value exceeds primary drinking water stanoard.

* - VALUE EXCEEDS PROPOSED PRIMARY ORINKING WATER STANDARD.

* - VALUE EXCEEDS SCREENING LEVEL FOR FURTHER INVESTIGATION.

+ - DETECTION LIMIT WAS NOT AVAILABLE FOR COMPARISON

NR - ANALYSIS NOT REQUESTED OR NOT YET REPORTED

VALUES IN \{\} ARE COUNTING ERRORS FOR RADIONUCLIDES

WATER STANOARD'(S) IN PARENTHESES ARE PROPOSED ONLY 
TABLE A.5. (contd)

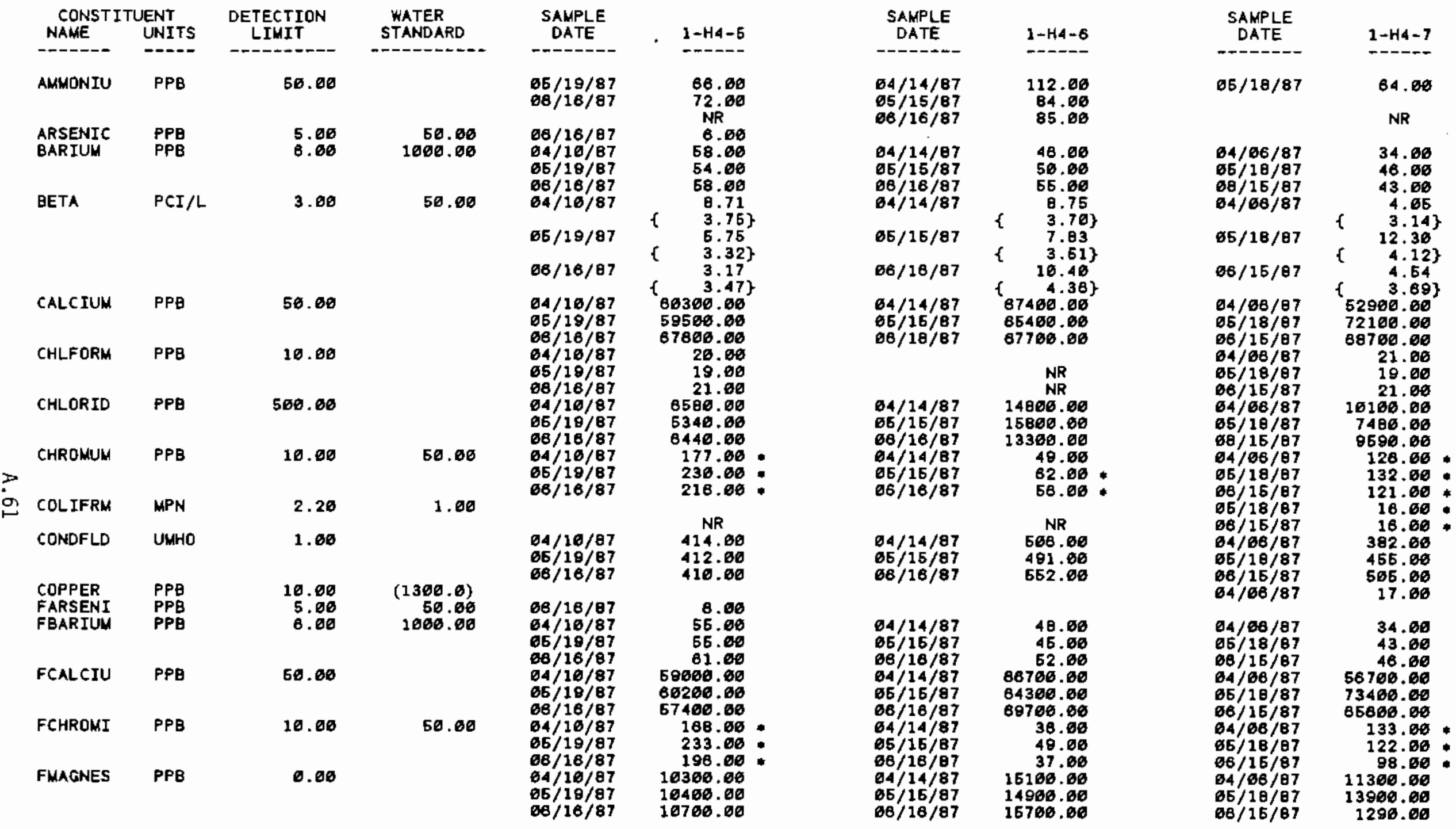

* - VALUE EXCEEDS PRIMARY DRINKING WATER STANDARD.

* - VALUE EXCEEDS PROPOSED PRIMARY DRINKING WATER STANDARD.

\# - VALUE EXCEEOS SCREENING LEVEL FOR FURTHER INVESTIGATION.

+ - DETECTION LIMIT WAS NOT AVAILABLE FOR COMPARISON

NR - ANALYSIS NOT REQUESTED OR NOT YET REPDRTED

VALUES IN \{ \} ARE COUNTING ERRORS FOR RADIONUCLIDES

WATER STANDARD(S) IN PARENTHESES ARE PROPOSED ONLY 
TABLE A.5. (cont $d$ )

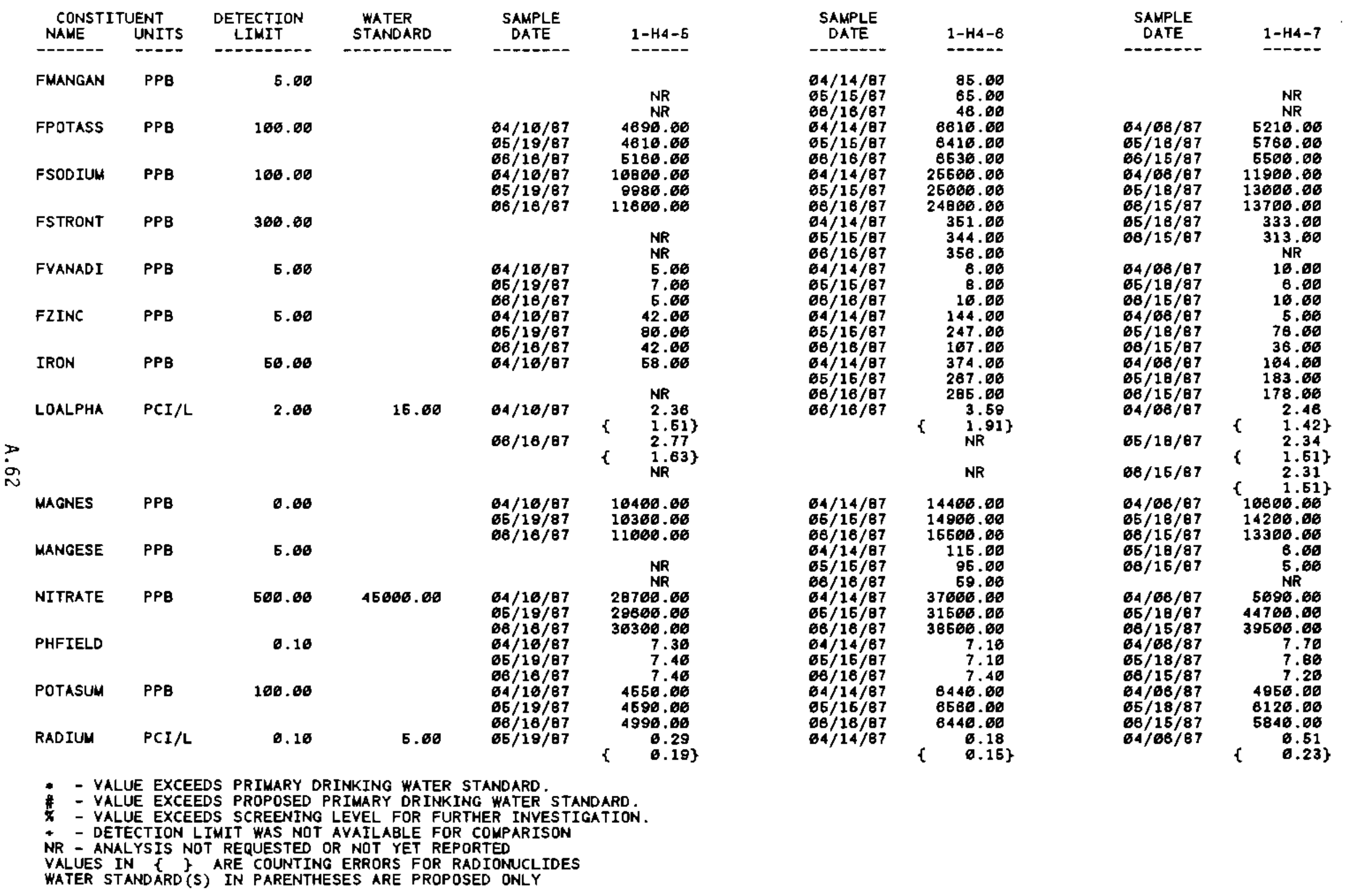


TABLE A.5. (contd)

\begin{tabular}{|c|c|c|c|c|c|c|c|c|c|}
\hline $\begin{array}{l}\text { CONSTI } \\
\text { NAME }\end{array}$ & $\begin{array}{l}\text { UENT } \\
\text { UNITS }\end{array}$ & $\begin{array}{l}\text { DETECTION } \\
\text { LIMIT }\end{array}$ & $\begin{array}{l}\text { WATER } \\
\text { STANDARD }\end{array}$ & $\begin{array}{c}\text { SAMPLE } \\
\text { DATE }\end{array}$ & $2-H 4-6$ & $\begin{array}{l}\text { SAMPLE } \\
\text { DATE }\end{array}$ & $1-\mathrm{H}_{4}-6$ & $\begin{array}{l}\text { SAMPLE } \\
\text { DATE }\end{array}$ & $1-H 4-7$ \\
\hline$-\infty-\infty$ & $\cdots-$ & 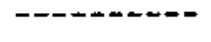 & $--n+20-0-1$ & $--\infty-\infty-2$ & ----- & $--\infty-\infty$ & 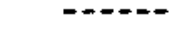 & $-\infty, \ldots-$ & $-----r$ \\
\hline \multirow[t]{2}{*}{ RADIUM } & $\mathrm{PCI} / \mathrm{L}$ & 0.10 & 5.60 & & & $05 / 15 / 87$ & 0.24 & $65 / 16 / 87$ & 0.34 \\
\hline & & & & & NR & $66 / 16 / 87$ & $\{\quad 0.13\}$ & $66 / 15 / 87$ & 0.38 \\
\hline SODIUM & PPE & 100.00 & & $\begin{array}{l}04 / 10 / 87 \\
05 / 19 / 87 \\
06 / 16 / 87\end{array}$ & $\begin{array}{r}11000.00 \\
9960.00 \\
19800.00\end{array}$ & $\begin{array}{l}64 / 14 / 87 \\
65 / 15 / 87 \\
66 / 18 / 87\end{array}$ & $\begin{array}{l}23800.00 \\
25600.00 \\
25600.00\end{array}$ & $\begin{array}{l}04 / 06 / 87 \\
65 / 18 / 87\end{array}$ & $\begin{array}{l}11200.00 \\
13700.00 \\
14000.00\end{array}$ \\
\hline STRONUM & PPB & 300.00 & & $06 / 16 / 87$ & $\begin{array}{c}361.06 \\
N R \\
N R\end{array}$ & $\begin{array}{l}64 / 14 / 87 \\
65 / 16 / 87 \\
66 / 16 / 87\end{array}$ & $\begin{array}{l}336.00 \\
368.00 \\
361.00\end{array}$ & $\begin{array}{l}65 / 18 / 87 \\
66 / 15 / 87\end{array}$ & $\begin{array}{l}361.00 \\
326.00 \\
\text { NR }\end{array}$ \\
\hline SULFATE & PPB & 600.00 & & $\begin{array}{l}04 / 10 / 87 \\
05 / 19 / 87 \\
06 / 16 / 87\end{array}$ & $\begin{array}{l}57000.00 \\
54600.00 \\
58000.00\end{array}$ & $\begin{array}{l}64 / 14 / 87 \\
65 / 16 / 87 \\
66 / 16 / 87\end{array}$ & $\begin{array}{r}92460.00 \\
86200.00 \\
118000.00\end{array}$ & $\begin{array}{l}04 / 08 / 87 \\
06 / 18 / 87 \\
06 / 16 / 87\end{array}$ & $\begin{array}{r}29100.00 \\
89000.00 \\
102000.00\end{array}$ \\
\hline TRITIUM & $P C I / L$ & 200.00 & 20000.00 & $\begin{array}{l}04 / 16 / 87 \\
65 / 19 / 87 \\
06 / 16 / 87 \\
64 / 10 / 87\end{array}$ & $\begin{array}{r}866.00 \\
723.00 \\
815.00 \\
1670.00\end{array}$ & $\begin{array}{l}64 / 14 / 87 \\
66 / 16 / 87 \\
66 / 16 / 87 \\
64 / 14 / 87\end{array}$ & $\begin{array}{r}990.00 \\
959.00 \\
1200.00 \\
4670.00\end{array}$ & $\begin{array}{l}64 / 06 / 67 \\
65 / 18 / 87 \\
06 / 16 / 87\end{array}$ & $\begin{array}{c}900.00 \\
928.00 \\
938.00 \\
\text { NR }\end{array}$ \\
\hline UANADUN & $\begin{array}{l}\mathrm{PCI} / \mathrm{L} \\
\mathrm{PPB}\end{array}$ & $\begin{array}{l}6.60 \\
5.80\end{array}$ & 800.06 & $\begin{array}{l}64 / 10 / 87 \\
64 / 10 / 87 \\
66 / 16 / 87\end{array}$ & $\begin{array}{l}2.07 \\
\text { E. } .00 \\
7.00 \\
\text { NR }\end{array}$ & $\begin{array}{l}64 / 14 / 87 \\
64 / 14 / 87 \\
65 / 16 / 87 \\
66 / 18 / 87\end{array}$ & $\begin{array}{r}2.48 \\
7.00 \\
8.00 \\
10.00\end{array}$ & $\begin{array}{l}64 / 66 / 87 \\
66 / 18 / 87 \\
66 / 16 / 87\end{array}$ & $\begin{array}{l}\text { NR } \\
6.00 \\
8.00 \\
7.00\end{array}$ \\
\hline ZINC & PPB & 5.60 & & $\begin{array}{l}64 / 10 / 87 \\
66 / 19 / 87 \\
66 / 16 / 87\end{array}$ & $\begin{array}{l}48.00 \\
78.00 \\
65.00\end{array}$ & $\begin{array}{l}64 / 14 / 87 \\
66 / 16 / 87 \\
66 / 16 / 87\end{array}$ & $\begin{array}{l}188.00 \\
198.00 \\
164.00\end{array}$ & $\begin{array}{l}64 / 66 / 87 \\
66 / 18 / 87 \\
66 / 16 / 87\end{array}$ & $\begin{array}{l}13.00 \\
80.00 \\
48.00\end{array}$ \\
\hline
\end{tabular}

* - VALUE EXCEEDS PRIMARY DRINKING water STANDARo.

* - VALUE EXCEEDS PROPOSED PRIMARY DRINGING WATER STANDARD.

* VALUE EXCEEDS SCREENING LEVEL FOR FURTHER INVESTIGATION.

* - DETECTION LIUIT WAS NOT AVAILABLE FOR COMPARISON

NR - ANALYSIS NOT REQUESTED OR NOT YET REPORTED

VALUES IN \{ \} ARE COUNTING ERRORS FOR RADIONUCLIDES 
TABLE A.5. (contd)

\begin{tabular}{|c|c|c|c|c|c|c|c|c|c|}
\hline $\begin{array}{l}\text { CONSTI } \\
\text { NAME }\end{array}$ & $\begin{array}{l}\text { JENT } \\
\text { UNI TS }\end{array}$ & $\begin{array}{c}\text { DETECTION } \\
\text { LIMIT }\end{array}$ & $\begin{array}{l}\text { WATER } \\
\text { STANDARD }\end{array}$ & $\begin{array}{l}\text { SAMPLE } \\
\text { DATE }\end{array}$ & $1-\mathrm{H} 4-8$ & $\begin{array}{l}\text { SAMPLE } \\
\text { DATE }\end{array}$ & $1-\mathrm{H} 4-9$ & $\begin{array}{l}\text { SAMPLE } \\
\text { DATE }\end{array}$ & $1-H 4-16$ \\
\hline ALUMNUIM & PPB & 150.00 & & $\begin{array}{l}05 / 18 / 87 \\
06 / 12 / 87\end{array}$ & $\begin{array}{r}1500.00 \\
924.00\end{array}$ & $\begin{array}{l}05 / 18 / 87 \\
06 / 16 / 87\end{array}$ & $\begin{array}{l}178.66 \\
248.68\end{array}$ & & NR \\
\hline $\begin{array}{l}\text { AMMONIU } \\
\text { ARSENIC }\end{array}$ & $\begin{array}{l}\mathrm{PPB} \\
\mathrm{PPB}\end{array}$ & $\begin{array}{r}50.00 \\
5.00\end{array}$ & 50.00 & & & $\begin{array}{l}65 / 18 / 87 \\
84 / 68 / 87\end{array}$ & $\begin{array}{r}52.06 \\
9.06\end{array}$ & & \\
\hline & & & & & $\begin{array}{l}\text { NR } \\
\text { NR }\end{array}$ & $\begin{array}{l}\theta 5 / 18 / 87 \\
66 / 16 / 87\end{array}$ & $\begin{array}{l}9.00 \\
\theta .00\end{array}$ & & $\begin{array}{l}\mathbf{N R} \\
\text { NR }\end{array}$ \\
\hline BARIUK & PPB & 6.66 & 1000.60 & $\begin{array}{l}64 / 09 / 87 \\
65 / 18 / 97 \\
68 / 12 / 97\end{array}$ & $\begin{array}{l}45.00 \\
65.06 \\
47.06\end{array}$ & $\begin{array}{l}64 / 6 \theta / 87 \\
05 / 18 / 87 \\
06 / 15 / 87\end{array}$ & $\begin{array}{l}59.00 \\
64.06 \\
49.06\end{array}$ & $\begin{array}{l}64 / 06 / 87 \\
65 / 14 / 87 \\
66 / 16 / 87\end{array}$ & $\begin{array}{l}72.00 \\
44.60 \\
37.60\end{array}$ \\
\hline BETA & PCI /L & 3.80 & 50.00 & $04 / 09 / 87$ & $\begin{array}{l}8.71 \\
3.63\}\end{array}$ & $64 / 06 / 87$ & $\begin{array}{c}128.60 \% \\
16.46\}\end{array}$ & $04 / 06 / 87$ & $\begin{array}{l}8.96 \\
3.71\}\end{array}$ \\
\hline & & & & $05 / 18 / 87$ & 11.86 & $05 / 18 / 87$ & $184.60 \times$ & $65 / 14 / 87$ & 5.26 \\
\hline & & & & $06 / 12 / 87$ & 8.18 & $06 / 15 / 07$ & $\begin{array}{l}12.165 \\
40.20\end{array}$ & $68 / 10 / 07$ & $\begin{array}{l}2.98\} \\
7.71\end{array}$ \\
\hline & & & 10.99 & & $3.87\}$ & $98 / 15197$ & 8.63\}) & & $3.71\}$ \\
\hline CALCIUN & $\mathrm{PPB}$ & 58.00 & 10.60 & $\begin{array}{l}64 / 00 / 87 \\
66 / 18 / 87\end{array}$ & $\begin{array}{l}63760.00 \\
67906.00\end{array}$ & $\begin{array}{l}64 / 68 / 67 \\
65 / 16 / 87\end{array}$ & $\begin{array}{l}01200.60 \\
88900.60\end{array}$ & $\begin{array}{l}04 / 08 / 87 \\
06 / 14 / 87\end{array}$ & $\begin{array}{l}45000.00 \\
31200.00\end{array}$ \\
\hline CHLFORM & PPB & 10.66 & & $\begin{array}{l}04 / 69 / 97 \\
05 / 18 / 87 \\
66 / 12 / 97\end{array}$ & $\begin{array}{r}68400.06 \\
20.00 \\
17.06 \\
18.00\end{array}$ & $\begin{array}{l}06 / 16 / 87 \\
04 / 68 / 87 \\
05 / 18 / 87 \\
66 / 16 / 87\end{array}$ & $\begin{array}{r}87906.00 \\
17.00 \\
26.06 \\
19.00\end{array}$ & $06 / 18 / 87$ & $\begin{array}{c}27406.60 \\
N R \\
N R\end{array}$ \\
\hline CHLORID & PPB & 500.80 & & $\begin{array}{l}04 / 08 / 87 \\
06 / 18 / 87 \\
06 / 12 / 87\end{array}$ & $\begin{array}{l}9068.00 \\
7620.00 \\
9198.06\end{array}$ & $\begin{array}{l}64 / 68 / 67 \\
65 / 16 / 87 \\
66 / 15 / 87\end{array}$ & $\begin{array}{l}8950.00 \\
9340 . .60 \\
8890.00\end{array}$ & $\begin{array}{l}64 / 66 / 87 \\
65 / 14 / 67 \\
66 / 16 / 87\end{array}$ & $\begin{array}{l}8340.06 \\
1400.06 \\
2070.00\end{array}$ \\
\hline CHROMUY & PPB & 10.00 & 56.00 & $\begin{array}{l}64 / 09 / 87 \\
66 / 18 / 87\end{array}$ & $\begin{array}{l}181.06 \\
113.06\end{array}$ & $\begin{array}{l}64 / 06 / 87 \\
65 / 16 / 87\end{array}$ & 163.06 & $\begin{array}{l}04 / 06 / 87 \\
05 / 14 / 87\end{array}$ & $\begin{array}{l}87.00 \\
18.00\end{array}$ \\
\hline CONDFLD & UMHO & 1.00 & & $\begin{array}{l}64 / 69 / 97 \\
06 / 19 / 87\end{array}$ & $\begin{array}{l}436.66 \\
396.60\end{array}$ & $\begin{array}{l}64 / 08 / 87 \\
65 / 18 / 87\end{array}$ & $\begin{array}{l}139.66 \\
678.06 \\
839.06\end{array}$ & $\begin{array}{l}68 / 10 / 87 \\
64 / 60 / 87 \\
65 / 14 / 87\end{array}$ & $\begin{array}{r}17.60 \\
332.06 \\
236.06\end{array}$ \\
\hline COPPER & PPB & 10.00 & $(1300.0)$ & $04 / 69 / 87$ & 16.00 & $08 / 10 / 81$ & 478.60 & $\begin{array}{l}06 / 16 / 87 \\
64 / 68 / 87\end{array}$ & $\begin{array}{r}14.68 \\
14.60\end{array}$ \\
\hline FARSENI & PPB & 5.00 & 50.00 & $00 / 18 / 8$ & 11.00 & $04 / 00 / 87$ & 8.66 & $68 / 16 / 87$ & 11.00 \\
\hline FBARIUM & PPB & 6.68 & 1000.00 & & $\begin{array}{l}\text { NR } \\
43.90\end{array}$ & $05 / 18 / 87$ & $B 0 \mathrm{ag}$ & & NR \\
\hline ronk10m & rro & 0.100 & שס. & $\begin{array}{l}66 / 19 / 67 \\
06 / 12 / 67\end{array}$ & $\begin{array}{l}40.000 \\
39.00\end{array}$ & $06 / 18 / 67$ & NR & $\begin{array}{l}05 / 14 / 87 \\
60 / 10 / 87\end{array}$ & $\begin{array}{l}12.06 \\
46.00\end{array}$ \\
\hline FCALCIU & PPB & 60.00 & & $\begin{array}{l}04 / 69 / 87 \\
06 / 19 / 87 \\
06 / 12 / 87\end{array}$ & $\begin{array}{l}64000.00 \\
76806.00 \\
73700.00\end{array}$ & $\begin{array}{l}64 / 08 / 87 \\
66 / 18 / 87\end{array}$ & $\begin{array}{l}84200.00 \\
\theta 0900.60 \\
\mathrm{NR}\end{array}$ & $\begin{array}{l}04 / 68 / 87 \\
66 / 14 / 87 \\
66 / 10 / 87\end{array}$ & $\begin{array}{l}47000.00 \\
29800.00 \\
29700.60\end{array}$ \\
\hline FCHROMI & PPB & 10.00 & 56.00 & $\begin{array}{l}84 / 69 / 87 \\
65 / 18 / 87 \\
68 / 12 / 87\end{array}$ & $\begin{array}{r}94.06 \\
111.06 \\
112.00\end{array}$ & $\begin{array}{l}04 / 08 / 87 \\
06 / 18 / 87\end{array}$ & $\begin{array}{c}164.00 \\
143.00 \\
\mathrm{NR}\end{array}$ & $\begin{array}{l}64 / 66 / 87 \\
65 / 14 / 67 \\
68 / 16 / 87\end{array}$ & $\begin{array}{l}91.68 \\
16.08 \\
18.08\end{array}$ \\
\hline
\end{tabular}

* - Value exceeds primary dRINking hater standard.

* - VALUE EXCEEDS PROPOSED PRIMARY DRINKINGG WATER STANDARD.

* VALUE EXCEEDS SCREENING LEVEL FOR FURTHER INVESTIGATION.

* - DETECTION LIMIT WAS NOT AVAILABLE FOR COMPARISON

NR - ANALYSIS NOT REQUESTED DR NOT YET REPORTED

VALUES IN $\{$ \& ARE COUNTING ERRORS FOR RADIONUCLIDES

WATER STANDARD (S) IN PARENTHESES ARE PROPOSED ONLY 
IABLE A.5. (cont d)

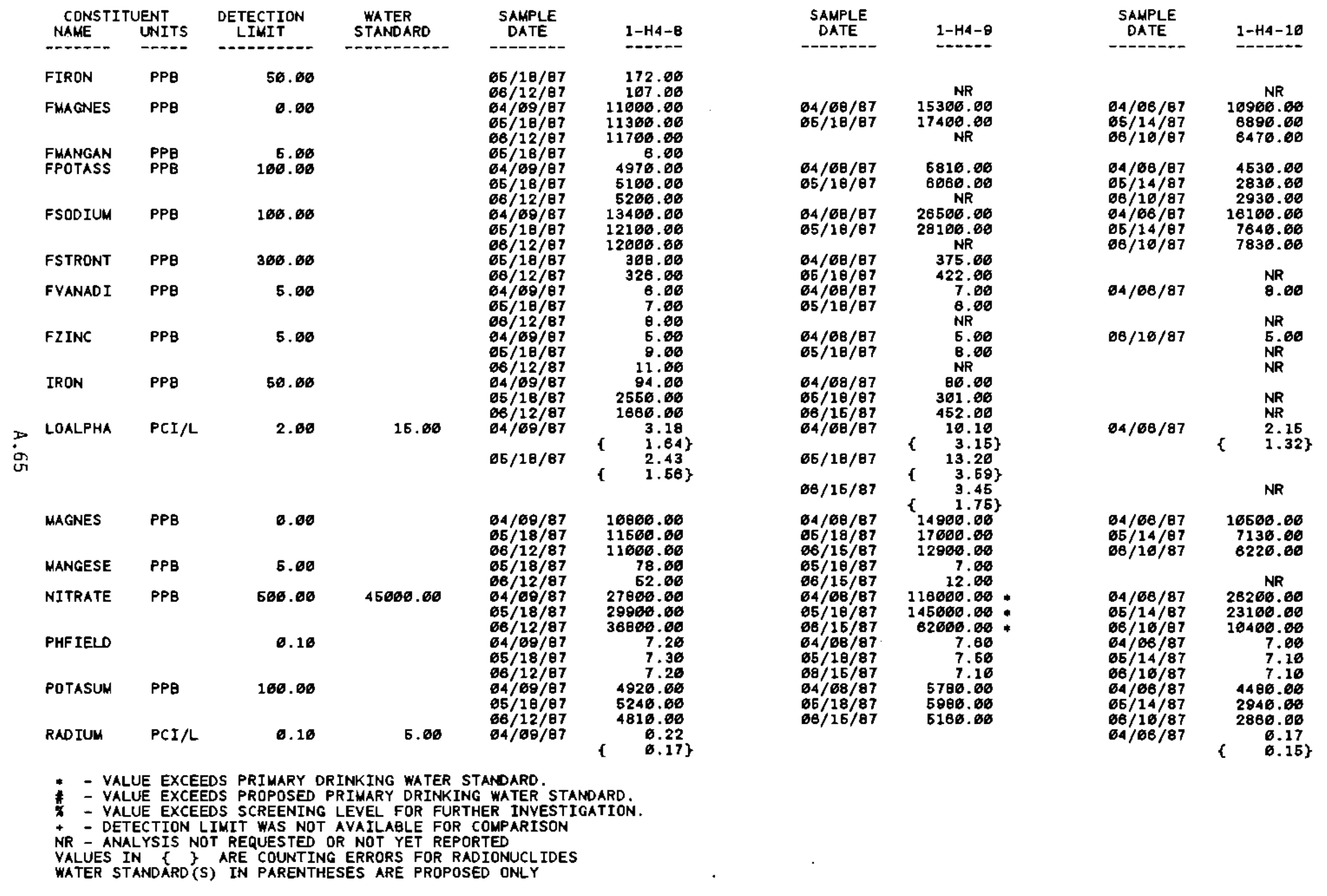


TABLE A.5. (contd)

\begin{tabular}{|c|c|c|c|c|c|}
\hline $\begin{array}{l}\text { CONSTI } \\
\text { NAME }\end{array}$ & $\begin{array}{l}\text { JENT } \\
\text { UNITS }\end{array}$ & $\begin{array}{l}\text { DETECTION } \\
\text { LIMIT }\end{array}$ & $\begin{array}{l}\text { WATER } \\
\text { STANDARD }\end{array}$ & $\begin{array}{l}\text { SAMPLE } \\
\text { DATE }\end{array}$ & I-H4-B \\
\hline RADIUM & $P C I / L$ & 0.10 & \multirow[t]{7}{*}{5.00} & $05 / 16 / 87$ & 0.28 \\
\hline SODIUH & PPB & 100.00 & & $\begin{array}{l}64 / 09 / 87 \\
65 / 18 / 87 \\
\emptyset 6 / 12 / 87\end{array}$ & $\begin{array}{l}13400.00 \\
12300.00 \\
10000.00\end{array}$ \\
\hline STRONUM & PPB & 300.00 & & $\begin{array}{l}05 / 18 / 87 \\
06 / 12 / 87\end{array}$ & $\begin{array}{c}323.00 \\
3 \emptyset 1.0 \emptyset \\
\text { NR }\end{array}$ \\
\hline SULFATE & PPE & 500.00 & & $\begin{array}{l}04 / 69 / 87 \\
85 / 18 / 87 \\
06 / 12 / 87\end{array}$ & $\begin{array}{l}58600.00 \\
62900.00 \\
68700.00\end{array}$ \\
\hline$T D C$ & PPB & 200.00 & & $\begin{array}{l}04 / 09 / 67 \\
06 / 18 / 87 \\
66 / 12 / 67\end{array}$ & $\begin{array}{r}915.00 \\
764.00 \\
1050.06\end{array}$ \\
\hline VANADUM & PPB & 5.00 & & $\begin{array}{l}64 / 09 / 87 \\
65 / 18 / 87 \\
68 / 12 / 87\end{array}$ & $\begin{array}{r}6.00 \\
15.00 \\
12.00\end{array}$ \\
\hline ZINC & PPB & 5.80 & & $\begin{array}{l}04 / 09 / 87 \\
05 / 10 / 87 \\
06 / 12 / 87\end{array}$ & $\begin{array}{l}13.00 \\
15.00 \\
10.00\end{array}$ \\
\hline
\end{tabular}

\begin{tabular}{|c|c|}
\hline $\begin{array}{l}\text { SAHPLE } \\
\text { DATE }\end{array}$ & I-H4-9 \\
\hline & NR \\
\hline $\begin{array}{l}04 / 08 / 87 \\
65 / 18 / \theta 7 \\
06 / 15 / 87 \\
64 / 08 / 87 \\
66 / 18 / 87 \\
66 / 15 / 87 \\
64 / 08 / 87 \\
65 / 18 / 87 \\
66 / 15 / 87 \\
64 / 68 / 87 \\
65 / 16 / 97 \\
\emptyset 6 / 15 / 87 \\
04 / 68 / 87 \\
66 / 18 / 87\end{array}$ & $\begin{array}{r}26960.00 \\
26800.00 \\
19660.00 \\
383.06 \\
428.06 \\
318.00 \\
64060.00 \\
71500.00 \\
70100.00 \\
651.00 \\
921.00 \\
904.00 \\
7.00 \\
9.00\end{array}$ \\
\hline $\begin{array}{l}04 / 08 / 87 \\
65 / 18 / 87 \\
68 / 15 / 87\end{array}$ & $\begin{array}{r}6.66 \\
5.66 \\
12.60\end{array}$ \\
\hline
\end{tabular}

\begin{tabular}{|c|c|}
\hline $\begin{array}{l}\text { SAMPLE } \\
\text { DATE }\end{array}$ & $1-\mathrm{H} 4-10$ \\
\hline $65 / 14 / 87$ & 6.11 \\
\hline $\begin{array}{l}64 / 68 / 87 \\
65 / 14 / 87 \\
68 / 16 / 87\end{array}$ & $\begin{array}{r}15860.06 \\
77.60 .06 \\
7890.00\end{array}$ \\
\hline & $\begin{array}{c}\text { NR } \\
\text { NR }\end{array}$ \\
\hline $\begin{array}{l}04 / 06 / 87 \\
06 / 14 / 87 \\
06 / 10 / 87 \\
64 / 06 / 87 \\
65 / 14 / 87 \\
66 / 16 / 87 \\
64 / 66 / 87 \\
66 / 14 / 87\end{array}$ & $\begin{array}{r}43200.00 \\
23200.00 \\
21700.00 \\
637.00 \\
801.00 \\
011.00 \\
8.00 \\
5.00\end{array}$ \\
\hline $\begin{array}{l}04 / 68 / 87 \\
06 / 14 / 87 \\
08 / 10 / 87\end{array}$ & $\begin{array}{r}12.00 \\
6.00 \\
11.00\end{array}$ \\
\hline
\end{tabular}

* - VALUE EXCEEDS PRIMARY DRINKING waTER STANDARD.

- VALUE EXCEEDS PROPOSED PRIMARY DRINKING WATER STANDARD.

- VALUE EXCEEDS SCREENING LEVEL FOR FURTHER INVESTIGATION.

* - DETECTION LIMIT WAS NOT AVAILABLE FOR COMPARISON

NR - ANALYSIS NOT REQUESTED OR NOT YET REPORTED

VALUES IN \{\} ARE COUNTING ERRORS FOR RADIDNUCLIDES

WATER STANDARO'(S) IN PARENTHESES ARE PROPOSED ONLY 
TABLE A.5. (cont $d$ )

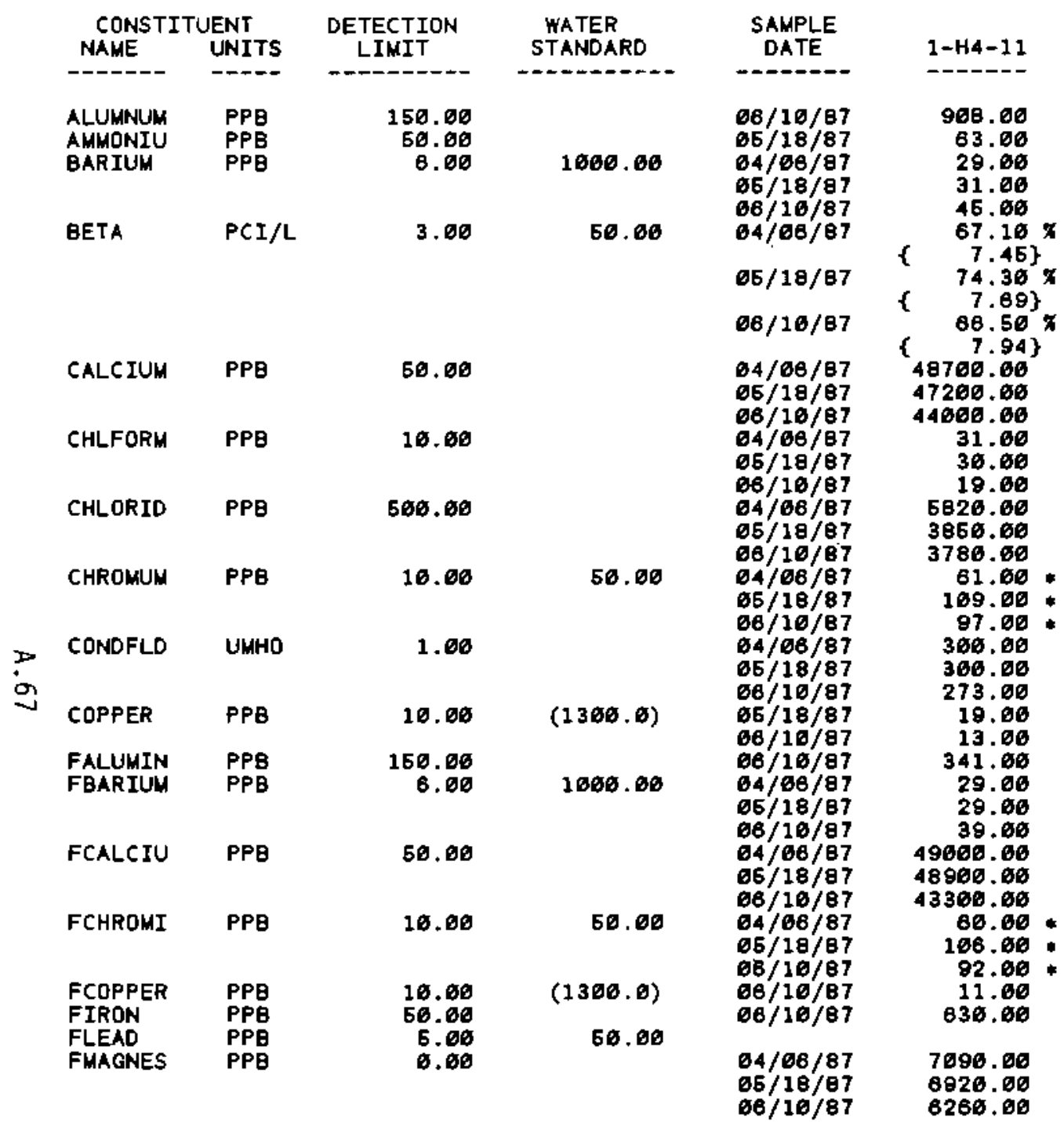

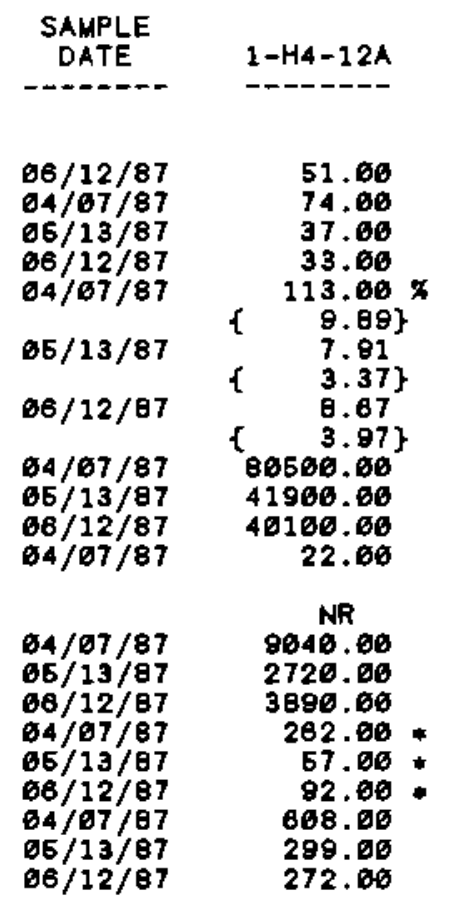

NR

04/07/87

$05 / 13 / 87$

$06 / 12 / 87$

$04 / 67 / 87$

$05 / 13 / 87$

$60 / 12 / 87$

$04 / 67 / 87$

$05 / 13 / 87$

$65 / 13 / 87$
$66 / 12 / 87$

$64 / 67 / 87$

$64 / 07 / 67$

$65 / 13 / 87$
$66 / 12 / 87$

77.0

34.00

000

80000.

40700.00

281.00

46.00

93.00

5.60

12200.60

7240.000
SAMPLE DATE

1-H4-12B
$9 / 67 / 87$
$6 / 13 / 67$ $6 / 12 / 87$

$04 / 07 / B 7$

$65 / 13 / 87$

$68 / 12 / 87$

$04 / 67 / 87$

$65 / 13 / 87$

$66 / 12 / 87$

$04 / 67 / 87$

$65 / 13 / 87$

$66 / 12 / 87$

$64 / 67 / 87$

$66 / 13 / 87$

$6 / 12 / 87$

$4 / 67 / 87$

$65 / 13 / 87$

$66 / 12 / 87$

$64 / 67 / 87$

$06 / 13 / 87$

$66 / 12 / 87$

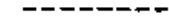

$04 / 07 / 07$

$5 / 13 / 87$

$66 / 12 / 87$

$04 / 07 / 87$

$65 / 13 / 87$

$06 / 12 / 87$

$44 / 07 / 8$

$06 / 12 / 87$

99.00

00.00

73.00
$54.86 \times$

$\{\quad 7.06\}$

$78.76 \%$

$\{\quad 7.92\}$

( 5.10 \}

73400.00

55406.00

5506.00

(1)

20.06

12.06

12.00

7540.06

4280.00

5090.00

220.00 *

162.00 *

158.00 .

639.06

351.00

331.60

NR

105.00

75.01

72.60

72900.00

54000.60

51500.60

229.00

168.00

$04 / 67 / 87$ $65 / 13 / 87$

\subsection{0 \\ 8660.00}

* - Value exceeds PRIMARY dRINking water STANDaro.

- VALUE EXCEEDS PROPOSED PRIMARY DRINKING WATER STANDARD.

- VALUE EXCEEDS SCREENING LEVEL FOR FURTHER INVESTIGATION.

* DETECTION LIMIT WAS NOT AVAILABLE FOR COMPARISON

NR - ANALYSIS NOT REQUESTED OR NOT YET REPORTED

VALUES IN \{\} ARE COUNTING ERRORS FOR RADIONUCLIDES

WATER STANDARD(S) IN PARENTHESES ARE PROPOSED ONLY 
TABLE A.5. (contd)

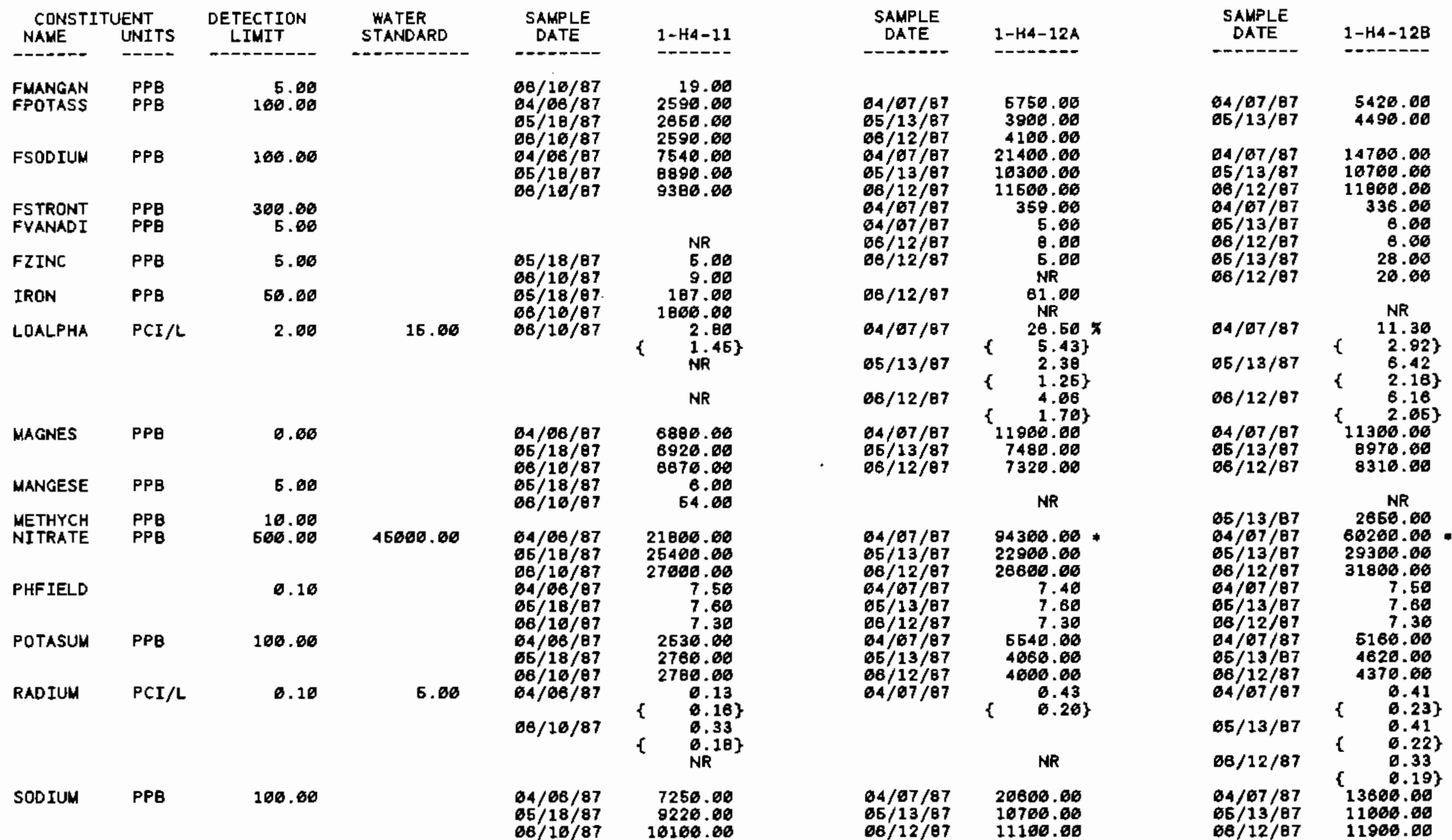

* - VAlUe EXCEeds PRIMARY DRINKING hater STANDARD.

* - VALUE EXCEEDS PROPOSED PRIMARY DRINKING WATER STANDARD.

* VALUE EXCEEDS SCREENING LEVEL FOR FURTHER INVESTIGATION.

* - DETECTION LIMIT WAS NOT AVAILABLE FOR COMPARISON

NR - ANALYSIS NOT REQUESTED OR NOT YET REPORTED

VALUES IN \{\} ARE COUNTING ERRORS FOR RADIONUCLIDES

WATER STANDARD (S) IN PARENTHESES ARE PROPOSED ONLY 
TABLE A.5. (contd)

\begin{tabular}{|c|c|c|c|c|c|c|c|}
\hline $\begin{array}{l}\text { CONSTI } \\
\text { NAME }\end{array}$ & $\begin{array}{l}\text { UENT } \\
\text { UNITS }\end{array}$ & $\begin{array}{l}\text { DETECTION } \\
\text { LIMIT }\end{array}$ & $\begin{array}{l}\text { WATER } \\
\text { STANDARD }\end{array}$ & $\begin{array}{l}\text { SAMPLE } \\
\text { DATE }\end{array}$ & $1-\mathrm{H} 4-11$ & $\begin{array}{l}\text { SAMPLE } \\
\text { DATE }\end{array}$ & $1-H 4-12 A$ \\
\hline$-\infty-\infty--$ & $-\infty .-$ & $-\cdots---\infty$ & $-\infty .-1-0-1$ & $-\infty \ldots-\infty$ & $--n--0$ & ------ & 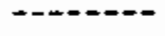 \\
\hline $\begin{array}{l}\text { STRONUM } \\
\text { SULFATE }\end{array}$ & $\begin{array}{l}\text { PPB } \\
\text { PPB }\end{array}$ & $\begin{array}{l}300.00 \\
500.00\end{array}$ & & $\begin{array}{l}64 / 06 / 87 \\
65 / 18 / 87 \\
06 / 10 / 87 \\
64 / 06 / 87 \\
65 / 18 / 87 \\
66 / 16 / 87\end{array}$ & $\begin{array}{r}38400.00 \\
37300.00 \\
32900.00 \\
735.00 \\
583.00 \\
988.00\end{array}$ & $\begin{array}{l}04 / 67 / 87 \\
64 / 67 / 87 \\
65 / 13 / 87 \\
66 / 12 / 87 \\
64 / 67 / 87 \\
66 / 13 / 87 \\
06 / 12 / 87\end{array}$ & $\begin{array}{r}357.00 \\
48300.00 \\
29400.00 \\
32400.00 \\
863.00 \\
942.00 \\
748.00\end{array}$ \\
\hline $\begin{array}{l}\text { TOX } \\
\text { VANADUM }\end{array}$ & $\begin{array}{l}\text { PPB } \\
\text { PPB }\end{array}$ & $\begin{array}{r}100.00 \\
5.00\end{array}$ & & $\begin{array}{l}64 / 66 / 87 \\
66 / 18 / 87 \\
66 / 10 / 87 \\
64 / 66 / 87 \\
65 / 18 / 87 \\
66 / 10 / 87\end{array}$ & $\begin{array}{r}6.00 \\
7.00 \\
6.00 \\
10.00 \\
15.00\end{array}$ & $\begin{array}{l}04 / 07 / 87 \\
05 / 13 / 87 \\
06 / 12 / 87 \\
06 / 12 / 87\end{array}$ & $\begin{array}{l}5.00 \\
10.00 \\
0.00 \\
9.00 \\
\text { NR } \\
\text { NR }\end{array}$ \\
\hline
\end{tabular}

SAMPLE DATE

$1-\mathrm{H} 4-12 \mathrm{~B}$

$64 / 07 / 87$

$04 / 67 / 87$

$65 / 13 / 87$

$08 / 12 / 87$

$64 / 67 / 87$

$65 / 13 / 87$

$65 / 13 / 87$

$04 / 67 / 87$

$05 / 13 / 87$

$66 / 12 / 87$

$64 / 67 / 87$

$05 / 13 / 87$

$65 / 13 / 87$
$68 / 12 / 87$

327.00

50600.00

38400.00

47160.00

824.06

719.00

839.00

3260.60

5.66

5.60
8.00

9.00

5.66

5.66

41.00
24.06

* - VALUE EXCEEDS PRIMARY DRINKING WATER STANDARD.

* - VALUE EXCEEDS PROPOSED PRIMARY DRINKING WATER STANDARO.

* - VALUE EXCEDDS SCREENING LEVEL FOR FURTHER INVESTIGATION.

+ - DETECTION LIMIT WAS NOT AVAILABLE FOR COMPARISON

NR - ANALYSIS NOT REQUESTED OR NOT YET REPORTED

VALUES IN \{ $\}$ ARE COUNTING ERRORS FOR RADIONUCLIDES

WATER STANDARD(5) IN PARENTHESES ARE PROPOSED ONLY 
IABLE A.5. (contd)

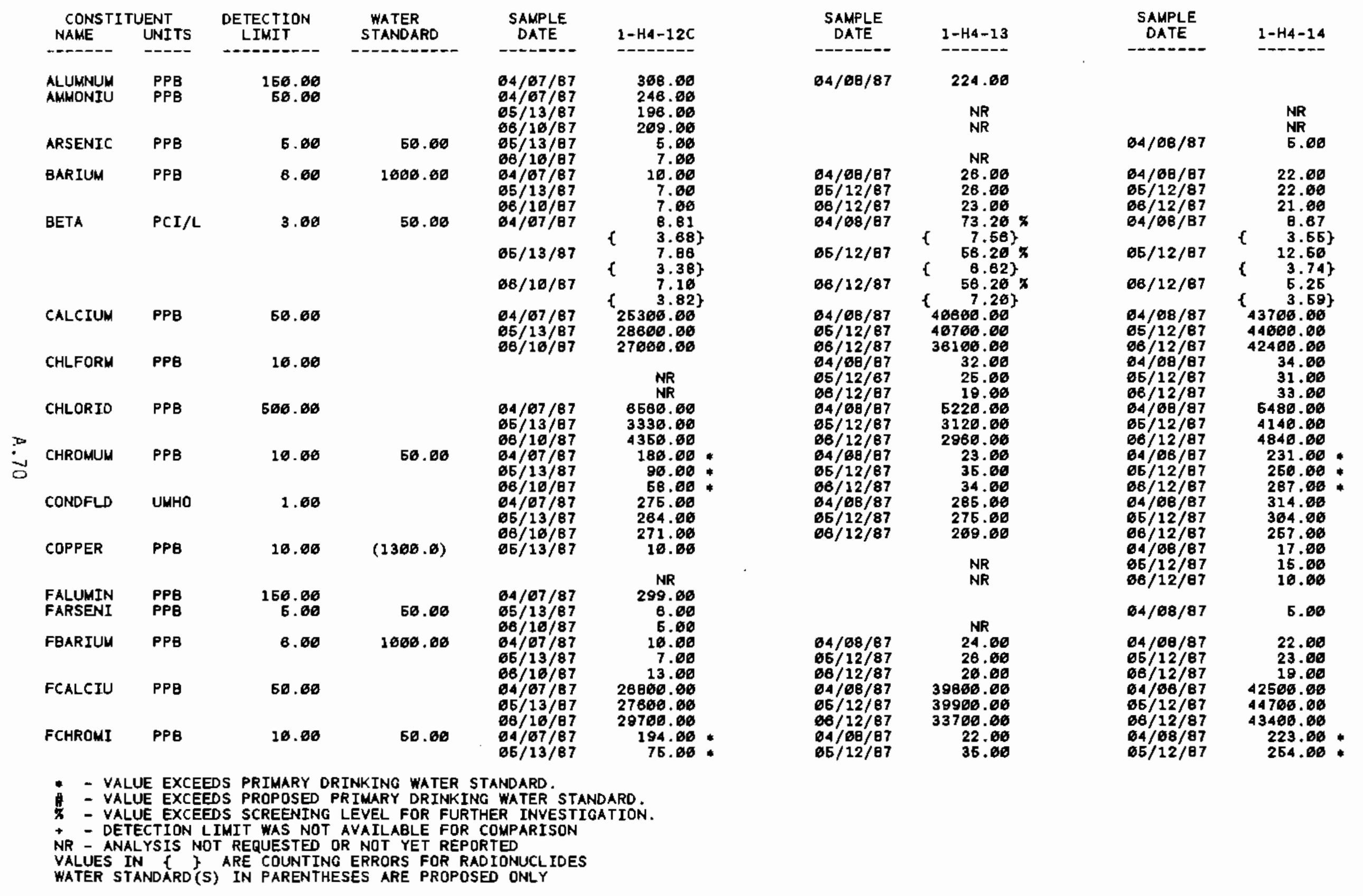


TABL.E A.5. (contd)

\begin{tabular}{|c|c|c|c|c|c|c|c|c|c|}
\hline $\begin{array}{l}\text { CONSTI } \\
\text { NAME }\end{array}$ & $\begin{array}{l}\text { UENT } \\
\text { UNITS }\end{array}$ & $\begin{array}{c}\text { DETECTION } \\
\text { LIMIT }\end{array}$ & $\begin{array}{l}\text { WATER } \\
\text { STANDARD }\end{array}$ & $\begin{array}{c}\text { SAMPLE } \\
\text { DATE }\end{array}$ & $1-\mathrm{H}_{4}-12 \mathrm{C}$ & $\begin{array}{l}\text { SAMPLE } \\
\text { OATE }\end{array}$ & $1-H 4-13$ & $\begin{array}{l}\text { SAMPLE } \\
\text { DATE }\end{array}$ & $1-H 4-14$ \\
\hline FCHROMI & $\begin{array}{l}P P B \\
P P A\end{array}$ & 16.00 & \multirow[t]{9}{*}{50.00} & \multirow{9}{*}{ 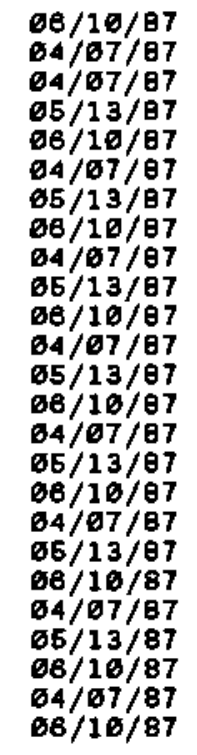 } & \multirow{4}{*}{$\begin{array}{r}54.00 \\
512.00 \\
11560.06 \\
11066.00 \\
11360.00 \\
45.00 \\
28.00 \\
35.06 \\
34.06 \\
11.06 \\
17.06\end{array}$} & \multirow{4}{*}{$\begin{array}{l}06 / 12 / 87 \\
04 / 60 / 87 \\
04 / 68 / 87 \\
06 / 12 / 87 \\
06 / 12 / 87\end{array}$} & 28.68 & $66 / 12 / 87$ & 268.00 \\
\hline $\begin{array}{l}\text { FIRON } \\
\text { FWAGNES }\end{array}$ & $\begin{array}{l}\mathrm{PPB} \\
\mathrm{PPB}\end{array}$ & $\begin{array}{r}50.00 \\
0.00\end{array}$ & & & & & $\begin{array}{l}6590.06 \\
6736.00 \\
5586.00\end{array}$ & $\begin{array}{l}04 / 98 / 87 \\
66 / 12 / 87 \\
06 / 12 / 87\end{array}$ & $\begin{array}{l}8200.06 \\
8630.06 \\
8010.00\end{array}$ \\
\hline FMANGAN & PPB & 5.00 & & & & & $\begin{array}{l}\text { NR } \\
\text { NR }\end{array}$ & & $\begin{array}{l}\text { NR } \\
\text { NR }\end{array}$ \\
\hline FNICKEL & PPB & 10.00 & & & & & $\begin{array}{l}\text { NR } \\
\text { NR }\end{array}$ & & NR \\
\hline FPOTASS & PPB & 100.00 & & & $\begin{array}{l}5336.08 \\
5320.06 \\
5286.00\end{array}$ & $\begin{array}{l}64 / 68 / 87 \\
65 / 12 / 87 \\
66 / 12 / 87\end{array}$ & $\begin{array}{l}2226.00 \\
2220.06 \\
2836.00\end{array}$ & $\begin{array}{l}04 / 00 / 87 \\
05 / 12 / 97 \\
06 / 12 / 87\end{array}$ & $\begin{array}{l}4190.00 \\
4250.00 \\
4000.00\end{array}$ \\
\hline FSODIUM & PPB & 100.00 & & & $\begin{array}{l}14600.00 \\
12200.06 \\
11200.00\end{array}$ & $\begin{array}{l}64 / 98 / 67 \\
65 / 12 / 87 \\
66 / 12 / 87\end{array}$ & $\begin{array}{l}7210.00 \\
8360.00 \\
5590.00\end{array}$ & $\begin{array}{l}04 / 08 / 87 \\
05 / 12 / \theta 7 \\
\theta 6 / 12 / 87\end{array}$ & $\begin{array}{l}8020.00 \\
8070.00 \\
7270.00\end{array}$ \\
\hline FVANADI & PPB & 6.60 & & & $\begin{array}{l}21.06 \\
14.00 \\
12.06\end{array}$ & $\begin{array}{l}64 / 08 / 87 \\
65 / 12 / 87\end{array}$ & $\begin{array}{l}6.00 \\
5.00\end{array}$ & $\begin{array}{l}04 / 08 / 87 \\
05 / 12 / \theta 7\end{array}$ & $\begin{array}{l}6.00 \\
8.00\end{array}$ \\
\hline FZINC & PPB & 5.00 & & & $\begin{array}{r}8 . \varnothing \varnothing \\
33 . \varnothing \varnothing \\
18.0 \varnothing\end{array}$ & $\begin{array}{l}65 / 12 / 87 \\
68 / 12 / 87\end{array}$ & $\begin{array}{l}17.00 \\
19.06 \\
\mathrm{NR}\end{array}$ & $66 / 12 / 87$ & $\begin{array}{c}10.00 \\
\text { NR } \\
\text { NR }\end{array}$ \\
\hline IRON & PPB & 50.06 & & & $\begin{array}{l}521.00 \\
124.60 \\
\text { NR }\end{array}$ & $\begin{array}{l}04 / 98 / 97 \\
65 / 12 / 97 \\
66 / 12 / 97\end{array}$ & $\begin{array}{l}488.06 \\
158.00 \\
105.00\end{array}$ & $\begin{array}{l}04 / 00 / 87 \\
65 / 12 / 87\end{array}$ & $\begin{array}{r}59.66 \\
52.06\end{array}$ \\
\hline LOALPHA & $\mathrm{PCI} / \mathrm{L}$ & 2.00 & 15.00 & $64 / 07 / 87$ & $\begin{array}{r}2.71 \\
1.387\end{array}$ & $64 / 88 / 87$ & $\begin{array}{r}100.60 \\
\quad 2.70 \\
\end{array}$ & $B 8 / 12 / 87$ & 221.80 \\
\hline MAGNES & PPB & 0.00 & & $\begin{array}{l}04 / 67 / 87 \\
65 / 13 / 87 \\
68 / 10 / 67\end{array}$ & $\begin{array}{l}11200.00 \\
11400.00 \\
10900.00\end{array}$ & $\begin{array}{l}04 / 08 / 97 \\
65 / 12 / 87 \\
06 / 12 / 87\end{array}$ & $\begin{array}{l}6820.06 \\
6856.60 \\
6140.60\end{array}$ & $\begin{array}{l}84 / 08 / 87 \\
65 / 12 / 67 \\
66 / 12 / 87\end{array}$ & $\begin{array}{l}8480.00 \\
8576.00 \\
7970.00\end{array}$ \\
\hline MANGESE & PPB & 5.00 & & $\begin{array}{l}04 / 07 / 87 \\
65 / 13 / 87 \\
08 / 16 / 87\end{array}$ & $\begin{array}{r}44.00 \\
29.00 \\
33.00\end{array}$ & $04 / 88 / 97$ & 13.00 & & $\begin{array}{l}\text { NR } \\
\text { NR }\end{array}$ \\
\hline $\begin{array}{l}\text { METHYCH } \\
\text { NICKEL }\end{array}$ & $\begin{array}{l}\mathrm{PPB} \\
\mathrm{PPB}\end{array}$ & $\begin{array}{l}10.00 \\
10.00\end{array}$ & & $\begin{array}{l}65 / 13 / 87 \\
64 / 67 / 87 \\
65 / 13 / 67 \\
66 / 10 / 67\end{array}$ & $\begin{array}{r}410.00 \\
29.00 \\
16.00 \\
22.00\end{array}$ & & $\begin{array}{l}\mathrm{NR} \\
\mathrm{NR}\end{array}$ & $\begin{array}{l}65 / 12 / 87 \\
66 / 12 / 07\end{array}$ & $\begin{array}{c}37.00 \\
11.00 \\
\text { NR } \\
\text { NR }\end{array}$ \\
\hline NITRATE & PPB & 500.00 & 46000.00 & $\begin{array}{l}64 / 67 / 67 \\
65 / 13 / 87 \\
66 / 16 / 87\end{array}$ & $\begin{array}{l}4780.08 \\
3810.08 \\
2640.08\end{array}$ & $\begin{array}{l}64 / 98 / 87 \\
86 / 12 / 87 \\
68 / 12 / 87\end{array}$ & $\begin{array}{l}22006.06 \\
19706.06 \\
12100.00\end{array}$ & $\begin{array}{l}04 / 08 / 87 \\
06 / 12 / 97 \\
06 / 12 / 87\end{array}$ & $\begin{array}{l}18400.06 \\
26506.06 \\
19900.06\end{array}$ \\
\hline PHFIELD & PPQ & $10 \varnothing . \varnothing 0$ & & $\begin{array}{l}64 / 67 / 87 \\
65 / 13 / 87 \\
66 / 16 / 87 \\
64 / 87 / 87\end{array}$ & $\begin{array}{r}7.16 \\
7.50 \\
73.20 \\
5300.00\end{array}$ & $\begin{array}{l}64 / 68 / 87 \\
65 / 12 / 87 \\
68 / 12 / 87 \\
64 / 68 / 87\end{array}$ & $\begin{array}{r}7.10 \\
7.26 \\
8.96 \\
2316.06\end{array}$ & $\begin{array}{l}64 / 60 / 87 \\
65 / 12 / 87 \\
68 / 12 / 67 \\
04 / 68 / 87\end{array}$ & $\begin{array}{r}7.80 \\
7.86 \\
7.46 \\
4360.06\end{array}$ \\
\hline 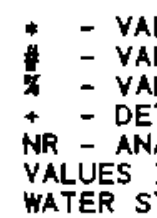 & $\begin{array}{l}\text { UE EXCEE } \\
\text { ECTION L } \\
\text { LYSIS NO } \\
\text { NS }\} \\
\text { ANDARD 's }\end{array}$ & $\begin{array}{l}\text { DS PRIMARY D } \\
\text { DS PROPOSED } \\
\text { DS SCREENING } \\
\text { IUIT WAS NOT } \\
\text { TREQUESTED } \\
\text { ARE COUNTIN } \\
\text { IN PARENTH }\end{array}$ & 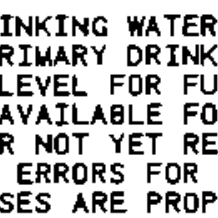 & $\begin{array}{l}\text { ANDARD. } \\
\text { WATER STA } \\
\text { ER INVESTI } \\
\text { OMPARISON } \\
\text { TED } \\
\text { IONUCLIDES } \\
\text { D ONLY }\end{array}$ & ARD. & & & & \\
\hline
\end{tabular}


TABLE A.5. (cont $d$ )

\begin{tabular}{llr}
$\begin{array}{c}\text { CONSTITUENT } \\
\text { NAKE }\end{array}$ & UNITS & \multicolumn{1}{r}{$\begin{array}{r}\text { DETECTION } \\
\text { LIMIT }\end{array}$} \\
\hline POTASUM & PPB & 100.00 \\
RADIUM & PCI/L & 0.10 \\
& & \\
SODIUM & PPB & 106.60 \\
SULFATE & PPB & 506.00 \\
TOC & PPE & 260.00 \\
TOX & PPE & 100.06 \\
VANADUM & PPB & 5.00 \\
ZINC & PPB & 5.60 \\
& &
\end{tabular}

WATER
STANDARO
5.06

SAMPLE DATE

$1-144-12 C$

อ5/13/87

$66 / 16 / 87$
$64 / 07 / 87$

6480.06

6280.60

0.26
$1 \quad 0.17$

$65 / 13 / 87$

$66 / 16 / 87$

$64 / 67 / 87$

$66 / 13 / 87$

$06 / 16 / 87$

$64 / 07 / 87$

$65 / 13 / 87$

$06 / 16 / 87$

$04 / 87 / 87$

$05 / 13 / 87$

$86 / 16 / 97$

$65 / 13 / 87$

$04 / 67 / 87$

$65 / 13 / 97$

66/18/87

$64 / 67 / 87$

$06 / 13 / 87$
$66 / 16 / 87$

0.17

$\{\quad 0.14\}$

$1 \quad .13\}$

14506.06

1160000

24206.00

24260.06

27760.68

547.00

603.00

616.60

312.00

22.00

18.00

10.00
6.00

5.06
44.00

44.00
27.00
SAMPLE DATE

1-H4-13

\section{$06 / 12 / 87$}

96/12/87

04/08/87

$\begin{array}{lll}05 / 12 / 97 & \{.33 \\ & \{\quad 0.18\}\end{array}$

2270.00
2250.60

0.10

$04 / 08 / 87 \quad 7356.06$

8490.00

$04 / 88 / 87 \quad 840.0$

$05 / 12 / 87$

$01 / 12 / 8727700.00$

$4 / 12 / 97 \quad 27700.00$

4787

$65 / 12 / 87$

$66 / 12 / 87$

869.06

$64 / 08 / 87$
$65 / 12 / 87$

5.00

$04 / 08 / 87$

$05 / 12 / 87$

$06 / 12 / \theta 7$

5.00

23.60
SAMPLE DATE

$1-\mathrm{H4}-14$

$06 / 12 / 87$
$04 / 69 / 87$

$05 / 12 / 87$

$66 / 12 / 8$ ?

$64 / 08 / 87$

$66 / 12 / 87$

$88 / 12 / 87$

$64 / 88 / 87$

$85 / 127$

$65 / 12 / 87$

$0412 / 87$

$95 / 127$

96/12/87

$04 / 08 / 87$

$65 / 12 / 87$

$08 / 12 / 87$

$64 / 68 / 87$

$66 / 12 / 87$

66/12/87

4180.00

4010.00

$\{\quad 0.15\}$

$\{\quad 0.13\}$

( 0.13

0286.06

7986.60

7280.60

1800

41800.00

38106.00

636.00

636.06 849.00
800.00

9.00

5.60

5.60

11.00

23.06
15.60
$05 / 12 / 87$

- - VALUE exceEDS PRIMARY DRINKINg water STANDARD.

* - VALUE EXCEEDS PROPOSED PRIMARY DRINKING WATER STANDARD.

- VALUE EXCEEDS SCREENING LEVEL FOR FURTHER INVESTIGATION.

+ - DETECTION LIMIT WAS NOT AVAILABLE FOR COMPARISON

NR - ANALYSIS NOT REQUESTED OR NOT YET REPORTED

VALUES IN \{\} ARE COUNTING ERRORS FOR RADIDNUCLIDES

WATER STANDARD(S) IN PARENTHESES ARE PROPOSED ONLY 
TABLE A.5. (contd)

\begin{tabular}{|c|c|c|c|c|c|c|c|c|c|}
\hline $\begin{array}{l}\text { CONST1 } \\
\text { NAME }\end{array}$ & $\begin{array}{l}\text { UENT } \\
\text { UNITS }\end{array}$ & $\begin{array}{l}\text { DETECTION } \\
\text { LIMIT }\end{array}$ & $\begin{array}{l}\text { WATER } \\
\text { STANDARD }\end{array}$ & $\begin{array}{l}\text { SAMPLE } \\
\text { DATE }\end{array}$ & $1-H 4-15 A$ & $\begin{array}{l}\text { SAMPLE } \\
\text { DATE }\end{array}$ & $1-H 4-15 B$ & $\begin{array}{l}\text { SAMPLE } \\
\text { DATE }\end{array}$ & $1-\mathrm{H} 4-16$ \\
\hline ALUMNUM & PPB & 150.00 & & $65 / 14 / 87$ & 549.00 & & NR & $\begin{array}{l}65 / 97 / 87 \\
68 / 15 / B 7\end{array}$ & $\begin{array}{r}1180.00 \\
822.00\end{array}$ \\
\hline $\begin{array}{l}\text { AMMONIU } \\
\text { BARIUM }\end{array}$ & $\begin{array}{l}\text { PPB } \\
\text { PPB }\end{array}$ & $\begin{array}{r}50.06 \\
6.00\end{array}$ & 1000.00 & $\begin{array}{l}64 / 69 / 87 \\
65 / 14 / 87 \\
68 / 11 / 87\end{array}$ & $\begin{array}{l}77.06 \\
67.06 \\
76.06\end{array}$ & $\begin{array}{l}04 / 09 / 87 \\
65 / 14 / 87 \\
06 / 11 / 87\end{array}$ & $\begin{array}{l}102.00 \\
167.00 \\
109.00\end{array}$ & $\begin{array}{l}06 / 15 / 87 \\
66 / 07 / 87 \\
66 / 15 / 87\end{array}$ & $\begin{array}{l}74.00 \\
39.06 \\
48.00 \\
\text { NR }\end{array}$ \\
\hline BETA & $P C I / L$ & 3.66 & 60.00 & $64 / 69 / 87$ & $\begin{array}{c}0.68 \\
3.74\}\end{array}$ & $04 / 69 / 87$ & $\begin{array}{c}16.96 \\
3.99\}\end{array}$ & $65 / 07 / 07$ & 18.96 \\
\hline & & & & $05 / 14 / 87$ & 6.68 & $65 / 14 / 87$ & 10.96 & $06 / 15 / 97$ & $71,70^{5}$ \\
\hline & & & & $00 / 11 / 87$ & $\begin{array}{r}3.223 \\
10.26 \\
4.147\end{array}$ & $06 / 11 / 87$ & $\begin{array}{r}3.71\} \\
\{\quad 3.63 \\
\{\quad 3.98\}\end{array}$ & & $\stackrel{B}{R} \cdot$ \\
\hline CALCIUM & PPB & 50.00 & & $\begin{array}{l}04 / 09 / 87 \\
05 / 14 / 87 \\
06 / 11 / 87\end{array}$ & $\begin{array}{l}48600.06 \\
42106.60 \\
53860.06\end{array}$ & $\begin{array}{l}64 / 69 / 87 \\
65 / 14 / 87 \\
66 / 11 / 87\end{array}$ & $\begin{array}{l}49000.00 \\
49400.00 \\
49200.00\end{array}$ & $\begin{array}{l}65 / 67 / 87 \\
66 / 15 / 87\end{array}$ & $\begin{array}{c}40000.00 \\
58606.00 \\
\mathrm{NR}\end{array}$ \\
\hline $\begin{array}{l}\text { CHLFORM } \\
\text { CHLORID }\end{array}$ & $\begin{array}{l}\text { PPB } \\
\text { PPB }\end{array}$ & $\begin{array}{r}10.00 \\
500.06\end{array}$ & & $\begin{array}{l}04 / 09 / 87 \\
64 / 09 / 87 \\
65 / 14 / 67\end{array}$ & $\begin{array}{r}16.00 \\
6286.60 \\
3670.00\end{array}$ & $\begin{array}{l}64 / 09 / 67 \\
64 / 09 / 87 \\
65 / 14 / 87\end{array}$ & $\begin{array}{r}15.00 \\
5690.06 \\
6380.06\end{array}$ & $\begin{array}{l}86 / 15 / 87 \\
66 / 15 / 87\end{array}$ & $\begin{array}{c}29.00 \\
5450.00 \\
\text { NR }\end{array}$ \\
\hline CHROMUM & PPB & 16.60 & 50.00 & $\begin{array}{l}66 / 11 / 87 \\
64 / 69 / 87 \\
65 / 14 / 87\end{array}$ & $\begin{array}{r}5710.60 \\
168.60 \\
80.00\end{array}$ & $\begin{array}{l}06 / 11 / 87 \\
04 / 08 / 87 \\
65 / 14 / 87\end{array}$ & $\begin{array}{c}5770.06 \\
184.00 \\
117.00\end{array}$ & $68 / 15 / 87$ & $\begin{array}{c}\text { NR } \\
258.90 \\
N R\end{array}$ \\
\hline CONDFLD & UMHO & 1.06 & & $\begin{array}{l}66 / 11 / 87 \\
64 / 69 / 87 \\
66 / 14 / 87 \\
66 / 11 / 87\end{array}$ & $\begin{array}{l}80.00 \\
403.60 \\
313.00 \\
372.00\end{array}$ & $\begin{array}{l}08 / 11 / 87 \\
64 / 89 / 87 \\
65 / 14 / 87 \\
06 / 11 / 87\end{array}$ & $\begin{array}{l}167.00 \\
416.00 \\
367.60 \\
337.00\end{array}$ & $06 / 16 / 87$ & $\begin{array}{c}\text { NR } \\
308, \Phi \theta \\
\text { NR } \\
\text { NR }\end{array}$ \\
\hline $\begin{array}{l}\text { COPPER } \\
\text { FALUMIN } \\
\text { FARSENI }\end{array}$ & $\begin{array}{l}\text { PPB } \\
P P B \\
P P B\end{array}$ & $\begin{array}{r}10.00 \\
150.00 \\
5.00\end{array}$ & $\begin{array}{r}(1300.0) \\
50.00\end{array}$ & $\begin{array}{l}04 / 69 / 87 \\
05 / 14 / 87 \\
68 / 11 / 87\end{array}$ & $\begin{array}{r}12.06 \\
202.06 \\
5.00\end{array}$ & & & $\begin{array}{l}06 / 87 / 87 \\
66 / 15 / 87\end{array}$ & $\begin{array}{r}12.00 \\
802.06\end{array}$ \\
\hline FBARIUM & PPB & 8.06 & 1000.00 & $\begin{array}{l}04 / 69 / 87 \\
65 / 14 / 97 \\
68 / 11 / 87\end{array}$ & $\begin{array}{l}78.06 \\
82.06 \\
77.06\end{array}$ & $\begin{array}{l}64 / 68 / 87 \\
65 / 14 / 87 \\
66 / 11 / 87\end{array}$ & $\begin{array}{l}100.00 \\
110.00 \\
107.00\end{array}$ & $06 / 15 / 87$ & $\begin{array}{c}44.60 \\
\mathrm{NR} \\
\mathrm{NR}\end{array}$ \\
\hline FCALCIU & PPB & 50.60 & & $\begin{array}{l}64 / 69 / 97 \\
65 / 14 / 87 \\
66 / 11 / 87\end{array}$ & $\begin{array}{l}50200.06 \\
44460.06 \\
53900.06\end{array}$ & $\begin{array}{l}64 / 09 / 87 \\
66 / 14 / 87 \\
06 / 11 / 67\end{array}$ & $\begin{array}{l}51900.00 \\
51900.00 \\
40300.00\end{array}$ & $6 B / 15 / 87$ & $\begin{array}{c}81100.00 \\
\text { NR } \\
\text { NR }\end{array}$ \\
\hline FCHROMI & PPB & 10.00 & 56.00 & $\begin{array}{l}64 / 69 / 97 \\
65 / 14 / 97 \\
66 / 11 / 87\end{array}$ & $\begin{array}{r}188.00 \\
62.66 \\
74.60\end{array}$ & $\begin{array}{l}04 / 09 / 87 \\
66 / 14 / 87 \\
08 / 11 / 87\end{array}$ & $\begin{array}{r}171.00 \\
121.00 \\
99.00\end{array}$ & $68 / 15 / 87$ & $\begin{array}{c}282.00 \\
\text { NR } \\
\text { NR }\end{array}$ \\
\hline FIRON & PPB & 50.00 & & $\begin{array}{l}05 / 14 / 87 \\
68 / 11 / 87\end{array}$ & $\begin{array}{l}307.06 \\
124.00\end{array}$ & $66 / 11 / 87$ & ${ }_{M R}^{\theta 8}$ & $06 / 15 / 87$ & 1106.00 \\
\hline FMAGNES & PPB & 0.00 & & $\begin{array}{l}64 / 69 / 87 \\
65 / 14 / 87 \\
66 / 11 / 67\end{array}$ & $\begin{array}{r}16400.66 \\
9846.06 \\
11600.06\end{array}$ & $\begin{array}{l}04 / 69 / 87 \\
65 / 14 / 87 \\
66 / 11 / 87\end{array}$ & $\begin{array}{l}10968.00 \\
11206.06 \\
10700.00\end{array}$ & $68 / 15 / 87$ & $\begin{array}{c}10106.06 \\
N R \\
N R\end{array}$ \\
\hline $\begin{array}{l}\text { FMANGAN } \\
\text { FNICKEL }\end{array}$ & $\begin{array}{l}P P B \\
P P B\end{array}$ & $\begin{array}{r}5.60 \\
10.06\end{array}$ & & $65 / 14 / 87$ & $\begin{array}{r}11000.000 \\
7.00\end{array}$ & & & $\begin{array}{l}06 / 15 / \theta 7 \\
06 / 15 / \theta 7\end{array}$ & $\begin{array}{l}61.00 \\
21.00\end{array}$ \\
\hline
\end{tabular}

- - VALUE EXCEEDS PRIMARY DRINKING waTER STANDARD.

* - VALUE EXCEEDS PROPOSED PRIMARY DRINKING WATER STANDARD.

* - VALUE EXCEEDS SCREENING LEVEL FOR FURTHER INVESTIGATION.

* - DETECTION LIMIT WAS NOT AVAILABLE FOR COMPARISON

NR - ANALYSIS NOT REQUESTED OR NOT YET REPORTED

VALUES IN \{ $\}$ ARE COUNTING ERRORS FOR RAD IONUCLIDES

WATER STANDARD(S) IN PARENTHESES ARE PROPOSED ONLY 
TABLE A.5. (contd)

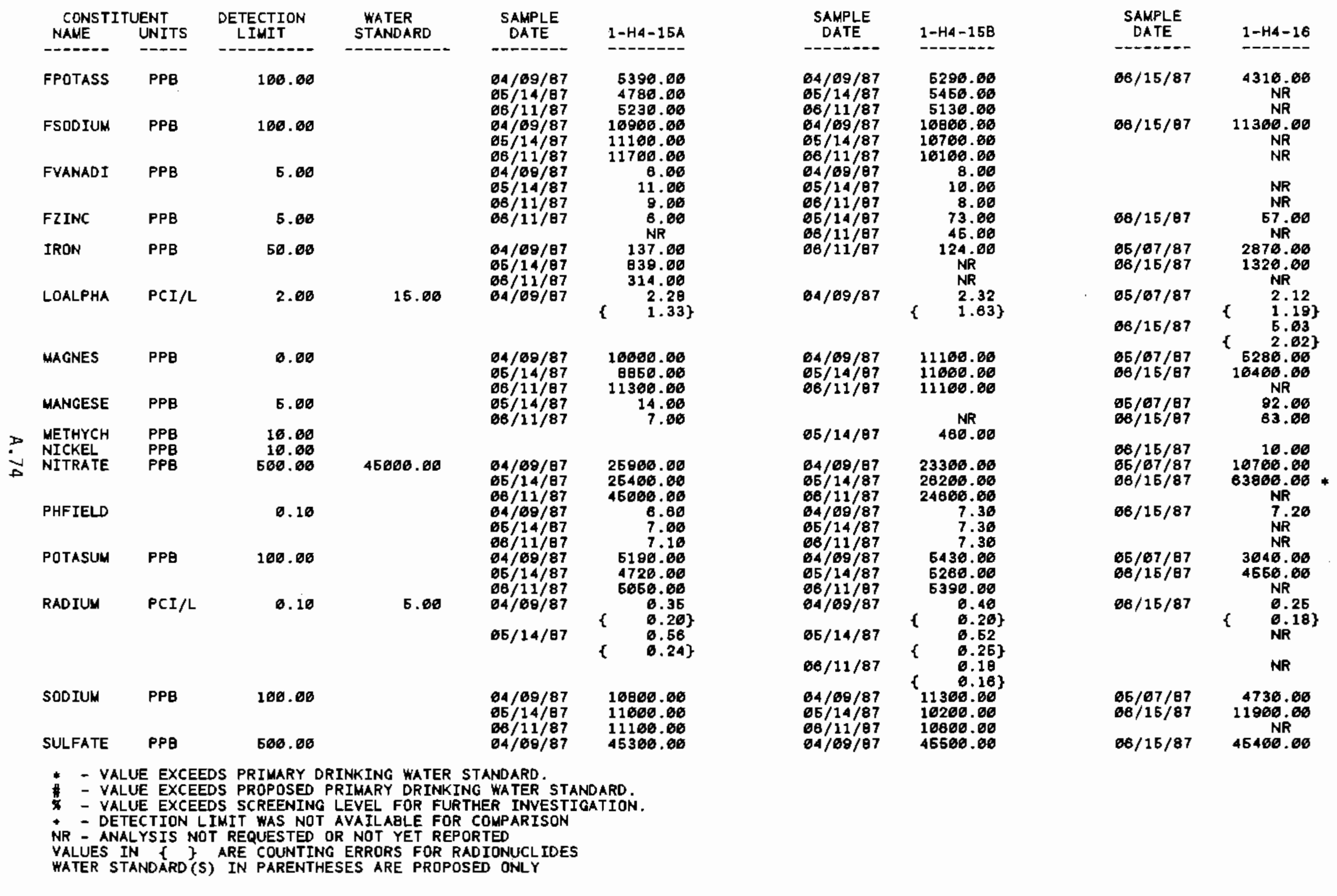


TABLE A.5. (contd)

\begin{tabular}{|c|c|c|c|c|c|c|c|c|c|}
\hline CONSTITUENT & $\begin{array}{l}\text { JENT } \\
\text { UNITS }\end{array}$ & $\begin{array}{l}\text { DETECTION } \\
\text { LIMIT }\end{array}$ & $\begin{array}{l}\text { WATER } \\
\text { STANOARO }\end{array}$ & $\begin{array}{l}\text { SAMPLE } \\
\text { DATE }\end{array}$ & $1-\mathrm{H} 4-16 \mathrm{~A}$ & $\begin{array}{l}\text { SAMPLE } \\
\text { DATE }\end{array}$ & $1-\mathrm{H}_{4}-15 B$ & $\begin{array}{l}\text { SAHPLE } \\
\text { DATE }\end{array}$ & $2-\mathrm{H} 4-16$ \\
\hline$-+-\infty-\infty$ & $-\infty-\infty$ & $-----\infty-0-1$ & $-----\infty-\infty$ & 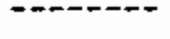 & ----- & ------- & $----2+-$ & $-+\infty+\infty-\infty$ & -----6 \\
\hline SULFATE & PPB & 500.00 & & $\begin{array}{l}05 / 14 / 87 \\
66 / 11 / 87\end{array}$ & $\begin{array}{l}32300.00 \\
52300.00\end{array}$ & $\begin{array}{l}85 / 14 / 87 \\
06 / 11 / 87\end{array}$ & $\begin{array}{l}41700.00 \\
47700.00\end{array}$ & & $\begin{array}{l}\text { NR } \\
\text { NR }\end{array}$ \\
\hline TOC & PPB & 200.00 & & $\begin{array}{l}64 / 69 / 87 \\
65 / 14 / 87 \\
66 / 11 / 87\end{array}$ & $\begin{array}{l}582.06 \\
681.00 \\
968.00\end{array}$ & $\begin{array}{l}64 / 09 / 87 \\
86 / 14 / 87 \\
66 / 11 / 87\end{array}$ & $\begin{array}{l}520.00 \\
478.00 \\
586.00\end{array}$ & $68 / 15 / 87$ & $\begin{array}{c}867.08 \\
N R \\
N R\end{array}$ \\
\hline $\begin{array}{l}\text { TDX } \\
\text { YANADUM }\end{array}$ & $\begin{array}{l}\text { PPB } \\
\text { PPB }\end{array}$ & $\begin{array}{r}100.00 \\
5.00\end{array}$ & & $04 / 69 / 87$ & 9.00 & $\begin{array}{l}B 5 / 14 / 87 \\
64 / 09 / 87\end{array}$ & $\begin{array}{r}414.00 \\
5.00\end{array}$ & $65 / 07 / 87$ & 6.00 \\
\hline ZINC & PPB & 5.00 & & $\begin{array}{l}65 / 14 / 87 \\
66 / 11 / 87 \\
64 / 89 / 87 \\
65 / 14 / 87 \\
66 / 11 / 87\end{array}$ & $\begin{array}{r}11.00 \\
7.00 \\
9.00 \\
8.00 \\
8.00\end{array}$ & $\begin{array}{l}\emptyset 5 / 14 / 87 \\
06 / 11 / 87 \\
04 / 09 / 87 \\
\square 5 / 14 / 87 \\
06 / 11 / 87\end{array}$ & $\begin{array}{r}9.00 \\
9.00 \\
8.00 \\
79.00 \\
55.00\end{array}$ & $\begin{array}{l}66 / 15 / 87 \\
85 / 67 / 87 \\
66 / 16 / 87\end{array}$ & $\begin{array}{c}9.00 \\
\text { NR } \\
9.00 \\
116.00 \\
\text { NR }\end{array}$ \\
\hline
\end{tabular}

* - VALUE EXCEEDS PROPOSED PRIMARY DRINKING WATER STANDARD.

* - VALUE EXCEEDS SCREENING LEVEL FOR FURTHER INVESTIGATION.

+ DETECTION LIMIT WAS NOT AVAILABLE FOR COMPARISON

NR - ANALYSIS NOT REQUESTED OR NOT YET REPORTED

VALUES IN \{ \} ARE COUNTING ERRORS FOR RADIONUCLIDES

WATER STANDARD (S) IN PARENTHESES ARE PROPOSED ONLY 
TABLE A.5. (contd)

\begin{tabular}{|c|c|c|c|c|c|}
\hline $\begin{array}{l}\text { CONSTI } \\
\text { NAME }\end{array}$ & $\begin{array}{l}\text { JENT } \\
\text { UNITS } \\
-----\end{array}$ & $\begin{array}{l}\text { DETECTION } \\
\text { LIMIT }\end{array}$ & $\begin{array}{l}\text { HATER } \\
\text { STANDARD }\end{array}$ & $\begin{array}{l}\text { SAMPLE } \\
\text { DATE }\end{array}$ & $1-\mathrm{H} 4-17$ \\
\hline $\begin{array}{l}\text { ALUMNUM } \\
\text { AMMONIU } \\
\text { BARIUM } \\
\text { BETA }\end{array}$ & $\begin{array}{l}\text { PPB } \\
\text { PPB } \\
\text { PPB } \\
\text { PCI/L }\end{array}$ & $\begin{array}{r}160.00 \\
50.00 \\
6.00 \\
3.00\end{array}$ & $\begin{array}{r}1000.00 \\
60.00\end{array}$ & $\begin{array}{l}06 / 15 / \theta 7 \\
06 / 15 / \theta 7 \\
66 / 15 / 87 \\
06 / 16 / \theta 7\end{array}$ & \multirow{3}{*}{ 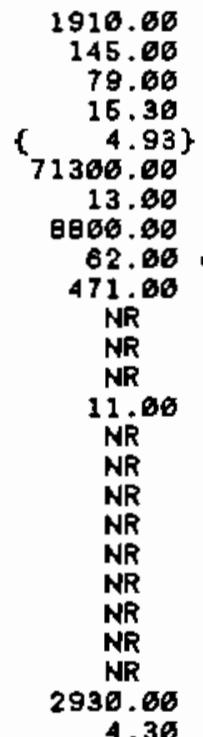 } \\
\hline $\begin{array}{l}\text { CALCIUM } \\
\text { CHLFORH } \\
\text { CHLORID } \\
\text { CHRONUN } \\
\text { CONDFLD }\end{array}$ & $\begin{array}{l}\text { PPB } \\
\text { PPB } \\
\text { PPB } \\
\text { PPE } \\
\text { UWHO }\end{array}$ & $\begin{array}{r}50.00 \\
10.00 \\
500.00 \\
10.00 \\
1.00\end{array}$ & 60.60 & $\begin{array}{l}66 / 15 / 87 \\
66 / 15 / \theta 7 \\
66 / 16 / 87 \\
66 / 16 / 87 \\
66 / 16 / 87\end{array}$ & \\
\hline $\begin{array}{l}\text { COPPER } \\
\text { FARSENI } \\
\text { FEARIUW } \\
\text { FCALCIU } \\
\text { FWAGNES } \\
\text { FHANGAN } \\
\text { FPOTASS } \\
\text { FSODIUH } \\
\text { FVANADI } \\
\text { FZINC } \\
\text { IRON } \\
\text { LOALPHA }\end{array}$ & $\begin{array}{l}\text { PPB } \\
\text { PPB } \\
\text { PPB } \\
\text { PPB } \\
\text { PPB } \\
\text { PPB } \\
\text { PPB } \\
\text { PPB } \\
\text { PPB } \\
\text { PPB } \\
\text { PPB } \\
\text { PCI/L }\end{array}$ & $\begin{array}{r}10.00 \\
5.00 \\
6.00 \\
60.00 \\
0.00 \\
5.00 \\
100.00 \\
100.00 \\
5.00 \\
5.00 \\
50.00 \\
2.00\end{array}$ & $\begin{array}{r}(1306.0) \\
58.08 \\
1000.00\end{array}$ & $\begin{array}{l}06 / 16 / 87 \\
06 / 15 / 87\end{array}$ & \\
\hline $\begin{array}{l}\text { MAGNES } \\
\text { MANGESE } \\
\text { NITRATE } \\
\text { PHFIELD }\end{array}$ & $\begin{array}{l}\text { PPB } \\
\text { PPB } \\
\text { PPB }\end{array}$ & $\begin{array}{r}0.60 \\
5.00 \\
500.00 \\
0.10\end{array}$ & 45060.06 & $\begin{array}{l}66 / 15 / 87 \\
06 / 15 / 97 \\
66 / 15 / 87 \\
66 / 16 / 87\end{array}$ & \multirow[t]{2}{*}{$\begin{array}{c}14100.00 \\
267.00 \\
43860.00 \\
6.90 \\
\text { NR } \\
\text { NR } \\
\text { NR } \\
7370.60\end{array}$} \\
\hline $\begin{array}{l}\text { POTASUH } \\
\text { RADIUM }\end{array}$ & $\begin{array}{l}\mathrm{PPB} \\
\mathrm{PCI} / \mathrm{L}\end{array}$ & $\begin{array}{r}100.00 \\
0.10\end{array}$ & \multirow[t]{3}{*}{5.00} & $\begin{array}{l}06 / 15 / 87 \\
08 / 15 / 87\end{array}$ & \\
\hline $\begin{array}{l}\text { SODIUH } \\
\text { STRONUN } \\
\text { SULFATE } \\
\text { TC }\end{array}$ & $\begin{array}{l}\text { PPB } \\
\text { PPB } \\
\text { PPB } \\
\text { PPB }\end{array}$ & $\begin{array}{l}100.00 \\
300.00 \\
500.00\end{array}$ & & $\begin{array}{l}06 / 15 / 97 \\
06 / 15 / 87 \\
06 / 15 / 87\end{array}$ & \multirow{2}{*}{$\begin{array}{c}0.69 \\
0.24\} \\
15200.00 \\
32 \theta .00 \\
81400.00 \\
\text { NR } \\
\text { NR } \\
775.60 \\
\text { NR } \\
\text { NR }\end{array}$} \\
\hline TOC & PPB & 200.00 & & $66 / 15 / 87$ & \\
\hline
\end{tabular}

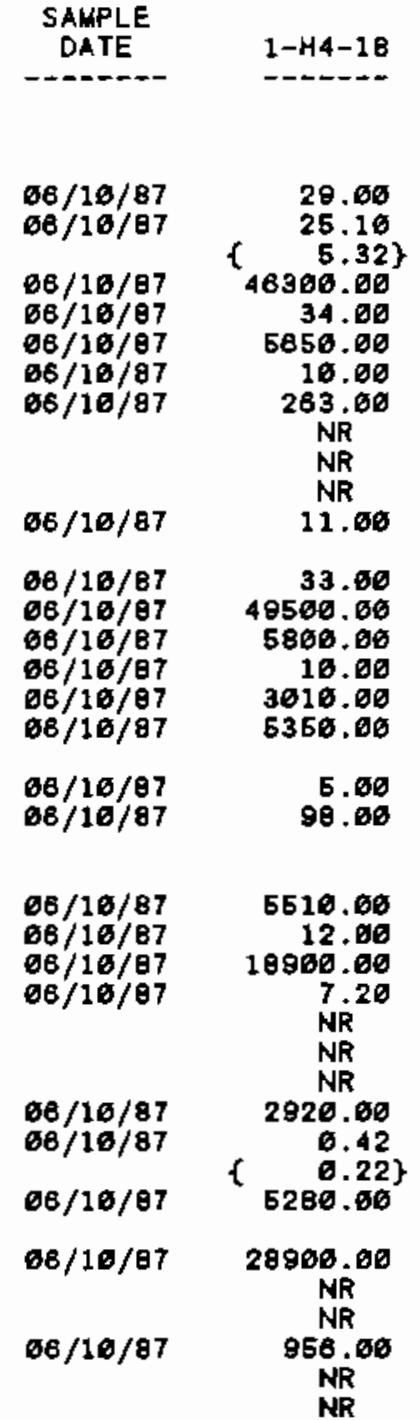
SAMPLE
DATE

2-E25-27

2-E2G-27

$86 / 30 / 87$

$68 / 36 / 87$

$66 / 36 / 87$

$66 / 30 / 87$

$68 / 36 / 87$

$66 / 30 / 87$

$06 / 30 / 87$

$08 / 30 / 87$

$26 / 36 / 87$

$66 / 36 / 87$

$66 / 36 / 87$

$86 / 36 / 87$

$06 / 36 / 87$

$06 / 30 / 87$

$66 / 36 / 87$

$66 / 36 / 87$

$68 / 30 / 87$

$66 / 36 / 87$

$66 / 30 / 87$

$06 / 30 / \theta 7$

$66 / 30 / 87$

$68 / 36 / 87$

$66 / 30 / 87$

$66 / 30 / 87$

$06 / 30 / 87$

$68 / 30 / 87$
$6 \theta / 36 / 87$

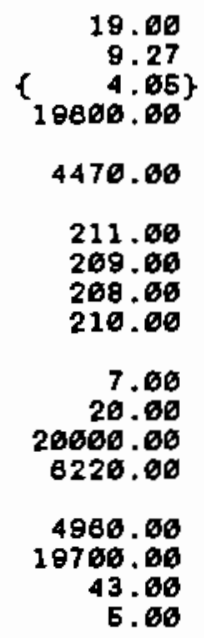

6150.00

6098.60

7.80

7.70

7.70

4920.00

19500.00

28700.00

21560.68 +

6960.66

674.00

694.06

820.00

* - VALUE EXCEEDS PRIMARY DRINKING WATER STANDARD.

\# - VALUE EXCEEDS PROPOSED PRIMARY DRINKING WATER STANDARD.

- VALUE EXCEEDS SCREENING LEVEL FOR FURTHER INVESTIGATION.

$+{ }^{+}-$DETECTION LIMIT WAS NOT AVAILABLE FOR COMPARISON

VALUES IN

VALUES IN \{ $\}$ ARE COUNTING ERRORS FOR RADIONUCLIDES

WATER STANDARD(S) IN PARENTHESES ARE PROPOSED ONLY 
TABLE A.5. (contd)

\begin{tabular}{|c|c|c|c|c|c|c|c|c|c|}
\hline $\begin{array}{l}\text { CONSTI } \\
\text { NAME }\end{array}$ & $\begin{array}{l}\text { UENT } \\
\text { UNITS }\end{array}$ & $\begin{array}{l}\text { OETECTION } \\
\text { LIMIT }\end{array}$ & $\begin{array}{l}\text { HATER } \\
\text { STANDARD }\end{array}$ & $\begin{array}{l}\text { SAMPLE } \\
\text { DATE }\end{array}$ & $1-\mathrm{H} 4-17$ & $\begin{array}{l}\text { SAMPLE } \\
\text { DATE }\end{array}$ & $1-H 4-18$ & $\begin{array}{l}\text { SAMPLE } \\
\text { DATE }\end{array}$ & $2-E 25-27$ \\
\hline $\begin{array}{l}\text { TOC } \\
\text { TOXLDL }\end{array}$ & $\begin{array}{l}\text { PPB } \\
\text { PPB }\end{array}$ & $\begin{array}{r}206.60 \\
20.06\end{array}$ & 20000.00 & & $\begin{array}{l}\text { NR } \\
\text { NR } \\
\text { NR } \\
\text { NR } \\
\text { NR }\end{array}$ & & $\begin{array}{l}\text { NR } \\
\text { NR } \\
\text { NR } \\
\text { NR } \\
\text { NR }\end{array}$ & $\begin{array}{l}66 / 36 / 87 \\
66 / 36 / 87 \\
66 / 36 / 87 \\
66 / 36 / 87 \\
66 / 36 / 87\end{array}$ & $\begin{array}{r}838.00 \\
25.60 \\
31.60 \\
26.00 \\
9520.00\end{array}$ \\
\hline $\begin{array}{l}\text { VANADUM } \\
\text { TINC }\end{array}$ & $\begin{array}{l}\text { PPE } \\
\text { PPB }\end{array}$ & $\begin{array}{l}5.60 \\
5.00\end{array}$ & & $\begin{array}{l}06 / 15 / 87 \\
66 / 16 / 87\end{array}$ & $\begin{array}{r}8.06 \\
17.00\end{array}$ & $66 / 16 / 87$ & 16.000 & $86 / 36 / 87$ & $\begin{array}{r}354.00 \\
43.00\end{array}$ \\
\hline
\end{tabular}

* - VALUE EXCEEDS PRIMARY DRINKING WATER STANDARD.

* - VALUE EXCEEDS PROPOSED PRIMARY DRINKING WATER STANDARO.

- VALUE EXCEEDS SCREENING LEVEL FOR FURTHER INVESTIGATION.

- DETECTION LIMIT WAS NOT AVAILABLE FOR COMPARISON

NR - ANALYSIS NOT REQUESTED OR NOT YET REPORTED

VALUES IN \{\} ARE COUNTING ERRORS FOR RADIONUCLIDES

WATER STANDARO (S) IN PARENTHESES ARE PROPOSED DNLY 
TABLE A.5. (contd)

\begin{tabular}{|c|c|c|c|c|c|}
\hline \multicolumn{2}{|c|}{$\begin{array}{l}\text { CONSTITUENT } \\
\text { NAME } \\
\text { UNITS }\end{array}$} & $\begin{array}{l}\text { DETECTION } \\
\text { LIUIT }\end{array}$ & $\begin{array}{l}\text { WATER } \\
\text { STANDARD }\end{array}$ & $\begin{array}{l}\text { SAMPLE } \\
\text { DATE }\end{array}$ & $2-E 25-28$ \\
\hline $\begin{array}{l}\text { ARSENIC } \\
\text { BARIUH }\end{array}$ & $\begin{array}{l}\text { PPB } \\
\text { PPB }\end{array}$ & $\begin{array}{l}6.00 \\
6.00\end{array}$ & $\begin{array}{r}50.00 \\
1000.00\end{array}$ & $\begin{array}{l}66 / 36 / 87 \\
06 / 36 / 87\end{array}$ & $\begin{array}{r}9.00 \\
22.00\end{array}$ \\
\hline BETA & $\mathrm{PCI} / \mathrm{L}$ & 3.00 & 50.00 & $66 / 36 / 87$ & $\begin{array}{l}8.28 \\
3.78 \text { \} } \\
\text { NR }\end{array}$ \\
\hline CALCIUM & PPB & 60.00 & & $68 / 30 / 87$ & $\begin{array}{c}23600.60 \\
\mathrm{NR}\end{array}$ \\
\hline CHLFORU & PPE & 10.00 & & & \\
\hline CHLORID & PPE & $6 \sigma 0.00$ & & $08 / 30 / 87$ & 4340.60 \\
\hline $\begin{array}{l}\text { COLIFRM } \\
\text { CONDFLD }\end{array}$ & $\begin{array}{l}\text { MPN } \\
\text { UMHO }\end{array}$ & $\begin{array}{l}2.20 \\
1.00\end{array}$ & 1.00 & $08 / 36 / 87$ & ${ }_{\mathrm{NR}}^{181.00}$ \\
\hline $\begin{array}{l}\text { COPPER } \\
\text { FARSENI } \\
\text { FBARIUM }\end{array}$ & $\begin{array}{l}\text { PPB } \\
\text { PPB } \\
\text { PPB }\end{array}$ & $\begin{array}{r}10.00 \\
5.00 \\
8.00\end{array}$ & $\begin{array}{r}(1300.0) \\
56.00 \\
1000.00\end{array}$ & $\begin{array}{l}06 / 30 / 87 \\
06 / 36 / 87\end{array}$ & $\begin{array}{l}11.00 \\
20.00\end{array}$ \\
\hline FCALCIU & PPB & 50.00 & & $06 / 30 / 87$ & ${ }_{N R}^{18800.60}$ \\
\hline $\begin{array}{l}\text { FCOPPER } \\
\text { FIRON } \\
\text { FMAGNES }\end{array}$ & $\begin{array}{l}\text { PPB } \\
\text { PPB } \\
\text { PPB }\end{array}$ & $\begin{array}{r}16.00 \\
60.00 \\
6.00\end{array}$ & $(1300.0)$ & $08 / 30 / 87$ & 5920.00 \\
\hline FPOTASS & PPB & 100.00 & & $06 / 30 / 87$ & 4096.66 \\
\hline FSODIUM & PPB & 160.00 & & $06 / 30 / 87$ & 13400.06 \\
\hline $\begin{array}{l}\text { FVANADI } \\
\text { FZINC } \\
\text { IRON }\end{array}$ & $\begin{array}{l}\text { PPB } \\
\text { PPB } \\
\text { PPB }\end{array}$ & $\begin{array}{r}5.60 \\
5.60 \\
50.60\end{array}$ & & $\begin{array}{l}06 / 36 / 87 \\
06 / 36 / 87 \\
06 / 30 / 87\end{array}$ & $\begin{array}{c}45.00 \\
67.00 \\
68.00 \\
\mathrm{NR}\end{array}$ \\
\hline $\begin{array}{l}\text { LEADGF } \\
\text { LOALPHA. }\end{array}$ & $\begin{array}{l}\mathrm{PPB} \\
\mathrm{PCI} / \mathrm{L}\end{array}$ & $\begin{array}{l}5.00 \\
2.00\end{array}$ & $\begin{array}{l}50.00 \\
16.60\end{array}$ & $66 / 30 / 87$ & $\begin{array}{l}2.66 \\
1.18\} \\
\mathrm{NR}^{-13}\end{array}$ \\
\hline MAGNES & PPB & 0.00 & & $86 / 30 / 87$ & 7430.60 \\
\hline NITRATE & PPB & 600.00 & 45006.00 & $06 / 30 / 87$ & $\underset{\substack{N R \\
N R}}{1390.00}$ \\
\hline
\end{tabular}

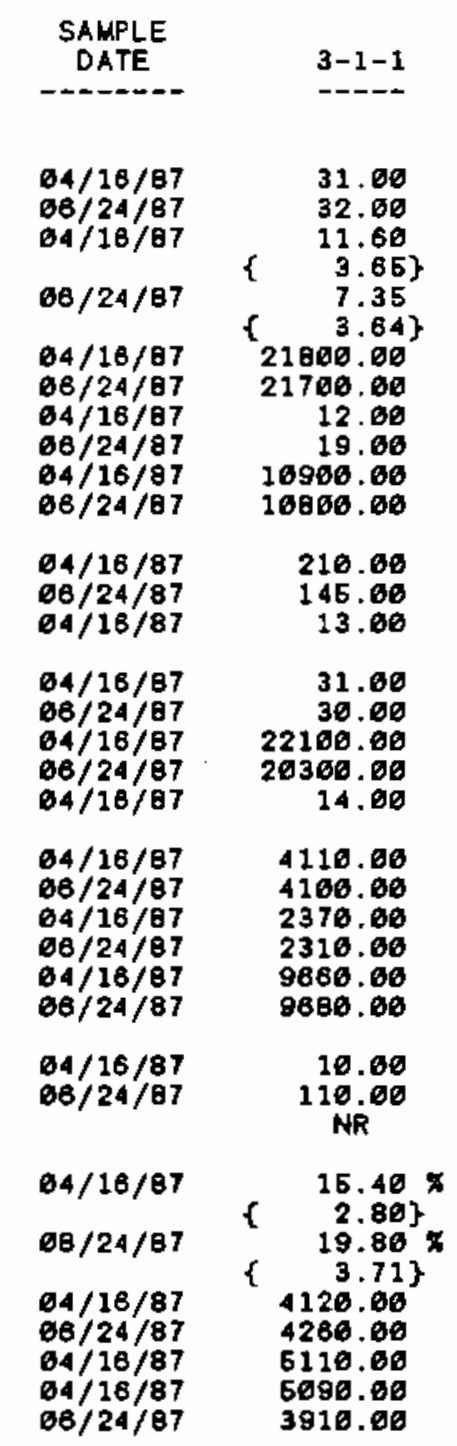

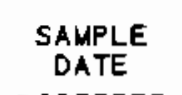

3-1-2

DATE

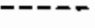

04/17/67 $06 / 24 / 87$ $04 / 17 / 87$

$06 / 24 / 87$

$04 / 17 / 87$

$06 / 24 / 87$

$04 / 17 / 87$

$06 / 24 / 87$

$64 / 17 / 87$

$68 / 24 / 87$

$04 / 17 / 87$

$68 / 24 / 87$

$64 / 17 / 67$

04/17/67

$06 / 24 / 87$

$04 / 17 / 87$

$06 / 24 / 87$

$04 / 17 / 87$

$04 / 17 / 87$

$04 / 17 / 87$
$08 / 24 / 87$

$08 / 24 / 87$

$04 / 17 / 87$
$06 / 24 / 87$

$08 / 24 / 87$

$68 / 24 / 87$

$66 / 24 / 87$

$04 / 17 / 87$

$06 / 24 / 87$

$06 / 24 / 87$

$08 / 24 / 87$

$06 / 24 / 87$

$64 / 17 / 87$

$66 / 24 / 87$

$04 / 17 / 87$

$04 / 17 / 67$
$06 / 24 / 67$

* - Value exceeds primary dRINKING water standard.

\# - VALUE EXCEEDS PROPOSED PRIMARY DRINKING WATER STANDARD.

* - VALUE EXCEEOS SCREENING LEVEL FOR FURTHER INVESTIGATION.

+ - DETECTION LIMIT WAS NOT AVAILABLE FOR COMPARISON

NR - ANALYSIS NDT REQUESTED OR NOT YET REPORTED

VALUES IN \{ $\}$ ARE COUNTING ERRORS FOR RADIONUCLIDES

WATER STANDARD'(S) IN PARENTHESES ARE PROPOSED ONLY 
TABLE A.5. (contd)

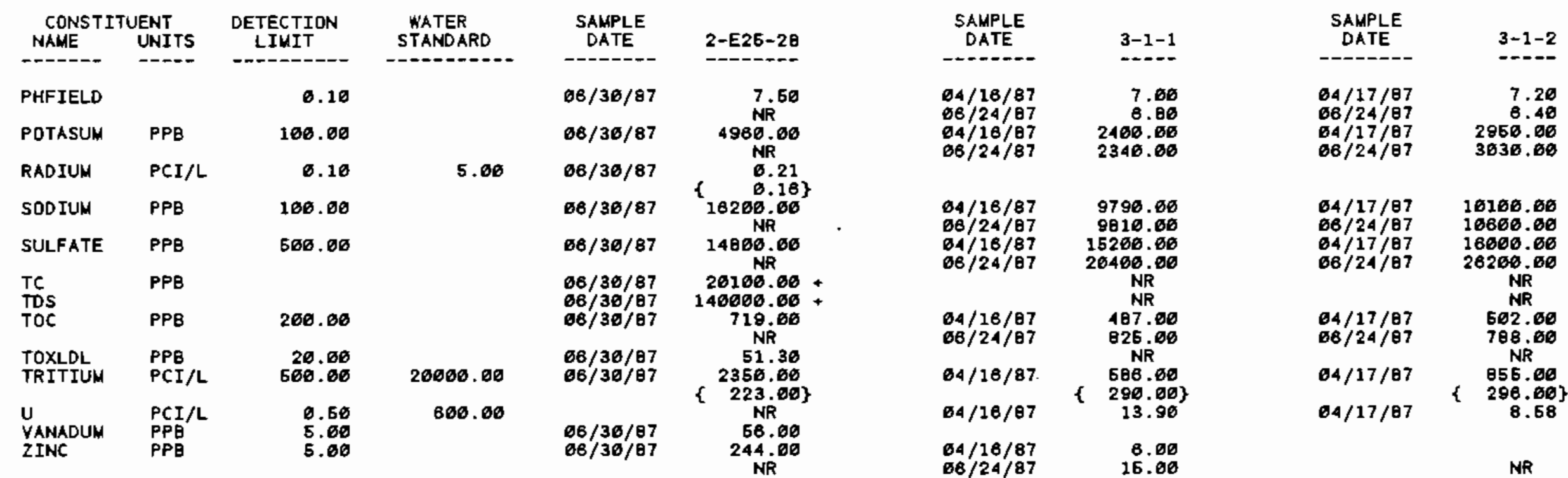

- - VAlUe exceEds PRIMARY dRINKING water STANDARo.

* - VALUE EXCEEDS PROPOSED PRIMARY DRINKING WATER STANDARD.

- VALUE EXTEEDS SCREENING LEVEL FOR FURTHER INVESTIGATION.

* - DETECTION LIMIT WAS NOT AVAILABLE FOR COMPARISON

NR - ANALYSIS NOT REQUESTED OR NOT YET REPORTED

VALUES IN \{\} ARE COUNTING ERRORS FOR RADIONUCLIDES

WATER STANDARO (S) IN PARENTHESES ARE PROPOSED ONLY 
TABLE A.5. (contd)

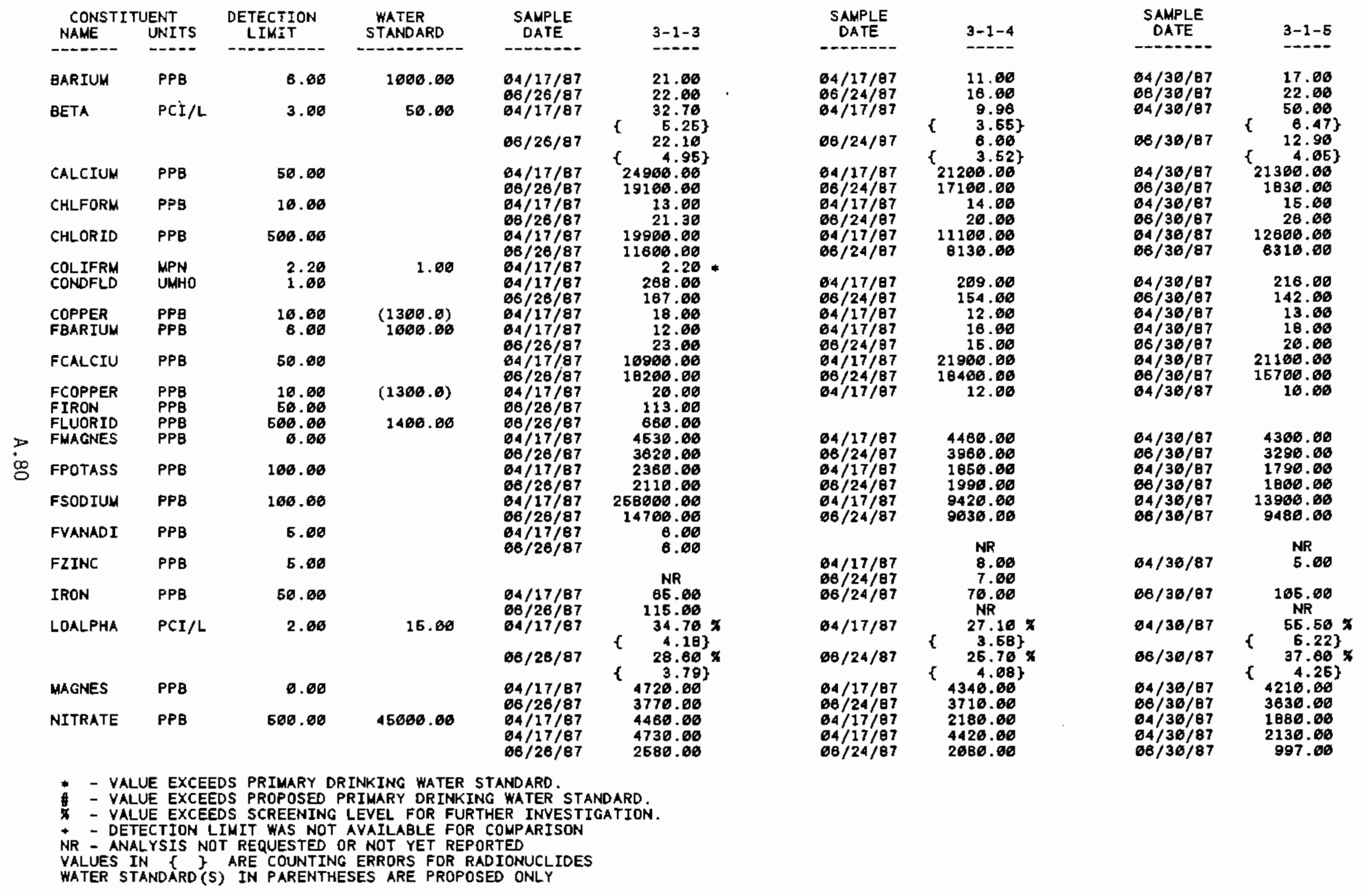


TABLE A.5. (contd)

\begin{tabular}{|c|c|c|c|c|c|c|c|c|c|}
\hline \multirow{2}{*}{\multicolumn{2}{|c|}{$\begin{array}{c}\text { CONSTI TUENT } \\
\text { NAKE } \\
\end{array}$}} & $\begin{array}{l}\text { DETECTIDN } \\
\text { LINIT }\end{array}$ & $\begin{array}{l}\text { WATER } \\
\text { STANDARD }\end{array}$ & $\begin{array}{l}\text { SAMPLE } \\
\text { DATE }\end{array}$ & $3-1-3$ & $\begin{array}{l}\text { SAMPLE } \\
\text { DATE }\end{array}$ & $3-1-4$ & $\begin{array}{l}\text { SAMPLE } \\
\text { DATE }\end{array}$ & $3-1-5$ \\
\hline & & - - & 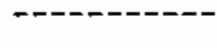 & 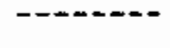 & 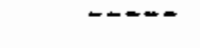 & ------ & --- & ----- &.---1 \\
\hline PHF IELD & & 0.16 & & $04 / 17 / 07$ & 7.10 & $64 / 17 / 67$ & 0.90 & $04 / 30 / 87$ & 6.30 \\
\hline POTASUM & PPB & 100.00 & & $64 / 17 / 67$ & 2470.00 & $04 / 17 / 87$ & 1820.00 & $04 / 36 / 87$ & 1090.00 \\
\hline SOD IUH & PPG & 100.00 & & $04 / 17 / 97$ & 15600.00 & $\begin{array}{l}66 / 24 / 67 \\
64 / 17 / 97\end{array}$ & $\begin{array}{l}1910.60 \\
9140.06\end{array}$ & $\begin{array}{l}68 / 36 / 87 \\
64 / 36 / 87\end{array}$ & $\begin{array}{r}2160.66 \\
13600.66\end{array}$ \\
\hline SULFATE & PPB & 560.00 & & $\begin{array}{l}66 / 26 / \theta 7 \\
04 / 17 / 87 \\
06 / 26 / 87\end{array}$ & $\begin{array}{l}14500.00 \\
17860.06 \\
19700.00\end{array}$ & $\begin{array}{l}66 / 24 / 87 \\
64 / 17 / 87 \\
66 / 24 / 87\end{array}$ & $\begin{array}{r}0820.00 \\
16000.06 \\
26400.00\end{array}$ & $\begin{array}{l}68 / 36 / 87 \\
64 / 36 / 87 \\
66 / 36 / 87\end{array}$ & $\begin{array}{l}16100.60 \\
17600.00 \\
16700.60\end{array}$ \\
\hline TOC & PPB & 200.00 & & $\begin{array}{l}04 / 17 / 97 \\
08 / 28 / 87\end{array}$ & $\begin{array}{l}712.00 \\
921.00\end{array}$ & $\begin{array}{l}64 / 17 / 87 \\
66 / 24 / 67\end{array}$ & $\begin{array}{l}692.00 \\
020.06\end{array}$ & $\begin{array}{l}64 / 36 / 87 \\
68 / 36 / 87\end{array}$ & $\begin{array}{r}754.06 \\
1330.00\end{array}$ \\
\hline TRITIUN & $P C I / L$ & 500.00 & 26006.00 & $04 / 17 / 67$ & $\begin{array}{l}567.00 \\
\{\quad 289.00\}\end{array}$ & & & & \\
\hline $\begin{array}{l}U \\
U-C H E M\end{array}$ & $\begin{array}{l}\mathrm{PCI} / \mathrm{L} \\
\text { UG/L }\end{array}$ & $\begin{array}{l}0.50 \\
0.73\end{array}$ & 866.66 & $\begin{array}{l}04 / 17 / 87 \\
04 / 17 / 87\end{array}$ & $\begin{array}{l}26.60 \\
47.60\end{array}$ & $64 / 17 / 07$ & ${ }_{\mathrm{NR}}^{23.20}$ & $04 / 36 / 87$ & ${ }_{\mathrm{NR}}^{50.20}$ \\
\hline $\begin{array}{l}\text { VANADUM } \\
\text { ZINC }\end{array}$ & $\begin{array}{l}\text { PPQ } \\
\text { PPE }\end{array}$ & $\begin{array}{l}5.00 \\
5.00\end{array}$ & & $\begin{array}{l}06 / 26 / 87 \\
04 / 17 / 87 \\
06 / 26 / 87\end{array}$ & $\begin{array}{l}7.00 \\
6.00 \\
8.00\end{array}$ & $\begin{array}{l}64 / 17 / 87 \\
68 / 24 / 87\end{array}$ & $\begin{array}{l}\theta .60 \\
\theta .06\end{array}$ & $\begin{array}{l}66 / 36 / 87 \\
64 / 36 / 87\end{array}$ & $\begin{array}{r}0.66 \\
10.06\end{array}$ \\
\hline
\end{tabular}

- - VALUE EXCEEDS PRIMARY DRINKING WATER STANDARO

- VALUE EXCEEDS PROPOSED PRIMARY DRINKING WATER STANDARD.

- VALUE EXCEEDS SCREENING LEVEL FOR FURTHER INVESTIGATION.

- DETECTION LIMIT WAS NOT AVAILABLE FOR COMPARISON

NR - ANALYSIS NOT REQUESTED OR NOT YET REPORTED

VALUES IN \{ $\}$ ARE COUNTING ERRORS FOR RADIONUCLIDES

WATER STANDARD(S) IN PARENTHESES ARE PROPOSED ONLY 
TABLE A.5. (contd)

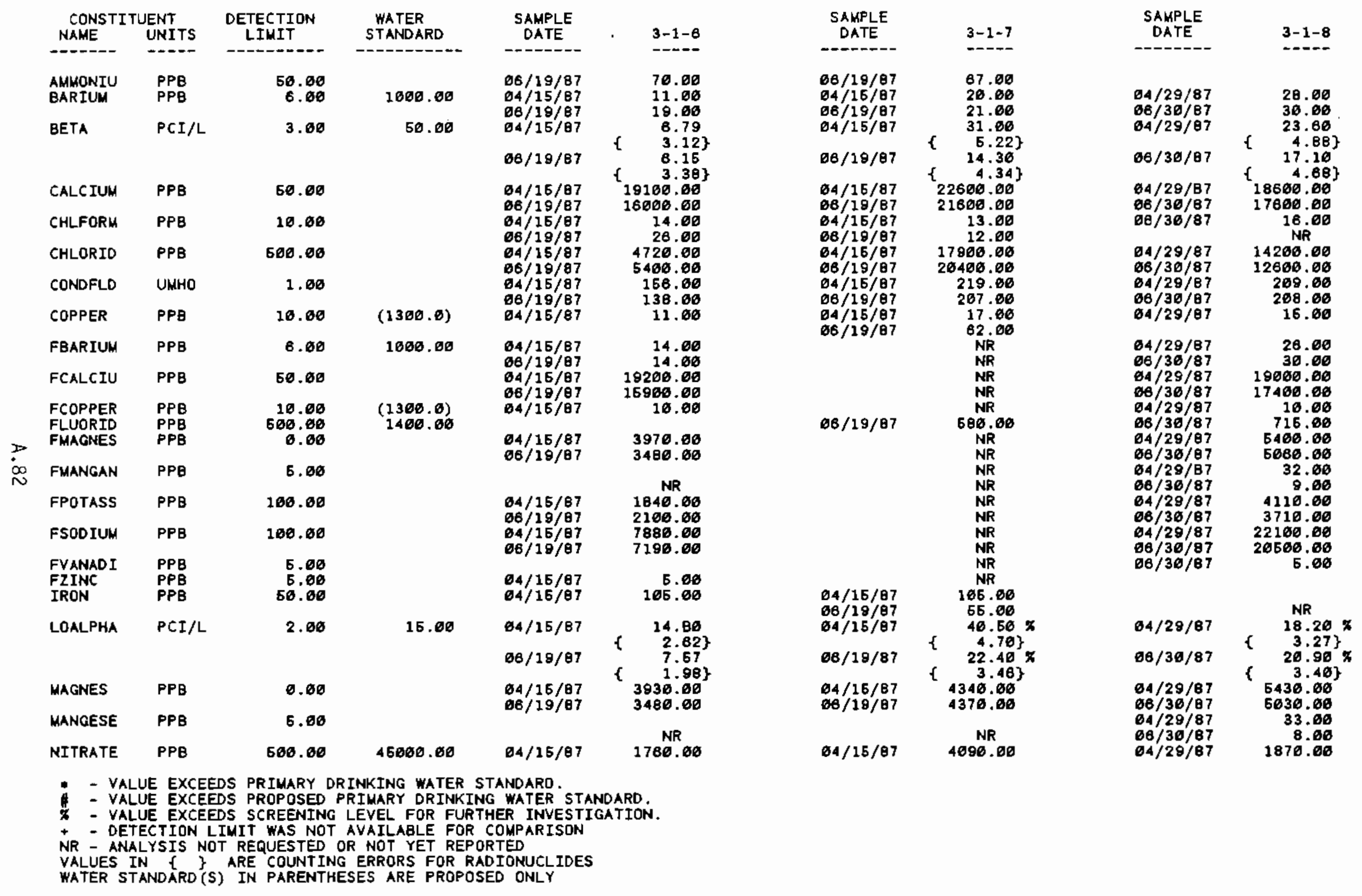


IABLE A.5. (contd)

\begin{tabular}{|c|c|c|c|c|c|c|c|c|c|}
\hline $\begin{array}{l}\text { CONSTI } \\
\text { NAWE }\end{array}$ & $\begin{array}{l}\text { UENT } \\
\text { UNITS }\end{array}$ & $\begin{array}{l}\text { OETECTION } \\
\text { LIMIT }\end{array}$ & $\begin{array}{l}\text { WATER } \\
\text { STANOARO }\end{array}$ & $\begin{array}{l}\text { SAMPLE } \\
\text { DATE }\end{array}$ & $3-1-6$ & $\begin{array}{l}\text { SAMPLE } \\
\text { DATE }\end{array}$ & $3-1-7$ & $\begin{array}{l}\text { SAMPLE } \\
\text { DATE }\end{array}$ & $3-1-8$ \\
\hline $\begin{array}{l}\text { NITRATE } \\
\text { PHFIELD } \\
\text { POTASUH } \\
\text { RADIUM }\end{array}$ & $\begin{array}{l}\text { PPE } \\
\text { PCI/L }\end{array}$ & $\begin{array}{r}600.00 \\
0.10 \\
100.00 \\
0.10\end{array}$ & 46060.60 & $\begin{array}{l}04 / 15 / 87 \\
06 / 19 / 87 \\
04 / 16 / 87 \\
06 / 19 / 87 \\
04 / 16 / 87 \\
08 / 19 / 87\end{array}$ & $\begin{array}{r}1990.00 \\
1470.00 \\
6.70 \\
6.70 \\
1810.00 \\
2150.00\end{array}$ & $\begin{array}{l}66 / 19 / 87 \\
64 / 15 / 87 \\
66 / 19 / 87 \\
64 / 15 / 87 \\
66 / 19 / 87\end{array}$ & $\begin{array}{c}2340.00 \\
\text { NR } \\
6.30 \\
6.80 \\
2280.00 \\
2380.00\end{array}$ & $\begin{array}{l}06 / 36 / 87 \\
04 / 28 / 87 \\
66 / 36 / 87 \\
64 / 29 / 87 \\
06 / 30 / 87 \\
04 / 29 / 87\end{array}$ & $\begin{array}{r}1560.60 \\
N R \\
7.50 \\
7.20 \\
4180.60 \\
4190.00 \\
0.12\end{array}$ \\
\hline $\begin{array}{l}\text { SODIUK } \\
\text { SULFATE } \\
\text { TOC } \\
\text { Z INC }\end{array}$ & $\begin{array}{l}\text { PPB } \\
\text { PPB } \\
\text { PPB } \\
\text { PCI/L } \\
\text { PPB }\end{array}$ & $\begin{array}{r}100.00 \\
500.00 \\
200.00 \\
6.50 \\
6.00\end{array}$ & $\theta 00.00$ & $\begin{array}{l}04 / 15 / 87 \\
06 / 19 / 87 \\
04 / 16 / 87 \\
66 / 19 / 87 \\
04 / 16 / 87 \\
06 / 19 / 87 \\
64 / 16 / 87 \\
04 / 15 / 87 \\
06 / 19 / 87\end{array}$ & $\begin{array}{r}7640.00 \\
7410.00 \\
15500.00 \\
17900.00 \\
854.00 \\
904.00 \\
14.00 \\
5.00 \\
6.00\end{array}$ & $\begin{array}{l}64 / 15 / 87 \\
66 / 19 / 87 \\
64 / 15 / 87 \\
66 / 18 / 87 \\
64 / 15 / 87 \\
66 / 19 / 87 \\
66 / 18 / 87\end{array}$ & $\begin{array}{c}14800.00 \\
16100.00 \\
15700.00 \\
19800.00 \\
618.00 \\
798.00 \\
\mathrm{NR} \\
184.00 \\
\mathrm{NR}\end{array}$ & $\begin{array}{l}64 / 28 / 87 \\
66 / 36 / 87 \\
64 / 29 / 87 \\
66 / 36 / 87 \\
64 / 29 / 87 \\
66 / 36 / 87\end{array}$ & $\begin{array}{c}22560.00 \\
21460.60 \\
18100.60 \\
18960.60 \\
478.06 \\
1490.60 \\
\text { NR } \\
\text { NR }\end{array}$ \\
\hline
\end{tabular}

- - VAlUe exceeos PRIMARY orinking water STANDARD.

* - VALUE EXCEEDS PROPOSED PRIMARY DRINKING WATER STANDARD.

* - VALUE EXCEEDS SCREENING LEVEL FOR FURTHER INVESTIGATION.

- DETECTION LIMIT WAS NOT AVAILABLE FOR COMPARISON

NR - ANALYSIS NOT REQUESTED OR NOT YET REPORTED

VALUES IN \{\} ARE COUNTING ERRORS FOR RADIONUCLIDES

WATER STANDARD (S) IN PARENTHESES ARE PROPOSED ONLY 
TABLE A.5. (contd)

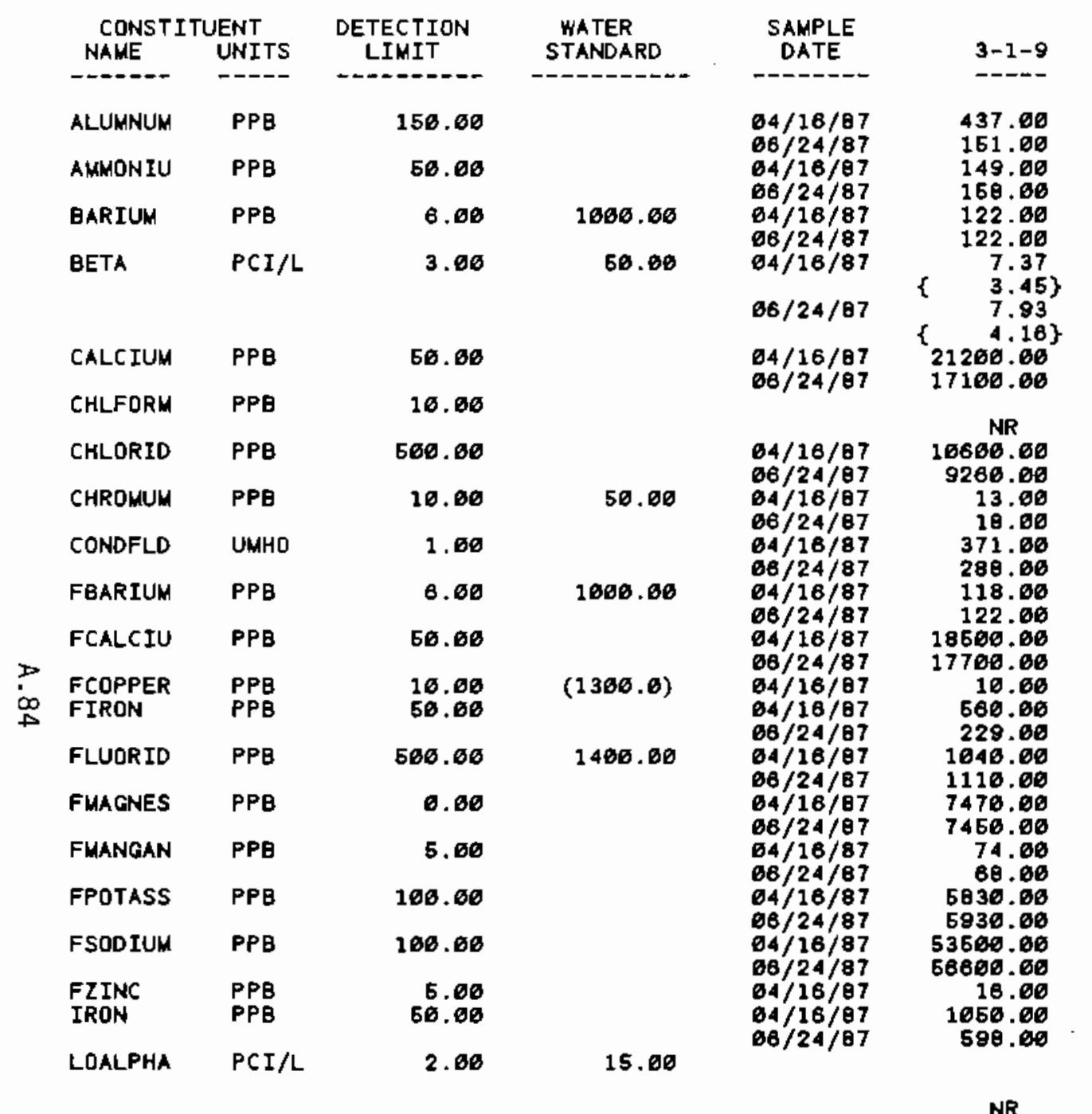

\begin{tabular}{|c|c|}
\hline $\begin{array}{l}\text { SAMPLE } \\
\text { DATE }\end{array}$ & $3-1-16$ \\
\hline $06 / 10 / 87$ & $\begin{array}{l}\text { NR } \\
73.06\end{array}$ \\
\hline $\begin{array}{l}04 / 29 / 87 \\
66 / 18 / 87 \\
04 / 29 / 87\end{array}$ & $\begin{array}{r}23.80 \\
21.00 \\
8.86\end{array}$ \\
\hline $08 / 18 / 07$ & 6.52 \\
\hline $\begin{array}{l}04 / 29 / 87 \\
06 / 18 / 67 \\
64 / 29 / 87 \\
66 / 18 / 97 \\
64 / 29 / 67 \\
68 / 18 / 97\end{array}$ & $\begin{array}{r}20600.06 \\
17360.06 \\
14.06 \\
23.06 \\
3810.06 \\
7360.08\end{array}$ \\
\hline $\begin{array}{l}04 / 29 / 87 \\
06 / 19 / 87 \\
64 / 29 / 87 \\
06 / 18 / 87 \\
04 / 29 / 67 \\
06 / 10 / 87 \\
64 / 29 / 87 \\
08 / 10 / 87\end{array}$ & $\begin{array}{r}\text { NR } \\
161.00 \\
152.06 \\
23.06 \\
21.06 \\
20806.60 \\
18106.66 \\
10.06 \\
71.66\end{array}$ \\
\hline $68 / 18 / 07$ & $\begin{array}{l}\text { NR } \\
656.68 \\
N R\end{array}$ \\
\hline $\begin{array}{l}04 / 28 / 87 \\
68 / 18 / 87\end{array}$ & $\begin{array}{l}4320.06 \\
3960.00\end{array}$ \\
\hline $\begin{array}{l}04 / 29 / 87 \\
66 / 18 / 97 \\
64 / 29 / 97 \\
66 / 18 / 97\end{array}$ & $\begin{array}{c}\text { NR } \\
2000.00 \\
2400.00 \\
8170.00 \\
8840.00\end{array}$ \\
\hline $04 / 28 / 87$ & $\begin{array}{c}N R \\
10.40\end{array}$ \\
\hline & $\begin{array}{l}\mathrm{t} \\
\mathrm{f} \\
\mathrm{f} \\
1.762\end{array}$ \\
\hline
\end{tabular}

\begin{tabular}{|c|c|}
\hline $\begin{array}{c}\text { SAMPLE } \\
\text { DATE }\end{array}$ & $3-1-11$ \\
\hline $04 / 29 / 87$ & $\begin{array}{c}\text { NR } \\
76.00\end{array}$ \\
\hline $\begin{array}{l}04 / 29 / 87 \\
06 / 30 / 87 \\
04 / 29 / 87\end{array}$ & $\begin{array}{l}12.06 \\
21.06 \\
58.68 \times\end{array}$ \\
\hline $06 / 30 / 87$ & $\begin{array}{l}6.48\} \\
7.62\end{array}$ \\
\hline $\begin{array}{l}64 / 29 / 87 \\
66 / 36 / 87 \\
64 / 29 / 67 \\
66 / 36 / 87 \\
64 / 29 / 67 \\
66 / 36 / 87\end{array}$ & $\begin{array}{r}18500.04 \\
18200.00 \\
18.06 \\
24.76 \\
3328.06 \\
5486.00\end{array}$ \\
\hline
\end{tabular}

$\begin{array}{ll}04 / 29 / 97 & 151.06 \\ 06 / 30 / 87 & 136.06\end{array}$

$4 / 29 / 87$

$06 / 36 / 87$

$34 / 29 / 87$

$68 / 36 / 87$

11.00

20.00

19200.00
16000.00

NR

NR

$64 / 28 / 97$

$68 / 30 / 87$

4286.00
3720.00

NR

$04 / 29 / 87 \quad 1320$

$06 / 30 / 87 \quad 1450.00$

041201972740.00

$04729 / 87 \quad 740.60$

04/30107 5240.00

$04 / 29 / 87$

$06 / 36 / 87$

$04 / 29 / 07$

5.00
56.06

NR.

TQ. $96 \%$

$08 / 30 / 07$

* - VAlue exCeeds primary dRINKINg water standard.

* - VALUE EXCEEDS PROPOSED PRIMARY DRINKING HATER STANDARD.

* - VALUE EXCEEDS SCREENING LEVEL FOR FURTHER INYESTIGATION.

* - DETECTION LIMIT WAS NOT AVAILABLE FOR COMPARISDN

NR - ANALYSIS NOT REQUESTED OR NOT YET REPORTED

VALUES IN \{ $\}$ ARE COUNTING ERRORS FOR RADIONUCLIDES

WATER STANDARD (S) IN PARENTHESES ARE PROPDSED ONLY 
TABLE A.5. (contd)

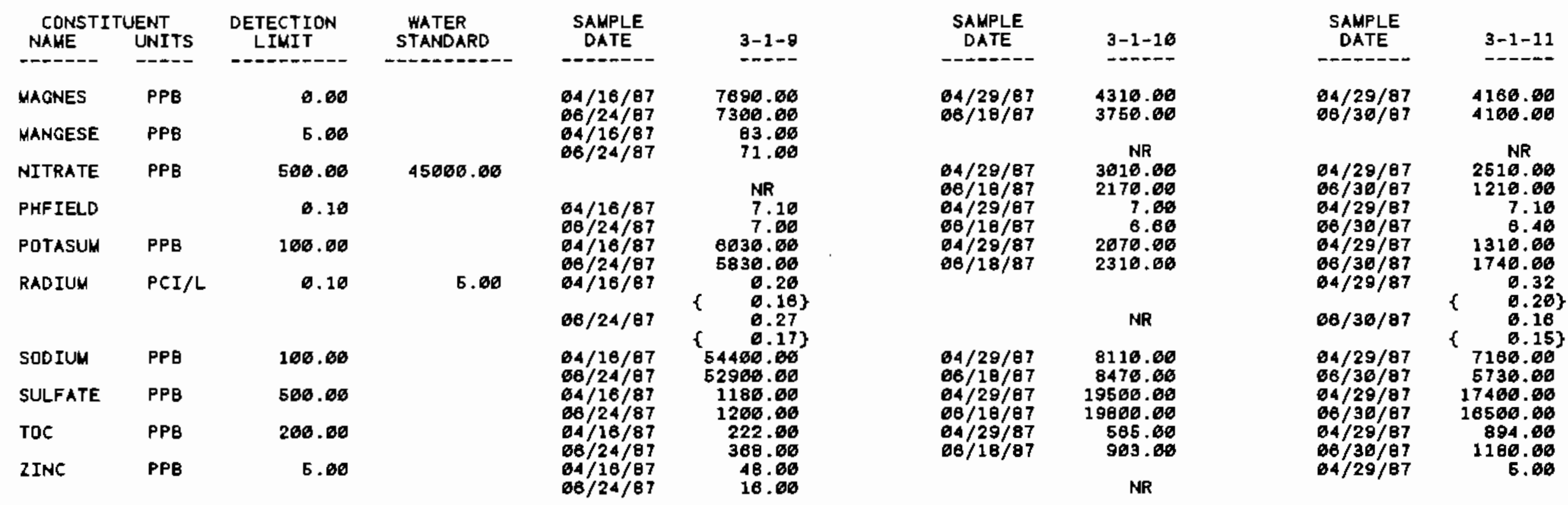

- - Value exceeds primary drinking water standaro.

\# - VALUE EXCEEDS PROPOSED PRIMARY DRINKING WATER STANDARD.

* - VALUE EXCEEDS SCREENING LEVEL FOR FURTHER INVESTIGATION.

- - DETECTION LIMIT WAS NOT AVAILABLE FOR COMPARISON

NR - ANALYSIS NOT REQUESTED OR NOT YET REPORTED

VALUES IN \{ $\}$ ARE COUNTING ERRORS FOR RADIONUCLIDES

WATER STANDARD (S) IN PARENTHESES ARE PROPOSED ONLY 
TABLE A.5. (cont d)

\begin{tabular}{|c|c|c|c|c|c|c|c|c|c|}
\hline $\begin{array}{l}\text { CONSTI } \\
\text { NAME }\end{array}$ & $\begin{array}{l}\text { JENT } \\
\text { UNITS }\end{array}$ & $\begin{array}{l}\text { DETECTION } \\
\text { LIMIT }\end{array}$ & $\begin{array}{l}\text { WATER } \\
\text { STANDARD }\end{array}$ & $\begin{array}{l}\text { SAMPLE } \\
\text { DATE }\end{array}$ & $3-1-12$ & $\begin{array}{l}\text { SAMPLE } \\
\text { DATE }\end{array}$ & $3-1-13$ & $\begin{array}{l}\text { SAMPLE } \\
\text { DATE }\end{array}$ & $3-1-14$ \\
\hline $\begin{array}{l}\text { AMMONIU } \\
\text { BARIUM }\end{array}$ & $\begin{array}{l}\text { PPB } \\
\text { PPB }\end{array}$ & $\begin{array}{r}50.00 \\
8.00\end{array}$ & 1000.00 & $\begin{array}{l}06 / 18 / 87 \\
64 / 27 / 87 \\
08 / 18 / 87\end{array}$ & $\begin{array}{r}102.00 \\
14.08 \\
18.00\end{array}$ & $\begin{array}{l}66 / 18 / 87 \\
64 / 27 / 87 \\
06 / 10 / 87\end{array}$ & $\begin{array}{l}78.06 \\
22.06 \\
23.06\end{array}$ & $\begin{array}{l}06 / 1 \theta / 87 \\
04 / 2 \theta / 87 \\
06 / 1 \theta / 87\end{array}$ & $\begin{array}{l}86.00 \\
11.6 \sigma \\
21.6 \theta\end{array}$ \\
\hline BETA & PCI/L & 3.00 & 60.00 & $64 / 27 / 87$ & 47.10 & $84 / 27 / 87$ & 13.46 & $64 / 29 / 87$ & 16.60 \\
\hline & & & & $06 / 18 / 87$ & $\begin{array}{r}16.18 \\
\quad 3.71\}\end{array}$ & $66 / 18 / 87$ & $\begin{array}{l}5.62 \\
3.45\}\end{array}$ & $68 / 18 / 87$ & $\begin{array}{l}3.94 \\
3.25\}\end{array}$ \\
\hline CALCIUM & PPB & 50.00 & & $\begin{array}{l}64 / 27 / 87 \\
86 / 18 / 87\end{array}$ & $\begin{array}{l}21800.71\} \\
18400.08\end{array}$ & $\begin{array}{l}64 / 27 / 97 \\
06 / 18 / 87\end{array}$ & $\begin{array}{l}2670 \theta .06\} \\
26500 . \theta 0\end{array}$ & $\begin{array}{l}04 / 29 / 87 \\
06 / 10 / 87\end{array}$ & $\begin{array}{l}23406.06\} \\
26000.00\end{array}$ \\
\hline CHLFORM & PPB & 10.00 & & $\begin{array}{l}04 / 27 / 87 \\
06 / 19 / 87\end{array}$ & $\begin{array}{r}14.0 B \\
24 . \boxminus B\end{array}$ & $\begin{array}{l}84 / 27 / 87 \\
86 / 18 / 87\end{array}$ & $\begin{array}{r}12.06 \\
15.06\end{array}$ & $\begin{array}{l}04 / 29 / 87 \\
66 / 16 / 87\end{array}$ & $\begin{array}{r}12.08 \\
. \quad 15.86\end{array}$ \\
\hline CHLORID & PPB & 500.06 & & $\begin{array}{l}64 / 27 / 87 \\
68 / 16 / 87 \\
64 / 27 / 87\end{array}$ & $\begin{array}{r}14866.06 \\
6366.06 \\
10.06\end{array}$ & $\begin{array}{l}64 / 27 / 87 \\
66 / 10 / 87\end{array}$ & $\begin{array}{r}9748.00 \\
11406.00\end{array}$ & $\begin{array}{l}64 / 28 / 87 \\
66 / 10 / 87\end{array}$ & $\begin{array}{r}9936.06 \\
13700.00\end{array}$ \\
\hline $\begin{array}{l}\text { CHROMUM } \\
\text { CONDFLD }\end{array}$ & $\begin{array}{l}\text { PPB } \\
\text { UMHO }\end{array}$ & $\begin{array}{r}10.00 \\
1.00\end{array}$ & 66.00 & $\begin{array}{l}64 / 27 / 87 \\
64 / 27 / 87 \\
68 / 18 / 87\end{array}$ & $\begin{array}{l}197.06 \\
149.06\end{array}$ & $\begin{array}{l}04 / 27 / 87 \\
06 / 18 / 97\end{array}$ & $\begin{array}{l}212.06 \\
222.00\end{array}$ & $\begin{array}{l}64 / 29 / 87 \\
66 / 10 / 87\end{array}$ & $\begin{array}{l}171.06 \\
294.06\end{array}$ \\
\hline $\begin{array}{l}\text { COPPER } \\
\text { FBARIUH }\end{array}$ & $\begin{array}{l}\text { PPB } \\
\text { PPB }\end{array}$ & $\begin{array}{r}10.00 \\
6.00\end{array}$ & $\begin{array}{c}(1300.0) \\
1000.00\end{array}$ & $\begin{array}{l}64 / 27 / 87 \\
64 / 27 / 87 \\
66 / 18 / 87\end{array}$ & $\begin{array}{l}14.60 \\
19.86 \\
20.06\end{array}$ & $\begin{array}{l}04 / 27 / 87 \\
04 / 27 / 87 \\
06 / 18 / 87\end{array}$ & $\begin{array}{l}17.06 \\
23.06 \\
21.00\end{array}$ & $\begin{array}{l}64 / 29 / 87 \\
64 / 29 / 87 \\
66 / 10 / 87\end{array}$ & $\begin{array}{l}12.06 \\
13.86 \\
21.06\end{array}$ \\
\hline FCALCIU & PPB & 50.00 & & $\begin{array}{l}64 / 27 / 87 \\
06 / 18 / 87\end{array}$ & $\begin{array}{l}2200 \theta .0 \emptyset \\
1720 \theta .06\end{array}$ & $\begin{array}{l}04 / 27 / 87 \\
06 / 18 / 87\end{array}$ & $\begin{array}{l}26706.00 \\
25100.00\end{array}$ & $\begin{array}{l}64 / 29 / 87 \\
06 / 18 / 87\end{array}$ & $\begin{array}{l}23406.06 \\
27600.66\end{array}$ \\
\hline $\begin{array}{l}\text { FCOPPER } \\
\text { FLUORID }\end{array}$ & $\begin{array}{l}\text { PPB } \\
\text { PPB }\end{array}$ & $\begin{array}{r}10.00 \\
500.00\end{array}$ & $\begin{array}{c}(1300.0) \\
1400.00\end{array}$ & $\begin{array}{l}64 / 27 / 87 \\
66 / 18 / 87\end{array}$ & $\begin{array}{r}11.06 \\
500.00\end{array}$ & $04 / 27 / 87$ & 20.00 & $04 / 29 / 87$ & 13.06 \\
\hline FMAGNES & PPB & 0.00 & & $\begin{array}{l}64 / 27 / 87 \\
66 / 18 / 87\end{array}$ & $\begin{array}{l}4670.60 \\
3719.60\end{array}$ & $\begin{array}{l}04 / 27 / 87 \\
08 / 18 / 87\end{array}$ & $\begin{array}{l}5716.00 \\
5870.00\end{array}$ & $\begin{array}{l}04 / 29 / 97 \\
86 / 18 / 67\end{array}$ & $\begin{array}{l}4856.06 \\
5706.06\end{array}$ \\
\hline FPOTASS & PPB & 100.00 & & $\begin{array}{l}64 / 27 / 87 \\
66 / 10 / 87\end{array}$ & $\begin{array}{l}1990.00 \\
1930.00\end{array}$ & $\begin{array}{l}04 / 27 / 87 \\
08 / 18 / 87\end{array}$ & $\begin{array}{l}3410.00 \\
3460.00\end{array}$ & $\begin{array}{l}64 / 29 / 97 \\
98 / 18 / 97\end{array}$ & $\begin{array}{l}2276.06 \\
3010.06\end{array}$ \\
\hline FSODIUM & PPB & 100.00 & & $\begin{array}{l}64 / 27 / 87 \\
86 / 18 / 87\end{array}$ & $\begin{array}{r}13706.00 \\
8410.00\end{array}$ & $\begin{array}{l}04 / 27 / 87 \\
66 / 18 / 87\end{array}$ & $\begin{array}{l}9070.00 \\
9268.00\end{array}$ & $\begin{array}{l}64 / 29 / 87 \\
66 / 18 / 87\end{array}$ & $\begin{array}{l}7860.06 \\
9780.06\end{array}$ \\
\hline $\begin{array}{l}\text { FYANADI } \\
\text { FIINC } \\
\text { IRON } \\
\text { LOALPHA }\end{array}$ & $\begin{array}{l}\text { PPB } \\
\text { PPB } \\
\text { PPE } \\
\text { PCI/L }\end{array}$ & $\begin{array}{r}5.00 \\
5.60 \\
58.00 \\
2.00\end{array}$ & 15.00 & $64 / 27 / 87$ & $63.30 \times$ & $\begin{array}{l}04 / 27 / 97 \\
04 / 27 / 67 \\
64 / 27 / 97 \\
04 / 27 / 97\end{array}$ & $\begin{array}{r}5.60 \\
6.00 \\
62.06 \\
13.26\end{array}$ & $\begin{array}{l}64 / 29 / 87 \\
6 / 18 / 87 \\
64 / 29 / 87\end{array}$ & $\begin{array}{r}6.00 \\
114.00 \\
10.60 \times\end{array}$ \\
\hline & & & & $06 / 18 / 87$ & $28.00 \times$ & & $\begin{array}{r}2.68\} \\
14.09 \\
2.84\}\end{array}$ & $68 / 18 / 87$ & $\begin{array}{c}3.82\} \\
18.46 \times \\
2.867\end{array}$ \\
\hline MAGNES & PPB & 0.00 & & $\begin{array}{l}04 / 27 / 87 \\
68 / 18 / 97\end{array}$ & $\begin{array}{l}4538.06 \\
3596.06\end{array}$ & $\begin{array}{l}04 / 27 / 87 \\
66 / 18 / 87\end{array}$ & $\begin{array}{l}5720.06 \\
5940.00\end{array}$ & $\begin{array}{l}64 / 29 / 87 \\
06 / 18 / 87\end{array}$ & $\begin{array}{l}4689.00 \\
5418.00\end{array}$ \\
\hline NITRATE & PPB & 506.06 & 45600.68 & $\begin{array}{l}64 / 27 / 87 \\
06 / 18 / 87\end{array}$ & $\begin{array}{l}1780.00 \\
1820.00\end{array}$ & $\begin{array}{l}04 / 27 / 87 \\
68 / 18 / 87\end{array}$ & $\begin{array}{l}4450.00 \\
5690.06\end{array}$ & $\begin{array}{l}64 / 29 / 87 \\
66 / 18 / 87\end{array}$ & 2316.00 \\
\hline PHFIELD & & 0.10 & & $\begin{array}{l}04 / 27 / 87 \\
08 / 18 / 87\end{array}$ & $\begin{array}{l}6.80 \\
8.48\end{array}$ & $\begin{array}{l}04 / 27 / 87 \\
06 / 18 / 87\end{array}$ & $\begin{array}{l}8.00 \\
7.16\end{array}$ & $\begin{array}{l}04 / 29 / 97 \\
08 / 18 / 97\end{array}$ & $\begin{array}{r}7.50 \\
8.60\end{array}$ \\
\hline POTASUM & PPB & 100.00 & & $\begin{array}{l}04 / 27 / 87 \\
66 / 18 / 87\end{array}$ & $\begin{array}{l}1870.06 \\
1920.06\end{array}$ & $\begin{array}{l}04 / 27 / 87 \\
08 / 18 / 87\end{array}$ & $\begin{array}{l}3450.06 \\
3620.06\end{array}$ & $\begin{array}{l}64 / 29 / 87 \\
96 / 18 / 87\end{array}$ & $\begin{array}{l}2340.60 \\
2876.06\end{array}$ \\
\hline
\end{tabular}

* - VALUE EXCEEDS PRIMARY DRINKING WATER STANDARD.

\# - VALUE EXCEEDS PROPOSED PRIMARY DRINKING WATER STANDARD.

* - VALUE EXCEEDS SCREENING LEYEL FOR FURTHER INVESTIGATION.

* DETECTION LIMIT WAS NOT AVATLABLE FOR COMPARISON

NR - ANALYSIS NOT REQUESTED OR NOT YET REPORTED

VALUES IN $f$ ? ARE COUNTING ERRORS FOR RADIONUCLIDES

WATER STANDARD (S) IN PARENTHESES ARE PROPOSED ONLY 
TABLE A.5. (contd)

\begin{tabular}{|c|c|c|c|c|c|c|c|c|c|}
\hline $\begin{array}{l}\text { CONSTI } \\
\text { NAME }\end{array}$ & $\begin{array}{l}\text { UENT } \\
\text { UNITS }\end{array}$ & $\begin{array}{l}\text { DETECTION } \\
\text { LIMIT }\end{array}$ & $\begin{array}{l}\text { WATER } \\
\text { STANDARD }\end{array}$ & $\begin{array}{l}\text { SAMPLE } \\
\text { DATE }\end{array}$ & $3-1-12$ & $\begin{array}{l}\text { SAMPLE } \\
\text { DATE }\end{array}$ & $3-1-13$ & $\begin{array}{l}\text { SAMPLE } \\
\text { DATE }\end{array}$ & $3-1-14$ \\
\hline----- & $\ldots$ & 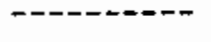 & 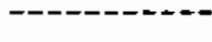 & $--\infty-\infty$ & $--\cdots-\infty$ & $---0-\infty$ & ----- & $---\ldots .$. & -ーーーா \\
\hline RADIUH & PCI /L & 0.10 & 5.00 & $64 / 27 / 87$ & $\left\{\begin{array}{l}6.23 \\
\quad 0.17\}\end{array}\right.$ & $64 / 27 / 87$ & $\left\{\begin{array}{l}0.12 \\
\{\quad 0.13\}\end{array}\right.$ & & \\
\hline SODIUM & PPB & 100.00 & & $\begin{array}{l}64 / 27 / 87 \\
68 / 18 / 87\end{array}$ & 13600.06 & $64 / 27 / 87$ & 9786.00 & $64 / 29 / 87$ & 7880.00 \\
\hline SULFATE & PPB & 500.00 & & $\begin{array}{l}64 / 27 / 87 \\
68 / 18 / \theta 7\end{array}$ & 18600.00 & $\begin{array}{l}84 / 27 / 87 \\
66 / 18 / 87\end{array}$ & 18000.00 & $04 / 29 / \theta 7$ & $\begin{array}{l}16106.00 \\
25200.08\end{array}$ \\
\hline TOC & PPB & 200.00 & & $\begin{array}{l}64 / 27 / 67 \\
66 / 18 / 87\end{array}$ & $\begin{array}{l}760.00 \\
805.00\end{array}$ & $\begin{array}{l}04 / 27 / 67 \\
06 / 18 / 87\end{array}$ & $\begin{array}{l}831.00 \\
813.00\end{array}$ & $\begin{array}{l}04 / 29 / 87 \\
06 / 10 / \theta 7\end{array}$ & $\begin{array}{l}628.00 \\
785.00\end{array}$ \\
\hline $\begin{array}{l}\text { VANADUM } \\
\text { ZINC }\end{array}$ & $\begin{array}{l}\text { PPB } \\
\text { PPB }\end{array}$ & $\begin{array}{l}6.60 \\
5.00\end{array}$ & & $04 / 27 / 87$ & 5.60 & $66 / 18 / 87$ & 5.00 & & \\
\hline
\end{tabular}

* - VAlUe eXCeEdS PRIMARY DRINKING hater STANDARD.

* - VALUE EXCEEDS PROPOSED PRIMARY DRINKING WATER STANDARD.

* - VALUE EXCEEDS SCREENING LEVEL FOR FURTHER INVESTIGATION.

- DETECTION LIMIT WAS NOT AVAILABLE FDR COMPARISON

NR - ANALYSIS NOT REQUESTED OR NOT YET REPORTED

VALUES IN \{\} ARE COUNTING ERRDRS FOR RADIONUCL IDES

WATER STANDARD (S) IN PARENTHESES ARE PROPOSED ONLY 
TABLE A.5. (contd)

\begin{tabular}{|c|c|c|c|c|c|}
\hline $\begin{array}{l}\text { CONSTI } \\
\text { NAME }\end{array}$ & $\begin{array}{l}\text { UENT } \\
\text { UNITS }\end{array}$ & $\begin{array}{l}\text { DETECTION } \\
\text { LIMIT }\end{array}$ & $\begin{array}{l}\text { HATER } \\
\text { STANDARD }\end{array}$ & $\begin{array}{l}\text { SAMPLE } \\
\text { DATE }\end{array}$ & $3-1-15$ \\
\hline AMMONIU & PPB & 56.00 & & $06 / 18 / 07$ & 76.00 \\
\hline BAR IUM & PPB & 8.00 & 1000.00 & $04 / 27 / 87$ & 44.60 \\
\hline BETA & $\mathrm{PCI} / \mathrm{L}$ & 3.00 & 50.00 & $64 / 27 / 67$ & 17.30 \\
\hline & & & & $06 / 1 B / B 7$ & 9.51 \\
\hline CALCIUM & PPB & 56.60 & & $04 / 27 / 87$ & 44900.00 \\
\hline CHLFORM & PPB & 10.00 & & & $\underset{N R}{N R}$ \\
\hline CHLORID & PPB & 560.00 & & $\begin{array}{l}64 / 27 / 87 \\
66 / 18 / 87\end{array}$ & $\begin{array}{l}15600.00 \\
27600.00\end{array}$ \\
\hline $\begin{array}{l}\text { CHROMUN } \\
\text { CONDFLD }\end{array}$ & $\begin{array}{l}\text { PPB } \\
\text { UMHO }\end{array}$ & $\begin{array}{r}10.00 \\
1.00\end{array}$ & 50.00 & $\begin{array}{l}64 / 27 / 87 \\
66 / 18 / 87\end{array}$ & $\begin{array}{l}350.00 \\
377.06 \\
{ }_{\mathrm{NR}}\end{array}$ \\
\hline $\begin{array}{l}\text { COPPER } \\
\text { FBARIUM }\end{array}$ & $\begin{array}{l}\mathrm{PPQ} \\
\mathrm{PPB}\end{array}$ & $\begin{array}{r}10.60 \\
8.00\end{array}$ & $\begin{array}{c}(1300.0) \\
1000.00\end{array}$ & $04 / 27 / 87$ & 41.00 \\
\hline FCALCIU & PPE & 50.00 & & $84 / 27 / 87$ & 43300.00 \\
\hline $\begin{array}{l}\text { FCOPPER } \\
\text { FIRON } \\
\text { FLUORID }\end{array}$ & $\begin{array}{l}\text { PPB } \\
P P B \\
P P B\end{array}$ & $\begin{array}{r}10.00 \\
56.06 \\
500.00\end{array}$ & $\begin{array}{r}(1300.6) \\
1400.00\end{array}$ & & \\
\hline FMAGNES & PPB & 6.00 & & $\begin{array}{l}04 / 27 / 87 \\
08 / 18 / 67\end{array}$ & $\begin{array}{l}16900.00 \\
11600.00\end{array}$ \\
\hline FMANGAN & PPB & 5.00 & & & \\
\hline FPOTASS & PPB & 160.00 & & $04 / 27 / 87$ & 5040.60 \\
\hline F50DIUM & PPB & 100.06 & & $\begin{array}{l}64 / 27 / 87 \\
68 / 18 / 87\end{array}$ & $\begin{array}{l}18400.00 \\
21300.00\end{array}$ \\
\hline FZINC & PPB & 5.00 & & & \\
\hline IRON & PPB & 50.00 & & $\begin{array}{l}04 / 27 / 87 \\
06 / 18 / 87\end{array}$ & $\begin{array}{r}132.68 \\
78.68\end{array}$ \\
\hline LOALPHA & $\mathrm{PCI} / \mathrm{L}$ & 2.00 & 15.00 & $04 / 27 / 87$ & 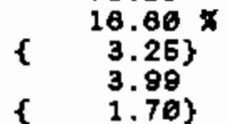 \\
\hline
\end{tabular}

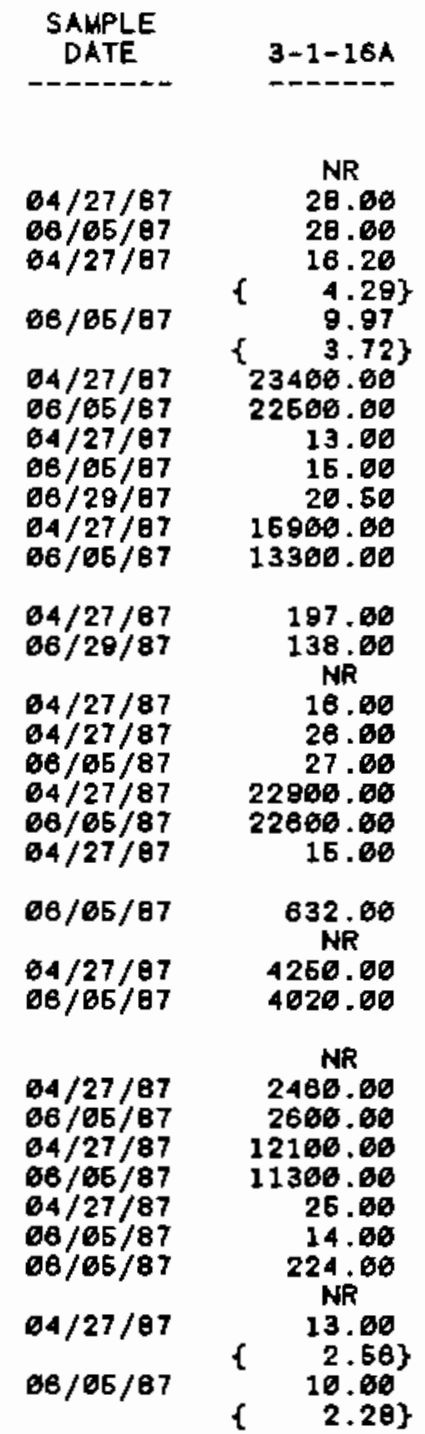

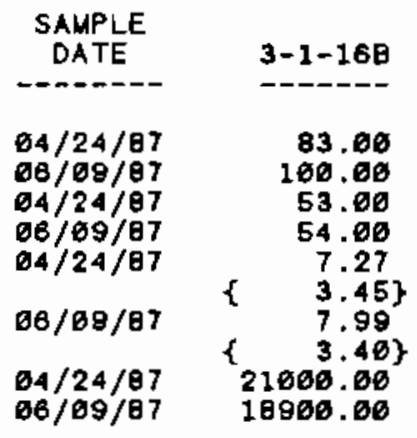

ถ6/09/8 7

$64 / 24 / 8$

$64 / 24 / 87$
$66 / 09 / 87$

$04 / 24 / 87$

$64 / 24 / 87$

$68 / 09 / 87$
$66 / 29 / 67$

$04 / 24 / 67$

$08 / 69 / 87$

$04 / 24 / 87$

$94 / 24 / 87$

$04 / 24 / 87$

$06 / 09 / 87$

$64 / 24 / 87$

66/09/87

$04 / 24 / 87$

$64 / 24 / 87$
$68 / 09 / 87$

$64 / 24 / 87$

$06 / 09 / 87$

$04 / 24 / 87$

$04 / 24 / 97$

$06 / 69 / 87$
$04 / 24 / 87$

$04 / 24 / 67$
$08 / 09 / 87$

$06 / 09 / 87$

$04 / 24 / 87$

$66 / 09 / 87$
$64 / 24 / 87$

$66 / 09 / 87$
NR

9590.00

9590.00
12100.00

14.00

386.08

320.00

247.00

52.00

61.60

20900.00

21500.00

98.00

1610.00 .

0900.00

6780.66

90.00

82.06

6490.00

6470.00

52860.00

60400.00

13.00
9.00

147.00

120.60

2.26

$1.41\}$

$1.41\}$
2.74
1.43

* - VALUE exceEds primary dRINKING water STANDARD.

* - VALUE EXCEEDS PROPOSED PRIMARY DRINKING WATER STANOARD.

* - VALUE EXCEEDS SCREENING LEVEL FOR FURTHER INVESTIGATION.

+ - DETECTION LIMIT WAS NOT AVAILABLE FOR COMPARISON

NR - ANALYSIS NOT REQUESTED OR NOT YET REPORTED

VALUES IN $f$ I ARE COUNTING ERRORS FOR RADIONUCLIDES

WATER STANDARD(S) IN PARENTHESES ARE PROPOSED ONLY 
IABLE A.5. (contd)

\begin{tabular}{|c|c|c|c|c|c|c|c|c|c|}
\hline $\begin{array}{l}\text { CONSTI } \\
\text { NAME }\end{array}$ & $\begin{array}{l}\text { JENT } \\
\text { UNITS }\end{array}$ & $\begin{array}{c}\text { DETECTION } \\
\text { LIMIT }\end{array}$ & $\begin{array}{l}\text { WATER } \\
\text { STANOARD }\end{array}$ & $\begin{array}{c}\text { SAMPLE } \\
\text { DATE }\end{array}$ & $3-1-15$ & $\begin{array}{l}\text { SAMPLE } \\
\text { DATE }\end{array}$ & $3-1-1 B A$ & $\begin{array}{l}\text { SAMPLE } \\
\text { DATE }\end{array}$ & $3-1-168$ \\
\hline MAGNES & PPB & 0.00 & & $\begin{array}{l}84 / 27 / 87 \\
68 / 18 / 87\end{array}$ & $\begin{array}{l}11100.00 \\
11800.00\end{array}$ & $\begin{array}{l}04 / 27 / 87 \\
66 / 65 / \theta 7\end{array}$ & $\begin{array}{l}4280.06 \\
4630.08\end{array}$ & $\begin{array}{l}64 / 24 / 87 \\
60 / 69 / 87\end{array}$ & $\begin{array}{l}7010.66 \\
8330.06\end{array}$ \\
\hline MANGESE & PPB & 5.00 & & & NR & $66 / 06 / 87$ & $\stackrel{5}{N R}^{00}$ & $\begin{array}{l}64 / 24 / 87 \\
68 / 89 / 67\end{array}$ & $\begin{array}{l}81.00 \\
84.00\end{array}$ \\
\hline $\begin{array}{l}\text { NICKEL } \\
\text { NITRATE }\end{array}$ & $\begin{array}{l}\text { PPB } \\
\text { PPB }\end{array}$ & $\begin{array}{r}10.00 \\
500.00\end{array}$ & 45000.00 & $\begin{array}{l}94 / 27 / 87 \\
06 / 18 / 87\end{array}$ & $\begin{array}{l}16900.00 \\
22800.06\end{array}$ & $\begin{array}{l}64 / 27 / 87 \\
6 \theta / 05 / \theta 7\end{array}$ & $\begin{array}{l}5770.08 \\
7810.06\end{array}$ & $04 / 24 / 87$ & $\begin{array}{l}10.00 \\
N R\end{array}$ \\
\hline PHFIELD & & 0.16 & & $\begin{array}{l}04 / 27 / 87 \\
08 / 18 / 87\end{array}$ & $\begin{array}{l}7.00 \\
7.20 \\
\mathrm{NR}\end{array}$ & $\begin{array}{l}04 / 27 / 87 \\
66 / 29 / 87\end{array}$ & $\begin{array}{l}6.16 \\
6.10 \\
\mathrm{NR}\end{array}$ & $\begin{array}{l}64 / 24 / 87 \\
66 / 09 / 87 \\
66 / 29 / 87\end{array}$ & $\begin{array}{l}7.10 \\
7.40 \\
6.40\end{array}$ \\
\hline POTASUM & PPE & 100.00 & & $\begin{array}{l}04 / 27 / 87 \\
66 / 18 / 87\end{array}$ & $\begin{array}{l}5030.60 \\
6040.00\end{array}$ & $\begin{array}{l}64 / 27 / 87 \\
66 / 85 / 87\end{array}$ & $\begin{array}{l}2486.06 \\
2560.00\end{array}$ & $\begin{array}{l}64 / 24 / 87 \\
66 / 69 / 87\end{array}$ & $\begin{array}{l}5586.00 \\
5050.00\end{array}$ \\
\hline RADIUL & $\mathrm{PCI} / \mathrm{L}$ & 0.10 & 5.00 & $04 / 27 / 87$ & $\begin{array}{ll}\{\quad 0.18 \\
\{\quad 0.16\}\end{array}$ & $\begin{array}{l}64 / 27 / 87 \\
86 / 06 / 87\end{array}$ & $\begin{array}{ll} & 0.29 \\
\{\quad & 0.19\} \\
\{\quad 0.24 \\
\{\quad 0.16\}\end{array}$ & $68 / 09 / 87$ & $\left\{\begin{array}{l}\qquad .28 \\
\{.17\} \\
N R\end{array}\right\}$ \\
\hline 500 IUM & PPB & 160.06 & & $64 / 27 / 87$ & 17000.00 & $04 / 27 / 87$ & 12300.00 & $64 / 24 / 87$ & 53480.00 \\
\hline SULFATE & PPB & 500.06 & & $04 / 27 / 67$ & 41700.60 & $04 / 27 / 87$ & 16100.00 & $64 / 24 / 87$ & 6920.00 \\
\hline TOC & PPB & 200.00 & & $\begin{array}{l}64 / 27 / 87 \\
68 / 18 / 87\end{array}$ & $\begin{array}{l}416.00 \\
533.00\end{array}$ & $\begin{array}{l}04 / 27 / 87 \\
06 / 05 / 97\end{array}$ & $\begin{array}{r}532.06 \\
599.06\end{array}$ & $\begin{array}{l}04 / 24 / 87 \\
06 / 09 / 67\end{array}$ & $\begin{array}{r}318.86 \\
365.00\end{array}$ \\
\hline TRANDCE & PPB & 10.06 & $(70.0)$ & & $\begin{array}{l}\text { NR } \\
\text { NR } \\
\text { NR }\end{array}$ & & $\begin{array}{l}\mathrm{NR} \\
\mathrm{NR}\end{array}$ & $\begin{array}{l}64 / 24 / 97 \\
68 / 69 / 87 \\
06 / 29 / 87\end{array}$ & $\begin{array}{l}71.60 \\
67.00 \\
72.00\end{array}$ \\
\hline TRICENE & PPB & 10.00 & $5.6)$ & & $\begin{array}{l}\text { NR } \\
\text { NR }\end{array}$ & & $\begin{array}{l}\text { NR } \\
\text { NR }\end{array}$ & $\begin{array}{l}04 / 24 / 97 \\
06 / 89 / 97 \\
66 / 29 / 67\end{array}$ & $\begin{array}{l}22.00 \\
20.00 \\
24.10\end{array}$ \\
\hline VANADUM & PPB & 6.00 & & $\begin{array}{l}64 / 27 / 87 \\
66 / 18 / 87\end{array}$ & $\begin{array}{r}7.00 \\
10.00\end{array}$ & & NR & & \\
\hline ZINC & PPB & 5.06 & & $04 / 27 / 87$ & 8.00 & $64 / 27 / 87$ & 29.00 & $\begin{array}{l}64 / 24 / 87 \\
66 / 89 / 87\end{array}$ & $\begin{array}{l}23.08 \\
13.06\end{array}$ \\
\hline
\end{tabular}

- - VALUe exceEds primary dRINKING water STANDARD.

- VALUE EXCEEDS PROPOSED PRIMARY DRINKING WATER STANDARD.

- VALUE EXCEEDS SCREENING LEVEL FOR FURTHER INVESTIGATION.

+ - DETECTION LIMIT WAS NOT AVAILABLE FOR COMPARISON

- ANALYSIS NOT REQUESTED OR NOT YET REPORTED

VALUES IN \{ $\}$ ARE COUNTING ERRORS FOR RADIONUCLIDES

WATER STANDARO(S) IN PARENTHESES ARE PROPOSED ONLY 
TABLE A.5. (cont d)

\begin{tabular}{|c|c|c|c|c|c|c|c|c|c|}
\hline $\begin{array}{l}\text { CONSTI } \\
\text { NAME }\end{array}$ & $\begin{array}{l}\text { UENT } \\
\text { UNITS }\end{array}$ & $\begin{array}{l}\text { DETECTION } \\
\text { LIMIT }\end{array}$ & $\begin{array}{l}\text { WATER } \\
\text { STANDARD }\end{array}$ & $\begin{array}{l}\text { SAMPLE } \\
\text { DATE }\end{array}$ & $3-1-16 C$ & $\begin{array}{c}\text { SAMPLE } \\
\text { DATE }\end{array}$ & $3-1-16 D$ & $\begin{array}{c}\text { SAMPLE } \\
\text { DATE }\end{array}$ & $3-1-17 A$ \\
\hline $\begin{array}{l}\text { ALUMNUM } \\
\text { AMMONIU }\end{array}$ & $\begin{array}{l}\mathrm{PPO} \\
\mathrm{PPB}\end{array}$ & $\begin{array}{r}150.00 \\
50.00\end{array}$ & & $04 / 24 / 87$ & 102.68 & $\begin{array}{l}66 / 29 / 87 \\
86 / 29 / 87\end{array}$ & $\begin{array}{c}175.00 \\
63.60\end{array}$ & & \\
\hline BARIUM & PPB & 6.00 & 1000.06 & $\begin{array}{l}04 / 24 / 87 \\
06 / 89 / 87\end{array}$ & 64.00 & $66 / 29 / 87$ & 8Q.60 & $\begin{array}{l}64 / 23 / 87 \\
98 / 64 / 87\end{array}$ & $\begin{array}{l}20 . \emptyset 0 \\
28.06\end{array}$ \\
\hline BETA & PCI/L & 3.60 & 50.00 & $\begin{array}{l}64 / 24 / 87 \\
68 / 69 / 87\end{array}$ & $\begin{array}{r}14.50 \\
\{\quad 4.68\} \\
16.98 \\
\{\quad 3.65\}\end{array}$ & $66 / 29 / 87$ & $\left\{\begin{array}{c}14.89 \\
4.68 \\
N R\end{array}\right\}$ & $\begin{array}{l}04 / 23 / 87 \\
06 / 04 / 87\end{array}$ & $\begin{array}{cc}56.267 \\
\{\quad 6.43\} \\
\{\quad 51.26\}\end{array}$ \\
\hline $\begin{array}{l}\text { CADMIUM } \\
\text { CALCIUM }\end{array}$ & $\begin{array}{l}\text { PPB } \\
\text { PPB }\end{array}$ & $\begin{array}{r}2.00 \\
58.00\end{array}$ & 16.00 & $\begin{array}{l}04 / 24 / 87 \\
08 / 69 / 87\end{array}$ & $\begin{array}{l}14860.00 \\
15906.00\end{array}$ & $\begin{array}{l}06 / 29 / 87 \\
06 / 29 / 87\end{array}$ & $\begin{array}{c}9.00 \\
13500.00 \\
\mathrm{NR}\end{array}$ & $\begin{array}{l}64 / 23 / 87 \\
66 / 94 / 87\end{array}$ & 19800.60 \\
\hline CHLFORM & PPB & 10.00 & & $04 / 24 / 87$ & $\begin{array}{c}\text { NR } \\
\text { NR } \\
10900.00\end{array}$ & $68 / 29 / 87$ & $\begin{array}{c}\text { NR } \\
\text { NR } \\
11100.00\end{array}$ & $\begin{array}{l}64 / 23 / 87 \\
66 / 64 / 87 \\
66 / 26 / 87 \\
64 / 23 / 87\end{array}$ & $\begin{array}{r}13.60 \\
19.60 \\
25.60\end{array}$ \\
\hline CHROMUM & PPB & 10.06 & 50.00 & $\begin{array}{l}68 / 09 / 87 \\
64 / 24 / 87\end{array}$ & $\begin{array}{r}13100.06 \\
20.66\end{array}$ & $100 /<8 / 81$ & NR & $08 / 04 / 87$ & 28960.60 \\
\hline CONDFLD & UMHO & 1.06 & & $\begin{array}{l}68 / 69 / 67 \\
64 / 24 / 67 \\
06 / 69 / 97 \\
68 / 29 / 67\end{array}$ & $\begin{array}{l}33.00 \\
302.06 \\
287.00 \\
287.00\end{array}$ & $06 / 29 / 87$ & $\begin{array}{c}N R \\
294, .60 \\
N R \\
N R \\
N R\end{array}$ & $\begin{array}{l}04 / 23 / 87 \\
06 / 04 / 67 \\
66 / 26 / 67\end{array}$ & $\begin{array}{l}\text { NR } \\
179.60 \\
216.60 \\
189.60\end{array}$ \\
\hline COPPER & PPB & 10.00 & $(1300.0)$ & & NR & & NR & $\begin{array}{l}64 / 23 / 87 \\
06 / 04 / 87\end{array}$ & 18.60 \\
\hline FBARIUM & PPB & 8.00 & 1000.00 & $\begin{array}{l}04 / 24 / 87 \\
68 / 69 / 87\end{array}$ & $\begin{array}{l}64.00 \\
50.00\end{array}$ & & $\begin{array}{l}\text { NR } \\
\text { NR }\end{array}$ & $\begin{array}{l}84 / 23 / 87 \\
66 / 64 / 87\end{array}$ & $\begin{array}{l}19.00 \\
28.06\end{array}$ \\
\hline FCALCIU & PPB & 60.00 & & $\begin{array}{l}64 / 24 / 87 \\
06 / 99 / 87\end{array}$ & $\begin{array}{l}16100.00 \\
15900.00\end{array}$ & & $\begin{array}{l}N R \\
N R\end{array}$ & $\begin{array}{l}64 / 23 / 87 \\
68 / 04 / 87\end{array}$ & $\begin{array}{l}19660.66 \\
23306.00\end{array}$ \\
\hline FCOPPER & PPB & 16.60 & $(1300.6)$ & & & & $\begin{array}{c}\text { NR } \\
\text { NR }\end{array}$ & $\begin{array}{l}64 / 23 / 87 \\
68 / 64 / 87\end{array}$ & 22.00 \\
\hline FLUORID & PPB & 500.00 & 1460.00 & $\begin{array}{l}64 / 24 / 87 \\
68 / 09 / 67\end{array}$ & $\begin{array}{l}1570.06 \\
1530.06\end{array}$ & $68 / 29 / 87$ & $1779.00 *$ & $06 / 84 / 87$ & $\begin{array}{l}\text { OBJ. } \\
\text { NR }\end{array}$ \\
\hline FMAGNES & PPB & 0.00 & & $\begin{array}{l}64 / 24 / 67 \\
06 / 89 / 87\end{array}$ & 5420.66 & & $\begin{array}{c}N R \\
N R\end{array}$ & $\begin{array}{l}64 / 23 / 87 \\
66 / 94 / 87\end{array}$ & 3970.00 \\
\hline FMANGAN & PPB & 5.00 & & $\begin{array}{l}04 / 24 / 87 \\
66 / 69 / 87\end{array}$ & $\begin{array}{l}48.06 \\
41.00\end{array}$ & & $\begin{array}{l}\mathbf{N R} \\
\mathbf{N R}\end{array}$ & & 7row \\
\hline FPOTASS & PPB & 100.00 & & $\begin{array}{l}04 / 24 / 87 \\
06 / 09 / 87\end{array}$ & $\begin{array}{l}6576.06 \\
5480.60\end{array}$ & & NR & $64 / 23 / 87$ & 17300.00 \\
\hline FSODIUM & PPB & 100.00 & & $\begin{array}{l}64 / 24 / 87 \\
68 / 69 / 87\end{array}$ & $\begin{array}{l}57860.00 \\
44800.00\end{array}$ & & NR & $\begin{array}{l}04 / 23 / 87 \\
68 / 64 / 97\end{array}$ & $\begin{array}{l}16766.66 \\
15666.06\end{array}$ \\
\hline FZINC & PPB & 5.60 & & & & & NR & $64 / 23 / 67$ & 8.00 \\
\hline IRON & PPB & 60.00 & & $\begin{array}{l}04 / 24 / 87 \\
08 / \varnothing 9 / 87\end{array}$ & $\begin{array}{l}128.66 \\
293.68\end{array}$ & $66 / 29 / 87$ & 1130.06 & $64 / 23 / 87$ & 129.60 \\
\hline \multicolumn{10}{|c|}{ 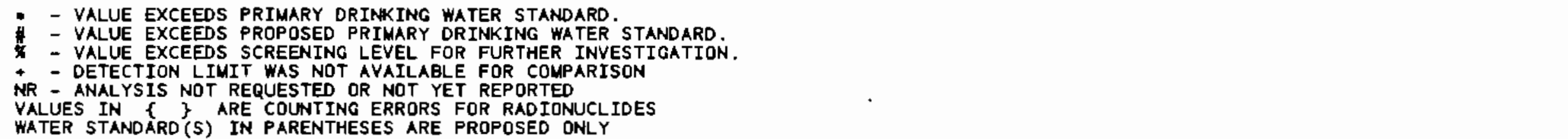 } \\
\hline
\end{tabular}


TABLE A.5. (contd)

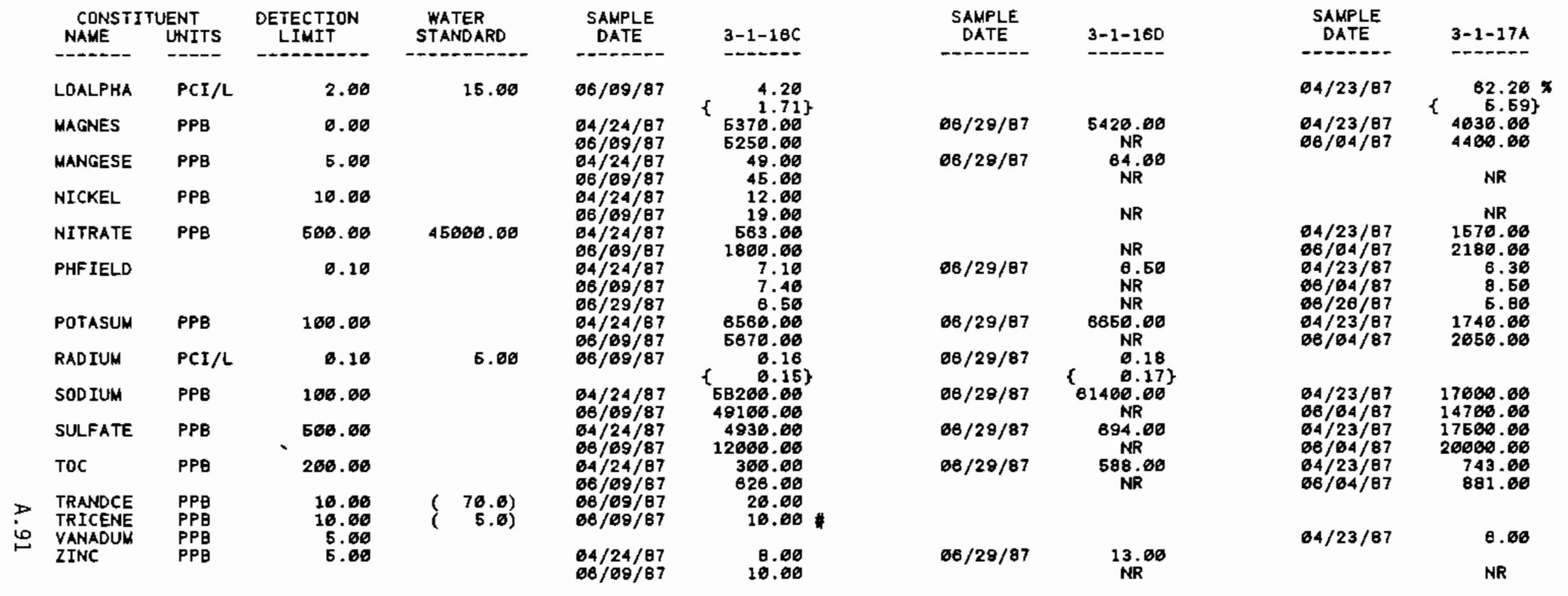

* - VALUE EXCEEDS PRIMARY DRINIING WaTER STANDARD.

* - VALUE EXCEEDS PROPOSED PRIMARY DRINKING WATER STANDARD.

- VALUE EXCEEDS SCREENING LEVEL FOR FURTHER INVESTIGATION.

* - DETECTION LIMIT WAS NOT AVAILABLE FOR COMPARISON

NR - ANALYSIS NOT REQUESTED OR NOT YET REPORTED

VALUES IN \{\} ARE COUNTING ERRORS FOR RADIONUCLIDES

WATER STANDARO(S) IN PARENTHESES ARE PROPOSED ONLY 
TABLE A.5. (contd)

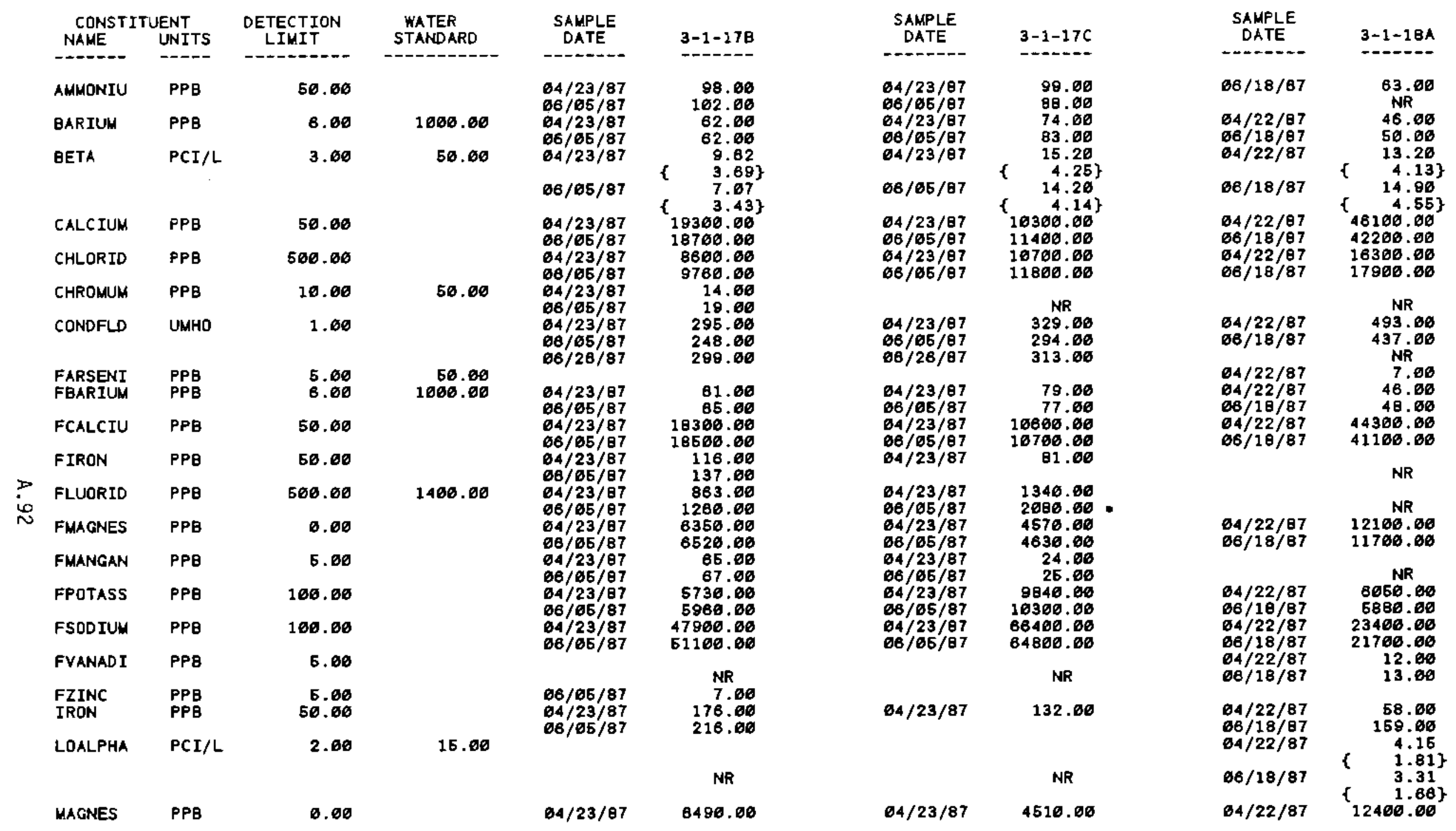

- - VALUE EXCEEDS PRIMARY DRINKING WATER STANDARD.

- VALUE EXCEEDS PROPOSED PRIMARY ORINKING HATER STANDARD.

* - VALUE EXCEEDS SCREENING LEVEL FOR FURTHER INVESTIGATION.

+ - DETECTION LIMIT WAS NOT AVAILABLE FOR COMPARISON

NR - ANALYSIS NOT REQUESTED OR NOT YET REPORTED

VALUES IN \{ $\}$ ARE COUNTING ERRORS FOR RADIONUCLIDES

WATER STANDARO (S) IN PARENTHESES ARE PROPOSED ONLY 
TABLE A.5. (contd)

\begin{tabular}{|c|c|c|c|c|c|c|c|c|c|}
\hline $\begin{array}{l}\text { CONSTI } \\
\text { NAME }\end{array}$ & $\begin{array}{l}\text { JENT } \\
\text { UNITS }\end{array}$ & $\begin{array}{c}\text { DETECTION } \\
\text { LIMIT }\end{array}$ & $\begin{array}{l}\text { WATER } \\
\text { STANDARD }\end{array}$ & $\begin{array}{c}\text { SAMPLE } \\
\text { DATE }\end{array}$ & $3-1-17 \theta$ & $\begin{array}{l}\text { SAMPLE } \\
\text { DATE }\end{array}$ & $3-1-17 C$ & $\begin{array}{c}\text { SAMPLE } \\
\text { DATE }\end{array}$ & $3-1-18 \mathrm{~A}$ \\
\hline $\begin{array}{l}\text { MAGNES } \\
\text { MANGESE }\end{array}$ & $\begin{array}{l}\text { PPB } \\
\text { PPB }\end{array}$ & $\begin{array}{l}0.00 \\
5.08\end{array}$ & & $\begin{array}{l}66 / 05 / 87 \\
04 / 23 / 87 \\
06 / \theta 5 / \theta 7\end{array}$ & $\begin{array}{r}6816.66 \\
89.06 \\
69.00\end{array}$ & $\begin{array}{l}66 / 05 / 87 \\
64 / 23 / 87 \\
66 / 95 / 87\end{array}$ & $\begin{array}{r}4990.00 \\
24.00 \\
26.00\end{array}$ & $66 / 18 / 87$ & $\begin{array}{c}11900.60 \\
\text { NR }\end{array}$ \\
\hline $\begin{array}{l}\text { METHYCH } \\
\text { NICKEL }\end{array}$ & $\begin{array}{l}\text { PPQ } \\
\text { PPB }\end{array}$ & $\begin{array}{l}10.00 \\
10.00\end{array}$ & & $\begin{array}{l}06 / 95 / 67 \\
68 / \boxminus 5 / 67\end{array}$ & $\begin{array}{l}16.66 \\
18.66\end{array}$ & & & & \\
\hline NITRATE & PPE & 500.00 & 45000.00 & & NR & & & $\begin{array}{l}64 / 22 / 87 \\
68 / 18 / 67\end{array}$ & 20000.000 \\
\hline PHFIELD & & 0.16 & & $\begin{array}{l}64 / 23 / 67 \\
06 / 06 / 97 \\
68 / 28 / 67\end{array}$ & $\begin{array}{l}6.80 \\
7.26 \\
8.86\end{array}$ & $\begin{array}{l}64 / 23 / 87 \\
66 / 65 / 87 \\
96 / 28 / 67\end{array}$ & $\begin{array}{l}7.50 \\
7.60 \\
7.20\end{array}$ & $\begin{array}{l}64 / 22 / 87 \\
66 / 18 / 87\end{array}$ & $\begin{array}{l}7.46 \\
7.76 \\
\mathrm{NR}\end{array}$ \\
\hline POTASUM & PPB & 100.00 & & $\begin{array}{l}04 / 23 / 67 \\
06 / 65 / 67\end{array}$ & $\begin{array}{l}5926.06 \\
6136.66\end{array}$ & $\begin{array}{l}64 / 23 / 87 \\
68 / 05 / 67\end{array}$ & $\begin{array}{r}9920.00 \\
11200.00\end{array}$ & $\begin{array}{l}64 / 22 / 87 \\
06 / 10 / 87\end{array}$ & $\begin{array}{l}8218.06 \\
5930.06\end{array}$ \\
\hline RADIUM & $\mathrm{PCI} / \mathrm{L}$ & 0.16 & 5.60 & $\begin{array}{l}64 / 23 / 87 \\
06 / 06 / 87\end{array}$ & 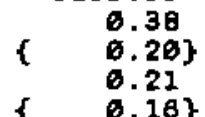 & $06 / 06 / 87$ & $\begin{array}{ll}\{\quad 0.17 \\
\left.\quad \mathrm{NR}^{0.15}\right\}\end{array}$ & & NR \\
\hline SODIUM & PPB & 160.60 & & $\begin{array}{l}64 / 23 / 87 \\
66 / 65 / 87\end{array}$ & 47806.06 & $\begin{array}{l}64 / 23 / 87 \\
66 / 95 / 87\end{array}$ & $\begin{array}{l}86400.00 \\
85809.00\end{array}$ & $64 / 22 / 87$ & 23400.000 \\
\hline SULFATE & PPB & 600.00 & & $04 / 23 / 87$ & 4620.60 & & & $04 / 22 / 87$ & 48900.00 \\
\hline TOC & PPB & 200.00 & & $\begin{array}{l}04 / 23 / 87 \\
86 / 05 / 87\end{array}$ & $\begin{array}{r}394.06 \\
297.08\end{array}$ & $04 / 23 / 87$ & 245.00 & $64 / 22 / 67$ & 286.00 \\
\hline TRANDCE & PPB & 10.00 & $(70.0)$ & $\begin{array}{l}64 / 23 / 87 \\
66 / 65 / 97 \\
66 / 26 / 97\end{array}$ & $\begin{array}{r}31.06 \\
27.06 \\
28.06\end{array}$ & & $\begin{array}{l}\text { NR } \\
\text { NR }\end{array}$ & & $\begin{array}{l}\mathrm{NR} \\
\mathrm{NR} \\
\mathrm{NR}\end{array}$ \\
\hline VANADUM & PPB & 5.00 & & & & & NR & $\begin{array}{l}04 / 22 / 07 \\
06 / 18 / 67\end{array}$ & $\begin{array}{l}12.00 \\
14.00\end{array}$ \\
\hline ZINC & PPE & 5.00 & & $\begin{array}{l}04 / 23 / 87 \\
66 / 65 / 87\end{array}$ & $\begin{array}{r}7.00 \\
10.00\end{array}$ & $\begin{array}{l}94 / 23 / 87 \\
66 / 65 / 87\end{array}$ & $\begin{array}{l}\theta .00 \\
5.00\end{array}$ & & NR \\
\hline
\end{tabular}

* - VALUE EXCEEDS PRIMARY DRINKING waTER STANDARD.

* - VALUE EXCEEDS PROPOSED PRIMARY DRINHING WATER STANDARD.

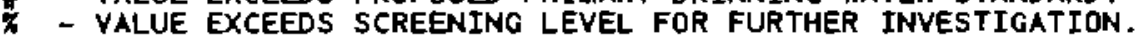

+ - DETECTION LIMIT WAS NOT AVAILABLE FOR COMPARISON

NR - ANALYSIS NOT REQUESTED OR NOT YET REPORTED

VALUES IN \{ $\}$ ARE COUNTING ERRORS FOR RADIONUCLIDES

WATER STANDARD'(S) IN PARENTHESES ARE PROPOSED ONLY 
TABLE A.5. (contd)

\begin{tabular}{|c|c|c|c|c|c|c|c|c|c|}
\hline $\begin{array}{l}\text { CONSTI } \\
\text { NAME }\end{array}$ & $\begin{array}{l}\text { UENT } \\
\text { UNITS }\end{array}$ & $\begin{array}{c}\text { DETECTION } \\
\text { LIMIT }\end{array}$ & $\begin{array}{l}\text { WATER } \\
\text { STANDARD }\end{array}$ & $\begin{array}{c}\text { SAMPLE } \\
\text { DATE }\end{array}$ & $3-1-18 \mathrm{~B}$ & $\begin{array}{c}\text { SAMPLE } \\
\text { DATE }\end{array}$ & $3-1-18 C$ & $\begin{array}{l}\text { SAMPLE } \\
\text { DATE }\end{array}$ & $3-1-19$ \\
\hline ALUUMNUM & PPB & 150.00 & & & NR & & NR & $04 / 02 / 87$ & $\begin{array}{r}1640.00 \\
285.00\end{array}$ \\
\hline AMMONIU & PPB & 56.00 & & $08 / 18 / 87$ & 125.66 & $04 / 22 / 87$ & 08.68 & & \\
\hline GARIUM & PPB & 6.00 & 1000.00 & $\begin{array}{l}84 / 22 / 87 \\
66 / 18 / 87\end{array}$ & $\begin{array}{l}46.60 \\
41.66 \\
N R\end{array}$ & $\begin{array}{l}64 / 22 / 87 \\
66 / 1 \theta / 87\end{array}$ & $\begin{array}{l}67 . .86 \\
65.86 \\
\mathrm{NR}\end{array}$ & $\begin{array}{l}84 / 62 / \theta 7 \\
64 / 27 / 67 \\
86 / 36 / \theta 7\end{array}$ & $\begin{array}{l}28.06 \\
23.06 \\
22.00\end{array}$ \\
\hline BETA & $\mathrm{PCI} / \mathrm{L}$ & 3.00 & 50.00 & $\begin{array}{l}04 / 22 / 87 \\
66 / 18 / 87\end{array}$ & $\left.\begin{array}{ll} & 13.90 \\
\{ & 4.15\} \\
& 9.26 \\
\{ & 4.06\} \\
& N R\end{array}\right\}$ & $\begin{array}{l}64 / 22 / \theta 7 \\
66 / 18 / \theta 7\end{array}$ & $\begin{array}{ll} & 7.81 \\
\{\quad 3.51\} \\
\left\{\begin{array}{l}3.83 \\
3.86\} \\
\mathrm{NR}\end{array}\right.\end{array}$ & \multicolumn{2}{|r|}{$\begin{array}{c}110.00 \\
9.07\}^{*} \\
121.06 \times \\
9.50\} \\
39.10 \\
5.96\end{array}$} \\
\hline CALCIUM & PPB & 50.00 & & $\begin{array}{l}64 / 22 / 87 \\
66 / 10 / 87\end{array}$ & $\begin{array}{c}13100.00 \\
11100.00 \\
\mathrm{NR}\end{array}$ & $\begin{array}{l}64 / 22 / 87 \\
06 / 18 / 97\end{array}$ & $\begin{array}{l}13500.00 \\
12000.00 \\
\mathrm{NR}\end{array}$ & $\begin{array}{l}04 / 62 / 87 \\
64 / 27 / 87 \\
06 / 30 / 87\end{array}$ & $\begin{array}{l}15400.06 \\
18606.00 \\
16008.00\end{array}$ \\
\hline CHLFORM & PPE & 10.00 & & $\begin{array}{l}64 / 22 / 87 \\
68 / 18 / 87\end{array}$ & $\begin{array}{c}\text { NR } \\
\text { NR } \\
10600.00 \\
11000.00 \\
\text { NR }\end{array}$ & $\begin{array}{l}64 / 22 / 87 \\
66 / 10 / 87\end{array}$ & $\begin{array}{c}\mathrm{NR} \\
\mathrm{NR} \\
\mathrm{NR} \\
11106.60 \\
16906.06 \\
\mathrm{NR}\end{array}$ & $\begin{array}{l}04 / 62 / 87 \\
64 / 27 / 87 \\
66 / 36 / 87 \\
64 / 62 / 87 \\
64 / 27 / 87 \\
68 / 36 / \theta 7\end{array}$ & $\begin{array}{r}15.00 \\
14.06 \\
20.46 \\
16000.00 \\
12780.06 \\
8870.00\end{array}$ \\
\hline CHROMUM & PPB & 10.00 & 50.00 & $04 / 22 / 87$ & 12.60 & & \multirow{2}{*}{$\begin{array}{c}N R \\
N R \\
407.08 \\
401.68 \\
N R\end{array}$} & \multirow{4}{*}{$\begin{array}{l}64 / 02 / 87 \\
64 / 92 / 87 / 87 \\
64 / 27 / 87 \\
66 / 36 / 87 \\
64 / 02 / 87 \\
64 / 27 / 87 \\
68 / 30 / 87 \\
64 / 02 / 87 \\
64 / 27 / 87 \\
64 / 62 / 87 \\
64 / 27 / 87\end{array}$} & \multirow{9}{*}{ 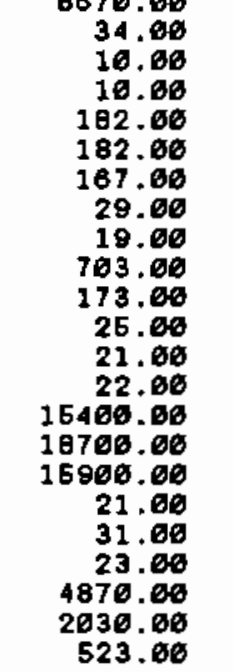 } \\
\hline CONDFLD & UMHO & 1.00 & & $\begin{array}{l}04 / 22 / \theta 7 \\
06 / 10 / 87\end{array}$ & $\begin{array}{c}\text { NR } \\
468.00 \\
374.00 \\
\text { NR }\end{array}$ & $\begin{array}{l}64 / 22 / 07 \\
68 / 18 / 87\end{array}$ & & & \\
\hline COPPER & PPB & 10.00 & $(1300.0)$ & & NR & & Tre & & \\
\hline FALUMIN & PPB & 150.00 & & & NR & & NR & & \\
\hline FBARIUM & $\mathrm{PPB}$ & 6.00 & 1000.60 & $\begin{array}{l}64 / 22 / 87 \\
86 / 18 / 97\end{array}$ & $\begin{array}{l}41.86 \\
42.06 \\
\mathrm{NR}\end{array}$ & $\begin{array}{l}04 / 22 / 87 \\
08 / 18 / 87\end{array}$ & $\begin{array}{c}69.00 \\
64.60 \\
\mathrm{NR}\end{array}$ & $\begin{array}{l}04 / 02 / 87 \\
64 / 27 / 87 \\
68 / 36 / 87\end{array}$ & \\
\hline FCALCIU & PPB & 50.06 & & $\begin{array}{l}04 / 22 / 87 \\
08 / 18 / 87\end{array}$ & $\begin{array}{c}12500.00 \\
11060.00 \\
N R\end{array}$ & $\begin{array}{l}04 / 22 / 87 \\
68 / 18 / 97\end{array}$ & $\begin{array}{c}13100.00 \\
12000.00 \\
N R\end{array}$ & $\begin{array}{l}64 / 62 / 87 \\
64 / 27 / 87 \\
06 / 30 / 67\end{array}$ & \\
\hline $\begin{array}{l}\text { FCHROMI } \\
\text { FCOPPER }\end{array}$ & $\begin{array}{l}\text { PPB } \\
\text { PPB }\end{array}$ & $\begin{array}{l}16.00 \\
10.00\end{array}$ & $\begin{array}{r}50.00 \\
(1300.0)\end{array}$ & & NR & & & $\begin{array}{l}04 / 02 / 87 \\
04 / 02 / 87 \\
04 / 27 / 87\end{array}$ & \\
\hline FIRON & PPB & 50.00 & & $\begin{array}{l}04 / 22 / 97 \\
06 / 18 / 87\end{array}$ & $\begin{array}{l}153.06 \\
143.06\end{array}$ & $\begin{array}{l}64 / 22 / 87 \\
66 / 18 / 87\end{array}$ & $\begin{array}{r}122.00 \\
84.00\end{array}$ & $\begin{array}{l}64 / 02 / 87 \\
64 / 27 / 87\end{array}$ & \\
\hline FLUORID & PPB & 506.00 & 1400.06 & $04 / 22 / 87$ & $1146.0 B$ & $64 / 22 / 87$ & $1580.00 *$ & $64 / 62 / 87$ & \\
\hline $\begin{array}{l}\text { * - VAL } \\
\#-V A L \\
*-V A L \\
+-D E \\
\text { NR - AN } \\
\text { VALUES } \\
\text { WATER S }\end{array}$ & $\begin{array}{l}\text { UE EXCEE } \\
\text { UE EXCEE } \\
\text { UE EXCEE } \\
\text { ECTION L } \\
\text { LYSIS NO } \\
\text { N \{ \} } \\
\text { ANDARD'S }\end{array}$ & $\begin{array}{l}\text { DS PRIMARY D } \\
\text { DS PROPOSED } \\
\text { DS SCREENING } \\
\text { IMIT WAS NDT } \\
\text { T REQUESTTED } \\
\text { ARE COUNTIN } \\
\text { IN PARENTH }\end{array}$ & $\begin{array}{l}\text { INKING WATER } \\
\text { RIMARY DRINKI } \\
\text { LEVEL FOR FUP } \\
\text { AVAILABLE FOF } \\
\text { R NOT YET REP } \\
\text { ERRORS FOR } \\
\text { SES ARE PROPC }\end{array}$ & $\begin{array}{l}\text { ANDARD. } \\
\text { WATER STA } \\
\text { ER INVESTI } \\
\text { OMPARISON } \\
\text { TED } \\
\text { IONUCLIDES } \\
\text { D ONLY }\end{array}$ & ATION. & & & & \\
\hline
\end{tabular}


TABLE A.5. (contd)

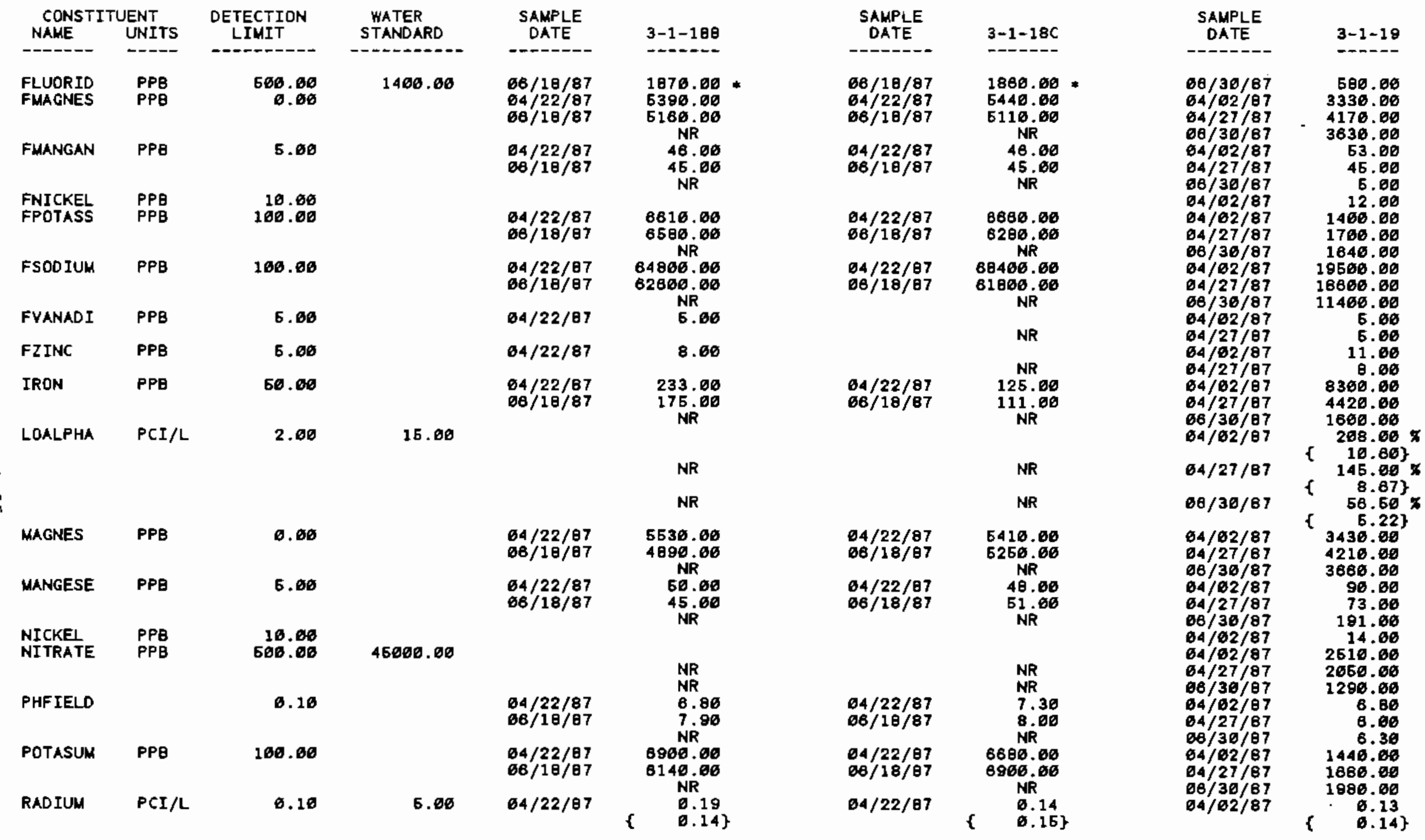

- - VALUE EXCEEDS PRIMARY DRINKING WATER STANDARD.

* - VALUE EXCEEDS PROPOSED PRIMARY DRINKING Hater STANDARD.

* - VALUE EXCEEDS SCREENING LEVEL FOR FURTHER INYESTIGATION.

* - DETECTION LIMIT WAS NOT AVAILABLE FOR COMPARISON

NR - ANALYSIS NDT REQUESTED OR NOT YET REPORTED

VALUES IN \{ $\}$ ARE CDUNTING ERRORS FOR RADIONUCLIDES

WATER STANDARO'(S) IN PARENTHESES ARE PROPOSED ONLY 
TABLE A.5. (contd)

\begin{tabular}{|c|c|c|c|c|c|c|c|c|c|}
\hline $\begin{array}{l}\text { CONSTI } \\
\text { NAKE }\end{array}$ & $\begin{array}{l}\text { UENT } \\
\text { UNITS }\end{array}$ & $\begin{array}{l}\text { DETECTION } \\
\text { LIMIT }\end{array}$ & $\begin{array}{l}\text { WATER } \\
\text { STANDARD }\end{array}$ & $\begin{array}{l}\text { SAHPLE } \\
\text { DATE }\end{array}$ & $3-1-18 \theta$ & $\begin{array}{l}\text { SAMPLE } \\
\text { DATE }\end{array}$ & $3-1-18 C$ & $\underset{\text { DATE }}{\text { SAMPLE }}$ & $3-1-19$ \\
\hline$---n+\infty$ & $\cdots$ & --------- & 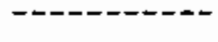 & - - & $\cdots+-\infty$ & 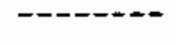 & 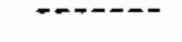 & ーー-ーーーー & $-\ldots--$ \\
\hline RAD IUN & PCI/L & 0.10 & 5.00 & & \multirow{3}{*}{$\begin{array}{c}65400.00 \\
60406.00 \\
\text { NR } \\
701.00\end{array}$} & $86 / 18 / 87$ & \multirow{6}{*}{$\begin{array}{c}0.22 \\
\begin{array}{c}0.16\} \\
66300.00\end{array} \\
65900.00 \\
\text { NR } \\
1210.00 \\
1910.00 \\
\text { NR } \\
262.00 \\
467.00 \\
N R \\
5.00 \\
N R \\
7.00 \\
5.00\end{array}$} & \multirow{6}{*}{$\begin{array}{l}04 / 27 / 87 \\
04 / 02 / 87 \\
04 / 27 / \theta 7 \\
06 / 30 / \theta 7 \\
04 / 02 / \theta 7 \\
04 / 27 / 67 \\
06 / 30 / 87 \\
04 / 62 / \theta 7 \\
04 / 27 / 97 \\
06 / 30 / \theta 7 \\
04 / 02 / 87 \\
06 / 30 / 87 \\
04 / 02 / 87 \\
04 / 27 / \theta 7\end{array}$} & \multirow{6}{*}{ 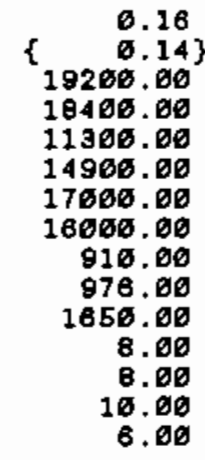 } \\
\hline SODIUH & PPB & 100.00 & & $\begin{array}{l}64 / 22 / 87 \\
66 / 18 / 87\end{array}$ & & $\begin{array}{l}04 / 22 / 87 \\
06 / 18 / 87\end{array}$ & & & \\
\hline SULFATE & PPB & 500.00 & & $84 / 22 / 87$ & & $\begin{array}{l}64 / 22 / 87 \\
66 / 18 / 87\end{array}$ & & & \\
\hline TOC & PPB & 200.00 & & $\begin{array}{l}64 / 22 / 87 \\
66 / 18 / 87\end{array}$ & $\begin{array}{c}\text { NR } \\
401.00 \\
323.00 \\
\text { NR }\end{array}$ & $\begin{array}{l}04 / 22 / 87 \\
66 / 18 / 87\end{array}$ & & & \\
\hline VANADUH & PPB & 5.00 & & & & $86 / 18 / 87$ & & & \\
\hline ZINC & PPB & 5.60 & & $\begin{array}{l}64 / 22 / 87 \\
66 / 18 / 87\end{array}$ & $\begin{array}{l}7.00 \\
5.00\end{array}$ & $\begin{array}{l}64 / 22 / 87 \\
66 / 18 / 87\end{array}$ & & & \\
\hline
\end{tabular}


IABLE A.5. (contd)

\begin{tabular}{|c|c|c|c|c|c|c|c|c|c|}
\hline $\begin{array}{l}\text { CONSTI } \\
\text { NAME }\end{array}$ & $\begin{array}{l}\text { UENT } \\
\text { UNI TS }\end{array}$ & $\begin{array}{l}\text { DETECTION } \\
\text { LIMIT }\end{array}$ & $\begin{array}{l}\text { WATER } \\
\text { STANDARD }\end{array}$ & $\begin{array}{l}\text { SAMPLE } \\
\text { DATE }\end{array}$ & $3-2-1$ & $\begin{array}{l}\text { SAMPLE } \\
\text { DATE }\end{array}$ & $3-3-7$ & $\begin{array}{c}\text { SAMPLE } \\
\text { DATE }\end{array}$ & $3-3-16$ \\
\hline BAR IUM & PPB & 0.66 & 1800.06 & $\begin{array}{l}84 / 21 / 87 \\
68 / 24 / 87\end{array}$ & $\begin{array}{l}46.66 \\
39.08\end{array}$ & $\begin{array}{l}04 / 2 \theta / 87 \\
06 / 24 / 87\end{array}$ & $\begin{array}{l}34.66 \\
42.80\end{array}$ & $\begin{array}{l}64 / 14 / 87 \\
66 / 25 / 67\end{array}$ & $\begin{array}{l}42.66 \\
42.6 \varnothing\end{array}$ \\
\hline BETA & PCI/L & 3.66 & 50.60 & $64 / 21 / 87$ & $\begin{array}{c}16.60 \\
4.3 \sigma\}\end{array}$ & $84 / 28 / 87$ & $\begin{array}{c}13.76 \\
4.11\}\end{array}$ & $04 / 14 / 87$ & $\begin{array}{r}15.46 \\
4.16\}\end{array}$ \\
\hline & & & & $68 / 24 / 87$ & $\begin{array}{r}9.62 \\
\{\quad 3.91\}\end{array}$ & $00 / 24 / 87$ & $\begin{array}{r}7.41 \\
\quad 3.97\}\end{array}$ & $68 / 25 / 87$ & $\begin{array}{r}8.17 \\
\{\quad 3.86\}\end{array}$ \\
\hline CALCIUM & PPB & 50.00 & & $\begin{array}{l}64 / 21 / 87 \\
66 / 24 / 87\end{array}$ & $\begin{array}{l}24300.06 \\
20000.00\end{array}$ & $\begin{array}{l}04 / 2 \theta / 87 \\
60 / 24 / 87\end{array}$ & $\begin{array}{l}40306.60 \\
38000.00\end{array}$ & $\begin{array}{l}04 / 14 / 07 \\
66 / 25 / 67\end{array}$ & $\begin{array}{l}36800.00 \\
25400.00\end{array}$ \\
\hline $\begin{array}{l}\text { CHLFORM } \\
\text { CHLORID }\end{array}$ & $\begin{array}{l}\text { PPB } \\
\text { PPB }\end{array}$ & $\begin{array}{r}10.60 \\
500.80\end{array}$ & & $\begin{array}{l}66 / 24 / 87 \\
64 / 21 / 87 \\
66 / 24 / 87\end{array}$ & $\begin{array}{r}12.00 \\
17200.06 \\
17606.00\end{array}$ & $\begin{array}{l}04 / 2 \theta / 87 \\
66 / 24 / 87\end{array}$ & $\begin{array}{r}9010.00 \\
16000.00\end{array}$ & $\begin{array}{l}66 / 25 / 97 \\
64 / 14 / 87 \\
66 / 25 / 87\end{array}$ & $\begin{array}{r}12.00 \\
10708.60 \\
8020.66\end{array}$ \\
\hline $\begin{array}{l}\text { COLIFRM } \\
\text { CONDFLD }\end{array}$ & $\begin{array}{l}\text { MPN } \\
\text { UMHO }\end{array}$ & $\begin{array}{l}2.20 \\
1.60\end{array}$ & 1.80 & $\begin{array}{l}66 / 24 / 87 \\
64 / 21 / 87 \\
66 / 24 / 87\end{array}$ & $\begin{array}{r}2.20 \\
211.06 \\
184.00\end{array}$ & $\begin{array}{l}64 / 28 / 87 \\
68 / 24 / 87\end{array}$ & $\begin{array}{l}329.00 \\
360.6 \varnothing\end{array}$ & $\begin{array}{l}66 / 25 / 87 \\
64 / 14 / 87 \\
66 / 25 / 87\end{array}$ & $\begin{array}{r}5.10 \\
248.00 \\
141.00\end{array}$ \\
\hline FBAR IUM & PPB & 8.00 & 1600.60 & $\begin{array}{l}04 / 21 / 87 \\
66 / 24 / 87\end{array}$ & $\begin{array}{r}10.00 \\
39.00\end{array}$ & $\begin{array}{l}64 / 29 / 87 \\
86 / 24 / 87\end{array}$ & $\begin{array}{r}32.00 \\
40.00\end{array}$ & $\begin{array}{l}84 / 14 / 67 \\
68 / 25 / 97\end{array}$ & $\begin{array}{r}48.66 \\
46.00\end{array}$ \\
\hline FCALCIU & PPB & 56.60 & & $\begin{array}{l}04 / 21 / 87 \\
08 / 24 / 67\end{array}$ & $\begin{array}{l}24600.00 \\
21606.06\end{array}$ & $\begin{array}{l}64 / 26 / 67 \\
66 / 24 / 87\end{array}$ & $\begin{array}{l}38400.00 \\
35700.00\end{array}$ & $\begin{array}{l}04 / 14 / 87 \\
06 / 25 / 87\end{array}$ & $\begin{array}{l}31300.06 \\
27800.06\end{array}$ \\
\hline FIRON & PPB & 56.80 & & & & & & $\begin{array}{l}04 / 14 / 87 \\
68 / 25 / 87\end{array}$ & $\begin{array}{r}127.60 \\
89.60\end{array}$ \\
\hline FMAGNES & PPB & 6.06 & & $\begin{array}{l}64 / 21 / 87 \\
68 / 24 / 87\end{array}$ & $\begin{array}{l}5600.06 \\
4700.00\end{array}$ & $\begin{array}{l}04 / 28 / 87 \\
\emptyset 6 / 24 / \theta 7\end{array}$ & $\begin{array}{l}7998.00 \\
7870.00\end{array}$ & $\begin{array}{l}04 / 14 / 87 \\
66 / 25 / 87\end{array}$ & $\begin{array}{l}6830.66 \\
6230.06\end{array}$ \\
\hline FMANGAN & PPB & 5.60 & & & NR & & NR & $\begin{array}{l}04 / 25 / 87 \\
06 / 25 / 87\end{array}$ & $\begin{array}{r}16.00 \\
6.06\end{array}$ \\
\hline FPOTASS & PPB & 100.60 & & $\begin{array}{l}64 / 21 / 87 \\
66 / 24 / 87\end{array}$ & $\begin{array}{l}3330.60 \\
3230.60\end{array}$ & $\begin{array}{l}04 / 20 / 87 \\
06 / 24 / 87\end{array}$ & $\begin{array}{l}4210.00 \\
4760.00\end{array}$ & $\begin{array}{l}04 / 14 / 87 \\
66 / 25 / 87\end{array}$ & $\begin{array}{l}3680.00 \\
3530.00\end{array}$ \\
\hline FSOOIUM & PPB & 100.00 & & $\begin{array}{l}64 / 21 / 87 \\
06 / 24 / 87\end{array}$ & $\begin{array}{l}13600.06 \\
12906.80\end{array}$ & $\begin{array}{l}04 / 20 / 87 \\
06 / 24 / 87\end{array}$ & $\begin{array}{l}16360.00 \\
19900.00\end{array}$ & $\begin{array}{l}64 / 14 / 87 \\
66 / 25 / 87\end{array}$ & $\begin{array}{l}12100.00 \\
12200.00\end{array}$ \\
\hline FVANADI & PPB & 5.60 & & & NR & $\begin{array}{l}04 / 2 \theta / 87 \\
66 / 24 / 87\end{array}$ & $\begin{array}{r}7.68 \\
8.66\end{array}$ & & NR \\
\hline FZINC & PPB & 5.06 & & & NR & $68 / 24 / 67$ & $\begin{array}{l}\text { N. } \\
\text { NR }\end{array}$ & $\begin{array}{l}64 / 14 / 87 \\
86 / 25 / 87\end{array}$ & $\begin{array}{l}5.00 \\
5.06\end{array}$ \\
\hline IRON & PPB & 56.60 & & $\begin{array}{l}94 / 21 / 87 \\
06 / 24 / 87\end{array}$ & $\begin{array}{r}94.00 \\
196.00\end{array}$ & & NR & $\begin{array}{l}04 / 14 / 87 \\
68 / 25 / 97\end{array}$ & $\begin{array}{l}288.06 \\
267.06\end{array}$ \\
\hline LOALPHA & $\mathrm{PCI} / \mathrm{L}$ & 2.60 & 15.00 & $\begin{array}{l}04 / 21 / 87 \\
68 / 24 / 87\end{array}$ & $\begin{array}{l}7.88 \\
\{\quad 2.12\} \\
\quad 9.88 \\
\quad 0.15\end{array}$ & $\begin{array}{l}64 / 2 \theta / 87 \\
06 / 24 / 87\end{array}$ & $\begin{array}{l}7.22 \\
\left\{\quad \begin{array}{r}2.80\} \\
16.30\end{array}\right. \\
\quad\end{array}$ & $\begin{array}{l}04 / 14 / 07 \\
06 / 26 / 87\end{array}$ & $\begin{array}{l}13.86 \\
2.79 \\
16.76 \\
\end{array}$ \\
\hline MAGNES & PPB & 0.60 & & $\begin{array}{l}64 / 21 / 87 \\
06 / 24 / 87\end{array}$ & $\begin{array}{l}5650.00 \\
4630.00\end{array}$ & $\begin{array}{l}04 / 28 / 87 \\
06 / 24 / 87\end{array}$ & $\begin{array}{l}8230.06 \\
8480.00\end{array}$ & $\begin{array}{l}04 / 14 / 87 \\
06 / 26 / 97\end{array}$ & $\begin{array}{l}6250.00 \\
5640.60\end{array}$ \\
\hline $\begin{array}{l}\text { NETHYCH } \\
\text { NITRATE }\end{array}$ & $\begin{array}{l}\text { PPB } \\
\text { PPB }\end{array}$ & $\begin{array}{r}10.60 \\
580.00\end{array}$ & 46600.60 & $\begin{array}{l}64 / 21 / 87 \\
64 / 21 / 87 \\
86 / 24 / 87\end{array}$ & $\begin{array}{l}15100.00 \\
17706.60 \\
10166.06\end{array}$ & $\begin{array}{l}04 / 2 \theta / 87 \\
04 / 29 / 87 \\
04 / 28 / 87 \\
06 / 24 / 87\end{array}$ & $\begin{array}{r}3000.00 \\
11560.00 \\
11560.00 \\
18560.00\end{array}$ & $\begin{array}{l}04 / 14 / 97 \\
64 / 14 / 87 \\
04 / 14 / 87 \\
06 / 25 / 87\end{array}$ & $\begin{array}{r}300.00 \\
24700.00 \\
25060.60 \\
17200.00\end{array}$ \\
\hline
\end{tabular}

- - value exceeds primary dRINKINg water standard.

\# - VALUE EXCEEDS PROPOSED PRIMARY DRINKING WATER STANDARO.

* - VALUE EXCEEDS SCREENING LEVEL FOR FURTHER INVESTIGATION.

+ - DETECTION LIMIT WAS NOT AVAILABLE FOR COMPARISON

NR - ANALYSIS NOT REQUESTED OR NOT YET REPORTED

VALUES IN $\left\{\begin{array}{l}f \\ \text { ARE COUNTING ERRORS FOR RADIONUCLIDES }\end{array}\right.$

WATER STANDARD (S) IN PARENTHESES ARE PROPOSED ONLY 
TABLE A.5. (contd)

\begin{tabular}{|c|c|c|c|c|c|c|c|c|c|}
\hline $\begin{array}{l}\text { CONST I } \\
\text { NAME }\end{array}$ & $\begin{array}{l}\text { JENT } \\
\text { UNITS }\end{array}$ & $\begin{array}{l}\text { OETECTION } \\
\text { LIMIT }\end{array}$ & $\begin{array}{l}\text { WATER } \\
\text { STANDARO }\end{array}$ & $\begin{array}{l}\text { SAMPLE } \\
\text { DATE }\end{array}$ & $3-2-1$ & $\begin{array}{l}\text { SAMPLE } \\
\text { DATE }\end{array}$ & $3-3-7$ & $\begin{array}{l}\text { SAMPLE } \\
\text { DATE }\end{array}$ & $3-3-16$ \\
\hline$--\infty-\infty$ & ----- & 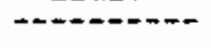 & --------- & $---\infty$ & $-\cdots$ & 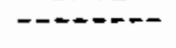 & ---- & ------ & $-\infty---\infty$ \\
\hline $\begin{array}{l}\text { PHFIELD } \\
\text { POTASUM }\end{array}$ & PPB & 100.00 & & $\begin{array}{l}64 / 21 / 87 \\
06 / 24 / 87 \\
04 / 21 / 87 \\
06 / 24 / 87\end{array}$ & $\begin{array}{r}5.90 \\
6.30 \\
3360.00 \\
3136.00\end{array}$ & $\begin{array}{l}64 / 28 / 07 \\
66 / 24 / 87 \\
64 / 28 / 67 \\
66 / 24 / 67\end{array}$ & $\begin{array}{r}7.30 \\
8.80 \\
4230.00 \\
5210.00\end{array}$ & $\begin{array}{l}04 / 14 / 87 \\
08 / 25 / 87 \\
04 / 14 / 87 \\
06 / 25 / 87\end{array}$ & $\begin{array}{r}6.00 \\
5.80 \\
3210.00 \\
3120.00\end{array}$ \\
\hline RAD IUM & PCI $/ L$ & 0.10 & 5.60 & $04 / 21 / 87$ & $\begin{array}{l}\quad 0.10 \\
\{\quad 0.14\}\end{array}$ & $84 / 28 / 07$ & $\left\{\begin{array}{l}0.10 \\
\quad 0.12\}\end{array}\right.$ & $04 / 14 / 87$ & $\left\{\begin{array}{l}0.13 \\
\quad 0.13\}\end{array}\right.$ \\
\hline SODIUM & PPQ & 106.00 & & $\begin{array}{l}04 / 21 / 87 \\
06 / 24 / 87\end{array}$ & $\begin{array}{l}13400.00 \\
12700.00\end{array}$ & $\begin{array}{l}64 / 28 / 97 \\
66 / 24 / 97\end{array}$ & $\begin{array}{l}17200.00 \\
21500.00\end{array}$ & $64 / 14 / 87$ & $\begin{array}{l}11400.00 \\
10000.00\end{array}$ \\
\hline SULFATE & PPB & 500.00 & & & $\begin{array}{l}23100.00 \\
18800.00\end{array}$ & $\begin{array}{l}64 / 28 / 67 \\
66 / 24 / 67\end{array}$ & $\begin{array}{l}27100.00 \\
31700.00\end{array}$ & & $\begin{array}{l}27100.00 \\
26700.00\end{array}$ \\
\hline TOC & PPB & 200.00 & & & $\begin{array}{l}969.06 \\
764.06\end{array}$ & $\begin{array}{l}04 / 28 / \theta 7 \\
06 / 24 / 67\end{array}$ & $\begin{array}{l}468.00 \\
579.00\end{array}$ & $\begin{array}{l}04 / 14 / 87 \\
06 / 25 / 87\end{array}$ & $\begin{array}{l}514.00 \\
700.00\end{array}$ \\
\hline $\begin{array}{l}\text { TOX } \\
\text { TRITIUM }\end{array}$ & $\begin{array}{l}\text { PPB } \\
\text { PCI/L }\end{array}$ & $\begin{array}{l}100.00 \\
500.00\end{array}$ & 20000.00 & $04 / 21 / 87$ & 1170.60 & $04 / 28 / 07$ & 2210.60 & $\begin{array}{l}64 / 14 / 87 \\
04 / 14 / 87\end{array}$ & $\begin{array}{r}225.00 \\
1290.00\end{array}$ \\
\hline $\begin{array}{l}\text { U } \\
\text { VANADUM }\end{array}$ & $\begin{array}{l}\mathrm{PCI} / \mathrm{L} \\
\mathrm{PPB}\end{array}$ & $\begin{array}{l}0.50 \\
5.00\end{array}$ & 600.00 & $84 / 21 / 87$ & $\begin{array}{c}6.63 \\
1 \quad 300.605\end{array}$ & $\begin{array}{l}04 / 28 / 87 \\
84 / 28 / 87\end{array}$ & $\begin{array}{l}7.36 \\
9.06\end{array}$ & $64 / 14 / 87$ & $\begin{array}{l}31.965 \\
13.90\end{array}$ \\
\hline ZINC & PPB & 5.00 & & $\begin{array}{l}64 / 21 / 87 \\
66 / 24 / 87\end{array}$ & $\begin{array}{l}\text { NR } \\
\text { B.00 } \\
8.00\end{array}$ & $\begin{array}{l}68 / 24 / 87 \\
64 / 28 / 87\end{array}$ & $\begin{array}{l}8.00 \\
5.00\end{array}$ & $\begin{array}{l}64 / 14 / 87 \\
66 / 25 / 87\end{array}$ & $\begin{array}{l}\text { NR } \\
6.60 \\
6.60\end{array}$ \\
\hline
\end{tabular}

* - VALUE EXCEEOS PRIMARY DRINHINg water STANDARD.

* - VALUE EXCEEDS PROPOSED PRIMARY DRINKING WATER STANDARD.

* - VALUE EXCEEDS SCREENING LEVEL FOR FURTHER INVESTIGATION.

* - DETECTION LIMIT WAS NOT AVAILABLE FOR COMPARISON

NR - ANALYSIS NOT REQUESTED OR NOT YET REPORTED

VALUES IN $\{$ f ARE COUNTING ERRORS FOR RADIONUCLIDES

WATER STANDARO(S) IN PARENTHESES ARE PROPOSED ONLY 
TABLE A.5. (contd)

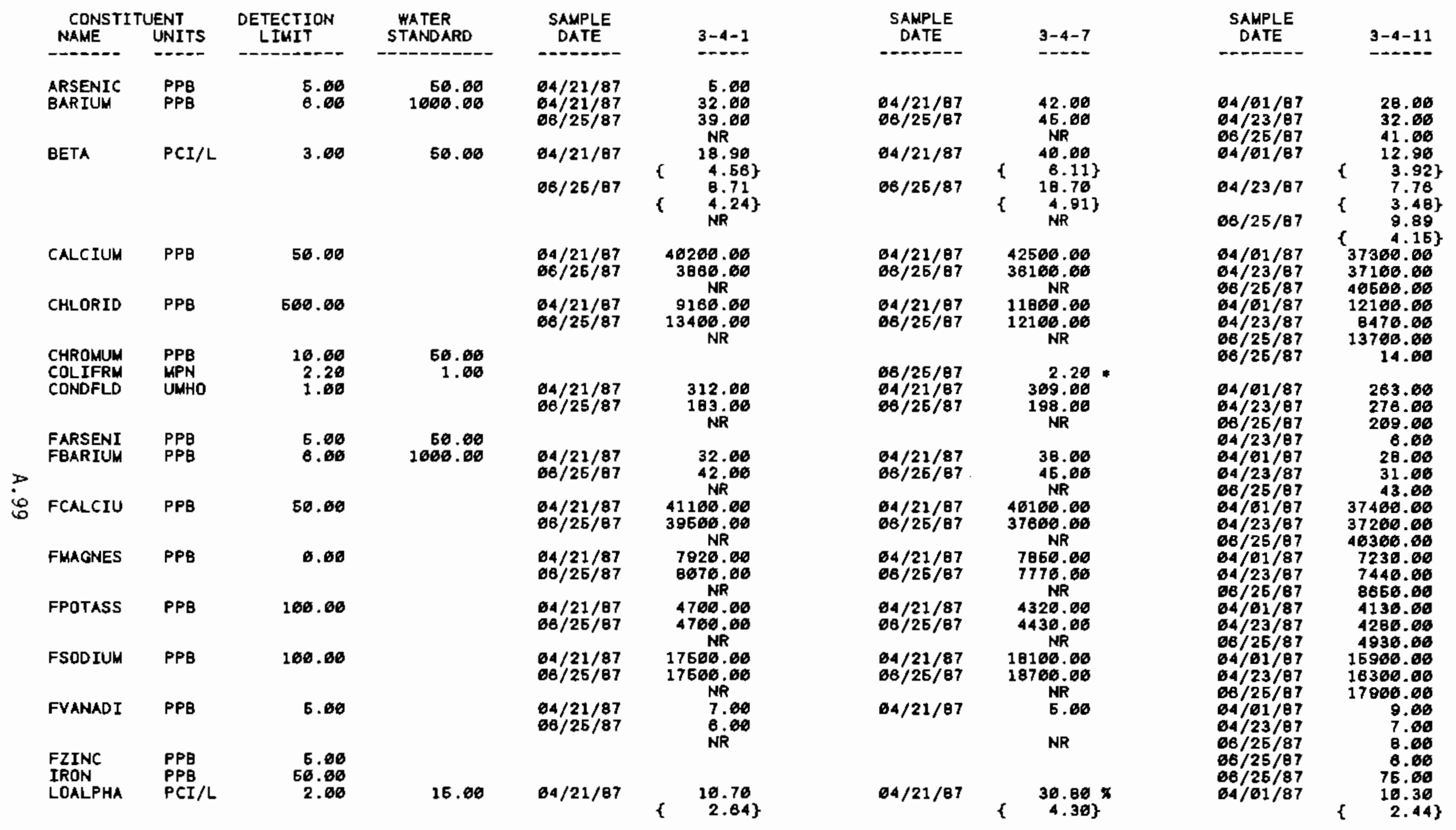

* - VALUE EXCEEDS PRIMARY DRINKING WATER STANDARD.

* - VALUE EXCEEDS PROPOSED PRIMARY DRINKING WATER STANDARD.

- VALUE EXCEEDS SCREENING LEVEI FOR FURTHER INVESTIGATION.

+ - DETECTION LIMIT WAS NOT AVAILABLE FOR COMPARISON

NR - ANALYSIS NOT REQUESTED OR NOT YET REPORTED

VALUES IN \{\} ARE COUNTING ERRORS FOR RAOIONUCLIDES

WATER STANDARD(S) IN PARENTHESES ARE PROPOSED ONLY 
TABLE A.5. (contd)

\begin{tabular}{|c|c|c|c|c|c|c|c|c|c|}
\hline $\begin{array}{l}\text { CONSTI } \\
\text { NAME }\end{array}$ & $\begin{array}{l}\text { UENT } \\
\text { UNITS }\end{array}$ & $\begin{array}{c}\text { DETECTION } \\
\text { LIMIT }\end{array}$ & $\begin{array}{l}\text { HATER } \\
\text { STANDARD }\end{array}$ & $\begin{array}{c}\text { SAMPLE } \\
\text { DATE }\end{array}$ & $3-4-1$ & $\begin{array}{l}\text { SAMPLE } \\
\text { DATE }\end{array}$ & $3-4-7$ & $\begin{array}{l}\text { SAMPLE } \\
\text { DATE }\end{array}$ & $3-4-11$ \\
\hline LOALPHA & $P C I / L$ & 2.60 & 15.60 & $06 / 25 / 87$ & $\begin{array}{c}11.80 \\
2.69\} \\
\text { NR }\end{array}$ & $\theta 6 / 25 / 87$ & $\begin{array}{c}34.207 \\
4.56\} \\
N R\end{array}$ & $\begin{array}{l}04 / 23 / 87 \\
06 / 25 / 87\end{array}$ & $\begin{array}{c}9.23 \\
2.30\} \\
11.80\end{array}$ \\
\hline MAGNES & PPB & 0.00 & & $\begin{array}{l}64 / 21 / 87 \\
66 / 25 / 87\end{array}$ & $\begin{array}{l}7800.00 \\
7800.60\end{array}$ & $\begin{array}{l}04 / 21 / 87 \\
06 / 25 / 87\end{array}$ & $\begin{array}{c}0146.60 \\
7336.60 \\
\mathrm{NR}\end{array}$ & $\begin{array}{l}04 / 61 / 87 \\
64 / 23 / 87 \\
06 / 25 / 87\end{array}$ & $\begin{array}{r}7210.00 \\
7550.00 \\
B 00.00\end{array}$ \\
\hline PHFIELD & PPB & 506.00 & 46000.00 & $\begin{array}{l}64 / 21 / 87 \\
64 / 21 / 87 \\
68 / 26 / 87 \\
64 / 21 / 87 \\
66 / 26 / 87\end{array}$ & $\begin{array}{r}16700.00 \\
15600.00 \\
15600.00 \\
7.46 \\
6.46\end{array}$ & $\begin{array}{l}04 / 21 / 97 \\
04 / 21 / 67 \\
06 / 25 / 67 \\
04 / 21 / 67 \\
06 / 25 / 67\end{array}$ & $\begin{array}{r}17606.00 \\
19400.00 \\
19606.00 \\
6.90 \\
6.30 \\
\end{array}$ & $\begin{array}{l}04 / 01 / 87 \\
64 / 23 / 87 \\
66 / 25 / 87 \\
64 / 01 / 87 \\
04 / 23 / 87 \\
06 / 25 / 87\end{array}$ & $\begin{array}{r}13700.00 \\
13706.00 \\
17606.00 \\
7.70 \\
7.00 \\
7.00\end{array}$ \\
\hline POTASUM & PPB & 100.00 & & $\begin{array}{l}64 / 21 / 87 \\
96 / 25 / 87\end{array}$ & $\begin{array}{c}4896.66 \\
4426.60 \\
\text { NR }\end{array}$ & $\begin{array}{l}04 / 21 / 87 \\
66 / 25 / 67\end{array}$ & $\begin{array}{c}4560.60 \\
4160.00 \\
\mathrm{NR}\end{array}$ & $\begin{array}{l}04 / 91 / 87 \\
64 / 23 / 87 \\
68 / 25 / 87\end{array}$ & $\begin{array}{l}4110.08 \\
4468.06 \\
4940.00\end{array}$ \\
\hline RADIUM & $\mathrm{PCI} / \mathrm{L}$ & 0.10 & 6.00 & & NR & $64 / 21 / 87$ & $\left\{\begin{array}{l}0.14 \\
\quad 0.16\}\end{array}\right.$ & $\begin{array}{l}04 / 23 / 87 \\
68 / 25 / 87\end{array}$ & $\begin{array}{r}0.39 \\
\{\quad 0.21\} \\
\quad 0.15 \\
\{\quad 0.14\}\end{array}$ \\
\hline SODIUM & PPB & 160.06 & & $\begin{array}{l}04 / 21 / 87 \\
86 / 26 / 87\end{array}$ & $\begin{array}{c}17460.00 \\
18366.00 \\
\mathrm{NR}\end{array}$ & $\begin{array}{l}64 / 21 / 87 \\
86 / 26 / 87\end{array}$ & $\begin{array}{c}19206.06 \\
17000.06 \\
\mathrm{NR}\end{array}$ & $\begin{array}{l}04 / 01 / 87 \\
64 / 23 / 87 \\
66 / 25 / 67\end{array}$ & $\begin{array}{l}16960.06 \\
16600.06 \\
17706.00\end{array}$ \\
\hline SULFATE & PPB & 566.60 & & $\begin{array}{l}04 / 21 / 87 \\
86 / 25 / 87\end{array}$ & $\begin{array}{c}27700.00 \\
28200.00 \\
\mathrm{NR}\end{array}$ & $\begin{array}{l}64 / 21 / 87 \\
66 / 25 / 87\end{array}$ & $\begin{array}{c}32800.06 \\
33600.66 \\
\mathrm{NR}\end{array}$ & $\begin{array}{l}64 / 61 / 87 \\
84 / 23 / 87 \\
86 / 25 / 67\end{array}$ & $\begin{array}{l}28800.00 \\
25400.06 \\
30000.00\end{array}$ \\
\hline TOC & PPB & 200.00 & & $\begin{array}{l}64 / 21 / 87 \\
66 / 25 / 87\end{array}$ & $\begin{array}{c}326.00 \\
692.60 \\
\mathrm{NR}\end{array}$ & $\begin{array}{l}64 / 21 / 87 \\
06 / 25 / 87\end{array}$ & $\begin{array}{c}489.06 \\
729.06 \\
\mathrm{NR}\end{array}$ & $\begin{array}{l}64 / 61 / 87 \\
64 / 23 / 87 \\
66 / 25 / 87\end{array}$ & $\begin{array}{l}489.06 \\
325.06 \\
848.00\end{array}$ \\
\hline $\begin{array}{l}\text { TOX } \\
\text { TRITIUN }\end{array}$ & $\begin{array}{l}\mathrm{PPB} \\
\mathrm{PCI} / \mathrm{L}\end{array}$ & $\begin{array}{l}100.00 \\
560.00\end{array}$ & 26000.60 & & & $\begin{array}{l}04 / 21 / 87 \\
04 / 21 / 87\end{array}$ & $\begin{array}{r}388.60 \\
1826.60 \\
\quad 368.00\end{array}$ & & NR \\
\hline UANADUM & $\begin{array}{l}\mathrm{PCI} / \mathrm{L} \\
\mathrm{PPB}\end{array}$ & $\begin{array}{l}0.60 \\
5.00\end{array}$ & 600.00 & $\begin{array}{l}64 / 21 / 87 \\
64 / 21 / 87 \\
06 / 25 / 87\end{array}$ & $\begin{array}{r}11.46 \\
7.60 \\
8.60\end{array}$ & $\begin{array}{l}04 / 21 / 97 \\
04 / 21 / 67 \\
66 / 25 / 67\end{array}$ & $\begin{array}{c}31.40 \\
7.00 \\
7.00 \\
\mathrm{NR}\end{array}$ & $\begin{array}{l}04 / 01 / 67 \\
04 / 23 / 87 \\
06 / 25 / 87\end{array}$ & $\begin{array}{l}\text { NR } \\
8.06 \\
9.06 \\
8.06\end{array}$ \\
\hline ZINC & PPB & 5.00 & & $04 / 21 / 87$ & 5.00 & $64 / 21 / 87$ & 5.08 & & \\
\hline
\end{tabular}

* - VALUe EXCEEDS PRIMARY DRINKING waTER STANDARD.

* - VALUE EXCEEDS PROPOSED PRIMARY dRINKING WATER STANDARD.

* - VALUE EXCEEDS SCREENING LEVEL FOR FURTHER INVESTIGATION.

+ DETECTION LIMIT WAS NOT AVAILABLE FOR COMPARISON

NR - ANALYSIS NOT REQUESTED OR NOT YET REPORTED

VALUES IN \{\} ARE COUNTING ERRORS FOR RADIONUCL IDES

WATER STANDARD'S) IN PARENTHESES ARE PROPOSED ONLY 
TABLE A.5. (contd)

\begin{tabular}{|c|c|c|c|c|c|c|c|c|c|}
\hline $\begin{array}{l}\text { CONST I } \\
\text { NAME }\end{array}$ & $\begin{array}{l}\text { UENT } \\
\text { UNITS }\end{array}$ & $\begin{array}{l}\text { DETECTION } \\
\text { LIMIT }\end{array}$ & $\begin{array}{l}\text { WATER } \\
\text { STANDARD }\end{array}$ & $\begin{array}{c}\text { SAMPLE } \\
\text { DATE }\end{array}$ & $3-\theta-2$ & $\begin{array}{l}\text { SAMPLE } \\
\text { DATE }\end{array}$ & B-S19-E13 & $\begin{array}{l}\text { SAMPLE } \\
\text { DATE }\end{array}$ & 8-S36E IEA \\
\hline $\begin{array}{l}\text { AMMONIU } \\
\text { ARSENIC }\end{array}$ & $\begin{array}{l}\mathrm{PPB} \\
\mathrm{PPB}\end{array}$ & $\begin{array}{r}50.00 \\
5.00\end{array}$ & 60.60 & $\begin{array}{l}06 / 19 / 87 \\
64 / 15 / 87\end{array}$ & $\begin{array}{r}70.60 \\
8.06\end{array}$ & & NR & & NR \\
\hline BARIUM & PPB & 8.00 & 1000.00 & $04 / 15 / 87$ & 40.00 & $04 / 30 / 87$ & 45.06 & $04 / 28 / 87$ & 56.00 \\
\hline BETA & $\mathrm{PCI} / \mathrm{L}$ & 3.00 & 50.60 & $\begin{array}{l}04 / 15 / 07 \\
06 / 19 / 87\end{array}$ & 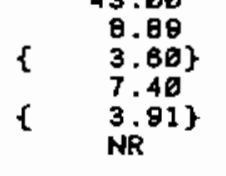 & $\begin{array}{l}64 / 30 / 87 \\
66 / 25 / 87\end{array}$ & 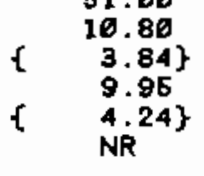 & $\begin{array}{l}04 / 28 / 87 \\
65 / 31 / 87 \\
06 / 25 / 87\end{array}$ & $\begin{array}{l}9.54 \\
\{\quad 3.83\} \\
6.11 \\
\{\quad 2.04\} \\
6.38 \\
3.00\}\end{array}$ \\
\hline CALCIUM & $\mathrm{PPB}$ & 50.06 & & $04 / 15 / 87$ & 42700.00 & $04 / 36 / 87$ & 41600.00 & $04 / 28 / 87$ & ${ }_{67700.00}$ \\
\hline CHLORID & PPB & 500.00 & & $\begin{array}{l}06 / 19 / 87 \\
84 / 15 / 97 \\
66 / 19 / 97\end{array}$ & $\begin{array}{l}46686.06 \\
10366.06 \\
11566.00\end{array}$ & $\begin{array}{l}06 / 25 / 87 \\
64 / 30 / 87 \\
66 / 26 / 87\end{array}$ & $\begin{array}{r}4220.00 \\
15800.00 \\
17700.00\end{array}$ & $\begin{array}{l}68 / 26 / 97 \\
64 / 28 / 97 \\
68 / 25 / 87\end{array}$ & $\begin{array}{r}62800.60 \\
4810.00 \\
6510.00\end{array}$ \\
\hline $\begin{array}{l}\text { COLIFRM } \\
\text { CONDFLD }\end{array}$ & $\begin{array}{l}\text { MPN } \\
\text { UMHO }\end{array}$ & $\begin{array}{l}2.20 \\
1.00\end{array}$ & 1.06 & $\begin{array}{l}06 / 19 / 87 \\
04 / 16 / 87 \\
08 / 10 / 87\end{array}$ & $\begin{array}{l}16.66 \\
386.66 \\
329.60\end{array}$ & $\begin{array}{l}64 / 36 / 87 \\
66 / 25 / 87\end{array}$ & $\begin{array}{l}450 . \varnothing \varnothing \\
258 . \varnothing \varnothing\end{array}$ & $\begin{array}{l}84 / 28 / 87 \\
66 / 25 / 87\end{array}$ & $\begin{array}{l}418.00 \\
259.96\end{array}$ \\
\hline $\begin{array}{l}\text { FARSENI } \\
\text { FBARIUM }\end{array}$ & $\begin{array}{l}P P B \\
P P B\end{array}$ & $\begin{array}{l}5.00 \\
8.00\end{array}$ & $\begin{array}{r}56.06 \\
1006.00\end{array}$ & $\begin{array}{l}04 / 15 / 87 \\
04 / 15 / 87\end{array}$ & $\begin{array}{l}10.06 \\
46.00\end{array}$ & $\begin{array}{l}04 / 36 / 87 \\
04 / 30 / 97\end{array}$ & $\begin{array}{r}7.00 \\
48.00\end{array}$ & $04 / 2 \theta / 87$ & 57.89 \\
\hline FCALCIU & PPB & 50.66 & & $04 / 15 / 87$ & 43300.00 & $64 / 30 / 87$ & $\begin{array}{r}53.00 \\
43800.00\end{array}$ & $\begin{array}{l}88 / 26 / 87 \\
04 / 28 / 87\end{array}$ & $\begin{array}{r}63.99 \\
86100.00\end{array}$ \\
\hline FMAGNES & PPB & 0.00 & & $\begin{array}{l}66 / 19 / 87 \\
64 / 16 / 87 \\
66 / 19 / 87\end{array}$ & $\begin{array}{r}40160.60 \\
92 \sigma 0 . \varnothing 6 \\
9600.66\end{array}$ & $\begin{array}{l}66 / 25 / 87 \\
64 / 36 / 87 \\
66 / 25 / 87\end{array}$ & $\begin{array}{l}42400.06 \\
12800.00 \\
12900.00\end{array}$ & $\begin{array}{l}68 / 25 / 97 \\
64 / 28 / 67 \\
68 / 25 / 87\end{array}$ & $\begin{array}{l}62900.00 \\
12500.00\end{array}$ \\
\hline FPOTASS & PPB & 100.00 & & $04 / 15 / 87$ & 5500.00 & $64 / 30 / 87$ & $8210 . \varnothing \varnothing$ & $64 / 28 / 87$ & 8190.00 \\
\hline FSODIUM & PPB & 100.60 & & $\begin{array}{l}64 / 15 / 87 \\
66 / 18 / 87\end{array}$ & $\begin{array}{l}19386.06 \\
18406.09\end{array}$ & $\begin{array}{l}04 / 30 / 87 \\
88 / 25 / 87\end{array}$ & 22500.08 & $04 / 28 / 87$ & 13360.66 \\
\hline FVANADI & PPB & 5.00 & & $\begin{array}{l}04 / 16 / 87 \\
06 / 19 / 87\end{array}$ & $\begin{array}{r}13.00 \\
10.60\end{array}$ & $64 / 38 / 87$ & $\begin{array}{r}15.06 \\
18.06\end{array}$ & $64 / 28 / 87$ & $\begin{array}{r}130100.000 \\
8.00\end{array}$ \\
\hline FZINC & PPB & 5.06 & & & & $68 / 26 / 87$ & 8,00 & $04 / 28 / 87$ & 79.06 \\
\hline $\begin{array}{l}\text { IRON } \\
\text { LEADGF } \\
\text { LOALPHA }\end{array}$ & $\begin{array}{l}\mathrm{PPB} \\
\mathrm{PPB} \\
\mathrm{PCI} / \mathrm{L}\end{array}$ & $\begin{array}{r}56.66 \\
5.06 \\
2.60\end{array}$ & $\begin{array}{l}60.00 \\
15.66\end{array}$ & $\begin{array}{l}86 / 19 / 87 \\
06 / 19 / 87\end{array}$ & $\begin{array}{l}5.00 \\
2.34 \\
1.42\} \\
N R\end{array}$ & $\begin{array}{l}66 / 26 / 87 \\
64 / 30 / 87 \\
66 / 26 / 87\end{array}$ & $\begin{array}{l}2.29 \\
\{\quad 1.47\} \\
2.81 \\
1.59\}\end{array}$ & & 102.80 \\
\hline $\begin{array}{l}\text { MAGNES } \\
\text { NITRATE }\end{array}$ & $\begin{array}{l}\text { PPB } \\
\text { PPB }\end{array}$ & $\begin{array}{r}6.00 \\
500.00\end{array}$ & $46 \sigma \sigma \sigma .66$ & $\begin{array}{l}64 / 15 / 87 \\
06 / 19 / 87 \\
04 / 16 / 87 \\
64 / 16 / 87 \\
06 / 19 / 87\end{array}$ & $\begin{array}{r}9006.00 \\
9160.00 \\
23100.00 \\
23160.00 \\
24260.00\end{array}$ & $\begin{array}{l}04 / 30 / 87 \\
66 / 25 / 87 \\
04 / 36 / 87 \\
65 / 31 / 87 \\
66 / 25 / 87\end{array}$ & $\begin{array}{l}12200.06 \\
12806.00 \\
22160.06 \\
19800.00 \\
23400.0 \varnothing\end{array}$ & $\begin{array}{l}64 / 28 / 87 \\
66 / 25 / 87 \\
64 / 29 / 87 \\
65 / 31 / 87 \\
66 / 25 / 87\end{array}$ & $\begin{array}{l}12600.00 \\
12160.00 \\
10960.00 \\
11700.00 \\
12700.00\end{array}$ \\
\hline
\end{tabular}

* - VAlUE EXCEEDS PRIMARY DRINKING waTER STANDARD.

- VXLUE EXCEEDS PROPOSED PRIMARY DRINKING WATER STANDARD.

- VALUE EXCEEDS SCREENING LEYEL FOR FURTHER INVESTIGATION.

+ - DETECTION LIMIT WAS NOT AVAILABLE FOR COMPARISON

NR - ANALYSIS NOT REQUESTED OR NOT YET REPORTED

VALUES IN \{\} ARE COUNTING ERRORS FOR RADIONUCLIDES

WATER STANDARD(S) IN PARENTHESES ARE PROPOSED ONLY 
TABLE A.5. (contd)

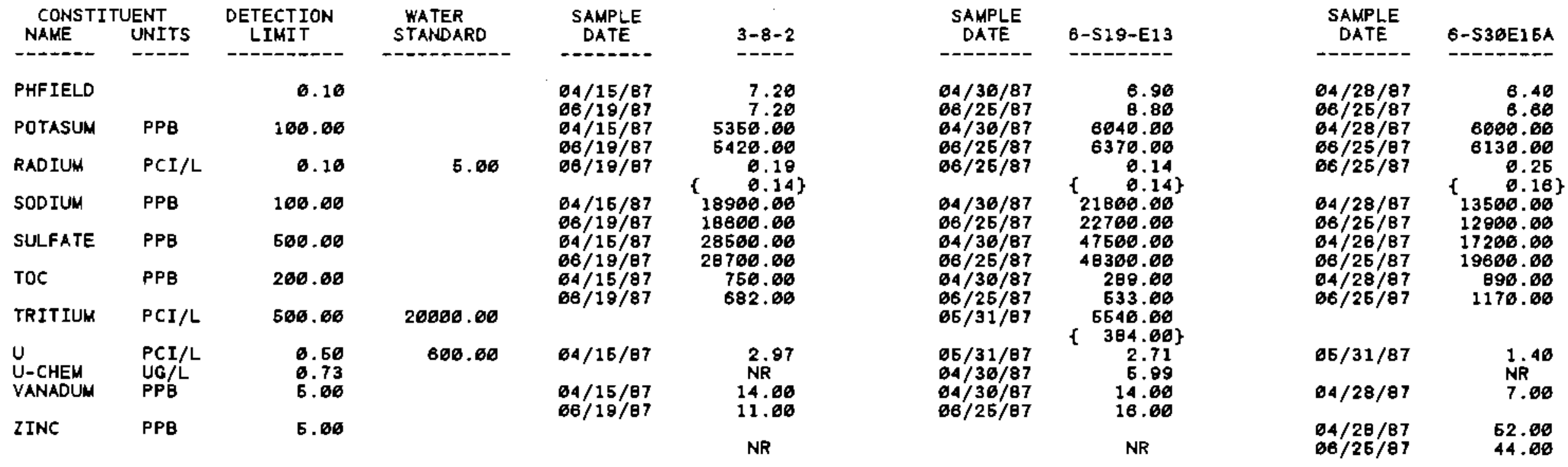

- - VALUE EXCEEDS PRIMARY DRINKING water STANDARD.

\# - VALUE EXCEEDS PROPOSED PRIMARY DRINKING WATER STANDARD.

* - VALUE EXCEEDS SCREENING LEVEL FOR FURTHER INVESTIGATION.

+ - DETECTION LIMIT WAS NOT AVAILABLE FOR COMPARISON

NR - ANALYSIS NOT REQUESTED OR NOT YET REPORTED

VALUES IN \& $\}$ ARE COUNTING ERRORS FOR RADIONUCLIDES

WATER STANDARD(S) IN PARENTHESES ARE PROPOSED ONLY 
TABLE A.5. (cont d)

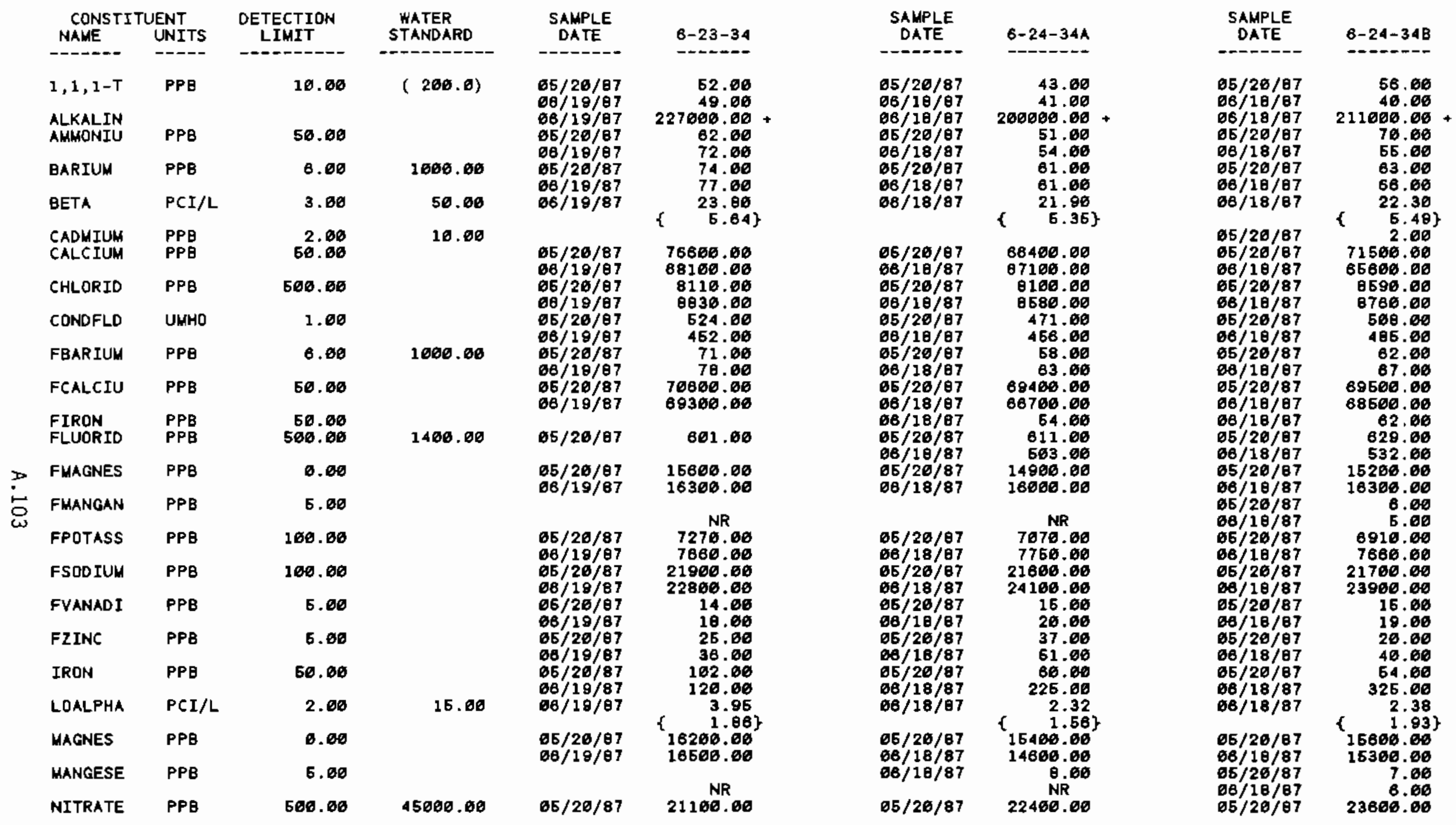

* - Value exceeds primary dRINKIng water standard.

* - VALUE EXCEEDS PROPOSED PRIMARY DRINIING WATER STANDARD.

* - VALUE EXCEEDS SCREENING LEVEL FOR FURTHER INVESTIGATION.

+ DETECTION LIMIT WAS NOT AVAILABLE FOR COMPARISON

NR - ANALYSIS NOT REQUESTED OR NOT YET REPORTED

VALUES IN \{ $\}$ ARE COUNTING ERRORS FOR RAOIONUCLIDES

WATER STANDARD'(S) IN PARENTHESES ARE PROPOSED ONLY 
TABLE A.5. (contd)

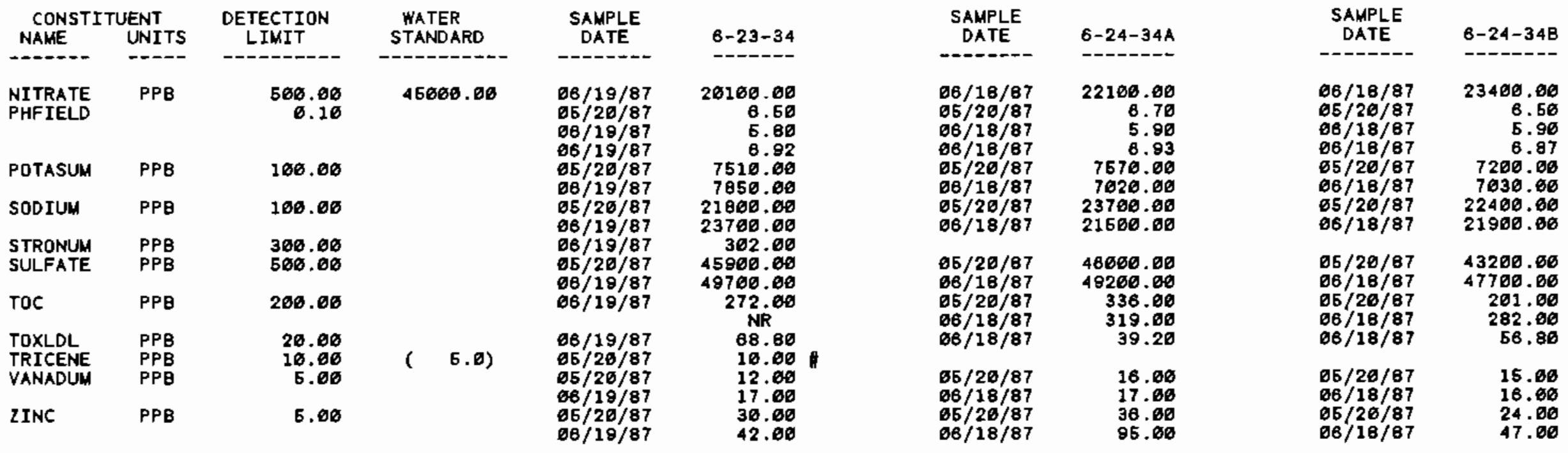

* - VALUE EXCEEDS PRIMARY DRINKING WATER STANDARD.

* - VALUE EXCEEDS PROPOSED PRIMARY DRINKING WATER STANDARD.

* - VALUE EXCEEDS SCREENING LEVEL FOR FURTHER INVESTIGATION.

+ - DETECTION LIMIT WAS NOT AVAILABLE FOR COMPARISON

NR - ANALYSIS NOT REQUESTED OR NOT YET REPORTED

VALUES IN \{\} ARE COUNTING ERRORS FOR RADIONUCLIDES

WATER STANDARD(S) IN PARENTHESES ARE PROPOSED ONLY 
TABLE A.5. (contd)

\begin{tabular}{|c|c|c|c|c|c|}
\hline $\begin{array}{l}\text { CONSTI } \\
\text { NAME }\end{array}$ & $\begin{array}{l}\text { UENT } \\
\text { UNITS }\end{array}$ & $\begin{array}{l}\text { DETECTION } \\
\text { LIMIT }\end{array}$ & $\begin{array}{l}\text { WATER } \\
\text { STANDARD }\end{array}$ & $\begin{array}{c}\text { SAMPLE } \\
\text { DATE }\end{array}$ & $6-24-34 C$ \\
\hline $1,1,1-T$ & PPB & 10.00 & $(200.0)$ & $65 / 20 / 87$ & 24.60 \\
\hline $\begin{array}{l}\text { ALKALIN } \\
\text { AMMONIU }\end{array}$ & PPB & 68.00 & & $\begin{array}{l}06 / 18 / 87 \\
06 / 20 / 87\end{array}$ & $\begin{array}{r}196060.00 \\
60.00\end{array}$ \\
\hline BARIUM & PPE & 6.00 & 1000.00 & $\theta 5 / 20 / 97$ & 48.00 \\
\hline BETA & $\mathrm{PCI} / \mathrm{L}$ & 3.60 & 60.00 & $08 / 18 / \theta 7$ & 27.86 \\
\hline $\begin{array}{l}\text { CADMIUM } \\
\text { CALCIUM }\end{array}$ & $\begin{array}{l}\mathrm{PPB} \\
\mathrm{PPB}\end{array}$ & $\begin{array}{r}2.60 \\
50.00\end{array}$ & 10.00 & $\begin{array}{l}65 / 20 / 87 \\
05 / 26 / 87\end{array}$ & $\begin{array}{r}2.06 \\
58400.60\end{array}$ \\
\hline CHLOR IO & PPB & 600.60 & & $\begin{array}{l}\sigma 5 / 26 / 87 \\
\sigma 8 / 1 B / 87\end{array}$ & $\begin{array}{l}7700.00 \\
8480.00\end{array}$ \\
\hline COLIFRM & MPN & 2.20 & 1.66 & & DO \\
\hline CONDFLD & UMHO & 1.00 & & $06 / 20 / 87$ & 505.00 \\
\hline FBAR IUM & PPB & 8.60 & 1000.00 & $05 / 20 / 87$ & 46.00 \\
\hline FCALCIU & PPB & 50.00 & & $65 / 26 / 67$ & $\begin{array}{l}63800.06 \\
56200.06\end{array}$ \\
\hline $\begin{array}{l}\text { FLEAD } \\
\text { FLUDRID }\end{array}$ & $\begin{array}{l}\text { PPB } \\
\text { PPG }\end{array}$ & $\begin{array}{r}5.60 \\
500.00\end{array}$ & $\begin{array}{r}50.06 \\
140 \sigma .00\end{array}$ & $65 / 20 / 87$ & 834.66 \\
\hline FMAGNES & PPE & 0.06 & & $65 / 26 / 87$ & 14900.00 \\
\hline FMANGAN & PPB & 5.06 & & $65 / 20 / 87$ & 35.00 \\
\hline FPOTASS & PPB & 160.06 & & $05 / 20 / 97$ & 6846.80 \\
\hline FSODIUM & PPB & 100.00 & & $66 / 26 / 67$ & 22100.08 \\
\hline FVANADI & PPB & 5.00 & & $65 / 26 / 87$ & 14.66 \\
\hline FZINC & PPB & 6.00 & & $06 / 20 / 87$ & 69.00 \\
\hline IRON & PPB & 50.00 & & $\begin{array}{l}05 / 26 / 87 \\
68 / 18 / 87\end{array}$ & $\begin{array}{l}66.00 \\
92.00\end{array}$ \\
\hline LOALPHA & $\mathrm{PCI} / \mathrm{L}$ & 2.60 & 15.60 & $66 / 18 / 87$ & \\
\hline MAGNES & PPB & 0.00 & & $\begin{array}{l}65 / 26 / \theta 7 \\
66 / 10 / 97 \\
65 / 26 / \theta 7\end{array}$ & $\begin{array}{r}14306.06 \\
15300.66 \\
37.00\end{array}$ \\
\hline
\end{tabular}

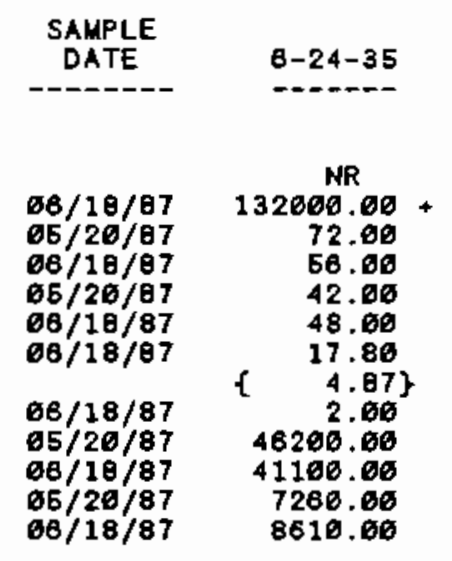

$65 / 26 / 87$

$08 / 10 / 97$
$65 / 20 / 67$

$66 / 16 / 87$

$05 / 20 / 97$

$05 / 20 / 87$

$68 / 18 / 87$

$65 / 20 / 87$

$06 / 18 / 87$

$65 / 26 / 97$

$66 / 18 / 97$

65/26/87

$68 / 18 / 87$

$66 / 20 / 87$

$66 / 18 / 87$

$86 / 18 / 87$

$68 / 18 / 87$

$68 / 18 / 87$

$05 / 20 / 87$

B8/18/87

362.06

41.06

51.00

43100.00

619.00

519.60

16900.60

11800.06

NR

6430.60

7210.00

21300.00

21.00

28.00

15.60

34.06

90.00

90.66
$\mathrm{NR}$
4.13
1.85

11100.00
SAMPLE DATE

$6-25-34 C$

\section{DATE-- -----}

$96 / 19 / 67 \quad$ NR 130000.00 $68 / 19 / 97 \quad 98.60$ $65 / 26 / 87 \quad 34.06$ $66 / 19 / 87 \quad 42.06$ $08 / 19 / 87 \quad 16.78$

\section{$65 / 26 / 87$ \\ 44000.00} $66 / 19 / 87$ $65 / 26 / 87$ $68 / 18 / 87$ $06 / 26 / 87$ 68/19/97 5/28/87 B $/ 10 / 87$ $05 / 28 / 87$ $66 / 18 / 07$ $05 / 20 / \theta 7$ $66 / 19 / 87$ $68 / 19 / 97$ $65 / 20 / 87$ $66 / 19 / 87$ $65 / 26 / 87$ $68 / 19 / 87$ $65 / 20 / 87$

$65 / 26 / 87$ $68 / 19 / 87$ (5) $85 / 29 / 87$ $65 / 20 / 87$ 65/20/87 (5) $86 / 18 / 87$ $65 / 20 / 87$ $66 / 19 / 97$

б5/26/87 $68 / 19 / 87$
$\{4.76\}$ 48000.00 7648.00 9120.00 5.10
2.20 370.00 336.00 34.00 40.00 41300.60 38060.00

5.00 836.60

560.00

11260.08

11700.60

6.00

6030.60

6360.00

22260.00

22860.06

22.00

19.00

32.00

49.06

96.00

90.00

3.68

\{ 1.50$\}$

11400.06

* - Value exceEdS PRIMARY DRINKING WaTER STANDARD.

* - VALUE EXCEEDS PROPOSED PRIMARY DRINKING WATER STANDARO

* - VALUE EXCEEDS SCREENING LEVEL FOR FURTHER INVESTIGATION.

+ - DETECTION LIMIT WAS NOT AVAILABLE FOR COMPARISON

NR - ANALYSIS NDT REQUESTED OR NOT YET REPORTED

VALUES IN \{\} ARE COUNTING ERRORS FOR RADIONUCLIDES

WATER STANDARD(S) IN PARENTHESES ARE PROPOSED ONLY 
TABLE A.5. (cont $d$ )

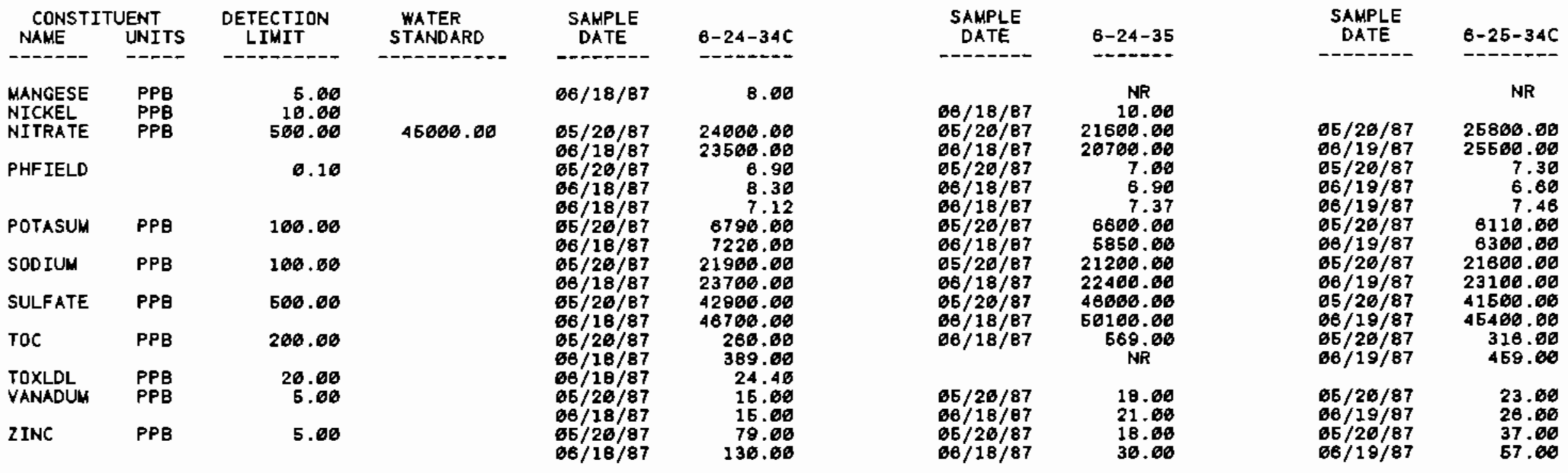

* - VALUE EXCEEDS PROPOSED PRIMARY DRINKING WATER STANDARD.

- VALUE EXCEEDS SCREENING LEVEL FOR FURTHER INVESTIGATION.

* - DETECTION LIMIT WAS NOT AVAILABLE FOR COMPARISON

NR - ANALYSIS NOT REQUESTED OR NOT YET REPORTED

VALUES IN \{\} ARE COUNTING ERRORS FOR RADIONUCLIOES

WATER STANDARD(S) IN PARENTHESES ARE PROPOSED ONLY 
TABLE A.5. (contd)

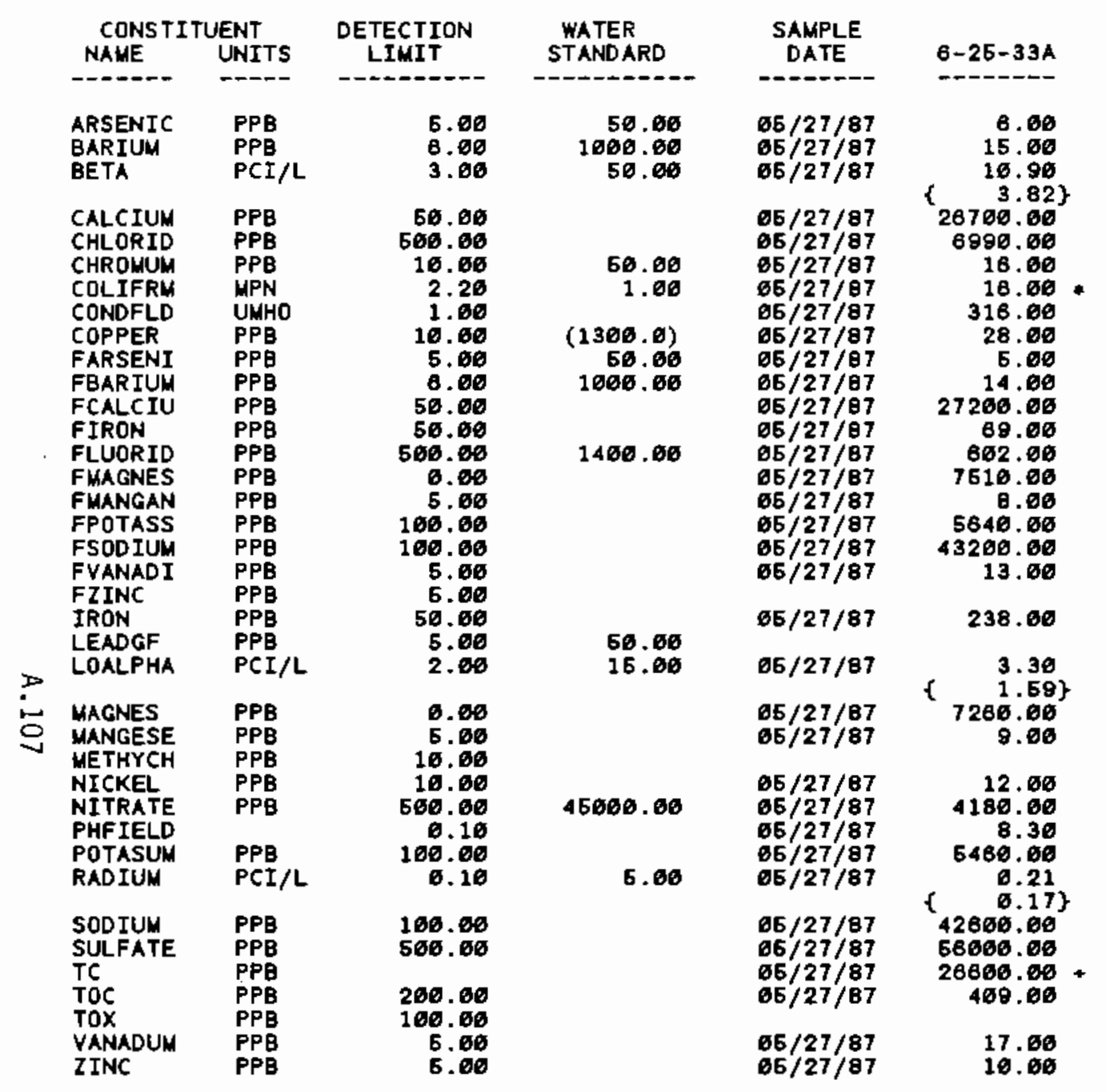

\begin{tabular}{|c|c|c|c|}
\hline $\begin{array}{l}\text { SAMPLE } \\
\text { DATE }\end{array}$ & $6-25-34 A$ & $\begin{array}{l}\text { SAMPLE } \\
\text { DATE }\end{array}$ & $6-25-348$ \\
\hline $\begin{array}{l}95 / 26 / 87 \\
85 / 26 / 87 \\
05 / 26 / 87\end{array}$ & $\begin{array}{r}5.60 \\
34.60 \\
28.70\end{array}$ & $\begin{array}{l}05 / 26 / 87 \\
65 / 26 / 87\end{array}$ & $\begin{array}{l}32.00 \\
34.30\end{array}$ \\
\hline $\begin{array}{l}06 / 26 / 87 \\
05 / 26 / 87\end{array}$ & $\begin{array}{c}38900.60 \\
7560.00\end{array}$ & $\begin{array}{l}05 / 26 / 87 \\
05 / 26 / 87\end{array}$ & $\begin{array}{c}38700.06 \\
8010.00\end{array}$ \\
\hline $65 / 26 / 87$ & 387.00 & $\theta 6 / 26 / \theta 7$ & 381.00 \\
\hline $\begin{array}{l}65 / 26 / 87 \\
65 / 26 / 97\end{array}$ & $\begin{array}{r}32.60 \\
36960.66\end{array}$ & $\begin{array}{l}65 / 26 / 87 \\
\varnothing 5 / 26 / 97\end{array}$ & $\begin{array}{r}31.00 \\
37760.00\end{array}$ \\
\hline $\begin{array}{l}65 / 26 / 87 \\
06 / 26 / 87\end{array}$ & $\begin{array}{r}677.00 \\
10100.00\end{array}$ & $\begin{array}{l}65 / 26 / 97 \\
65 / 26 / 97\end{array}$ & $\begin{array}{r}732.00 \\
10806.60\end{array}$ \\
\hline $\begin{array}{l}05 / 26 / 87 \\
85 / 26 / 87 \\
65 / 26 / 87 \\
65 / 26 / 87\end{array}$ & $\begin{array}{r}8050.60 \\
22600.06 \\
27.60 \\
6.06\end{array}$ & $\begin{array}{l}65 / 26 / 87 \\
\sigma 6 / 26 / \theta 7 \\
65 / 26 / 67\end{array}$ & $\begin{array}{r}8076.00 \\
22906.06 \\
23.06\end{array}$ \\
\hline $65 / 26 / 87$ & $\left\{\begin{array}{c}3.93 \\
1.71\} \\
10800.00\end{array}\right.$ & $\begin{array}{l}05 / 26 / 87 \\
05 / 26 / 87 \\
65 / 26 / 87\end{array}$ & 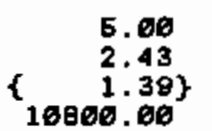 \\
\hline & & $05 / 26 / 87$ & 220.00 \\
\hline $\begin{array}{l}05 / 26 / 87 \\
65 / 26 / 87 \\
65 / 26 / 87\end{array}$ & $\begin{array}{r}28706.06 \\
7.56 \\
8200.06\end{array}$ & $\begin{array}{l}\sigma 5 / 26 / 87 \\
\sigma 5 / 26 / 97 \\
\sigma 5 / 26 / 87\end{array}$ & $\begin{array}{r}27700.00 \\
7.48 \\
6280.00\end{array}$ \\
\hline $\begin{array}{l}65 / 26 / 87 \\
65 / 26 / 87 \\
65 / 26 / 87 \\
65 / 26 / 87\end{array}$ & $\begin{array}{c}23200.60 \\
42160.00 \\
27606.00 \\
286.00\end{array}+$ & $\begin{array}{l}06 / 26 / 87 \\
65 / 26 / 87 \\
66 / 26 / 87 \\
06 / 26 / 87\end{array}$ & $\begin{array}{r}23260.66 \\
41466.06 \\
27966.06 \\
331.66\end{array}$ \\
\hline $\begin{array}{l}06 / 26 / 87 \\
06 / 26 / 87\end{array}$ & $\begin{array}{r}25.66 \\
7.00\end{array}$ & $\begin{array}{l}05 / 26 / 87 \\
05 / 26 / 97\end{array}$ & $\begin{array}{r}24.06 \\
5.00\end{array}$ \\
\hline
\end{tabular}

- - Value exceeds primary drinking water standard.

* - VALUE EXCEEDS PROPOSED PRTMARY DRINKING WATER STANDARD.

* - VALUE EXCEEDS SCREENING LEVEL FOR FURTHER INVESTIGATION.

+ DETECTION LIMIT WAS NOT AVAILABLE FOR COMPARISON

NR - ANALYSIS NOT REQUESTED OR NOT YET REPORTED

VALUES IN \& \& ARE COUNTING ERRORS FOR RADIONUCLIDES

WATER STANDARD(S) IN PARENTHESES ARE PROPOSED ONLY 
TABLE A.5. (contd)

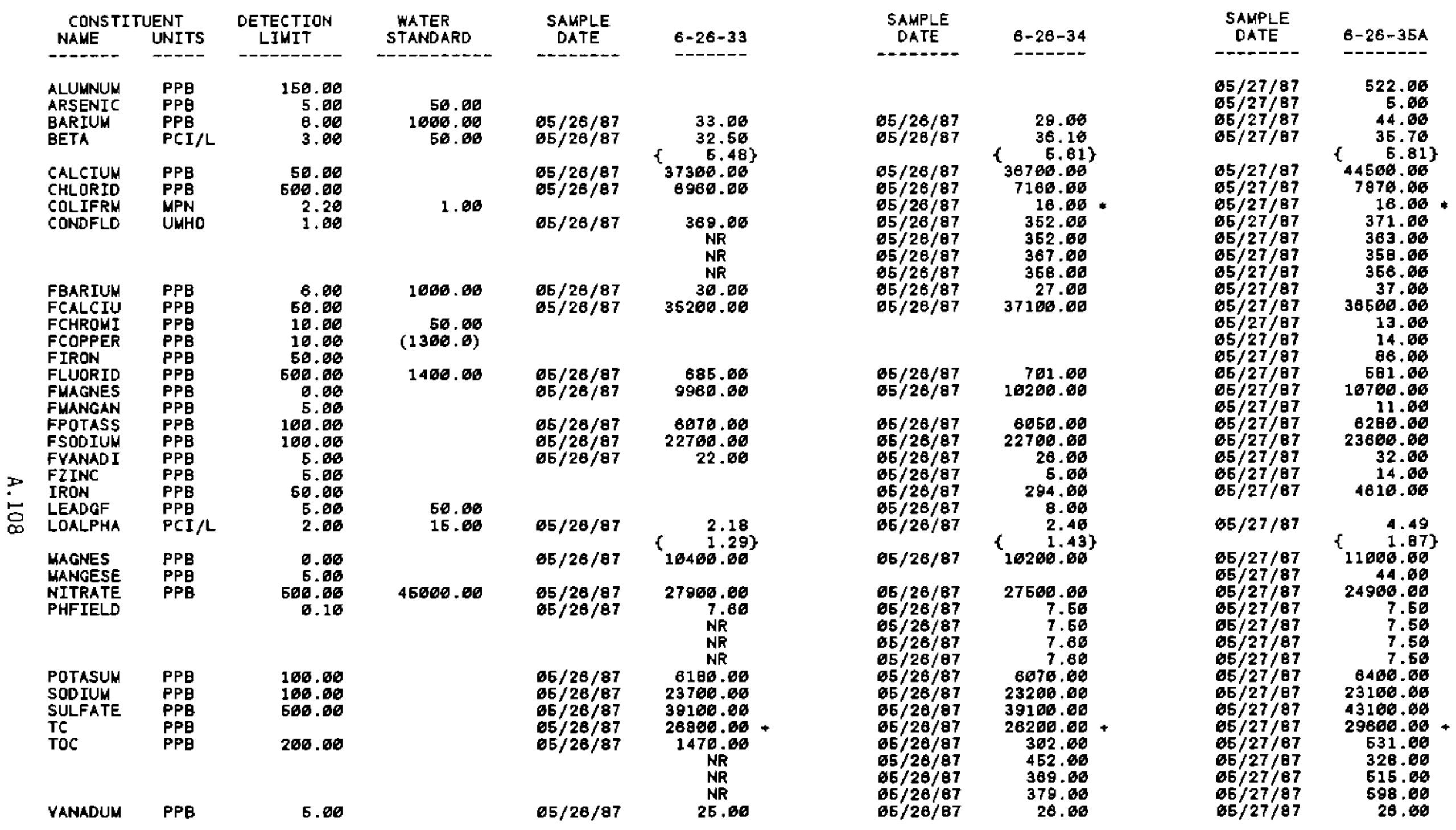

* - value exceeds primary drinking water standard.

- VALUE EXCEEDS PROPOSED PRIMARY DRINKING HATER STANDARD.

* VALUE EXCEEDS SCREENING LEVEL FOR FURTHER INVESTIGATION.

+ - DETECTION LIMIT WAS NOT AVAILABLE FOR COMPARISON

NR - ANALYSIS NOT REQUESTED OR NOT YET REPORTED

VALUES IN \{\} ARE COUNTING ERRORS FOR RADIONUCLIDES

WATER STANDARO(S) IN PARENTHESES ARE PROPOSED ONLY 
TABLE A.5. (cont $d)$

\begin{tabular}{|c|c|c|c|c|c|c|c|c|c|}
\hline $\begin{array}{l}\text { CONST } \\
\text { NAME }\end{array}$ & $\begin{array}{l}\text { UENT } \\
\text { UNITS }\end{array}$ & $\begin{array}{c}\text { DETECTION } \\
\text { LIMIT }\end{array}$ & $\begin{array}{l}\text { WATER } \\
\text { STANDARO }\end{array}$ & $\begin{array}{l}\text { SAMPLE } \\
\text { DATE }\end{array}$ & $6-26-33$ & $\begin{array}{c}\text { SAMPLE } \\
\text { DATE }\end{array}$ & $6-26-34$ & $\begin{array}{c}\text { SAMPLE } \\
\text { DATE }\end{array}$ & $6-26-36 A$ \\
\hline$-\cdots-1$ & 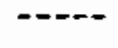 & $----0-1-1$ & -1--1-non & $--\infty \ldots$ & $--\infty-\infty$ & -...-- & ------- & $-\cdots--$ & ------- \\
\hline ZINC & PPE & 5.00 & & $86 / 28 / 87$ & $6.0 B$ & $05 / 26 / 07$ & 8.60 & $05 / 27 / 87$ & 17.60 \\
\hline
\end{tabular}

* - VALUE excEeds PRIMARY dRINHING hater STANDARD

- VALUE EXCEEDS PROPOSED PRIMARY DRINKING WATER STANDARD.

VALUE EXCEEDS SCREENING LEVEL FOR FURTHER INVESTIGATION.

* DETECTION LIMIT WAS NOT AVAILABLE FOR COMPARISON

MR - ANALYSIS NOT REQUESTED OR NOT YET REPORTED

VALUES IN \{\} ARE COUNTING ERRORS FOR RADIONUCLIDES

WATER STANDARD (S) IN PARENTHESES ARE PROPOSED ONLY 
TABLE A.5. (contd)

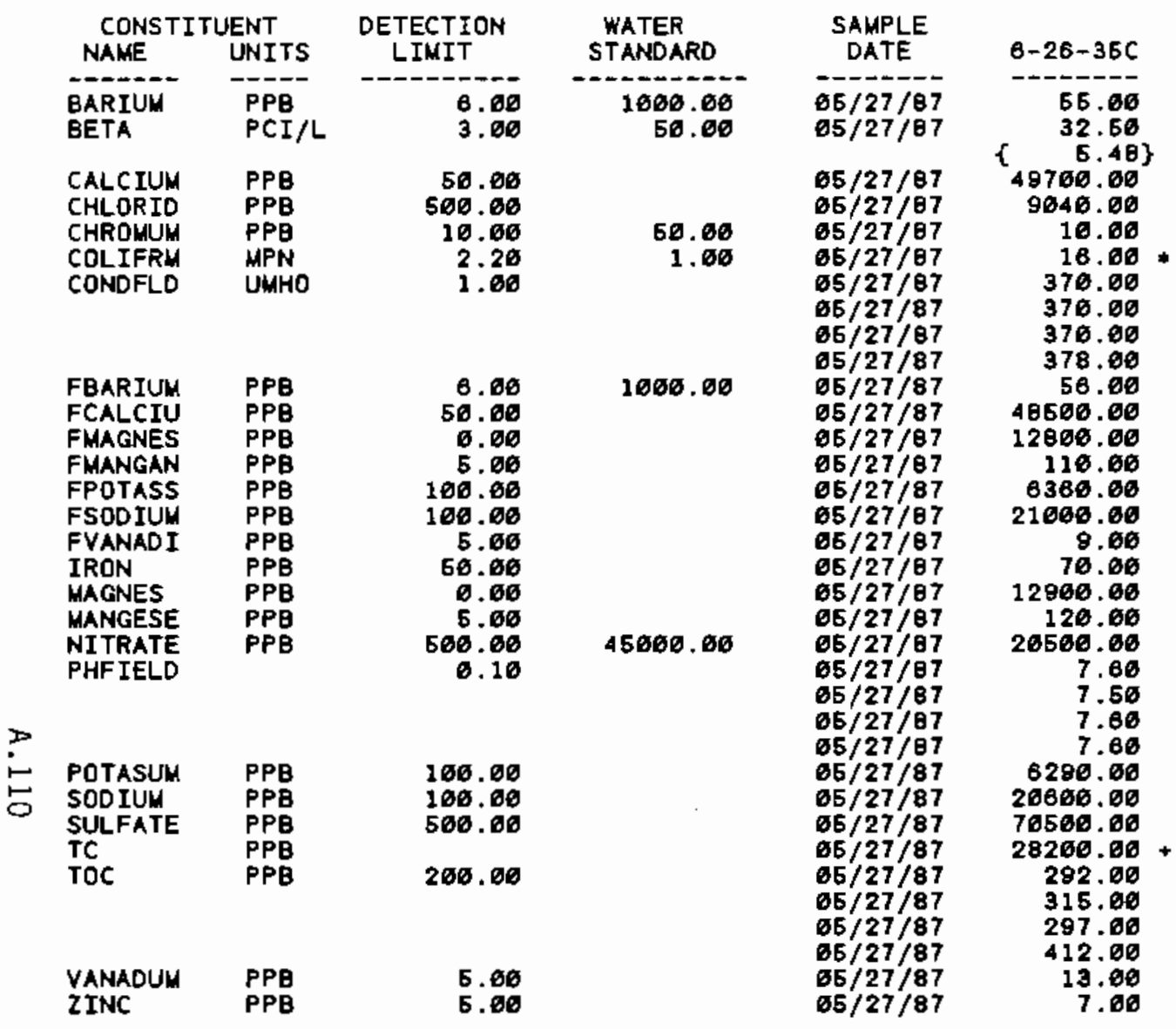

* - VALUE EXCEEDS PRIMARY DRINKING WATER STANDARD.

* - VALUE EXCEEDS PROPOSED PRIMARY DRINKING WATER STANDARD.

- VALUE EXCEEDS SCREENING LEVEL FOR FURTHER INVESTIGATION.

- - DETECTION LIMIT WAS NOT AVAILABLE FOR COMPARISON

NR - ANALYSIS NOT REQUESTED OR NOT YET REPORTED

VALUES IN \{ \} ARE COUNTING ERRORS FOR RADIONUCLIDES

WATER STANDARD(S) IN PARENTHESES ARE PROPOSED ONLY 
IABLE A.6. Results for Site-Wide Radiological and Nitrate Monitoring

Wells Not Included in Table A.4 or A.5

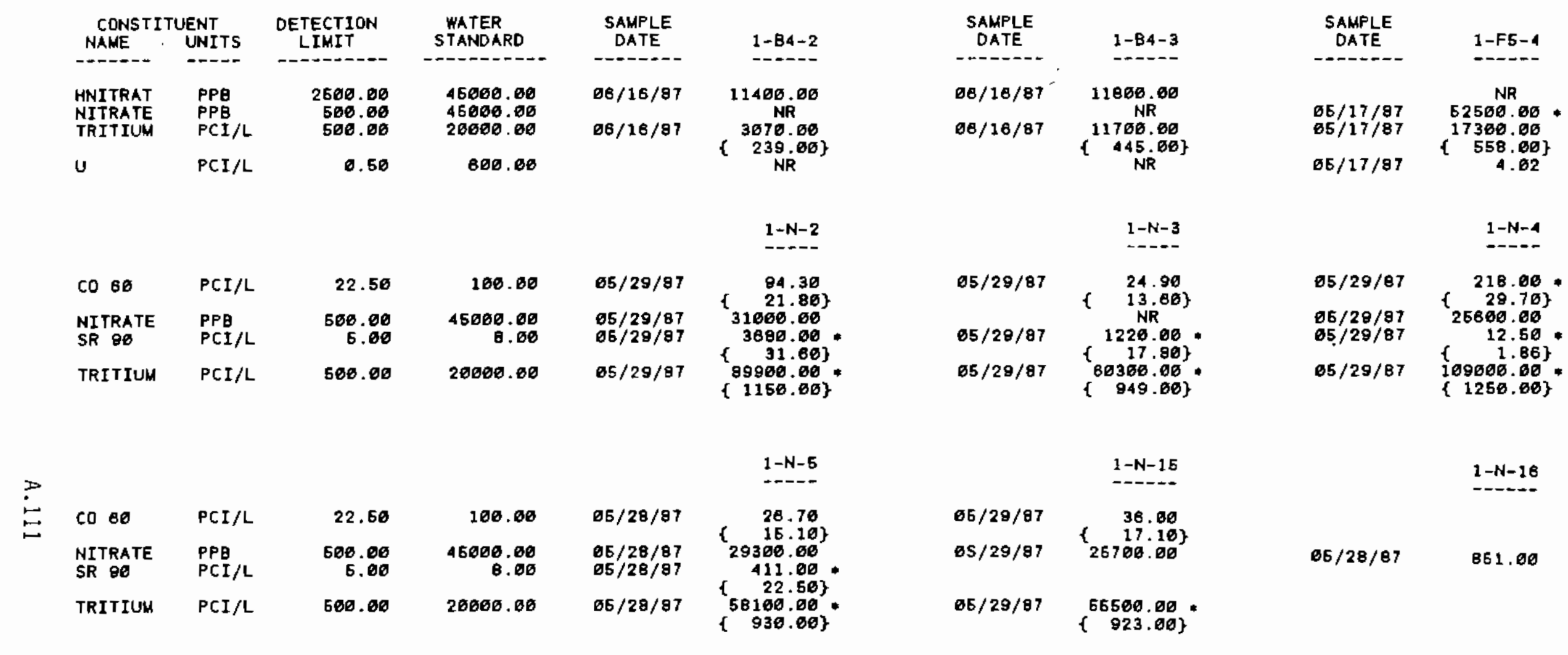

* - VALUE EXCEEOS PRIMARY DRINKING WaTER STANDARD.

* - VALUE EXCEEDS PROPOSED PRIMARY OR INKING WATER STANDARO.

* - VALUE EXCEEOS SCREENING LEVEL FOR FURTHER INYESTIGATION.

+ - DETECTION LIMIT WAS NOT AVAILABLE FOR COMPARISON

NR - ANALYSIS NOT REQUESTED OR NOT YET REPORTED

VALUES IN $\{$ \& ARE COUNTING ERRORS FOR RADIONUCLIDES
WATER STANDARO'(S) IN PARENTHESES ARE PROPOSED ONLY 
IABLE A.6. (contd)

\begin{tabular}{|c|c|c|c|c|c|}
\hline \multicolumn{2}{|c|}{ CONSTITUENT } & $\begin{array}{l}\text { DETECTION } \\
\text { LIMIT }\end{array}$ & $\begin{array}{l}\text { WATER } \\
\text { STANDARD }\end{array}$ & $\begin{array}{c}\text { SAMPLE } \\
\text { DATE }\end{array}$ & $1-N-1 \theta$ \\
\hline------ & --- & - - & $-\infty+\infty-0-0-0$ & ------- & 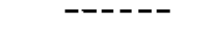 \\
\hline $\begin{array}{l}\text { NITRATE } \\
\text { SR } 90\end{array}$ & $\begin{array}{l}\text { PPB } \\
\text { PCI/L }\end{array}$ & $\begin{array}{r}506.00 \\
6.00\end{array}$ & $\begin{array}{r}45606.00 \\
0.00\end{array}$ & $\begin{array}{l}05 / 28 / 87 \\
06 / 28 / 87\end{array}$ & $\begin{array}{l}514.06 \\
256.00 .\end{array}$ \\
\hline TRITIUN & PCI/L & 500.00 & 20000.06 & $65 / 28 / 87$ & $\begin{array}{r}33160.00 \\
\{\quad 723.06\}\end{array}$ \\
\hline
\end{tabular}

\begin{tabular}{|c|c|c|c|c|c|}
\hline $\cos \theta$ & PCI $/ \mathrm{L}$ & 22.50 & 100.00 & & \\
\hline $\begin{array}{l}\text { NITRATE } \\
\text { RU } 163\end{array}$ & $\begin{array}{l}\text { PPB } \\
\mathrm{PCI} / \mathrm{L}\end{array}$ & $\begin{array}{r}500.06 \\
20.00\end{array}$ & $\begin{array}{l}45000.00 \\
50000.00\end{array}$ & $\begin{array}{l}05 / 28 / 87 \\
05 / 28 / 87\end{array}$ & $\begin{array}{c}13000.00 \\
13000.00 \\
\text { NR }\end{array}$ \\
\hline TRI I IUM & $\mathrm{PCI} / \mathrm{L}$ & 600.00 & 20000.06 & $\begin{array}{l}06 / 28 / 87 \\
65 / 28 / 87\end{array}$ & $\begin{array}{r}2320.00 \\
319.00\} \\
2110.00 \\
314.00\}\end{array}$ \\
\hline
\end{tabular}

篎

$\begin{array}{llrr}\text { CO } 60 & \text { PCI/L } & 22.50 & 100.00 \\ \text { NITRATE } & \text { PPB } & 560.00 & 45000.00 \\ \text { RU 103 } & \text { PCI/L } & 20.00 & 56000.00 \\ \text { SB } 125 & \text { PCI/L } & 48.00 & 56000.00 \\ \text { SR } 90 & \text { PCI/L } & 5.00 & 8.00 \\ \text { TRITIUM } & \text { PCI/L } & 560.00 & 20000.00\end{array}$

1-N-21

\begin{tabular}{|c|c|}
\hline $\begin{array}{c}\text { SAMPLE } \\
\text { DATE }\end{array}$ & $1-N-19$ \\
\hline 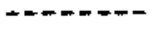 & ------ \\
\hline $\begin{array}{l}05 / 28 / 87 \\
65 / 28 / 87\end{array}$ & $\begin{array}{r}17500.00 \\
129.00\end{array}$ \\
\hline $65 / 28 / 87$ & $\begin{array}{c}5.99\} \\
0050.00 \\
\{\quad 437.00\}\end{array}$ \\
\hline
\end{tabular}

$$
\text { 1-N-22 }
$$

$\begin{array}{cc}65 / 20 / 87 & 5260.66 \\ & \text { NR } \\ & \text { NR }\end{array}$

ø5/28/87

\section{1-N-25}

ต6/28/67

$\left\{\begin{array}{r}27.46\} \\ 18090.09\end{array}\right.$
SAMPLE DATE 1-N-20

$66 / 28 / 87 \quad 14100.00$ $05 / 28 / 87 \quad 41.50$. $05 / 28 / 87$ \{ $\begin{array}{r}3.53\} \\ 3910.00\end{array}$ ( 364.06$)$

$1-\mathrm{N}-23$

$05 / 28 / 87$

$65 / 28 / 87$ $65 / 28 / 87$
$65 / 28 / 87$

$65 / 2 \theta / 87$

$85 / 28 / 87$

C.-.-

23.80 \{ 11.606$\}$ 26300.06 46.06

$\{\quad 16.30\}$

8690.00

( $\begin{array}{r}392.06) \\ 5840.00\end{array}$

$$
\text { NR }
$$

1-N-27

$$
-\infty-2-
$$

$06 / 17 / 87$

$05 / 17 / 87$

อ5/17/67

$65 / 17 / 87$

$65 / 17 / 87$

$65 / 17 / 87$
18060.60

85.36
15.60

241.00

$40.26\}$
93.56

\{ 4.00$\}$

03360.60
(380.00\}

$1-N-36$

$06 / 61 / 87$

$66 / 01 / 87$ $66 / 01 / 87$

190.00

\{r31.26\}

67.50

$\{\quad 18,40\}$

NR

$66 / 01 / 87 \quad 167000.00$

* - VALUE EXCEEDS PRIMARY ORINKING WATER STANDARD.

- VALUE EXCEEDS PROPOSED PRINARY DRINKING WATER STANDARD.

* - VALUE EXCEEDS SCREENING LEVEL FOR FURTHER INVESTIGATION.

* - DETECTION LIMIT WAS NOT AVAILABLE FOR COMPARISON

NR - ANALYSIS NOT REQUESTED OR NOT YET REPORTED

VALUES IN \& 3 ARE COUNTING ERRORS FOR RADIONUCLIDES 
TABLE A.6. (contd)

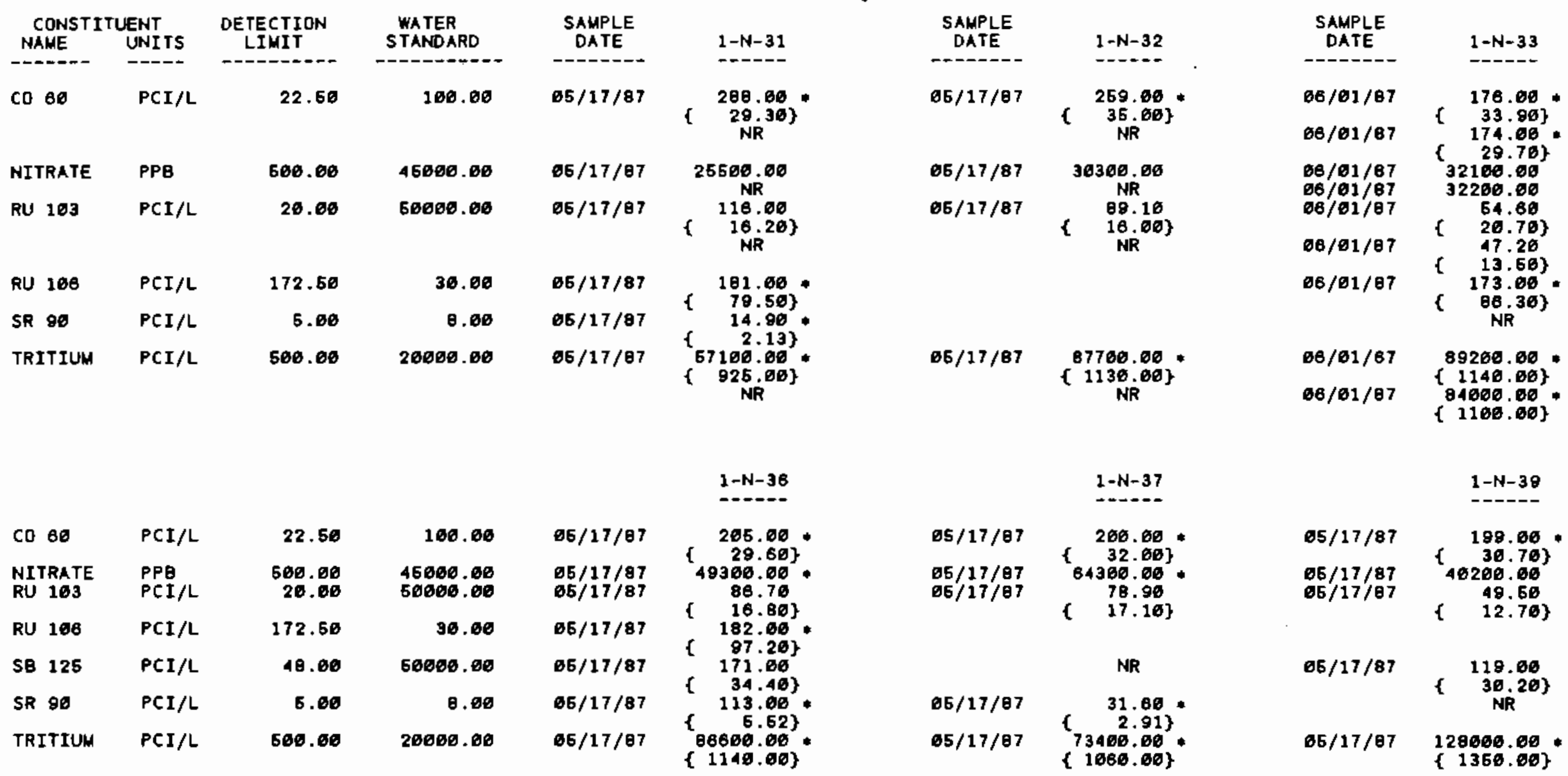

* - VALUE EXCEEDS PRIMARY DRINKING WATER STANDARD.

- VALUE EXCEEDS PROPOSED PRIMARY ORINKING WATER STANDARO.

- VALUE EXCEEDS SCREENING LEVEL FOR FURTHER INVESTIGATION.

+ - DETECTION LIMIT WAS NOT AVAILABLE FOR COMPARISON

NR - ANALYSIS NOT REQUESTED OR NOT YET REPORTED

VALUES IN \{ $\}$ ARE COUNTING ERRORS FOR RADIONUCLIDES

WATER STANDARO (S) IN PARENTHESES ARE PROPOSED ONLY 
IABLE A.6. (contd)

\begin{tabular}{|c|c|c|c|c|c|c|c|c|c|}
\hline CONST ITUENT & $\begin{array}{l}\text { JENT } \\
\text { UNITS }\end{array}$ & $\begin{array}{l}\text { DETECTION } \\
\text { LIHIT }\end{array}$ & $\begin{array}{l}\text { WATER } \\
\text { STANDARD }\end{array}$ & $\begin{array}{l}\text { SAMPLE } \\
\text { DATE }\end{array}$ & \multicolumn{2}{|r|}{$\begin{array}{l}\text { SAMPLE } \\
\text { DATE }\end{array}$} & $1-N-48$ & $\begin{array}{l}\text { SALPLE } \\
\text { DATE }\end{array}$ & $1-\mathrm{N}-60$ \\
\hline$C O B 6$ & $\mathrm{PCI} / \mathrm{L}$ & 22.50 & 100.00 & $05 / 29 / 97$ & $\left\{\begin{array}{c}138.00 \\
25.90\}\end{array}\right\}^{*}$ & & NR & & NR \\
\hline $\begin{array}{l}\text { NITRATE } \\
\text { RU } 103\end{array}$ & $\begin{array}{l}\text { PPB } \\
\text { PCI/L }\end{array}$ & $\begin{array}{r}500.00 \\
20.00\end{array}$ & $\begin{array}{l}45000.00 \\
50000.00\end{array}$ & $\begin{array}{l}06 / 29 / 87 \\
05 / 29 / 67\end{array}$ & $\begin{array}{c}80700.00 \\
62.00 \\
\{\quad 13.26\}\end{array}$ & $65 / 17 / 07$ & $\begin{array}{c}43000.00 \\
N R\end{array}$ & $05 / 17 / 87$ & $\underset{\mathrm{NR}}{28006.00}$ \\
\hline SB 125 & $\mathrm{PCI} / \mathrm{L}$ & 48.80 & 50000.00 & $05 / 29 / 87$ & $\begin{array}{r}197.06 \\
+\quad 38.40\}\end{array}$ & & NR & & NR \\
\hline SR 96 & $\mathrm{PCI} / \mathrm{L}$ & 5.60 & 0.00 & $06 / 29 / 87$ & $\begin{array}{r}2870.00 \\
05.70)\end{array}$ & & & & \\
\hline \multirow[t]{2}{*}{ TRIT IUM } & $\mathrm{PCI} / \mathrm{L}$ & 500.00 & 26000.00 & $65 / 29 / 87$ & $\begin{array}{c}84200.00 \\
(1110.06)\end{array}$ & $05 / 17 / 07$ & $\begin{array}{l}197000.00 * \\
\{1670.00\}\end{array}$ & $06 / 17 / 87$ & $\begin{array}{c}81100.00 \\
\{1000.00\}\end{array}$ \\
\hline & & & & & $1-N-51$ & & $1-N-52$ & & $2-E 13-\theta$ \\
\hline BETA & $\mathrm{PCI} / \mathrm{L}$ & 3.00 & 50.00 & & NR & & NR & $05 / 22 / 07$ & 0.93 \\
\hline $\begin{array}{l}\text { NITRATE } \\
\text { TRITIUN }\end{array}$ & $\begin{array}{l}\mathrm{PPB} \\
\mathrm{PCI} / \mathrm{L}\end{array}$ & $\begin{array}{l}500.00 \\
500.00\end{array}$ & $\begin{array}{l}46000.00 \\
20000.00\end{array}$ & $\begin{array}{l}06 / 17 / 87 \\
05 / 17 / 67\end{array}$ & $\begin{array}{c}24000.00 \\
60400.00 \\
\left\{\begin{array}{c}842.00\}\end{array}\right.\end{array}$ & $\begin{array}{l}65 / 68 / 87 \\
65 / 60 / 87\end{array}$ & $\begin{array}{c}21300.00 \\
120000.00 \\
\{1310.00\}\end{array}$ & & $\begin{array}{l}2.12\} \\
\text { NR } \\
\text { NR }\end{array}$ \\
\hline
\end{tabular}

- - VALUE EXCEEDS PRIMARY DRINKING WATER STANDARD.

- VALUE EXCEEDS PROPOSED PRIMARY DRINKING WATER STANDARD.

+ - DETECTION LIMIT WAS NOT AVAILABLE FDR COMPARISON

NR - ANALYSIS NOT REQUESTED OR NOT YET REPORTED

VALUES IN \& \& ARE COUNTING ERRORS FOR RADIONUCLIDES

WATER STANDARD(S) IH PARENTHESES ARE PROPOSED ONLY 
TABLE A.6. (contd)

\begin{tabular}{|c|c|c|c|c|c|c|}
\hline $\begin{array}{l}\text { CONSTI } \\
\text { NAME }\end{array}$ & $\begin{array}{l}\text { UENT } \\
\text { UNITS }\end{array}$ & $\begin{array}{l}\text { DETECTION } \\
\text { LIMIT }\end{array}$ & $\begin{array}{l}\text { WATER } \\
\text { STANDARD }\end{array}$ & $\begin{array}{c}\text { SAMPLE } \\
\text { DATE }\end{array}$ & & $13-19$ \\
\hline - - & $-\cdots$ & $------\infty$ & -..---n-- & 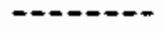 & & ---- \\
\hline BETA & $P C I / L$ & 3.06 & 60.00 & $05 / 22 / 87$ & \{ & $\begin{array}{l}90.4 \theta \times \\
6 . \theta 0\} \\
N R\end{array}$ \\
\hline & & & & & & NR \\
\hline LOALPHA & PCI/L & 2.60 & 16.00 & & & NR \\
\hline & & & & & & NR \\
\hline NITRATE & PPE & 500.00 & 15000.00 & & & $\begin{array}{l}\text { NR } \\
\text { NR } \\
N R\end{array}$ \\
\hline TRITIUM & $P C I / L$ & 500.00 & 20000.00 & & & NR \\
\hline & & & & & & NR \\
\hline & & & & & & NR \\
\hline
\end{tabular}

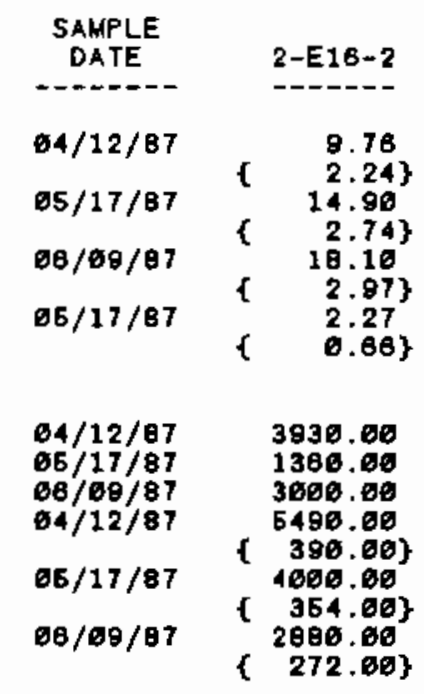

\begin{tabular}{|c|c|}
\hline $\begin{array}{l}\text { SAMPLE } \\
\text { DATE }\end{array}$ & 2-E17-2 \\
\hline $06 / 16 / 87$ & 448.00 \\
\hline $66 / 68 / 87$ & 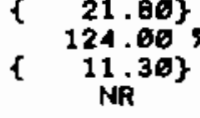 \\
\hline $06 / 16 / 87$ & 7.71 \\
\hline $68 / 08 / 87$ & \\
\hline $\begin{array}{l}65 / 16 / 87 \\
66 / 68 / 87\end{array}$ & $\begin{array}{l}175000.00 \\
120000.00\end{array}$ \\
\hline $\begin{array}{l}66 / 16 / 87 \\
66 / 68 / 87\end{array}$ & $\begin{array}{c}91400.00 \\
2480.00\} \\
43406.00 \\
\{1890.00\} \\
\mathrm{NR}\end{array}$ \\
\hline
\end{tabular}

- - Value exceeds primary orinking water standaro.

- VALUE EXCEEDS PROPOSED PRIMARY DRINKING WATER STANDARD.

- VALUE EXCEEDS SCREENING LEVEL FOR FURTHER INVESTIGATION.

+ - DETECTION LIMIT WAS NOT AVAILABLE FOR COMPARISON

NR - ANALYSIS NOT REQUESTED OR NOT YET REPORTED

VALUES IN \{\} ARE COUNTING ERRORS FOR RADIONUCLIDES

WATER STANDARD'S) IN PARENTHESES ARE PROPOSED ONLY 
TABLE A.6. (contd)

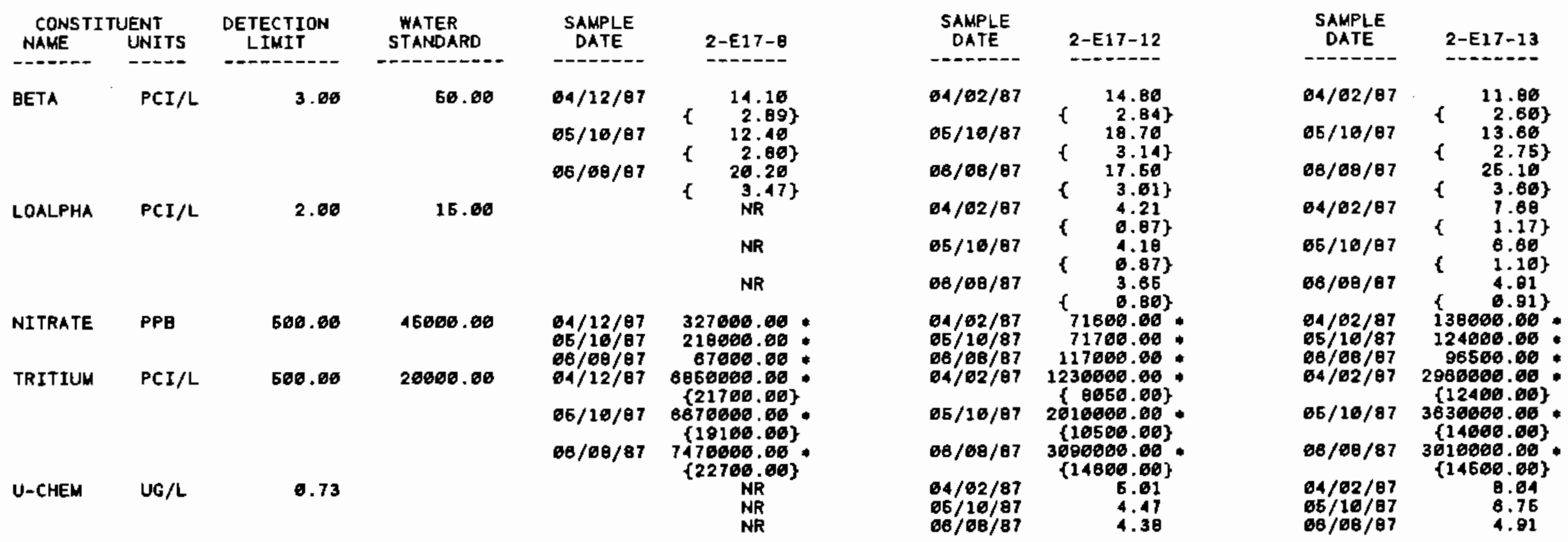

* - VALUE EXCEEDS PRIMARY DRINKING WATER STANDARD.

- VALUE EXCEEOS PROPOSED PRIMARY ORINKING WATER STANDARD

* - VALUE EXCEEDS SCREENING LEVEL FOR FURTHER INVESTIGATION.

* - DETECTION LIMIT WAS NOT AVAILAGLE FOR COMPARISON

NR - ANALYSIS NOT REQUESTED OR NOT YET REPORTED

VALUES IN \{ $\}$ ARE COUNTING ERRORS FOR RAOIONUCLIDES

WATER STANDARO (S) IN PARENTHESES ARE PROPOSED ONLY 
TABLE A.6. (contd)

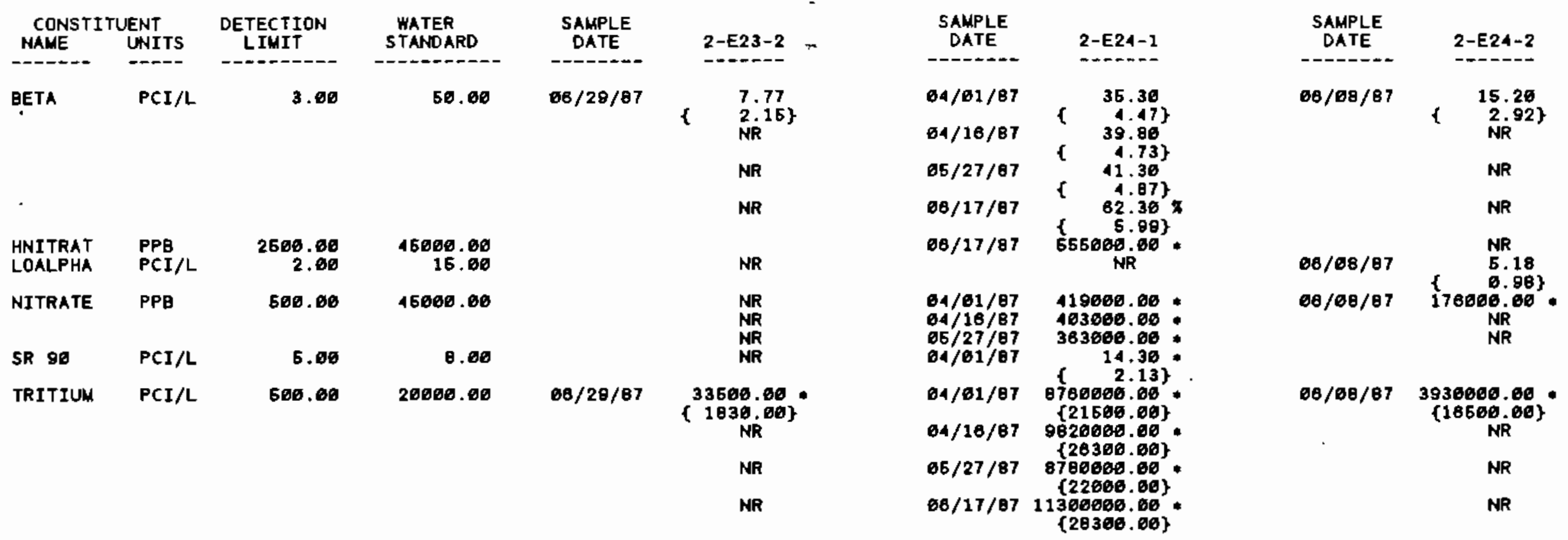

- - VALUE EXCEEDS PRIMARY DR INKING WATER STANDARO.

- - VALUE EXCEEDS PROPOSED PRIMARY DRINKING WATER STANDARD.

* - VALUE EXCEEDS SCREENING LEVEL FOR FURTHER INVESTIGATION.

* - DETECTION LIMIT WAS NOT AVAILAELE FOR COMPARISON

NR - ANALYSIS NOT REQUESTED OR NOT YET REPORTED

YALUES IN $T$ ARE COUNTING ERRORS FOR RADIONUCLIDES

WATER STANDARD(S) IN PARENTHESES ARE PROPOSED ONLY 
IABLE A.6. (contd)

\begin{tabular}{|c|c|c|c|c|c|c|c|c|c|}
\hline $\begin{array}{l}\text { CONSTI } \\
\text { NAME }\end{array}$ & $\begin{array}{l}\text { UENT } \\
\text { UNITS }\end{array}$ & $\begin{array}{l}\text { DETECTION } \\
\text { LIMIT }\end{array}$ & $\begin{array}{l}\text { WATER } \\
\text { STANDARO }\end{array}$ & $\begin{array}{l}\text { SAMPLE } \\
\text { DATE }\end{array}$ & $2-E 24-4$ & $\begin{array}{c}\text { SAMPLE } \\
\text { DATE }\end{array}$ & $2-E 24-11$ & $\begin{array}{c}\text { SAMPLE } \\
\text { DATE }\end{array}$ & $2-E 24-13$ \\
\hline BETA & PCI/L & 3.00 & 50.00 & $\begin{array}{l}04 / 02 / 87 \\
64 / 12 / 87 \\
65 / 16 / 87\end{array}$ & $\begin{array}{ll} & 6.74 \\
\{\quad 1.97\} \\
\{\quad \\
\{.57 \\
1.81\} \\
4.82 \\
1.75\}\end{array}$ & $\begin{array}{l}04 / 12 / 87 \\
65 / 10 / 87 \\
66 / 68 / 87\end{array}$ & $\begin{array}{cc} & 14.40 \\
\{\quad 3.02\} \\
\{\quad 11.80 \\
\{\quad 2.78\} \\
\{\quad 16.19 \\
\{\quad 3.17\}\end{array}$ & $\begin{array}{l}64 / 02 / 87 \\
08 / 29 / 87\end{array}$ & $\begin{array}{l}8.03 \\
2.12\} \\
6.89 \\
2.01\} \\
\text { NR }\end{array}$ \\
\hline $\begin{array}{l}\text { HNI TRAT } \\
\text { NITRATE }\end{array}$ & $\begin{array}{l}\text { PPB } \\
\text { PPB }\end{array}$ & $\begin{array}{r}2500.00 \\
500.00\end{array}$ & $\begin{array}{l}45000.00 \\
45000.00\end{array}$ & $\begin{array}{l}04 / 12 / 87 \\
65 / 16 / 87 \\
06 / 08 / 87\end{array}$ & $\begin{array}{c}\text { NR } \\
2778.00 \\
2796.06 \\
4280.08\end{array}$ & $04 / 12 / 87$ & 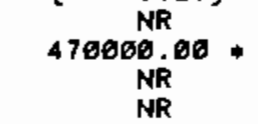 & $\begin{array}{l}66 / 29 / 87 \\
64 / 62 / 87\end{array}$ & $\begin{array}{c}4216.60 \\
2958.60 \\
\mathrm{NR} \\
\mathrm{NR}\end{array}$ \\
\hline TRITIUM & $\mathrm{PCI} / \mathrm{L}$ & 500.00 & 20000.00 & $\begin{array}{l}04 / 62 / 87 \\
64 / 12 / 87 \\
66 / 16 / 87 \\
66 / 68 / 87\end{array}$ & 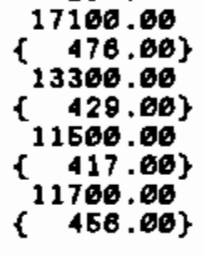 & $04 / 12 / 87$ & $\begin{array}{c}13906060.06 \\
\{31400.00\} \\
\text { NR } \\
\text { NR } \\
\text { NR }\end{array}$ & $64 / 02 / 87$ & $\begin{array}{c}\left\{\begin{array}{c}2200.00 \\
\text { (NR.06) }\end{array}\right. \\
\text { NR } \\
\text { NR }\end{array}$ \\
\hline & & & & & 2-E25-3 & & 2-E2 Б-6 & & 2-E25-8 \\
\hline BETA & $\mathrm{PCI} / \mathrm{L}$ & 3.06 & 50.00 & $06 / 29 / 87$ & $\begin{array}{l}3.88 \\
1.65\} \\
\text { NR }\end{array}$ & $\begin{array}{l}05 / 17 / 87 \\
06 / 99 / 87\end{array}$ & $\begin{array}{ll} & 3.68 \\
\{\quad 1.56\} \\
\quad 5.21 \\
\{\quad 1.77\}\end{array}$ & $\begin{array}{l}04 / 12 / 87 \\
05 / 17 / 87\end{array}$ & 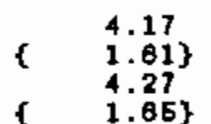 \\
\hline $\begin{array}{l}\text { NITRATE } \\
\text { TRITIUM }\end{array}$ & $\begin{array}{l}\mathrm{PPB} \\
\mathrm{PCI} / \mathrm{L}\end{array}$ & $\begin{array}{l}500.00 \\
500.00\end{array}$ & $\begin{array}{l}45000.00 \\
26000.00\end{array}$ & & $\begin{array}{l}\text { NR } \\
\text { NR } \\
\text { NR } \\
\text { NR }\end{array}$ & $\begin{array}{l}04 / 12 / 87 \\
64 / 12 / 87 \\
06 / 17 / 87 \\
06 / 09 / 87\end{array}$ & $\begin{array}{c}1880.66 \\
31500.00 \\
\{1680.00\} \\
6840.06 \\
354.00\} \\
8590.06 \\
\{\quad 462.00\}\end{array}$ & $\begin{array}{l}64 / 12 / 87 \\
04 / 12 / 87 \\
05 / 17 / 87\end{array}$ & $\begin{array}{c}1426.60 \\
3116.66 \\
345.06\} \\
3506.06 \\
345.66\} \\
\text { NR }\end{array}$ \\
\hline
\end{tabular}

- - VALUE EXCEEDS PRIMARY DRINKING waTER STANDARO.

- VALUE EXCEEDS PROPOSED PRIMARY DRINKING WATER STANOARO.

* - VALUE EXCEEDS SCREENING LEVEL FOR FURTHER INVESTIGATION.

+ - DETECTION LIMIT WAS NOT AVAILABLE FOR COMPARISON

NR - ANALYSIS NOT REQUESTED OR NOT YET REPORTED

VALUES IN \{ I ARE COUNTING ERRORS FOR RADIONUCLIDES
WATER STANDARD(S) IN PARENTHESES ARE PROPOSED ONLY 
IABLE A.6. (contd)

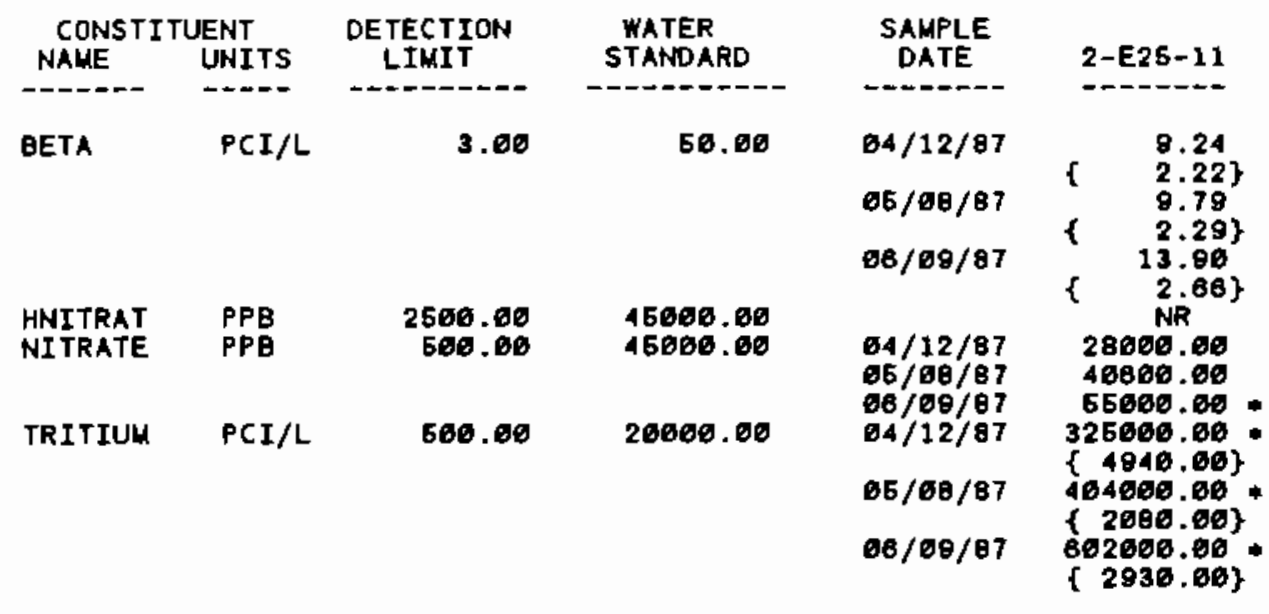

官
50.60

BETA

PCI/L

3.00

600.00

45000.60

NITRATE PPB

TRITIUM PCI/L

506.06

26000.00
2-E25-19

\begin{tabular}{|c|c|c|}
\hline $\begin{array}{l}\text { SAHPLE } \\
\text { DATE }\end{array}$ & \multicolumn{2}{|c|}{ 2-E2E-13 } \\
\hline & & 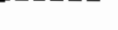 \\
\hline $64 / 62 / 87$ & & 11.10 \\
\hline $66 / 28 / 87$ & 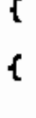 & $\begin{array}{c}2.98\} \\
10.00 \\
3.08\} \\
N R\end{array}$ \\
\hline
\end{tabular}

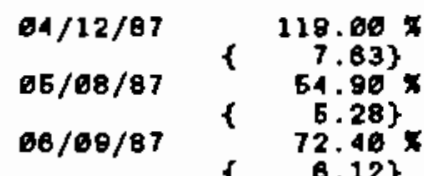

$66 / 69 / 87$

$04 / 12 / 97$

$68 / 09 / 97$

241090.12\}

158000.00 .

206000.06 .

(10000.00

$65 / 08 / 87$ 5200600.

$06 / 09 / \theta 7 \quad 5296600.06$.
$66 / 29 / 87$ $04 / 02 / 67$

\subsection{0 \\ 300060.00 .}

NR

NR

NP

NR

2-E25-23

08/69/87

SALPLE DATE

2-E25-17

$04 / 12 / 87$

$66 / 00 / 87$

$66 / 69 / 87$

$04 / 12 / \theta 7$

$65 / 98 / 97$

$68 / 09 / 87$
$64 / 12 / 87$

$05 / 06 / 87$

$06 / 09 / 87$

\{ 9.33

$\left\{\begin{array}{r}2.19\} \\ 10.90\end{array}\right.$

$\{\quad 2.38\}$

$\{\quad 2.25\}$

26500.00

18300.00

17160.00

502000.00

\{5296.00\}

374060.00.

\{ 4830.00$\}$

$\left\{\begin{array}{l}11060.66 \\ 5620.66\}\end{array}\right.$

2-E25-24

2.73 )

NR

66/09/87 4630.60

NR

$06 / 69 / 87$

678.60

NR

NR
$66 / 99 / 87$

22.20
$\{\quad 3.29\}$
NR

NR

66/69/87

$06 / 09 / 8$ NR 1026.66 NR

NR

- VALUE EXCEEDS PRIMARY DRINKING WATER STANDARD.

- VALUE EXCEEDS PROPOSED PRIMARY DRINKING WATER STANDARD.

- VALUE EXCEEDS SCREENING LEVEL FOR FURTHER INVESTIGATION.

* - DETECTION LIMIT WAS NOT AVAILABLE FOR COMPARISON

NR - ANALYSIS NOT REQUESTED OR NOT YET REPORTED

VALUES IN $f\}$ ARE COUNTING ERRORS FOR RADIONUCLIDES

WATER STANDARD(S) IN PARENTHESES ARE PROPOSED ONLY 
IABLE A.6. (contd)

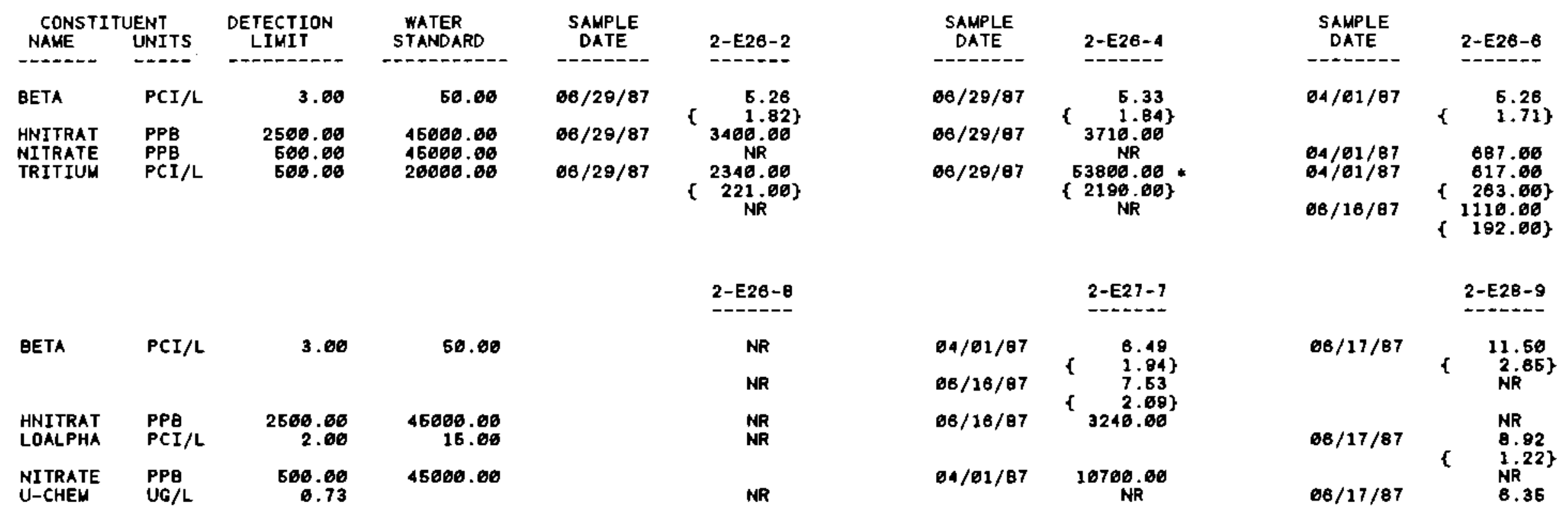

* - VALUE EXCEEDS PRIMARY DRINKING water STANDARD.

- VALUE EXCEEDS PROPOSED PRIMARY DRINKING WATER STANDARD.

- VALUE EXCEEDS SCREENING LEVEL FOR FURTHER INVESTIGATION.

+ - DETECTION LIMIT WAS NOT AVAILABLE FOR COMPARISON

NR - ANALYSIS NOT REQUESTED OR NOT YET REPORTED

VALUES IN \{ $\}$ ARE COUNTING ERRORS FOR RADIONUCLIDES
WATER STANDARD (S) IN PARENTHESES ARE PROPOSED ONLY 
TABLE A.6. (contd)

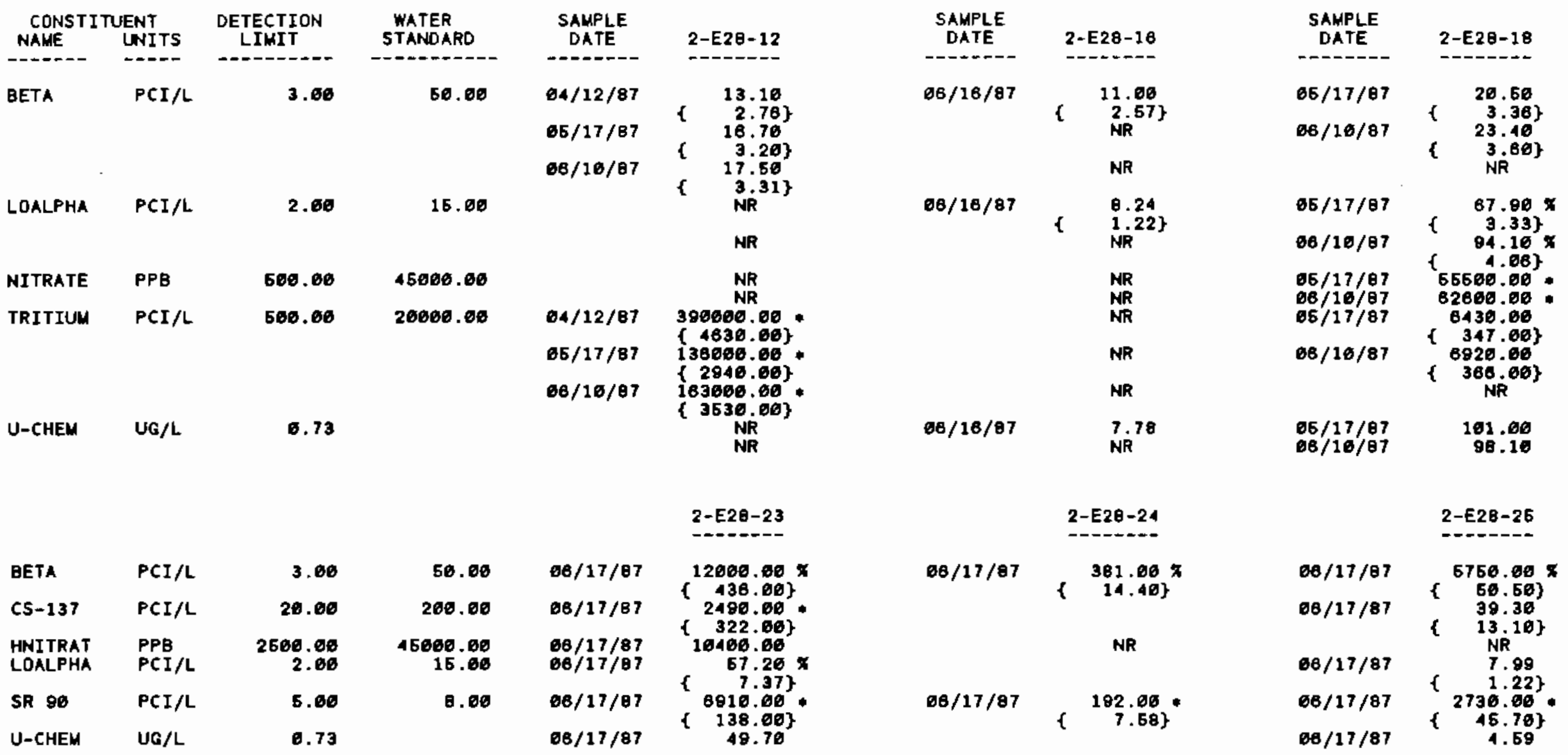

- - VALUe EXCEEDS PRIMARY DRINKING WaTER STANDARD.

- VALUE EXCEEDS PROPOSED PRIMARY DRINKING WATER STANDARD.

* - VAlUE EXCEEDS SCREENING LEVEL FoR FURTHER INVESTIGATION.

- - DETECTION LIMIT WAS NOT AVAILAELE FOR COMPARISON

NR - ANALYSIS NOT REQUESTED OR NOT YET REPORTED

VALUES IN $\{?$ ARE COUNTING ERRORS FOR RADIONUCLIDES

WATER STANDARD (S) IN PARENTHESES ARE PROPOSED ONLY 
TABLE A.6. (contd)

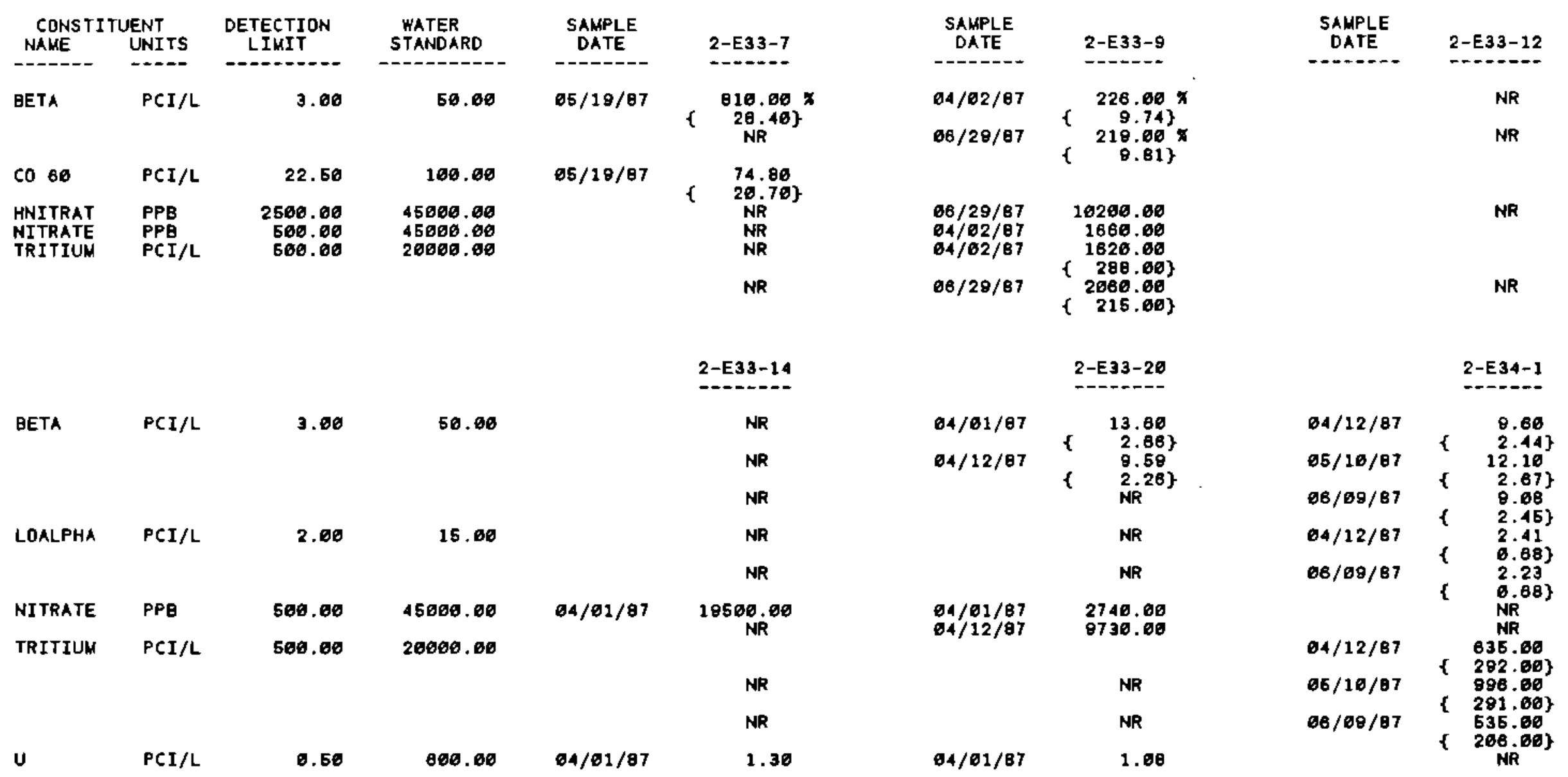

- - VALUE exceEdS PRIMARY dRINKINg water STANDARD.

- VALUE EXCEEDS PROPOSED PRIMARY DRINKING WATER STANDARD.

* - VALUE EXCEEDS SCREENING LEVEL FOR FURTHER INVESTIGATION.

- DETECTION LIMIT WAS NOT AVAILABLE FOR COMPARISON

NR - ANALYSIS NOT REQUESTED OR NOT YET REPORTED

VALUES IN \{ \} ARE COUNTING ERRORS FOR RADIONUCLIDES

WATER STANDARO(S) IN PARENTHESES ARE PROPOSED ONLY 
IABLE A.6. (contd)

\begin{tabular}{|c|c|c|c|c|c|c|c|c|c|}
\hline $\begin{array}{l}\text { CONSTI } \\
\text { NAWE }\end{array}$ & $\begin{array}{l}\text { JENT } \\
\text { UNITS }\end{array}$ & $\begin{array}{l}\text { DETECTION } \\
\text { LIUIT }\end{array}$ & $\begin{array}{l}\text { WATER } \\
\text { STANDARD }\end{array}$ & $\begin{array}{l}\text { SAMPLE } \\
\text { DATE }\end{array}$ & $2-W 11-23$ & $\begin{array}{c}\text { SAMPLE } \\
\text { DATE }\end{array}$ & $2-W 11-24$ & $\begin{array}{l}\text { SAMPLE } \\
\text { DATE }\end{array}$ & $2-w 14-10$ \\
\hline$----\infty$ & ---- & 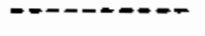 & $-x-0-0-0-1$ & $---c--$ & $---n$ & $---1-\infty$ & $--\ldots-n$ & $-\infty-\infty-\infty$ & --n-co- \\
\hline \multirow[t]{2}{*}{ BETA } & PCI / L & 3.00 & БD. . DE & $05 / 17 / 87$ & $\begin{array}{l}32.40 \\
\operatorname{NR}^{3.56\}}\end{array}$ & $65 / 17 / 87$ & $\begin{array}{l}8.63 \\
2.60\} \\
\text { NR }\end{array}$ & $\begin{array}{l}04 / 19 / 87 \\
06 / 10 / 87\end{array}$ & $\begin{array}{l}6.75 \\
2.11\} \\
6.94\end{array}$ \\
\hline & & & & & NR & & NR & $D 8 / 09 / 87$ & 6.62 \\
\hline \multirow[t]{2}{*}{ LOALPHA } & PCI /L & 2.00 & 15.60 & $65 / 17 / 87$ & 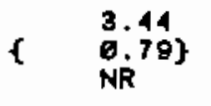 & & NR & $\begin{array}{l}64 / 19 / 87 \\
65 / 10 / 87\end{array}$ & $\begin{array}{l}3.98 \\
6.85\} \\
5.11\end{array}$ \\
\hline & & & & & NR & & NR & $06 / 09 / 87$ & 4.77 \\
\hline NITRATE & PCI/L & 500.00 & 20060.00 & $05 / 17 / 67$ & $\begin{array}{c}151000.00 \\
\text { NR } \\
\text { NR } \\
\text { NR }\end{array}$ & $05 / 17 / 87$ & $\begin{array}{c}221000,00 \\
\text { NR } \\
\text { NR } \\
\text { NR }\end{array}$ & $\begin{array}{l}64 / 19 / 87 \\
65 / 10 / 87 \\
06 / 69 / 87 \\
04 / 19 / 87\end{array}$ & $\begin{array}{c}83000.00 \\
99800.00 \\
99100.00 \\
1670.00 \\
314.001\end{array}$ \\
\hline \multirow{3}{*}{ TRITIUM } & & & & & NR & & MR & $06 / 10 / 87$ & 1840.60 \\
\hline & & & & & NR & & NR & $08 / 69 / 87$ & $\begin{array}{r}1826.00 \\
239.00\}\end{array}$ \\
\hline & & & & \multicolumn{2}{|r|}{$2-w 1 B-1$} & \multicolumn{2}{|r|}{ 2-w11-11 } & & $2-K 11-18$ \\
\hline \multirow[t]{2}{*}{ BETA } & $\mathrm{PCI} / \mathrm{L}$ & 3.00 & 50.00 & $08 / 29 / 87$ & $\begin{array}{c}33.00 \\
\{\quad 4.56\}\end{array}$ & $B 6 / 29 / 87$ & $\begin{array}{c}43.40 \\
4.963\end{array}$ & $06 / 17 / 87$ & $\begin{array}{c}47.06 \\
5.12\}\end{array}$ \\
\hline & & & & \multicolumn{2}{|r|}{$2-w 16-6$} & \multicolumn{2}{|r|}{ 2-W18-5 } & & $2-W 18-9$ \\
\hline BETA & $\mathrm{PCI} / \mathrm{L}$ & 3.00 & 60.00 & $05 / 17 / 97$ & \multirow{2}{*}{$\begin{array}{c}5.12 \\
1.84\} \\
N R \\
11306.60\end{array}$} & $68 / 15 / 67$ & \multirow{2}{*}{$\begin{array}{l}3.94 \\
1.67\} \\
\text { NR } \\
\text { NR }\end{array}$} & $66 / 28 / 67$ & \multirow{2}{*}{$\begin{array}{c}3.97 \\
1.62\} \\
6900.00 \\
N R\end{array}$} \\
\hline $\begin{array}{l}\text { HNITRAT } \\
\text { NITRATE }\end{array}$ & $\begin{array}{l}\text { PPB } \\
\text { PPB }\end{array}$ & $\begin{array}{r}2500.00 \\
500.00\end{array}$ & $\begin{array}{l}45000.60 \\
45000.00\end{array}$ & $05 / 17 / 67$ & & & & $06 / 29 / 87$ & \\
\hline
\end{tabular}

- - value exceeds primary drinking water standaro.

- VALUE EXCEEDS PROPOSED PRIHARY DRINKING WATER STAMDARD.

- VALUE EXCEEDS SCREENING LEVEL FOR FURTHER INVESTIGATION.

- - DETECTION LIMIT WAS NOT AVAILABLE FOR COMPARISON

NR - ANALYSIS NOT REQUESTED OR NOT YET REPORTED

VALUES IN \& \} ARE COUNTING ERRORS FOR RADIONUCL IDES
WATER STANOARD'(S) IN PARENTHESES ARE PROPOSED ONLY 
IABLE A.6. (contd)

\begin{tabular}{|c|c|c|c|c|c|c|c|c|c|}
\hline $\begin{array}{l}\text { CONSTI } \\
\text { NAME }\end{array}$ & $\begin{array}{l}\text { WENT } \\
\text { UNITS }\end{array}$ & $\begin{array}{l}\text { DETECTION } \\
\text { LIMIT }\end{array}$ & $\begin{array}{l}\text { WATER } \\
\text { STANDARD }\end{array}$ & $\begin{array}{l}\text { SAMPLE } \\
\text { DATE }\end{array}$ & $2-W 18-15$ & $\begin{array}{l}\text { SAMPLE } \\
\text { DATE }\end{array}$ & $2-\$ 18-17$ & $\begin{array}{l}\text { SAMPLE } \\
\text { DATE }\end{array}$ & $2-W 1 \theta-1 B$ \\
\hline \multirow[t]{3}{*}{ BETA } & $\mathrm{PCI} / \mathrm{L}$ & 3.00 & 50.00 & $84 / 19 / 87$ & 16.40 & $06 / 29 / 87$ & 4.72 & $05 / 15 / 87$ & $\begin{array}{l}4.39 \\
1.64\}\end{array}$ \\
\hline & & & & $05 / 15 / 87$ & 12.00 & & $\mathrm{NR}$ & & $\mathrm{NR}^{1.04}$ \\
\hline & & & & $08 / 15 / 87$ & 28.38 & & NR & & NR \\
\hline \multirow[t]{3}{*}{$\begin{array}{l}\text { HNITRAT } \\
\text { LOALPHA }\end{array}$} & $\begin{array}{l}\mathrm{PPB} \\
\mathrm{PCI} / \mathrm{L}\end{array}$ & $\begin{array}{r}2500.00 \\
2.00\end{array}$ & $\begin{array}{r}45006.00 \\
16.00\end{array}$ & $\begin{array}{l}06 / 15 / 87 \\
04 / 19 / 87\end{array}$ & $\begin{array}{r}2780.60 \\
49.60 \times\end{array}$ & & NR & $06 / 15 / 87$ & 3010.00 \\
\hline & & & & $05 / 15 / 87$ & $51.08 x$ & & NR & & NR \\
\hline & & & & $08 / 15 / 87$ & $52.60 \%$ & & NR & & NR \\
\hline \multirow[t]{3}{*}{ NITRATE } & PPB & 500.00 & 45000.00 & $04 / 19 / 07$ & 967.06 & & NR & $B 5 / 15 / 87$ & 772.80 \\
\hline & UG/L & 6.73 & & $\begin{array}{l}64 / 19 / 87 \\
65 / 16 / 97 \\
06 / 15 / 87\end{array}$ & $\begin{array}{l}82.18 \\
70.78 \\
78.18\end{array}$ & & $\begin{array}{l}\text { NR } \\
\text { NR } \\
\text { NR }\end{array}$ & & $\begin{array}{l}\mathrm{NR} \\
\mathrm{NR} \\
\mathrm{NR}\end{array}$ \\
\hline & & & & & $2-\$ 18-28$ & & $2-\$ 19-2$ & & $2-W 19-12$ \\
\hline BETA & $\mathrm{PCI} / \mathrm{L}$ & 3.00 & 50.00 & $68 / 29 / 87$ & 3.73 & $05 / 17 / 87$ & $89.80 \times$ & $65 / 17 / 87$ & 13.60 \\
\hline $\begin{array}{l}\text { HNITRAT } \\
\text { LOALPHA }\end{array}$ & $\begin{array}{l}\mathrm{PPB} \\
\mathrm{PCI} / \mathrm{L}\end{array}$ & $\begin{array}{r}2500.00 \\
2.00\end{array}$ & $\begin{array}{r}45000.06 \\
15.00\end{array}$ & $66 / 29 / 87$ & 3170.06 & $05 / 17 / 87$ & $\begin{array}{l}\text { NR } \\
20.70 \times\end{array}$ & $05 / 17 / 87$ & $\begin{array}{l}N R \\
4.27\end{array}$ \\
\hline $\begin{array}{l}\text { NITRATE } \\
\text { SR } 96\end{array}$ & $\begin{array}{l}\mathrm{PPB} \\
\mathrm{PCI} / \mathrm{L}\end{array}$ & $\begin{array}{r}500.06 \\
5.00\end{array}$ & $\begin{array}{r}45000.00 \\
8.06\end{array}$ & & $\begin{array}{l}\mathrm{NR} \\
\mathrm{NR}\end{array}$ & $\begin{array}{l}05 / 17 / 87 \\
05 / 17 / 87\end{array}$ & 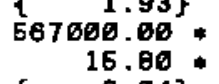 & $05 / 17 / 87$ & 3170.00 \\
\hline TC-99 & PCI/L & 15.00 & 100000.00 & & NR & $05 / 17 / 87$ & 289.60 & & NR \\
\hline TRITIUM & $\mathrm{PCI} / \mathrm{L}$ & 500.06 & 20000.06 & & & $05 / 17 / 87$ & $129600.00^{\circ}$ & & \\
\hline U-CHEM & UG/L & 6.73 & & & $N R$ & $05 / 17 / 07$ & 31.00 & $05 / 17 / 87$ & 8.88 \\
\hline
\end{tabular}

- - VALUE EXCEeds PRIMARY DRINKING water STANDARD.

- VALUE EXCEEDS PROPOSED PRIMARY DRINKING WATER STANDARD.

- VALUE EXCEEDS SCREENING LEVEL FOR FURTHER INVESTIGATION.

+ - DETECTION LIMIT WAS NOT AVAILABLE FOR COMPARISON

NR - ANALYSIS NOT REQUESTED OR NOT YET REPORTED

VALUES IN \{ $\{$ ARE COUNTING ERRORS FOR RADIONUCLIDES
WATER STANDARD(S) IN PARENTHESES ARE PROPOSED ONLY 
TABLE A.6. (contd)

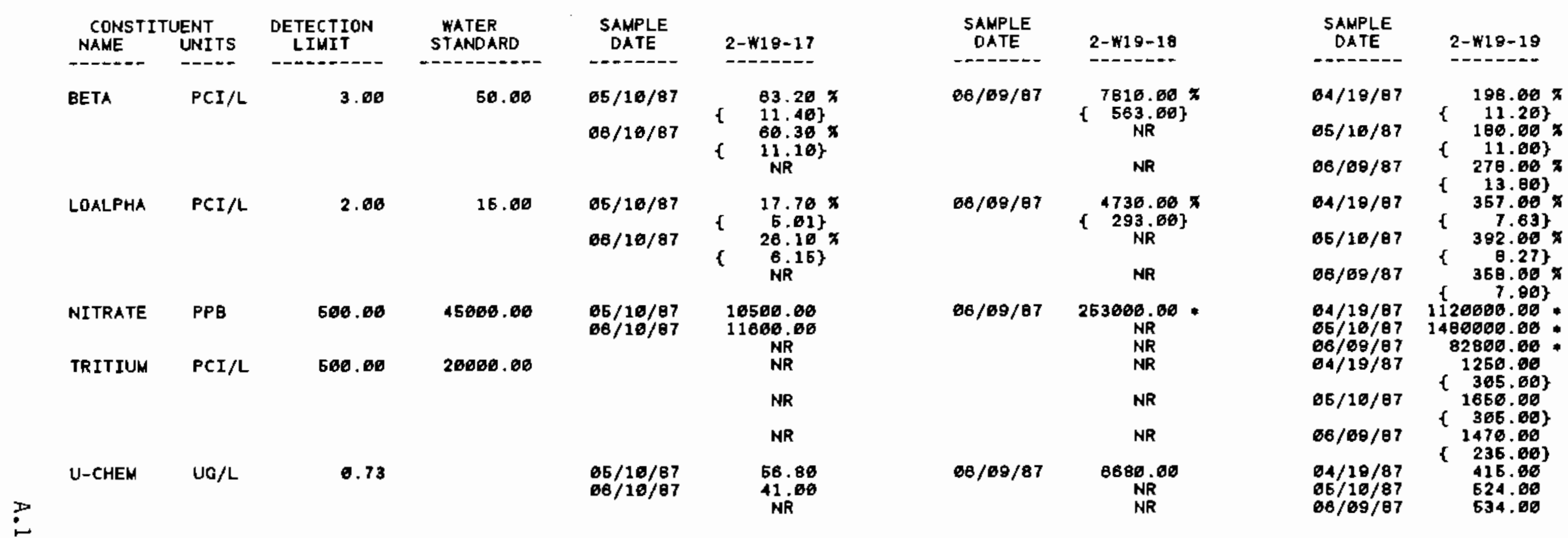

- - ValUe exceEds primary dRINKINg water STANDARo.

- - VALUE EXCEEDS PROPOSED PRIMARY DRINKING WATER STANDARD.

- VALUE EXCEEDS SCREENING LEVEL FOR FURTHER INVESTIGATION.

* - DETECTION LIMIT WAS NOT AVAILABLE FOR COMPARISON

NR - ANALYSIS NOT REQUESTEO OR NOT YET REPORTED

VALUES IN \{\} ARE COUNTING ERRORS FOR RADIONUCLIDES

WATER STANDARD'(S) IN PARENTHESES ARE PROPOSED ONLY 
IABLE A.6. (contd)

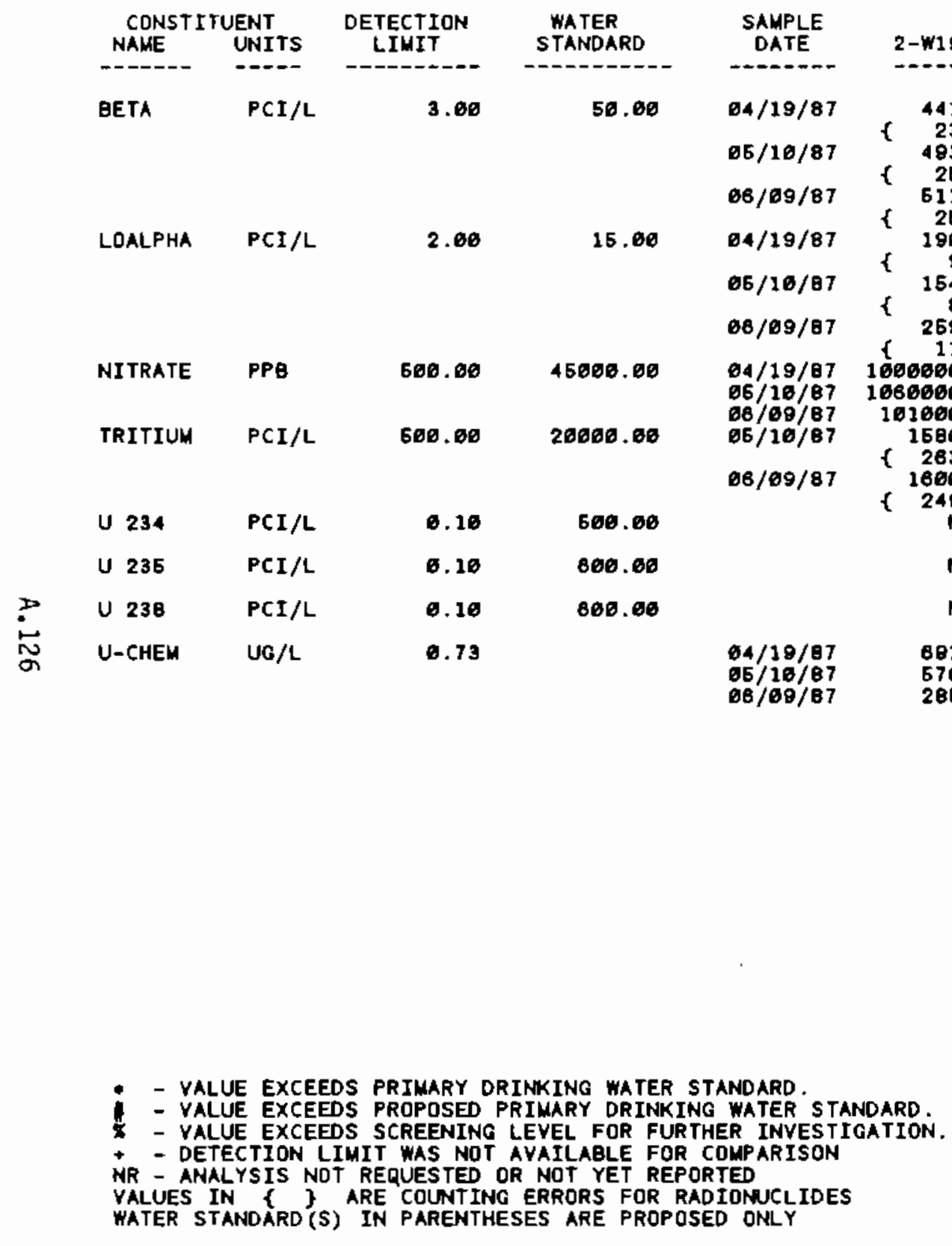

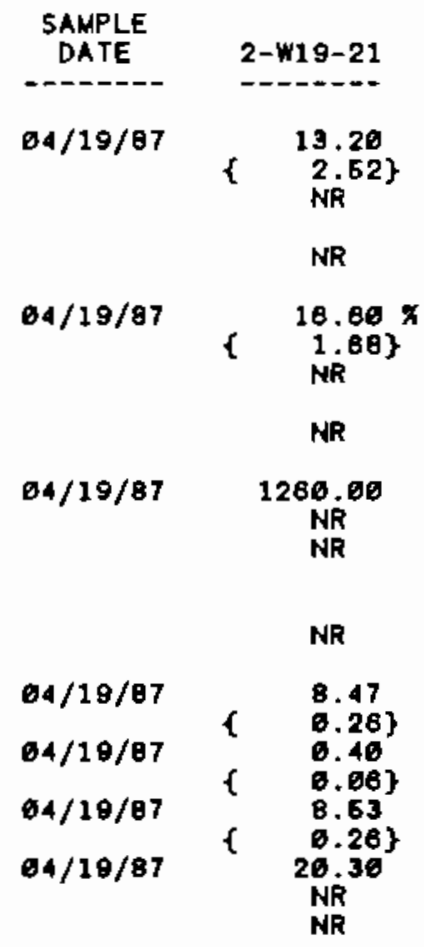

SAMPLE

DATE

2-พ19-23

$441.00 \%$

$23.80\}$

$483.60 \%$

25.46 )

$190.00 x$

$154.00 \%$

$8.66\}$
$259.06 \%$

$11.40\}$

.

$101000.00 *$

1580.00

263.663
1600.00

$249.60)$

NR

NR

NR

801.00

576.00

285.00
-..-...-

$05 / 19 / 87 \quad 127.60 \%$

$08 / 09 / 87$

$\{\quad 0.76\}$

( 7.19$\}$

NR

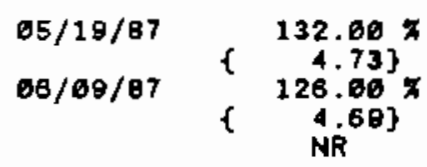

$05 / 18 / 87 \quad 166006.06$

$68 / 69 / 87$ 57500B.00

( 298.06$\}$

68/08/87 1036.06

$66 / 09 / 87<224.00$

$08 / 09 / 87\left\{\begin{array}{l}3.18 \\ 2\end{array}\right.$

$\left\{\begin{array}{l}2.88 \\ (93.18)\end{array}\right.$

66/69/87 73.10

( 3.23$\}$

$65 / 18 / 87 \quad 230.60$ 
IABLE A.6. (contd)

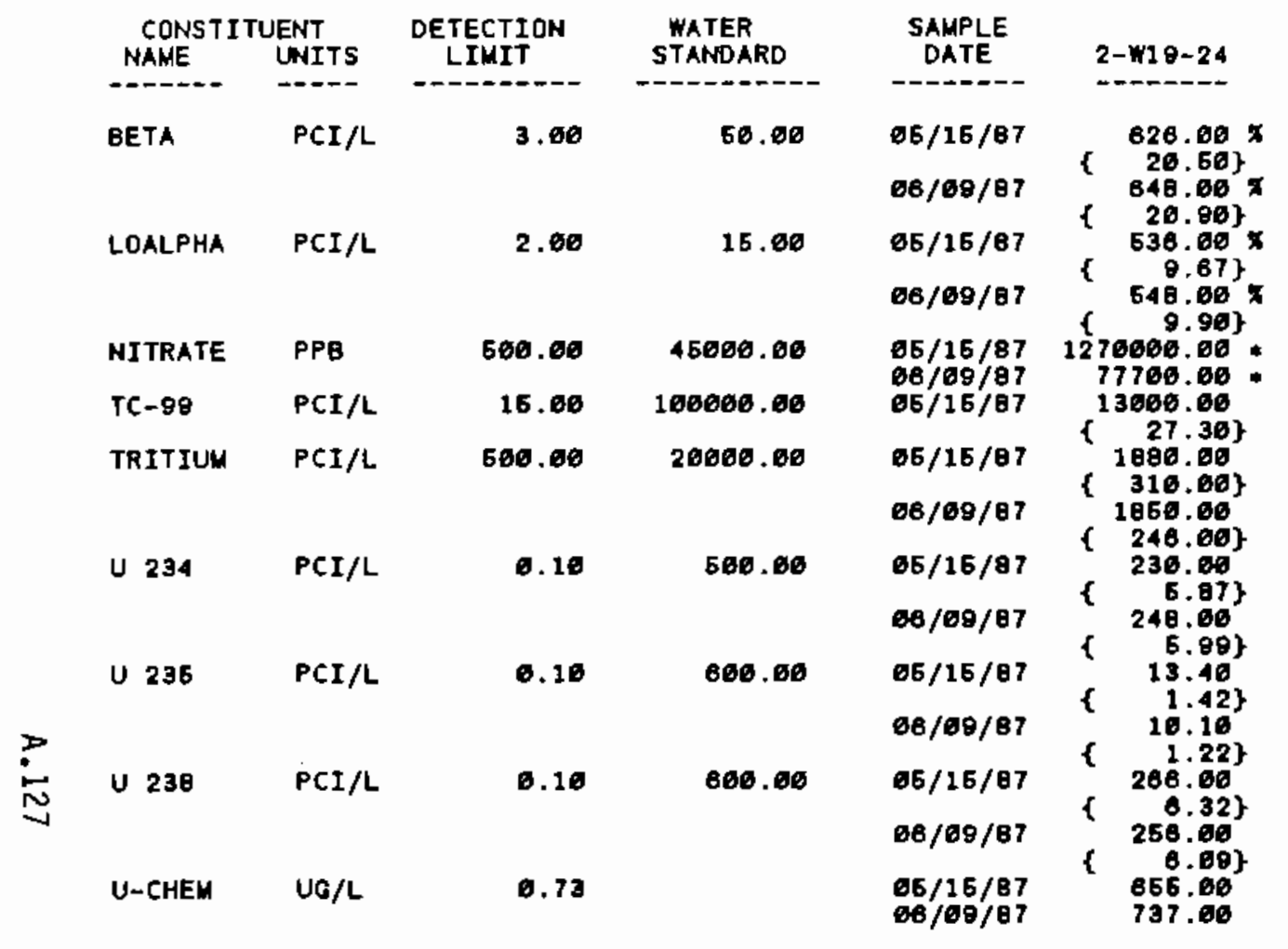

\begin{tabular}{|c|c|}
\hline $\begin{array}{l}\text { SAMPLE } \\
\text { DATE }\end{array}$ & $2-W 19-25$ \\
\hline $65 / 15 / 97$ & $848.60 \times$ \\
\hline $06 / 09 / 87$ & $637.06 \times$ \\
\hline $65 / 16 / 87$ & $\begin{array}{c}19.38\} \\
283.06 \times\end{array}$ \\
\hline $06 / 09 / \theta 7$ & $\begin{array}{l}7.24\} \\
73.68 \times\end{array}$ \\
\hline $\begin{array}{l}96 / 15 / 87 \\
66 / 69 / 87 \\
65 / 15 / 87\end{array}$ & $\begin{array}{l}728060.60 \\
149606.66 \\
18366.66\end{array}$ \\
\hline $85 / 16 / 87$ & $\begin{array}{r}1536.00 \\
30200\end{array}$ \\
\hline $86 / 89 / 87$ & $\begin{array}{r}1470.06 \\
237.08\}\end{array}$ \\
\hline $66 / 16 / 87$ & 131.66 \\
\hline$\theta 0 / 8 \theta / 87$ & 134.80 \\
\hline $96 / 16 / 87$ & 8 \\
\hline $86 / 6 \theta / 87$ & 8 \\
\hline $65 / 16 / 87$ & 148.60 \\
\hline $06 / 69 / 87$ & 8 \\
\hline $\begin{array}{l}06 / 16 / 87 \\
68 / 69 / 87\end{array}$ & $\begin{array}{l}223.06 \\
344.00\end{array}$ \\
\hline
\end{tabular}

SAMPLE

-.-.-...

$06 / 10 / 87$

$65 / 15 / 87$

$68 / 10 / 87$

$05 / 15 / 87$

$9 B / 16 / 87$

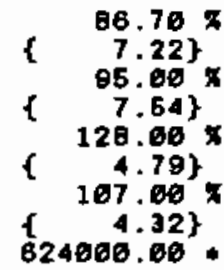

882666.86 .

$65 / 15 / 87 \quad 1300.60$

$06 / 10 / 87$ \{ 287.00

$\{231.00\}$

(61.10

60/10/07 I

\{ 3.38$\}$

$\left\{\begin{array}{l}2.16 \\ 0.82\end{array}\right.$

$68 / 16 / 87$ f 3.82

$05 / 26 / 87\left\{\begin{array}{c}0.78\} \\ 08 / 18 / 87\end{array}\right.$

( 2.13$\}$

$68 / 10 / 87, \begin{array}{r}80.50 \\ 3.39\end{array}$

$66 / 15 / 87.124 .06$
$00 / 16 / 87 . \quad 145.60$

- - VALUE EXEEEDS PRIMARY dRINKING water STANDARD.

- VALUE EXCEEDS PROPOSED PRIMARY DRINHING WATER STANDARD.

* - VALUE EXCEEDS SCREENING LEVEL FOR FURTHER INVESTIGATION.

- DETECTION LIMIT WAS NOT AVAILABLE FOR COMPARISON

NR - ANALYSIS NOT REQUESTED OR NOT YET REPORTED

VALUES IN \{ \} ARE COUNTING ERRORS FOR RADIONUCLIDES

WATER STANDARD'(S) IN PARENTHESES ARE PROPOSED ONLY 
IABLE A.6. (contd)

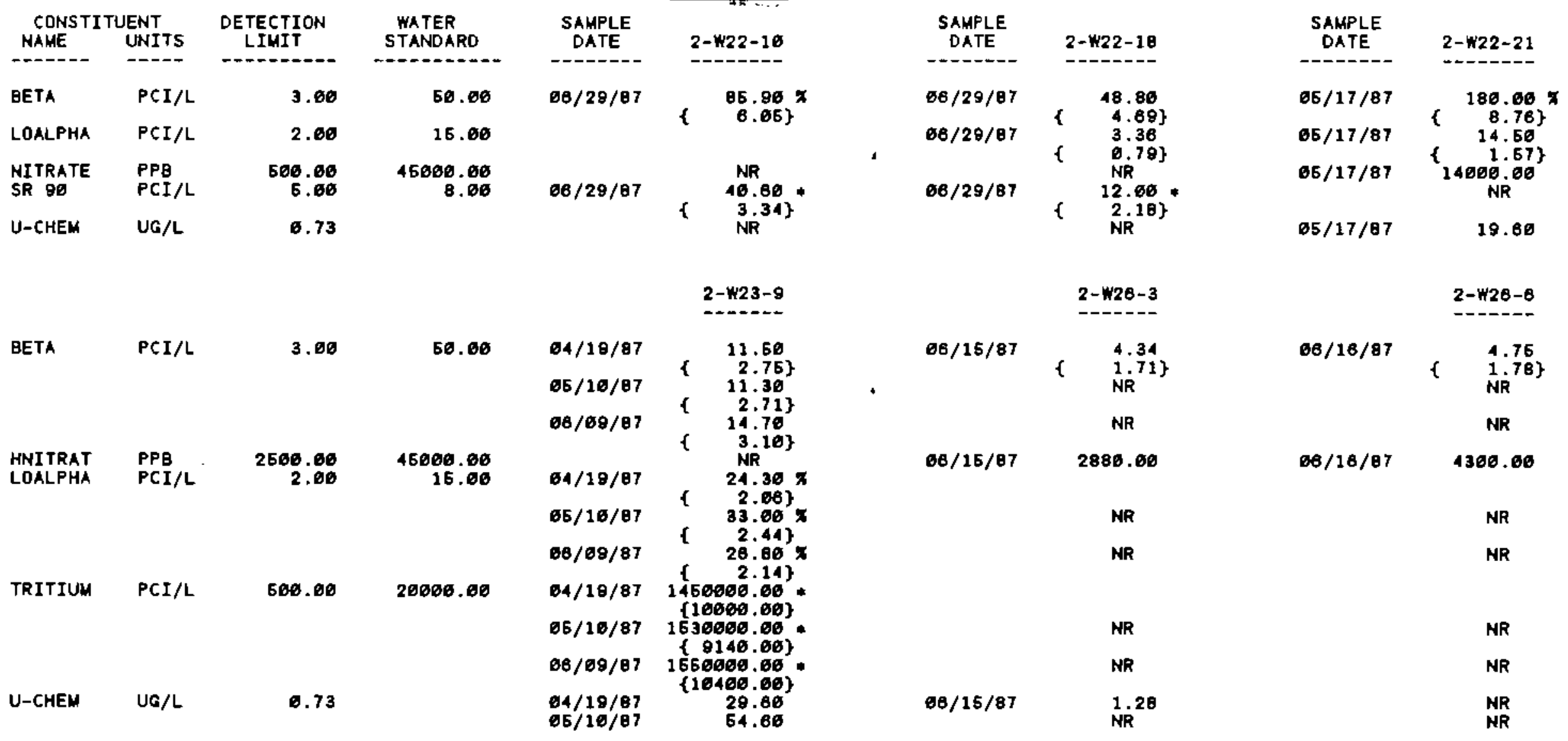

* - VALUE EXCEEDS PRIMARY DRINKING WATER STANDARD.

- VALUE EXCEEDS PROPOSED PRIMARY DRINKING WATER STANOARO.

\$ - VALUE EXCEEDS SCREENING LEVEL FOR FURTHER INVESTIGATION.

+ - DETECTION LIMIT WAS NOT AVAILABLE FOR COMPARISON

MR - ANALYSIS NOT REQUESTED OR NOT YET REPORTED

VALUES IN \{ ARE COLNTING ERRORS FOR RADIONUCLIDES

WATER STANOARO'(S) IN PARENTHESES ARE PROPOSED ONLY 
TABLE A.6. (contd)

\begin{tabular}{|c|c|c|c|c|c|c|c|c|c|}
\hline \multicolumn{2}{|c|}{ CONSTITUENT } & $\begin{array}{c}\text { DETECTION } \\
\text { LIMIT }\end{array}$ & $\begin{array}{l}\text { WATER } \\
\text { STAMDARD }\end{array}$ & $\begin{array}{l}\text { SAMPLE } \\
\text { DATE }\end{array}$ & $3-2-2$ & $\begin{array}{l}\text { SAMPLE } \\
\text { DATE }\end{array}$ & $3-2-3$ & $\begin{array}{l}\text { SAMPLE } \\
\text { DATE }\end{array}$ & $3-3-1$ \\
\hline$-\infty---$ & ----- & - - - & 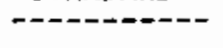 & - n-c-- & 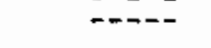 & $---\cdots---$ & $\cdots-$ & $-\infty+\infty-\infty$ & $-\cdots$ \\
\hline $\begin{array}{l}\text { NITRATE } \\
\text { TRITIUM }\end{array}$ & $\begin{array}{l}\mathrm{PPB} \\
\mathrm{PCI} / \mathrm{L}\end{array}$ & $\begin{array}{l}500.00 \\
600.00\end{array}$ & $\begin{array}{l}45000.00 \\
20000.00\end{array}$ & $\begin{array}{l}64 / 62 / 87 \\
64 / 02 / 87\end{array}$ & $\begin{array}{r}6870.00 \\
580.00\end{array}$ & $\begin{array}{l}64 / 02 / 07 \\
04 / 02 / 87\end{array}$ & $\begin{array}{r}19160.00 \\
1260.06\end{array}$ & $\begin{array}{l}04 / 02 / 87 \\
04 / 02 / \theta 7\end{array}$ & $\begin{array}{r}22360.00 \\
1660.00\end{array}$ \\
\hline \multirow[t]{2}{*}{$\mathbf{U}$} & $\mathrm{PCI} / \mathrm{L}$ & 6.60 & 600.00 & $04 / 02 / 87$ & $\begin{array}{r}22.10 \\
\end{array}$ & $04 / 02 / 87$ & $\begin{array}{c}1 \quad 8.48 \\
1008\end{array}$ & $04 / 02 / \theta 7$ & $\begin{array}{r}314.813 \\
6.91\end{array}$ \\
\hline & & & & & $3-3-2$ & & $3-3-3$ & & $3-3-6$ \\
\hline \multirow[t]{2}{*}{$\begin{array}{l}\text { NITRATE } \\
\text { U }\end{array}$} & $\begin{array}{l}\text { PPB } \\
\mathrm{PCI} / \mathrm{L}\end{array}$ & $\begin{array}{r}500.60 \\
0.60\end{array}$ & $\begin{array}{r}45060.00 \\
660.00\end{array}$ & $04 / 02 / 87$ & $0.8 \theta$ & $\begin{array}{l}04 / 67 / 87 \\
04 / 07 / 87\end{array}$ & $\begin{array}{r}9220.00 \\
7.72\end{array}$ & $\begin{array}{l}64 / 02 / 87 \\
64 / 62 / 67\end{array}$ & $\begin{array}{r}18000.00 \\
12.80\end{array}$ \\
\hline & & & & & $3-3-\theta$ & & $3-3-11$ & & $3-3-12$ \\
\hline \multirow{4}{*}{$\begin{array}{l}\text { NITRATE } \\
\text { SR } 90 \\
\text { TRITIUN } \\
\text { U }\end{array}$} & $\begin{array}{l}\text { PPB } \\
\text { PCI } / L\end{array}$ & $\begin{array}{r}600.00 \\
5.00\end{array}$ & $\begin{array}{r}45000.00 \\
8.00\end{array}$ & $04 / 67 / 87$ & 25360.60 & $\begin{array}{l}04 / 07 / 87 \\
04 / 07 / 87\end{array}$ & $\begin{array}{r}15100.00 \\
\quad 0.92 \\
\quad 2.09\}\end{array}$ & $05 / 07 / 87$ & 12700.60 \\
\hline & $\mathrm{PCI} / \mathrm{L}$ & 500.00 & 20000.00 & $04 / 07 / 87$ & 1420.60 & $04 / 07 / 07$ & 2216.00 & $05 / 07 / 07$ & 2650.00 \\
\hline & $\mathrm{PCI} / \mathrm{L}$ & 6.50 & 806.00 & $04 / 07 / 87$ & $\begin{array}{c}300.005 \\
10.20\end{array}$ & $04 / 07 / 87$ & $\begin{array}{c}323.06\} \\
26.56\end{array}$ & $95 / 67 / 87$ & 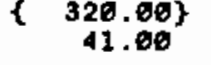 \\
\hline & & & & & $3-4-8$ & & $3-4-16$ & & $3-6-1$ \\
\hline $\begin{array}{l}\text { NITRATE } \\
\text { TRITIUM }\end{array}$ & $\begin{array}{l}\text { PPB } \\
\text { PCI/L }\end{array}$ & $\begin{array}{l}560.00 \\
560.00\end{array}$ & $\begin{array}{l}45000.00 \\
20000.00\end{array}$ & $\begin{array}{l}64 / 07 / 87 \\
04 / 07 / 87\end{array}$ & $\begin{array}{r}22800.00 \\
2320.00\end{array}$ & $\begin{array}{l}06 / 97 / 87 \\
65 / 07 / 87\end{array}$ & $\begin{array}{r}16466.06 \\
1426.00\end{array}$ & $64 / 87 / \dot{8} 7$ & 45700.00 \\
\hline U & $\mathrm{PCI} / \mathrm{L}$ & 0.60 & 600.00 & $04 / 07 / 87$ & 25.00 & 65/07/87 & $\begin{array}{c}307.603 \\
17.60\end{array}$ & $64 / 07 / 87$ & 3.48 \\
\hline
\end{tabular}

- - value exceeds primary drikiking water stamdard.

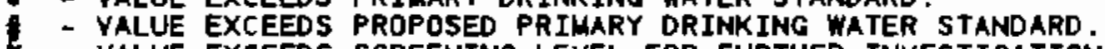

- VALUE EXCEEDS SCREENING LEVEL FOR FURTHER INVESTIGATION.

* - DETECTION LIMIT WAS NOT AVAILAGLE FOR COMPARISON

NR - ANALYSIS NOT REQUESTED OR NOT YET REPORTED

WATER IN ( $)$ ARE COUNTING ERRORS FOR RADIONUCLIDES 
IABLE A.6. (contd)

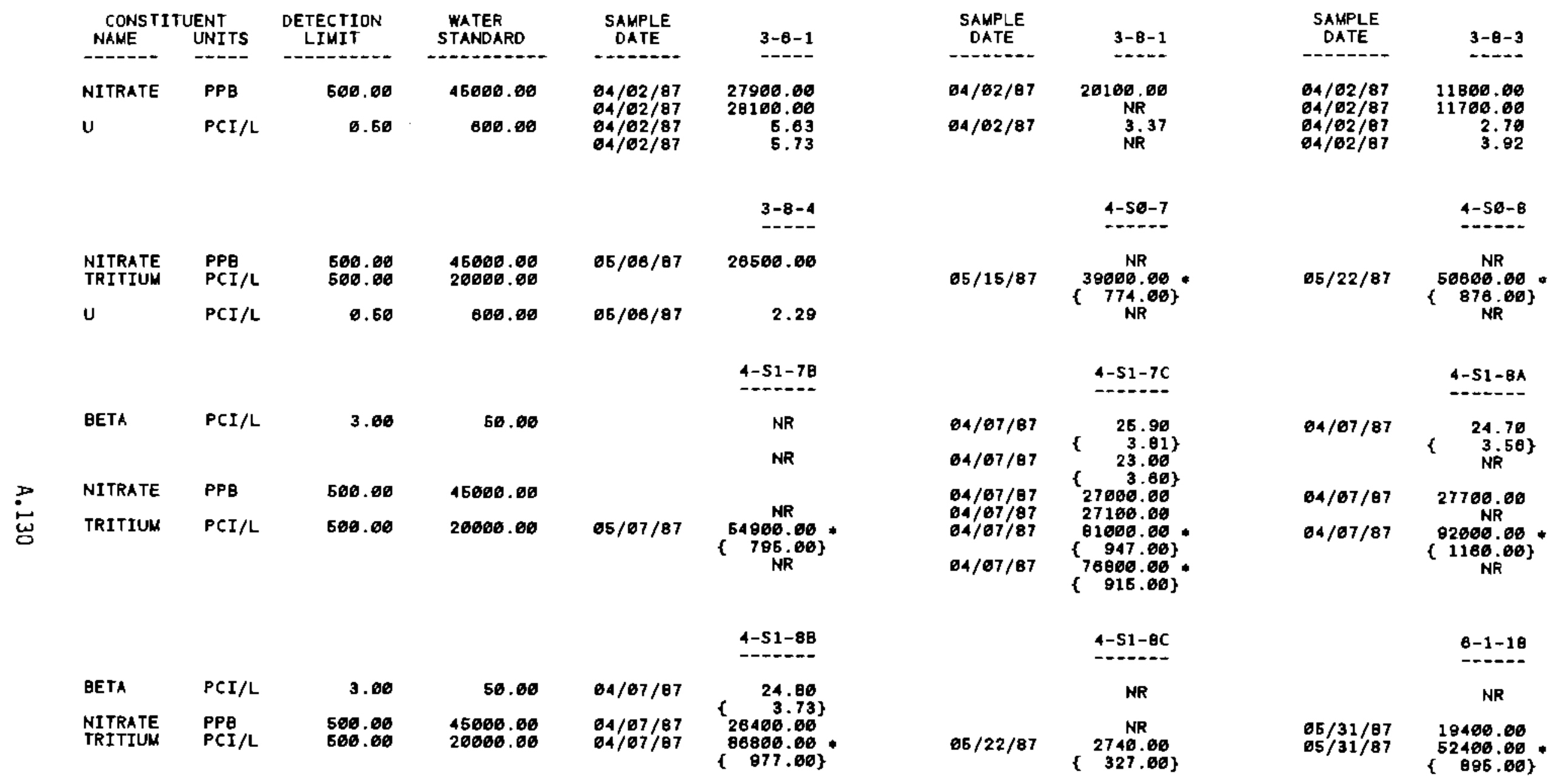

- - VAlue eXceeds primary orinking mater standaro.

- VALUE EXCEEDS PROPOSED PRIMARY ORINKING WATER STANDARO.

- VALUE EXCEEOS SCREENING LEVEL FOR FURTHER INVESTIGATION.

+ DETECTION LIMIT WAS NOT AVAILABLE FOR CDMPARISON

NR - DETECTION LIMIT WAS NOT AVAILABLE FOR CDMPAR

VALUES IN I I RRE COUNTING ERRORS FOR RADIONUCLIDES

WATER STANDARO'(S) IN PARENTHESES ARE PROPOSED ONLY 
TABLE A.6. (contd)

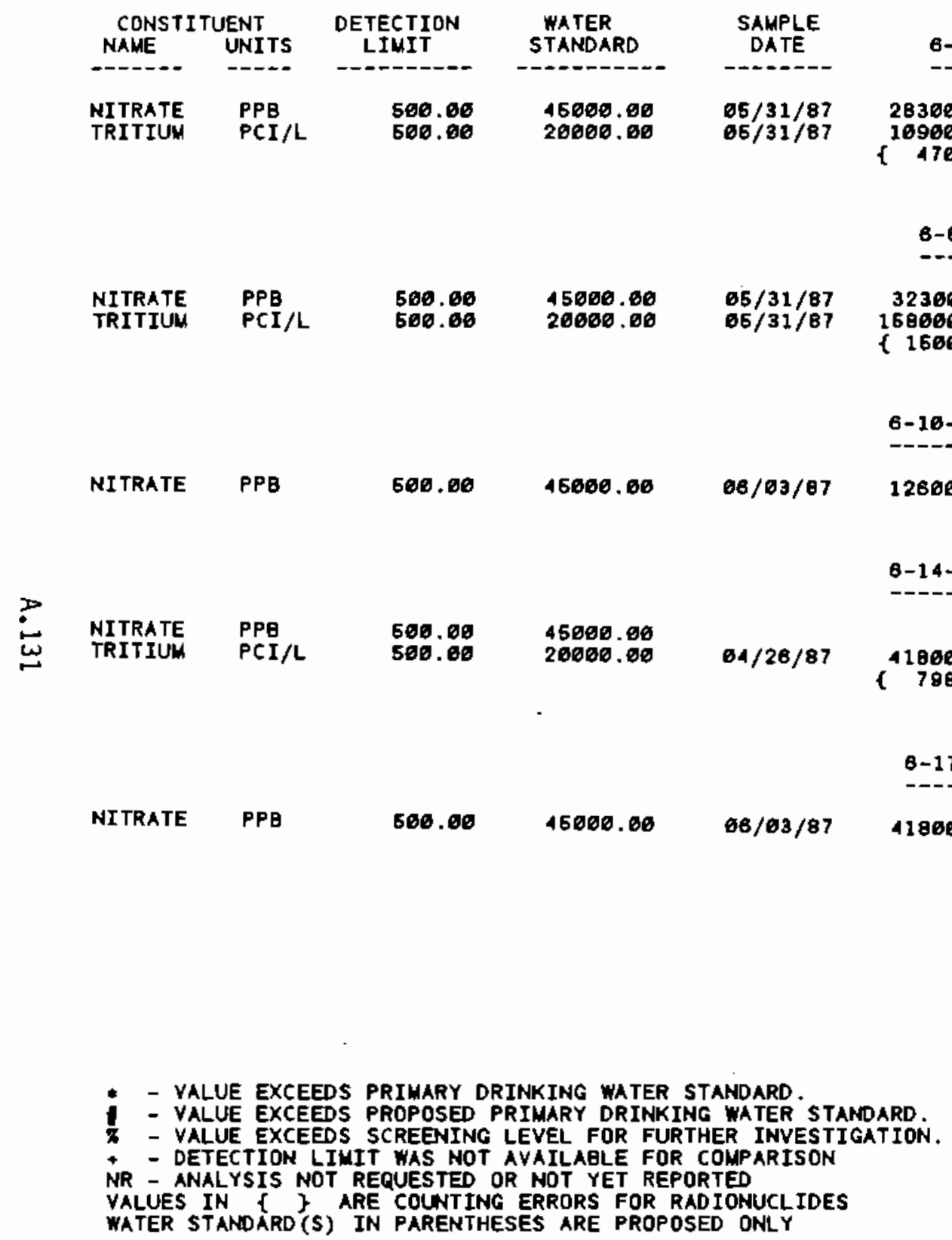

$\begin{array}{ll}\text { SAMPLE } & \\ \text { DATE } & 6-3-45 \\ - & ---1\end{array}$

$08 / 02 / 87 \quad 918.08$

6-8-32

5830.60

$06 / 04 / 07$

32360.60

(1586.06)

$6-16-54 A$

2600.00

$6-12-4 \theta$

NR

8-14-30

4760.00

$06 / 04 / 87$

7000.00
$790.00\}$

$6-17-70$

$6-18-43$

11300.60

\section{SAMPLE DATE} $6-4-E 6$

$66 / 03 / 87$

13100.00

8-10-E12

$66 / 03 / 87$ $06 / 03 / 87$

20300.00 14500.00 \{ 488.00$\}$

3-13-64 087.06

$66 / 03 / 97$

$6-14-47$ - $-14----$

$06 / 02 / 67 \quad 968.60$ $8-19-68$

NO REPORTABLE DATA

WATER STANDARD'(S) IN PARENTHESES ARE PROPOSED ONLY 
TABLE A.6. (contd)

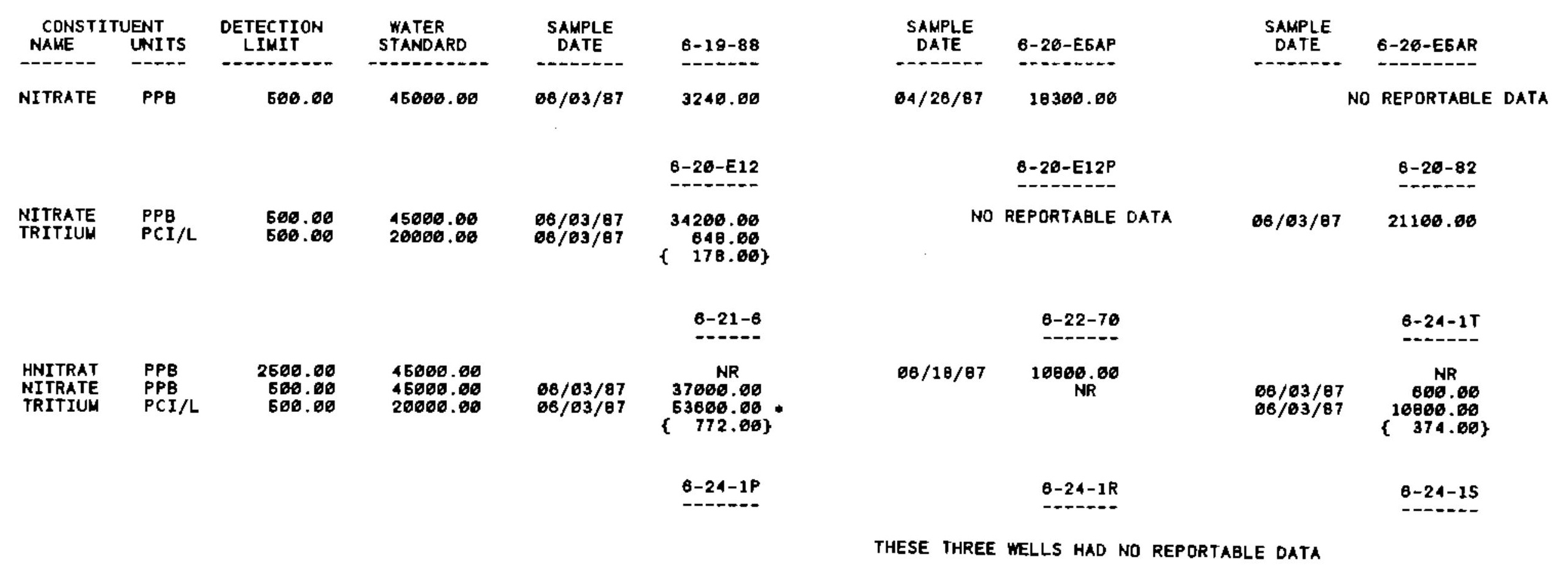

* - VALUE EXCEEDS PRIMARY DRINKING WATER STANDARD.

- VALUE EXCEEDS PROPOSED PRIMARY DRINKING WATER STANDARD.

- VALUE EXCEEDS SCREENING LEVEL FOR FURTHER INVESTIGATION.

+ - DETECTION LIMIT WAS NOT AVAILABLE FOR COMPARISON

NR - ANALYSIS NOT REQUESTED OR NDT YET REPORTED

VALUES IN \& \} ARE COUNTING ERRORS FOR RADIONUCLIDES

WATER STANDARD(S) IN PARENTHESES ARE PROPOSED ONLY 
IABLE A.6. (contd)

\begin{tabular}{|c|c|c|c|c|c|c|c|c|c|}
\hline $\begin{array}{l}\text { CONSTI } \\
\text { NAME }\end{array}$ & $\begin{array}{l}\text { JENT } \\
\text { UNITS }\end{array}$ & $\begin{array}{l}\text { DETECTION } \\
\text { LIUIT }\end{array}$ & $\begin{array}{l}\text { WATER } \\
\text { STANDARD }\end{array}$ & $\begin{array}{l}\text { SAHPLE } \\
\text { DATE }\end{array}$ & $6-24-46$ & $\begin{array}{l}\text { SAHPLE } \\
\text { DATE }\end{array}$ & $6-25-56$ & $\begin{array}{l}\text { SAMPLE } \\
\text { DATE }\end{array}$ & $6-25-7 \theta$ \\
\hline 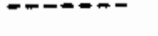 & $---\infty$ & -+ & $-\infty---\infty-\infty-0$ & -------- & $------\infty$ & $--\infty-\infty$ & $-\infty-\infty--$ & $----1-\infty$ & -ーーー-ー- \\
\hline $\begin{array}{l}\text { NITRATE } \\
\text { TRITIUM }\end{array}$ & $\begin{array}{l}\mathrm{PPE} \\
\mathrm{PCI} / \mathrm{L}\end{array}$ & $\begin{array}{l}500.00 \\
500.00\end{array}$ & $\begin{array}{l}45000.00 \\
20000.00\end{array}$ & $06 / 04 / 87$ & 6770.00 & $06 / 04 / 07$ & 14500.60 & $\begin{array}{l}06 / 02 / \theta 7 \\
06 / 02 / \theta 7\end{array}$ & $\begin{array}{r}12606.06 \\
889.06 \\
\{\quad 188.06\}\end{array}$ \\
\hline & & & & & $6-26-15 A$ & & $6-28-89$ & & $6-27-8$ \\
\hline $\cos 6$ & $\mathrm{PCI} / \mathrm{L}$ & 22.50 & 100.00 & $05 / 01 / 87$ & 23.20 & & & & \\
\hline $\begin{array}{l}\text { NITRATE } \\
\text { TRITIUM }\end{array}$ & $\begin{array}{l}\text { PPg } \\
\text { PCI/L }\end{array}$ & $\begin{array}{l}500.00 \\
500.00\end{array}$ & $\begin{array}{l}45000.00 \\
20000.00\end{array}$ & $\begin{array}{l}65 / 61 / 87 \\
65 / 61 / 87\end{array}$ & $\begin{array}{l}42506.00 \\
387600.06 \\
\{2260.00\}\end{array}$ & $06 / 63 / 87$ & $\underset{\mathrm{NR}}{4110.00}$ & $\begin{array}{l}06 / 03 / 87 \\
66 / 03 / 87\end{array}$ & $\begin{array}{c}37600.00 \\
329000.00 \\
\{1080.06\}\end{array}$ \\
\hline & & & & & $6-2 \theta-4 \theta P$ & & $8-2 \theta-52 A$ & & $8-28-7 \theta$ \\
\hline
\end{tabular}

THESE THREE WELLS HAD NO REPORTABLE DATA

- - value exceeds primary orinking water standaro.

- VALUE EXCEeDS PROPOSED PRIMARY DRINKING WATER STANDARD.

* - VALUE EXCEEDS SCREENING LEVEL FOR FURTHER INVESTIGATION.

+ DETECTION LIMIT WAS NOT AVAILABLE FOR COMPARISON

ANALYSIS NOT REQUESTED OR NOT YET REPORTED

VALUES IN ( 3 ARE COUNTING ERRORS FOR RADIONUCL IDES

WATER STANOARD'(S) IN PARENTHESES ARE PROPOSED ONLY 
TABLE A.6. (contd)

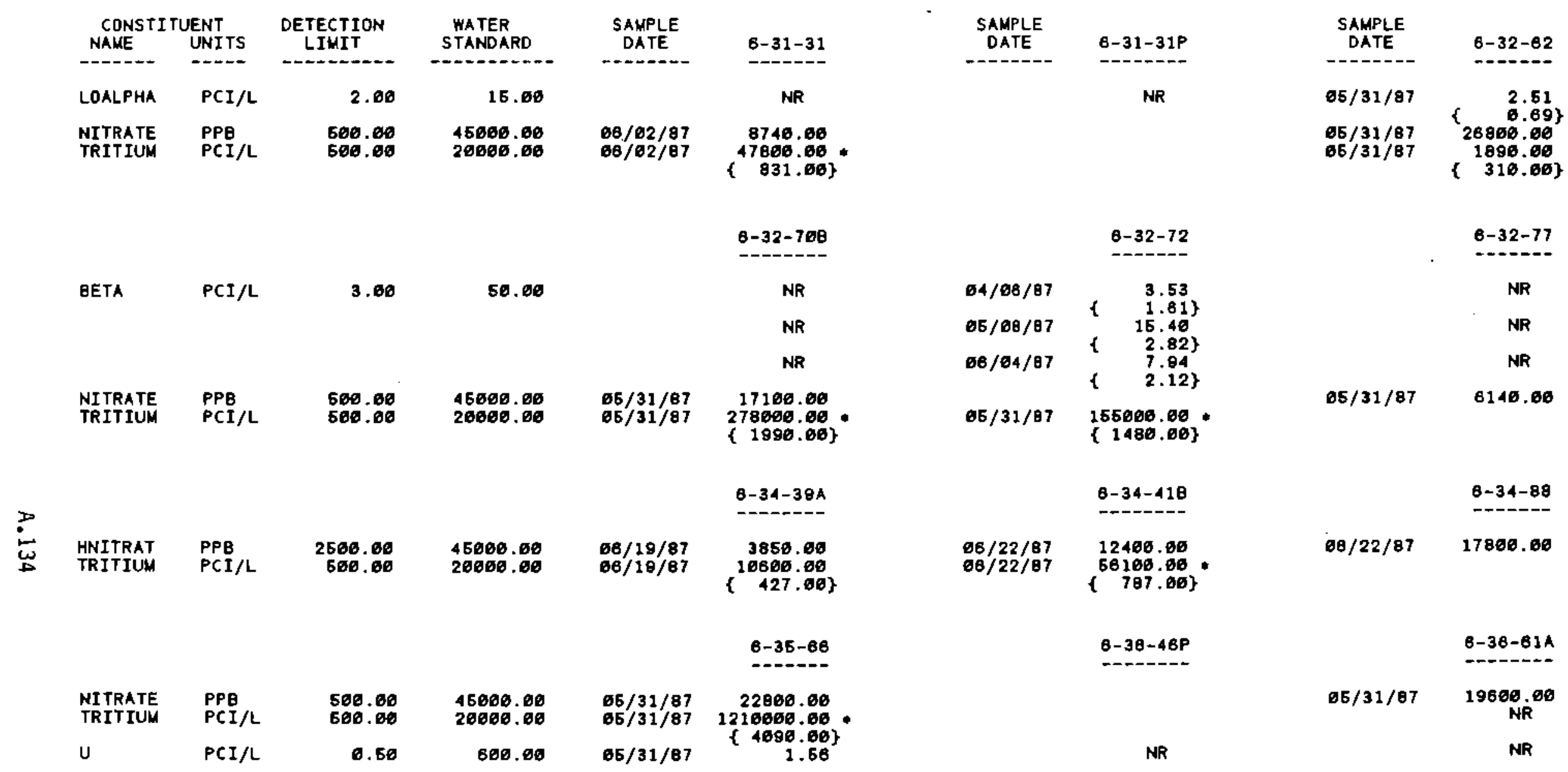

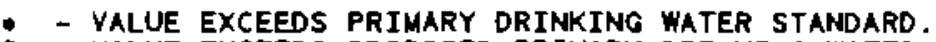

- VALUE EXCEEDS PROPOSED PRIMARY DRINKING WATER STANDARD.

- YALUE EXCEEDS SCREENING LEVEL FOR FURTHER INVESTIGATION.

* - DETECTION LIMIT WAS NOT AYAILABLE FOR COMPARISON

NR - ANALYSIS NOT REQUESTED OR NOT YET REPORTED

VALUES IN \{\} ARE COUNTING ERRORS FOR RADIONUCLIDES

WATER STANDARD(S) IN PARENTHESES ARE PROPOSED ONLY 
IABLE A.6. (contd)

$\begin{array}{ccc}\text { CONSTITUENT } & \text { DETECTION } \\ \text { NAME } & \text { UNITS } & \text { LIMIT } \\ - & - & \end{array}$

NITRATE PPQ 500.00 TRITIUM PCI/L 568.00
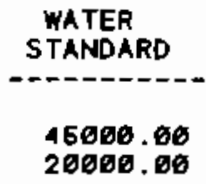

\begin{tabular}{lr}
$\begin{array}{c}\text { SAMPLE } \\
\text { DATE }\end{array}$ & $6-30-818$ \\
\hline $05 / 31 / 87$ & 677.00
\end{tabular}

$6-3 \theta-16$

$\begin{array}{llr}\text { CO } B E & \text { PCI/L } & 22.50 \\ \text { NITRATE } & \text { PPB } & 500.00 \\ \text { TRITIUM } & \text { PCI/L } & 586.00\end{array}$

100.00
45000.00
20000.00

$04 / 28 / 87$ $04 / 28 / 87$
$04 / 28 / 87$

$$
6-38-16
$$

53600.00.

\section{SAMPLE} DATE

$06 / 04 / 87$
$06 / 04 / 87$

6-37-E4

23506.06

$\left\{\begin{array}{c}43460.06 \\ \{796.00\}\end{array}\right.$

$6-38-7 \theta$

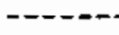
457000.00
$\{2510.00\}$

6-39-39

2790.06

NR

NR

45000.00

$68 / 22 / 87$ 500.06

20000.00

NITRATE PPI

$\overbrace{\substack{\omega \\ \omega}}$

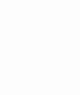

\begin{tabular}{|c|c|c|c|}
\hline $\begin{array}{l}\text { HNITRAT } \\
\text { NITRATE } \\
\text { TRITIUM }\end{array}$ & $\begin{array}{l}P P B \\
P P B \\
P C I / L\end{array}$ & $\begin{array}{r}2506.06 \\
506.80 \\
506.00\end{array}$ & $\begin{array}{l}45060.06 \\
45006.66 \\
20000.00\end{array}$ \\
\hline & PCI/L & 0.50 & 600.60 \\
\hline
\end{tabular}

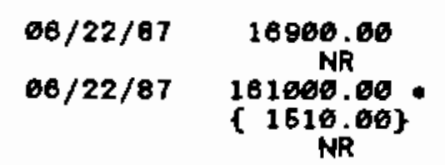

$60 / 22 / 87$

\section{NR $\left\{\begin{array}{r}1200.06 \\ 196.06\end{array}\right.$}

B-46-82

\section{NR}

$06 / 31 / 87$
$65 / 31 / 87$

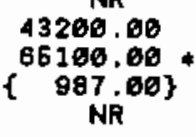

$$
\text { 6-42-2 }
$$

\section{NR}

$06 / 19 / 97$
$65 / 19 / 87$

NR
$\begin{gathered}32400.00 \\ 214000.00 \\ (1760.00\} \\ \text { NR }\end{gathered}$

SAMPLE DATE

$65 / 31 / 87$ $65 / 31 / 87$ 11700.00 \{1000.06\}."

\section{6-39-6}

$06 / 04 / 87$ $06 / 64 / 87$

37900.00 228000.00.

$$
\text { 6-4l-1 }
$$

$05 / 19 / 87$ $05 / 19 / 97$

$05 / 19 / 87$

\section{NR}

34100.00 242600.60. $\{1040.66\}$ \{31000.00*

$6-42-12 A$ (4)

NP

$06 / 64 / 67$ $68 / 64 / 97$

41000.06 322000.00 .

$06 / 64 / 87$ \{ 1860.06\}

- - VALUE EXCEEDS PRIMARY DRINKING WATER STANDARD.

- VALUE EXCEEDS PROPOSED PRIMARY ORINKING WATER STANDARD.

- VALUE EXCEEDS SCREENING LEVEL FOR FURTHER INVESTIGATION.

* - DETECTION LIMIT WAS NOT AVAILABLE FOR COMPARISON

NR - ANALYSIS NOT REQUESTED OR NOT YET REPORTED

WALUES IN $\{$ F ARE COUNTING ERRORS FOR RADIONUCLIOES 
IABLE A.6. (contd)

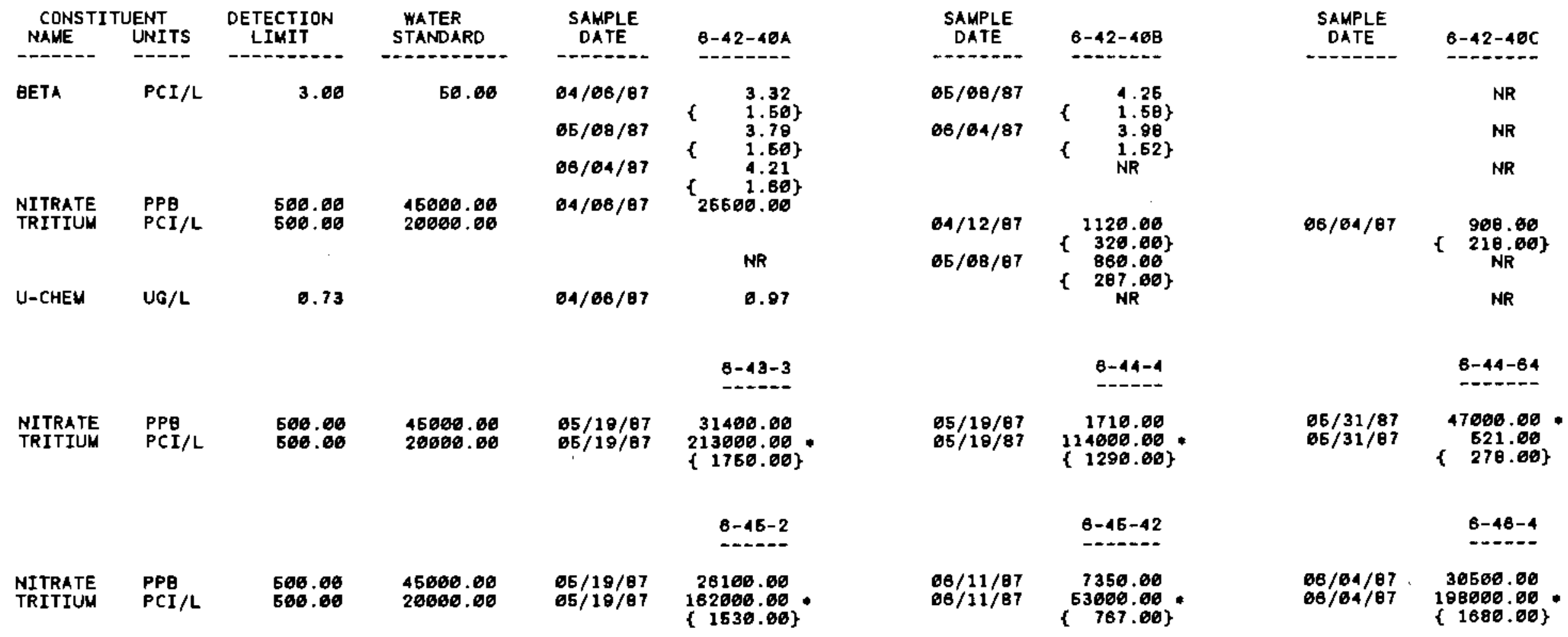

- - VALUE EXCEeds PRIMARY DRINKING waTER STANDARO.

- VALUE EXCEEDS PROPOSED PRIMARY DRINIING WATER STANDARD.

* - VALUE EXCEEDS SCREENING LEVEL FOR FURTHER INVESTIGATION.

* - DETECTION LIMIT WAS NOT AVAILABLE FOR COMPARISON

NR - ANALYSIS NOT REQUESTED OR NOT YET REPORTED

VALUES IN $\{$ \& ARE COUNTING ERRORS FOR RADIONUCLIDES

WATER STANDARD(S) IN PARENTHESES ARE PROPOSED ONLY 
IABLE A.6. (contd)

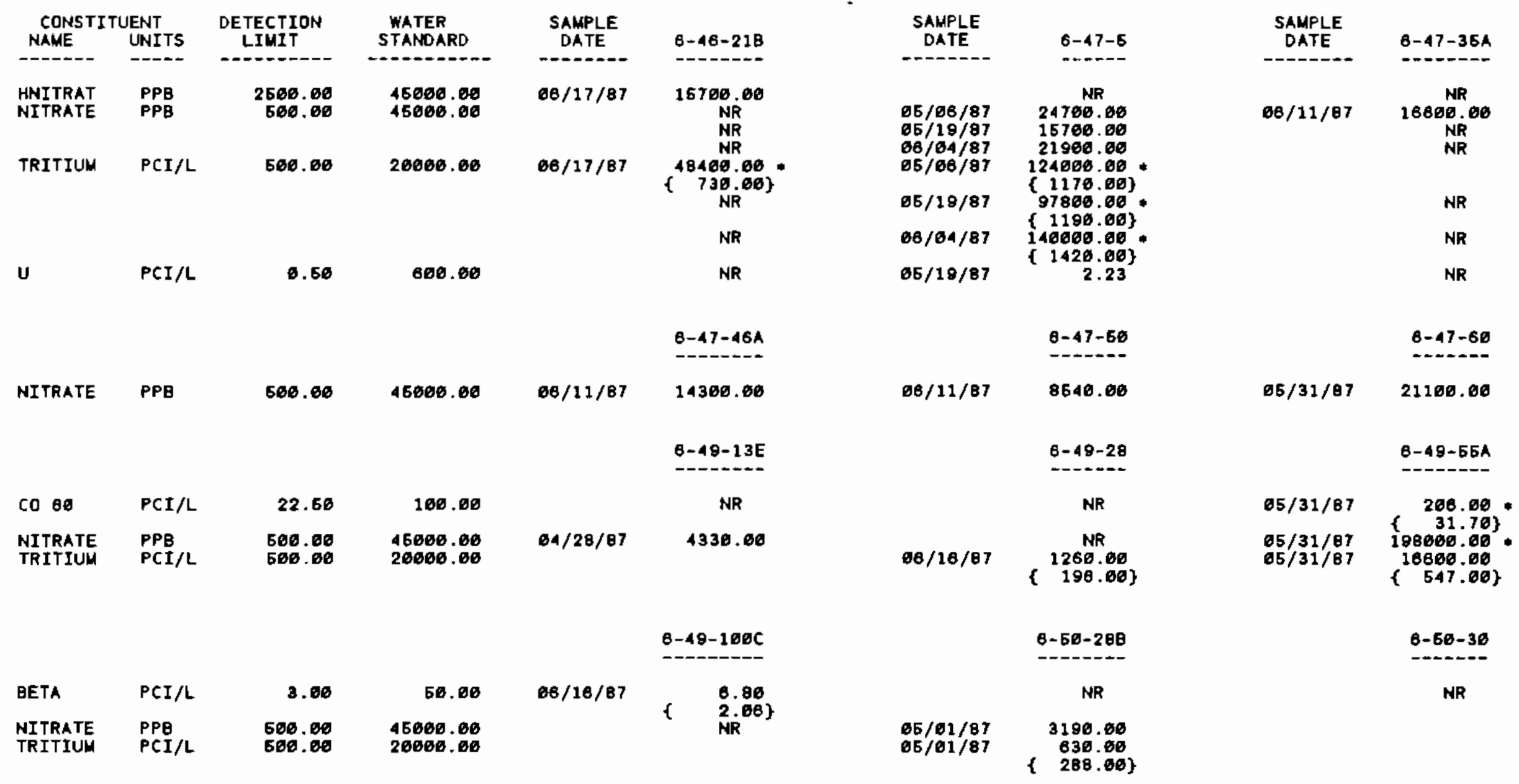

- - Value exceeds primary dRINKING water standard.

- VALUE EXCEEDS PROPOSED PRIMARY DRINKING WATER STANDARO.

* - VALUE EXCEEDS SCREENING LEVEL FOR FURTHER INVESTIGATION.

* - DETECTION LIMIT WAS NOT AVAILABLE FOR COMPARISON

ALYSIS NOT REQUESTED OR NOT YET REPORTED

VALUES IN \& ) ARE COUNTING ERRORS FOR RADIONUCLIDES

WATER STANDARD (S) IN PARENTHESES ARE PROPOSED ONLY 
IABLE A.6. (contd)

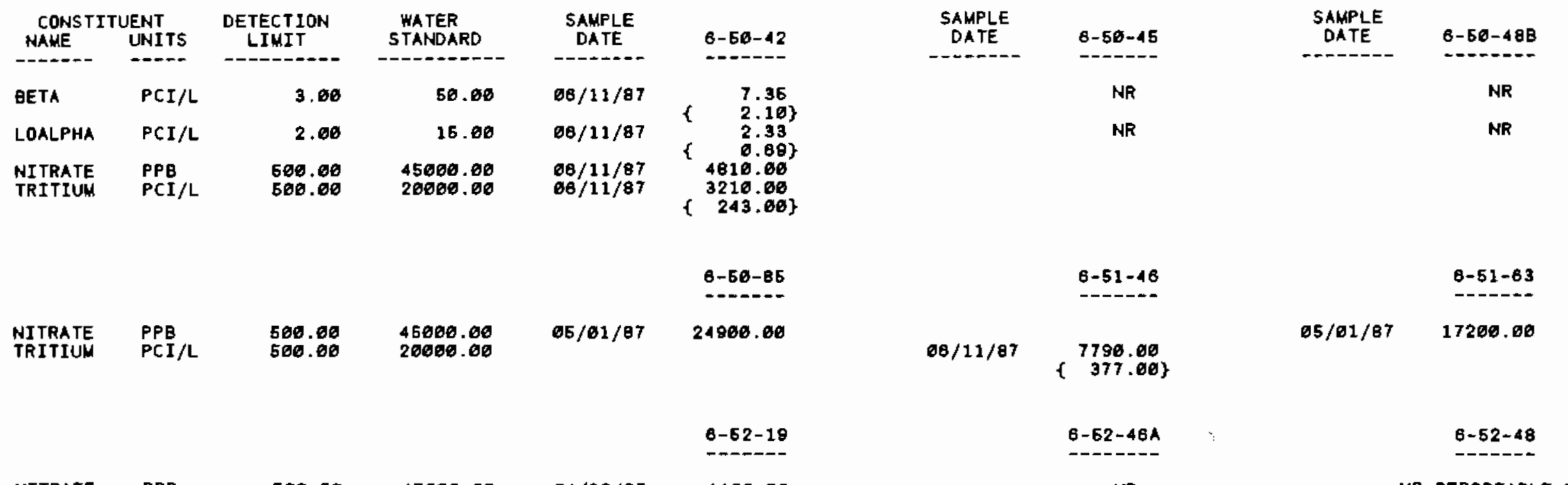

$\begin{array}{lllllll} & \text { NITRATE } & \text { PPB } & 500.60 & 46600.00 & 04 / 28 / 87 & 4400.60 \\ 2 & & & & & \\ 0 & & & & & \end{array}$

- VALUE EXCEEDS PRIMARY DRIMKING WATER STANDARD.
- VALUE EXCEEDS PROPOSED PRIMARY DRINKING WATER STAMDARD.

* VALUE EXCEEDS SCREENING LEVEL FOR FURTHER INVESTIGATION.

+ - DETECTION LIMIT WAS NOT AVAILABLE FOR COMPARISON

NR - ANALYSIS NOT REQUESTED OR NOT YET REPORTED

VALUES IN \{\} ARE COUNTING ERRORS FOR RADIONUCLIDES
WATER STANDARD (S) IN PARENTHESES ARE PROPOSED ONLY 
IABLE A.6. (contd)

\begin{tabular}{|c|c|c|c|c|c|c|c|c|c|}
\hline $\begin{array}{l}\text { CONSTI } \\
\text { NAME }\end{array}$ & $\begin{array}{l}\text { UENT } \\
\text { UNITS }\end{array}$ & $\begin{array}{l}\text { DETECTION } \\
\text { LIMIT }\end{array}$ & $\begin{array}{l}\text { WATER } \\
\text { STANDARD }\end{array}$ & $\begin{array}{c}\text { SAMPLE } \\
\text { DATE }\end{array}$ & $6-53-35$ & $\begin{array}{l}\text { SAMPLE } \\
\text { DATE }\end{array}$ & $6-63-47 A$ & $\begin{array}{c}\text { SAMPLEE } \\
\text { DATE }\end{array}$ & $\theta-53-47 B$ \\
\hline \multirow[t]{3}{*}{ BETA } & $\mathrm{PCI} / \mathrm{L}$ & 3.00 & 50.06 & & NR & $04 / 06 / 87$ & $127.60 \times$ & $04 / 06 / 87$ & $143.06 \times$ \\
\hline & & & & & NR & $06 / 07 / 87$ & $120.00 \times$ & $05 / 07 / 87$ & $183.06 x$ \\
\hline & & & & & NR & $06 / 04 / 87$ & $122.00 \times$ & $66 / 04 / 87$ & $162.06 \times$ \\
\hline \multirow[t]{3}{*}{ LOALPHA } & $\mathrm{PCI} / \mathrm{L}$ & 2.06 & 15.00 & & NR & $04 / 06 / 87$ & $\begin{array}{r}3.8 B \\
\quad 8.93\end{array}$ & $04 / 06 / 87$ & 3.43 \\
\hline & & & & & NR & $05 / 07 / 87$ & $\begin{array}{r}3.16 \\
+\quad 77\}\end{array}$ & $65 / 67 / 87$ & 3.81 \\
\hline & & & & & NR & $06 / 04 / 87$ & $\begin{array}{l}3.86 \\
0.78\end{array}$ & $08 / 04 / 87$ & $\begin{array}{r}4.06 \\
+\quad 0.07\}\end{array}$ \\
\hline \multirow[t]{4}{*}{$\begin{array}{l}\text { NITRATE } \\
\text { SR }\end{array}$} & $\begin{array}{l}\mathrm{PPB} \\
\mathrm{PCI} / \mathrm{L}\end{array}$ & $\begin{array}{r}608.06 \\
5.60\end{array}$ & $\begin{array}{r}45000.00 \\
B .96\end{array}$ & $68 / 11 / 87$ & $\underset{N R}{3000,08}$ & $04 / 08 / 87$ & $\begin{array}{c}\mathrm{NR} \\
79.90\end{array}$ & $04 / 60 / 87$ & $\begin{array}{ll}\mathrm{NR} \\
80.60\end{array}$ \\
\hline & & & & & NR & $05 / 67 / 87$ & $\begin{array}{l}5.25\} \\
83.40 .\end{array}$ & $06 / 67 / 87$ & 77.86 \\
\hline & & & & & NR & $08 / 04 / 87$ & $\begin{array}{cc}\{ & 4.64\} \\
& 33.58 \\
\{ & 4.09\}\end{array}$ & $06 / 04 / 87$ & 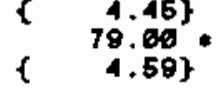 \\
\hline & & & & & 6-53-48A & & $6-53-488$ & & $8-53-60$ \\
\hline \multirow[t]{3}{*}{ BETA } & PCI /L & 3.06 & 60.00 & $64 / 06 / 87$ & $\begin{array}{l}8.54 \\
1 \quad 3.68\}\end{array}$ & $64 / 06 / 87$ & $495.06 \times$ & & NR \\
\hline & & & & $06 / 07 / 87$ & $\begin{array}{r}7.82 \\
3.58\}\end{array}$ & $66 / 97 / 87$ & $531.08 \times$ & & NR \\
\hline & & & & $66 / 04 / 87$ & 16.28 & $68 / 04 / 87$ & $517.00 \times$ & & NR \\
\hline \multirow[t]{2}{*}{ LOALPHA } & PCI $/ L$ & 2.00 & 15.00 & $65 / 67 / 87$ & $\begin{array}{l}2.52 \\
0.67\end{array}$ & & & & NR \\
\hline & & & & $06 / 04 / 87$ & $\begin{array}{l}0.41 \\
1.28\}\end{array}$ & & NR & & NR \\
\hline \multirow[t]{3}{*}{ SR 90} & $\mathrm{PCI} / \mathrm{L}$ & 6.00 & 8.00 & & & $04 / 06 / \theta 7$ & 326.00 & & NR \\
\hline & & & & & NR & $65 / 07 / 87$ & 265.06 & & NR \\
\hline & & & & & NR & $08 / 04 / 07$ & $\left\{\begin{array}{c}308.00 \\
\{1.06\}\end{array}\right.$ & & NR \\
\hline
\end{tabular}

* - value exceeds primary drinking mater standaro.

- VALUE EXCEEDS PROPOSED PRIMARY DRINKING WATER STANDARD.

- YALUE EXCEEDS SCREENING LEVEL FDR FURTHER INVESTIGATION.

4 - DETECTION LIMIT WAS NOT AVAILABLE FOR COMPARISON

NR - ANALYSIS NOT REQUESTED OR NOT YET REPORTED

VALUES IN \{\} ARE COUNTING ERRORS FOR RADIONUCLIDES

WATER STANDARO(S) IN PARENTHESES ARE PROPOSED ONLY 
TABLE A.6. (contd)

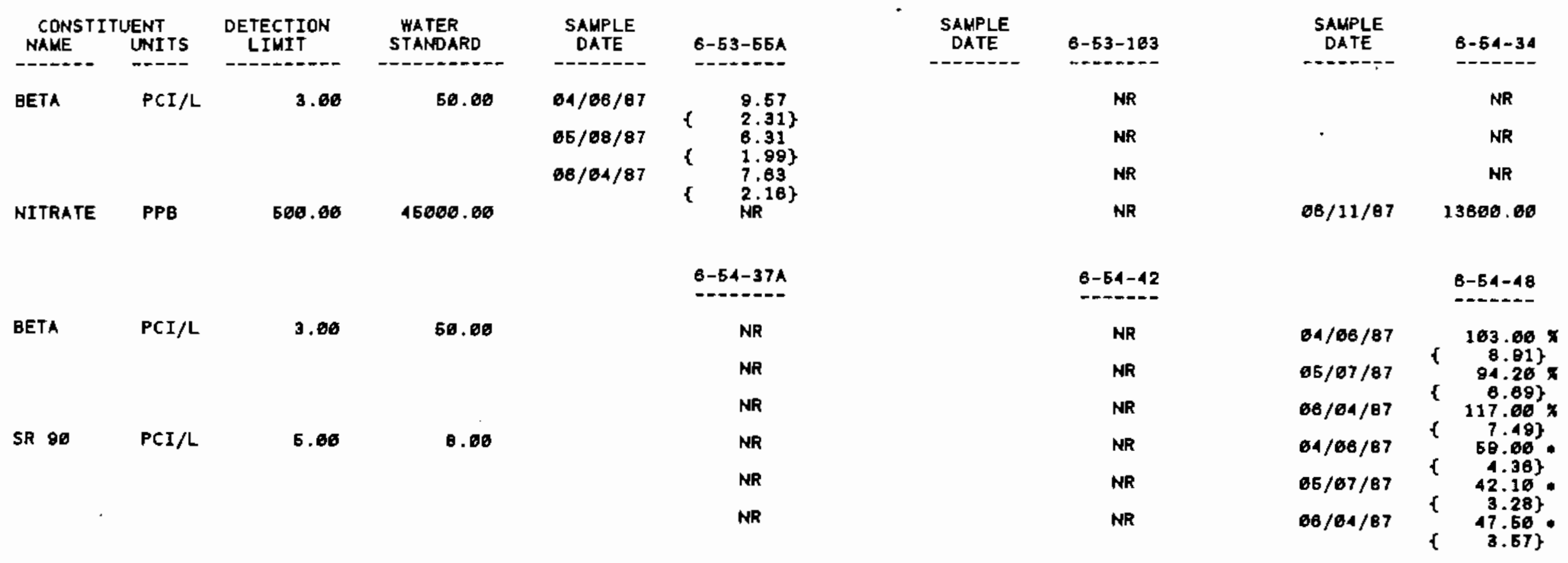

* - VALUE EXCEEDS PRIMARY DRINKING WATER STANDARD.

- VALUE EXCEEDS PROPOSED PRIMARY DRINKING waTeR STANDARD.

* - VALUE EXCEEDS SCREENING LEVEL FOR FURTHER INVESTIGATION.

+ - DETECTION LIMIT WAS NOT AVAILABLE FOR COMPARISON

NR - ANALYSIS NOT REQUESTED OR NOT YET REPORTED

VALUES IN \& $)$ ARE COUNTING ERRORS FOR RADIONUCLIDES

WATER STANDARD'(S) IN PARENTHESES ARE PROPOSED ONLY 
TABLE A.6. (contd)

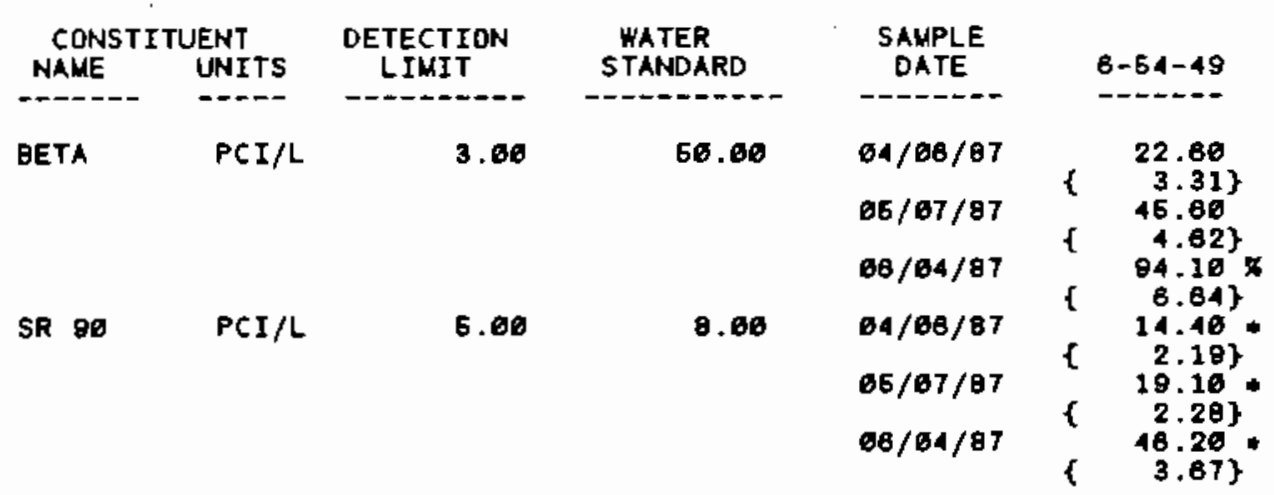

$\begin{array}{llrr}\text { EETA } & \text { PCI/L } & 3.00 & 50.00 \\ \text { NITRATE } & \text { PPB } & 500.00 & 46000.00\end{array}$

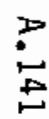

BETA

PCI/L

PPB

3.00

50.00

600.60

45060.60

NITRATE PPB

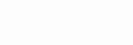

HNITRAT PPE 2600.60

45060.60

\section{8-66-44}

NR

-

SAMPLE
DATE
NR
NR
NR
NR
NR
NR

8-65-60A

6-66-70

$08 / 11 / 87 \quad\left\{\quad \begin{array}{c}4.80 \\ 1.80\}\end{array}\right.$

$06 / 11 / 97 \quad 7636.60$

6-65-89

4826.66
NR

NR

NR

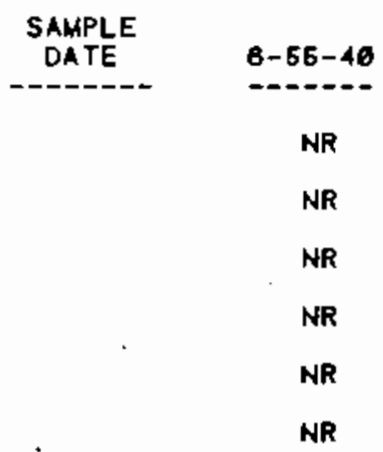

8-55-50C -.-.-.-

$68 / 11 / 87$

$66 / 11 / 67$

$\left\{\begin{array}{r}4.72 \\ 1.77\} \\ 3200.00\end{array}\right.$

$6-65-76$

NR

$65 / 61 / 87$

7260.06

$6-56-43$

NR

\section{6-56-63}

NO REPORTABLE DATA

- VALUE EXCEEDS PRIMARY DRINKING WATER STANDARD.

- VALUE EXCEEDS PROPOSED PRIMARY DRINKING WATER STANDARO.

* - VALUE EXCEEDS SCREENING LEVEL FOR FURTHER INVESTIGATION.

+ - DETECTION LIMIT WAS NOT AVAILABLE FOR COMPARISON

NR - ANALYSIS NOT REQUESTED OR NOT YET REPORTED

VALUES IN \{\} ARE COUNTING ERRORS FOR RADIONUCLIDES

WATER STANDARD(S) IN PARENTHESES ARE PROPOSED ONLY 
TABLE A.6. (contd)

\begin{tabular}{|c|c|c|c|c|c|}
\hline \multicolumn{2}{|c|}{ CONSTITUENT } & $\begin{array}{c}\text { DETECTION } \\
\text { LINIT }\end{array}$ & $\begin{array}{l}\text { WATER } \\
\text { STANDARD }\end{array}$ & $\begin{array}{c}\text { SAMPLE } \\
\text { DATE }\end{array}$ & $6-67-25 A$ \\
\hline$--\infty-0$ & 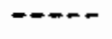 & $--1-0-1$ & $-+x--0--1$ & 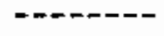 & - \\
\hline $\begin{array}{l}\text { HNITRAT } \\
\text { NITRATE }\end{array}$ & $\begin{array}{l}\text { PPB } \\
\text { PPB } \\
\text { PCI }\end{array}$ & $\begin{array}{r}2500.06 \\
560.00\end{array}$ & $\begin{array}{l}45000.00 \\
45600.06 \\
26066.00\end{array}$ & $64 / 36 / 87$ & $\begin{array}{c}N R \\
3200.00\end{array}$ \\
\hline
\end{tabular}$$
3200.00
$$

$$
64 / 30 / 87
$$

$8-68-24$

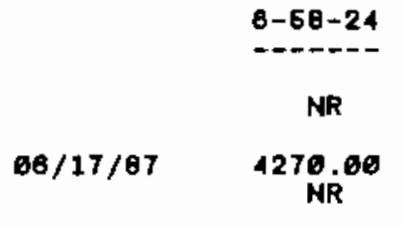

\section{BETA}

PCI/L

3.00

HNITRAT NITRATE $\begin{array}{lr}\text { PPB } & 2600.00 \\ \text { PPB } & 500.00\end{array}$ PCI/L

50.00

45600.00 46000.00 20000.00

$66 / 17 / 07$
6-59-80B

NR
NR

$\begin{array}{llll}\text { NITRATE } & \text { PPB } & 600.00 & 45000.00 \\ \text { TRITIUM } & \text { PCI/L } & 600.60 & 20000.00\end{array}$

(n)

NI TRAT

TRITIUH

PPB
PCI L

586.00
560.60

20000.00

$\begin{array}{cc}\text { SAMPLE } & \\ \text { DATE } & 6-67-29 A \\ -04 / 36 / 87 & 2460.06 \\ 04 / 30 / 87 & 639.60 \\ & \left\{\begin{array}{c}208.66\} \\ \end{array}\right.\end{array}$

$6-69-32$

NR

NR

$04 / 30 / 87$

$04 / 36 / 87$

083.09

(292.00\}

$6-60-32$

5280.06

761.00

$64 / 30 / 87$

$\{\quad 289.06\}$

6-81-37

6-60-60

- $-1-2-1$

$04 / 26 / 87$

8150.00
$430.06)$

SAMPLE DATE

$66 / 16 / 87$

6-67-83A

3090 .

NR
NR

6-69-68

$0-1-\cdots$

$04 / 26 / 87$

( $\quad{ }_{N R}^{4.64}$

$04 / 26 / 87$

769.00

( 288.06)

$6-80-57$

$64 / 26 / 87$

615.00

$6-61-41$

$04 / 36 / 07$

- VALUE EXCEEDS PRIMARY DRINKING WATER STANDARO

- VALUE EXCEEDS PROPOSED PRIMARY DRINKING WATER STANDARD.

- VALUE EXCEEDS SCREENING LEVEL FOR FURTHER INVESTIGATIDN.

* - DETECTION LIHIT WAS NOT AVAILABLE FOR COMPARISON

NR - ANALYSIS NOT REQUESTED OR NOT YET REPORTED

VALUES IN I ? ARE COUNTING ERRORS FOR RADIONUCLIDES

WATER STANDARD'(S) IN PARENTHESES ARE PROPOSED ONLY 
IABLE A.6. (contd)

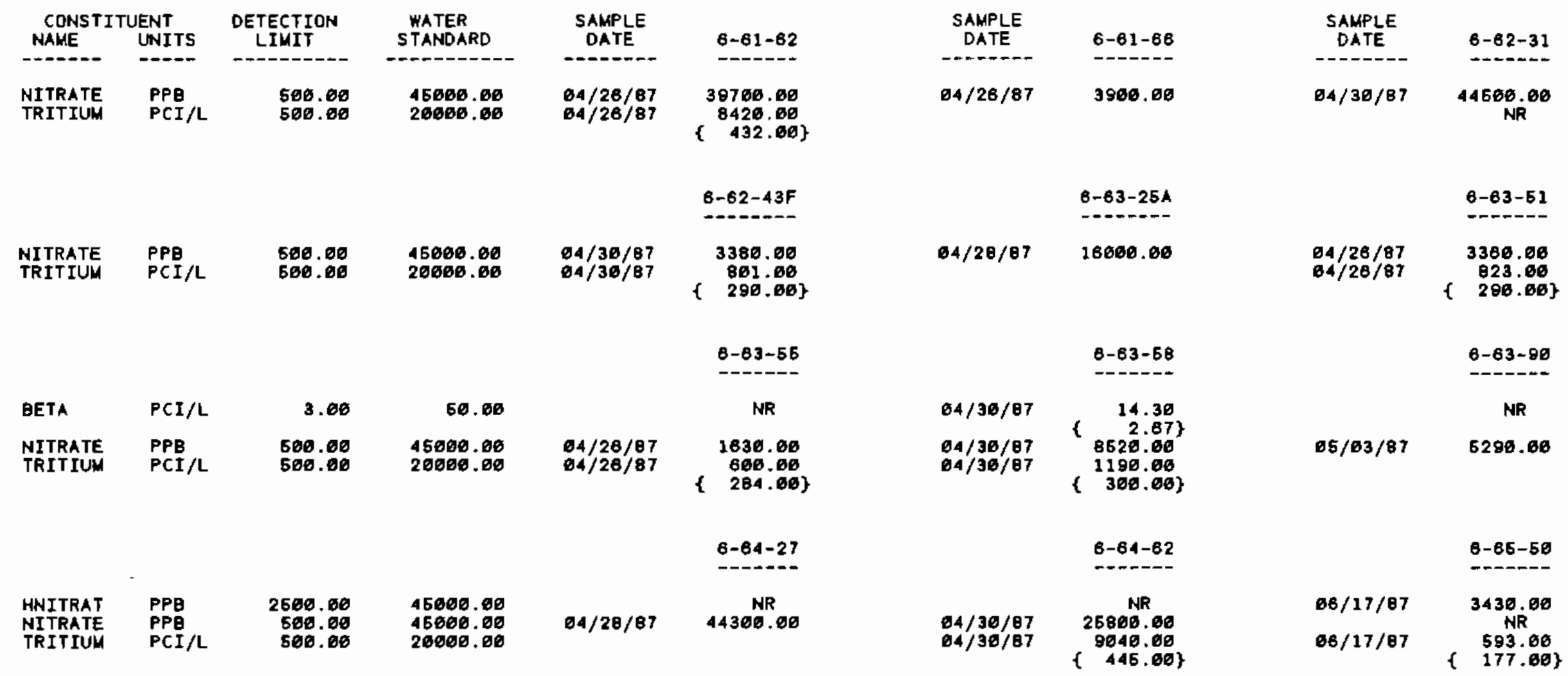

* - VAlue exceeds primary orinking water standard.

- VALUE EXCEEDS PROPOSED PRIMARY DRINKING WATER STANDARD.

* - VALUE EXCEEDS SCREENING LEVEL FOR FURTHER INVESTIGATION.

- DETECTION LIMIT WAS NOT AVAILABLE FOR COMPARISON

NR - ANALYSIS NOT REQUESTED OR NOT YET REPORTED

VALUES IN \& $\}$ ARE COUNTING ERRORS FOR RADIONUCLIDES

WATER STANDARD (S) IN PARENTHESES ARE PROPOSED ONLY 
IABLE A.6. (contd)

\begin{tabular}{|c|c|c|c|c|c|}
\hline $\begin{array}{l}\text { CONSTI } \\
\text { NAME }\end{array}$ & $\begin{array}{l}\text { UENT } \\
\text { UNITS }\end{array}$ & $\begin{array}{l}\text { DETECTION } \\
\text { LIMIT }\end{array}$ & $\begin{array}{l}\text { WATER } \\
\text { STANOARO }\end{array}$ & $\begin{array}{c}\text { SAMPLE } \\
\text { DATE }\end{array}$ & $6-65-58$ \\
\hline $\begin{array}{l}\text { HNITRAT } \\
\text { NITRATE } \\
\text { TRITIUH }\end{array}$ & $\begin{array}{l}\text { PPB } \\
\text { PPB } \\
\text { PCI/L }\end{array}$ & $\begin{array}{r}2500.00 \\
500.00 \\
500.00\end{array}$ & $\begin{array}{l}15000.00 \\
45000.00 \\
20000.00\end{array}$ & $\begin{array}{l}06 / 17 / 87 \\
06 / 17 / 87\end{array}$ & $\begin{array}{c}3500.00 \\
N R \\
619.00 \\
177.00\}\end{array}$ \\
\hline
\end{tabular}

$6-66-23$

$\begin{array}{llllll}\text { NITRATE PPB } & 600.60 & 46000.00 & 64 / 28 / 07 & 42008.60\end{array}$

\begin{tabular}{|c|c|c|c|c|c|}
\hline & & & & & $6-68-58$ \\
\hline $\begin{array}{l}\text { HNITRAT } \\
\text { NITRATE } \\
\text { TRITIUM }\end{array}$ & $\begin{array}{l}\text { PPB } \\
\text { PPB } \\
\text { PCI/L }\end{array}$ & $\begin{array}{r}2500.06 \\
560.00 \\
600.00\end{array}$ & $\begin{array}{l}45000.00 \\
45000.00 \\
26000.00\end{array}$ & $\begin{array}{l}64 / 28 / 87 \\
64 / 26 / 87\end{array}$ & $\begin{array}{c}N R \\
1250.00 \\
613.00 \\
\{\quad 286.00\}\end{array}$ \\
\hline
\end{tabular}

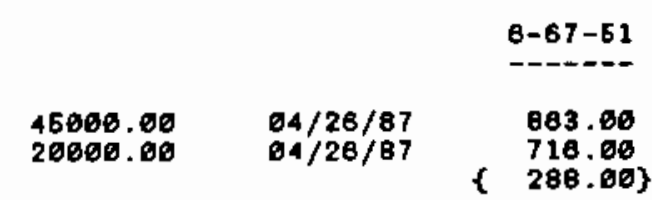
$\begin{array}{llrr}\text { HNITRAT } & \text { PPB } & 2500.00 & 45000.00 \\ \text { NITRATE } & \text { PPB } & 560.00 & 46000.00\end{array}$ $\begin{array}{llll}\text { NITRATE } & P P B & 560.00 & 46000.00 \\ \text { TRITIUM } & P C I / L & 600.00 & 20000.00\end{array}$
6-88-105 - - - - -

NR

$\begin{array}{cc} & 6-68-106 \\ 05 / 83 / 87 & N R \\ & 1990.00\end{array}$

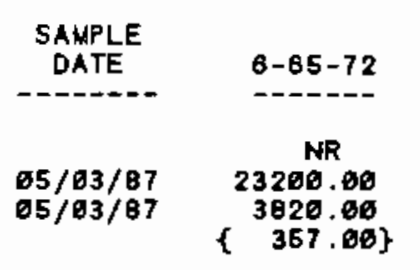

\section{$6-68-3 \theta$}

THESE TWO WELLS HAD NO REPORTABLE DATA

6-66-84

$66 / 17 / 87 \quad 14206.00$

ø8/17/87

$$
\text { NR }
$$

6510.00
$306.00\}$

6-67-88

2770.90

$05 / 03 / 87$

$66 / 63 / 67$

1070.6

\{ 298.00\}

6-69-36

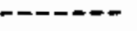

NR
885.60

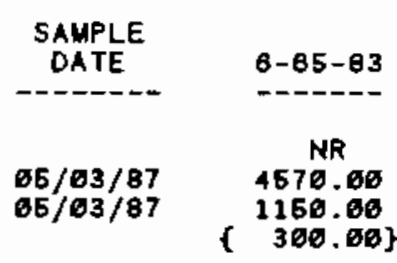

6-66-39
$04 / 27 / 87$

$0.66-103$
$06 / 03 / 67 \quad$ NR
596.00

$6-87-88$ $\begin{array}{ll}05 / 03 / 87 & 4740.00 \\ 06 / 03 / 87 & 1980.00\end{array}$ \{ 318.00$\}$

6-70-88

66/16/87

$66 / 16 / 87$ $-\cdots-1-$

4370.00 NR

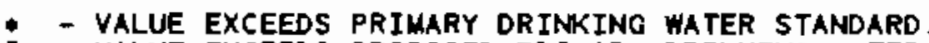

- VALUE EXCEEDS PROPOSED PRIMARY DRINKING WATER STANDARD.

* - VALUE EXCEEDS SCREENING LEVEL FOR FURTHER INVESTIGATION.

+ - DETECTION LIMIT WAS NOT AVAILAELE FOR COMPARISON

NR - ANALYSIS NOT REQUESTED OR NOT YET REPORTED

VALUES IN \{\} ARE COUNTING ERRORS FOR RAOIONUCLIOES

WATER STANDARD(S) IN PARENTHESES ARE PROPOSED ONLY 
IABLE A.6. (contd)

\begin{tabular}{|c|c|c|c|c|c|}
\hline $\begin{array}{l}\text { CONSTI } \\
\text { NAME }\end{array}$ & $\begin{array}{l}\text { UENT } \\
\text { UNITS }\end{array}$ & $\begin{array}{l}\text { DETECTION } \\
\text { LIMIT }\end{array}$ & $\begin{array}{l}\text { WATER } \\
\text { STANDARD }\end{array}$ & $\begin{array}{l}\text { SAMPLE } \\
\text { DATE }\end{array}$ & $\theta-71-3 \theta$ \\
\hline --ー-ー- & 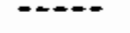 & 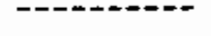 & 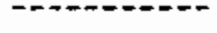 & & \\
\hline $\begin{array}{l}\text { HNITRAT } \\
\text { NITRATE } \\
\text { TRITIUM }\end{array}$ & $\begin{array}{l}\text { PPB } \\
\text { PPB } \\
\text { PCI/L }\end{array}$ & $\begin{array}{r}2600.00 \\
560.00 \\
500.00\end{array}$ & $\begin{array}{l}45000.00 \\
45000.00 \\
20000.00\end{array}$ & $04 / 27 / 87$ & $\begin{array}{c}\text { NR } \\
25100.00\end{array}$ \\
\hline
\end{tabular}

$6-72-73$

$\begin{array}{llrr}\text { HNITRAT } & \text { PPB } & 2600.00 & 45000.00 \\ \text { NITRATE } & \text { PPB } & 600.00 & 46000.00\end{array}$

$\begin{array}{llll}\text { NITRATE } & \text { PPB } & 600.00 & 46000.00 \\ \text { TRITIUM } & \text { PCI/L } & 600.00 & 20000.00\end{array}$

๑6/16/87

$06 / 16 / 87$

\begin{tabular}{|c|c|c|}
\hline & & $6-73-61$ \\
\hline $\begin{array}{l}45600.00 \\
45000.00\end{array}$ & $04 / 27 / 87$ & $\begin{array}{c}\text { NR } \\
7050.00\end{array}$ \\
\hline
\end{tabular}

$6-78-62$

$\begin{array}{lllll}\text { NITRATE PPB } & 500.60 & 46000.00 & 04 / 27 / 87 & 7480.00\end{array}$
$6-72-88$

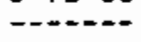

$\begin{array}{lc} & N R \\ 05 / 63 / 87 & 4640.00 \\ 05 / 63 / 87 & 2640.00\end{array}$

2640.00
$330.00\}$

$8-74-44$

$------$

ø6/17/87 3680.00

NR

$6-80-43 F$

……-

THESE TWO WELLS HAD NO REPORTABLE DATA

SAMPLE

DATE

$66 / 16 / 87$

$66 / 16 / 87$

7770.06

NR

$05 / 03 / 87$

$65 / 63 / 87$

6210.00

NR

$64 / 28 / 67$

$\begin{array}{cc}\text { SAMPLE } & \\ \text { DATE } & 6-71-52 \\ & \text { NR } \\ 64 / 27 / 87 & 6600.06 \\ 64 / 27 / 87 & 911.06 \\ & 292.06\}\end{array}$

6-71-77

3170.00
241.00

$6-72-82$

\{ 313.00$\}$

$6-77-36$

67306.60

$6-86-43 R$

* - VALUE EXCEEDS PRIMARY DRINKING WATER STANDARD.

- VALUE EXCEEDS PROPOSED PRIMARY DRINKING WATER STANDARD.

* - VALUE EXCEEDS SCREENING LEVEL FOR FURTHER INVESTIGATION.

* - DETECTION LIMIT WAS NOT AVAILABLE FOR COMPARISON

NOT REQUESTED OR NOT YET REPORTED

VALUES IN \{ \} ARE COUNTING ERRORS FOR RADIONUCLIDES 
TABLE A.6. (contd)

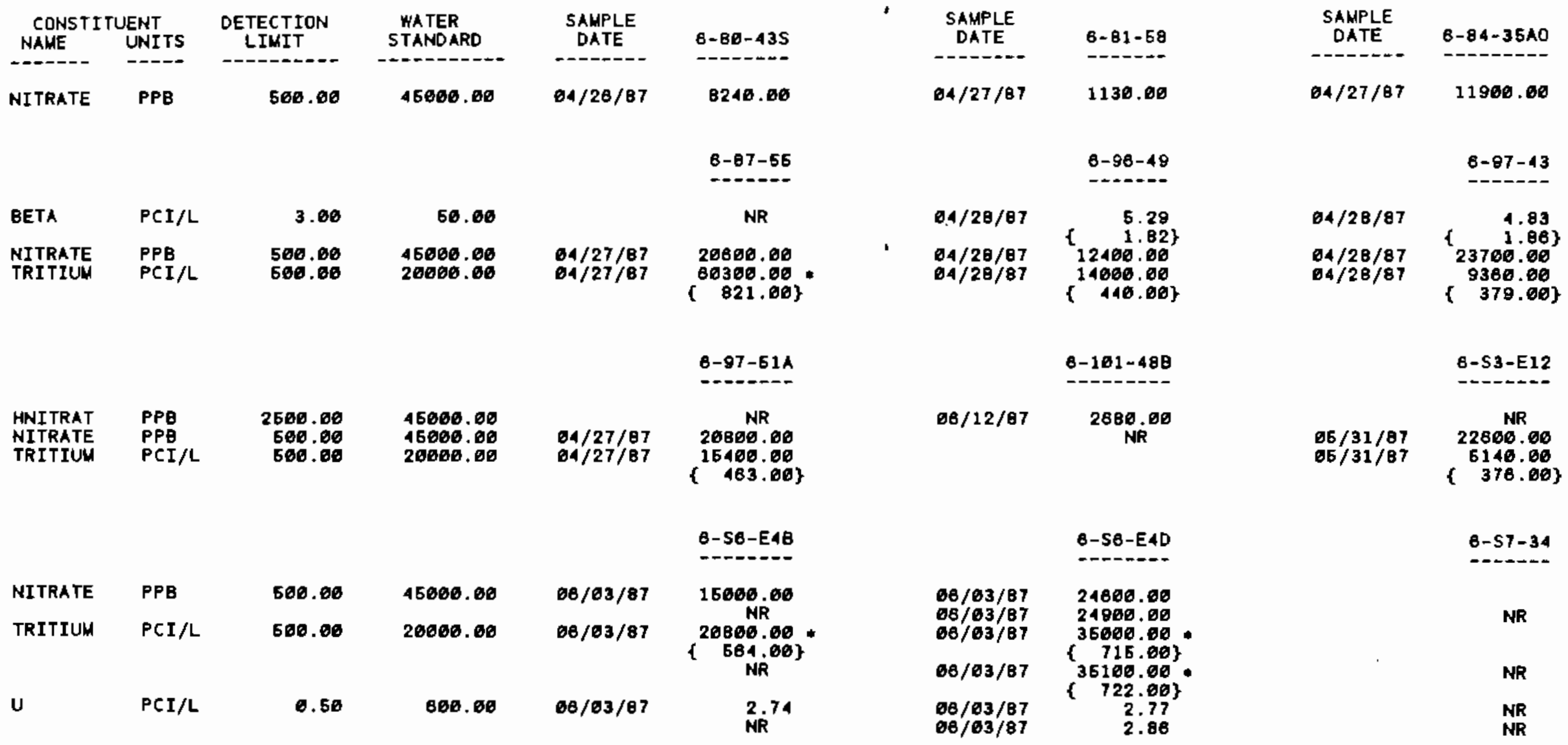

- - VAlUe exceeds primary dRINKINg water 5TANDARD.

- VALUE EXCEEDS PROPOSED PRIMARY DRINKING WATER STANDARO.

* - VALUE EXCEEDS SCREENING LEVEL FOR FURTHER INVESTIGATION.

+ - DETECTION LIMIT WAS NOT AVAILABLE FDR COMPARISON

NR - ANALYSIS NOT REQUESTED OR NOT YET REPORTED

VALUES IN $f$ I ARE COUNTING ERRORS FOR RADIONUCLIDES

WATER STANDARD'(S) IN PARENTHESES ARE PROPOSED ONLY 
IABLE A.6. (contd)

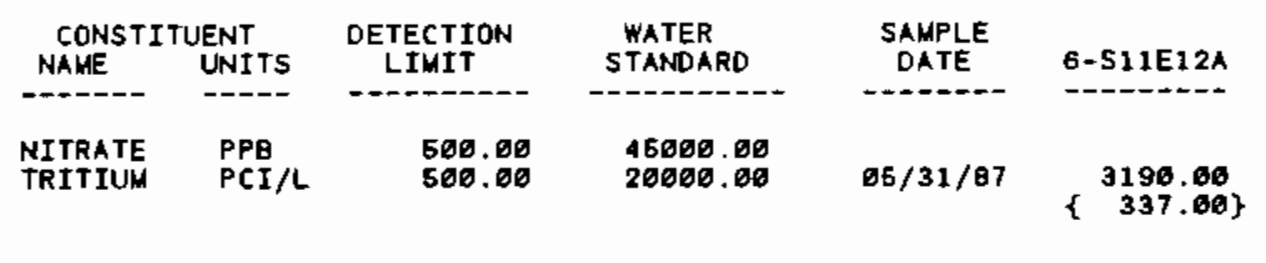

6-514-28A

$\begin{array}{llrr}\text { BETA } & \text { PCI/L } & 3.00 & 66.00 \\ \text { NITRATE } & \text { PPB } & 506.00 & 15060.00 \\ \text { TRITIUM } & \text { PCI/L } & 606.00 & 20000.66 \\ U & \text { PCI/L } & 0.60 & 600.66\end{array}$

2780.66

6-524-18

NR

NITRATE

PPB

560.60

45000.60

$65 / 31 / 87$

NR
NR
NR
NR
NR
NR

$6-531-1 P$

NO REPORTABLE DATA

\begin{tabular}{lr}
$\begin{array}{l}\text { SAMPLE } \\
\text { DATE }\end{array}$ & $6-511 E 12 A P$ \\
\hline $65 / 31 / 07$ & 20000.00
\end{tabular}

SAMPLE

DATE

8-512-29

$06 / 02 / 87 \quad 18700.00$

$6-518-61$

$6-518-11$

05/31/87

9580.00

$66 / 62 / 87$

1030.66

Q-527-E14

NR

$05 / 07 / 87 \quad 28400.00$ $00 / 04 / 87 \quad 28000.00$ $88 / 04 / 97$ פ5/07/87

פ5/07/97 $06 / 64 / 87$ ๑6/64/87

863.60
$312.08]$

2.83
3.41

3.41

(2)

6-528-E0

06/03/87

$06 / 03 / 87$ $\begin{array}{r}8.17 \\ 1 \quad 2.22\} \\ \hline\end{array}$

$\left\{\begin{array}{r}2.22 \\ 16100.06\end{array}\right.$

NR
NR

NR

\footnotetext{
- - VALUE EXCEEDS PRIMARY DRINKING WATER STANDARD.

- VALUE EXCEEDS PROPOSED PRIMARY DRINKING WATER STANDARD.

- VALUE EXCEEDS SCREENING LEVEL FOR FURTHER INVESTIGATION.

+ - DETECTION LIMIT WAS NOT AVAILABLE FOR COUPARISON

NR - ANALYSIS NOT REQUESTED OR NOT YET REPORTED

VALUES IN \{\} ARE COUNT ING ERRORS FOR RADIONUCLIDES

WATER STANDARO(S) IN PARENTHESES ARE PROPOSED ONLY
} 
IABLE A.7. Key to Constituent Names in Tables A.4, A.5, and A.6

\begin{tabular}{|c|c|c|c|}
\hline Name in Tables & Units & Group & Full Name \\
\hline $1,1,1-\mathrm{T}$ & $\mathrm{ppb}$ & VOLORG & $1,1,1$-trichioroethane \\
\hline ACETONE & $\mathrm{ppb}$ & & Acetone \\
\hline ALKALIN & $\mathrm{ppb}$ & & Total alkalinity in ppb $\mathrm{CaCO}_{3}$ \\
\hline ALUMNUM & ppb & ICPMT & Aluminum \\
\hline AMMONIU & $\mathrm{ppb}$ & & Ammonium jon \\
\hline ARSENIC & $\mathrm{ppb}$ & & Arsenic \\
\hline BARIUM & $\mathrm{ppb}$ & ICPMT & Barium, unfiltered \\
\hline BETA & $\mathrm{pCi} / \mathrm{L}$ & & Gross beta \\
\hline BIS2EPH & $\mathrm{ppb}$ & SEMVOLE & Bis(2-ethylhexyl) phthalate \\
\hline CADMIUM & $\mathrm{ppb}$ & ICPMT & Cadmium, unf $i$ tered \\
\hline CALCIUM & $\mathrm{ppb}$ & ICPMT & Calcium, unfiltered \\
\hline CHLFORM & $\mathrm{ppb}$ & VOLORG & Chioroform \\
\hline CHLORID & $\mathrm{ppb}$ & IONS & Chloride \\
\hline CHROMUM & $\mathrm{ppb}$ & ICPMT & Chromium, unfiltered \\
\hline CO 60 & $\mathrm{pCi} / \mathrm{L}$ & GAMMA & Cobalt-60 \\
\hline $\begin{array}{l}\text { COLIFRM } \\
\text { CONDFLD }\end{array}$ & mpn & & Coliform bacteria \\
\hline COPPER & Dob & ICPMT & copper, unfiltered \\
\hline CS-137 & $\mathrm{pCi} / \mathrm{L}$ & GAMMA & Ces ium-137 \\
\hline CYANIDE & $\rho p b$ & & Cyanide \\
\hline FALUMIN & $\mathrm{ppb}$ & ICPMTF & Aluminum, filtered \\
\hline FARSENI & $\mathrm{ppb}$ & & Arsenic, filtered \\
\hline FBARIUM & $\mathrm{ppb}$ & ICPMTF & Barium, filtered \\
\hline FCADMIU & ppb & ICPMTF & Cadmium, filtered \\
\hline FCALCIU & ppb & ICPMTF & Calcium, filtered \\
\hline FCHROMI & $\mathrm{ppb}$ & ICPMTF & Chromium, filtered \\
\hline FCOPPER & $\mathrm{ppb}$ & ICPMTF & Copper, filtered \\
\hline FIRON & $\mathrm{ppb}$ & ICPMTF & Iron, filtered \\
\hline FLEAD & $\mathrm{ppb}$ & & Lead, filtered \\
\hline FLUORID & $\mathrm{ppb}$ & IONS & Fluoride \\
\hline FMAGNES & $\mathrm{ppb}$ & ICPMTF & Magnesium, filtered \\
\hline FMANGAN & ppb & ICPMTF & Manganese, filtered \\
\hline FMERCUR & $\mathrm{ppb}$ & & Mercury, filtered \\
\hline FNICKEL & $\mathrm{ppb}$ & ICPMTF & Nickel, filtered \\
\hline FPOTASS & $\mathrm{ppb}$ & ICPMTF & Potassium, filtered \\
\hline FSELENI & $\mathrm{ppb}$ & & Selenium, filtered \\
\hline FSILVER & $\mathrm{ppb}$ & ICPMTF & Silver, filtered \\
\hline FSODIUM & $\mathrm{ppb}$ & ICPMTF & Sodium, filtered \\
\hline FSTRONT & ppb & ICPMTFE & Strontium, filtered \\
\hline FVANADI & $\mathrm{ppb}$ & ICPMTF & Vanadium, filtered \\
\hline FZINC & $\mathrm{ppb}$ & ICPMTF & Zinc, filtered \\
\hline HNITRAT & $\mathrm{ppb}$ & & Nitrate, high detection limit \\
\hline HXDECAC & $\mathrm{ppb}$ & & Hexadeconoic aid \\
\hline IRON & $\mathrm{ppb}$ & ICPMT & Iron, unfiltered \\
\hline $\begin{array}{l}\text { LEADGF } \\
\text { LAI PHA }\end{array}$ & $\mathrm{ppb}$ & & Lead (graphite furnace) \\
\hline $\begin{array}{l}\text { LOALPHA } \\
\text { MAGNES }\end{array}$ & $\mathrm{pCj} / \mathrm{L}$ & & Gross alpha \\
\hline $\begin{array}{l}\text { MAGNES } \\
\text { MANGESE }\end{array}$ & $\mathrm{ppb}$ & ICPMI & Magnesium, unfiltered \\
\hline MANGESE & ppb & ICРMT & Manganese, unfiltered \\
\hline
\end{tabular}


TABLE A.7. (contd)

\begin{tabular}{|c|c|c|c|}
\hline Name in Tables & Units & Group & Full Name \\
\hline METHONE & $\mathrm{ppb}$ & VOLORG & Methyl ethyl ketone \\
\hline METHYCH & $\mathrm{ppb}$ & VOLORG & Methylene chloride \\
\hline MOLSULF & $\mathrm{ppb}$ & & Molecular sulfur \\
\hline NICKEL & $\mathrm{ppb}$ & ICPMT & Nickel, unfiltered \\
\hline NITRATE & $\mathrm{ppb}$ & IONS & Nitrate \\
\hline NNIDIME & $\mathrm{ppb}$ & SEMVOLE & $\mathrm{N}$-nitrosodimethylamine \\
\hline NONACD & $\mathrm{ppb}$ & & Nonadeconic acid \\
\hline PHFIELD & & & pH (measured in field) \\
\hline PHOSPHA & $\mathrm{ppb}$ & IONS & Phosphate \\
\hline POTASUM & $\mathrm{ppb}$ & ICPMT & Potassium, unfiltered \\
\hline $\begin{array}{l}\text { PU39-40 } \\
\text { RADIUM }\end{array}$ & $\begin{array}{l}\mathrm{pCi} / \mathrm{L} \\
\mathrm{pCi} / \mathrm{L}\end{array}$ & PU-ISO & $\begin{array}{l}\text { Pluton ium-239, } 240 \\
\text { Radium-226 }\end{array}$ \\
\hline RU 103 & $\mathrm{pCi} / \mathrm{L}$ & & Ruthen i um-103 \\
\hline RU 106 & $\mathrm{pCi} / \mathrm{L}$ & GAMMA & Ruthen i um- 106 \\
\hline SB 125 & $\mathrm{pCi} / \mathrm{L}$ & & Ant inony-125 \\
\hline SODIUM & $\mathrm{ppb}$ & ICPMT & Sodium, unfiltered \\
\hline SR 90 & $\mathrm{pCi} / \mathrm{L}$ & 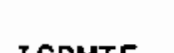 & Strontium-90 \\
\hline $\begin{array}{l}\text { STRONUM } \\
\text { SULFATE }\end{array}$ & $\begin{array}{l}\mathrm{ppb} \\
\mathrm{ppb}\end{array}$ & $\begin{array}{l}\text { ICPMTE } \\
\text { IONS }\end{array}$ & $\begin{array}{l}\text { Strontium, unfiltered } \\
\text { Sulfate }\end{array}$ \\
\hline TC & $\mathrm{ppb}$ & & Total carbon \\
\hline IC-99 & $\mathrm{pCi} / \mathrm{L}$ & & Technet ium-99 \\
\hline TDS & $\mathrm{ppm}$ & & Total dissolved solids \\
\hline TETRANE & $\mathrm{ppb}$ & VOLORG & $\begin{array}{l}\text { Tetrachloromethane (Carbon } \\
\text { tetrachloride) }\end{array}$ \\
\hline TOC & $\mathrm{ppb}$ & & Total organic carbon \\
\hline TOX & $\mathrm{ppb}$ & & Total organic halogen \\
\hline TOXLDL & $\mathrm{ppb}$ & & $\begin{array}{l}\text { Total organic halogen, low detection } \\
\text { limit }\end{array}$ \\
\hline TRANDCE & $\mathrm{ppb}$ & VOLORGE & Trans-1,2-dich Toroethene \\
\hline TRICENE & $\mathrm{ppb}$ & VOLORG & Trichloroethylene (1,2-trichloroethene) \\
\hline TRITIUM & $\mathrm{pCi} / \mathrm{L}$ & & Tritium \\
\hline U & $\mathrm{pCj} / \mathrm{L}$ & & Total uranium \\
\hline U 234 & $\mathrm{PCi} / \mathrm{L}$ & & Uranium-234 \\
\hline U 235 & $\mathrm{pCi} / \mathrm{L}$ & & Uranium-235 \\
\hline $\begin{array}{l}\text { U } 238 \\
\text { U-CHEM }\end{array}$ & $\begin{array}{l}\mathrm{PCi} / \mathrm{L} \\
\mu \mathrm{a} / \mathrm{L}(\mathrm{a})\end{array}$ & & Uranium-238 \\
\hline UNKNOWN & $\begin{array}{ll}\mathrm{ppb} \\
\mathrm{ppb}\end{array}$ & & lotal uranium \\
\hline VANADUM & $\mathrm{ppb}$ & ICPMT & Vanadium, unfiltered \\
\hline ZINC & $\mathrm{ppb}$ & ICPMT & Zinc, unfiltered \\
\hline
\end{tabular}

(a) $\mu g / L=p p b$. 


\section{REFERENCES}

Law, A. G., J. A. Serkowski, and A. L. Schatz. 1987. Results of the Separations Area Ground-Water Monitoring Network for 1986 .

RHO-RE-SR-87-24P, Rockwell Hanford Operations, Richland, washington. 


\section{APPENDIX B}

DRINKING WATER STANDARDS AND

DERIVED CONCENTRATION GUIDELINES 
TABLE B.1. Radiological Drinking Water Standards: U.S. Environmental Protection Agency, National Interim Primary Drinking Water Regulations (OFR 1986) and State of Washington, Rules and Regulations of the State Board of Health Regarding Public Water Systems

Contaminant

Gross alpha (excluding uranium)

Combined radium-226 and radium-228

Radium-226 (State of Washington only)

Gross beta and gamma radioactivity from manmade radionuclides
Limit

\section{$15 \mathrm{pCi} / \mathrm{L}$}

$5 \mathrm{pCi} / \mathrm{L}$

$3 \mathrm{pCi} / \mathrm{L}$

Annual average concentration shall not produce an annual dose from manmade radionuclides equivalent to the total body or any internal organ dose greater than $4 \mathrm{mrem} / \mathrm{yr}$. If two or more radionuclides are present, the sum of their annual dose equivalent shall not exceed $4 \mathrm{mrem} / \mathrm{yr}$.

Compliance may be assumed if annual average concentrations for gross beta activity, tritium, and strontium-90 are less than $50 \mathrm{pCi} / \mathrm{L}, 20,000 \mathrm{pCi} / \mathrm{L}$, and $8 \mathrm{pC} i / L$, respectively. It should be noted that these "screening levels" are conservatively calculated and not directly equivalent to an annual dose of 4 mrem.

The following list provides the annual average concentrations, with respect to the Columbia River, for selected manmade radionuclides of interest. These radionuclides are assumed to yield an annual dose of 4 mrem to the indicated organ. Data are taken from the National Interim Primary Water Regulations, Table IV-2A (USEPA 1976 and OFR 1986).

\begin{tabular}{|c|c|c|}
\hline Radionuclide & Critical Organ & Concentration, $\mathrm{pCi} / \mathrm{L}$ \\
\hline Antimony-125 & GI (LLI) & 300 \\
\hline Ces ium- 134 & GI (s) & 20,000 \\
\hline Cesium-137 & Whole body & 200 \\
\hline Cobalt-60 & GI (LLI) & 100 \\
\hline Iodine -129 & Thyroid & 1 \\
\hline Iodine-131 & Thyroid & 3 \\
\hline Niobium-95 & GI (LLI) & 300 \\
\hline Ruthenium-106 & GI (LLI) & 30 \\
\hline Strontium-89 & Bone & 20 \\
\hline Stront ium-89 & Bone marrow & 80 \\
\hline Strontium-90 & Bone marrow & 8 \\
\hline Technetium-99 & GI (LLI) & 900 \\
\hline Tritium & Whole body & 20,000 \\
\hline Zirconium-95 & GI $(\mathrm{LLI})$ & 200 \\
\hline
\end{tabular}

B.1 
TABLE B.2. Chemical Drinking Water Standards: U.S. Environmental Protection Agency, National Interim Primary Drinking Water Regulations (OFR 1986) and State of Washington, Rules and Regulations of the State Board of Health Regarding Public water Systems (USEPA 1976)

Chemical Constituent
Arsenic
Barium
Cadmium
Carbon tetrachloride
Chromium
Copper
Fluorine
Lead
Mercury
Nitrate ion
Selenium

Concentration

(a) $\mathrm{ppb}=\mathrm{ppm} / 1000$.

$\begin{array}{cl}50 & \mathrm{ppb}^{(\mathrm{a})} \\ 1 & \mathrm{ppm} \\ 10 & \mathrm{ppb} \\ 5 & \mathrm{ppb} \\ 50 & \mathrm{ppb} \\ 1.3 & \mathrm{ppm} \\ 2 & \mathrm{ppm} \\ 50 & \mathrm{ppb} \\ 2 & \mathrm{ppb} \\ 45 & \mathrm{ppm} \\ 10 & \mathrm{ppb}\end{array}$


TABLE B.3. Proposed Derived Concentration Guides (a)

Radionuclide
Antimony-125
Cerium-144
Cesium-137
Chronium-51
Cobalt-60
Iodine-129
Iodine-131
Mongonese-54
Plutonium-238
Plutonium-239,240
Ruthenium-103
Ruthenium-106
Stront ium-89
Stront ium-90
Technetium-99
Tritium
Uranium-234
Uranium-235
Uranium-238
Zirconium-65

$p C i / L$
60,000
7,000
3,000
$1,000,000$
5,000
500
3,000
50,000
400
300
50,000
6,000
20,000
1,000
100,000
$2,000,000$
500
600
600
9,000

(a) Concentrations of radionuclides in water that could be continuously consumed and not exceed an effective dose equivalent of $100 \mathrm{mrem} / \mathrm{yr}$. 


\section{REFERENCES}

U.S. Department of Energy (USDOE). 1981. "Environmental Protection, Safety, and Health Protection Information Reporting Requirements." In DOE Order 5481.1, Washington, D.C.

U.S. Environmental Protection Agency (USEPA). 1976. National Interim Primary Drinking Water Regulations. EPA-570/976-003, Washington, $\overline{D . C}$.

Office of the Federal Register (OFR). 1986. Code of Federal Regulations, Protection of Environment. 40 CFR Parts 100 to 149. U.S. Government Printing office, Washington, D.C. 
PNL-6315-1

UC- 41,11

\section{DISTRIBUTION}

No. of

Copies

OFFSITE

2 DOE Technical Information Center

B. Blake

133 lst Ave. North

Minneapolis, MN 55401

ONSITE

7 DOE Richland 0perations Office

M. J. Anthony

J. D. Goodenough

P. J. Krupin

R. J. Nevarez

M. W. Shupe

J. J. Sutey/D. L. Sours

M. W. Tiernan

22 Westinghouse Hanford Company

M. R. Adams

L. C. Brown

G. D. Carpenter

M. A. Christie

M. L. Cramer

L. P. Diedicker

J. J. Dorian

G. W. Egert

K. A. Gano

K. A. Gasper

E. M. Greagor

R. B. Kasper

M. J. Lauterbach

A. G. Law

S. P. Luttrell

P. S. Peacock

K. M. Probasko

P. S. Schaus

J. A. Serkowski
No. of

Copies

D. M. Tulburg

R. E. Wheeler

S. A. Wiegman

69 Pacific Northwest Laboratory

D. J. Bates

C. S. Cline

L. J. Criscenti

D. I. Dennison (5)

D. W. Dragnich

P. A. Eddy

C. E. Elderkin

J. C. Evans (10)

M. D. Freshley

J. S. Fruchter

R. M. Fruland

M. J. Graham

R. H. Gray

L. K. Grove (11)

J. M. Hales

M. S. Hanson (2)

P. C. Hays

N. W. Hinman

E. J. Jensen

K. M. Krupka

W. W. Laity

T. L. Liikala

P. J. Mitchell

R. W. Nelson

J. R. Raymond

J. T. Rieger

R. Schalla

D. R. Sherwood (5)

C. S. Simmons

R. L. Skaggs

R. M. Smith

G. P. Streile

R. E. Wildung

Publishing Coordination (2)

Technical Report Files (5) 


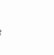

